An In Vitro Investigation of the Flow Fields Through Bileaflet and Polymeric Prosthetic Heart Valves

\author{
A Dissertation \\ Presented to \\ the Academic Faculty
}

by

Hwa Liang Leo

\author{
In Partial Fulfillment \\ Of the Requirements for the Degree \\ Doctor of Philosophy in Biomedical Engineering
}

Georgia Institute of Technology

August 2005 


\section{An In Vitro Investigation of the Flow Fields Through Bileaflet and Polymeric Prosthetic Heart Valves}

Approved by :

Dr. Ajit P. Yoganathan, Advisor College of Engineering

Georgia Institute of Technology

Dr. Stephen R. Hanson

College of Engineering

Oregon Health and Science University

Dr. Hong Tong Low

College of Engineering

National University of Singapore
Dr. Don P. Giddens

College of Engineering

Georgia Institute of Technology

Dr. John Oshinksi

College of Engineering

Georgia Institute of Technology

Date Approved: April 14 ${ }^{\text {th }}, 2005$ 


\section{ACKNOWLEDGEMENTS}

Many people supported me during the completion of this thesis with criticism, helpful assistance and references. This thesis would have never been possible without them.

First, I would like to thank my immediate family for encouraging me to pursue my dream, my fiancée, Melanie, and my friends for their constant support over the past six years. Words cannot express my gratitude for every time they were there to listen and encourage.

I would like to thank my advisor, Dr. Ajit P. Yoganathan, for his constant advice, support, and encouragement from the very beginning of this project. Dr. Yoganathan has provided me the opportunities to work directly with leading experts in the heart valve industry during the course of my research. My thesis would not have been possible without the collaboration and industrial support we received. I would like to express my gratitude to St. Jude Medical and Aortech, Inc for providing us the heart valve prostheses for this project. In particular, I would like to thank Dr. Yi-Ren Woo of the St. Jude Medical Heart Valve Division and Dr. O'Connor, formerly the engineer of the Aortech Inc Heart Valve Division, for their advice and discussions throughout this project.

My gratitude is also expressed to the members of my thesis reading committee, Dr. Don Giddens, Dr. Stephen Hanson, Dr. Dr. John Oshinski, and Dr. Hong Tong Low for giving me the solid advice on the research that I performed in these studies. I deeply appreciate 
the guidance that they have provided throughout this project. I would like to express my sincere appreciation and gratitude to Mr Tom and Shirley Gurley for their unwavering financial support throughout this project. My sincere appreciation is also extended to the Agency for Science, Technology and Research (ASTAR), Singapore for giving me the scholarship.

The amount of support I received from the administrative staff was greatly appreciated. Time and again, they were always ready to help. My deepest appreciation goes to Chris Ruffin, Michelle Mayberry, Pat Fowler, Arnold Rachel, Joanne Wheatley, and Trudy Walker for their tireless efforts to help keep our offices, labs, and lives running smoothly. I would also like to thank Steven Marzec for ensuring the computers and networks in our lab are running smoothly, free from the hassle of the hackers and viruses.

I am very grateful for the support, encouragement, professionalism, and entertainment provided by all of my colleagues, past and present, in the Cardiovascular Fluid Mechanics Research Laboratory. I would especially like to thank Dr. Brandon Travis, Dr. Timothy Healy, and Ann Ensley for taking the time to acclimate me when I first joined our group, and Chris Conrad for teaching me the art of 'cacking' and many unique phrases of the American, like 'taking the brown to the superbowl'. I would also like to thanks Dr. Lakshmi, Hélène Simon (yes, I would never forget the accent on the 'e'), Anna Fallon, and Chang for the many technical advises they gave me throughout my thesis writing. 


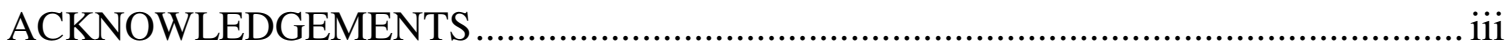

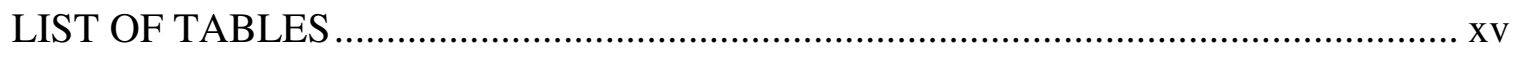

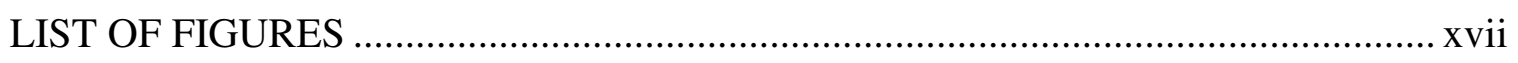

LIST OF SYMBOLS AND ABBREVIATIONS ....................................................... xliii

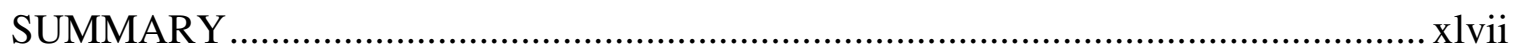

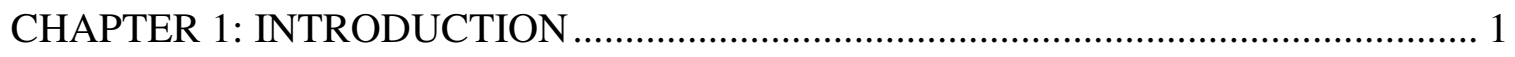

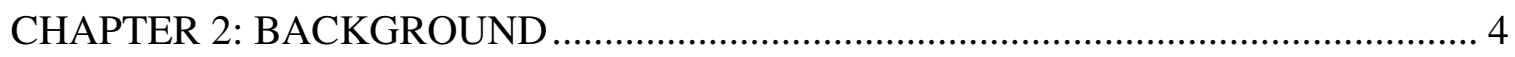

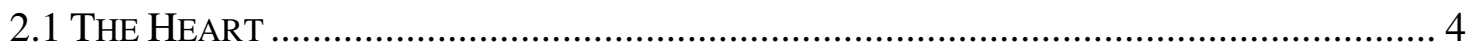

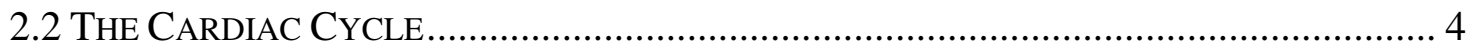

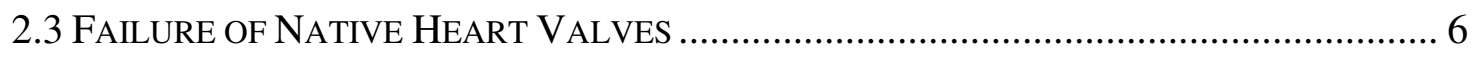

2.4 Prosthetic Heart Valves ............................................................................. 7

2.4.1 Valve Types and Their Complications ……………………........................ 9

2.5 Characteristics Of Human BLOOD ................................................................ 10

2.5.1 Platelet Activation and Aggregation .............................................................. 11

2.5.2 Red Blood Cells and Hemolysis .................................................................... 12

2.6 Patient Related Complications With Mechanical Heart Valves ............... 13

2.6.1 Thrombus Formation In Mechanical Heart Prostheses..................................... 14

2.7 IN Vitro InVESTIgations of Mechanical Heart Valves ................................... 15

2.7.1 Leakage Flow Through Heart Valve Prostheses.............................................. 18

2.7.1.1 Various Bileaflet Hinge Designs............................................................ 19

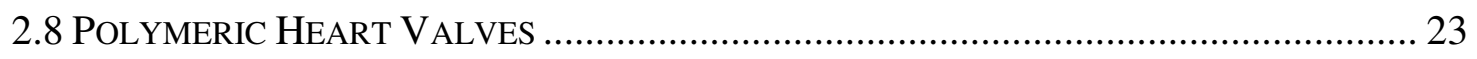

2.8.1 Biomaterials of Polymeric Heart Valves ……………….............................. 23 


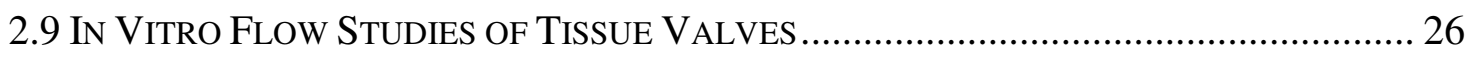

2.10 IN Vitro InVESTIGATIONS OF Polymeric Heart VALVES....................................... 29

2.10.1 Mechanical Fatigue and Calcification of Polymeric Heart Valves................. 30

2.11 ANIMAL ModelS AND CALCIFICATION............................................................... 33

2.12 Leaflet ThicknesS of Polymeric Heart VALVES............................................ 36

2.13 Fabrication of Polymeric Heart Valves...................................................... 37

CHAPTER 3: HYPOTHESES AND SPECIFIC AIMS …………………………….... 38

3.1 Hinge Flow StUdies IN Mitral Position........................................................... 38

3.1.1 1st Hypothesis ................................................................................................ 40

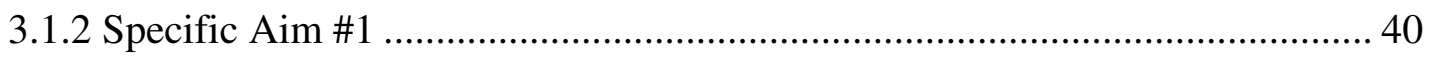

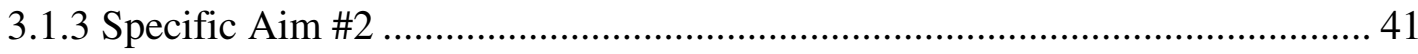

3.2 Aortic Polymeric Heart VAlve StUdies ....................................................... 42

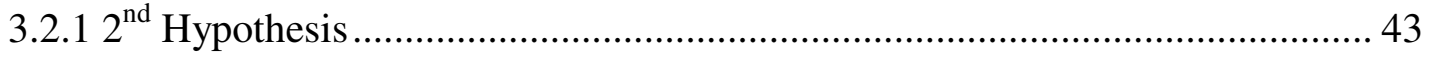

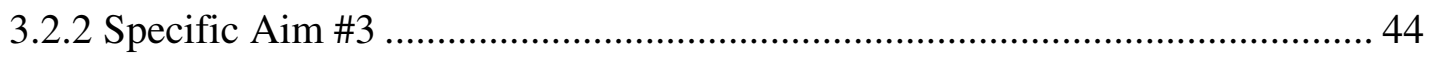

CHAPTER 4: EQUIPMENT AND MATERIALS ……………………………….... 46

4.1 Bileaflet Mechanical Heart Valve Prostheses ............................................. 46

4.1.1 St. Jude Medical Bileaflet Mechanical Heart Valve ……................................. 47

4.1.2 Carbomedics Bileaflet Mechanical Heart Valve............................................... 51

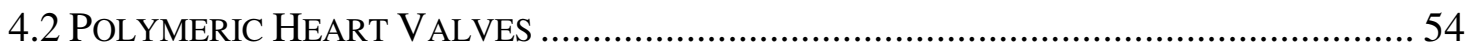

4.2.1 Aortech Polymeric Heart Valve ....................................................................... 56

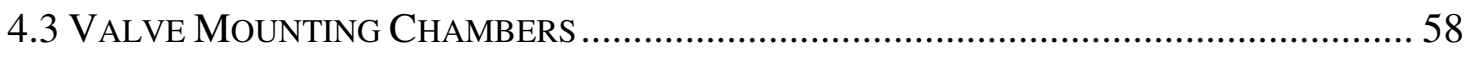

4.3.1 Mounting Chamber For The Three $27 \mathrm{~mm}$ Transparent Bileaflet Heart

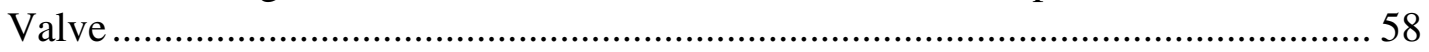

4.3.2 Mounting Chamber For The Carbomedics Transparent Bileaflet Heart Valve60 4.3.3 Mounting Chamber For Aortech Polymeric Heart Valves ................................ 61

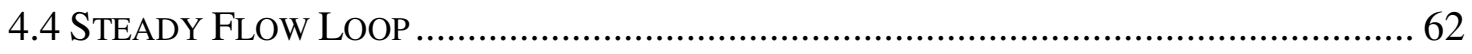


4.4.1 Static Leakage Loop For Bileaflet Mechanical and Polymeric Heart Valves . 62

4.4.2 Temperature-Dependent Study in Polymeric Heart Valves............................ 63

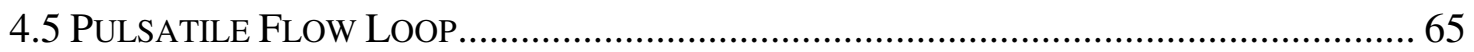

4.5.1 Mitral Hinge Flow Studies: CM 23 mm and SJM 27 mm Standard............... 65

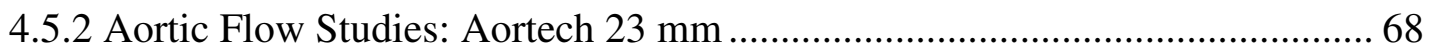

4.6 TEMPERATURE-DEPENDENT STUDY ......................................................... 70

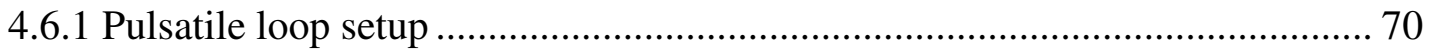

4.6.2 Markers and High Speed Camera Setup ..................................................... 72

4.6.3 Obtaining Markers' 2D and 3D co-ordinates........................................ 75

4.6.4 Leaflet Surface Reconstruction.......................................................... 76

4.7 Description of Working Medium And Particle SeEdingS ........................... 78

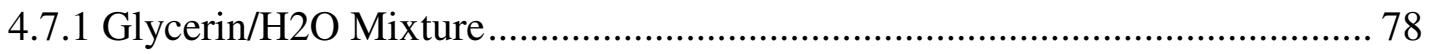

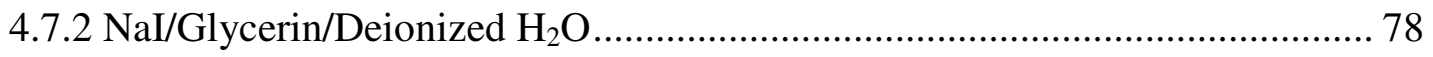

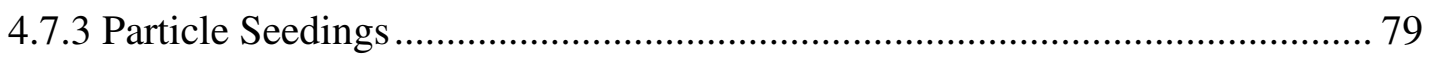

4.8 MEASUREMENT EquiPMENT AND TheIR CALIBRATION ................................... 80

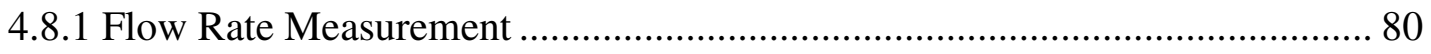

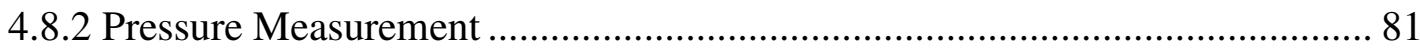

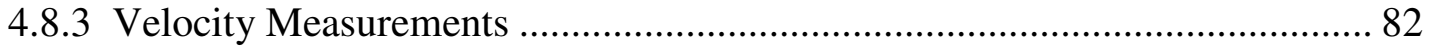

4.8.3.1 Laser Doppler Velocimetry ....................................................... 82

4.8.3.2 Particle Imaging Velocimetry …................................................. 88

4.8.3.4 Important Considerations for PIV Experiments .................................. 93

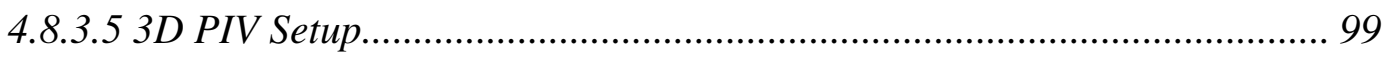

4.8.3.6. White Light Interferometry ......................................................... 104

4.8.3.7 Principle of White Light Interferometer .......................................... 106

CHAPTER 5: EXPERIMENTAL PROCEDURES/PROTOCOLS ............................ 109 


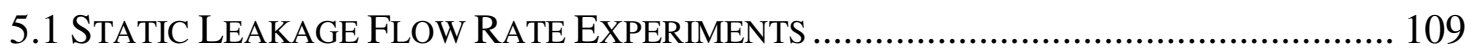

5.1.1 Bileaflet Mechanical Heart Valves ............................................................... 109

5.1.2 Polymeric Heart Valves With Temperature Effect........................................ 109

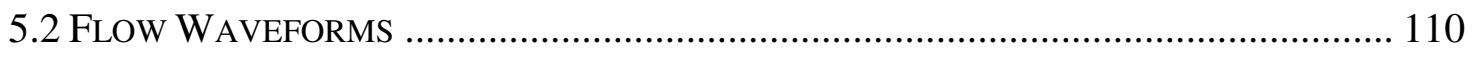

5.2.1 Mitral Flow And Pressure Waveforms …………........................................... 110

5.2.2 Aortic Flow And Pressure Waveforms ......................................................... 111

5.2.3 The Effect of Resistant and Compliant Units on The Waveforms ................. 112

5.3 LASER DOPPLER VELOCIMETRY EXPERIMENTS................................................... 114

5.3.1 Pulsatile Mitral Hinge Flow Experiments....................................................... 114

5.3.1.1 Measurement Sites Inside The Hinge Regions........................................ 118

5.3.2 Pulsatile Aortic Flow Experiments ............................................................ 124

5.4 PARTICle IMAGing Velocimetry EXPERIMENTS ............................................... 128

5.4.1 Pulsatile Aortic Flow Experiments .............................................................. 128

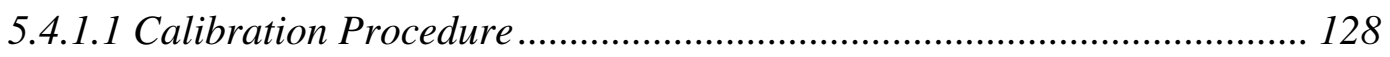

5.4.1.2 Measurement Sites For Polymeric Valves ........................................... 133

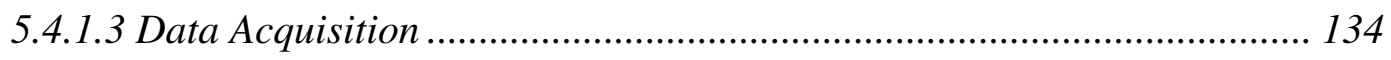

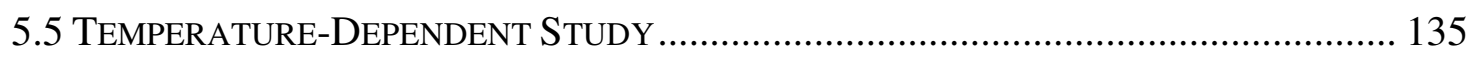

5.6 SuRface ToPogRaPhy StUdieS WITH DifFERENT HEART VALVES ....................... 136

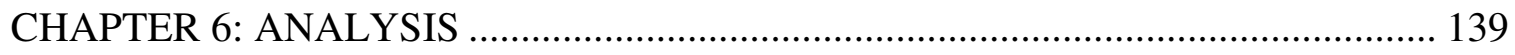

6.1 LASER DOPPLER VELOCIMETRY.................................................................... 139

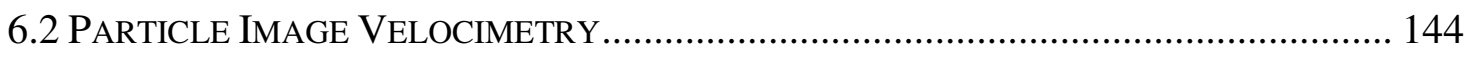

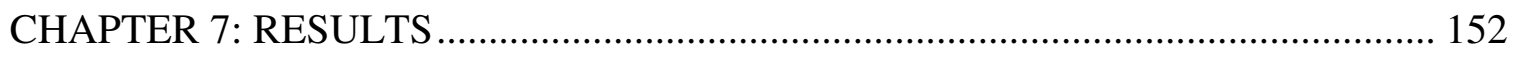

7.1 EFFect Of Hinge Gap width Tolerance On Hinge Flow Dynamics (Mitral

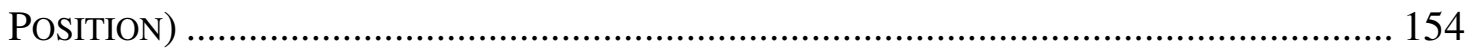

7.1.1 Static Leakage Rate Experiments On The Three $27 \mathrm{~mm}$ Bileaflet Heart Valves And The CM 23 mm Bileaflet Heart Valve............................................................ 154 
7.1.2 2D LDV Mitral Hinge Studies With The Three Prototype $27 \mathrm{~mm}$ Bileaflet Clear Housing Valves 155

7.1.2.1 SJM 27 mm Standard ...................................................................... 155

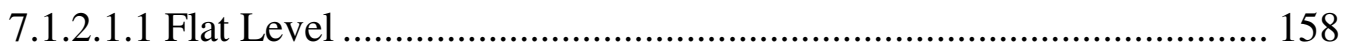

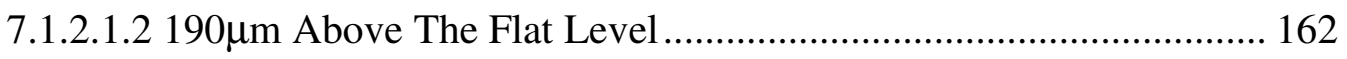

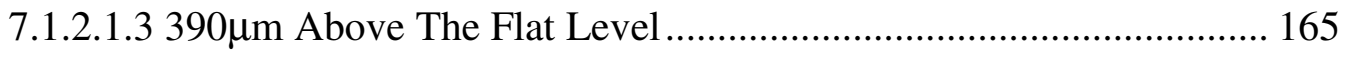

7.1.2.1.4 585

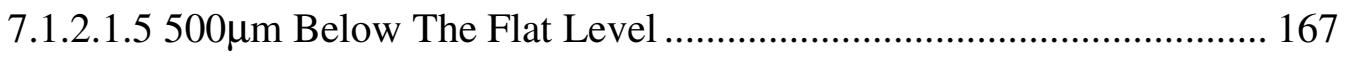

7.1.2.1.6 1 mm Below The Flat Level ............................................................ 171

7.1.2.1.7 3 mm Below The Flat Level ........................................................ 175

7.1.2.1.8 Downstream of The Thumbnail Region ......................................... 177

7.1.2.2 27 mm Prototype Low Leaker............................................................... 180

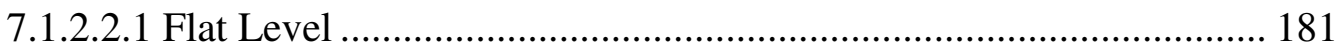

7.1.2.2.2 190

7.1.2.2.3 390 $\mu \mathrm{m}$ Above The Flat Level........................................................... 189

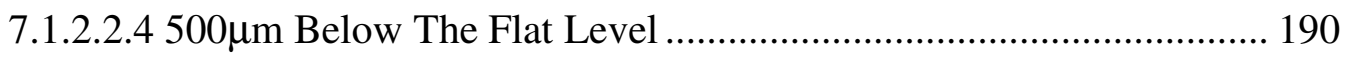

7.1.2.2.5 1 mm Below The Flat Level ........................................................... 194

7.1.2.2.6 3 mm Below The Flat Level ........................................................... 197

7.1.2.2.7 Downstream of The Thumbnail Region .......................................... 199

7.1.2.3 27 mm Prototype High Leaker .............................................................. 201

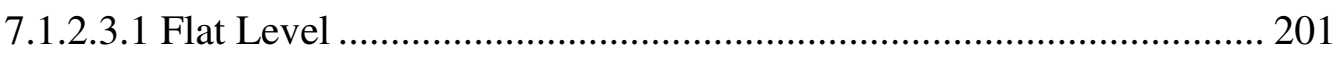

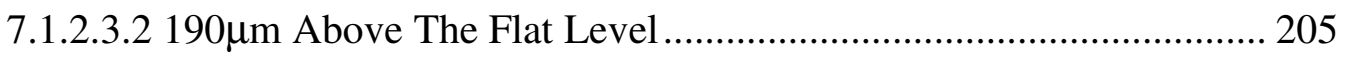

7.1.2.3.3 390 $\mu \mathrm{m}$ Above The Flat Level........................................................... 209

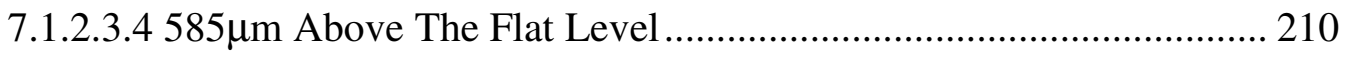

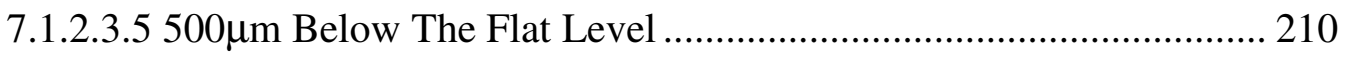


7.1.2.3.6 $1 \mathrm{~mm}$ Below The Flat Level ....................................................... 214

7.1.2.3.7 3 mm Below The Flat Level ............................................................ 216

7.1.2.3.8 Downstream of The Thumbnail Region .......................................... 218

7.2 Effect of Valve Design on Hinge Flow Dynamics (Mitral Position)........ 220

7.2.1 2D LDV Mitral Hinge Studies With The CM 23 mm Bileaflet Clear Housing

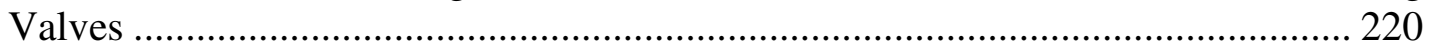

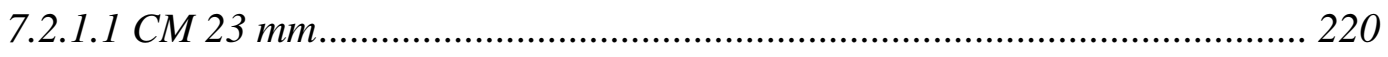

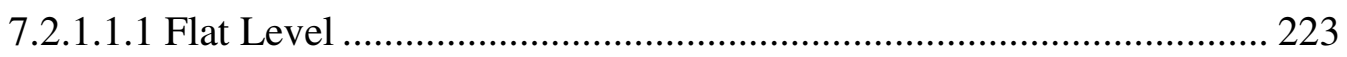

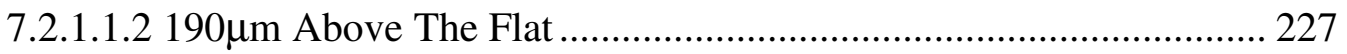

7.2.1.1.3 390

7.2.1.1.4 1mm Below The Flat Level ......................................................... 235

7.3 Flow Quantification in Polymeric Heart Valves in the Aortic Position 238

7.3.1 Temperature Dependent Leakage Rate Studies ............................................ 238

7.3.1.1 Static Leakage Rate Experiments On The Three Aortech 23 mm Polymeric

Heart Valves..................................................................................................... 238

7.3.1.2 Pulsatile Leakage Rate Experiments On The Prototype Polymeric Valve

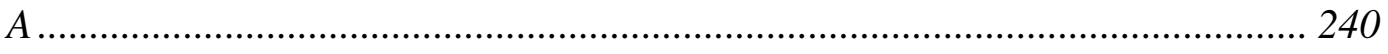

7.3.2 3D High-Speed Video and Marker Experiments ............................................ 240

7.3.3 2D LDV Measurements On The Three Prototype Aortech $23 \mathrm{~mm}$ Polymeric

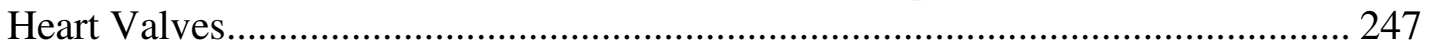

7.3.3.1 Closed Commissure Valve With $80 \mu \mathrm{m}$ Leaflet Thickness (Prototype A)248

7.3.3.1.1 Flow Fields Downstream of the Valve ……….................................. 248

7.3.3.1.2 Flow Downstream of the Stent .................................................... 252

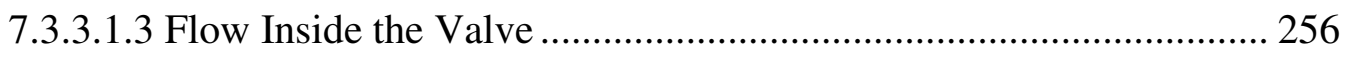

7.3.3.2 Open Commissure Valve With $120 \mu \mathrm{m}$ Leaflet Thickness (Prototype B) 261

7.3.3.2.1 Flow Fields Downstream of the Valve .............................................. 261

7.3.3.2.2 Flow Downstream of the Stent ........................................................ 264 
7.3.3.2.3 Flow Inside the Valve 268

7.3.3.3 Semi-Open Commissure Valve With $120 \mu$ m Leaflet Thickness (Prototype C). 273

7.3.3.3.1 Flow Fields Downstream of the Valve .......................................... 273

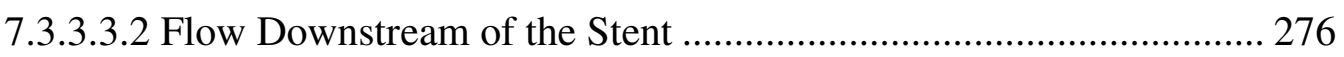

7.3.3.3.3 Flow Inside the Valve .................................................................. 280

7.3.4 3D LDV Measurements On The Prototype Polymeric Valve A.................... 284

7.3.4.1 Closed Commissure Valve With 80 um Leaflet Thickness (Prototype A)284

7.3.4.1.1 Flow Fields Downstream of the Valve 284

7.3.5 3D PIV Measurements On The Three Prototype Aortech $23 \mathrm{~mm}$ Polymeric Heart Valves 289

7.3.5.1 Closed Commissure Valve With 80 um Leaflet Thickness (Prototype A)291

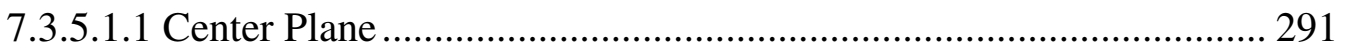

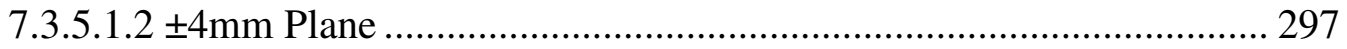

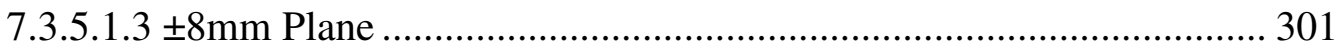

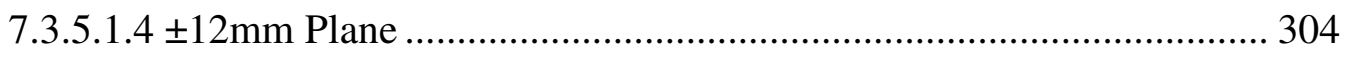

7.3.5.2 Open Commissure Valve With $120 \mu$ m Leaflet Thickness (Prototype B) 308

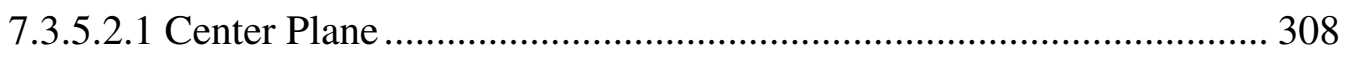

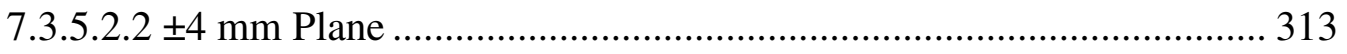

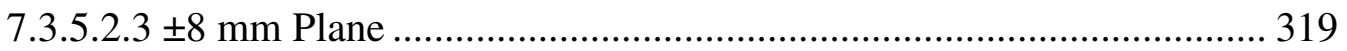

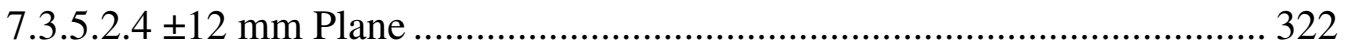

7.3.5.3 Semi-Open Commissure Valve With $120 \mu$ m Leaflet Thickness

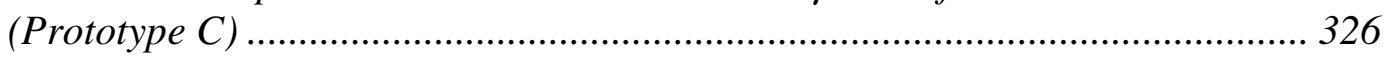

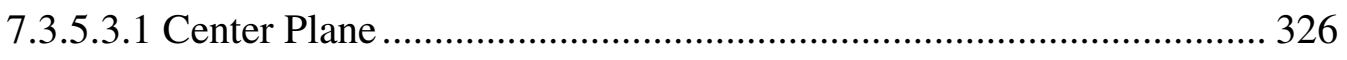

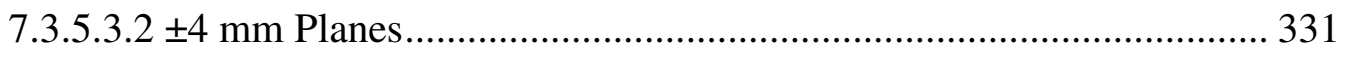

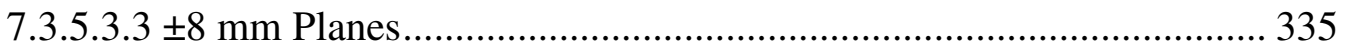


7.3.5.3.4 $\pm 12 \mathrm{~mm}$ Planes 339

7.3.6 Leaflets Surface Topography Study............................................................ 343

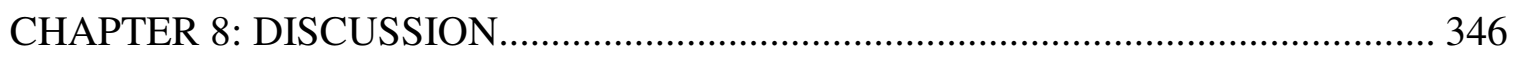

8.1 EFFeCt of Hinge Gap Width Tolerance On Hinge Flow .................................... 347

8.1.1 Effect On Static Leakage Flow ………………………….......................... 347

8.1.2 Effect On Flow Structures In Hinge ............................................................ 348

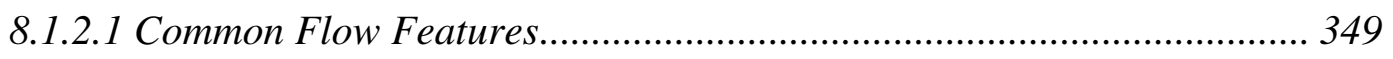

8.1.2.2 Differences In Flow Features And Reynolds Shear Stresses ................... 357

8.1.3 Comparison Of Current $27 \mathrm{~mm}$ Prototype Valves Studies With Previous Hydrogen Bubble Experiments ............................................................................... 363

8.1.4 Integration Of Previous Blood Experiments With Observed Flow

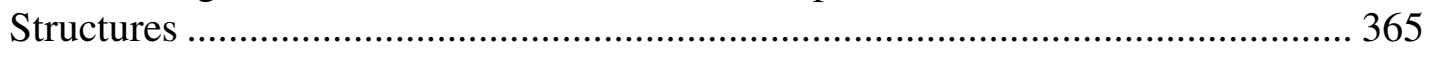

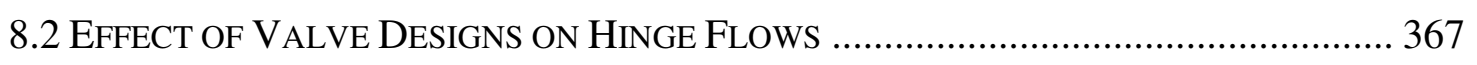

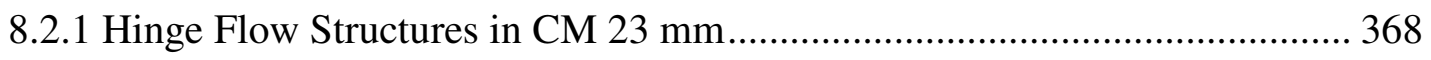

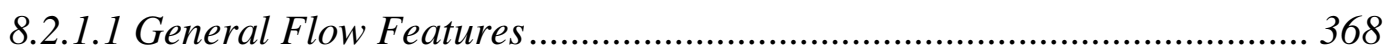

8.2.2.1 Comparison of The CM $23 \mathrm{~mm}$ With The Three $27 \mathrm{~mm}$ Bileaflet Heart

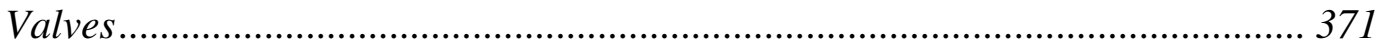

8.2.2.2 Comparison Of Current Hinge Studies With Previous Experiments...... 375

8.3 Flow Quantification in Aortic Polymeric Valves ....................................... 379

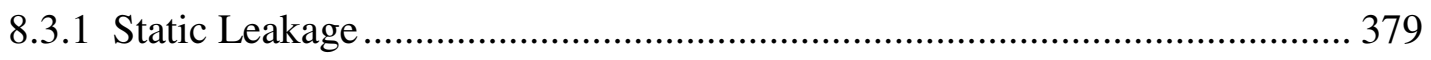

8.3.2 Effect Of Valve Design on Flow Fields Inside and in the Vicinity of the

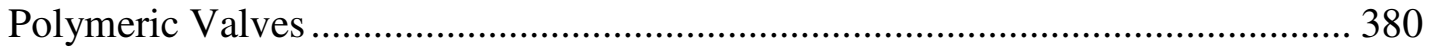

8.3.2.1 Common Flow Structures …………………………………………….... 381

8.3.2.1.1 Common Flow Features Outside and Downstream of Aortic

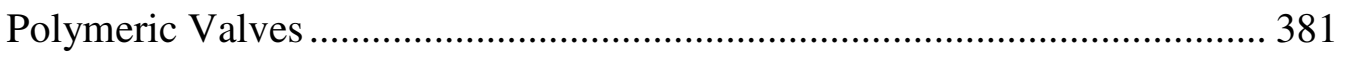

8.3.2.1.2 Common Flow Features Inside the Aortic Polymeric Valves ......... 385

8.3.2.1.3 Reynolds Shear Stress Distribution ............................................... 386 
8.3.2.3.1 Flow Features Downstream of the Aortic Polymeric Valves 389

8.3.2.3.2 Flow Features Inside the Aortic Polymeric Valves

8.3.2.4 Comparison Of Current Aortic Polymeric Valves Studies With Previous Experiments. 395

8.3.3 Integration of Previous In Vivo Studies with Observed Flow Structures ...... 400

8.4 COMPARISON OF THE LDV AND PIV TECHNIQUES................................................. 407

8.4.1 Comparison of Velocity Magnitude and Reynolds Shear Stress Profiles at Immediate Downstream of the Valve 408

8.4.2 Comparison of Velocity Magnitude, Reynolds Shear Stress $(-\overline{\rho u v})$ and $U_{r m s}$ Profiles $40 \mathrm{~mm}$ Downstream of the Three Polymeric Heart Valves 409

8.4.3 Comparison of Velocity Magnitude, Reynolds Shear Stress (- $\overline{\rho u v})$ and $U_{r m s}$ Inside the Three Polymeric Heart Valves ................................................................ 415

8.4.4 Review of the LDV and PIV Techniques .................................................... 421

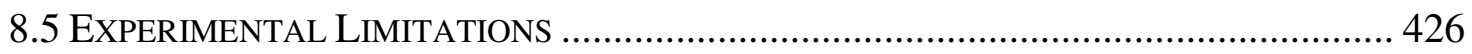

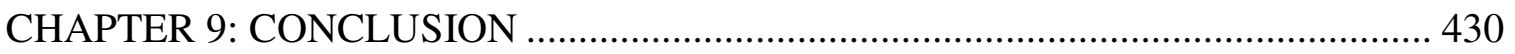

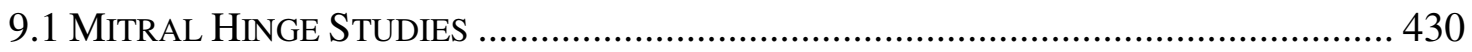

9.2 Aortic Polymeric VAlve STUdies ................................................................ 432

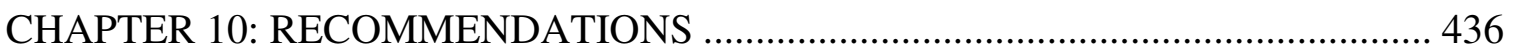

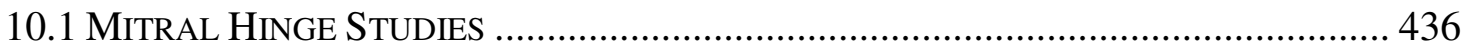

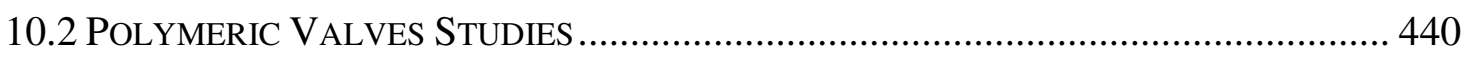

APPENDIX A: PROGRAM CODE FOR AUTOTRACKING.M ................................ 443

APPENDIX B: PROGRAM CODE FOR MARKERCHECK.M ……………............... 445

APPENDIX C: PROGRAM CODE FOR SW3D7.M ……………………………..... 446

APPENDIX D: PROGRAM CODE FOR SURFACEPLOT.M....................................... 447

APPENDIX E: PROGRAM CODE FOR VELOCITYPLOT.M …………………....... 448 
APPENDIX F: COMPARISON OF PHASE-AVERAGING AND CYCLIC REMOVAL

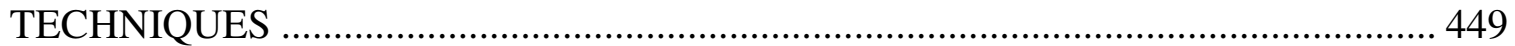

APPENDIX G: TABULATED DATA FOR THE STATIC LEAKAGE

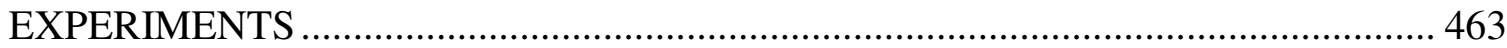

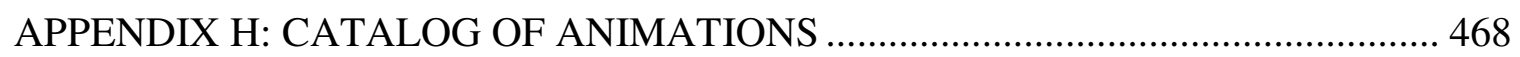

APPENDIX I: LEAFLET MOTION RESULTS AT PHYSIOLOGICAL TEMPERATURE …………………………………......................................... 472

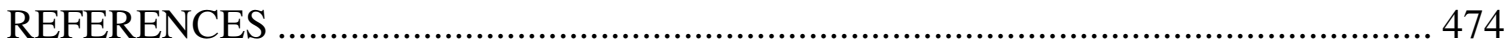




\section{LIST OF TABLES}

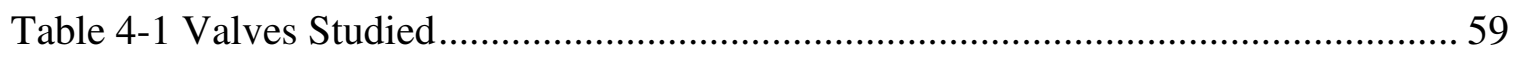

Table 4-2 Description of working medium for different experiments............................. 78

Table 5-1 Measurement levels inside the $\mathrm{CM}$ valve and the corresponding number of

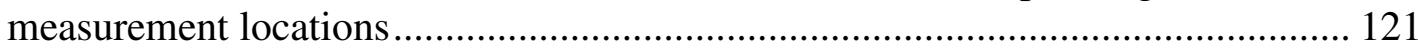

Table 5-2 Various measurement levels in the three $27 \mathrm{~mm}$ transparent bileaflet valves and corresponding number of measurement locations .................................................. 122

Table 5-3 Measurement planes inside and proximal to the polymeric heart valve and corresponding number of measurement locations ................................................ 127

Table 5-4 Pulse separation setting for the three polymeric heart valve ......................... 135

Table 7-1 Tabulated peak velocity of the marker point during the opening and closing phases at both the room $\left(20.5^{\circ} \mathrm{C}\right)$ and physiological $\left(37.0^{\circ} \mathrm{C}\right)$ temperatures. The number in the parenthesis refer to the position of the marker

Table 7-2 The $R_{a}$ and $R_{q}$ values of the leaflet surfaces of the two heart valve prostheses

Table 8-1 Peak phase averaged velocities $(\mathrm{m} / \mathrm{s})$ measured within the hinge region of the three $27 \mathrm{~mm}$ bileaflet valves. $\mathrm{NA}=$ not available

Table 8-2 Maximum phased averaged Reynolds shear stresses $\left(\mathrm{dynes} / \mathrm{cm}^{2}\right)$ measured within the hinge region of the three $27 \mathrm{~mm}$ valves. $\mathrm{NA}=$ not available

Table 8-3 Comparison of the number of measurement locations in the atrial and adjacent leakage jets at the flat level with their corresponding velocity and RSS range....... 359

Table 8-4 Comparison of the number of measurement locations in the atrial and adjacent leakage jets at the $190 \mu \mathrm{m}$ above flat level with their corresponding velocity and RSS range

Table 8-5 Comparison of the calculated maximum viscous shear stress inside the hinge recess for the three $27 \mathrm{~mm}$ bileaflet mechanical heart valves

Table 8-6 Peak phase averaged velocities and RSS measured within the hinge region of the CM $23 \mathrm{~mm}$ clear housing valve 370 
Table 8-7 Comparison of the velocity magnitudes and Reynolds shear stresses from various valve designs

Table 8-8 Maximum phased averaged velocity magnitudes $(\mathrm{m} / \mathrm{s})$ inside and in the immediate vicinity of the three polymeric heart valves during systole and diastole (2D LDV). Refer to section 5.3.2 for the locations of the 2D LDV measurement planes (parenthisis refer to the distance from the edge of the valve sewing ring marked as $\mathrm{x}=0$, see Figure 7-93)....

Table 8-9 Maximum Reynolds shear stresses $\left(\right.$ dynes $\left./ \mathrm{cm}^{2}\right)$ inside and in the immediate vicinity of the three polymeric heart valves (2D LDV). Refer to section 5.3.2 for the locations of the 2D LDV measurement planes (parenthisis refer to the distance from the edge of the valve sewing ring marked as $\mathrm{x}=0$, see Figure 7-93).... 382

Table 8-10 Maximum phased averaged velocity magnitudes $(\mathrm{m} / \mathrm{s})$ and corresponding RSS values (dynes/cm2) inside and downstream of prototype A during peak systole and diastole (3D PIV). Refer to section 5.4.1 for the locations of the 3D PIV measurement planes (parenthisis refer to the distance from the edge of the valve sewing ring marked as $\mathrm{x}=0$, see Figure 7-93) ....

Table 8-11 Maximum velocity magnitudes $(\mathrm{m} / \mathrm{s})$ and corresponding RSS values $\left(\right.$ dynes $/ \mathrm{cm}^{2}$ ) inside and downstream of prototype B during peak systole and diastole (3D PIV). Refer to section 5.4.1 for the locations of the 3D PIV measurement planes (parenthisis refer to the distance from the edge of the valve sewing ring marked as $\mathrm{x}=0$, see Figure 7-93)....

Table 8-12 Maximum velocity magnitudes (m/s) and corresponding RSS values $\left(\right.$ dynes $/ \mathrm{cm}^{2}$ ) inside and downstream of prototype C during peak systole and diastole (3D PIV). Refer to section 5.4.1 for the locations of the 3D PIV measurement planes (parenthisis refer to the distance from the edge of the valve sewing ring marked as $\mathrm{x}=0$, see Figure 7-93). 384

Table 8-13 Comparison of the velocity magnitudes and Reynolds shear stresses from various trileaflet valve designs 


\section{LIST OF FIGURES}

Figure 2-1 Anatomy of the Heart; the heart is made up of four chambers responsible for the regulation of the blood flow and pressure through the body

Figure 2-2 Various heart valve prosthetheses (a) Starr-Edwards ball and cage valve, (b) Medtronic-Hall tilting disc valve, (c) St. Jude Medical bileaflet valve, (d) CarboMedics bileaflet valve, (e) Hancock porcine valve, and (f) Carpentier-Edward pericardial valve

Figure 2-3 Flow Velocity Profile of Various Valve Prostheses: a) ball and caged valve, b) tissue valve, c) tilting disk, and d) bileaflet valve. 16

Figure 2-4 Various Hinge Geometry Designs. The SJM hinge geometry is similar to that of the CM valve except the latter has a sharper corner and a flatter hinge recess. The MP hinge design is characterized by a series of expansion and contraction, and the MA hinge design incorporate a secondary flat level to enhance washout characteristics

Figure 4-1 Picture of a typical mechanical bileaflet heart valve 47

Figure 4-2 Top view of a SJM bileaflet heart valve at the open position 48

Figure 4-3 Comparison of different SJM valve designs. Larger effective orifice area is the major design improvement among the three valve types.

Figure 4-4 Close-up cutaway side view illustrating geometry of SJM hinge recess. The leakage gap is defined as the distance between the tip of the leaflet ear and the top side of the hinge socket

Figure 4-5 Front and side views of the prototype SJM clear valve housing. The clear valve housing facilitate the optical access to the hinge region .............................. 51

Figure 4-6 Top view of the CM bileaflet heart valve at open position........................... 53

Figure 4-7 Comparison of the CM and SJM valve designs ....................................... 53

Figure 4-8 Front and side view of the cm clear valve housing ................................... 54

Figure 4-9 Computer aided design of the polymeric heart valves; (a) three stent aortic design, (b) two stent mitral design.... 
Figure 4-10 $23 \mathrm{~mm}$ tri-leaflet polymeric heart valve. The leaflet has the same refractive index as the working solution (aqueous Sodium Iodide), thus allowing velocity measurements by means of laser Doppler Velocimetry inside the polymeric valve 56

Figure 4-11 The three different designs of the polymeric heart valve; (a) prototype A, (b)

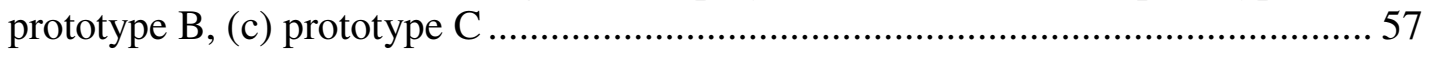

Figure 4-12 Mitral valve holder for the three $27 \mathrm{~mm}$ transparent bileaflet heart valves; one SJM $27 \mathrm{~mm}$ Standard and the two prototype leaker valves ..............................6 60

Figure 4-13 Valve holder for the CM 23 mm bileaflet heart valve .............................. 61

Figure 4-14 Schematic of the valve holder for the $23 \mathrm{~mm}$ polymeric heart valves.......... 62

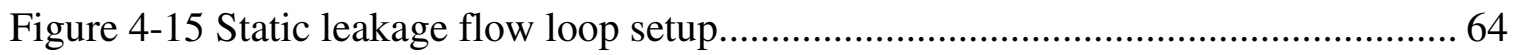

Figure 4-16 Pulsatile loop setup for the hinge flow experiments in mitral position......... 67

Figure 4-17 Pulsatile loop setup for the aortic valve experiments................................ 69

Figure 4-18 Pulsatile loop setup for the temperature dependent experiments................ 71

Figure 4-19 Dogleg section for use of acquiring upstream images of the polymeric aortic valve

Figure 4-20 Labeling of the marker array on high speed camera image of the polymeric valve leaflet.

Figure 4-21 Upstream image of the polymeric heart valve through dogleg section......... 74

Figure 4-22 Calibration cube as viewed by the two high speed cameras. For the camera calibration, the Surfaceplot.m asks for the two-dimensional coordinates of reference cube obtained from the two high speed cameras, as well as the three-dimensional known dimensions of the cube.

Figure 4-23 Principle of delaunay triangulation and dirichlet regions .......................... 77

Figure 4-24 Calibration Curve for the Flow Meter................................................. 81

Figure 4-25 Calibration Curve for Pressure Transducer.......................................... 82

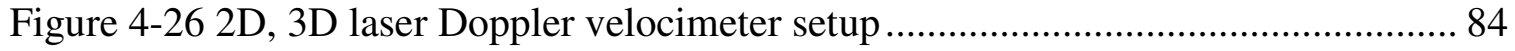

Figure 4-27 Direction of vector relative to the primary beam. The direction of the velocity vectors is determined by the direction of the crossing between the primary

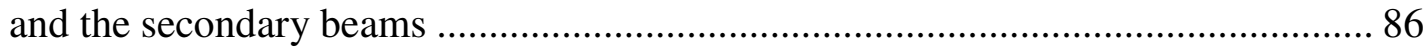

Figure 4-28 Interference pattern of probe volume as result of beams crossing ............... 87 
Figure 4-29 3D particle image velocimeter setup

Figure 4-30 Processing sequence of PIV raw images. Each image is divided into smaller rectangular interrogation windows where the intensity (I) of the particles is crosscorrelated with that of another image to provide an average of the particle displacement

Figure 4-31 Q-switch control of the lasing cavity. Setting the Q-switch slightly after the peak laser power can boost the laser output power and improves the consistency of the output from pulse to pulse.

Figure 4-32 Percentage error in measuring the principle Reynolds shear stress at a point with maximum turbulence intensity............................................................. 97

Figure 4-33 3D PIV camera configuration employed in this research ......................... 99

Figure 4-34 Schematic of scheimpflug rule. A scheimpflug mechanism is usually attached at the back of the CCD cameras to help increase the depth of the view so that more area could be brought into focus....

Figure 4-35 The calibration target used in the 3D PIV experiments. The thickness of the acrylic block should be similar to that of the test section to ensure accurate setup of the calibration file 102

Figure 4-36 Timing sequence of a single camera. The CCD camera open longer during the second frame because of the delay in sending the first frame to the computer 103

Figure 4-37 Setup for non-contact, three-dimensional, scanning white light and optical

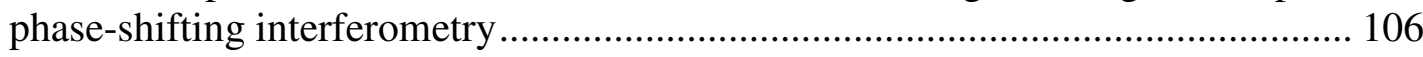

Figure 4-38 Interferences as a result of destructive and constructive wave interactions. Interference can be visualized as the addition of two waves. Depending upon the wave size (amplitude) and the degree to which they are in or out of step with each other (phase), the effects are either additive or subtractive

Figure 5-1 Mitral valve flowrate and pressure waveforms 111

Figure 5-2 Aortic valve flowrate and pressure waveforms..... 112

Figure 5-3 The effect of increasing resistant on the aortic pressure waveform. 113

Figure 5-4 The effect of increasing compliance on the aortic pressure waveform......... 113

Figure 5-5 Position of 2D LDV probe with respect to the valve chamber 115

Figure 5-6 Positioning of the laser probe volume with respect to the location of the hinge region; red cross signify the various locations in which to place the probe volume to find the flat level at the hinge region 
Figure 5-7 The edge of the hinge geometry was mapped by moving across the hinge in a predefined way to obtain an approximate co-ordinate of the hinge outline 117

Figure 5-8 Approximated outline of the hinge geometry 117

Figure 5-9 The size of the probe volume is smaller than the incremental resolution of the measurement 119

Figure 5-10 CM $23 \mathrm{~mm}$ hinge geometry as mapped by laser at primary flat level 120

Figure 5-11 The various measurements levels both inside and outside the hinge pocket of the four transparent bileaflet heart valves 120

Figure 5-12 Measurement locations at the flat level of SJM $27 \mathrm{~mm}$ Standard. 123

Figure 5-13 Hinge geometry of the three $27 \mathrm{~mm}$ transparent bileaflet clear valves as mapped by laser at primary flat level.

Figure 5-14 Measurement locations at the downstream of the thumbnail region and the below the flat level.

Figure 5-15 Position of the 2D LDV probe with respect to the valve chamber 125

Figure 5-16 Position of the 2D LDV and 1D LDV probes with respect to the receiver and the test section. The violet beam is a much weaker beam compared to the green and blue laser beams, therefore the $1 \mathrm{D}$ violet laser probe is position across the receiver at a frontscattering mode to maximize the data rate 126

Figure 5-17 Measurement locations at the downstream of the polymeric heart valves.. 126

Figure 5-18 Measurement locations both inside and at the immediate downstream of the polymeric heart valve 127

Figure 5-19 Translation of the calibration target during the calibration 129

Figure 5-20 The mapping of the calibration target by Davis to calculate displacement function

Figure 5-21 Mapping function is obtained from calibration target for each camera for the processing of velocity vectors

Figure 5-22 Vectors calculated from each camera are combined to produce 3D velocity map.

Figure 5-23 3D velocity vector map was obtained from the correlated images of the two CCD cameras. (a) The $1^{\text {st }}$ camera exposure, (b) the $2^{\text {nd }}$ camera exposure, and (c) the $3 \mathrm{D}$ velocity vectors from the two CCD cameras 132 
Figure 5-24 Application of masking to selectively process only the region of interest; (a) before masking, (b) after the application of masking

Figure 5-25 Measurement planes for the polymeric valves in the 3D PIV experiments 134

Figure 5-26 Surface measurement locations on prosthetic valves. Surface topography measurements were obtained on both sides (upstream and downstream facing surfaces) of the heart valves leaflets. The measurement location with respect to the upstream an downstream surfaces of the leaflet is specified by the cross (downstream) and the shaded circle (upstream) 137

Figure 6-1 The step sequences for processing of vectors in 3D PIV experiments 146

Figure 7-1 The leakage rate of the four clear valve models. The leakage rate of the SJM $\mathrm{mm}$ Standard valve was between that of the $27 \mathrm{~mm}$ prototype high and low leaker valves. The $27 \mathrm{~mm}$ prototype high leaker had similar leakage rate as that of the CM $23 \mathrm{~mm}$ bileaflet valve. 155

Figure 7-2 Pertinent terminology of SJM 27 mm Standard clear hinge design..... 156

Figure 7-3 Leaflet position at (a) valve closure, mid systole, and (b) mid acceleration, peak diastole, within the SJM $27 \mathrm{~mm}$ Standard clear housing valve.

Figure 7-4 SJM $27 \mathrm{~mm}$ Standard mitral hinge flow field at early systole, time bin 1, flat level; leakage jets issuing from atrial, adjacent and lateral corners of the hinge geometry (avi, 680k, reg_flat_vmag.avi).....

Figure 7-5 SJM $27 \mathrm{~mm}$ Standard mitral hinge flow field approaching mid systole, time bin 4, flat level; the diversion of flow by the leaflet ear at the ventricular side (avi, 680k, reg_flat_vmag.avi)

Figure 7-6 Reynolds shear stress distribution of the SJM 27 mm Standard at time bin 4, flat level; elevated Reynolds shear stress levels observed at the atrial and lateral corners of the hinge geometry (avi, 697k, reg_flat_rss.avi) 160

Figure 7-7 SJM $27 \mathrm{~mm}$ Standard mitral hinge flow field at early diastole, time bin 15, flat level; low flow persisted for 40-60 ms at start of diastole (avi, 680k, reg_flat_vmag.avi)

Figure 7-8 SJM $27 \mathrm{~mm}$ Standard mitral hinge flow field at mid diastole, time bin 27, flat level; recirculation flow appeared at the adjacent corner (avi, 680k, reg_flat_vmag.avi)..... 161

Figure 7-9 SJM $27 \mathrm{~mm}$ Standard mitral hinge flow field at early systole, time bin 1, 190 $\mu \mathrm{m}$ above flat level; leakage flow between the leaflet ear and the hinge wall (avi, 656k, reg_190um_above_flat_vmag.avi) 164 
Figure 7-10 SJM $27 \mathrm{~mm}$ Standard mitral hinge flow field approaching mid systole, time bin 4, $190 \mu \mathrm{m}$ above flat level; strong leakage flow at the atrial corner (avi, 656k, reg_190um_above_flat_vmag.avi).....

Figure 7-11 SJM $27 \mathrm{~mm}$ Standard mitral hinge flow field at mid diastole, time bin 27, $190 \mu \mathrm{m}$ above flat level; recirculation flow visible at the adjacent corner (avi, 656k, reg_190um_above_flat_vmag.avi) .............................................................. 165

Figure 7-12 SJM 27 mm Standard mitral hinge flow field at early systole, time bin 2, 390 $\mu \mathrm{m}$ above flat level; low flow was evident throughout the cardiac cycle (avi, 603k, reg_390um_above_flat_vmag.avi) 166

Figure 7-13 SJM $27 \mathrm{~mm}$ Standard mitral hinge flow field at early systole, time bin 1, 500 $\mu \mathrm{m}$ below flat level; low leakage flow evident at the hinge pockets and the between the closing leaflets (avi, 702k, reg_500um_below_flat_vmag.avi).... 169

Figure 7-14 SJM $27 \mathrm{~mm}$ Standard mitral hinge flow field approaching mid systole, time bin $4,500 \mu \mathrm{m}$ below flat level; the leakage jets emanating from the hinge regions (avi, 702k, reg_500um_below_flat_vmag.avi)....

Figure 7-15 Reynolds shear stress distribution of the SJM $27 \mathrm{~mm}$ Standard at time bin 4, $500 \mu \mathrm{m}$ above flat level; elevated Reynolds shear stress levels coincided with regions of the leakage jets (avi, 708k, reg_500um_below_flat_rss.avi)..... 170

Figure 7-16 SJM $27 \mathrm{~mm}$ Standard mitral hinge flow field at mid diastole, time bin 27, $500 \mu \mathrm{m}$ below flat level; forward flow was characterized by one central jet and two lateral jets (avi, 702k, reg_500um_below_flat_vmag.avi)

Figure 7-17 Reynolds shear stress distribution of the SJM $27 \mathrm{~mm}$ Standard at time bin 27, $500 \mu \mathrm{m}$ above flat level; Reynolds shear stress of 1,000 dynes $/ \mathrm{cm}^{2}$ was observed inside the central forward flow jet (avi, 708k, reg_500um_below_flat_rss.avi)... 171

Figure 7-18 SJM $27 \mathrm{~mm}$ Standard mitral hinge flow field at early systole, time bin 1, 1 $\mathrm{mm}$ below flat level; leakage flow evident between the closing leaflets (avi, 691k, reg_1mm_below_flat_vmag.avi) 173

Figure 7-19 SJM $27 \mathrm{~mm}$ Standard mitral hinge flow field approaching mid systole, time bin 4, $1 \mathrm{~mm}$ below flat level; weak leakage jet was seen issuing from a single hinge pocket (avi, 691k, reg_1mm_below_flat_vmag.avi).....

Figure 7-20 Reynolds shear stress distribution of the SJM $27 \mathrm{~mm}$ Standard at time bin 43, $1 \mathrm{~mm}$ above flat level; elevated Reynolds shear stress levels of 100-1,000 dynes $/ \mathrm{cm}^{2}$ were observed at valve closure (avi, 697k, reg_1mm_below_flat_rss.avi) 174

Figure 7-21 SJM $27 \mathrm{~mm}$ Standard mitral hinge flow field at mid diastole, time bin 27, 1 mm below flat level; elevated Reynolds shear stress observed between the central jet and the lateral jets (avi, 691k, reg_1mm_below_flat_vmag.avi).... 174 
Figure 7-22 SJM $27 \mathrm{~mm}$ Standard mitral hinge flow field at early systole, time bin 43, 3 $\mathrm{mm}$ below flat level; disturbed flow at valve closure evident between the hinge mechanism (avi, 634k, reg_3mm_below_flat_vmag.avi).

Figure 7-23 SJM $27 \mathrm{~mm}$ Standard mitral hinge flow field at mid diastole, time bin 27, 3 $\mathrm{mm}$ below flat level; three forward jets observed during diastole (avi, 634k, reg_3mm_below_flat_vmag.avi)....

Figure 7-24 SJM 27 mm Standard mitral hinge flow field at early systole, time bin 1, downstream of thumbnail region, flat level; flow was relatively uniform during the $1^{\text {st }}$ part of systole (avi, 761k, reg_thumbnail_vmag.avi) 179

Figure 7-25 SJM $27 \mathrm{~mm}$ Standard mitral hinge flow field at accelerating phase, time bin 23 , downstream of thumbnail region, flat level; this cardiac phase marked the beginning of the inversion of the lateral flows (avi, 761k, reg_thumbnail_ vmag.avi)

Figure 7-26 Pertinent terminology of the $27 \mathrm{~mm}$ prototype low and high leaker clear hinge designs.

Figure 7-27 $27 \mathrm{~mm}$ prototype low leaker mitral hinge flow field at early systole, time bin 2, flat level; strong leakage jet appearing at the atrial corner of the hinge geometry (avi, 782k, low_flat_vmag.avi) 183

Figure 7-28 $27 \mathrm{~mm}$ prototype low leaker mitral hinge flow field approaching mid systole, time bin 4, flat level; second leakage jet became visible at the adjacent corner of the hinge geometry (avi, 782k, low_flat_vmag.avi) 184

Figure 7-29 Reynolds shear stress distribution of the $27 \mathrm{~mm}$ prototype low leaker at time bin 4, flat level; elevated Reynolds shear stress levels observed at the atrial and lateral corners of the hinge geometry (avi, 740k, low_flat_rss.avi) 184

Figure 7-30 $27 \mathrm{~mm}$ prototype low leaker mitral hinge flow field at early diastole, time bin 21 , flat level; forward jets observed in both adjacent and lateral corner of the hinge during the acceleration phase (avi, 782k, low_flat_vmag.avi) 185

Figure 7-31 $27 \mathrm{~mm}$ prototype low leaker mitral hinge flow field at mid diastole, time bin 27 , flat level; recirculation flow and a strong forward flow appeared at the adjacent and lateral corners, respectively (avi, 782k, low_flat_vmag.avi) 185

Figure 7-32 $27 \mathrm{~mm}$ prototype low leaker mitral hinge flow field at early systole, time bin 2, $190 \mu \mathrm{m}$ above flat level; leakage jets emanating from the atrial and adjacent corners (avi, 672k, low_190um_above_flat_vmag.avi)

Figure 7-33 $27 \mathrm{~mm}$ prototype low leaker mitral hinge flow field approaching mid systole, time bin 4, $190 \mu \mathrm{m}$ above flat level (avi, 672k, low_190um_above_flat_ vmag.avi) 188 
Figure 7-34 Reynolds shear stress distribution of the $27 \mathrm{~mm}$ prototype low leaker at time bin 4, $190 \mu \mathrm{m}$ above flat level; Reynolds shear stress levels in the adjacent corner were higher than those in the atrial corner (avi, 684k, low_190um_above_flat_rss.avi)

Figure 7-35 $27 \mathrm{~mm}$ prototype low leaker mitral hinge flow field at mid diastole, time bin 27, $190 \mu \mathrm{m}$ above flat level; recirculation visible at the adjacent corner (avi, 672k, low_190um_above_flat_vmag.avi) 189

Figure 7-36 $27 \mathrm{~mm}$ prototype low leaker mitral hinge flow field approaching mid systole, time bin 5, $390 \mu \mathrm{m}$ above flat level; low flow was visible throughout the cardiac cycle (avi, 618k, low_390um_above_flat_vmag.avi)....

Figure 7-37 $27 \mathrm{~mm}$ prototype low leaker mitral hinge flow field at early systole, time bin $43,500 \mu \mathrm{m}$ below flat level; flow mixing occurred at between hinge mechanism at valve closure (avi, 712k, low_500um_below_flat_vmag.avi).

Figure 7-38 $27 \mathrm{~mm}$ prototype low leaker mitral hinge flow field approaching mid systole, time bin 4, $500 \mu \mathrm{m}$ below flat level; two prominent leakage jets issuing from the hinge pockets (avi, 712k, low_500um_below_flat_vmag.avi)....

Figure 7-39 Reynolds shear stress distribution of the $27 \mathrm{~mm}$ prototype low leaker at time bin 4, $500 \mu \mathrm{m}$ below flat level; elevated Reynolds shear stress levels observed at the leakage jets emanating from the hinge region and the ventricular side of the hinge mechanism (avi, 708k, low_500um_below_flat_rss.avi) 193

Figure 7-40 $27 \mathrm{~mm}$ prototype low leaker mitral hinge flow field at peak diastole, time bin $27,500 \mu \mathrm{m}$ below flat level; the opened leaflets split the forward flow into three separate forward jets (avi, 712k, low_500um_below_flat_vmag.avi). 193

Figure 7-41 $27 \mathrm{~mm}$ prototype low leaker mitral hinge flow field at early systole, time bin $1,1 \mathrm{~mm}$ below flat level; leakage flow visible at the gap between closing leaflets (avi, 703k, low_1mm_below_flat_vmag.avi)....

Figure 7-42 $27 \mathrm{~mm}$ prototype low leaker mitral hinge flow field toward mid systole, time bin 4, $1 \mathrm{~mm}$ below flat level; single leakage issuing from hinge pocket (avi, 703k, low_1mm_below_flat_vmag.avi) 196

Figure 7-43 $27 \mathrm{~mm}$ prototype low leaker mitral hinge flow field at mid diastole, time bin $27,1 \mathrm{~mm}$ below flat level; the velocity of the central stream reached $1.3 \mathrm{~m} / \mathrm{s}$ at mid diastole, while that at the shear region was low at $0.05 \mathrm{~m} / \mathrm{s}$ (avi, 703k, low_1mm_below_flat_vmag.avi) .... 196

Figure 7-44 $27 \mathrm{~mm}$ prototype low leaker mitral hinge flow field at early systole, time bin 42, $3 \mathrm{~mm}$ below flat level; disturb flow at valve closure (avi, 643k, low_3mm_below_flat_vmag.avi)..... 198 
Figure 7-45 $27 \mathrm{~mm}$ prototype low leaker mitral hinge flow field at mid diastole, time bin $27,3 \mathrm{~mm}$ below flat level; maximum forward velocity of $1.35 \mathrm{~m} / \mathrm{s}$ was measured at the central flow stream at mid diastole (avi, 643k, low_3mm_below_flat_ vmag.avi) 198

Figure 7-46 $27 \mathrm{~mm}$ prototype low leaker mitral hinge flow field at early systole, time bin 1 , downstream of thumbnail region, flat level; disturbed flow at the lower part of the measurement field (avi, 733k, low_thumbnail_vmag.avi) 200

Figure 7-47 $27 \mathrm{~mm}$ prototype low leaker mitral hinge flow field approaching mid diastole, time bin 23, downstream of thumbnail region, flat level; inversion of the lateral jets into leakage flow (avi, 733k, low_thumbnail_vmag.avi) 200

Figure 7-48 $27 \mathrm{~mm}$ prototype high leaker mitral hinge flow field at early systole, time bin 1 , flat level; leakage jets at both the atrial and adjacent corner appeared to merge into a single jet occupying a large part of the atrial side (avi, 719k, high_flat_vmag.avi) 203

Figure 7-49 $27 \mathrm{~mm}$ prototype high leaker mitral hinge flow field approaching mid systole, time bin 4, flat level; the leakage jets at atrial and adjacent corners appeared to merge into a larger jet (avi, 719k, high_flat_vmag.avi) 204

Figure 7-50 Reynolds shear stress distribution of the $27 \mathrm{~mm}$ prototype high leaker at time bin 4, flat level; elevated Reynolds shear stress levels observed at the atrial and lateral corners of the hinge geometry (avi, 734k, high_flat_rss.avi) 204

Figure 7-51 $27 \mathrm{~mm}$ prototype high leaker mitral hinge flow field at mid diastole, time bin 27, flat level; recirculation flow appeared at the adjacent corner of hinge geometry (avi, 719k, high_flat_vmag.avi)..... 205

Figure 7-52 $27 \mathrm{~mm}$ prototype high leaker mitral hinge flow field at early systole, time bin $1,190 \mu \mathrm{m}$ above flat level; low flow of less than $0.2 \mathrm{~m} / \mathrm{s}$ observed close to the atrial corner (avi, 694k, high_190um_above_flat_vmag.avi).....

Figure 7-53 $27 \mathrm{~mm}$ prototype high leaker mitral hinge flow field toward mid systole, time bin 4, $190 \mu \mathrm{m}$ above flat level; leakage flow appeared to flow along the edge of the leaflet ear (avi, 694k, high_190um_above_flat_vmag.avi) 207

Figure 7-54 Reynolds shear stress distribution of the $27 \mathrm{~mm}$ prototype high leaker at time bin 4, $190 \mu \mathrm{m}$ above level; elevated Reynolds shear stress levels of at least 500 dynes $/ \mathrm{cm}^{2}$ observed throughout at the hinge region (avi, 710k, high_190um_above_flat_rss.avi)..... 208

Figure 7-55 $27 \mathrm{~mm}$ prototype high leaker mitral hinge flow field at mid diastole, time bin 27, $190 \mu \mathrm{m}$ above flat level; low recirculation flow observed at the adjacent corner (avi, 694k, high_190um_above_flat_vmag.avi)..... 208 
Figure 7-56 $27 \mathrm{~mm}$ prototype high leaker mitral hinge flow field at mid systole, time bin 9, $390 \mu \mathrm{m}$ above flat level (avi, 615k, high_390um_above_flat_vmag.avi).... 209

Figure 7-57 $27 \mathrm{~mm}$ prototype high leaker mitral hinge flow field at early systole, time bin 43, $500 \mu \mathrm{m}$ below flat level; strong leakage jets emanating from the hinge pockets and between the leaflet gap (avi, 714k, high_500um_below_flat_vmag.avi) ........ 212

Figure 7-58 $27 \mathrm{~mm}$ prototype high leaker mitral hinge flow field approaching mid systole, time bin 4, $500 \mu \mathrm{m}$ below flat level; the leakage jet issuing from the hinge accelerated to velocity of $1.0 \mathrm{~m} / \mathrm{s}$ at mid systole (avi, 714k, high_500um_below_flat_vmag.avi) 212

Figure 7-59 Reynolds shear stress distribution of the $27 \mathrm{~mm}$ prototype high leaker at time bin 4, $500 \mu \mathrm{m}$ below flat level; elevated Reynolds shear stress levels observed at the leakage jets issuing from the hinge regions (avi, 722k, high_500um_below_flat_rss.avi).

Figure 7-60 $27 \mathrm{~mm}$ prototype high leaker mitral hinge flow field at mid diastole, time bin $27,500 \mu \mathrm{m}$ below flat level; three forward jets observed during diastole with maximum velocity $1.3 \mathrm{~m} / \mathrm{s}$ in the central jet (avi, 714k, high_500um_below_flat_vmag.avi)

Figure 7-61 $27 \mathrm{~mm}$ prototype high leaker mitral hinge flow field at mid systole, time bin 9, $1 \mathrm{~mm}$ below flat level; leakage jet of $0.2 \mathrm{~m} / \mathrm{s}$ was observed issuing from the hinge (avi, 703k, high_1mm_below_flat_vmag.avi) 215

Figure 7-62 $27 \mathrm{~mm}$ prototype high leaker mitral hinge flow field at mid diastole, time bin 29, $1 \mathrm{~mm}$ below flat level; three forward jets were observed during systole with a maximum velocity of $1.4 \mathrm{~m} / \mathrm{s}$ recorded in the central jet (avi, 703k, high_1mm_below_flat_vmag.avi).... 215

Figure 7-63 $27 \mathrm{~mm}$ prototype high leaker mitral hinge flow field at early systole, time bin 43, $3 \mathrm{~mm}$ below flat level; flow at the atrial side of the closed leaflets was drawn towards a small central leakage jet between the closed leaflets (avi, 666k, high_3mm_below_flat_vmag.avi).....

Figure 7-64 $27 \mathrm{~mm}$ prototype high leaker mitral hinge flow field at mid diastole, time bin 27, $3 \mathrm{~mm}$ below flat level; three forward jets were observed during systole with a peak velocity of $1.4 \mathrm{~m} / \mathrm{s}$ measured in the central forward jet (avi, 666k, high_3mm_below_flat_vmag.avi)....

Figure 7-65 $27 \mathrm{~mm}$ prototype high leaker mitral hinge flow field at early systole, time bin 1, downstream of thumbnail region, flat level; uniform retrograde flow of 0.2 $\mathrm{m} / \mathrm{s}$ was observed immediately downstream of the thumbnail (avi, 716k, high_thumbnail_vmag.avi) 
Figure 7-66 $27 \mathrm{~mm}$ prototype high leaker mitral hinge flow field approaching mid diastole, time bin 23, downstream of thumbnail region, flat level; lateral flow increased in magnitude during the acceleration phase (avi, 716k, high_thumbnail_vmag.avi).....

Figure 7-67 Pertinent terminology of CM $23 \mathrm{~mm}$ clear hinge design. 221

Figure 7-68 Leaflet position at (a) valve closure, mid systole, and (b) mid acceleration, peak diastole, within the CM $23 \mathrm{~mm}$ clear housing valve

Figure 7-69 CM $23 \mathrm{~mm}$ mitral hinge flow field at early systole, time bin 2, flat level; leakage jet emanating from the atrial and lateral corners of the hinge geometry (avi, 656k, CM_flat_vmag.avi). 225

Figure 7-70 Reynolds shear stress distribution of the CM $23 \mathrm{~mm}$ at early systole, time bin 2, flat level; elevated Reynolds shear stress levels observed at the atrial and lateral corners of the hinge geometry (avi, 673k, CM_flat_rss.avi) 225

Figure 7-71 CM $23 \mathrm{~mm}$ mitral hinge flow field approaching mid systole, time bin 4, flat level; the leakage jets were still apparent at the two locations (avi, 656k, CM_flat_vmag.avi)..... 226

Figure 7-72 CM $23 \mathrm{~mm}$ mitral hinge flow field at early diastole, time bin 13, flat level. The flow is predominantly in the forward direction with flow velocities much lower than those observed during systole (avi, 656k, CM_flat_vmag.avi) 226

Figure 7-73 CM $23 \mathrm{~mm}$ mitral hinge flow field at peak diastole, time bin 23, flat level; recirculation zone visible at the adjacent corner (avi, 656k, CM_flat_vmag.avi).. 227

Figure 7-74 CM $23 \mathrm{~mm}$ mitral hinge flow field at early systole, time bin 2, $190 \mu \mathrm{m}$ above flat level; leakage jets visible at the atrial and lateral corners of the hinge (avi, 713k, CM_190um_above_flat_vmag.avi) 229

Figure 7-75 Reynolds shear stress distribution of the CM $23 \mathrm{~mm}$ at early systole, time bin 2, $190 \mu \mathrm{m}$ above flat level; elevated Reynolds shear stress levels observed at the atrial and lateral corners of the hinge (avi, 729k, CM_190um_above_flat_rss.avi) 229

Figure 7-76 CM $23 \mathrm{~mm}$ mitral hinge flow field at mid systole, time bin 5, $190 \mu \mathrm{m}$ above flat level. The leakage velocities of the two leakage jets were lower than those measured during early systole (avi, 713k, CM_190um_above_flat_vmag.avi) ..... 230

Figure 7-77 CM $23 \mathrm{~mm}$ mitral hinge flow field at early diastole, time bin 15, $190 \mu \mathrm{m}$ above flat level; no recirculation is visible at the adjacent corner (avi, 713k, CM_190um_above_flat_vmag.avi)

Figure 7-78 CM $23 \mathrm{~mm}$ mitral hinge flow field at mid diastole, time bin 22, $190 \mathrm{um}$ above flat level. A maximum forward velocity of $0.77 \mathrm{~m} / \mathrm{s}$ was measured at the adjacent corner of the hinge (avi, 713k, CM_190um_above_flat_vmag.avi) . 
Figure 7-79 CM $23 \mathrm{~mm}$ mitral hinge flow field at early systole, time bin 43, $390 \mu \mathrm{m}$ above flat level; leakage flow appeared along the outer edge of the hinge geometry (avi, 628k, CM_390um_above_flat_vmag.avi) 233

Figure 7-80 CM 23 mm mitral hinge flow field approaching mid systole, time bin 3, 390 $\mu \mathrm{m}$ above flat level. Peak velocity of $2.52 \mathrm{~m} / \mathrm{s}$ was observed at the lateral corner with low flow at the central region(avi, 628k, CM_390um_above_flat_vmag.avi)233

Figure 7-81 Reynolds shear stress distribution of the CM $23 \mathrm{~mm}$ at early systole, time bin 3, $390 \mu \mathrm{m}$ above flat level; elevated Reynolds shear stress levels were recorded in the atrial and lateral corners (avi, 644k, CM_390um_above_flat_rss.avi)............. 234

Figure 7-82 CM $23 \mathrm{~mm}$ mitral hinge flow field approaching mid diastole, time bin 16, $390 \mu \mathrm{m}$ above flat level. Low flow of less than $0.3 \mathrm{~m} / \mathrm{s}$ was observed throughout the hinge (avi, 628k, CM_390um_above_flat_vmag.avi) 234

Figure 7-83 CM $23 \mathrm{~mm}$ mitral hinge flow field approaching mid diastole, time bin 14, 1 $\mathrm{mm}$ below flat level; the influence of the leaflets is apparent at the below flat measurement levels (avi, 1,030k, CM_1mm below_flat_vmag.avi) 236

Figure 7-84 CM $23 \mathrm{~mm}$ mitral hinge flow field at peak diastole, time bin 23, $1 \mathrm{~mm}$ below flat level; acceleration of the central and two laterals jets is observed (avi, 1,030k, CM_1mm below_flat_vmag.avi)..... 236

Figure 7-85 CM $23 \mathrm{~mm}$ mitral hinge flow field at early systole, time bin 43, $1 \mathrm{~mm}$ below flat level; leakage jet appeared at the gap between the closing leaflets (avi, 1,030k, CM_1mm below_flat_vmag.avi)..... 237

Figure 7-86 Reynolds shear stress distribution of the CM $23 \mathrm{~mm}$ at early systole, time bin 1, $1 \mathrm{~mm}$ below flat level; elevated Reynolds shear stress levels between gap of the closing leaflets (avi, 1,015k, CM_1mm below_flat_rss.avi)

Figure 7-87 The leakage rate of the three polymeric heart valves was tested at both room $\left(20.5^{\circ} \mathrm{C}\right)$ and physiological $\left(37.0^{\circ} \mathrm{C}\right)$ temperatures. An additional set of leakage testing was performed with the oscillation (osc) of the valves to take into account the actual dynamics of the valve under pulsatile settings 239

Figure 7-88 Comparison of the leakage flow rate for the prototype polymeric valve A between the room $\left(20.5^{\circ} \mathrm{C}\right)$ and physiological $\left(37.0^{\circ} \mathrm{C}\right)$ temperatures in the aortic position

Figure 7-89 Velocity of marker \# 13 at the high central region of the leaflet of prototype polymeric valve A tested at a temperature of $20.5^{\circ} \mathrm{C}$. The graph shows two peaks, which corresponded to the valve opening and closing phases

Figure 7-90 Velocity of marker \# 13 at high central region of the leaflet of prototype polymeric valve A tested at a temperature of $37^{\circ} \mathrm{C}$. The two peaks corresponded to the valve opening and closing phases 
Figure 7-91 The motion of the leaflet through a single cardiac cycle (temperature $20.5^{\circ} \mathrm{C}$ ). (a) The beginning of the opening phase; (b) Leaflet at the verge of opening; (c) Leaflet at fully opened position; (d) The beginning of the closing phase; (e) The closing phase; and (f) Leaflet at fully closed position (wmv, 3,764k, 3D_reconstructed_leaflet_motion_room_temp.wmv)

Figure 7-92 Upstream high speed camera image of the valve through the dogleg (temperature $-20.5^{\circ} \mathrm{C}$ ). (a) The beginning of opening phase; (b) valve opening; (c) valve when full open; (d) valve closing; (e) valve when closed; and e) oscillation of valve leaflet during diastolic phase. (wmv, 1,850k, animation high_speed_camera_upstream_room_temp.wmv)

Figure 7-93 Pertinent terminology for the three Aortech $23 \mathrm{~mm}$ trileaflet polymeric heart valves. All downstream measurements were made with reference from the valve sewing ring, which is marked $\mathrm{x}=0$. 248

Figure 7-94 Velocity fields downstream of prototype A at acceleration phase, time bin 5, center plane; a developing central orifice jet of $1.8 \mathrm{~m} / \mathrm{s}$ was seen issuing from the orifice of the polymeric valve with formation of recirculation zone between the edge of the valve and $75 \mathrm{~mm}$ downstream from the edge of the valve sewing ring (avi, 586k, A_parallel_downstream_vmag.avi) 251

Figure 7-95 Velocity fields downstream of prototype A at peak systole, time bin 9, center plane; (a) Velocity vectors acquired along the parallel-axis plane at peak systole, (b) Schematic of the flow field

Figure 7-96 Reynolds shear stress distribution of prototype A at peak systole, time bin 9, center plane; elevated Reynolds shear stress levels were observed at the region of flow contraction, edge of the central orifice jet and $60 \mathrm{~mm}$ downstream from the edge of the valve (avi,600k, A_parallel_downstream_rss.avi)....

Figure 7-97 Velocity fields downstream of prototype A at mid diastole, time bin 21, center plane; the flow field during diastole was low at around $0.04 \mathrm{~m} / \mathrm{s}$ (avi, 586k, A_parallel_downstream_vmag.avi). 252

Figure 7-98 Velocity fields downstream of the stent of prototype A during acceleration phase, time bin 5 ; high forward jet of $0.87 \mathrm{~m} / \mathrm{s}$ was observed to emanate from the deep channel at the commissural region of the prototype A (avi, 589k, A_stent_downstream_vmag.avi)

Figure 7-99 Velocity fields downstream of the stent of prototype A during peak systole, time bin 9; high velocity jet emanates from the gap channel at the commissural region (avi, 589k, A_stent_downstream_vmag.avi).....

Figure 7-100 Reynolds shear stress distribution of prototype A during peak systole, time bin 9; elevated Reynolds shear stress of more than 1,000 dynes $/ \mathrm{cm}^{2}$ was observed at the stent tip, which coincided with the location of the deep channel as observed in Figure 7-92c (avi, 601k, A_stent_downstream_rss.avi) 255 
Figure 7-101 Velocity fields downstream of the stent of prototype A during mid diastole, time bin 21; the leakage jet has a flow velocity of approximately $0.7 \mathrm{~m} / \mathrm{s}$ (avi, 589k, A_stent_downstream_vmag.avi) ..... 256

Figure 7-102 Velocity fields inside prototype A during acceleration phase, time bin 5; high velocity of approximately $2.0 \mathrm{~m} / \mathrm{s}$ was observed along the trailing edge of the leaflet. An area of stagnation occurred at the commissural region with a region of lower flow $(1.0 \mathrm{~m} / \mathrm{s})$ along the stent inflow region (avi, 586k,

A_inside_valve_vmag.avi) 258

Figure 7-103 Velocity fields inside prototype A at peak systole, time bin 9; a peak forward velocity of $2.6 \mathrm{~m} / \mathrm{s}$ was observed at the edge of the leaflet above the commissural region (avi, 586k, A_inside_valve_vmag.avi)

Figure 7-104 Reynolds shear stress distribution of prototype A at peak systole, time bin 9; maximum Reynolds shear stress of more than 5,500 dynes $/ \mathrm{cm}^{2}$ occurred at the trailing edge of the valve. The Reynolds shear stress levels further upstream inside the valve range between 600 to 4,000 dynes $/ \mathrm{cm}^{2}$. The shear stress levels at the stagnation area were less than $5 \mathrm{dynes} / \mathrm{cm}^{2}$ (avi, 610k, A_inside_valve_rss.avi) .. 259

Figure 7-105 Velocity fields inside prototype A during mid diastole, time bin 21; the leakage jet was 3-4 mm from the coaptation region (avi, 586k, A_inside_valve_vmag.avi)

Figure 7-106 Velocity fields inside prototype A during mid diastole, time bin 21; flow during diastole was directed toward the stent inflow region and the high central region inside the valve (avi, 586k, A_inside_valve_vmag.avi).....

Figure 7-107 Velocity fields downstream of prototype B at acceleration phase, time bin 5, center plane; a developing central orifice jet of $1.5 \mathrm{~m} / \mathrm{s}$ was seen issuing from the orifice of the polymeric valve with formation of a recirculation zone between the edge of the valve and $75 \mathrm{~mm}$ downstream from the valve sewing ring (avi, 586k, B_parallel_downstream_vmag.avi).....

Figure 7-108 Velocity fields downstream of prototype B at peak systole, time bin 9, center plane; a maximum velocity of $2.2 \mathrm{~m} / \mathrm{s}$ was acquired along the parallel-axis plane at peak systole (avi, 586k, B_parallel_downstream_vmag.avi).

Figure 7-109 Reynolds shear stress distribution of prototype B at peak systole, time bin 9, center plane; elevated Reynolds shear stress levels of more than 1,000 dynes $/ \mathrm{cm}^{2}$ were observed at edge of the central orifice jet and $60 \mathrm{~mm}$ downstream from the edge of the valve (avi, 604k, B_parallel_downstream_rss.avi).....

Figure 7-110 Velocity fields downstream of prototype B at mid diastole, time bin 21, center plane; the flow field during diastole was lesser than $0.1 \mathrm{~m} / \mathrm{s}$. A retrograde flow of $0.25 \mathrm{~m} / \mathrm{s}$ was observed at the trailing edge of the leaflet throughout diastole (avi, 586k, B_parallel_downstream_vmag.avi) 
Figure 7-111 Velocity fields downstream of the stent of prototype B during acceleration phase, time bin 5; high forward flow of $1.9 \mathrm{~m} / \mathrm{s}$ was seen emanating from the commissural region of the prototype B (avi, 613k, B_stent_downstream_ vmag.avi) 266

Figure 7-112 Velocity fields downstream of the stent of prototype B during peak systole, time bin 9; maximum velocity of $2.3 \mathrm{~m} / \mathrm{s}$ was observed issuing from the orifice of the valve. Three distinct flow regions were observed throughout the systole (avi, 613k, B_stent_downstream_vmag.avi).....

Figure 7-113 Reynolds shear stress distribution of prototype B during peak systole, time bin 9; elevated Reynolds shear stress of more than 1,000 dynes $/ \mathrm{cm}^{2}$ was observed at transition flow between the high and low flow regions (avi, 618k, B_stent_downstream_rss.avi) 267

Figure 7-114 Velocity fields downstream of the stent of prototype B during mid diastole, time bin 21; leakage flow was observed through the channel gap (see Figure 7-92e for the definition) at the commissure region (avi, 613k, B_stent_downstream_vmag.avi)... 268

Figure 7-115 Velocity fields inside prototype B during acceleration phase, time bin 5; high velocity of approximately $2.0 \mathrm{~m} / \mathrm{s}$ was observed along the trailing edge of the leaflet. No area of stagnation was observed in commissural region of prototype B (avi, 594k, B_inside_valve_vmag.avi)

Figure 7-116 Velocity fields inside prototype B during peak systole, time bin 9; flow velocity closer to the trailing edge of the valve was typically higher than that inside the valve (avi, 594k, B_inside_valve_vmag.avi)

Figure 7-117 Reynolds shear stress distribution of prototype B during peak systole, time bin 9; Reynolds shear stress level during systole was typically less than 100 dynes $/ \mathrm{cm}^{2}$ (avi, 592k, B_inside_valve_rss.avi)

Figure 7-118 Velocity fields inside prototype B during late diastole, time bin 38; a leakage jet of more than $3.5 \mathrm{~m} / \mathrm{s}$ was observed $2 \mathrm{~mm}$ upstream from the trailing edge of the leaflet and extended $5 \mathrm{~mm}$ inside the valve (avi, 594k, B_inside_valve_vmag.avi). 272

Figure 7-119 Reynolds shear stress distribution of prototype B during late diastole, time bin 38; maximum Reynolds shear stress of more than 10,000 dynes $/ \mathrm{cm}^{2}$ occurred at the leakage jet inside the valve during diastole. The Reynolds shear stress levels at other measurement locations inside the valve were less than $50 \mathrm{dynes} / \mathrm{cm}^{2} \mathrm{c}(\mathrm{avi}$, 592k, B_inside_valve_rss.avi)..... 272

Figure 7-120 Velocity fields downstream of prototype $\mathrm{C}$ at acceleration phase, time bin 5, center plane; a developing central orifice jet of $1.5 \mathrm{~m} / \mathrm{s}$ was seen issuing from the orifice of the polymeric valve (avi, 586k, C_parallel_downstream_vmag.avi) ..... 275 
Figure 7-121 Velocity fields downstream of prototype $\mathrm{C}$ at peak systole, time bin 9, center plane; a peak velocity of $2.1 \mathrm{~m} / \mathrm{s}$ was measured in the central orifice jet (avi, 586k, C_parallel_downstream_vmag.avi) 275

Figure 7-122 Reynolds shear stress distribution of prototype $\mathrm{C}$ at peak systole, time bin 9, center plane; elevated Reynolds shear stress levels of more than 1,000 dynes $/ \mathrm{cm}^{2}$ were observed at edge of the central orifice jet and $60 \mathrm{~mm}$ downstream from the edge of the valve (avi, 599k, C_parallel_downstream_rss.avi) ............................ 276

Figure 7-123 Velocity fields downstream of prototype $\mathrm{C}$ at mid diastole, time bin 21 , center plane; the flow field during diastole was low at around $0.04 \mathrm{~m} / \mathrm{s}$ (avi, 586k, C_parallel_downstream_vmag.avi)

Figure 7-124 Velocity fields downstream of the stent of prototype $\mathrm{C}$ during acceleration phase, time bin 5; two forward jets $(1.59 \mathrm{~m} / \mathrm{s})$ were seen issuing from the commissural region during the acceleration phase (avi, 605k, C_stent_downstream_vmag.avi). 278

Figure 7-125 Velocity fields downstream of the stent of prototype $\mathrm{C}$ during peak systole, time bin 9; high velocity jet of $1.9 \mathrm{~m} / \mathrm{s}$ was seen emanating from the commissural region at peak systole (avi, 605k, C_stent_downstream_vmag.avi)

Figure 7-126 Reynolds shear stress distribution of prototype $\mathrm{C}$ during peak systole, time bin 9; elevated Reynolds shear stress of more than 1,000 dynes $/ \mathrm{cm}^{2}$ was observed in the smaller forward jet, while the upper jet recorded a Reynolds shear stress of less than 130 dynes $/ \mathrm{cm}^{2}$ (avi, 619k, C_stent_downstream_rss.avi)

Figure 7-127 Velocity fields downstream of the stent of prototype $\mathrm{C}$ during mid diastole, time bin 21; leakage jets of approximately $0.61 \mathrm{~m} / \mathrm{s}$ were seen at the commissural region during mid diastole (avi, 605k, C_stent_downstream_vmag.avi)..... 280

Figure 7-128 Velocity fields inside prototype $\mathrm{C}$ during acceleration phase, time bin 5; high velocity of approximately $1.4 \mathrm{~m} / \mathrm{s}$ was observed along the trailing edge of the leaflet. No area of stagnation was observed in commissural region (avi, 684k, C_inside_valve_vmag.avi)....

Figure 7-129 Velocity fields inside prototype $\mathrm{C}$ at peak systole, time bin 9; a maximum velocity of $2.1 \mathrm{~m} / \mathrm{s}$ was recorded along the edge of the valve during peak systole (avi, 684k, C_inside_valve_vmag.avi) 282

Figure 7-130 Velocity fields inside prototype $\mathrm{C}$ during diastole, time bin 23; retrograde flow of approximately $1.8 \mathrm{~m} / \mathrm{s}$ appeared along leaflet trailing edge, while a leakage jet of $2.0 \mathrm{~m} / \mathrm{s}$ appeared at the high central region of the valve (avi, 684k, C_inside_valve_vmag.avi).

Figure 7-131 Reynolds shear stress distribution of prototype $\mathrm{C}$ during diastole, time bin 23; elevated Reynolds shear stress of more than 1,000 dynes $/ \mathrm{cm}^{2}$ occurred along the edge of the valve during diastole (avi, 706k, C_inside_valve_rss.avi) 283 
Figure 7-132 3D LDV velocity fields downstream of prototype $\mathrm{A}$ at acceleration phase, time bin 5, center plane; a developing central orifice jet of $1.3 \mathrm{~m} /$. A vortex was observed inside the top sinus region (avi, 454k,

A_parallel_downstream_3DLDV_vmag.avi).... 286

Figure 7-133 3D LDV Velocity fields downstream of prototype A at peak systole, time bin 9 , center plane; flow velocity of $2.0 \mathrm{~m} / \mathrm{s}$ was observed inside the central orifice jet at location coinciding with that of the flow constriction observed $21 \mathrm{~mm}$ downstream from the valve sewing ring (avi, 454k,

A_parallel_downstream_3DLDV_vmag.avi).... 287

Figure 7-134 3D LDV Velocity fields downstream of prototype A at deceleration phase, time bin 14, center plane; flow velocity in the central orifice jet reduced from 2.0 to $0.5 \mathrm{~m} / \mathrm{s}$. The recirculation zone expanded into the center of the flow chamber (avi, 454k, A_parallel_downstream_3DLDV_vmag.avi).... 287

Figure 7-135 3D LDV Reynolds shear stress distribution downstream of prototype A at peak systole, time bin 9, center plane; elevated Reynolds shear stress levels were observed along the edge of the central orifice jet and at the distal region of the flow chamber (avi, 468k, A_parallel_downstream_3DLDV_rss.avi) 288

Figure 7-136 3D LDV Reynolds shear stress distribution downstream of prototype A at deceleration phase, time bin 14, center plane; elevated Reynolds shear stress levels were observed at the distal region of the flow chamber (avi, 468k,

A_parallel_downstream_3DLDV_rss.avi)

Figure 7-137 3D LDV velocity fields downstream of prototype A at mid diastole, time bin 21, center plane; the flow field during diastole was less than $0.08 \mathrm{~m} / \mathrm{s}$ (avi, 454k, A_parallel_downstream_3DLDV_vmag.avi)..... 289

Figure 7-138 Pertinent terminology for the three Aortech $23 \mathrm{~mm}$ trileaflet polymeric heart valves. All downstream measurements were made with reference from the valve sewing ring, which is marked $\mathrm{x}=0$

Figure 7-139 Velocity fields at the center plane of prototype A during acceleration phase, time bin 5; vortex ring was observed at the edge of the central orifice jet (avi, 615k, A_center_plane_vmag.avi) 294

Figure 7-140 Velocity fields at the center plane of prototype A during peak systole, time bin 9; velocity central orifice jet of $2.3 \mathrm{~m} / \mathrm{s}$ was observed issuing from the valve orifice with flow separation occurring at the trailing edge of the leaflet. Plug flow profile was evident at the immediate downstream of leaflet trailing edge and became more parabolic at the distal end of the flow chamber (avi, 615k, A_center_plane_vmag.avi)

Figure 7-141 Iso-surface of velocity magnitude of prototype A at peak systole, time bin 9; the flow profile at peak systole had a three-lobe configuration (avi, 915k, A_isosurface_vmag.avi) 295 
Figure 7-142 Reynolds shear stress contour plot of the center plane of prototype A during peak systole, time bin 9; high Reynolds shear stress values coincided with regions of high velocity gradient typically observed at the edge of the central orifice jet and at the distal part of the flow chamber (avi, 821k, A_center_plane_rss.avi).... 295

Figure 7-143 Velocity fields at the center plane of prototype A during mid diastole, time bin 21; leakage jet appeared at the coaptation region of the valve. See Figure 7-144 for the flow fields inside the enlarged red dotted box (avi, 615k,

A_center_plane_vmag.avi) 296

Figure 7-144 Velocity fields inside prototype A during mid diastole, time bin 21, center plane; split flow phenomenon evident inside the valve; one directed toward the lower stent and another toward the leakage jet at the high central region

Figure 7-145 Reynolds shear stress contour plot of the center plane of prototype A during mid diastole, time bin 21; elevated Reynolds shear stress of 5,000 dynes $/ \mathrm{cm}^{2}$ was observed in the leakage jet at the high central region of the valve (avi, 821, A_center_plane_rss.avi).

Figure 7-146 Velocity fields at the $+4 \mathrm{~mm}$ offset plane of prototype A acceleration phase, time bin 5; velocity of $1.5 \mathrm{~m} / \mathrm{s}$ was measured inside the central orifice jet (avi, $623 \mathrm{k}$, A_+4mm_plane_vmag.avi) 299

Figure 7-147 Velocity fields at the $+4 \mathrm{~mm}$ offset plane of prototype A during peak systole, time bin 9; a maximum velocity of $2.4 \mathrm{~m} / \mathrm{s}$ was observed inside the central orifice jet at a location coinciding with the contraction of flow $20 \mathrm{~mm}$ from the valve sewing ring (avi, 623k, A_+4mm_plane_vmag.avi).

Figure 7-148 Reynolds shear stress contour plot of the $+4 \mathrm{~mm}$ offset plane of prototype A during peak systole, time bin 9; high Reynolds shear stress values coincided with regions of high velocity gradient typically observed at the edge of the central orifice jet and at the distal part of the flow chamber (avi, 652k, A_+4mm_plane_rss.avi)300

Figure 7-149 Velocity fields at the $+4 \mathrm{~mm}$ offset plane of prototype A during mid diastole, time bin 21; leakage jet appeared at the near the coaptation region of the valve (avi, 623k, A_+4mm_plane_vmag.avi).....

Figure 7-150 Reynolds shear stress contour plot of the $+4 \mathrm{~mm}$ offset plane of prototype A during mid diastole, time bin 21; elevated Reynolds shear stress of more than 1,000 dynes $/ \mathrm{cm}^{2}$ was observed in the leakage jet inside the valve (avi, 652k, A_+4mm_plane_rss.avi) 301

Figure 7-151 Velocity fields at the $+8 \mathrm{~mm}$ offset plane of prototype A during the acceleration phase, time bin 5; part of the central orifice jet downstream of the valve and that inside the valve were visible at this offset plane (avi, 602k, A_+8mm_plane_vmag.avi) 
Figure 7-152 Velocity fields at the $+8 \mathrm{~mm}$ offset plane of prototype A during peak systole, time bin 9; the central orifice jet reached the velocity of $2.1 \mathrm{~m} / \mathrm{s}$ at peak systole. Elevated velocity of $2.0 \mathrm{~m} / \mathrm{s}$ was also observed inside the valve near the trailing edge of the leaflet (avi, 602k, A_+8mm_plane_vmag.avi).

Figure 7-153 Reynolds shear stress contour plot of the $+8 \mathrm{~mm}$ offset plane of prototype A during peak systole, time bin 9; high Reynolds shear stress values coincided with regions of high velocity gradient typically observed at the edge of the central orifice jet (avi, 666k, A_+8mm_plane_rss.avi)... 304

Figure 7-154 Velocity fields at the $+8 \mathrm{~mm}$ offset plane of prototype A during mid diastole, time bin 21; a small leakage jet of $0.3 \mathrm{~m} / \mathrm{s}$ was observed near the top stent post of the valve and persisted throughout the diastole (avi, $602 \mathrm{k}$, A_+8mm_plane_vmag.avi) 304

Figure 7-155 Velocity fields at the $+12 \mathrm{~mm}$ offset plane of prototype A during the acceleration phase, time bin 5; flow velocity downstream from the leaflet trailing edge was typically less than $0.03 \mathrm{~m} / \mathrm{s}$ during the acceleration phase (avi, 569k, A_+12mm_plane_vmag.avi)

Figure 7-156 Velocity fields at the $+12 \mathrm{~mm}$ offset plane of prototype A during peak systole, time bin 9; the central orifice jet of $0.75 \mathrm{~m} / \mathrm{s}$ was observed at location 35 $\mathrm{mm}$ downstream from the valve sewing ring (avi, 569k, A_+12mm_plane_ vmag.avi)

Figure 7-157 Reynolds shear stress contour plot of the $+12 \mathrm{~mm}$ offset plane of prototype A peak systole, time bin 9; elevated Reynolds shear stress was observed $45 \mathrm{~mm}$ downstream of the valve sewing ring at the location where the central orifice jet mix with the surrounding flow (avi, 663k, A_+12mm_plane_rss.avi).... 307

Figure 7-158 Velocity fields at the $+12 \mathrm{~mm}$ offset plane of prototype A during mid diastole, time bin 21; vortex structure was observed inside the edge of the sinus region throughout the diastole (avi, 569k, A_+12mm_plane_vmag.avi). 308

Figure 7-159 Velocity fields at the center plane of prototype B during acceleration phase, time bin 5; central orifice jet of $1.5 \mathrm{~m} / \mathrm{s}$ was observed emanating from the valve orifice. Vortex ring was seen along the edge of the central jet (avi, 594k, B_center_plane_vmag.avi)

Figure 7-160 Velocity fields at the center plane of prototype B during peak systole, time bin 9; recirculation flow observed at the upper region of the chamber wall (avi, 594k, B_center_plane_vmag.avi)

Figure 7-161 Iso-surface of velocity magnitude of prototype B at peak systole, time bin 9. The iso-surface of velocity had a three lobe configuration (avi, 945k, B_isosurface_vmag.avi). 
Figure 7-162 Reynolds shear stress contour plot of the center plane of prototype B during peak systole, time bin 9; high Reynolds shear stress values observed along the edge of the central orifice jet and at the commissural region inside the valve (avi, 728k, B_center_plane_rss.avi).

Figure 7-163 Velocity fields at the center plane of prototype B during mid diastole, time bin 21; leakage jets observed at the both the coaptation and commissural regions of the valve (avi, 594k, B_center_plane_vmag.avi) 313

Figure 7-164 Reynolds shear stress contour plot of the center plane of prototype B during mid diastole, time bin 21; elevated Reynolds shear stress levels of more than 5,000 dynes $/ \mathrm{cm}^{2}$ were observed in the leakage jets at the coaptation region and the commissural region (avi, 728k, B_center_plane_rss.avi).

Figure 7-165 Velocity fields at the $+4 \mathrm{~mm}$ offset plane of prototype B acceleration phase, time bin 5; velocity of $1.4 \mathrm{~m} / \mathrm{s}$ was measured inside the central orifice jet (avi, 602k, B_+4mm_plane_vmag.avi)..... 316

Figure 7-166 Velocity fields at the $+4 \mathrm{~mm}$ offset plane of prototype B during peak systole, time bin 9; maximum flow velocity of $2.1 \mathrm{~m} / \mathrm{s}$ was reached inside the central orifice jet at peak systole (avi, 602k, B_+4mm_plane_vmag.avi). 316

Figure 7-167 Reynolds shear stress contour plot of the $+4 \mathrm{~mm}$ offset plane of prototype B during peak systole, time bin 9; high Reynolds shear stress values observed along the edge of the central orifice jet, the trailing edge of the leaflet, and at the commissural region of the valve (avi, 818k, B_+4mm_plane_rss.avi)

Figure 7-168 Velocity fields at the $+4 \mathrm{~mm}$ offset plane of prototype B during mid diastole, time bin 20; leakage jet of $0.95 \mathrm{~m} / \mathrm{s}$ appeared at the near the coaptation region of the valve (avi, 602k, B_+4mm_plane_vmag.avi)....

Figure 7-169 Velocity vectors inside prototype B during mid-diastole, time bin $20,+4$ $\mathrm{mm}$ offset plane; vortex structures were observed at two location inside the valve; one near the top stent post next to the leakage jet and another near the rear of the valve

Figure 7-170 Reynolds shear stress contour plot of the $+4 \mathrm{~mm}$ offset plane of prototype B during mid-diastole, time bin 20; elevated Reynolds shear stress levels of more than $1,900 \mathrm{dyne} / \mathrm{cm}^{2}$ were observed in the leakage jet at the high central region of the valve (avi, 602k, B_+4mm_plane_vmag.avi).

Figure 7-171 Velocity fields at the $+8 \mathrm{~mm}$ offset plane of prototype B during the acceleration phase, time bin 5; a velocity of $1.5 \mathrm{~m} / \mathrm{s}$ was observed inside the central orifice jet (avi, 614k, B_+8mm_plane_vmag.avi) 321

Figure 7-172 Velocity fields at the $+8 \mathrm{~mm}$ offset plane of prototype B during peak systole, time bin 9; a region of reverse flow of $0.6 \mathrm{~m} / \mathrm{s}$ was evident at the lower edge of the central orifice jet (avi, 614k, B_+8mm_plane_vmag.avi) 
Figure 7-173 Reynolds shear stress contour plot of the $+8 \mathrm{~mm}$ offset plane of prototype B during peak systole, time bin 9; high Reynolds shear stress values coincided with regions of high velocity gradient typically observed along the edge of the central orifice jet (avi, 629k, B_+8mm_plane_rss.avi)

Figure 7-174 Velocity fields at the $+8 \mathrm{~mm}$ offset plane of prototype B during mid diastole, time bin 21; a small leakage jet of $0.6 \mathrm{~m} / \mathrm{s}$ was observed near the top stent post of the valve and persisted throughout the diastole (avi, 614k, B_+8mm_plane_vmag.avi).....

Figure 7-175 Velocity fields at the $\pm 12 \mathrm{~mm}$ offset plane of prototype B during the acceleration phase, time bin 5; forward flow velocity of $0.46 \mathrm{~m} / \mathrm{s}$ was observed 35 $\mathrm{mm}$ downstream from the valve sewing ring (avi, 559k, B_+12mm_plane_ vmag.avi)

Figure 7-176 Velocity fields at the $+12 \mathrm{~mm}$ offset plane of prototype B during peak systole, time bin 9; opposing flows of approximately $0.90 \mathrm{~m} / \mathrm{s}$ evident at the downstream region of the valve (avi, 559k, B_+12mm_plane_vmag.avi).

Figure 7-177 Reynolds shear stress contour plot of the $+12 \mathrm{~mm}$ offset plane of prototype B peak systole, time bin 9; elevated Reynolds shear stress was observed $45 \mathrm{~mm}$ downstream of the valve sewing ring at the location where the forward flow mix with the surrounding flow (avi, 610k, B_+12mm_plane_rss.avi) .... 325

Figure 7-178 Velocity vectors at the $+12 \mathrm{~mm}$ offset plane of prototype B during diastole, time bin 21; flow was typically lower than $0.09 \mathrm{~m} / \mathrm{s}$ downstream from the leaflet trailing edge and $0.03 \mathrm{~m} / \mathrm{s}$ inside the sinus region (avi, 559k, B_+12mm_plane_vmag.avi)

Figure 7-179 Velocity fields at the center plane of prototype $\mathrm{C}$ during acceleration phase, time bin 5; central orifice jet of $1.35 \mathrm{~m} / \mathrm{s}$ was observed emanating from the valve orifice with the formation of vortex ring was seen along the edge of the central jet (avi, 599k, C_center_plane_vmag.avi)

Figure 7-180 Velocity fields at the center plane of prototype $\mathrm{C}$ during peak systole, time bin 9; a maximum velocity of $2.1 \mathrm{~m} / \mathrm{s}$ was recorded inside the central orifice jet during peak systole (avi, 599k, C_center_plane_vmag.avi).....

Figure 7-181 Iso-surface of velocity magnitude of prototype $\mathrm{C}$ at peak systole, time bin 9; the velocity iso-surface of prototype $\mathrm{C}$ did not have the distinct three-lobe feature as seen in prototype A and B. The flow profile was much more circular as the central jet approached the distal end of the flow chamber (avi, 929k, C_isosurface_ vmag.avi) 330

Figure 7-182 Reynolds shear stress contour plot of the center plane of prototype C during peak systole, time bin 9; high Reynolds shear stress values observed along the edge of the central orifice jet and at the commissural region inside the valve (avi, 635k, C_center_plane_rss.avi).... 330 
Figure 7-183 Velocity fields at the center plane of prototype $\mathrm{C}$ during diastole, time bin 21; leakage jets of more than $0.7 \mathrm{~m} / \mathrm{s}$ were seen at both the coaptation and the commissural region in the valve (avi, 599k, C_center_plane_vmag.avi).....

Figure 7-184 Reynolds shear stress contour plot of the center plane of prototype $\mathrm{C}$ during diastole, time bin 21; elevated Reynolds shear stress levels of more than 4,000 dynes $/ \mathrm{cm}^{2}$ observed along the trailing edge of the valve (avi, $635 \mathrm{k}$, C_center_plane_rss.avi)....

Figure 7-185 Velocity fields at the $+4 \mathrm{~mm}$ offset plane of prototype $\mathrm{C}$ acceleration phase, time bin 5; velocity of $1.5 \mathrm{~m} / \mathrm{s}$ was measured inside the central orifice jet (avi, 600k, C_+4mm_plane_vmag.avi). 334

Figure 7-186 Velocity fields at the $+4 \mathrm{~mm}$ offset plane of prototype $\mathrm{C}$ during peak systole, time bin 9; maximum flow velocity of $2.1 \mathrm{~m} / \mathrm{s}$ was reached inside the central orifice jet at peak systole (avi, 600k, C_+4mm_plane_vmag.avi)..... 334

Figure 7-187 Reynolds shear stress contour plot of the $+4 \mathrm{~mm}$ offset plane of prototype $\mathrm{C}$ during peak systole, time bin 9; high Reynolds shear stress values observed along the edge of the central orifice jet and at the top stent post of the valve (avi, 767k, C_+4mm_plane_rss.avi)

Figure 7-188 Velocity fields at the $+4 \mathrm{~mm}$ offset plane of prototype $\mathrm{C}$ during mid diastole, time bin 21 ; leakage jets of approximately $0.3 \mathrm{~m} / \mathrm{s}$ were seen at both the coaptation region and near the rear part of the valve during diastole (avi, 600k, C_+4mm_plane_vmag.avi). 335

Figure 7-189 Velocity fields at the $+8 \mathrm{~mm}$ offset plane of prototype $\mathrm{C}$ during the acceleration phase, time bin 5; a velocity of $1.2 \mathrm{~m} / \mathrm{s}$ was observed inside the central orifice jet with vortex ring formation at the edge of the jet (avi, 576k, C_+8mm_plane_vmag.avi)..... 337

Figure 7-190 Velocity fields at the $+8 \mathrm{~mm}$ offset plane of prototype $\mathrm{C}$ during peak systole, time bin 9; a maximum velocity of $2.0 \mathrm{~m} / \mathrm{s}$ was recorded inside the central orifice jet at peak systole (avi, 576k, C_+8mm_plane_vmag.avi) .................................... 338

Figure 7-191 Reynolds shear stress contour plot of the $+8 \mathrm{~mm}$ offset plane of prototype $\mathrm{C}$ during peak systole, time bin 9; high Reynolds shear stress values coincided with regions mixing between the central orifice jet and the surrounding fluid (avi, 667k, C_+8mm_plane_rss.avi) 338

Figure 7-192 Velocity fields at the $+8 \mathrm{~mm}$ offset plane of prototype $\mathrm{C}$ during mid diastole, time bin 21; a small leakage jet of $0.26 \mathrm{~m} / \mathrm{s}$ was observed near the top stent post of the valve and persisted throughout the diastole (avi, 576k, C_+8mm_plane_vmag.avi)..... 339

Figure 7-193 Reynolds shear stress contour plot of the $+8 \mathrm{~mm}$ offset plane of prototype $\mathrm{C}$ during mid diastole, time bin 21; elevated Reynolds shear stress values were 
observed at the top stent post coinciding with the location of the leakage jet (avi, $667 \mathrm{k}$, C_+8mm_plane_rss.avi)

Figure 7-194 Velocity fields at the $+12 \mathrm{~mm}$ offset plane of prototype $\mathrm{C}$ during the acceleration phase, time bin 5; vortex was observed at the edge of the sinus region during the acceleration phase (avi, 550k, C_+12mm_plane_vmag.avi)....

Figure 7-195 Velocity fields at the $+12 \mathrm{~mm}$ offset plane of prototype $\mathrm{C}$ during peak systole, time bin 9; maximum flow velocity of $0.65 \mathrm{~m} / \mathrm{s}$ was recorded at location 55 $\mathrm{mm}$ from the valve sewing ring (avi, 550k, C_+12mm_plane_vmag.avi).

Figure 7-196 Reynolds shear stress contour plot of the $+12 \mathrm{~mm}$ offset plane of prototype C peak systole, time bin 9; elevated Reynolds shear stress was observed $45 \mathrm{~mm}$ downstream of the valve sewing ring at the location where the forward flow mix with the surrounding flow (avi, 602k, C_+12mm_plane_rss.avi)

Figure 7-197 Velocity vectors at the $+12 \mathrm{~mm}$ offset plane of prototype $\mathrm{C}$ during diastole, time bin 21; flow velocity was typically lower than $0.1 \mathrm{~m} / \mathrm{s}$ throughout the entire measurement plane during diastole (avi, 550k, C_+12mm_plane_vmag.avi)........ 343

Figure 7-198 3D surface topography of the leaflet of heart valve prostheses; (a) the leaflet surface of the pyrolytic carbon in the bileaflet mechanical valve, (b) the leaflet surface of the polymeric heart valve (prototype B)

Figure 7-199 The inner surface roughness of the polymeric heart valve 345

Figure 8-1 Flow features inside the hinge pocket of the three $27 \mathrm{~mm}$ bileaflet clear housing valve models; (a) leakage jets at the atrial side of the hinge socket during systole; (b) recirculation near the adjacent corner during diastole 353

Figure 8-2 Location of the lockup in the SJM bileaflet mechanical heart valve

Figure 8-3 Sketch of the flow characteristics of the SJM bileaflet mechanical heart valve as observed in the hydrogen bubble experiments [Shu, 2003] 364

Figure 8-4 The comparison of the washout flow characteristics during $\mathrm{s}$ in the mitral hinge studies; (a) The $27 \mathrm{~mm}$ prototype high leaker valve had a less efficient forward washout and a higher propensity for air bubbles to recirculate inside the pivot region, (b) The CM $23 \mathrm{~mm}$ valve had better washout due to a flatter hinge profile.

Figure 8-5 The opening configuration of the polymeric valve's leaflets during the acceleration phase; (a) gradual opening of the leaflets, (b) the edge of the leaflets opens faster than the middle portion, and (c) the formation of a crest at peak systole. Dotted arrows show the motion of the leaflets and bold arrows the fluid motion/behavior 388 
Figure 8-6 Three-lobe flow features common in the three prototype polymeric heart valves. This flow feature was more distinct in the prototype A than prototypes B and $\mathrm{C}$ as a result of the closed commissural design in prototype $\mathrm{A}$ 390

Figure 8-7 Schematic of the flow field at the stent during the acceleration and early diastolic phases for prototypes A. The closed commissural design of prototype A produced a forward and leakage jets at the gap channel during systole and diastole, respectively. The cross-hatched areas depict the regions where the adjacent leaflets at the commissural region come together 392

Figure 8-8 Schematic of the flow field at the stent during the acceleration and early diastolic phases for prototypes B. An unobstructed forward flow was observed at the commissural region in prototype B during systole, while a leakage jet was observed next to the gap channel during diastole. The cross-hatched areas depict the regions where the adjacent leaflets at the commissural region come together. 393

Figure 8-9 Schematic of the flow field at the stent during the acceleration and early diastolic phases for prototypes C. A small forward and leakage jet observed during systole and diastole, respectively, corresponded to the flow at the gap channel. The cross-hatched areas depict the regions where the adjacent leaflets at the commissural region come together. 393

Figure 8-10 Comparison of flow patterns and leaflet configuration at peak systole among the three heart valve prostheses: (a) porcine bioprosthesis, (b) pericardial bioprosthesis, and (c) Aortech polymeric heart valve [Black, 1991; Woo, 1983a] 398

Figure 8-11 Thrombi observed at the commissural region and along the stent inflow region of implanted polymeric heart valves; (a) aortic position [Daebritz, 2004], (b) mitral position [Daebritz, 2003]. 401

Figure 8-12 Stream traces of the flow inside prototype A during diastole 402

Figure 8-13 High speed images of prototype A illustrating the closing motion of the valve. (a) The valve closes first at the lateral side of the leaflets (dotted arrows) (b) forcing fluid 403

Figure 8-14 Crest and trough pattern in the inner surface of polymeric heart valve ...... 405

Figure 8-15 Comparison of velocity magnitude profile obtained by the 2D LDV, 3D LDV and the 3D PIV immediately downstream from the leaflet trailing edge of prototype A at peak systole, time bin 9 408

Figure 8-16 Comparison of Reynolds shear stress profile obtained by the 2D LDV, 3D LDV and the 3D PIV immediately downstream of the leaflet trailing edge of prototype $\mathrm{A}$ at peak systole, time bin 9 409 
Figure 8-17 Comparison of velocity magnitude profile obtained by the 2D LDV, 3D LDV and the 3D PIV $40 \mathrm{~mm}$ downstream from the sewing ring of prototype A at peak systole, time bin 9 410

Figure 8-18 Comparison of Reynolds shear stress profile obtained by the 2D LDV, 3D LDV and the 3D PIV $40 \mathrm{~mm}$ downstream from the sewing ring of prototype A at peak systole, time bin 9 .

Figure 8-19 Comparison of $U_{r m s}$ profile obtained by the 2D LDV, 3D LDV and the 3D PIV $40 \mathrm{~mm}$ downstream from the sewing ring of prototype $A$ at peak systole, time bin 9

Figure 8-20 Comparison of velocity magnitude profile obtained by the 2D LDV and the 3D PIV $40 \mathrm{~mm}$ downstream from the sewing ring of prototype B at peak systole, time bin 9

Figure 8-21 Comparison of Reynolds shear stress profile obtained by the 2D LDV and the 3D PIV $40 \mathrm{~mm}$ downstream from the sewing ring of prototype $\mathrm{B}$ at peak systole, time bin 9

Figure 8-22 Comparison of $U_{r m s}$ profile obtained by the 2D LDV and the 3D PIV $40 \mathrm{~mm}$ downstream from the sewing ring of prototype $B$ at peak systole, time bin 9 ...... 413

Figure 8-23 Comparison of velocity magnitude profile obtained by the 2D LDV and the 3D PIV $40 \mathrm{~mm}$ downstream from the sewing ring of prototype $\mathrm{C}$ at peak systole, time bin 9

Figure 8-24 Comparison of Reynolds shear stress profile obtained by the 2D LDV and the 3D PIV $40 \mathrm{~mm}$ downstream from the sewing ring of prototype $\mathrm{C}$ at peak systole, time bin 9 414

Figure 8-25 Comparison of $U_{r m s}$ profile obtained by the 2D LDV and the 3D PIV $40 \mathrm{~mm}$ downstream from the sewing ring of prototype $C$ at peak systole, time bin 9 ...... 415

Figure 8-26 Comparison of velocity magnitude profile obtained by the 2D LDV and the 3D PIV along the trailing edge of prototype A at peak systole, time bin 9

Figure 8-27 Comparison of Reynolds shear stress profile obtained by the 2D LDV and the 3D PIV along the leaflet trailing edge of prototype $A$ at peak systole, time bin 9

Figure 8-28 Comparison of $U_{r m s}$ profile obtained by the 2D LDV and the 3D PIV along the leaflet trailing edge of prototype $\mathrm{A}$ at peak systole, time bin 9 . 417

Figure 8-29 Comparison of velocity magnitude profile obtained by the 2D LDV and the $3 \mathrm{D}$ PIV along the trailing edge of prototype B at peak systole, time bin 9 418 
Figure 8-30 Comparison of Reynolds shear stress profile obtained by the 2D LDV and the 3D PIV along the leaflet trailing edge of prototype B at peak systole, time bin 9

Figure 8-31 Comparison of $U_{r m s}$ profile obtained by the 2D LDV and the 3D PIV along the leaflet trailing edge of prototype B at peak systole, time bin 9

Figure 8-32 Comparison of velocity magnitude profile obtained by the 2D LDV and the $3 \mathrm{D}$ PIV along the trailing edge of prototype $\mathrm{C}$ at peak systole, time bin 9

Figure 8-33 Comparison of Reynolds shear stress profile obtained by the 2D LDV and the 3D PIV along the leaflet trailing edge of prototype $\mathrm{C}$ at peak systole, time bin 9

Figure 8-34 Comparison of $U_{r m s}$ profile obtained by the 2D LDV and the 3D PIV along the leaflet trailing edge of prototype $\mathrm{C}$ at peak systole, time bin 9

Figure 8-35 Shows the overlapping of field of view for 2D, 3D LDV and 3D PIV. The different field of view constitute one of the main reasons for the observed different in the different flow features inside and in the vicinity of the polymeric heart valves

Figure 9-1 Clot locations inside an explanted polymeric valve (prototype B). Figure was obtained from preliminary animal trials performed with sheep by Dr Wheatley DJ of the University of Glasgow. Courtesy of Aortech, Inc. 434

Figure 10-1 Raw 2D PIV image inside a SJM $23 \mathrm{~mm}$ Regent clear valve housing. About half of the hinge region was captured at: a) $200 \mu \mathrm{m}$ above the flat level and b) at the primary flat level. 438

Figure 10-2 Velocity map inside the hinge region of a SJM $23 \mathrm{~mm}$ Regent clear valve housing; a) at $200 \mu \mathrm{m}$ above the flat level and b) at the primary flat level 439

Figure 10-3 Micro CT scan image of the hinge region of a SJM 23 mm Regent valve. 440

Figure 10-4 CT-scan image of polymeric valve A 441 


\title{
LIST OF SYMBOLS AND ABBREVIATIONS
}

\author{
$a \quad$ Number of cycles or constant (dimensionless) \\ $f \quad$ Aperture number \\ $g$ \\ Acceleration of gravity $\left(\mathrm{m} / \mathrm{s}^{2}\right)$ \\ $G_{i o} \quad$ Gate time averaged over cycle $i(\mathrm{~s})$ \\ $G_{i} \quad$ Specific gate time measurement (s) \\ $G_{i j} \quad$ Specific gate time measurement within cycle $i(\mathrm{~s})$ \\ $h \quad$ Height (m) \\ L $\quad$ Length (m) \\ $M_{\text {cal }}, N_{\text {cal }} \quad$ Grid size on CCD camera (pixels) \\ $m \quad$ Total number of measurement locations studied (dimensionless) \\ $N \quad$ Number of PIV image pairs \\ $N_{v} \quad$ Array of totally independent vectors \\ $N_{\text {int }} \quad$ Side of the interrogation area (pixels)
}


$n$

V

$p$

$\rho$

$R_{a}$

$R_{q}$

$R_{1} R_{2} \quad$ Correlation functions of CCD camera image pairs

$\overline{\sigma_{p 1}} \overline{\sigma_{p 2}}$

$T, t$

$U$

$\bar{U}, u_{o}, v_{o} \quad$ Average velocity $(\mathrm{m} / \mathrm{s})$

$u_{i o}, v_{i o} \quad$ Velocity averaged over cycle $i(\mathrm{~m} / \mathrm{s})$

$u_{o o}, v_{o o} \quad$ Velocity averaged over all cycles $(\mathrm{m} / \mathrm{s})$

$u_{i o}, v_{i o} \quad$ Sum of velocity measurements over cycle $i(\mathrm{~m} / \mathrm{s})$

$u^{\prime} v^{\prime} \quad$ Fluctuating velocity $(\mathrm{m} / \mathrm{s})$

Kinematic viscosity (cSt, 1/1000,000 m²/s)

Pressure $(\mathrm{mm} \mathrm{Hg})$

Density $\left(\mathrm{kg} / \mathrm{m}^{3}\right)$

Surface roughness average $(\mathrm{nm})$

Root-mean-square of surface roughness (nm)

Time (s)$$
\text { Velocity averaged over cycle } i(\mathrm{~m} / \mathrm{s})
$$

Number of measurements or normal vector (dimensionless)

Time averaged principal normal stresses (dynes $/ \mathrm{cm}^{2}, 10 \times \mathrm{N} / \mathrm{m}^{2}$ )

Instantaneous axial velocity $(\mathrm{m} / \mathrm{s})$ 

$u_{i}, v_{i} \quad$ Specific velocity measurement $(\mathrm{m} / \mathrm{s})$
$u_{i j}, v_{i j} \quad$ Specific velocity measurement within cycle $i(\mathrm{~m} / \mathrm{s})$
uv Correlation between two orthogonal fluctuating velocity components $(\mathrm{m} / \mathrm{s})$
$\tau \quad$ Reynolds shear stress (dynes $\left./ \mathrm{cm}^{2}, 10 \times \mathrm{N} / \mathrm{m}^{2}\right)$
Maximum time averaged Reynolds shear stress $\left(\right.$ dynes $/ \mathrm{cm}^{2}, 10 \times \mathrm{N} / \mathrm{m}^{2}$ )
$X \quad$ Pixel coordinate on CCD camera (pixels)
$\Delta x_{c a l} \quad$ Calibration displacement in $\mathrm{x}$ axis
$Y \quad$ Pixel coordinate on CCD camera (pixels)
$\Delta y_{c a l} \quad$ Calibration displacement in y axis
$\Delta z_{c a l} \quad$ Calibration displacement in y axis

ATS Advancing The Standard

CFD Computational fluid dynamics

CM CarboMedics

HITS High intensity transient signals

LDV Laser Doppler velocimetry 
MA Medtronic Advantage

MP Medtronic Parallel

PIV Particle image velocimetry

RSS Reynolds shear stress (dynes $/ \mathrm{cm}^{2}$ )

SJM St. Jude Medical 


\section{SUMMARY}

Current designs of bileaflet mechanical heart valves (BMHVs) and trileaflet polymeric heart valves(TPHVs) are plagued by unacceptable levels of hemolysis and thrombus formation in critical areas thereby producing mediocre clinical performance. The objective of this study is: (1) to investigate the influence of BMHV designs on hinge flow characteristics, (2) to quantify the influence of hinge gap width tolerance in a BMHV design, and (3) to investigate the influence of TPHV design on flow characteristics.

St. Jude Medical (SJM) provided four transparent mitral BMHVs: one $23 \mathrm{~mm}$ CarboMedics (CM), one $27 \mathrm{~mm}$ SJM Standard and two $27 \mathrm{~mm}$ prototype BHMVs with altered hinge gap widths. Aortech Inc. provided three $23 \mathrm{~mm}$ aortic prototype TPHVs. Laser Doppler velocimetry and Particle Image velocimetry were used to measure flow velocity inside these valve prostheses. The flows through the valves were maintained within physiological limits.

All valves revealed Reynolds shear stress (RSS) levels greater than 200 Pa far exceeding the threshold for platelet activation and hemolysis. MHV hinge flows in the mitral position were characterized by a strong recirculation during ventricular diastole while leakage jets over and adjacent to leaflets were prominent during ventricular systole. CM hinge flow had higher RSS than in the SJM hinge. The large gap width hinge had the largest leakage jet size and highest RSS ( $>400 \mathrm{~Pa}$ ) during ventricular diastole. The 
Standard gap width hinge showed better washout during systole and provided optimum hemodynamic performance than the prototype designs. In aortic prototype PHVs, elevated RSS conducive to hemolysis was observed along the central jet during systole and the leakage jet at the high central region inside the valve during diastole.

This study showed that hinge geometry designs and hinge gap width tolerance governed the success of the bileaflet MHV design. Also the performance of the three aortic PHVs is dependent on commissural designs and leaflet thicknesses. Owing to the critical nature flow fields on clinical outcomes studies such as the current study should be conducted in the pre-clinical evaluation phase for all new MHV or PHVs. 


\section{CHAPTER 1: INTRODUCTION}

Heart valve diseases are a major component of heart diseases that affect more than a million people every year worldwide. Since the 1960s, the replacement of defective heart valves with prosthetic valves has been a routine surgical treatment option for this condition. Over 250,000 heart valve replacements are performed worldwide every year [Black, 1994; Vongpatanasin, 1996]. Nearly 65\% of these replacements opted for mechanical heart valves (MHV) due to their superior durability and satisfactory bulk flow hemodynamics as opposed to non-mechanical options such as the bioprosthetic heart valves. Nevertheless, all mechanical heart valves are prone to thromboembolic complications thus necessitating lifelong anti-coagulation therapy for improved prognosis [Jamieson, 2002; Turitto, 1998]. Although the mechanisms that lead to thromboembolic complications and thrombus formation in these valves are still not well understood it is theorized that the materials of the valve and the turbulent flow patterns in the valve contribute to hemolysis and platelets activation, thus leading to thrombosis.

The most widely implanted mechanical heart valve has a bileaflet valve design with more than 130,000 of them implanted every year worldwide. A certain degree of regurgitant flow is ensured by the bileaflet design for sufficient washout of certain critical areas of the valve, such as the leaflet hinges and the region between leaflet edges and the valve housing. This washout is intended to prevent flow stasis that may be created in the vicinity of the hinge regions and upstream of the valve housing during valve closure and 
minimize the formation of thrombi. Recent studies have shown that the bileaflet MHV hinge geometry governs the leakage flow characteristics such as the leakage velocities and Reynolds shear stresses (RSS), within and near the hinge region [Ellis, 1996a; Ellis, 2000a; Ellis, 2000b]. The constricted flow area at the hinge region of these MHVs coupled with the high-pressure difference across the bileaflet valves during diastole causes the fluid to be subjected to high turbulent shear stresses. These studies suggest that the hinge as the most critical part of the bileaflet MHV since it directly influences the valve durability, functionality, and thrombus formation.

Another promising direction of artificial heart valve research is the development of polymeric heart valves built from polymeric material so as to mimic the function of the human heart valve. Compared to existing MHVs, the polymeric valve should have the potential to reduce or prevent thrombosis-related complications, thus eliminating or reducing the need for anticoagulant therapy [Wheatley, 2001]. Furthermore, polymeric valves have the potential to reduce calcification related problems currently experienced by bioprosthetic valves. However, recent in vivo animals experiments reported that the polymeric heart valve was plagued by thrombus formation at the leaflet/stent juncture [Daebritz, 2003; Daebritz, 2004]. Currently, very few in vitro studies have been performed to characterize the flow through the polymeric valves. With the absence of detailed flow information, the assessment of how valve design affects blood elements is difficult. Therefore, a detailed characterization of the flow fields inside and in the vicinity of the polymeric heart valve may provide important information regarding the relationship between flow structures and the observed blood clots. 
The objective of this study is two fold. The first goal is to investigate the hinge and nearhinge flow fields of two bileaflet mechanical heart valve designs: the CarboMedics (CM) and the St Jude Medical (SJM) bileaflet heart valves. In addition, the effect of various hinge gap tolerances of the SJM valve on the flow field is also studied. The second goal of this study is to conduct a detailed investigation of the fluid dynamic performance of three polymeric tri-leaflet valve designs provided by Aortech, Inc. The study focuses on the performance of the three prototype valves based on their different commissural designs and leaflet thicknesses as well as the influence of various observed flow structures on the potential for thrombus formation.

The broader objective of this study is to provide an improved quantitative and qualitative understanding of the functionality and potential thrombogenicity of bileaflet MHVs and polymeric heart valves beyond that available from previous studies. The results of this work should provide new insight into the roles the subtle design features, such as the commissural designs and leaflet thickness of the polymeric heart valve and the hinge tolerance gap width of the bileaflet valve, have on the potential for blood damage. This data is particularly relevant to heart valve manufacturers when considering new or modified valve designs that may have less damaging flow fields. 


\section{CHAPTER 2: BACKGROUND}

\section{$\underline{2.1 \text { The Heart }}$}

The human heart is a four chamber pump that pumps blood through both the systemic and pulmonary circulatory systems. The upper two chambers, the right and left atria, are the chambers that receive the blood from the veins, whereas the lower two pumping chambers, the right and left ventricles, propel blood through the body and the lungs. There are four valves within the heart that control the flow direction and help regulate blood pressure (Figure 2-1). The atrioventricular valves are located between the atria and ventricles; the tricuspid valve resides between the right atrium and the right ventricle, and the mitral valve is between the left atrium and the left ventricle. The semilunar valves are located between the ventricles and arteries; the pulmonary valve resides between the right ventricle and pulmonary artery, and the aortic valve is between the left ventricle and aorta. All of the heart valves consist of three tissue flaps (leaflets), except for the mitral valve (also known as the bicuspid valve), which is comprised of only two leaflets. The tricuspid and mitral valves regulate, respectively, the blood returning from the body and the lungs, while the pulmonary and aortic valves regulate the blood flowing into the lungs and the body, respectively.

\subsection{The Cardiac Cycle}

The time period between every heartbeat encompasses a sequence of events collectively called the cardiac cycle which lasts approximately 0.8 seconds. Each cardiac cycle 
consists of two phases: systole and diastole. Systole refers to the contraction of the heart muscle, whereas diastole refers to the relaxation of the heart muscle. Essentially, there are three major pumping stages in a single cardiac cycle: the atrial systole, the ventricular systole, and the ventricular diastole. During atrial systole, the atrial chambers contracts and pumps the blood into the ventricles. As the ventricles contract during ventricular systole, the pressure inside them exceeds that within the atria, thus forcing the mitral and tricuspid valves to close and the pulmonary and aortic valves to open so that blood is ejected from the ventricles into the aorta and pulmonary artery, respectively. During ventricular diastole, the pressure within the ventricles decreases, resulting in the closing of the pulmonary and aortic valves, and the opening of the mitral and tricuspid valves, which allow blood from the atria to flow back into the ventricles. The ventricles are filled to about 80 percent of their capacity before the atria begin to contract, and the cardiac cycle is repeated. A typical cardiac cycle in an adult male pumps blood at an average flow rate of 5 liters/minute. With a life span of about 76 years, the heart beats approximately 2.8 billion times and pumps about 169 million liters (179 million quarts) of blood. 


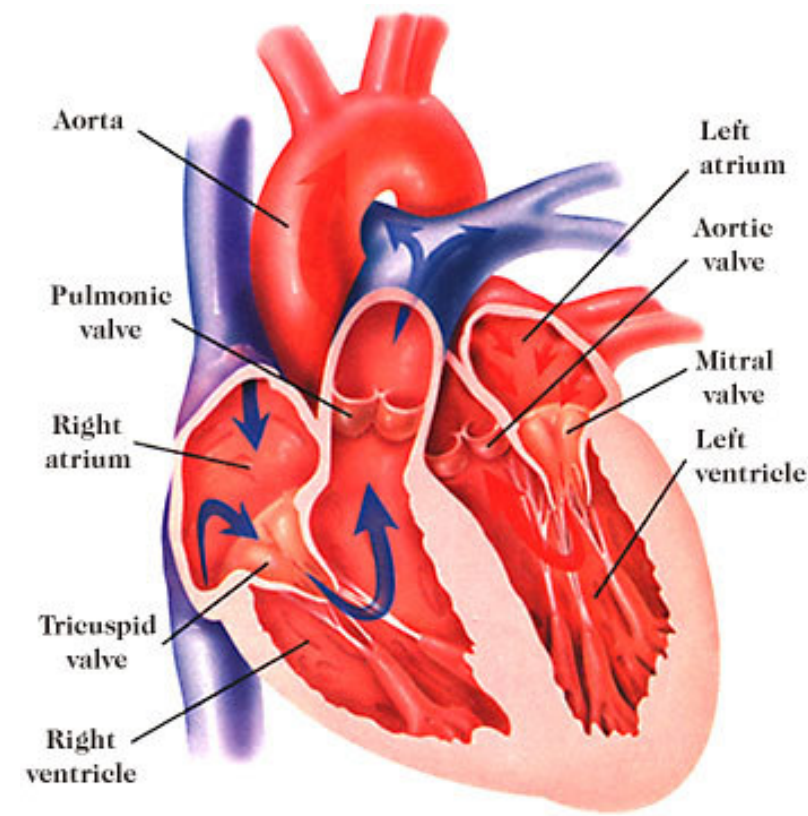

Figure 2-1 Anatomy of the Heart; the heart is made up of four chambers responsible for the regulation of the blood flow and pressure through the body

\subsection{Failure of Native Heart Valves}

Heart valve disease can be caused by a variety of factors such as rheumatic fever, ischemic heart disease, bacterial and fungal infection, connective tissue disorders, trauma, and malignant carcinoid [Black, 1994; Cebi, 2004; Goldsmith, 2002; Korossis, 2000]. They can be categorized into two types, namely, regurgitation and stenosis. The former, also known as insufficiency or incompetence, happens when a valve does not close properly, and blood begins to leak backwards. Such a condition causes the heart to pump harder and over time become enlarged and less efficient. Stenosis occurs when the leaflets do not open wide enough allowing only a small amount of blood through the valve due to the increased resistance to forward flow [Black, 1994; Korossis, 2000]. Stenosis is typically the result of stiffening and hardening of calcified valve leaflets usually observed in older patients [Black, 1994; Korossis, 2000]. In general both the 
mitral and aortic valves have a higher failure rate than the valves on the right side of the heart. This is because they encounter flow conditions that are much "harsher" than on the right side of the heart. For instance the mitral and the aortic valves encounter blood pressure differences of $0-120 \mathrm{mmHg}$ and $80-120 \mathrm{mmHg}$, respectively, whereas the blood pressure fluctuations on the right side of the heart are typically less than $40 \mathrm{mmHg}$.

\subsection{Prosthetic Heart Valves}

Existing commercial prosthetic heart valve designs can be divided into two groups: bioprosthetic, or tissue valves, and mechanical heart valves. The former is made from a combination of synthetic materials and chemically treated animal tissue mainly porcine in origin, whereas the latter is manufactured entirely from synthetic materials. One of the early successful mechanical heart valve surgeries was performed by Dr Charles Hufnagel in 1961 in which six of the eight patients who received a caged ball heart valve survived the operation [Hufnagel, 1966]. This surgery, enabled by the invention of the heart bypass machine, marked the beginning of the design of the artificial heart valve for long term implantation. During over 40 years of heart valve research and development, the design of heart valves has since diversified into more than 60 models each varying in mode of operation [Starr, 2002] (Figure 2-2). The most commonly used designs are the Starr-Edward caged-ball valve, the Bjork-Shiley and Medtronic-Hall tilting disc valves, the St. Jude Medical (SJM) and CarboMedics (CM) bileaflet valves, the CarpentierEdwards and Hancock porcine valves, the Ionescu-Shiley and the Carpentier-Edwards pericardial valves. Today the most widely implanted design is the mechanical bileaflet valve with well over 500,000 implants worldwide [Blackman, 2000]. 


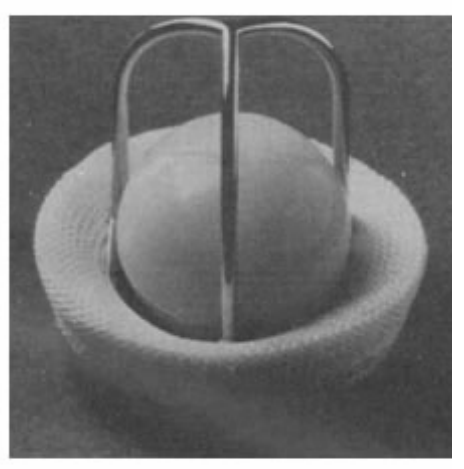

(a)

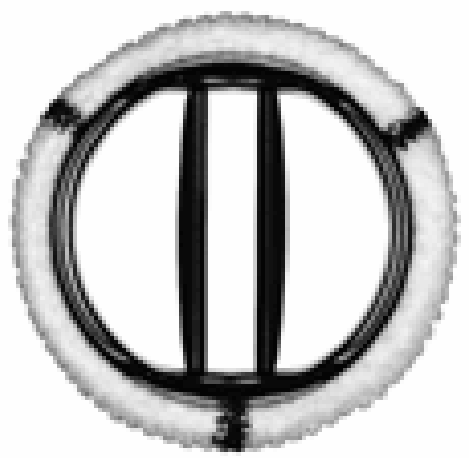

(c)

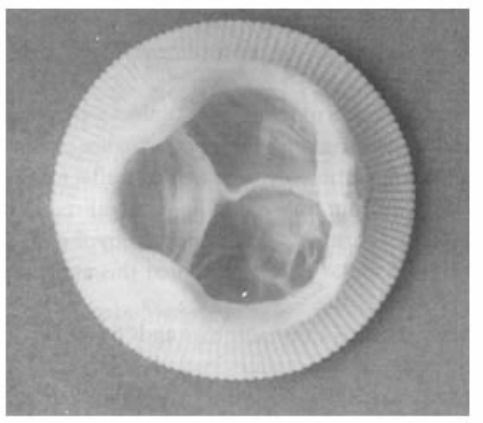

(e)

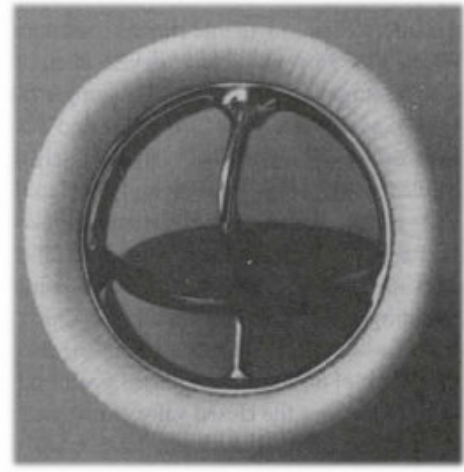

(b)

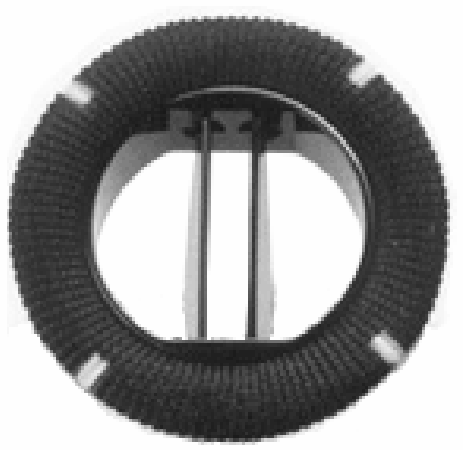

(d)

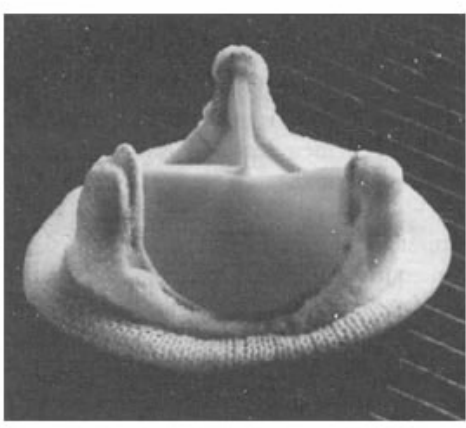

(f)

Figure 2-2 Various heart valve prosthetheses (a) Starr-Edwards ball and cage valve, (b) Medtronic-Hall tilting disc valve, (c) St. Jude Medical bileaflet valve, (d) CarboMedics bileaflet valve, (e) Hancock porcine valve, and (f) Carpentier-Edward pericardial valve

Unfortunately, current artificial heart valves are still plagued by thrombosis and thromboembolism, tissue overgrowth, infection, anti-coagulant related hemorrhage, and valve failure due to material fatigue or chemical change [Black, 1994; Korossis, 2000; Starr, 2002; Yoganathan, 2000]. 


\subsubsection{Valve Types and Their Complications}

The major disadvantage associated with the implantation of mechanical heart valves (MHVs) is the need for chronic anticoagulation therapy to prevent thrombosis and thromboembolic complications. These patients are plagued by an increased risk of bleeding, infection, and/or autoimmune response [Walker, 1992]. Blood flow through mechanical prostheses can lead to elevated turbulent stresses that may damage and/or activate blood elements and initiate platelet aggregation. Platelet aggregation can lead to thrombus formation with disastrous consequences for the patient. Thrombi can detach from the valve and become lodged in a downstream blood vessel, thus reducing or even cutting off the blood supply to vital tissues.

Tissue valves provide an alternative to mechanical valves utilizing the concept of a trileaflet configuration with one central orifice that mimics the design of the native valve. These valves have a lower potential for blood element damage than their mechanical counterparts, however, the mechanical properties of tissue valves degrade more rapidly, and they are prone to calcification [Black, 1994]. Often, implanted tissue valves fail in less than ten years, and reoperation is necessary. In contrast, some mechanical valves, such as the St. Jude Medical bileaflet and the Medtronic-Hall tilting disc, have been used as implants for almost two decades [Baudet, 1995; Black, 1994]. At present, approximately 250,000 prosthetic heart valves are implanted worldwide every year with 60,000 to 75,000 implanted in the United States [Black, 1994]. Worldwide, about 55 percent of these are mechanical and 45 percent are biological [Vongpatanasin, 1996]. According to a recent study, the use of MHVs is increasing at the rate of 3-5 percent per 
year, and the use of bioprosthetic valves is increasing at a rate of 8-11 percent per year [Vongpatanasin, 1996].

\subsection{Characteristics of Human Blood}

Human blood is a liquid tissue, and its major function is to transport oxygen to organs and tissues throughout the body. Blood contains many enzymes and hormones, supplies the tissues with nutrients and removes waste products. Blood is composed of plasma and cells, or corpuscles. The corpuscles include the red blood cells, or erythrocytes (99 percent), platelets, or thrombocytes (0.6-1.0 percent), and the white blood cells, or leukocytes (0.2 percent) [Fung, 1984]. Plasma is essentially an aqueous solution made up of 99 percent water by weight, 7 percent plasma proteins, 1 percent inorganic substances, and 1 percent other organic substances. Human red blood cells are disk-shaped and have a diameter of $7.6 \mu \mathrm{m}$ and a thickness of $2.8 \mu \mathrm{m}$. Platelets are much smaller with a diameter of about $2.5 \mu \mathrm{m}$. Blood is essentially a two-phase flow medium and its nonNewtonian character has been manifested when tested in a viscometer. Since experiments have shown that normal plasma is Newtonian, there is no doubt that that the nonNewtonian features of the blood can be attributed to its cellular constituents. The ability of red blood cells to deform at high shear rates and aggregate (rouleaux formation) at low shear rate has been illustrated and contribute to its non-Newtonian characteristics [Fung, 1984]. An important feature associated with this characteristic is that the flow profile of blood is similar to that of a plug flow, which means that low shear conditions are transiently present over greater regions. Such abrupt changes in the flow profile lead to greater shear rates close to the wall than for the same mean flow rate in Poiseuille flow. 
Hemostasis is the physiologic process which results in the cessation of bleeding in the body by the formation of tightly regulated, localized thrombus to seal the wound without the onset of gross thrombosis. The ability of blood to clot is an important feature of the body to heal wounds and protect the body from foreign substances. When blood comes into contact with an injured tissue or a foreign surface, a biochemical cascade is initiated that eventually leads to thrombus formation.

\subsubsection{Platelet Activation and Aggregation}

Various studies have reported that platelet activation and aggregation can occur in response to shear stresses as low as 50 dynes $/ \mathrm{cm}^{2}$, and irreversible platelet aggregation can occur at a shear stress of 100 dynes/cm² [Hung, 1976; Ramstack, 1979; Slack, 1993] Physiological shear has been shown to regulate the expression of proteins which influence the aggregation of platelets. A study by Diamon et al showed that shear stresses of 15 and 25 dynes $/ \mathrm{cm}^{2}$ elicit a response from the endothelium to secrete factors that augment clot dissolution and dilate narrow vessels [Diamond, 1989]. Researchers working with canine arteriovenous shunts containing both turbulent flow and laminar flow inducing devices attached to the femoral artery showed that thrombus deposits in the turbulence inducing shunt were 3.5 times heavier than those in the laminar shunt [Smith, 1972]. The authors observed that blood elements in the turbulent flow encountered higher shear and experienced a higher rate of collision with the wall of the tubing and thus prolonged contact with the foreign surface. The higher collision rate between activated and/or damaged blood elements was caused by the flow eddies and recirculation flow 
formed near the wall as a result of the turbulent flow. Platelet suspensions studies suggested that platelet aggregates would form in the vortex regions. Platelets are more than likely to enter a vortex than other formed elements due to their lower kinetic energy. As a consequence of the low rate of shear in a stationary vortex, which tends to retard inward migration of particles toward the center of stream and the accumulation of platelets, the activated platelets in the vortex encounter a higher rate of collision. In addition, the low return velocity in the vortex close to the wall suggests that once formed aggregates would stick to the wall. Researchers have since demonstrated that longer time exposures and higher shear increases platelet activation and platelet microparticle formation [Hung, 1976; Ramstack, 1979].

\subsubsection{Red Blood Cells and Hemolysis}

Human red blood cells are better adapted to withstand large fluid and mechanical shear than platelets. However, under severe non-physiological conditions, such as those encountered in MHVs, red blood cells can be irreversibly damage. A study by William et al showed that red blood cells (RBCs) exposed to shear stress of 500 dynes $/ \mathrm{cm}^{2}$ were able to maintain their integrity, but a gradual increment of shear stress from 700 to more than 3000 dynes $/ \mathrm{cm}^{2}$ leads to increased hemolysis [Williams, 1973]. Studies have shown that RBCs plays an important role in hemostasis, and that RBCs can potentiate shearinduced platelet activation [Joist, 1998]. Experiments revealed that red blood cells concentrated in the center of the flow actually facilitate the transport of platelets towards the vessel wall [Eckstein, 1991; Goldsmith, 1986]. This phenomenon may increase the 
platelet concentration near the wall thus increasing the likelihood of platelet collisions that may lead to aggregation.

\subsection{Patient Related Complications With Mechanical Heart Valves}

The implantation of a prosthetic heart valve introduces non-physiological flow phenomena such as stagnation, vortices, cavitations, boundary layer separation and turbulence that can activate platelets resulting in thromboembolic events and induce hemolysis. Mechanical valves lack a central flow across the valve annulus, which lead to an increased resistance to blood flow. Studies have shown that high intensity transient signals (HITS) in the cerebral perfusion representing microemboliztion to the brain have been demonstrated in patients carrying a mechanical aortic valve [Kleine, 2000; Raco, 1999]. However, it remains unknown whether HITS represent gaseous (which may be caused by cavitations when the leaflets contact the valve housing at high speeds during closing) or solid emboli (usually associated with platelet aggregates that are formed in regions of high and low shear in the valve) [Kleine, 2000]. Microemboli can be detected in about 75 percent of patients using transcranial Doppler sonography and may explain the observation of higher incidence of stroke in patients with mechanical valve implants [Kleine, 2000]. Recently, some studies have demonstrated that these HITS might also lead to silent brain infarction in some patients with high HITS [Daffertshofer, 1996; Lievense, 1998]. Some studies have demonstrated that the valve size can influence the number of microemboli to the brain of patients following valve replacement [Laas, 2003; Rambod, 1999]. Gencbay et al showed that microbubbles are associated more with the bileaflet and the monoleaflet valves [Gencbay, 1998]. Kleine et al compared the HITS 
counts between the Medtronic Hall monoleaflet and SJM bileaflet and reported that the bileaflet valves have significantly greater HITS counts than the monoleaflet valves [Kleine, 2000]. The authors believe the superior downstream characteristics of the Medtronic Hall are the contributing factor for its low HITS count. Using porcine blood, Steegers et al compared leakage flow through various bileaflet mechanical valves prostheses [Steegers, 1999]. They observed significant differences with regard to blood damage caused by leakage in various mechanical heart valves; the Sorin Bicarbon valve had the lowest and SJM the highest hemolysis in vitro compared to CarboMedics and Duromedics-Edwards valves, whereas Duromedics-Edwards had the highest index for platelet damage.

\subsubsection{Thrombus Formation In Mechanical Heart Prostheses}

Thrombosis remains one of the major complications in patients with mechanical heart valve prostheses [Black, 1994]. Thrombus formation generally occurs either from sewing ring to annulus or on the leading edge of tissue growth (pannus growth) and extends along the struts and hinge points in disc and bileaflet-type valves [Black, 1994]. These thrombi can be identified with regions in the valve where there is increased regurgitation and flow separation, as well as in regions where any strut protrudes into the flow orifice. These regions are typified by high and low shear. For instance, explanted caged-ball valves tend to have clots occurring on the struts, with a thickened clot at its apex, and this is often seen extending downstream of the valve [Christy, 1989; Christy, 2000; Keggen, 1996; Roudaut, 2003]. Similarly in the tilting disc designs, clots were found downstream on supporting struts, which extend into the main flow orifice [Roudaut, 2003]. In addition, 
clots can also be seen extending into the minor orifice region and into the pivot points, as well as formed on the center of the disc [Roudaut, 2003]. For the bileaflet valves, clots are usually found in both the upstream and downstream regions of the hinge geometry [Christy, 2000; Roudaut, 2003]. Studies have found that thrombi in bileaflet mechanical heart valves tend to originate in the hinge region and extend to the downstream outer edge of the leaflets [Christy, 2000; Gregoric, 2004; Roudaut, 2003]. It has been documented that extensive thrombus formation observed in some patients leads to significant impairment of the leaflet motion [Roudaut, 2003] since clots formed inside the hinge region affects the leaflet sweeping motion by decreasing the leaflet opening angle [DeWall, 2000; Okazaki, 2003; Vongpatanasin, 1996]. Studies have indicated that shearinduced platelet damage is cumulative in nature and this is particularly relevant to heart valves [Slack, 1993; Travis, 2001b; Turitto, 1998]. During an individual excursion through the replacement valve, the combination of shear magnitude and exposure time may not lead to platelets aggregation, however, after multiple journeys though the artificial valve, shear-induced damage may accumulate to an extent where it may eventually lead to thrombosis and subsequent embolization. All of the aortic and mitral valve designs (mechanical and tissue) studied created mean Reynolds shear stresses in

excess of 200 dyne/ $\mathrm{cm}^{2}$ during the major portion of systole and diastole, which could lead to damage of blood elements [Yoganathan, 2000].

\subsection{In Vitro Investigations of Mechanical Heart Valves}

Figure 2-3 shows the various flow profiles downstream of different valve types. One of the early in vitro investigations of MHV flow fields was performed by Figliola and 
Mueller (1977). Using a steady flow system and hot-wire anemometry, the investigators were able to quantify the flow characteristics downstream of the Kay-Shiley caged disk, the Starr-Edwards caged ball, and the Bjork-Shiley tilting disk valves in the aortic position [Figliola, 1981]. They observed that the flow separation downstream of the caged ball and disk valves was much larger than that downstream of the tilting disk valve, and the calculated Reynolds shear stresses in these valves exceeded 500 dynes $/ \mathrm{cm}^{2}$.
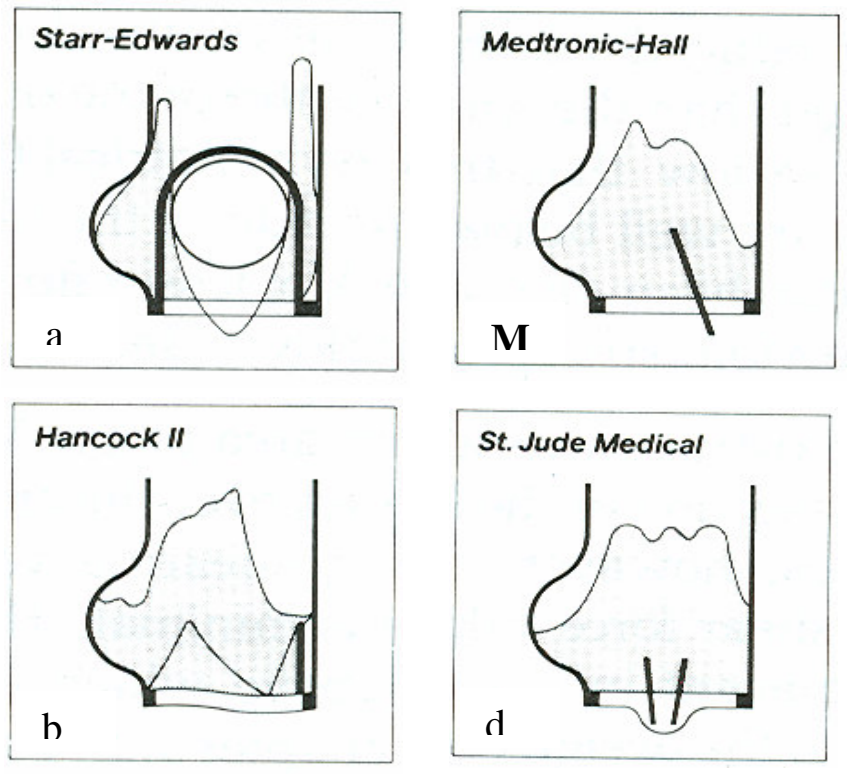

Figure 2-3 Flow Velocity Profile of Various Valve Prostheses: a) ball and caged valve, b) tissue valve, c) tilting disk, and d) bileaflet valve

Chandran et al used one-component laser Doppler velocimetry (LDV) to investigate the flow past a stellite Starr-Edwards silastic ball caged valve, Bjork-Shiley convexoconcave valve, and a Hall-Kaster tilting disc valve [Chandran, 1985a; Chandran, 1985b]. They demonstrated that the increased opening angle of the tilting disc valve leads to a larger, locally confined vortex along the wall in the minor flow region during peak 
systole and throughout diastole. They observed similar reverse flow downstream of the caged ball valves, which persisted throughout the cardiac cycle, and claimed that this flow phenomenon might be conducive to thrombus formation at the apex of the cage. Using one-component LDV, Schoephoerster et al studied the flow past various mitral valve prostheses (a polyurethane Starr-Edwards cage-ball, Bjork-Shiley tilting disc, and SJM bileaflet valves) in a left ventricle model [Schoephoerster, 1991]. They showed that all prosthetic heart valves produced vortex flow structures inside the ventricle model, especially the caged ball valve, which had a vortex that engulfed the entire ventricle. However, the calculated turbulent shear stresses in these valves did not exceed the value of 1500 dynes $/ \mathrm{cm}^{2}$ determined by Sutera et al to produce lysis of red blood cells [Sutera, 1988]. However, the reported turbulent shear stress values were near to or exceeded the critical value for platelet lysis $\left(100\right.$ dynes $\left./ \mathrm{cm}^{2}\right)$. Shipkowitz et al used PIV and Computational Fluid Dynamics (CFD) to investigate how the opening angles of the SJM and ATS bileaflet valves affect their downstream flow characteristics [Shipkowitz, 2000]. The PIV results showed that the smaller opening angle of the ATS leaflets produced a larger wake region behind the leaflets with impingement of the lateral jets on the wall of the test chamber. They concluded that the pivot region positioned farther upstream with respect to the orifice trailing edge of the valve provided a more favorable pressure distribution across the leaflet. Zhao et al used PIV to investigate flow past a simplified 2D model of a bileaflet heart valve [Zhao, 2001]. They observed that the hinge position could significantly influence the valvular flow characteristics. For instance, pivot hinges positioned close to each other led to a larger velocity gradient downstream where the high velocity lateral jets mix with the slower moving center jet. This higher gradient results in 
energy losses and high turbulent shear stresses. On the other hand, hinge regions placed too far apart would induce a fluttering motion in the leaflets, which caused an unstable flow pattern in all three orifices. This also produced more asynchronous closing and opening of the leaflets than the other hinge placements.

\subsubsection{Leakage Flow Through Heart Valve Prostheses}

Retrograde flow in mechanical prostheses is composed of the "squeeze flow," which occurs during valve closure, and the leakage flow, which occurs after valve closure. The former, which can be observed in bileaflet and tilting disk prosthetic designs, is a result of the rising fluid pressure across the valve and the closing occluder. These conditions "squeeze" blood through the interstitial gap between the closing occluder and the valve housing. The latter flow is comprised of the flow through the hinge region, which is found in the bileaflet designs only, and the leakage flow that can be observed through the perimeter of the closed occluder. Hinge flow is rapidly constricted from a large chamber to the small area of the hinge region, and is powered by the elevated transvalvular pressure after valve closure. These retrograde flow phenomena are characterized by elevated fluid velocities $(2-3 \mathrm{~m} / \mathrm{s})$ and high Reynolds shear stresses $(1,500-8,000$ dynes $/ \mathrm{cm}^{2}$ ) through gaps an average of $0.5 \mathrm{~mm}$ in width. The presence of a leakage phase is a distinctive feature of diseased and prosthetic heart valves. Healthy native valves do not leak, hence leakage magnitude is one of the important parameters for quantifying and studying diseased regurgitant heart valves. 
Most previous research has focused on the forward phase of heart valve prostheses; however, during the second half of the 1990s hinge flow was recognized as being potentially damaging to the blood. A certain amount of leakage flow is permitted in bileaflet mechanical heart valve design to enable washout of thrombus material in the critical areas of the valve, such as the hinges and the region between the leaflet edges and the housing. Recent studies have shown the importance of the hinge geometry in influencing the characteristics of the leakage flow structures, such as the leakage velocities and Reynolds shear stresses, within and near the hinge region [Ellis, 1996b; Ellis, 2000a; Ellis, 2000b; Gao, 1999; Steegers, 1999]. These studies have suggested the hinge mechanism as the most critical part of the bileaflet MHV since it directly influences valve durability, functionality, and thrombus formation [Ellis, 1996a; Ellis, 1996b; Ellis, 2000a; Ellis, 2000b; Leo, 2002].

\subsubsection{Various Bileaflet Hinge Designs}

Medtronic Parallel - Although the Medtronic Parallel (MP) MHV was discontinued while it was in clinical trials, several hinge flow studies were still performed on it, and the results were compared to those from other MHV hinge studies to illuminate the effects of hinge geometry on the leakage flow characteristics [Ellis, 1996a; Ellis, 1996b; Ellis, 2000a; Ellis, 2000b; Gross, 1996; Healy, 1997]. Its washout at the hinge regions is considered inferior to that of the St Jude Medical (SJM) Standard, Hemodynamic Plus, the Regent bileaflet MHV designs and the CM bileaflet MHV design [Ellis, 1996a; Ellis, 1996b; Ellis, 2000a; Ellis, 2000b; Leo, 2002]. Figure 2-4 compares the different hinge geometry designs. The MP valve leaflets are held in place by four cylindrical projections 
on the leaflets. The leaflet projections are placed into the four recesses, which are machined into the valve housing near the flat surface on the inflow side of the valve.

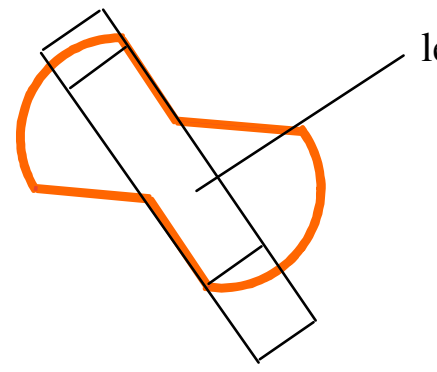

St Jude Medical (SJM)

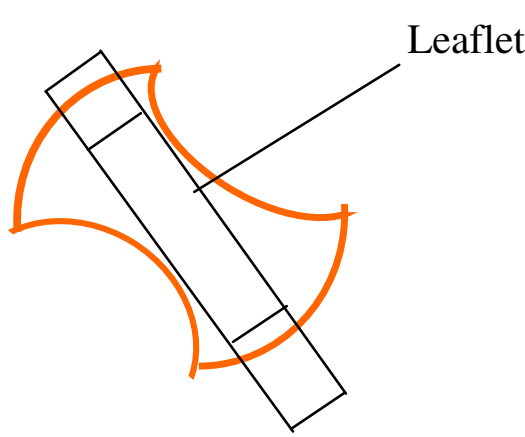

CarboMedics (CM)

eaflets

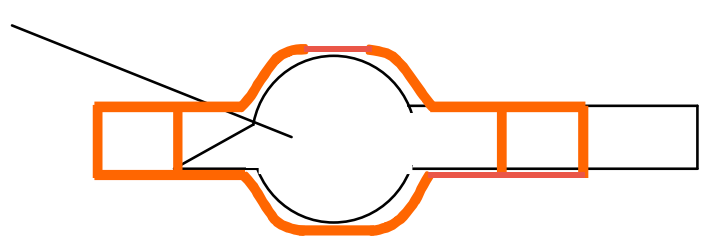

Medtronic Parallel (MP)

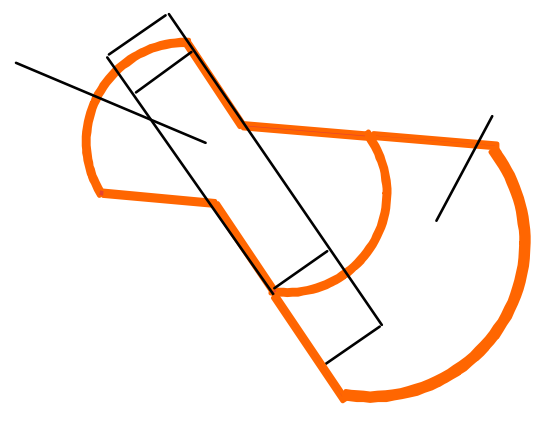

Medtronic Advantage (MA)

Figure 2-4 Various Hinge Geometry Designs. The SJM hinge geometry is similar to that of the CM valve except the latter has a sharper corner and a flatter hinge recess. The MP hinge design is characterized by a series of expansion and contraction, and the MA hinge design incorporate a secondary flat level to enhance washout characteristics

The geometry of the MP valve hinge recess is characterized by sudden expansion and contraction zones, which give rise to flow that is dominated by unsteady flow, vortex structure, and stagnation zones. These are the flow phenomena usually associated with elevated levels of shear stress. Reynolds shear stresses recorded near the hinge wall in the inflow channel were greater than 6,000 dynes $/ \mathrm{cm}^{2}$, which is sufficient to damage or activate blood elements and lead to thrombus formation [Ellis, 1996a; Ellis, 1996b]. The 
less effective washout of these damaged blood elements entrapped by these vortical fluid structures in the hinge region of the MP valve contributes to the potential buildup of more damaged blood elements.

St. Jude Medical - In contrast to the MP valve, the SJM bileaflet valve has shown over time better performance in terms of overall thrombogenicity and valve related events. The SJM Standard bileaflet valve is the 'gold' standard against which other mechanical valves are compared. It is the most frequently implanted MHV prosthesis worldwide [Black, 1994]. In vitro studies have shown that flow through all SJM mechanical valves is symmetric and relatively non-turbulent [Woo, 1985; Yoganathan, 1984]. Rashtian et al showed that the in vivo mean pressure gradients range from 3.0 to $5.2 \mathrm{mmHg}$ and 1.4 to $7.0 \mathrm{mmHg}$ in the aortic and mitral positions, respectively. The study also demonstrated that the regurgitant volume ranges from 7.6 to $10.6 \mathrm{~cm}^{3} / \mathrm{stroke}$ for aortic valves and 4.3 to $6.4 \mathrm{~cm}^{3} /$ stroke for mitral valves [Rashtian, 1986]. The highest peak leakage velocity and Reynolds shear stress recorded for the SJM $25 \mathrm{~mm}$ Standard was $3.5 \mathrm{~m} / \mathrm{s}$ and 7,200 dynes $/ \mathrm{cm}^{2}$, respectively [Ellis, 2000a], while an improved equivalent valve model, the SJM $23 \mathrm{~mm}$ Regent, achieved a lower leakage velocity and Reynolds shear stress of 1.5 $\mathrm{m} / \mathrm{s}$ and 2,600 dynes $/ \mathrm{cm}^{2}$, respectively. The SJM Regent valve has an expansion expansion, called the thumbnail, situated downstream of the hinge mechanism. Studies have shown that the thumbnail region is characterized by two recirculation zones, which bound the forward flow and prevent complete washout in this region [Ellis, 2000a; Ellis, 2000b]. In addition to the complex flow in the thumbnail region, flow separation and 
secondary flow were also observed downstream of the thumbnail. These areas of complex flow may be vulnerable to the formation of thrombus.

Carbomedics (CM) - Ever since its inception, the CM bileaflet valve has demonstrated a good clinical history. A study by Dalrymple-Hay and coworkers demonstrated that the operative mortality rate of the $\mathrm{CM}$ valves compared favorably to those of other mechanical valves, including St. Jude Medical, Medtronic Hall and Omniscience [Dalrymple-Hay, 2000]. They were also able to show that the CM valves exhibited a low incidence of valve-related complications. In another study, Jamieson and coworkers showed that CM valves implanted in the mitral, aortic and multiple positions had satisfactory overall clinical performance [Jamieson, 2000]. In a study by Aagaard and colleagues done over a time frame of 12 years, the authors showed that the CM bileaflet valves are highly reliable, as evidenced by no mechanical failures and a low incidence of valve-related complications [Aagaard, 2001].

Although statistically insignificant, a higher incidence of valve thrombosis in early and mid-term follow up studies has been reported for the CM valve when compared to that of the SJM valve [Akins, 1995]. However, many clinical valve studies have not placed much emphasis on the influence of the valve's geometric design, instead focusing mainly on clinical factors such as the preoperative defective valve seen in native endocarditic patients and non-clinical factors such as management of anticoagulation. These clinical studies leave many questions about the influence of valve design unanswered. 
Medtronic Advantage (MA) - The basic design features of the MA are similar to those of the SJM valves. Each leaflet of the MA valve is constrained and retained within the valve housing by a pair of bi-level butterfly pivots. The bi-level pivot consists of a primary flat, and a butterfly recess similar to those seen in the SJM valve, and a secondary flat. The secondary flat is recessed into the valve housing by approximately $1 / 3$ of the depth of the pivot recess to provide a flow channel over the secondary flat. Studies conducted recently demonstrated that the performance of the MA valve is equivalent to that of the SJM valve [Saxena, 2003; Shu, 2004]. However, the Reynolds shear stress levels recorded inside the hinge region of the MA valves $\left(>5,000\right.$ dynes $\left./ \mathrm{cm}^{2}\right)$ were higher than those observed in the SJM valves $\left(<2,000\right.$ dynes $\left./ \mathrm{cm}^{2}\right)$. These studies also demonstrated that the flow channel over the secondary flat of the MA valve enhances the flow washout at the pivot recess. Study by Saxena et al comparing the hinge flow characteristics of the MA and SJM valves showed that the flow patterns seen within the two hinge designs exhibited similar features, with more dynamic flow patterns observed in the MA hinge during the forward flow phase [Saxena, 2003]. In addition, the velocities within the SJM hinge were slightly higher than those measured in the MA valves. The MA valve is commercially available in countries of the European Union for use in aortic valve replacement. It is currently in clinical evaluation in the United States, Canada, Japan and Australia.

\section{$\underline{2.8 \text { Polymeric Heart Valves }}$}

\subsubsection{Biomaterials of Polymeric Heart Valves}

The first reported human implantation of polymeric heart valves were in 1958 [Roe, 1958] followed by a single unit trileaflet silicone rubber (SR) aortic valve implanted in several 
patients between 1960 and 1962 [Roe, 1966]. The trials were discontinued as a result of high incidences of thromboembolic events. Over the years various designs and materials have been tested, including Teflon, SR with the Silastic compound, SR-impregnated Dacron mesh and polytetrafluoroethylene (Teflon). However, these valves had limited success largely due to calcification, material failure and thrombogenicity [Cooley, 1977; Ghista, 1977; Hessel, 1966; Mori, 1973].

A breakthrough came in 1982 when Wisman and colleagues created a trileaflet valve fabricated from segmented polyurethane (SPU) [Wisman, 1982]. These valves were similar to tissue valves with a large central flow orifice, which helps to reduce turbulence and blood trauma as a result of a lower transvalvular pressure drop and better hydraulic efficiency. SPU is known to exhibit high flexure endurance, great strength, and inherent nonthrombogenic characteristics and, consequently, is especially well-suited for cardiovascular applications in which a constant, nonthrombogenic interface with circulating blood is of paramount importance.

Polyurethanes elastomers are copolymers containing blocks of low molecular weight polyesters or polyethers linked together by a urethane group. These elastomers are based on three monomers: (1) an isocyanate source, (2) a macroglycol, and (3) a chain extender, or curative. The isocyanate can either be aromatic or aliphatic. An example of an aromatic isocyanate is the methylene diphenyl diisocyanate or MDI, which is a very reactive isocyanate, and in many cases a does not require a catalyst for synthesis if a diamine chain extender is used. An example of an aliphatic isocyanate is the cyclo- 
aliphatic diisocyanate $\mathrm{H}_{12} \mathrm{MDI}$. The aromatic isocyanate containing polymers have a tendency to yellow upon exposure to ultraviolet radiation, and some aromatic polyurethanes, such as those made from methylene dianiline, or MDA, are known carcinogens and display immediate cell toxicity. However, elastomers produced from less toxic aliphatic isocyanates have inferior physical properties and are significantly more expensive.

The macroglycol is the most important part of the polymer as it determines the final physical and biological properties usually associated with medical-grade elastomers. Polyether-based polyurethane is typically used for medical applications such as heart valve prostheses because it is insensitive to hydrolysis and more stable in the physiological environment. One example of a polyether-based polymer is the polytetramethylene ether glycol (PTMEG) used extensively in medical applications. Chain extenders can be either diamines or diols. A commonly used chain extender for medical used is the 1,4 butane diol since it confers upon the polymers improved physical properties, excellent processing conditions, and a transparent appearance.

Polyurethane elastomers are two-phased structures in which the hard segments are separated to form discrete domains in a matrix of soft segments. The hard segment confers structural strength, and the soft segment imparts flexibility and elasticity to the material. This segmented arrangement leads to the term segmented polyurethanes, or SPU. The hard segment acts as bridge and filler particle, thus reinforcing the soft segment. The attractive forces between the hard segments are much stronger than those between 
the soft segments due to a higher concentration of polar groups in the former. This property of the hard segments can significantly affect the mechanical properties of the polymer, particularly the modulus, hardness, and tear strength. Polymer research has shown that the quantities and molecular weights of the chemicals used for polymer synthesis have significant effects on the properties of each specific SPU, which account for the major differences between the subtypes. Medical grade SPUs have been applied successfully in several blood-contacting devices, including artificial hearts, ventricle assist devices and blood pumps [Bernacca, 1997a; Bernacca, 2002b].

Recent studies have yielded a wide range of SPUs for polymeric heart valve leaflets [Alferiev, 2001; Bernacca, 1997a; Seifalian, 2003; Wheatley, 2001]. Bernacca et al used polyetherurethane (PEU) for both the frame and the valve leaflet. This polymer has a polyether soft segment with a hard segment composed of a 4,4'-dpiphenylmethane diisocyanante (MDI) chain extended with butanediol [Bernacca, 1998]. Another variant of polyurethane developed by the same group called polyetherurethane, or PEUE, has a soft segment of polyether and a hard segment formed from an MDI chain extended with ethylene diamine. PEUE contains urea groups rather than the butanediol found within PEU, and it has a higher proportion of soft segments than PEU.

\subsection{In Vitro Flow Studies of Tissue Valves}

Both porcine and bovine pericardial bioprosthetic valves provide a satisfactory alternative to mechanical heart valves. Given their superior hemodynamics performance, these valves have provided the patients an excellent quality of life with low rate of 
thromboembolism and thrombosis, and freedom from anti-coagulant-related hemorrhage. Typically, the bioprosthetic valves are low-pressure $(1.5 \mathrm{mmHg})$ treated with glutaraldehyde for the fixation of the biological tissue. The primary concern with these bioprostheses is limited durability as a result of fatigue- and biological related degeneration of the tissue. The majors factors influencing the long-term durability of the tissue valves include calcification, thrombus formation, endocarditis, fibrous tissue overgrowth, cuspal rupture and cuspal hemotomas.

Research for the past years have shown in the design of the bioprosthetic valves a trend toward lower transvalvular pressure by optimizing (increasing) the effective flow area; a more stenotic valve (mechanical or tissue) tend to be have higher incidence rate of hemolysis and thrombosis. The transvalvular gradient for an aortic $(23 \mathrm{~mm})$ and a mitral $(30 \mathrm{~mm})$ prostheses is approximately 25 and $4 \mathrm{mmHg}$, respectively. The construction of the bioprosthetic valves is typically that of the trileaflet configuration with the tissue mounted onto a totally flexible Dacron-covered Elgiloy (cobalt-nickel alloy) stent. An alternative to the stented bioprosthetic valve is the stentless type, which utilizes the native root as in the aortic valve, and the chordal unit as in the mitral valve, as the supporting structures for the leaflets thus maintaining a functional unit in the valve complex. Hence, stentless valves do not require supportive frames (stents) that occupy annular space and therefore have larger flow area. By minimizing resistance to flow, the mean pressure gradients achieved with stentless valves are near normal. 
The general flow profile of the bioprostheses valves is characterized by a flat center orifice flow with vortex formation on the edge of the center jet. A series of in vitro studies on the various tissue valves in aortic position were conducted by Yoganathan et al using 1D LDV [Yoganathan, 1986a]. The authors observed a forward velocity jet of 2.2 $\mathrm{m} / \mathrm{s}$ in a $27 \mathrm{~mm}$ Carpentier-Edwards porcine valve during peak systole with no regions of flow separation observed throughout the systolic period. However, the annular region between the outflow surfaces of the leaflets and the flow chamber wall was relatively stagnant throughout systole. A forward velocity of $3.3 \mathrm{~m} / \mathrm{s}$ was observed in the $25 \mathrm{~mm}$ Hancock Modified Orifice porcine valve during peak systole with relatively stagnant flow in the annular region between the outflow surfaces of the leaflets and the flow chamber wall. The flow profile of a $27 \mathrm{~mm}$ Carpentier-Edwards pericardial valve is comparatively flatter than those observed in the two previous valves, with a measured forward velocity of $1.8 \mathrm{~m} / \mathrm{s}$ during peak systole. A region of flow separation which extended about $6 \mathrm{~mm}$ from the wall was observed at peak systole and during the deceleration phase. The maximum Reynolds shear stress observed in the CarpentierEdwards porcine, the Hancock Modified Orifice porcine and the Carpentier-Edwards pericardial valves are 4,500, 2,900 and 1,000 dynes $/ \mathrm{cm}^{2}$, respectively. Studies have shown that the pericardial valve is superior to the porcine valve in terms of hemodynamics performance [Cosgrove, 1985; Fisher, 1986; Yoganathan, 1986a]. Testing in vitro six different types of tissue valves and six mechanical valves, Fisher et al showed that the porcine valves have much higher forward flow pressure gradients than the pericardial, tilting disk, or bileaflet mechanical valves [Fisher, 1986]. On the other hand, the porcine valves showed the least regurgitation, with the pericardial valves having less 
regurgitation than the mechanical valves. The authors concluded that the pericardial valves are less obstructive than the porcine valves.

\subsection{In Vitro Investigations of Polymeric Heart Valves}

Multiple in vitro studies have demonstrated that polymeric heart valves generate a centralized flow with a relatively flat profile similar to that of bioprostheses and native heart valves. Using one-component LDV, Herold et al compared the flow dynamics of four different trileaflet polyurethane heart valves to the Ionescu-Shirley bovine pericardial and Hancock porcine heart valves in the aortic position [Herold, 1987]. They observed that the velocity distributions at peak systole were very similar for the polyurethane valve and the porcine bioprosthesis. Both of the flow fields could be characterized as triangular jet-flow, and recirculation areas were located near the right aortic wall downstream of the stent post and in the opposite sinus cavity. On the other hand, the flow profile for the bovine bioprosthesis was a plug flow with recirculation in the sinus cavity and one small transient recirculating vortex near the right aortic wall. Overall, the flat flow profile of polyurethane valves is similar to those of the native and bioprostheses valves with vortex formation observed behind the leaflets in the sinus cavity. De'Souza et al characterized the velocity and turbulence waveforms distal to several trileaflet polymeric heart valves with varying degrees of stenosis [D'Souza, 2003]. They observed that the shape and orientation of the spatial velocity profile in a polyurethane heart valve depicts the effect of the constriction caused by the valve leaflet in a similar manner to that observed in bioprosthetic valves. Using two component LDV, Chandran et al investigated the flow dynamics distal to two tri-leaflet polyurethane 
valves: one with leaflets bound to the edge of the supporting stents (PUI) and the second with the leaflets mounted outside the stents (PUII) [Chandran, 1989]. The latter had a less obstructed flow orifice available for blood flow, which is thought to be more advantageous. Furthermore, the turbulent shear stress in the PUII valve was half that in the PUI valve showing that the increase in orifice diameter can help reduce the turbulent shear stresses and corresponding damage to blood elements. When compared to tissue valves, polymeric valves have larger turbulent shear stresses. The orifice diameters in polymer valves are usually smaller than those in bioprostheses, and as a result, polyurethane valves have larger observed velocity magnitudes and larger velocity gradients than the bioprostheses. Recent studies have shown that the trileaflet design is more efficient than double or quadruple arrangements [Chetta, 1980; Jansen, 1991; Leat, 1995; Mackay, 1996], and that the maximal stresses in these trileaflet configurations were found near the commissural attachment, which is consistent with other synthetic and bioprosthetic valves [Leat, 1995].

\subsubsection{Mechanical Fatigue and Calcification of Polymeric Heart Valves}

Mechanical failure in trileaflet prosthetic heart valves, including bioprostheses, usually occurs at the commissural region. Also, tears in the leaflets are observed to start at the free edge leading to separation of the leaflet from the stent. Butterfield et al have shown that polymeric valve leaflets at the fully open and closed positions experience bending deformation and resultant high levels of strain at the free edge of the leaflet, particularly at the commissural region [Butterfield, 2000]. Corden et al showed that in the closed position, the resultant stresses are caused by the high pressure difference across the valve 
while in the open position the stresses are due to high level of curvature at the leaflet surface, especially at the free edge close to the stent post of the valve [Corden, 1995a; Corden, 1995b].

The formation of nodular deposits of calcium phosphate or other calcium-containing compounds on cardiovascular devices is calcification. Calcification is one of the major failure mechanisms for polymeric heart valves in animal studies [Akutsu, 1959]. The unintended deposition of calcium phosphate on polymeric heart valves surface can interfere with the proper hemodynamics function of the valves leading to valve obstruction and/or incompetency. The most common mineral deposit found on biomaterials is a poorly crystalline calcium phosphate known as apatite, which is related to calcium hydroxyapatite, the mineral that is responsible for the structural integrity in the bones. Calcification, besides occurring at regions exposed to intense mechanical deformation such as flexure points in the polymeric valve leaflets, may also occur on the surface of an implant where it is often associated with attached tissue or cells or within the structural components. Calcification has been shown to occur preferentially in the commissural region of the leaflets, and this is believed to be accelerated by either the high bending deformations and associated bending strains that occur in this region when the valve is fully open or by high tensile stresses when the valve is fully closed [Corden, 1995a; Corden, 1995b]. Calcium deposits are frequently associated with microscopic defects on the leaflet surface perhaps as a result of environmental stress or mechanical effects, which can lead to cracking. It is hypothesized that these microscopic defects, or cracks, can serve as attachment points for calcific deposits in smooth polyurethane 
leaflets; however, this is not yet proven. Calcification in polymeric heart valves can either be intrinsic or extrinsic. If damaged tissue or cellular components become trapped in these surface defects, they may eventually calcify leading to extrinsic calcification. Some studies have also observed calcium deposits forming within the leaflet surfaces (intrinsic calcification) in the absence of any surface defects [Bernacca, 1995; Herold, 1987]. Studies have shown that surface defects that occur during the fabrication process can lead to extrinsic calcification whereas material composition has more influence on the occurrence of intrinsic calcification [Bernacca, 1997b; Bernacca, 2002b]. However, at present it is still debatable whether calcification is due to the intrinsic polymer properties, is externally mediated, or is a combination of both [Schoen, 1988]. Bernacca et al assessed the calcification of six different polyurethane valves (PEU) using an in vitro setup. Their results indicated a strong association between areas of material failure and calcific deposits [Bernacca, 1997b]. In another study, Bernacca et al compared the durability and calcification of both PEU and PEUE [Bernacca, 1996]. Their accelerated fatigue testing showed that PEUE is more durable than the PEU. However, in vitro (polymers were placed in vials containing calcium phosphate) and in vivo (polymers implanted in rat subcutaneous model) calcification tests did not show any marked differences between the two variants. The authors also reported that PEUE calcifies to a lesser extent than the bovine pericardial control sample when tested in vivo in the rat subcutaneous model. The authors also detected a high concentration of silicon in close proximity to failure areas, and they suggested that the silicone oil used for lubricating the surface of the injection molds used during valve manufacture may play a contributing role in polyurethane valve calcification . In their in vitro calcification tests, calcification 
was observed to form along the free edge of the leaflets as well as at the coaptation region of the valves.

Recently, researchers have attempted to modify the surface of polyurethane valves to alter their thrombogenicity [Alferiev, 2001; Bernacca, 1998; Zdrahala, 1999]. These modifications include the covalent attachment of heparin, taurine (a platelet membrane glycoprotein fragment), polyethylene oxide (PEO), 3-aminopropyltiethoxysilane, glucose, or glucosamine. Experiments showed that all surface modified polyurethane performed better than unmodified polyurethane at reducing thrombogenic complications [Zdrahala, 1999]. It was demonstrated that the glucose-modified surface achieved the greatest reduction in platelet and complement activation [Bernacca, 1998]. However, the mechanism by which glucose helps reduce platelet activation is still unknown. A recent study by Alferiev et al investigated the used of a covalent modification of PSU with biphosphonate derivatization [Alferiev, 2001]. They demonstrated that the modified PSU is able to resist calcium buildup and thus help maintain valve functionality. The biphosphonates act to resist calcification by disrupting calcium phosphate crystal growth.

\section{$\underline{2.11 \text { Animal Models and Calcification }}$}

Animal models remain the best means for evaluating anticalcification strategies in polymeric heart valves. Typical animal studies involve both subdermal (rat) and circulatory (sheep or calf) implants. Thromboembolic complications are a major concern in many animal studies using the sheep model since sheep is considered less thrombogenic than a human recipient. On the other hand, a growing calf represents an 
extreme calcification model, and, moreover, calves are much more thrombogenic than sheep, which can tolerate mechanical valves without anticoagulants. One of the early animal studies by Herold et al comparing various polyurethane valves with two IonescusShirley bovine pericardial valves in New Jersey calves demonstrated that polyurethane valves have similar advantages to bioprostheses in term of thrombus formation [Herold, 1987]. However, all explanted valves were calcified and thrombosed. Typical polymeric valve failure was related to valve dysfunction in which the leaflets became immobilized because of massive calcium deposits on the upstream and downstream sides of the leaflets. Failure in both bioprosthetic valves was caused by occlusion of the valves by massive thrombus extending from the atrial to the ventricular side. Similar studies by Wisman et al on 17 Holstein calves with tricuspid implants and 4 calves with mitral implants showed that all explanted valves had calcium deposits concentrated mainly on the free edges of the leaflets [Wisman, 1982]. Adherent thrombus was also seen when calcification was prominent on the leaflet surface. Animals studies with sheep performed over a period of six months by Wheatley and coworkers demonstrated that the SPU valve was superior to the porcine bioprosthesis in terms of hemodynamics performance and to the bileaflet mechanical valves in terms of lower thrombogenicity [Wheatley, 2000]. The authors reported that high intensity transient signals (HITS) were much more frequent in the mechanical valve group than in either the porcine or the polyurethane valve groups. The authors also found a correlation between the HITS count and platelet aggregation in the valves suggesting that these phenomenon are related and that changes in valve factors, such as reduction in flow orifice area, that tend to increase valve thrombogenicity could also increase the number of HITS. Three of the eight polyurethane valves were found to 
be seriously affected by thrombus and fibrous attachment to the leaflets making them significantly more stenotic than the remaining five polymeric valves. Calcification was mostly extrinsic with some penetration of the calcified deposits into the underlying leaflet polymer as a result of polymer degradation. Other studies have shown that fatigueinduced damage to the polymer seems to accelerate material degradation since no intrinsic calcification was found within intact areas of the polymer [Bernacca, 1995; Bernacca, 1997b]. Wheatley et al studied the biostability of two variant SPU valves implanted in the mitral position in six sheep. The two biostable SPU valves were based on MDI hard segments and polysiloxane soft segments. One used a diol chain extender and the other used a diamine chain extender [Wheatley, 2001]. No traces of intrinsic calcification were observed, but some traces of extrinsic calcification in thrombus and fibrin deposits located at the leaflet/frame junction of the inflow surface of the valve were noted. In two separate studies conducted by Daebritz et al valve prosthesis made of polycarbonaturethane (PCU) were implanted in the aortic and mitral positions of New Jersey calves [Daebritz, 2003]. These polymeric valves were compared to Perimount pericardial and Medtronic Mosiac porcine valves. In these two studies most of the animals with the SPU valve implants survived to the end of the study (20 weeks). Minor thrombus deposits were observed at the commissural regions of the SPU valves, but the bioprostheses showed moderate to severe thickening and deformation of the leaflets. One bioprosthesis in the mitral position was completely covered by a huge thrombus. 


\subsection{Leaflet Thickness of Polymeric Heart Valves}

Although SPU leaflets can be manufactured as thin as 100 microns to be both flexible and durable, there are concerns at thicknesses less than 150 microns [Mackay, 1996]. A valve that must undergo repeated cyclical flexion must be manufactured from a material with a low elastic modulus to reduce the imparted bending stresses. One of the major advantages of SPU is that it enables easier flexing, and hence provides less resistance and disturbance to blood flow. On the other hand, the ease of flexion must be counterbalanced by the ability to withstand the high reverse pressures and mechanical stresses present when the valve opens and closes in a normal physiologic cycle. Bernacca and coworkers examined the hydrodynamic behavior of biostable polyurethane valves varying Young's modulus from 5 to $63.6 \mathrm{MPa}$ [Bernacca, 2002a]. They demonstrated that valve hydrodynamic function is not significantly affected by the material modulus; however, the leaflet thickness is a highly significant factor. Another study by Bernacca et al showed that polymer valve durability is directly related to the leaflet thickness with good durability achieved with a median leaflet thickness of approximately $150 \mu \mathrm{m}$ [Bernacca, 1997a]. In this study, polymeric valve with a leaflet thickness of 40-60 $\mu \mathrm{m}$ lasted about 400 million cycles, or 11 years, while a valve with a leaflet thickness of up to 200 micron lasted 800 million cycles, or 20 years [Bernacca, 2002a]. Most polymeric valves tested today usually last between 400 and 648 million cycles (between 11 years and 17 years). More recent in vivo fatigue testing found that the durability of polyurethane valves with leaflet thicknesses varying between 100 and $300 \mu \mathrm{m}$ was between 600 million and 1 billion cycles, or 15.8 to 26 years [Daebritz, 2003]. 


\subsection{Fabrication of Polymeric Heart Valves}

The fabrication of the polymeric valve typically involves coating a semi-rigid stent made of poly-ether-ether-ketone (PEEK) in polyurethane. Traditionally, SPU valves have been manufactured by dip-coating, which involves multiple dips into weak SPU solutions [Herold, 1987; Wisman, 1982]. Jansen et al have shown that the dip-coating method enables better control of leaflet thickness at different regions of the leaflet surface so that leaflet thickness at regions of high flexure stress can be greater [Jansen, 1991]. However, the leaflet thickness of valves produced by this method was nonreproducible. An alternative technique is the film-fabricated method, in which pre-cast SPU film is solvent-bonded to the valve frame and then thermally formed to the leaflet shape [Leat, 1995]. However, this method yielded a valve that was inferior in term of durability due to an inconsistent leaflet-frame interface. Recently, polymeric valves fabricated from a single dip-coating process resulted in consistently tolerable uniform leaflet thickness distributions [Mackay, 1996]. These valves were tested and found to exhibit pressure gradients similar to those for a bioprosthesic valve with considerably lower levels of regurgitation and leakage than bileaflet MHVs or bioprosthesic valves [Mackay, 1996]. 


\section{CHAPTER 3: HYPOTHESES AND SPECIFIC AIMS}

The objectives of the current study are to relate flow structures to the observed thrombus locations in the hinge region of bileaflet mechanical heart valve designs and inside prototype trileaflet polymeric heart valves. These objectives are divided into the following set of hypotheses and specific aims related to the hinge flow studies of bileaflet mechanical heart valves and flows in aortic polymeric heart valves.

\subsection{Hinge Flow Studies in Mitral Position}

Despite the success of bileaflet mechanical heart valves (MHVs), thromboembolic and bleeding complications remain one of the major clinical problems.. Recent studies have shown that the regurgitant (retrograde) flow in these valve prostheses as the primary contributor to the observed failures [Ellis, 1996a; Ellis, 1996b; Ellis, 2000a; Ellis, 2000b; Gross, 1996]. Retrograde flow is incorporated into the bileaflet valve design as a washout mechanism. However, it is possible that with certain combinations of hinge design and gap width, the hinge leakage flow field could be traumatic to blood elements and lead to the buildup of thrombotic materials in the hinge region. Indeed, recent clinical studies have demonstrated that blood clots tend to localize inside the hinge regions of the bileaflet heart valves thus impairing their performance [Christy, 1989; Roudaut, 2003]. In addition, previous in vitro hinge studies have revealed quantitatively that the retrograde flow fields of bileaflet mechanical heart valves are characterized by high velocities and elevated turbulent stresses [Ellis, 1996a; Ellis, 1996b; Ellis, 2000a; Ellis, 2000b]. 
The CM and SJM bileaflet heart valves, which have both enjoyed good clinical history, constitute two of the most popular choices for heart valve replacements [Vongpatanasin, 1996]. Clinical studies have shown that both these valves have comparable hemodynamics performance even though some studies have reported that the CM valves have slightly higher transvalvular pressure gradient and increased incidence of thromboembolism, especially in the mitral position [Akins, 1995]. The primary difference in the two valve designs is the hinge geometry: the CM pivot is similar to that of the SJM valve except that it has a flatter and more angular geometry that is purported to increase efficiency of the back flushing when the valve is closed. Some studies have suggested that the sharper corner of the CM hinge geometry may restricts complete sweeping by the leaflet appendage. This is because the CM valve has lesser contact in the hinge area between the leaflet and the casing, leaving comparatively more unswept areas in its hinge region than that in the SJM, and thus more space for the accumulation of thrombotic materials [Hashimoto, 1996]. The characteristics of the hinge flow fields inside the SJM valves have been well elucidated in previous studies [Ellis, 2000a; Ellis, 2000b]. However, the hemodynamics benefits of the modification in the CM pivot design have never been experimentally investigated. It is unknown how the flatter hinge profile in the CM valve may influence the leakage flow and washout efficiency, and if the hinge design may lead to elevated Reynolds shear stresses.

In addition to the influence of the hinge geometry, previous hinge studies have also suggested the possible effect of the hinge gap width on the success of bileaflet MHVs [Ellis, 1999; Travis, 2001c]. Based on in vitro leakage experiments with two prototype 
models of the SJM $25 \mathrm{~mm}$ valve with differing hinge gap widths, Ellis et al demonstrated that the prototype high leaker valve had larger volumetric washout and higher RSS compared to the prototype low leaker valve [Ellis, 1999]. The study suggested that a smaller nominal leakage gap width would cause less blood damage than a larger nominal leakage gap width. In addition, based on in vitro blood studies with three prototype carbon models of the SJM $27 \mathrm{~mm}$ valve, Travis et al suggested the possible existence of an optimum gap width [Travis, 2001c]. However, both these studies did not provide any direct quantitative flow information inside the hinge recess concerning the effect of various hinge gap widths on the hemodynamics performance of bileaflet valves.

\subsubsection{1st Hypothesis}

The first part of this study addresses the hypothesis that the hinge profile and the hinge gap width influence the thrombogenic potential of bileaflet heart valves. The specific aims designed to test this hypothesis are:

\subsubsection{Specific Aim \#1}

To study the flow fields in the hinge region of different bileaflet mechanical heart valve designs and to evaluate their potential to cause thrombus formation. The objective is to identify micro flow structures within the hinge region of the CarboMedics bileaflet MHV. The evaluation of the flow fields in the CarboMedics bileaflet MHV hinge region is done by comparing the micro flow fields between the CarboMedics (CM), St. Jude Medical (SJM) Regent, Medtronic Advantage (MA), and Medtronic Parallel (MP) bileaflet MHVs. To accomplish this, two-component Laser Doppler Velocimetry (LDV) 
measurements were conducted in the hinge region of a $\mathrm{CM}$ valve placed in the mitral position. These measurements enabled the reconstruction and animation of detailed flow maps that revealed both the spatial and temporal variations of the hinge micro flow fields. These maps of the hinge micro flow fields illuminate regions of high shear stress where blood damage could potentially occur, and stagnation sites where damaged blood elements could accumulate and develop into a micro-thrombus. This study is the first detailed in vitro investigation of the $\mathrm{CM}$ bileaflet hinge design conducted under physiological mitral conditions. This study also provides a direct comparison of the micro flow field characteristics from three different valves with the same valve internal diameter but different hinge designs, thereby allowing the assessment of the influence of various hinge geometries on the micro flow fields. In this study two regions of the hinge are of special focus as previous studies have speculated higher chances of thrombus formation [Ellis, 1996a; Ellis, 1996b; Ellis, 2000a; Ellis, 2000b]: 1) the leaflet projections inside the hinge pocket where separate zones of high Reynolds shear stress and flow stasis may be created during the systolic phase; and 2) the atrial side of the hinge geometry where high Reynolds shear stress near the hinge wall may cause damage to the platelets or red blood cells. In contrast to previous studies a higher spatial resolution of the LDV measurements was achieved in this study to provide a detailed assessment of the hinge flow.

\subsubsection{Specific Aim \#2}

To study the flow fields in the hinge region of a bileaflet mechanical heart valve design with varying hinge gap widths and relate these flow fields to their respective 
thrombogenic potentials. The objective is to demonstrate the influence of hinge gap width on the spatial and temporal characteristics of the hinge micro flow fields. To accomplish this goal, two-component LDV measurements are conducted in the hinge flow regions of three transparent $27 \mathrm{~mm}$ bileaflet mechanical heart valves, namely, the SJM $27 \mathrm{~mm}$ Standard (standard hinge gap width), the prototype $27 \mathrm{~mm}$ high leaker (large hinge gap width) and the prototype $27 \mathrm{~mm}$ low leaker (small hinge gap). The experiments were conducted in a pulsatile loop, with the valves placed in the mitral position. Flow maps of these measurements are reconstructed and animated in time to reveal both the spatial and temporal variation of the hinge flow fields. These hinge flow field maps help illuminate the influence of hinge gap width on the locations of high shear stress where blood damage could potentially occur, and stagnation sites where damaged blood elements could accumulate and lead to thrombus formation.

\subsection{Aortic Polymeric Heart Valve Studies}

The polyurethane trileaflet polymeric heart valve constitutes the latest development in prosthetic heart valve research [Hyde, 1999]. The design is based on the natural aortic valve and is inherently appealing from a hemodynamic viewpoint. Even though this particular valve design is still in a developmental stage, studies have shown it to possess excellent forward flow hemodynamic properties equivalent to that of a tissue heart valve and promise a durability comparable to that of a mechanical heart valve [Bernacca, 1995; Jansen, 1991]. Nevertheless, recent animal trials involving polymeric valves have reported problems mainly associated with tearing of the leaflets and thrombus formation occuring along the stent region of the valve [Wheatley, 2001]. In addition, results of 
long-term in vivo evaluation have suggested that calcification could be a limitation to long-term function of polymeric valves [Bodnar, 1991]. The leaflets and basal attachments, such as the commissural region of the polymer valves, have experienced extrinsic calcification associated with surface microthrombi that appear to be independent of structural defects suggesting that the flow characteristics inside the polymeric valve may be a contributor to the observed blood clots. Currently, no study have been conducted to investigate the flow inside a trileaflet heart valve prosthesis. Such a study would provide new insight into the roles that design features such as leaflet thickness and commissural designs have on the potential for blood damage in a trileaflet heart valve.

Another aspect of the valve that has never been investigated in the past is the effect of temperature on polymeric valve materials. Polymer properties are generally temperature dependent, and tend to be more flexible at higher temperatures. However, in vitro investigations involving fatigue and hemodynamics performance are usually performed at room temperature [D'Souza, 2003; Herold, 1987]. Polymeric valves implanted in animals are exposed to physiological temperature, and the effect of the elevated temperature on the valves' structural integrity and hemodynamic performance has not been addressed.

\subsection{1 $2^{\text {nd }}$ Hypothesis}

The second part of this work addresses the hypothesis that leaflet thickness and the commissural design of a tri-leaflet polymeric heart valve influence the valve's functionality and thrombogenic potential. The specific aims designed to address this hypothesis are: 


\subsubsection{Specific Aim \#3}

To quantify and analyze the flow fields in the immediate vicinity of a polymeric heart valve with different leaflet thicknesses and commissural designs. The flow fields are then related to their potential to cause blood damage and thromboembolic complications, including thrombus deposition on the valve super structure. To accomplish this goal, a pulsatile flow loop was constructed and two- and threecomponent particle image Velocimetry (PIV) and LDV measurements were conducted on three prototype tri-leaflet polymeric heart valves placed in the aortic position. The three prototype valves are differentiated by their commissural designs and leaflet thicknesses. Prototype A is characterized by an open commissural design with $80 \mu \mathrm{m}$ thick leaflets; prototype B by the closed commissure design with $120 \mu \mathrm{m}$ thick leaflets; and prototype C by a semi-closed commissure with $120 \mu \mathrm{m}$ thick leaflets. The PIV and LDV measurements are reconstructed and animated in time and space to reveal both the spatial and temporal variations of the flow fields in the three polymeric heart valves. The velocity maps of the polymeric heart valves reveal regions of high shear and stagnation sites where blood damage could potentially occur. The study provides the first detailed in vitro investigation of the flow characteristics of the trileaflet polymeric heart valves designs and helps assess: (i) how the generated local flow structures are influenced by the different valve design parameters; and (ii) the effects of these flow fields on the potential to cause thrombus formation on the valve.

In addition, static and pulsatile leakage rate studies studies were conducted at both room $\left(20.5^{\circ} \mathrm{C}\right)$ and physiological temperatures $\left(37^{\circ} \mathrm{C}\right)$, on prototype A to assess the effect of 
temperature on the valve's hemodynamic performance. Dual Camera Stereo Photogrammetry (DCSP) was used to study the leaflet kinematics dynamics of prototype A. Data obtained from two high speed cameras were processed using "in-house" software to numerically reconstruct the three dimensional movement of the leaflet and also to calculate kinematics parameters for this movement. Results from this study are intended to establish a basic understanding of the influence of temperature on the kinematics of the polymeric valve by visualizing the dynamic motion of the leaflet. This knowledge could subsequently be used by valve manufacturers to improve the durability and hemodynamics of polymeric valves. In addition, this study is intended to act as a platform for numerical simulation studies of polymeric heart valves at Georgia Tech. 


\section{CHAPTER 4: EQUIPMENT AND MATERIALS}

\section{$\underline{4.1 \text { Bileaflet Mechanical Heart Valve Prostheses }}$}

A typical bileaflet mechanical heart valve prosthesis is shown in Figure 4-1. The essential design features of a bileaflet valve consists of two mobile leaflets that are shaped as semicircular disks, hence the name 'butterfly valve', retained within the ring or valve annular housing by four hinges. The hinge mechanism is such that a slight protrusion called the ear on either end of each leaflet mates the recess of the hinge profile machined inside the valve annular housing. Due to the nature of the hinge geometry, blood may flow (leak) through the hinge which the valve is either closed or open. The two leaflets move independent of each other and open to allow unimpeded flow through both the central and lateral areas of the orifice. The leaflets in the open position forms a large opening angle with respect to the plane of the valve housing, typically between $77^{\circ}$ and $90^{\circ}$. The leaflets open and close passively in response to the forces exerted by blood flow through the valve. In the closed position, the two leaflets meet while leaving a narrow opening of approximately $100 \mu \mathrm{m}$ between the edges allowing for some amount of leakage. This opening as well as those in the hinge regions allows blood to regurgitate during ventricular systole when the valve is in the mitral or tricuspid position and during ventricular diastole when in the aortic position. This leakage flow ensures continuous flushing of all the component surfaces and parts of the prosthesis during the cardiac cycle. All prosthetic valves use a rigid, sewing ring or cuff to aid the attachment of the valve to the surrounding tissue during implantation. The sewing cuff is made of the 
nonthrombogenic Dacron cloth or polyethylene terephthalate. The cloth ensures the encapsulation of endothelialized tissue or pannus growth and prevents the tissue from interfering with the leaflets motion. The leaflets and valve housing are made from a pyrolytic carbon material known to be blood compatible and has extremely high strength and wear resistance. The use of the pyrolytic carbon in all current mechanical valves as leaflets, valve housing, and strut-covering materials has virtually eliminated abrasive wear as a long term complication of heart valve replacement.

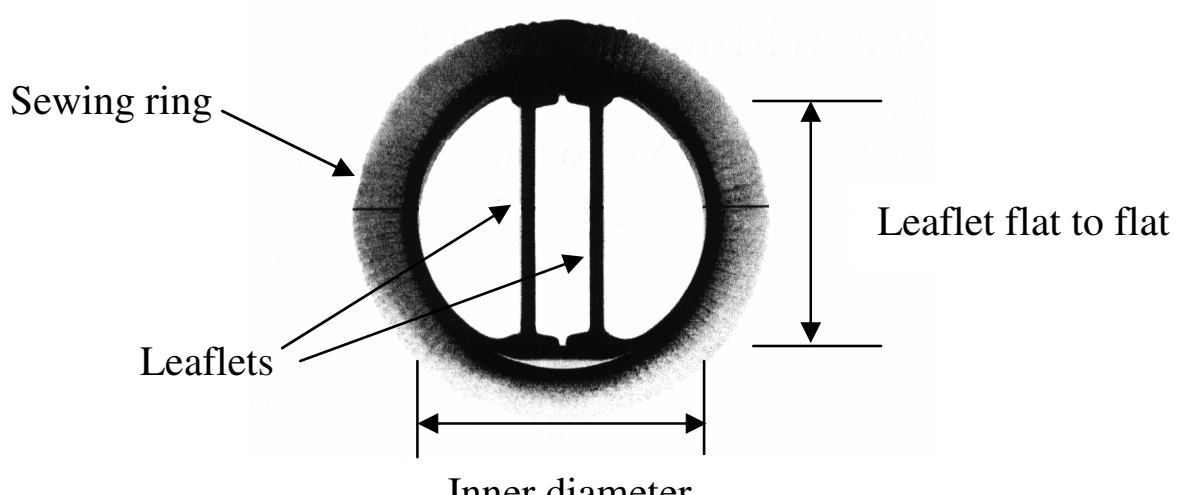

Figure 4-1 Picture of a typical mechanical bileaflet heart valve

\subsubsection{St. Jude Medical Bileaflet Mechanical Heart Valve}

The St. Jude Medical (SJM) Standard bileaflet mechanical heart valve was introduced for clinical use in the United States in 1977. Figure 4-2 shows the top view of the SJM bileaflet heart valve. The leaflet angle relative to the plane of the orifice is $85^{\circ}$ and approximately $35^{\circ}$ at the open and closed positions respectively. The leaflets therefore have a travel arc of $55^{\circ}$ to $60^{\circ}$. The sewing cuff is made of polyester polyethylene terephthalate (PET) or polytetrafluoroethylene (PTFE). 
The valve housing has an extension upstream known as the pivot guard designed to reduce exposure and interference with subvalvular structures, and also to reduce the working profile by allowing the leaflet to close entirely within the valve orifice. The SJM hinge is a butterfly-shaped depression. The leaflet is seated in the hinge by a hemispherical projection on the leaflet that mates to a similarly shaped recess around the center of the hinge. The geometry of the hinge is streamlined to provide a smooth hinge flow and minimize unwanted vortical structures and complex flow patterns as seen in the MP valve [Ellis, 1996b]. The SJM bileaflet design also includes at the downstream of the hinge mechanism a region of slight expansion, known as the thumbnail, machined into the valve housing.

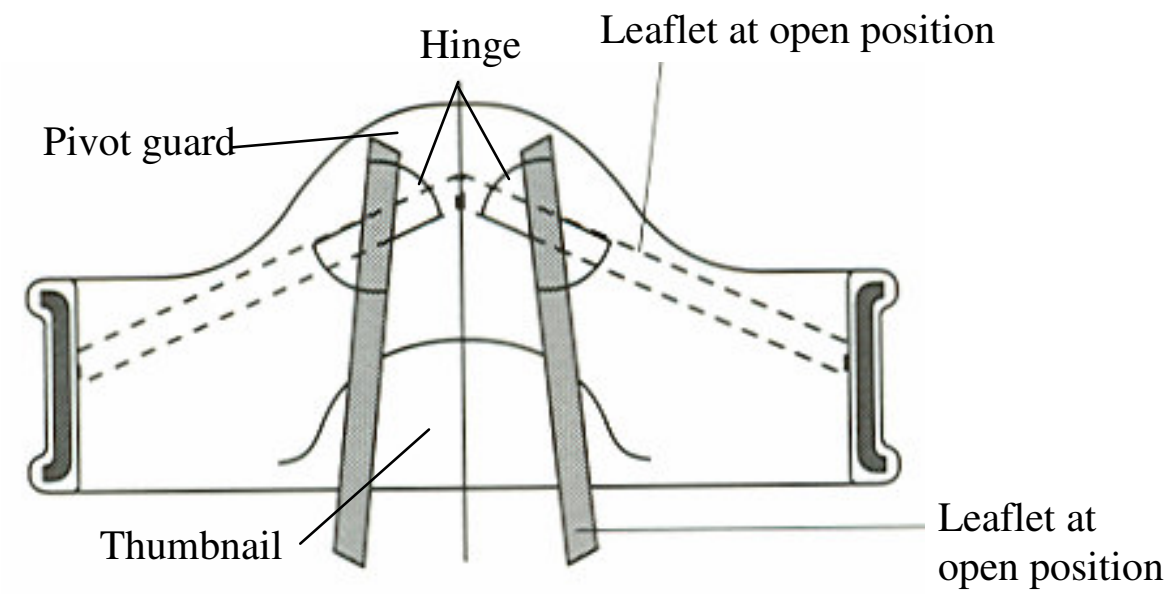

Figure 4-2 Top view of a SJM bileaflet heart valve at the open position

A series of improvements are now being incorporated into the newer St. Jude Medical bileaflet valves. The Hemodynamic Plus (SJM HP) series was introduced in 1993 to address problems associated with the mismatch between the size of the valve and the vessel of patients with small aortic annuli. The geometry and construction of the SJM HP 
is similar to that of the SJM Standard, except that the former includes a larger effective orifice area compared with the latter, with an equivalent valve annulus diameter (Figure 4-3). The annulus diameter is the same as the patient tissue annulus, while the orifice diameter constitutes the actual area of the orifice encountered by the blood. It was claimed that the hemodynamic performance of the SJM HP series is equivalent to that of the geometrically larger SJM Standard. For example, the orifice area of a SJM HP 17 mm valve is approximately equal to that of an SJM Standard $19 \mathrm{~mm}$ valve.
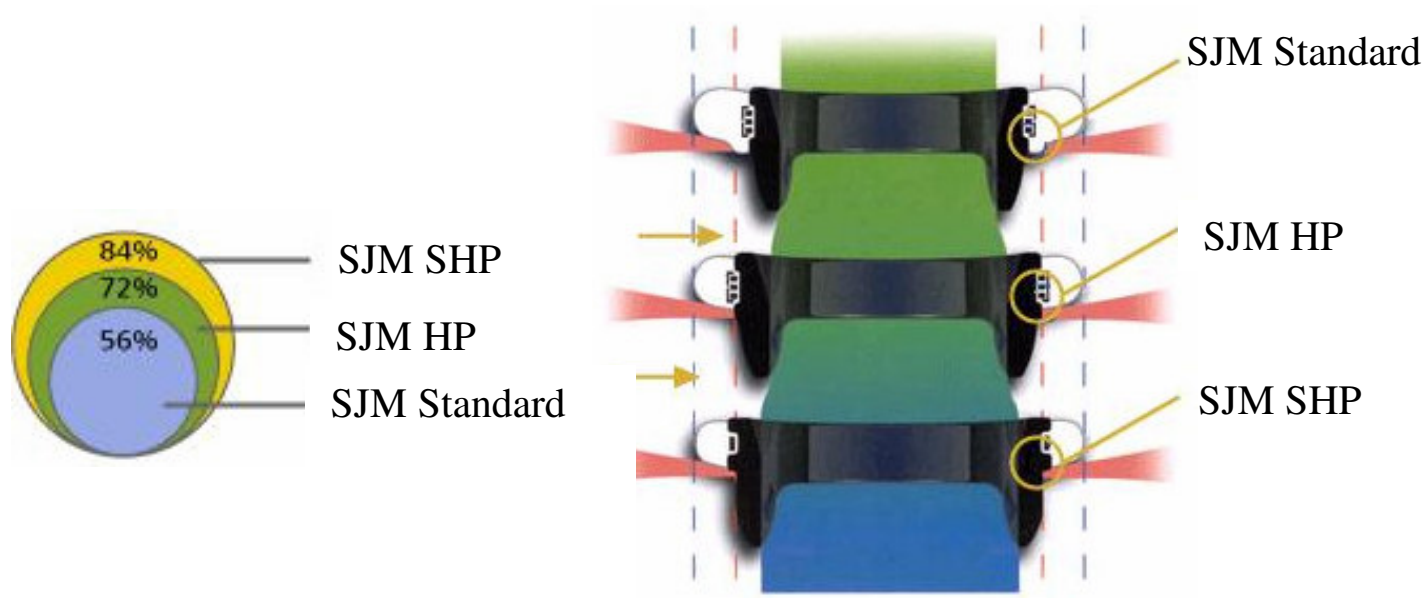

Figure 4-3 Comparison of different SJM valve designs. Larger effective orifice area is the major design improvement among the three valve types

The third and latest design of the SJM bileaflet valve series is the Super Hemodynamic Plus (SJM SHP) bileaflet mechanical valve, which is a modification of the currently marketed SJM HP bileaflet valve. The SJM SHP or Regent series incorporates a further increment in the orifice diameter over the SJM HP series and additional reinforcement of the housing to preserve mechanical integrity. The SJM SHP series retains the essentials 
of the original design of SJM HP series. Comparison of the three designs showed that each subsequent series has a larger flow orifice to tissue annulus ratio (Figure 4-3).

In this research the hinge flow fields from a clear SJM $27 \mathrm{~mm}$ Standard valve and two prototype valves with varying gap width tolerances were measured in the mitral position in order to study the effect of hinge gap width. The $27 \mathrm{~mm}$ SJM Standard valve is chosen as it is the most commonly implanted size of this valve in the mitral positions. The 27 mm SJM Standard valve has a gap width tolerance between the two prototype valves. The three valves are correspondingly designated as the ' $27 \mathrm{~mm}$ prototype low leaker, 'SJM 27 mm Standard and '27 mm prototype high leaker'. Leakage gap width is defined as the distance between the tip of the leaflet ear and the top of the hinge recess inside the housing (Figure 4-4).

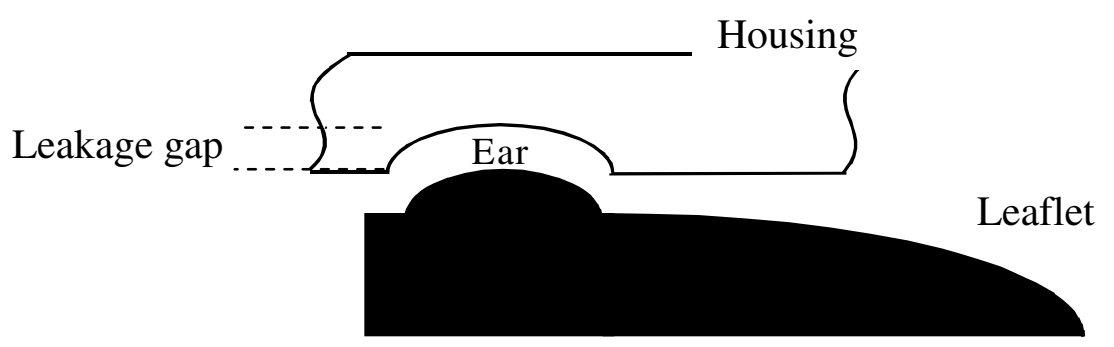

Figure 4-4 Close-up cutaway side view illustrating geometry of SJM hinge recess. The leakage gap is defined as the distance between the tip of the leaflet ear and the top side of the hinge socket

The control of the hinge gap width in these valves is determined by the tolerance of the distance between the ear of the leaflets and the depth of the hinge recess which is about $500 \mu \mathrm{m}$. The leakage gap width for the three clear valves are not specified but can only 
be differentiated by their static leakage rates performed at the St Jude Medical. However, the leakage gap widths were measured using micro-computer tomography and was determined to be approximately $50 \mu \mathrm{m}, 100 \mu \mathrm{m}$, and $200 \mu \mathrm{m}$ for the $27 \mathrm{~mm}$ prototype low leaker, SJM $27 \mathrm{~mm}$ Standard, and the $27 \mathrm{~mm}$ prototype high leaker, respectively. In order to gain optical access into the hinge volume of the three clear valve models, custom valves were machined from a clear housing at St. Jude Medical (Figure 4-5). For the clear valve housing models, the leaflets were made of Delrin, which is less rigid than the pyrolytic carbon and has been used in many commercially available valves.

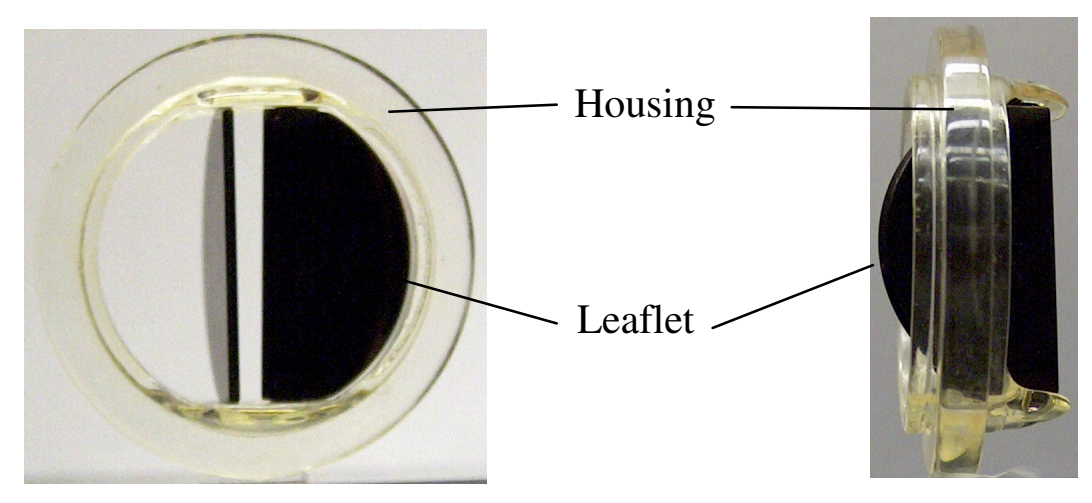

Figure 4-5 Front and side views of the prototype SJM clear valve housing. The clear valve housing facilitate the optical access to the hinge region

\subsubsection{Carbomedics Bileaflet Mechanical Heart Valve}

Another widely used heart valve is the Carbomedics ${ }^{\circledR}$ prosthetic heart valve $(\mathrm{CM})$, which shares many similar design features of the SJM bileaflet valve (Figure 4-6). The $\mathrm{CM}$ bileaflet valve is a second-generation pyrolytic carbon bileaflet valve with rotatable 
sewing ring. It was first introduced into clinical use in 1986 as the third bileaflet valve to reach clinical application after the St. Jude Medical and Edwards-Duromedics valves.

Like the SJM mechanical valves, the CM valve is a low-profile bileaflet heart valve prosthesis with pyrolytic carbon leaflets. Both SJM and CM valves contain tungsten to enhance radiographic imaging quality. Unlike the SJM valves, the CM bileaflet valve leaflets open to 78 degrees with a travel arc of $53^{\circ}$. The sewing cuff is constructed of a knitted polyester material coated with Biolite ${ }^{\circledR}$ carbon. The $\mathrm{CM}$ valve lacks the thumbnail and the pivot guard designs. The ear of the CM bileaflet valve leaflet is flatter than that of the SJM valves and it mates with a flatter and angulated hinge recess as shown in Figure 4-7. The CM valve has the similar 'butterfly' hinge geometry of the SJM valve except that its geometric profile has sharper corners. The hinge configuration in the $\mathrm{CM}$ valve is intended to enhance the washout of stagnant regions inside the hinge when the valve closed. Typically, the CM valves have smaller orifice to annulus ratio than the St Jude Medical heart valves. 


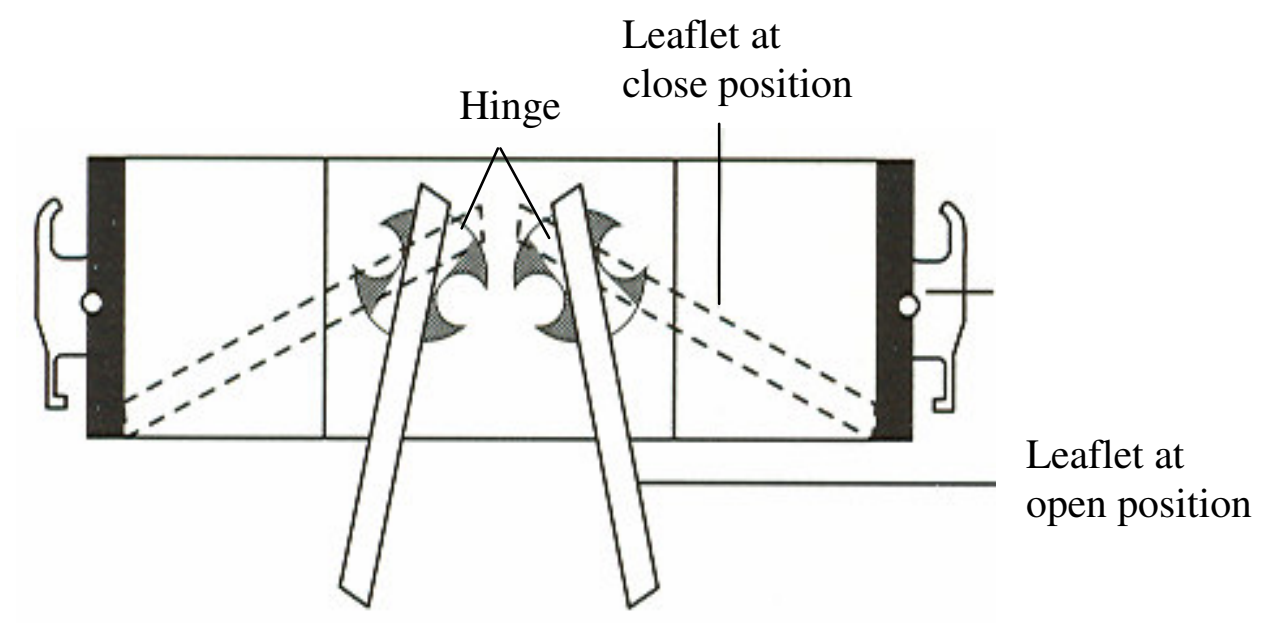

Figure 4-6 Top view of the CM bileaflet heart valve at open position

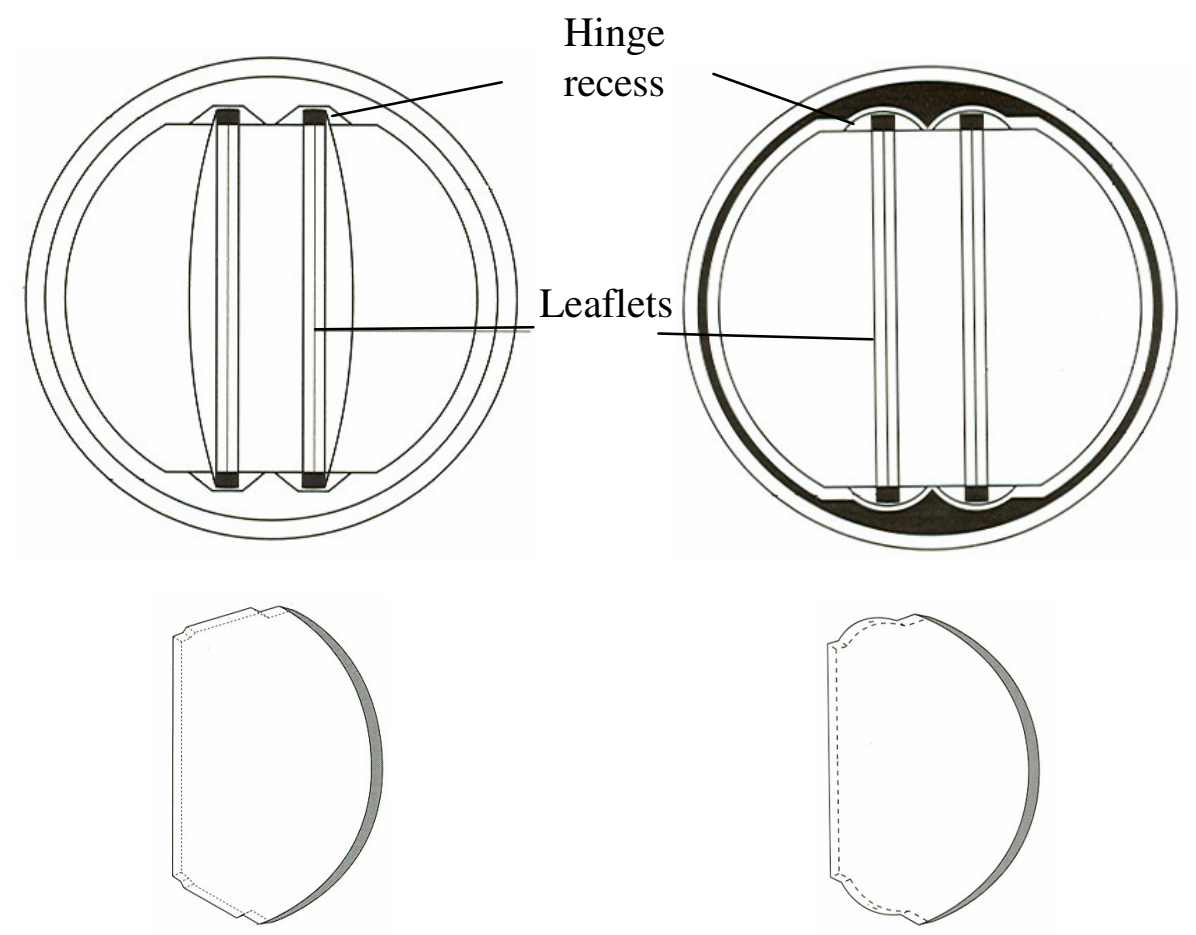

Figure 4-7 Comparison of the CM and SJM valve designs 
In this research the hinge flow fields from a CM $23 \mathrm{~mm}$ clear valve in the mitral position was studied in order to study the effects of different hinge designs. The $23 \mathrm{~mm}$ size was chosen to facilitate the evaluation and comparison of hinge flow fields of this valve with that of the St. Jude Medical $23 \mathrm{~mm}$ Regent and Medtronic Parallel performed earlier by Ellis et al [Ellis, 1996a; Ellis, 1996b]. St. Jude Medical provided a reverse engineered 23 mm CarboMedics standard bileaflet mechanical heart valve (Figure 4-8). The reproduction of the clear valve housing is of clinical quality to ensure accurate representation of hinge flow profile to be studied. The leaflets were made of Delrin.

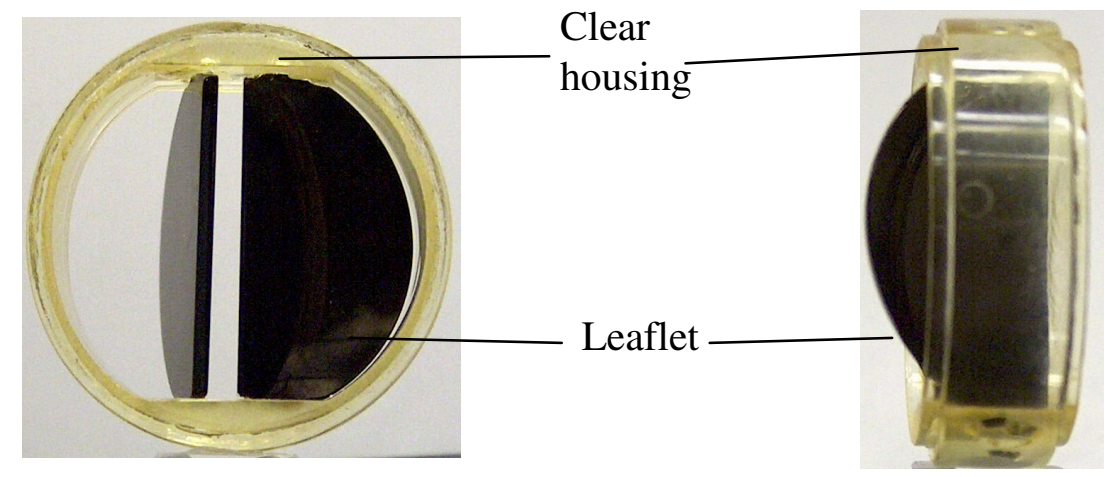

Figure 4-8 Front and side view of the $\mathrm{cm}$ clear valve housing

\subsection{Polymeric Heart Valves}

Attempts to manufacture leaflet valves from polymers date back to the 1950s and as early as the inception of the first mechanical heart valve prostheses. However, the first realistic solution was not realized until recently. The major hurdles for the success of the polymeric valves pertained to valve design and its chemical composition to eliminate thrombogenicity, calcification, mechanical stress, and durability. The current design of polyurethane valve under study is like the stent-supported bioprosthetic heart valve. Here 
the polymeric leaflets are mounted onto flexible stents that lead to a circular orifice during forward flow. However, instead of each leaflet being sewn separately onto the stents as in the bioprosthetic valve, the leaflets, stent, and the sewing ring in polymeric valves are made of a multilayered cohesively bonded single material and are available at various degrees of hardness [Daebritz, 2004]. The leaflets have a semi-open position during unstressed state to minimize membrane stresses during diastole and systole. Stent posts are flexible to some extent to ensure tight leaflet closure during diastole. All current bioprosthetic heart valves are tri-leaflet, however polymeric valves have also been designed with a bileaflet configuration specifically for mitral position implantation (Figure 4-9) [Daebritz, 2004]. Today, all polymeric heart valves are made entirely of polyurethane, with a combination of solution casting and injection molding. The stents or frames are injection molded and typically have a thickness of approximately $3 \mathrm{~mm}$. The polyurethane frames are then molded onto steel formers of ellipto-hyperbolic leaflet shape [Mackay, 1996] and dipped into a concentrated polyurethane solution so that they coat the whole valve to form the leaflets. The polymer valve is then allowed to dry while hanging free edge downward. The leaflet edge is later cut and trimmed by precision a laser cutting tool. The thickness of the leaflet ranges from 80 to $300 \mu \mathrm{m}$ [Bernacca, 1997a; Daebritz, 2003; Daebritz, 2004; Mackay, 1996]. Some polyurethane valves contain a stiffening ring of radio-opaque MRI compatible titanium alloy to facilitate radiographic imaging. 


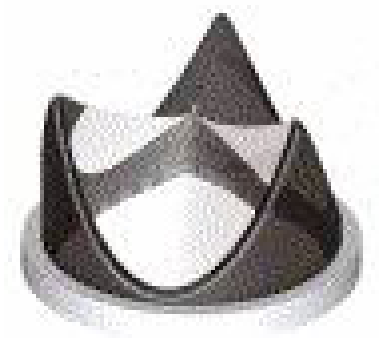

(a)

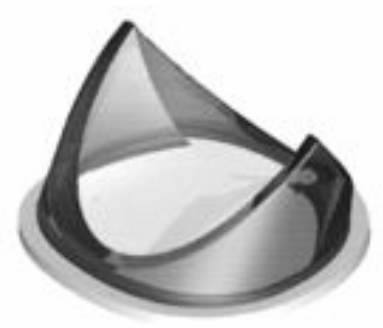

(b)

Figure 4-9 Computer aided design of the polymeric heart valves; (a) three stent aortic design, (b) two stent mitral design

\subsubsection{Aortech Polymeric Heart Valve}

Figure 4-10 shows one of the three $23 \mathrm{~mm}$ tri-leaflet polymeric valves investigated in this research. The commissural region is defined as where the leaflets meet near the stent post. The coaptation region is the center region where the three leaflets meet and the high central region of the valve is located at the upper portion of the each leaflet near its free edge.

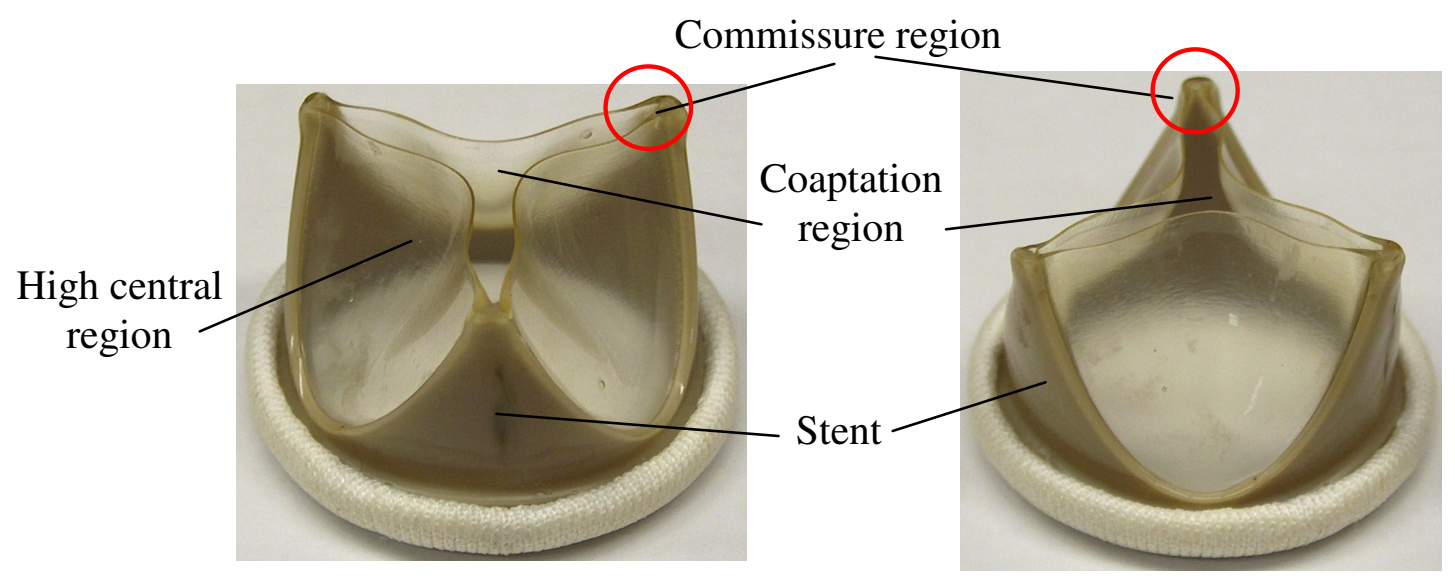

Figure 4-10 $23 \mathrm{~mm}$ tri-leaflet polymeric heart valve. The leaflet has the same refractive index as the working solution (aqueous Sodium Iodide), thus allowing velocity measurements by means of laser Doppler Velocimetry inside the polymeric valve 
Figure 4-11 shows the three prototype valves used in the current study. The prototype A valve, shown in Figure 4-11a, has a closed commissure design and a leaflet thickness of $80 \mu \mathrm{m}$; prototype $\mathrm{B}$ valve in Figure $4-11 \mathrm{~b}$ has an open commissure design and a leaflet thickness of $120 \mu \mathrm{m}$; prototype $\mathrm{C}$ in Figure 4-11c has a semi-open commissure and a leaflet thickness of $120 \mu \mathrm{m}$. A closed commissure design has the characteristics of two adjacent leaflets formed closer to each others near the stent region. On the other hand, in an open commissure design the adjacent leaflets did not come together near the stent but were well separated. A leaflet thickness range from 80 to $150 \mu \mathrm{m}$ was considered optimum for good valve durability and hydrodynamic performance.

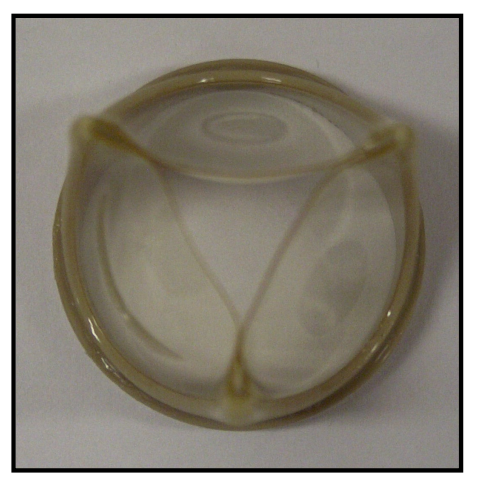

(a)

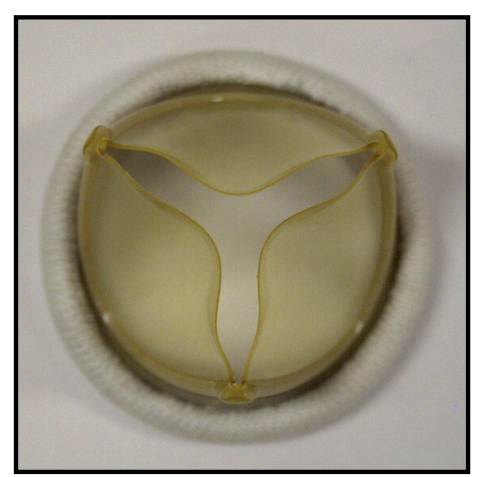

(b)

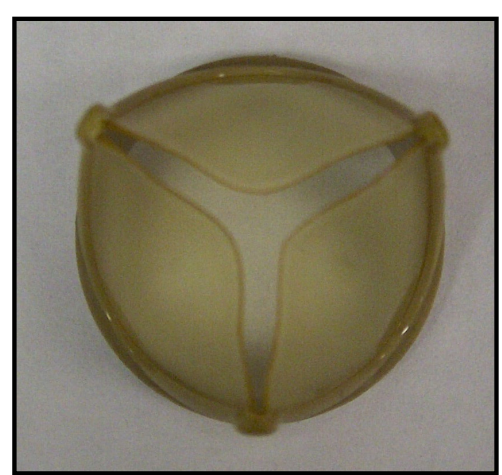

(c)

Figure 4-11 The three different designs of the polymeric heart valve; (a) prototype A, (b) prototype $\mathrm{B}$, (c) prototype $\mathrm{C}$

A comparison of leaflet thickness from $80 \mu \mathrm{m}$ to $120 \mu \mathrm{m}$ provide a demonstration of how leaflet thickness could influence the flow through the valves. The valves are prototype designs provided by AorTech Europe with leaflets manufactured from high silicone content polyurethane copolymers (Elast-Eon ${ }^{\mathrm{TM}}$ ) and the valve frame and stents were 
machined from PEEK (Poly-etheretherketone). The polymeric valve was fabricated by dip-coating the PEEK frame with a thin layer of polyurethane leaflet. Table 4-1 tabulates the various valve types that were investigated in this research

\section{$\underline{4.3 \text { Valve Mounting Chambers }}$}

\subsubsection{Mounting Chamber For The Three $27 \mathrm{~mm}$ Transparent Bileaflet Heart Valve}

Figure 4-12 shows the clear valve chamber provided by St Jude Medical to hold the three $27 \mathrm{~mm}$ clear valves (one SJM $27 \mathrm{~mm}$ Standard and the two prototype leaker valves). The chamber was fabricated from a solid acrylic block and can be divided into four sections. The valve seats within a recess in section (2) and is held in place when the four sections are fastened together by four bolts. The four sections have a 1" internal diameter. Sections (1) and (2) together form a 1.78" straight inlet section upstream of the valve housing while sections (3) and (4) form a 1.84" straight section downstream of the valve housing. The interface between sections (2) and (3) restricts optical access to certain parts of the valve housing. Depending on the region of interest (i.e. thumbnail or hinge region) the valve needs to be reversed to gain optical access. Gaskets were used between the interfaces of the four sections to prevent fluid leakage. The inlet and outlet of the model were connected to PVC piping with a outer and inner diameter of 1.25", and 1.00" respectively. This valve chamber was used for the mitral hinge flow and static leakage experiments for the SJM valves. 
Table 4-1 Valves Studied

\begin{tabular}{|c|c|}
\hline Valve types & Experiments \\
\hline CM $23 \mathrm{~mm}$ & $\begin{array}{c}\text { Pulsatile hinge and vicinity regions - 2D } \\
\text { LDV, mitral } \\
\text { Leakage Flow Rate }\end{array}$ \\
\hline SJM 27 mm Standard valve & $\begin{array}{c}\text { Pulsatile hinge and vicinity regions - 2D } \\
\text { LDV, mitral } \\
\text { Leakage Flow Rate }\end{array}$ \\
\hline $27 \mathrm{~mm}$ prototype low leaker valve & $\begin{array}{c}\text { Pulsatile hinge and vicinity regions - 2D } \\
\text { LDV, mitral } \\
\text { Leakage Flow Rate }\end{array}$ \\
\hline $27 \mathrm{~mm}$ prototype high leaker valve & $\begin{array}{c}\text { Pulsatile hinge and vicinity regions - 2D } \\
\text { LDV, mitral } \\
\text { Leakage Flow Rate }\end{array}$ \\
\hline $\begin{array}{c}\text { Aortech } 23 \mathrm{~mm} \text { prototype A polymeric } \\
\text { valve }\end{array}$ & $\begin{array}{c}\text { Temperature-dependent experiment } \\
\text { High speed marker for 3D reconstruction } \\
\text { of leaflet motion } \\
\text { Pulsatile inside valve and vicinity regions } \\
\text { - 2D LDV, aortic } \\
\text { Pulsatile valve downstream region - 3D } \\
\text { LDV, aortic } \\
\text { Pulsatile inside valve and vicinity regions } \\
\text { - 3D PIV, aortic } \\
\text { Leakage Flow Rate (room and } \\
\text { physiological temperature) }\end{array}$ \\
\hline $\begin{array}{c}\text { Aortech } 23 \mathrm{~mm} \text { prototype B polymeric } \\
\text { valve }\end{array}$ & $\begin{array}{c}\text { 3D Topography using White Light } \\
\text { Interferometer } \\
\text { Pulsatile inside valve and vicinity regions } \\
\text { - 2D LDV, aortic } \\
\text { Pulsatile inside valve and vicinity regions } \\
\text { - 3D PIV, aortic } \\
\text { Leakage Flow Rate (room and } \\
\text { physiological temperature) }\end{array}$ \\
\hline $\begin{array}{c}\text { Aortech } 23 \mathrm{~mm} \text { prototype } \mathrm{C} \text { polymeric } \\
\text { valve }\end{array}$ & $\begin{array}{l}\text { Pulsatile inside valve and vicinity regions } \\
\qquad \text {-2D LDV, aortic } \\
\text { Pulsatile inside valve and vicinity regions } \\
\text { - 3D PIV, aortic } \\
\text { Leakage Flow Rate (room and } \\
\text { physiological temperature) }\end{array}$ \\
\hline
\end{tabular}




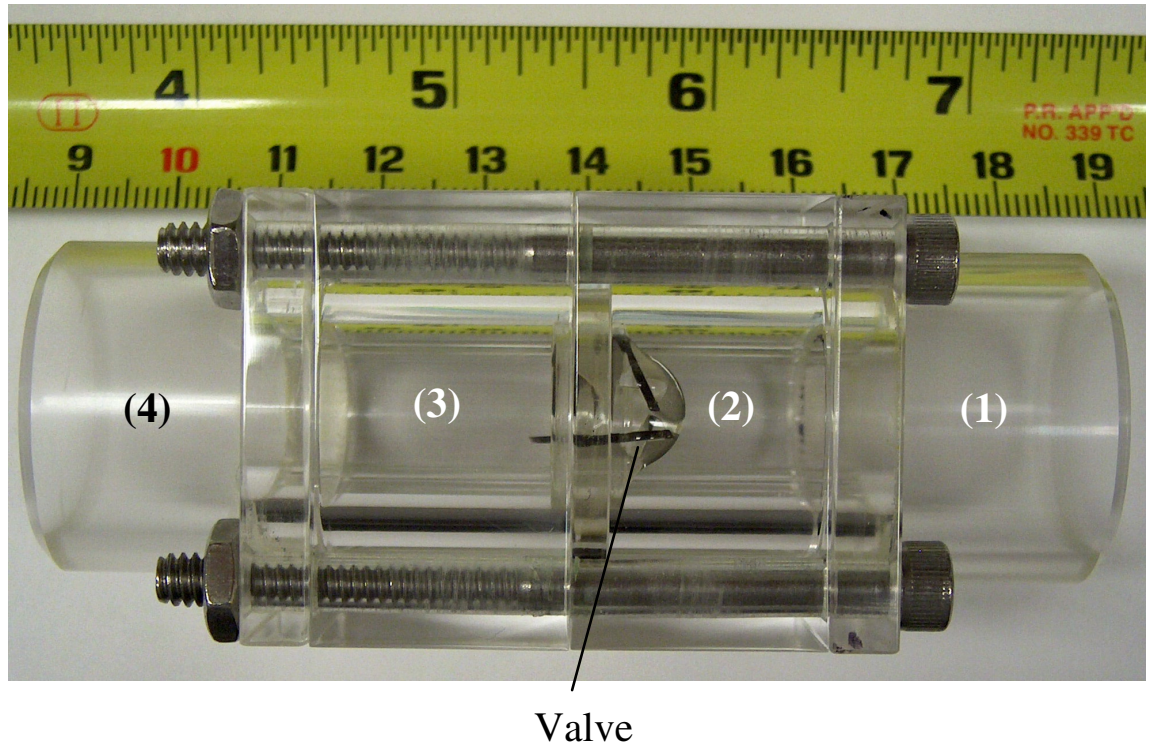

Figure 4-12 Mitral valve holder for the three $27 \mathrm{~mm}$ transparent bileaflet heart valves; one SJM $27 \mathrm{~mm}$ Standard and the two prototype leaker valves

\subsubsection{Mounting Chamber For The Carbomedics Transparent Bileaflet Heart Valve}

The valve chamber provided by St. Jude Medical was designed to hold the CM $23 \mathrm{~mm}$ clear valve (Figure 4-13). The valve chamber is divided into three sections fabricated from solid acrylic material. The 1.4" thick middle section (2) has an internal bore diameter of 0.78 " and a recess of 0.84 ". The valve housing fits into the recess of section (2) and is held in place by a 0.52 " long stopper, which has an internal and external diameter of 0.78 ", and 0.84 "respectively. In addition to the stopper, a threaded screw in the middle section of the section (2) is used to fix the valve and prevent it from rotating. The three sections are fastened together by bolts. The valve chamber has an equal upstream and downstream length of approximately 1.61 " with respect to the position of the valve. A step up tubing facilitates the mating of the smaller inlet and outlet sections to the 1.25 " PVC piping. Two seal gaskets were used to prevent fluid leakage at the 
interface of the three sections. This valve chamber was used for the mitral hinge, and static leakage experiments for the CM valve.

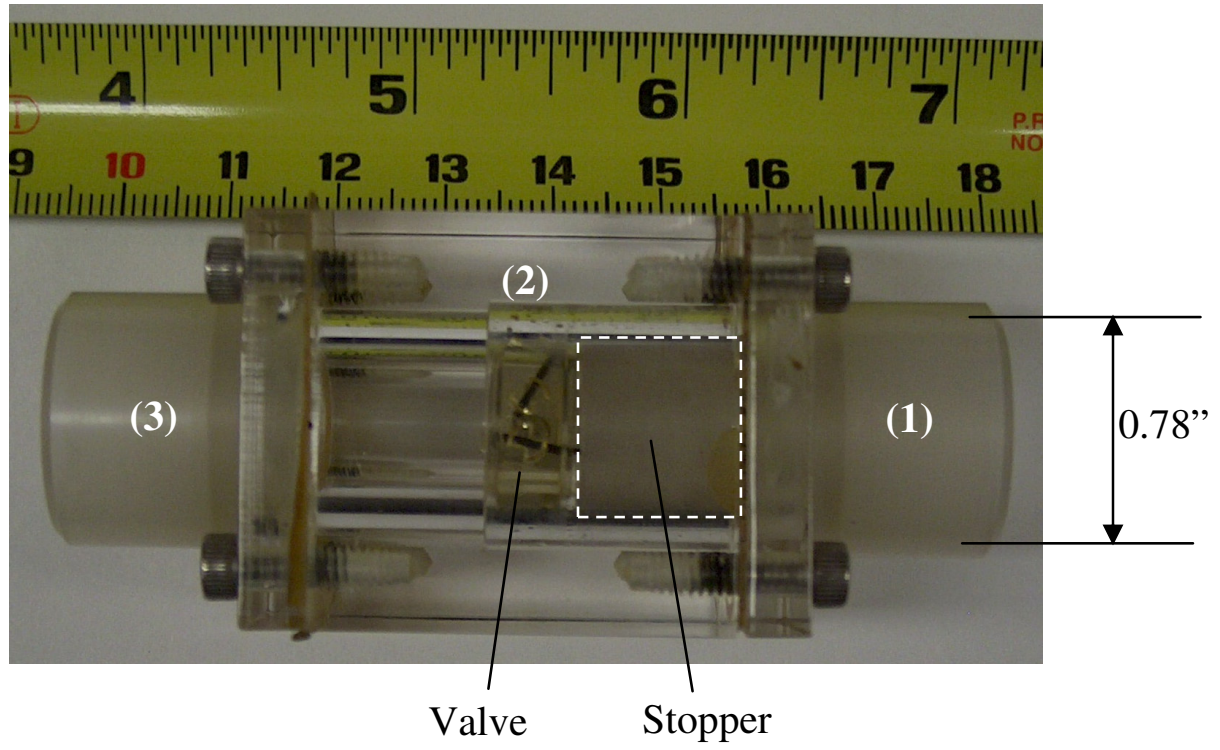

Figure 4-13 Valve holder for the CM $23 \mathrm{~mm}$ bileaflet heart valve

\subsubsection{Mounting Chamber For Aortech Polymeric Heart Valves}

The valve chamber for the polymeric valves was fabricated from solid block acrylic shown in Figure 4-14. The model was polished to allow optical access necessary to obtain the velocity measurements. The valve chamber is comprised of two sections; the distal section of the model is a straight, axisymmetric representation of the human aortic root. The diameter of the distal model section and length of the sinus region within this section were similar to the geometry of that in a human. The upstream section has a straight bore of 1 " diameter with an inlet length of 4.1". The polymeric valve is placed between the two sections of the valve chamber and the entire test section is held together by two metal plates, at both end of the valve chamber, bolted by four threaded rods . Two 
O-rings were placed between the sections to prevent leakage. This valve chamber was used for the aortic and static leakage experiments for the polymeric heart valves, at room temperature and physiological temperature.

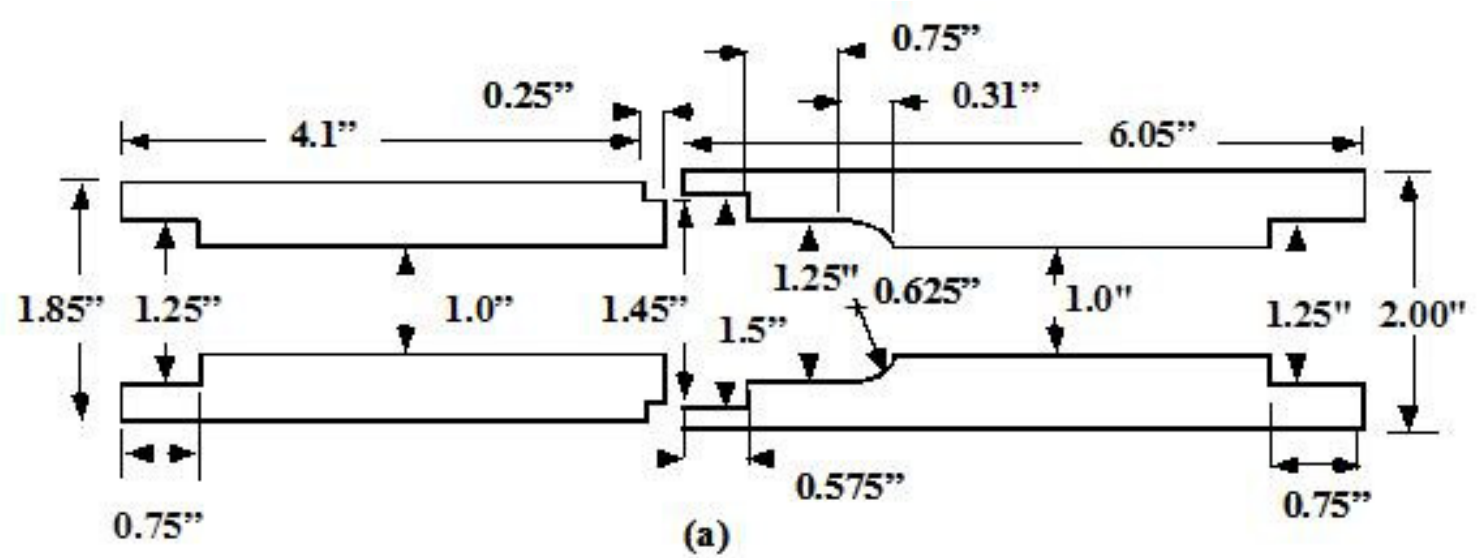

Figure 4-14 Schematic of the valve holder for the $23 \mathrm{~mm}$ polymeric heart valves

\section{$\underline{4.4 \text { Steady Flow Loop }}$}

\subsubsection{Static Leakage Loop For Bileaflet Mechanical and Polymeric Heart Valves}

The leakage setup, shown in Figure 4-15, was used to determine the leakage flow rates of the CM $23 \mathrm{~mm}$, the SJM $27 \mathrm{~mm}$ Standard, the two $27 \mathrm{~mm}$ prototype leakers, and the three Aortech $23 \mathrm{~mm}$ prototype polymeric heart valves. The prosthetic heart valves were subjected to a constant fluid pressure difference of $120 \mathrm{mmHg}$ across the valve as expected during diastole and systole. The flow setup mainly comprised of sections of $3 / 4$ " PVC tubing fitted together via rubber tubing and hose clamps. A plastic bucket served as the fluid reservoir. The vertical distance, $h$, between the level of the fluid inside the reservoir and the closed valve determines the pressure difference across the valve. The following relation is used to calculate $h$ for a given pressure drop $p$. 


$$
\begin{aligned}
& p=\rho g h \\
& h=\frac{16,000}{1,100 \times 9.81}=1.61 \mathrm{~m}
\end{aligned}
$$

where $p$ is the required pressure of $120 \mathrm{mmHg}$ or $16,000 \mathrm{kPa}$ and $\rho$ is the density of the glycerin/water solution used in these experiments which was determined to be 1,100

$\mathrm{kg} / \mathrm{m}^{3}$. Two PVC ball valves were placed between the reservoir and the prosthesis to regulate the flow through the circuit and facilitate the dissembling of the static loop. A third check valve was placed between the valve chamber and the collecting bucket.

\subsubsection{Temperature-Dependent Study in Polymeric Heart Valves}

The setup for the static leakage rate of the polymeric heart valves at physiological temperature was similar to leakage flow rate setup described in section 4.4.1, except for the addition of a water bath unit placed underneath the reservoir to heat the glycerin/water solution to temperature of $37^{\circ}$. The water bath comprised of a $1.5^{\prime} \times 1^{\prime} \mathrm{x}$ 4" metal tray (Polar Ware, WI) filled half way with water. The reservoir is placed within the metal tray, which was in turn placed on top of a hot plate stirrer (PC-351, Corning). Temperature effect was also investigated in the pulsatile mock loop later described in section 4.5.1. 


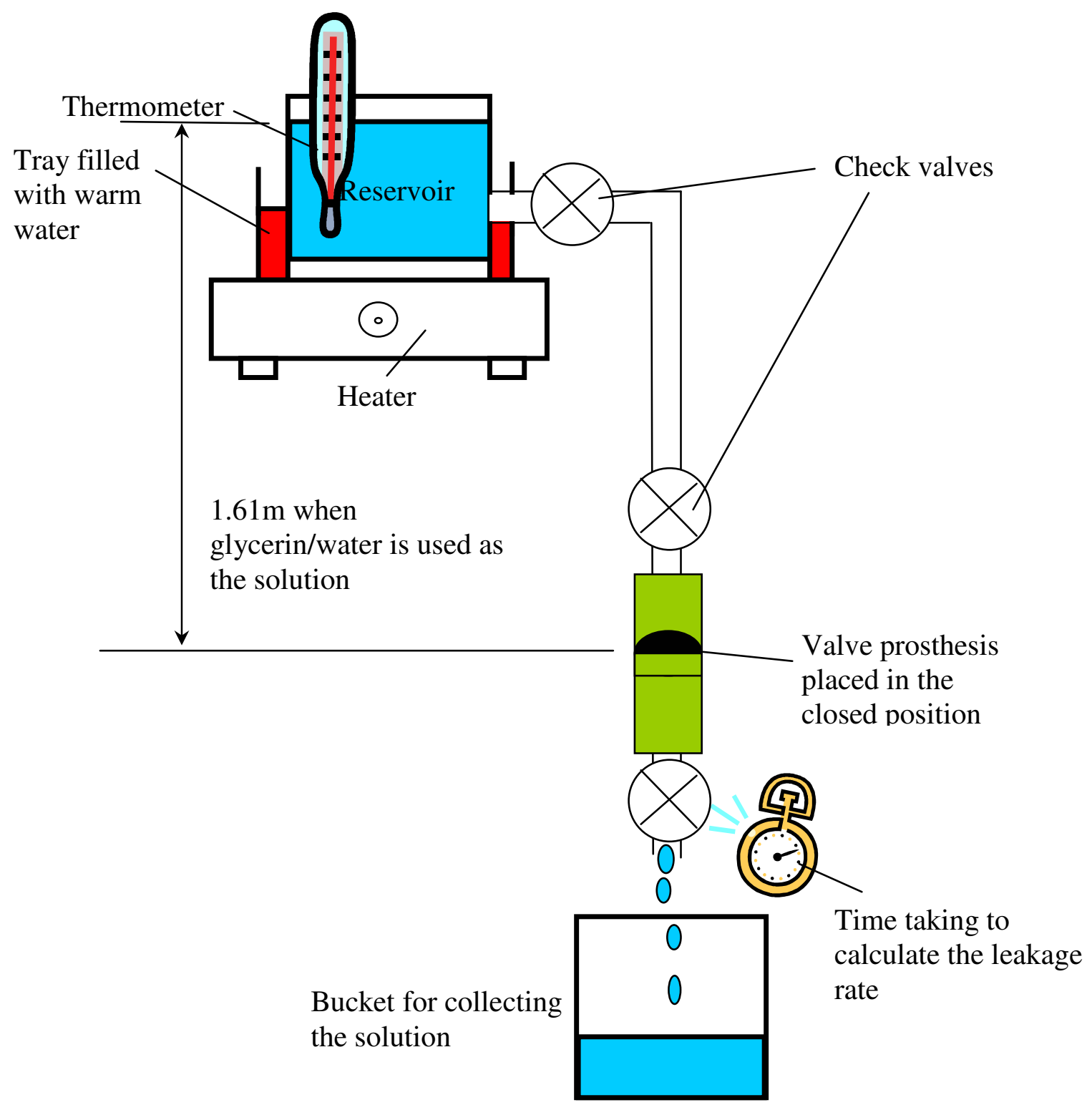

Figure 4-15 Static leakage flow loop setup 


\section{$\underline{4.5 \text { Pulsatile Flow Loop }}$}

\subsubsection{Mitral Hinge Flow Studies: CM $23 \mathrm{~mm}$ and SJM $27 \mathrm{~mm}$ Standard}

Figure 4-16 shows a schematic of the pulsatile loop used to investigate the flow field in the hinge region of the CM $23 \mathrm{~mm}$, the SJM $27 \mathrm{~mm}$ Standard, and the two $27 \mathrm{~mm}$ prototype valves. The loop, also called the Georgia Tech Left Heart simulator, was used to simulate the physiological conditions corresponding to the left side of the human heart. The loop consists of two parts; a) the pulse generation and delivery system, and b) the flow loop.

The pulse generation and delivery system consists of an air compressor, a pneumatic control board, and a pulse generator. The air compressor (Model T-2820P, Thomas, Detroit, MI) delivered an approximate pressure of $276 \mathrm{kN} / \mathrm{m}^{2}$ (40 PSI) of compressed air to the pneumatic control board, which consists of two pneumatic valves and a throttle valve. The length and time delay between the opening and closing of each pneumatic valve was controlled by a custom made computer-interfaced pulse generator (Model 56C12-111CA, MAC, Wixom, MI), a computer (Model 5150, IBM, Armonk, NY) that has an accuracy of $1 \mathrm{~ms}$. The throttle valve controlled the power of each stroke, and was used to regulate the flow and pressures across the valve prosthesis.

The flow loop comprised of a silicone pumping bulb, a flow transducer, two pressure transducers, a compliance unit,, resistance unit, a reservoir, and the two valve chambers with prostheses. The loop is connected by a 3/4" PVC tubing and 1" flexible rubber hose at joints. Compressed air was delivered from the pneumatic loop to the flexible silicone 
bulb sealed within an airtight acrylic chamber that was placed between the aortic and mitral chambers. The air compressed the silicone bulb thereby raising the pressure of the fluid between the mitral valve and the aortic valve. The onset of flow caused the mitral valve to close while fluid flowed through the aortic valve. During diastole, the relaxation of the bulb caused the aortic valve to close and drew the fluid through the open mitral valve into the bulb. The compliant and resistant units placed downstream of the aortic valve were adjusted to obtain accurate simulation of the mitral flow, ventricular and atrial pressures. This was accomplished by adjusting the clamp on the flexible rubber tubing to control the resistance, and the level of the solution inside the cylindrical compliance chamber. Ventricular pressure was measured between the mitral and the aortic valves, while the atrial pressure and the flow rate were obtained at the immediate upstream of the mitral valve. Both pressures were measured with disposable pressure transducers (Type 041-500503A, Argon, Athens, TX) while flow rate was measured with a $24 \mathrm{~mm}$ in-line ultrasonic flow probe (Model 24NSB12, Transonic Inc., Ithaca, NY). The three transducers were interfaced with CardioMed Amplifier CM 4008 (Medi-Stim AS, Norway) for real-time visualization. 


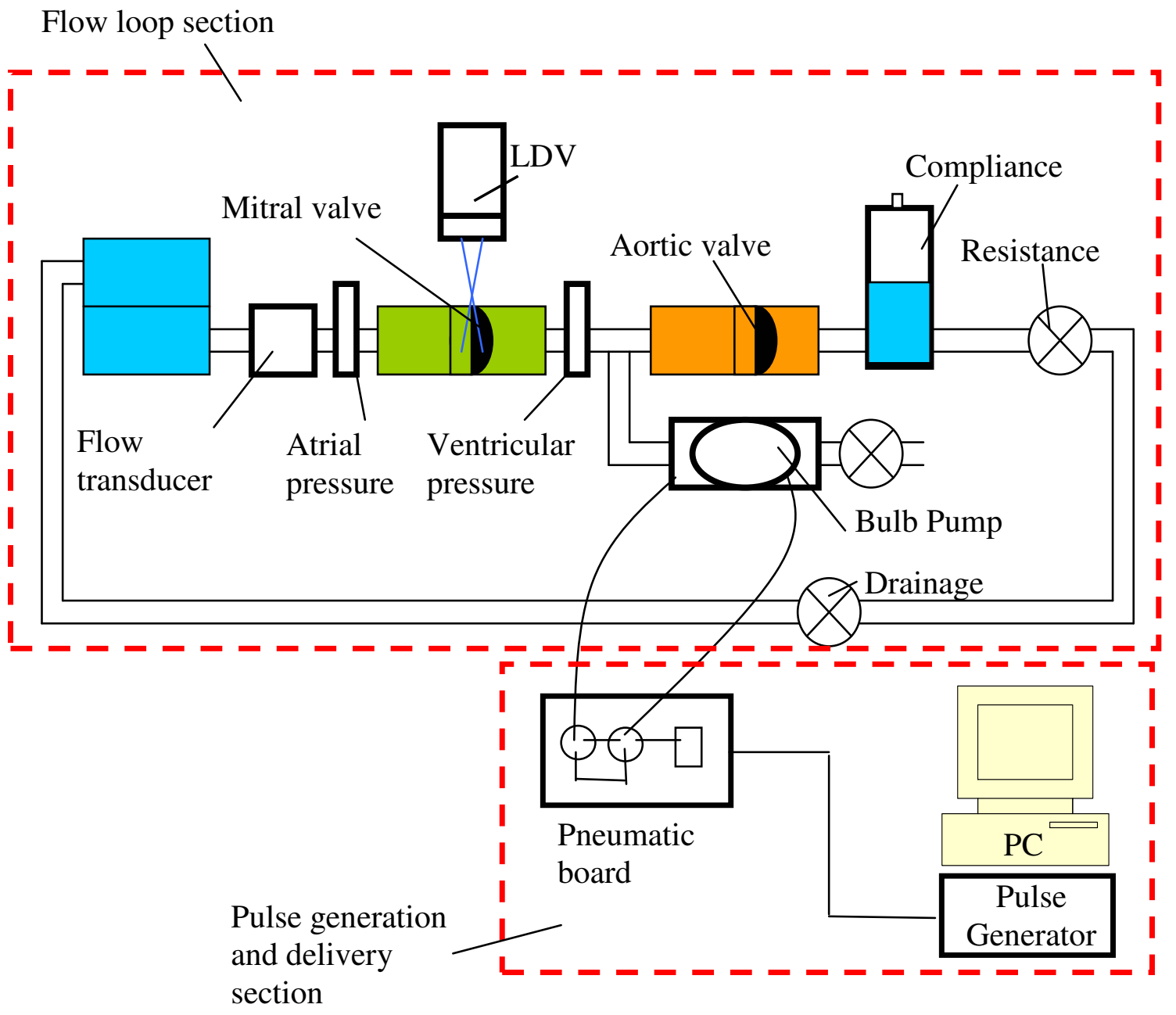

Figure 4-16 Pulsatile loop setup for the hinge flow experiments in mitral position 


\subsubsection{Aortic Flow Studies: Aortech 23 mm}

Figure 4-17 show the schematic of the pulsatile loop used to investigate the flow field through the three Aortech polymeric heart valves. The setup of this loop is similar to the one used for the hinge flow field measurements discussed in section 4.5.1. The main difference is that the focus of the study is now the valve prosthesis in the aortic position. Due to the different nature of polymeric valves compared to mechanical heart valves, several changes were incorporated into the loop in order to obtain the correct physiological waveforms corresponding to the flow rate, ventricular, and atrial pressure. The changes include an extra compliant unit downstream of the aortic valve, higher/lower elevation of the reservoir, and increased compressed air pressure to $345 \mathrm{kN} / \mathrm{m}^{2}$.

Ventricular pressure was measured between the mitral and the aortic valves, while the flow rate and aortic pressure was measured immediately downstream of the aortic valve. The pressures were measured with disposable pressure transducers (Type 041-500503A, Argon, Athens, TX) while the flow rate was measured with a $24 \mathrm{~mm}$ in-line ultrasonic flow probe (Model 24NSB12, Transonic Inc., Ithaca, NY). These three transducers were interfaced with CardioMed Amplifier CM 4008 (Medi-Stim AS, Norway). The loop was used for both LDV and PIV experiments when studying flow through the polymeric heart valves. 
Flow loop section

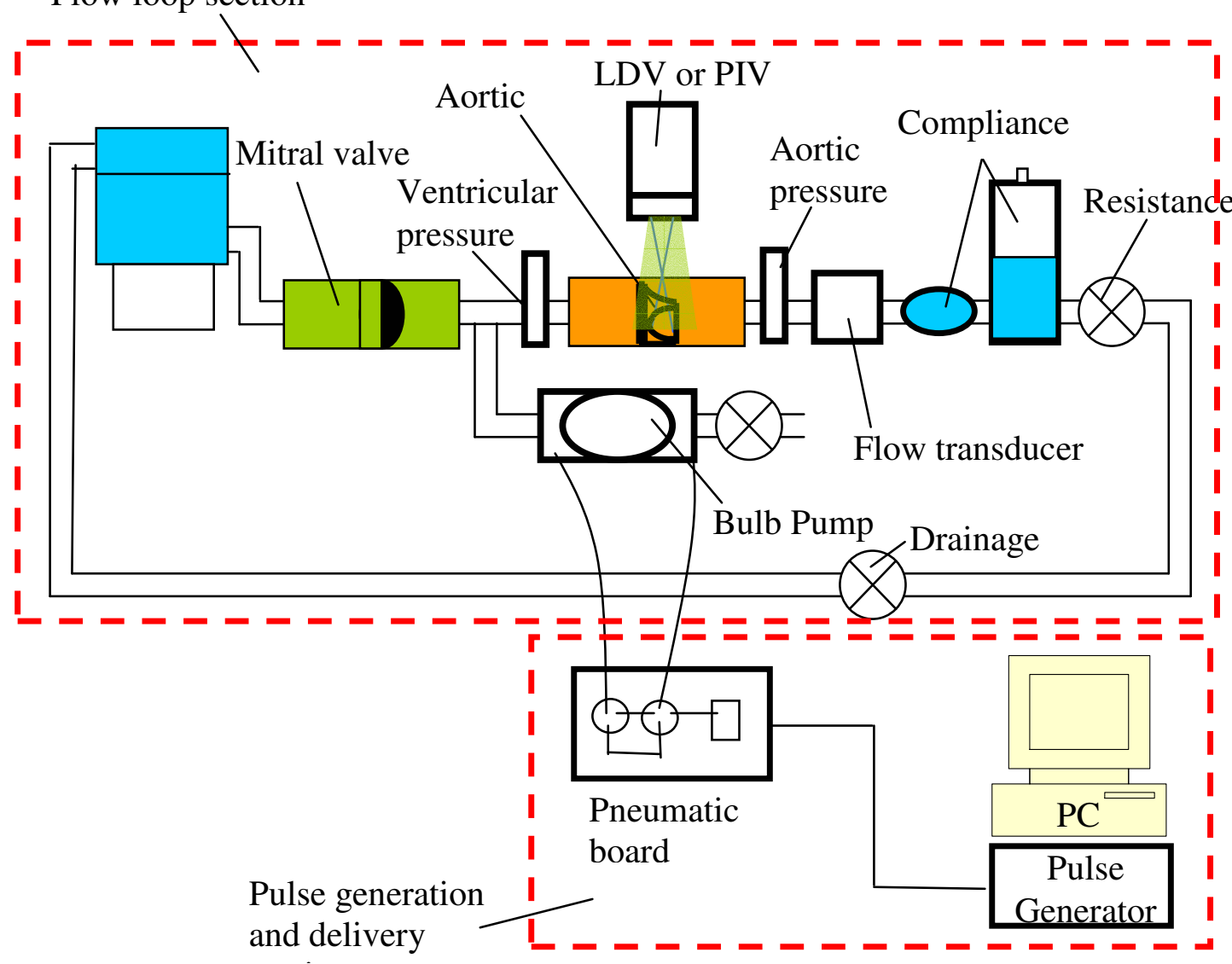

Figure 4-17 Pulsatile loop setup for the aortic valve experiments 


\subsection{Temperature-Dependent Study}

\subsubsection{Pulsatile loop setup}

Figure 4-18 shows a schematic of the setup for the Aortech polymeric valve. For this experiment, only polymeric valve A was investigated. The setup for this experiment was similar to the pulsatile setup discussed in section 4.5.2. Changes that were incorporated include (1) a water bath unit at the reservoir to raise the solution to physiological temperature, and (2) a dogleg section downstream of the valve chamber to facilitate optical access of the high speed camera (Figure 4-19). The temperature study setup comprised of three parts; a) the pulse generation and delivery, b) the flow loop, and c) the high speed camera setup. Ventricular pressure was measured between the mitral and the aortic valves, and the aortic flow rate and pressure were obtained at the immediate downstream of the aortic valve. Both pressures were measured with disposable pressure transducers (Type 041-500503A, Argon, Athens, TX) while flow rate was measured with a $24 \mathrm{~mm}$ in-line ultrasonic flow probe (Model 24NSB12, Transonic Inc., Ithaca, NY). The three transducers are interfaced with CardioMed Amplifier CM 4008 (Medi-Stim AS, Norway). The mock loop was used for both LDV and PIV experiments when studying flow through the polymeric heart valves. 


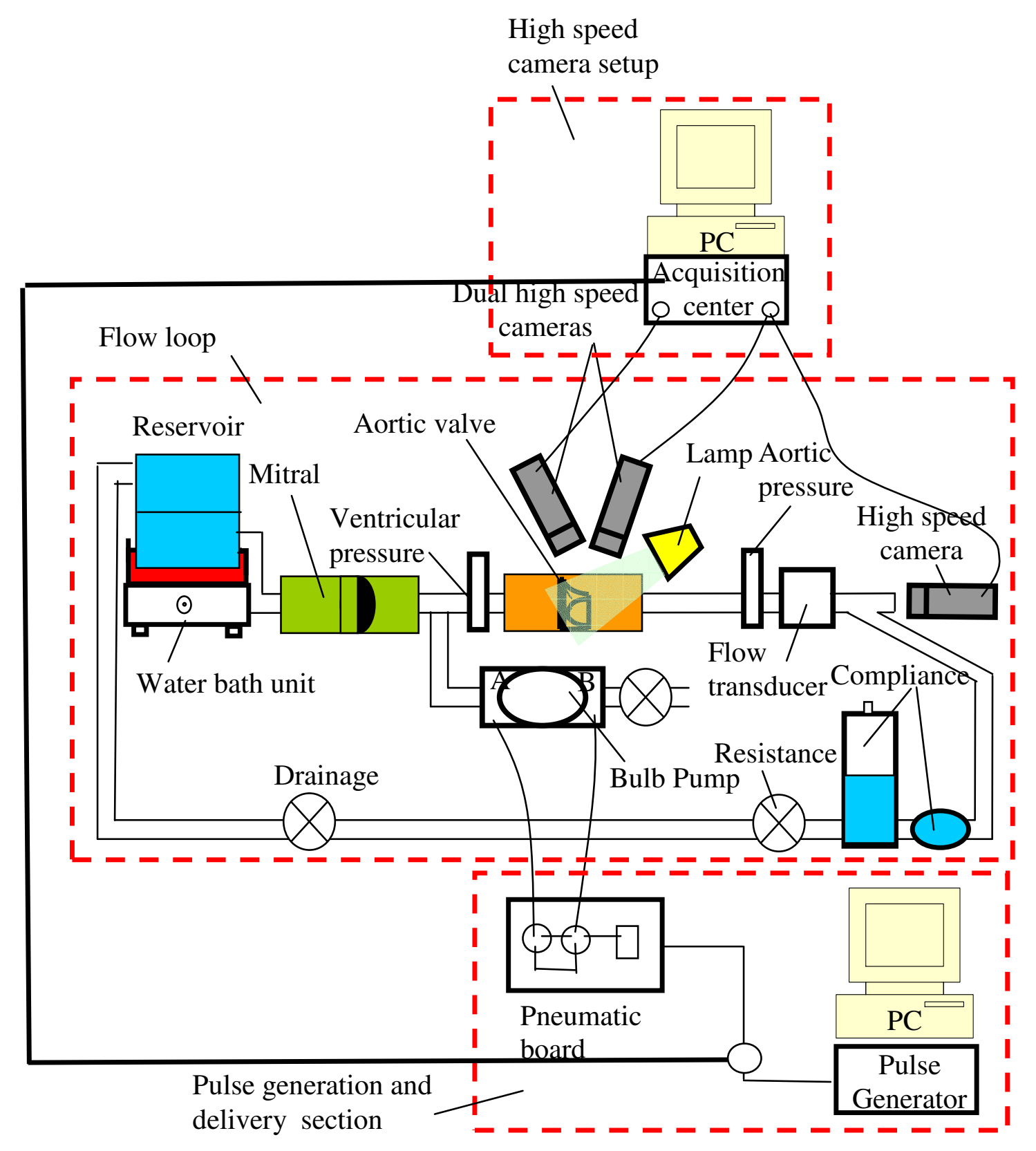

Figure 4-18 Pulsatile loop setup for the temperature dependent experiments 


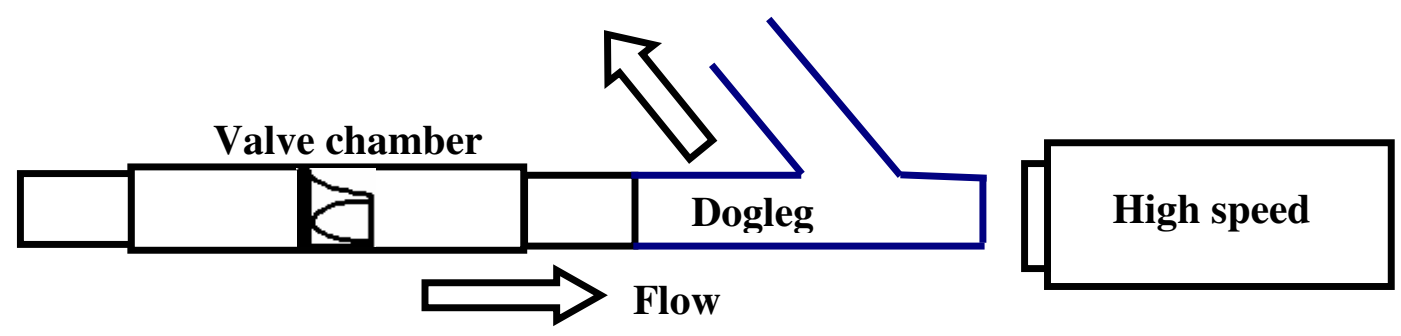

Figure 4-19 Dogleg section for use of acquiring upstream images of the polymeric aortic valve

\subsubsection{Markers and High Speed Camera Setup}

An array of 30 markers, each approximately $1 \times 1 \mathrm{~mm}^{2}$, was applied to the entire leaflet surface to allow detection of the leaflet by the camera (Figure 4-20). Instead of using graphite as marker, transparent tapes were cut into small pieces of $2 \times 2 \mathrm{~mm}^{2}$ and then pasted to the leaflet surface. A black sharpie pen was used to improve contrast on the marker tapes. The idea of using tapes instead of carbon carbide was to prevent damage to the leaflet surface from the adhesive glue.

The dynamic motion of the marker array was recorded using Dual Camera Stereo Photogrammetry (DCSP). Two high-speed cameras (Basler a504k, Basler Vision Technologies, PA) were focused on one side of the polymeric valve to simultaneously record the leaflet motion during the entire cardiac cycle at a recording speed of 500 frames per second with a resolution of 1280x1023 pixels ( 8 bit gray-scale). 
Figure 4-20 shows the labeling of the markers on the polymeric valve leaflet. Label \#1 corresponds to the marker on the bottom right corner of the leaflet with the label index increasing in the vertical direction. The index increases through marker \#9 located at the top stent post and continues with marker \#10 that is adjacent to marker \#1. The two leftmost markers were not able to be tracked by the autotracking software, due to their proximity to the dark vertical line (which is the opposite side of the valve housing) shown in Figure 4-20. These markers were later tracked manually whenever the Autotracking method was unable to resolve their positions.

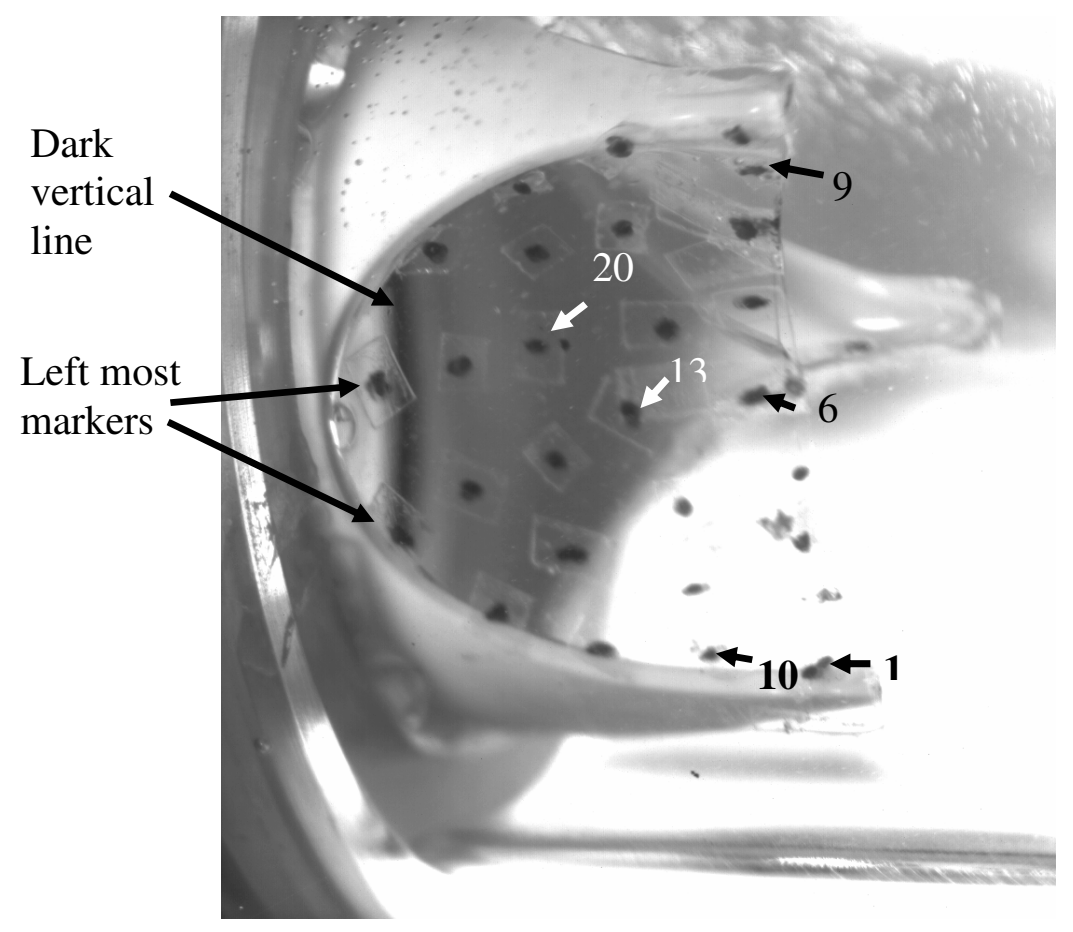

Figure 4-20 Labeling of the marker array on high speed camera image of the polymeric valve leaflet 
The two cameras were synchronized with the data acquisition system to match the images with the corresponding pressures and flow rates. The cameras were positioned on tripods ( $\sim 0.5 \mathrm{~m}$ away from valve) such that they had a view of the valve leaflet and were $\sim 30^{\circ}$ relative to each other with the valve as the vertex. The illumination and camera lenses were adjusted to provide maximum contrast between the markers and the leaflet for both cameras. Both cameras were connected to synchronized EPIX high-speed framegrabber cards (PIXCI CL3SD imaging board, EPIX Inc., Buffalo Grove, Illinois), which were fully controlled by software. A trigger signal was sent from the pulse generator to the image processor during the cardiac cycle to generate sets of paired images.

Leaflet motion downstream of the valve was captured in $2 \mathrm{D}$ using a dogleg section (Figure 4-21). One of the high speed cameras was positioned $0.02 \mathrm{~m}$ from the end of the dogleg, and the lighting next to the valve chamber was adjusted to obtain optimal contrast. Three evenly spaced marker tapes (without ink dots) were applied to the trailing edge of each leaflet to facilitate capture of the leaflet motion.

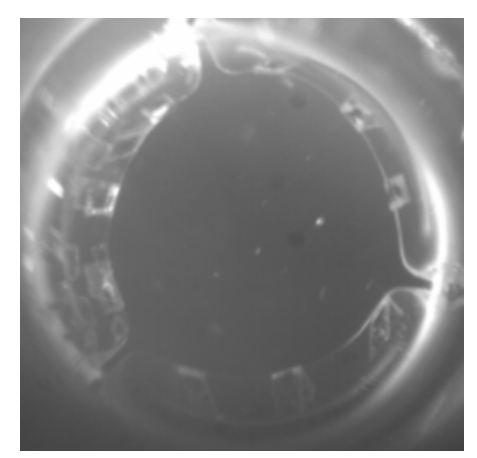

Figure 4-21 Upstream image of the polymeric heart valve through dogleg section 


\subsubsection{Obtaining Markers' 2D and 3D co-ordinates}

The 3D spatial coordinates of the dynamic leaflet surface were generated in the second phase of the DCSP by tracking the motion of the markers in a transient image sequence using two Matlab codes, Autotracking.m and Markercheck.m, developed 'in-house' by Dennis Soerensen (Appendix A and B). The Autotracking program followed the motion of the markers on the leaflet by tracking the areas of dark pigmentation on the leaflet surface. In this program, the user creates a small tracking area or window (in the MATLAB program) around each marker of interest to allow the Autotracking program to track the motion of the marker and then perform calculations to interpolate the motion of the associated tracking area. Each marker window had a mean size of 40 pixels, and the 2D position of each marker was determined from the centre of each marker with a mean error of 0.5 pixels. The second Matlab program was used to detect errors in either the tracking mechanism or in the image files (e.g. program failure due to poor contrast between a marker and the nearby valve housing). It also extrapolated the paired $2 \mathrm{D}$ coordinates of the applied markers to output a data file with these coordinates.

The $2 \mathrm{D}$ coordinates from cameras $\mathrm{A}$ and $\mathrm{B}$ were converted into a single set of $3 \mathrm{D}$ coordinates by the program SW3D7.m (Appendix C) using Direct Linear Transforms (DLT). The program also referred to the functions DLTFU.m and RECONFU.m, both of which were originally written by Christoph Reinschmidt from the University of Calgary. The parameters for the DLT were obtained by digitizing corner positions of a calibration cube with a known spatial size from images taken by both cameras. This yielded 3D coordinates represented in discrete domains. 


\subsubsection{Leaflet Surface Reconstruction}

In the third phase of the DCSP, a Matlab code called Surfaceplot.m (Appendix D) was used to construct a continuous leaflet surface from the 3D spatial coordinates generated from DLT (Appendix C). This program employs triangle-based cubic interpolation to convert the discrete data points into a smooth, contiguous surface (Figure 4-22). The surface to be reconstructed can be split into domains by associating each point with its nearest neighbors, thereby creating a Dirichlet region/Voronoi diagram, as shown in Figure 4-23. Superimposing this partitioned space onto an evenly spaced mesh results in a further subdivided region by drawing lines between any two points whose regions touch thus creating a set of tetrahedrons, known as the Delaunay triangulation. This process is then repeated for all the regions of the surface using a triangle-based cubic interpolation. This method is not only fast, but also preserves the folds and valleys of the surface, which are often lost via polynomial fits. However, cubic interpolation can result in the appearance of jagged contours when data points either disappear or become sparse. To better estimate the concavity of such regions, estimated gradient information was also incorporated into the reconstruction to help determine the concavity of the surface. Calculations were later performed in Velocityplot.m (Appendix E) to output velocity and acceleration information for each marker. 

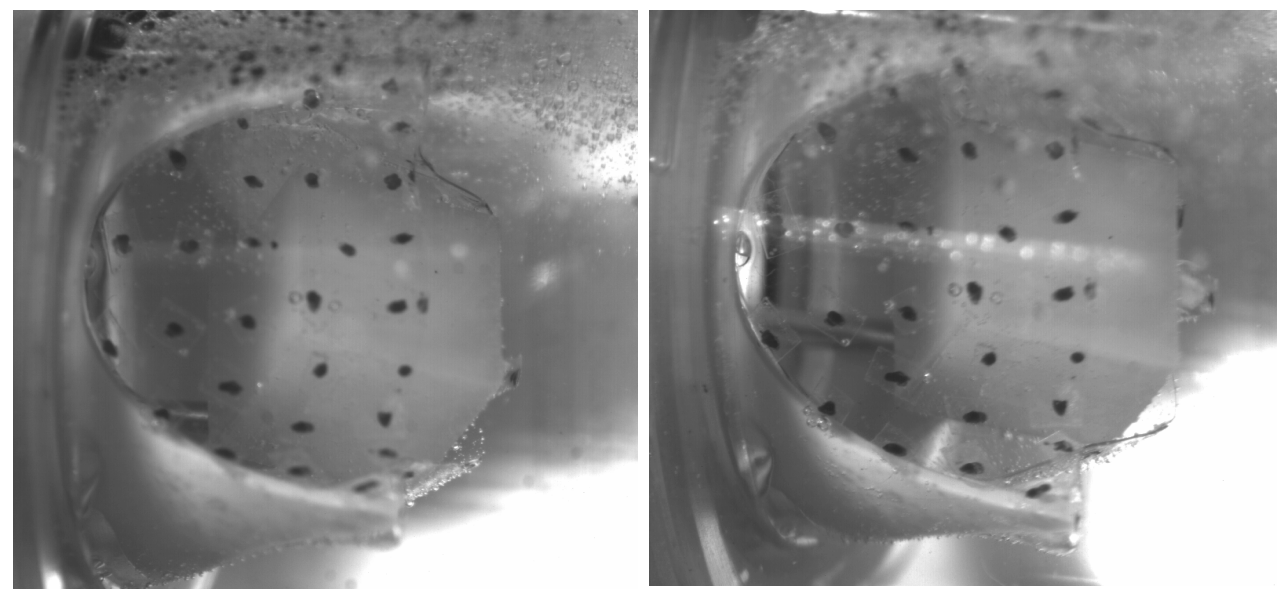

Figure 4-22 Calibration cube as viewed by the two high speed cameras. For the camera calibration, the Surfaceplot.m asks for the two-dimensional coordinates of reference cube obtained from the two high speed cameras, as well as the three-dimensional known dimensions of the cube

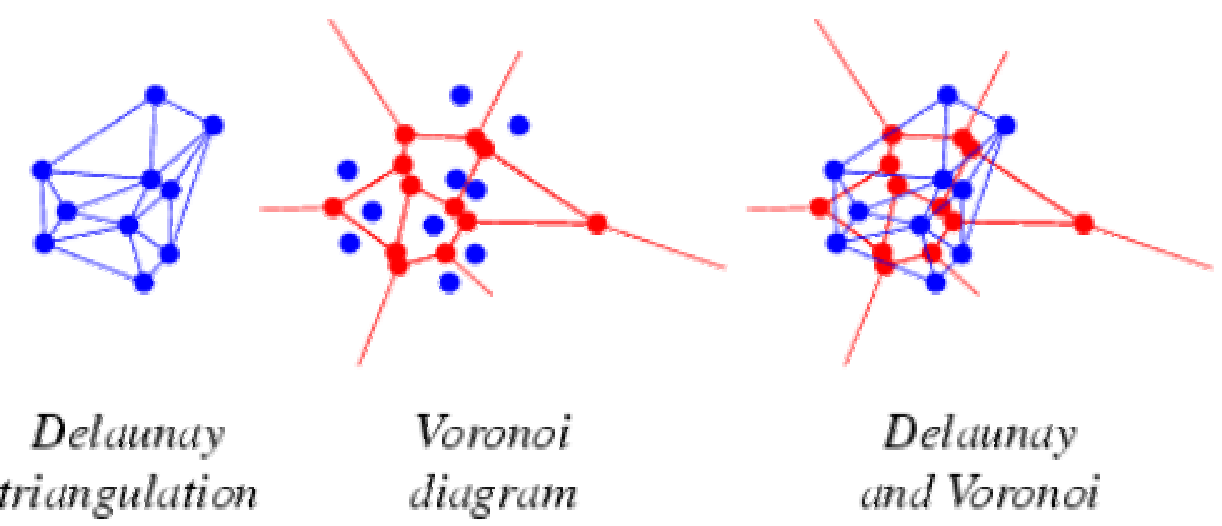

Figure 4-23 Principle of delaunay triangulation and dirichlet regions 


\section{$\underline{4.7 \text { Description of Working Medium and Particle Seedings }}$}

Table 4-2 Description of working medium for different experiments

\begin{tabular}{|c|c|}
\hline Experiments & Fluid Description \\
\hline Static Leakage Rate Study & Glycerin/ $\mathrm{H}_{2} \mathrm{O}$ \\
\hline Pulsatile Temperature-Dependent Study & Glycerin/ $\mathrm{H}_{2} \mathrm{O}$ \\
\hline Pulsatile (2D and 3D, LDV and PIV) & NaI/Glycerin/Deionized $\mathrm{H}_{2} \mathrm{O}$ \\
\hline
\end{tabular}

\subsubsection{Glycerin/H2O Mixture}

All static leakage rate and time-dependent studies employed Glycerin/ $\mathrm{H}_{2} \mathrm{O}$ in a 40/60 volumetric ratio as the working medium. The density of this mixture is about $1080 \mathrm{~kg} / \mathrm{m}^{3}$ or $1.08 \mathrm{~g} / \mathrm{cm}^{3}$, and the kinematic viscosity, $\mathrm{v}$, was approximately $3.5 \mathrm{cSt}$ or $3.5 \times 10^{-6}$ $\mathrm{m}^{2} / \mathrm{s}$ to match the kinematic viscosity of blood at high shear. On the other hand, the solution used for high temperature experiments $\left(37^{\circ} \mathrm{C}\right)$ had a kinematic viscosity of 6.25 $\mathrm{cSt}$ at room temperature and $3.5 \mathrm{cSt}$ at $37^{\circ} \mathrm{C}$. The viscosity of the working medium is determined by using a glass Cannon-Fenske routine viscometer (Viscometer No.75, N663, Cannon Instruments Company, PA) and the refractive index by means of a Extech transparent liquid refractometer (model 2129, Extech Instruments Corporation, MA).

\subsubsection{NaI/Glycerin/Deionized $\mathrm{H}_{2} \mathrm{O}$}

The fluid used for the pulsatile experiments, which include all 2D and 3D LDV and PIV experiments, was a solution of saturated sodium iodide, glycerin, and deionized water in a volumetric ratio of $79 / 20 / 1$. Although the density of this solution $\left(1.62 \mathrm{~g} / \mathrm{cm}^{3}\right)$ differed 
from blood $\left(1.05 \mathrm{~g} / \mathrm{cm}^{3}\right)$, the combined density and dynamic viscosity of the mixture matched the kinematic viscosity of blood at high shear rates $(3.5-4.0 \mathrm{cSt})$, and the refractive index (1.49) matched that of the polycarbonate valve mounting chambers. Matching the kinematic viscosity of blood enabled the assumption of dynamic similarity between the fluid flow created by the prosthesis within the mounting chamber and the blood flow created by the prosthesis within the human aorta. Matching the refractive index of the solution minimized optical distortion of the laser beams used in these studies.

A typical batch of NaI saturated solution has a life span of approximately 6-8 months depending on the frequency of usage because of the oxidation of the $\mathrm{NaI}$ constituent of the working medium, which causes it to turn dark brown after contact with the atmosphere. To rectify this problem, the solution was filtered regularly once every two weeks with a $5 \mu \mathrm{m}$ carbon impregnated filter cartridge (USFilter, Plymouth Products, MD) to deoxidize the solution and also to remove any contaminants. Furthermore, the water in the solution evaporates when exposed to the atmosphere. Thus, the solution's viscosity and refractive index had to be corrected regularly.

\subsubsection{Particle Seedings}

Neutrally buoyant silicone particles (TSI, Inc., St. Paul, MN) were used in all of the LVD experiments to seed the flow. These particles had a mean diameter of $1.5 \mu \mathrm{m}$, comparable to that of a human platelet. Fluorescent polymer particles (FPP-RhB-10, Dantec Dynamics, Denmark) based on melamine resin were used in all of the PIV experiments to seed the flow. The fluorescent particles had a diameter of $1-20 \mu \mathrm{m}$ and were delivered 
in suspension with water. These fluorescent particles were used to eliminate the laser glare off the polymeric valve surface. The particles absorb the laser light at a wavelength of $532 \mathrm{~nm}$ and emit light at above $560 \mathrm{~nm}$. An orange lens filter (Quantaray, $60 \mathrm{~mm}$, Wolf Camera) mounted on the camera lens only allowed wavelengths of $560-600 \mathrm{~nm}$ to pass, thus blocking the laser light that was reflected from the valve surface.

\subsection{Measurement Equipment and Their Calibration}

\subsubsection{Flow Rate Measurement}

Flow rate was measured with a $24 \mathrm{~mm}$ in-line ultrasonic flow probe (Model 24NSB12, Transonic Inc., Ithaca, NY) with a custom-made interface to the CardioMed acquisition terminal (Medi-Stim AS, Norway). The voltage signal from the flow probe was calibrated using a centrifugal pump to drive the sodium iodide solution through the flow probe for 30 seconds at various speeds. At each pump speed, the flow through the probe was collected in a bucket and the voltage output of the flow meter was recorded. The flow rate was calculated as the collected volume divided by the time over which it was collected (30 seconds). This flow rate was plotted against the voltage output from the flow meter to generate a calibration curve (Figure 4-24). A linear regression equation determined from this curve was used to determine the flow rate for a given voltage output from the flow meter. 


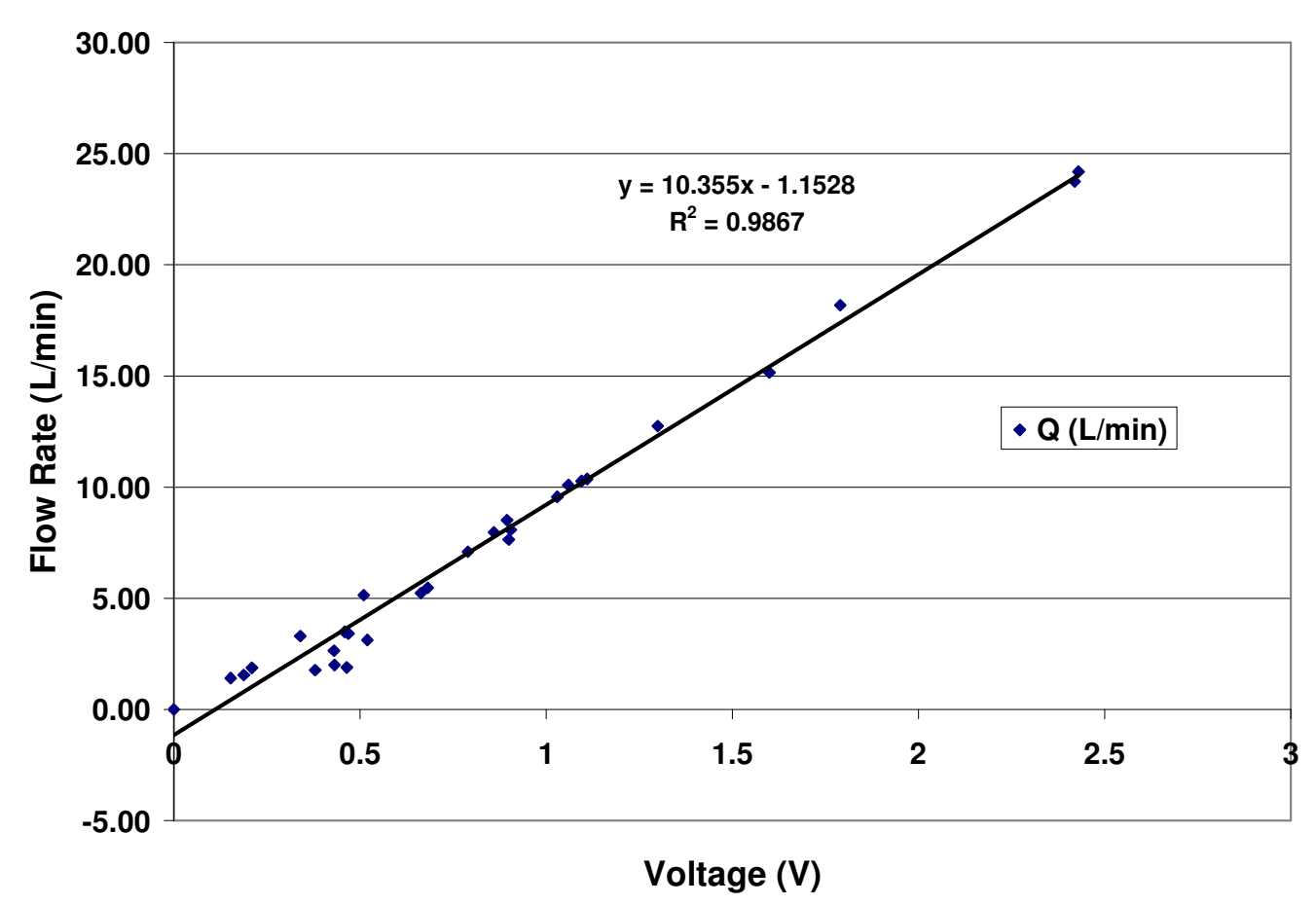

Figure 4-24 Calibration Curve for the Flow Meter

\subsubsection{Pressure Measurement}

Aortic, ventricular and atrial pressures were measured with disposable pressure transducers (Type 041-500503A, Argon, Athens, TX) with custom-made interfaces to the CardioMed acquisition terminal (Medi-Stim AS, Norway). The voltage signal from the pressure transducer was calibrated by placing water columns of varying height on the transducer membrane and recording the voltage output. Water column height was plotted against the voltage output to generate a calibration curve. A linear regression equation determined from this curve was used to determine the pressure for a given voltage output from the amplifier. Figure 4-25 shows a sample pressure transducer calibration curve. 


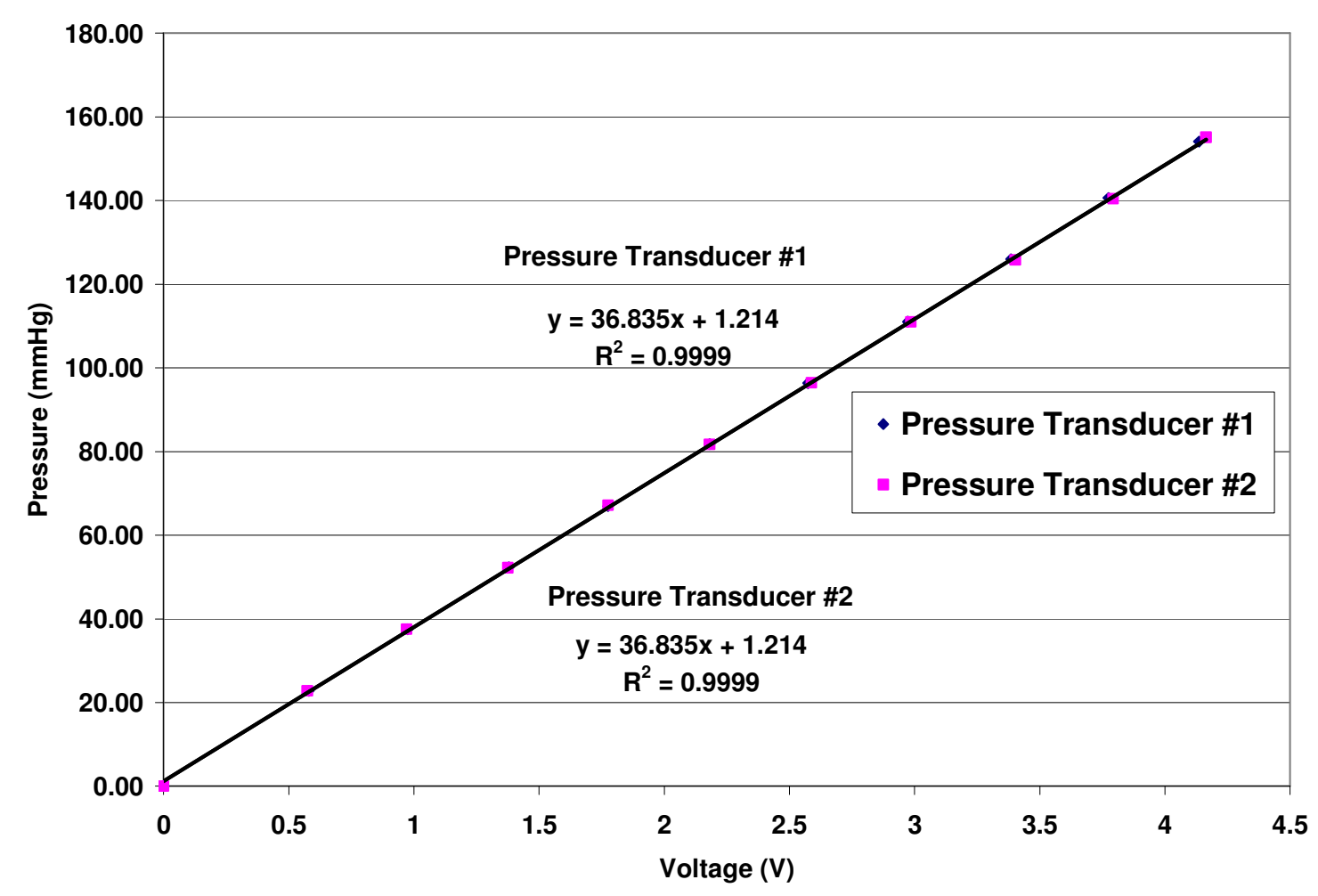

Figure 4-25 Calibration Curve for Pressure Transducer

\subsubsection{Velocity Measurements}

The velocity was measured using Laser Doppler Velocimetry (LDV) and Particle Imaging Velocimetry (PIV) techniques. The following sections describe the different working principles and components of the two velocity measurement techniques.

\subsubsection{Laser Doppler Velocimetry}

The Laser Doppler Velocimetry system has the following components (Figure 4-26):

1) Plasma tube and fiber drive

2) Transmitter and Receiver

3) Computer terminal and velocity information output 
Plasma tube - A 5 W, Argon-ion laser plasma tube (Model 70 Innova, Coherent, Santa Clara, CA) was used to generate the primary multi-line laser beam, which was subsequently passed through a fiber optic drive (Aerometrics, Sunnyvale, CA). The multi-line laser beam, which consists of different wavelength laser beams, was split by a beam splitter within the fiber drive into two beams of approximately equal intensity. The frequency of one of these beams was shifted by $40 \mathrm{MHz}$ using a Bragg cell within the fiber drive, and it is referred to as the secondary beam. The two beams were separated into their green $(514.5 \mathrm{~nm})$, blue $(488 \mathrm{~nm})$, and violet $(476.4 \mathrm{~nm})$ components by a prism inside the fiber drive. Each of the three pairs of resulting beams, i.e. two green, two blue, and two violet, was channeled into a beam coupler, which is linked to two transceivers by a series of fiber optic cables.

Transmitter and Receivers - Depending on whether it was 2D or 3D LDV, different scattering techniques were used for the velocity measurements. For 2D LDV measurements (using only green and blue beams), only one transceiver acting as both a transmitter and receiver was used, and the acquisition mode was backscattering. The naming convention for the different scattering mode is referenced from the receiver in relation to the direction of the incident beams. For 3D LDV, in addition to the use of two transceivers, a separate receiver was employed for receiving the reflected beams from the two transceivers. The transceiver with the weaker violet beams was placed directly across from the receiver to optimize the data rate from the violet beams, and the measurement mode is termed frontscattering. The second transceiver consisting of the green and blue beams was placed orthogonal to the first transceiver in sidescattering mode. 
Plasma tube and fiber drive

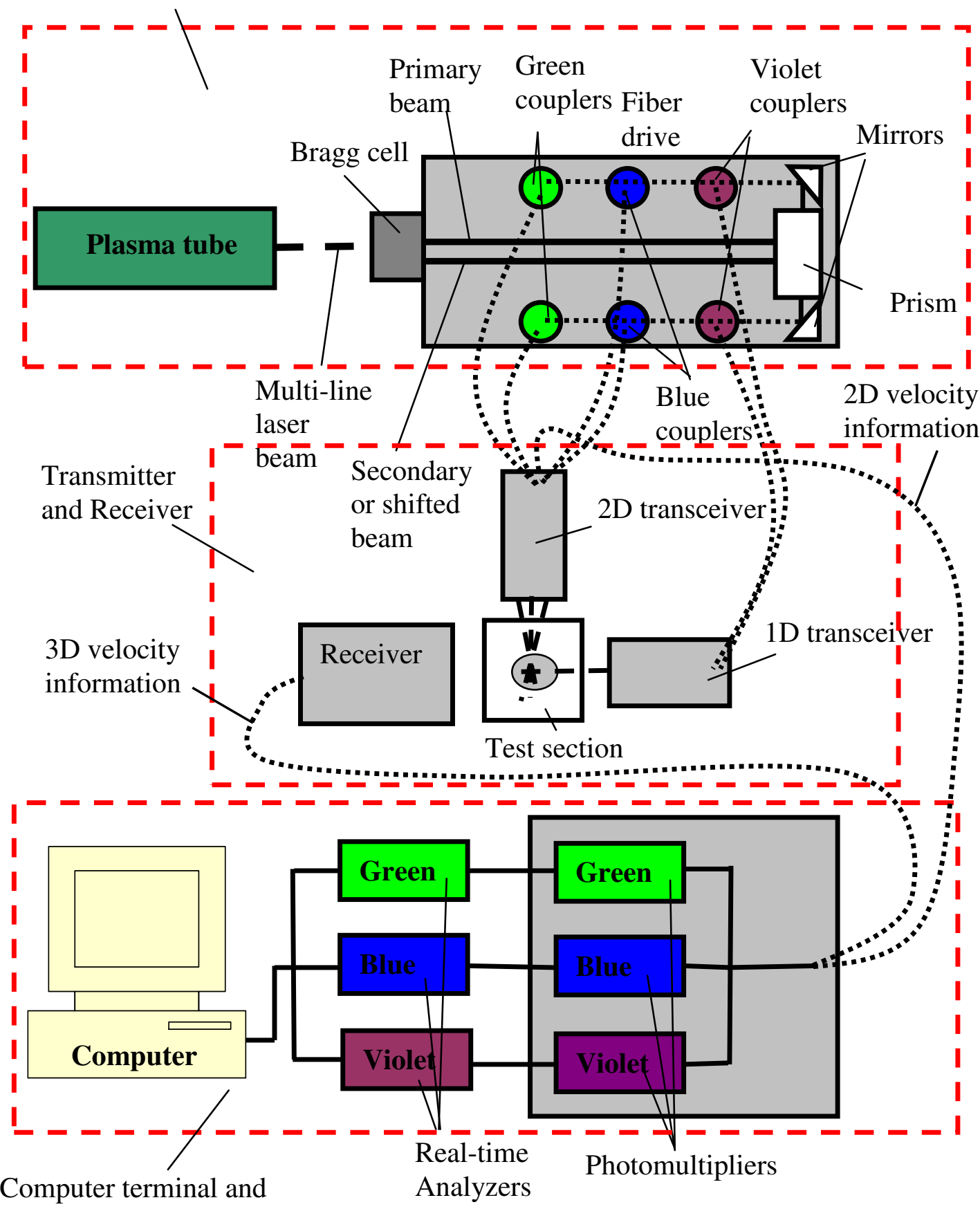

velocity information output

Figure 4-26 2D, 3D laser Doppler velocimeter setup 
Within the transceivers, each of the beams was passed through a lens with a $100 \mathrm{~mm}$ focal length. The sample volumes created by the intersection of the three color paired beams in the sodium iodide solution were ellipsoids with a major axis of $137.7 \pm 5.3 \mu \mathrm{m}$ and a minor axis of $18.3 \pm 0.7 \mu \mathrm{m}$. To measure the three orthogonal components of velocity, the three sample volumes of each color were positioned orthogonal to each other and spatially superimposed.

The plane of the calculated 2D velocity vectors map was orthogonal to the direction of the incident beams as illustrated in Figure 4-27. The velocity vectors measured by the green beams moved from left to right, while those measured by the blue beams moved into or out of the page depending on the direction of the intersecting primary and secondary blue beams. Figure 4-27 illustrates how the position of the intercrossing primary and secondary beams determines the direction of the velocity components. 


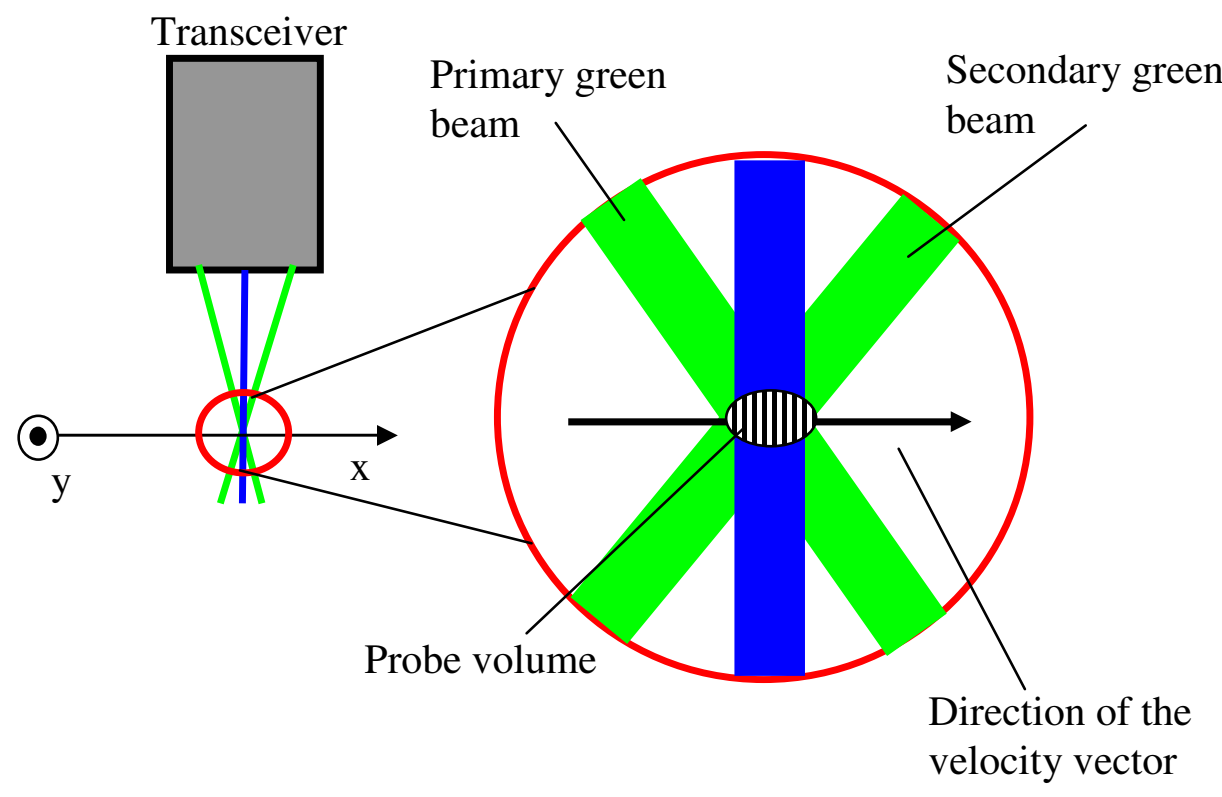

Figure 4-27 Direction of vector relative to the primary beam. The direction of the velocity vectors is determined by the direction of the crossing between the primary and the secondary beams

The intersection of the two beams created an interference pattern of bright and dark bands of light within the probe volume (Figure 4-28). Because one of the intersected beams in each color pair has a frequency shifted by $40 \mathrm{MHz}$ relative to the other, the interference pattern moved with a characteristic velocity. Thus, the reflective particles used to seed the flow generated a light signal called a Doppler burst as they passed through this interference pattern. When the particle crossed the bright bands of the interference pattern, a peak in the light signal was observed. Conversely, a valley in the Doppler burst was observed when the particle passed through the dark bands. The mobile interference pattern of the sample volume enabled particles of near-zero velocity to generate a Doppler burst and allowed differentiation between particles of positive and negative velocity. 


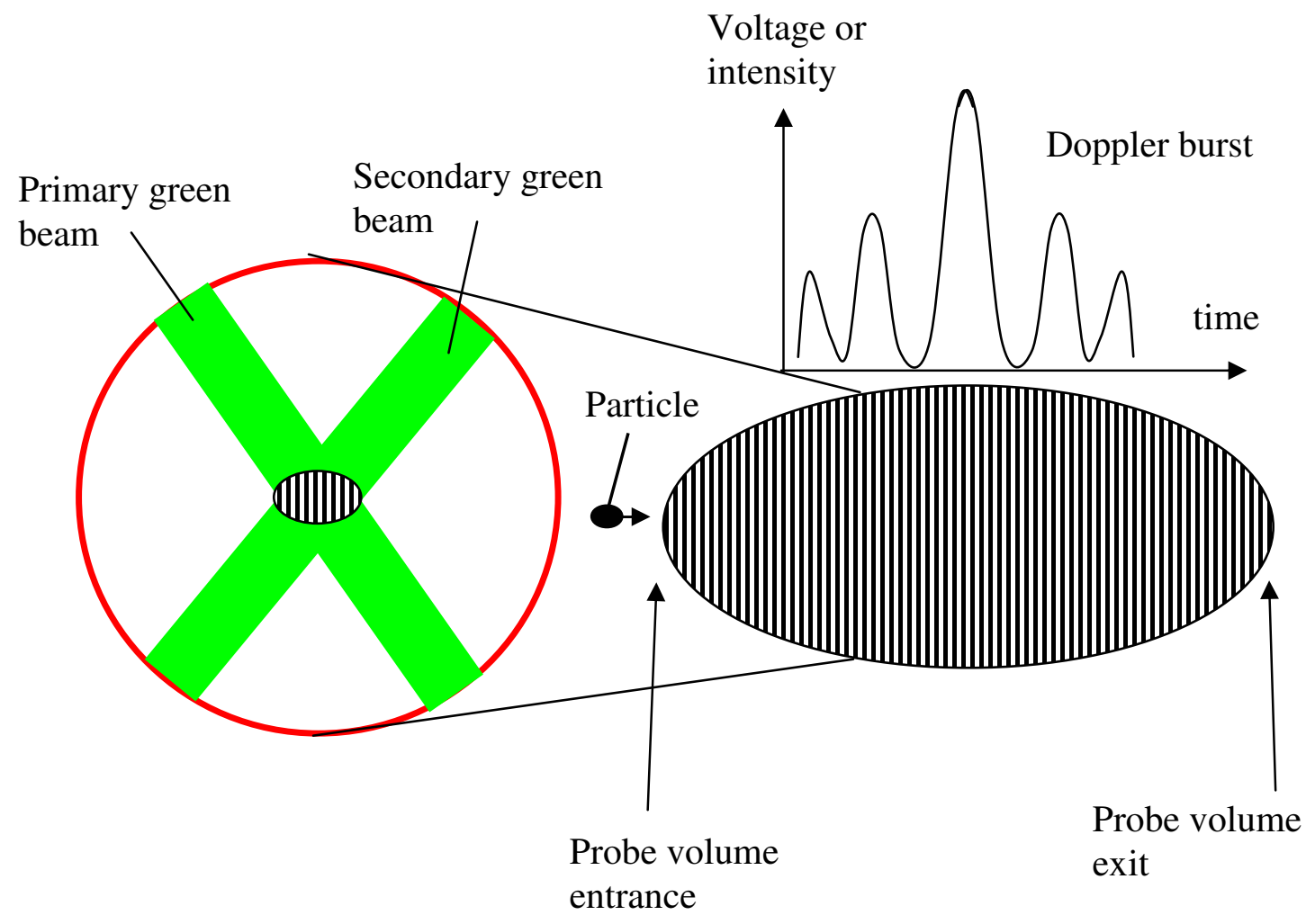

Figure 4-28 Interference pattern of probe volume as result of beams crossing

Computer terminal and velocity information output - The Doppler bursts from the passing particles were picked up by the fiber optic receiver that was focused on the superimposed probe volumes. These Doppler signals were transmitted through a series of fiber optics cables to three photomultiplier tubes. A band pass optical filter, allowing the passage of only green, blue, or violet light, covered the optical inlet to each of these tubes. The photomultiplier tubes amplify and convert the light signals into voltages for signal processing. This signal processing was performed by three real time, fast Fourier transform Doppler signal analyzers. The signal processors calculated the velocity of each particle that generated a Doppler burst using the distance between the interference fringes and the frequency with which the particle crossed the fringes. The fringe crossing 
frequency of a particle was calculated as an average of the inverse of the time between successive peaks in the Doppler burst. The distance between successive bright bands within the sample volume was derived from basic optical theory. The laser Doppler velocimeter required no calibration, as the velocities measured were calculated from known superposition properties of light. The cycle time of the velocity measurements was recorded by a 16 bit resettable clock (Aerometrics, Sunnyvale, CA). This clock was triggered by a signal from the pulse generator, and this same signal also opened the pneumatic solenoid valve for the compression of the flexible bulb. The resettable clock created a linear increase in voltage in response to increasing cycle times. This increase, when sampled digitally, effectively divided the $860 \mathrm{~ms}$ cycle time used in the pulsatile flow experiments into 55255 discrete intervals.

\subsubsection{Particle Imaging Velocimetry}

The PIV system (Figure 4-29) consisted of two Nd:YAG lasers (Model MiniLaser-I, New Wave Research, CA) with an energy of $17 \mathrm{~mJ}$ per pulse and maximum repetition rate of $15 \mathrm{~Hz}$ and a light arm (Model 610015, TSI, MN) to transfer the laser beam to the area of investigation. Two 1600 X 1200 CCD cameras (Model 1101MPRO, LaVision, Germany) equipped with $60 \mathrm{~mm}$ AF lenses (1:2.8D, Nikon) and orange filters (Quantaray, Wolf Camera) and a Scheimpflug mount (Model 1108196, LaVision, Germany) were used for image acquisition. A programmable timing unit (Model 1108013, LaVision, Germany) was used to synchronize image capture initiation with the laser pulse illumination of the particles, and an A/D converter (Model 1108033, LaVision, Germany) for integrated sampling control and data logging of the images. All of these components were 
connected to a host computer, which controlled the measurement and post-processing using image capture and analysis software (Davis 7.0, LaVision, Germany).

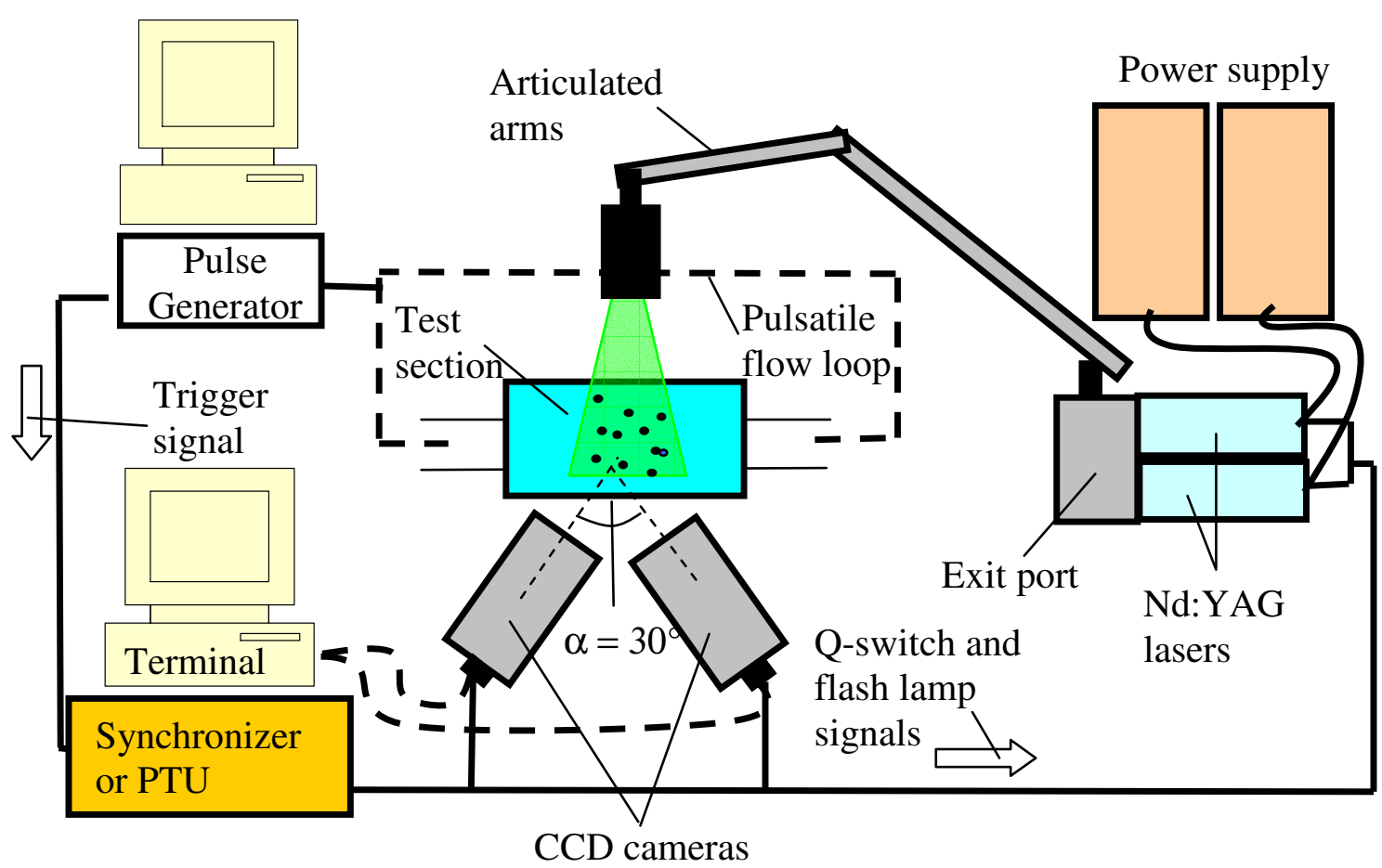

Figure 4-29 3D particle image velocimeter setup

The basis of velocity measurement using PIV is the simple tracking of particle displacement using paired images taken over a fixed time interval. Thus, particles in the solution - in this case fluorescent polymer particles with a mean diameter of $1-20 \mu \mathrm{m}-$ are illuminated by a pulsed laser light sheet approximately $1 \mathrm{~mm}$ in width that is produced by diffracting the laser beam with a cylindrical lens combined with a spherical lens. At the instant of illumination by the first laser, an image is captured by the CCD camera placed orthogonal to the light sheet followed by a second image capture after a set time interval at the instant of illumination by the second laser. These paired images 
are stored in the RAM of the host computer, and shortly afterwards (860 ms in these pulsatile flow experiments) the same procedure is repeated. With these paired images and the known time step, it is possible to determine the velocities in the field of investigation using a correlation algorithm. The final results of the PIV measurements are velocity fields, which describe the flow fields in the area of interest chosen by the investigator. With the known velocity field, it is possible to calculate quantities such as the total kinetic energy, the vorticity, and the viscous dissipation of energy due to turbulence. The following section describes in detail the components of the PIV system, which can be separated into several subsystems:

1) Solid state YAG laser

2) Laser transmitting articulated arm

3) Receiving cameras

4) Velocity calculation software

Solid state YAG lasers -The two solid state Nd:YAG or Neodimium Doped Yttrium Aluminum Garnet (LaserPulse Mini YAG 12) lasers are mounted on a Breadboard (SA series, Newport, CA). Each laser consists of a flash lamp and a YAG crystal placed inside a mirrored elliptical cavity to maximize the energy transfer from the flash lamp to the crystal. The flash lamp in the laser has an excitation duration of approximately $250 \mu \mathrm{s}$, and a repetition frequency of $15 \mathrm{~Hz}$. Each YAG laser is equipped with a Q-switch, which acts as a shutter to quickly open and close the laser cavity. The Q-switch is used to control the duration of the laser emission from the cavity so that the cavity is closed for the majority of the time and is only allowed to open for a brief period. The Q-switch 
mechanism allows the YAG rod to build up the lasing energy so that a high energy pulse can be released. The laser also contains a harmonic generator device to halve the wavelength of the emitted YAG laser light to $532 \mathrm{~nm}$ to improve the efficiency of the cameras, which are more sensitive to the blue-green spectrum. An additional advantage of having a visible light beam is to facilitate the alignment of the beam path. The doublepulsed laser beams pass through an exit port unit, which consists of a series of mirrors used to align the beams to ensure that they are overlapped.

Laser transmitting articulated arm- The articulated arm assembly was coupled to the exit port of the YAG lasers. The assembly consists of a series of mirrors and joints connected by hollow tubes to direct the laser beams from one mirror to the next irrespective of the orientation of the joint. At the end of the arm assembly is an output ring, which allows the coupling of various optics configurations. In these experiments, a cylindrical lens $(f=-25.4 \mathrm{~mm})$ and a spherical lens $(f=500 \mathrm{~mm})$ were mounted in series to the arm assembly to produce a laser sheet with a thickness (also known as waist) of approximately $1 \mathrm{~mm}$. The output end of the arm was mounted on a traversing mechanism to enable movement of the illumination volume.

Receiving Cameras -The term CCD stands for charge-coupled device. A CCD camera comprises an array of detectors called pixels. Each pixel is a MOS capacitor that is charged by converting incident photons of light into electrons, as in a photodiode. The charge falling on the individual pixels is transformed to a voltage during read-out of the CCD chip, and the value of the voltage is seen as a grey scale distribution on the PIV 
image map. Ideally, images should have a high charge i.e. appear white, and the background noise level of the CCD chip should have a low charge i.e. appear dark.

Velocity calculation software - The cross correlation of the digital image pairs to calculate the velocity fields was performed using commercially available PIV software (DaVis 7.0, LaVision, Germany). In the cross correlation program, each of the camera images are divided into rectangular regions called interrogation areas, and for each of these interrogation areas the particle images captured during the first and second pulse of the light-sheet are correlated to produce an average particle displacement vector (Figure 4-30). Essentially the cross correlation function statistically measures the degree of match between the two samples. The highest value in the correlation plane can then be used as a direct estimate of the particle image displacement. It is assumed that all particles within one interrogation region move homogeneously between two laser pulses.

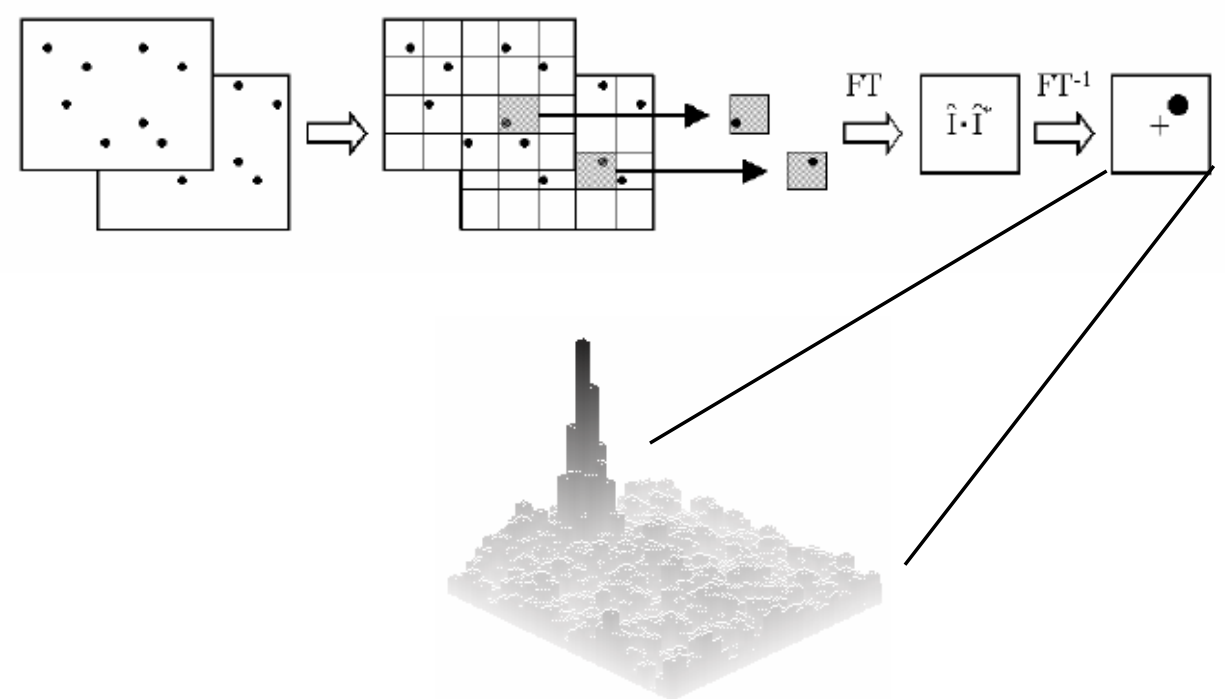

Figure 4-30 Processing sequence of PIV raw images. Each image is divided into smaller rectangular interrogation windows where the intensity (I) of the particles is crosscorrelated with that of another image to provide an average of the particle displacement 
Performing this calculation for all interrogation regions produces a vector map of average particle displacements. The displacement vectors are divided by the known pulse separation time and thus converted into a map of raw velocity vectors. FFT-processing is used to increase the speed of the cross correlation. With FFT-processing it is important to note that there is always be an outcome whether the input is meaningful or not. Since PIV is an instantaneous measurement technique, all spatial information is sampled at the same time. Consequently, there is a finite statistical probability that there are some regions where there is really no meaningful input. Therefore, it is necessary to validate the PIV vector map. The validation algorithms in $\mathrm{DaVis}$ can be applied to the raw vector maps so that outliers, the term for erroneous vectors, can be detected and removed.

\subsubsection{Important Considerations for PIV Experiments}

Illumination - Illumination of the particles constitutes an important aspect of the PIV setup. The laser sheet must be strong enough to illuminate the tracer particles in the flow so that sufficient scattered light reaches the CCD cameras. Ideally, the waist of the light sheet should be as thin as possible to reduce the effect of out-of-plane particles, and the test section should be located where the light sheet is the thinnest; for these experiments, this region is approximately $500 \mathrm{~mm}$ from the lens. Another way to maximize the laser power is through the Q-switch control, which enables the opening and closing of the shutter to the laser cavity. The firing time in this research was set at $210 \mu \mathrm{s}$, which corresponds to the time slightly after the peak of the laser power (Figure 4-31). There is a trade-off between allowing the energy to build up by the action of the flash lamp and the natural dissipation rate of the cavity so the Q-switch is normally fired $150-200 \mu$ s after 
the start of the flash lamp. The big advantage of the Q-switch is that all of the energy is released in a high energy pulse, and since the duration of the laser beam is short, the seeding particles move very little during illumination.

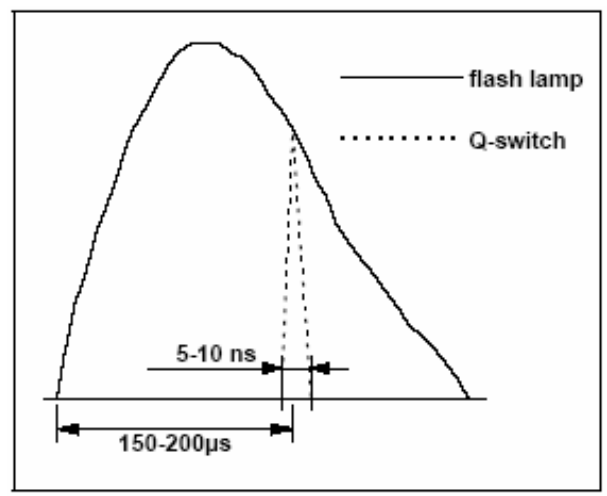

Figure 4-31 Q-switch control of the lasing cavity. Setting the Q-switch slightly after the peak laser power can boost the laser output power and improves the consistency of the output from pulse to pulse

Particle size and seeding density - The need to utilize larger particles because of their better light scattering is in contradiction to the demand to have the smallest particles possible so that they follow the flow faithfully. In general particles used to represent the flow of a fluid continuum should be (1) able to follow the flow, (2) good light scatterers, (3) cheap and conveniently generated, and (4) chemically inactive and clean. Typical particle size ranges between 1- $100 \mu \mathrm{m}$, and these are considered sufficient for most PIV applications. Fluorescent polymer particles with a mean diameter between $1-20 \mu \mathrm{m}$ were used for these studies. Particles with less variation in the mean diameter are considerably more expensive. 
Time delay between illumination pulses - The time delay between the illumination pulses must be long enough to enable determination of the displacement between image pairs with sufficient resolution and short enough to prevent particles with an out-of-plane velocity component from leaving the light sheet between pulses. The complex flows encountered in the polymeric valve experiments complicate the time delay setting. For each image pair, there was a mix of both very high and low velocity flows, thus, a time delay had to be used that could capture both types of flow but was not optimal for either. In addition, the flow changes throughout the entire cardiac cycle, necessitated having a different time delay setting at each particular point in the cycle. The typical time delay for the whole cardiac cycle ranged between $25-500 \mu$ s, and could also be influenced by the particle size as well as the size of the interrogation window. See Table 5-4 for the pulse separation used in the 3D PIV study of polymeric heart. Typically, a pulse separation of 75 and $500 \mu$ s were set for the systolic and diastolic phases, respectively.

Size of interrogation area - Besides the particle density and size and the camera resolution, the size of the interrogation area also affects the spatial resolution of the vector maps. In fluid flow experiments, 32 × 32 pixels is a typical interrogation window size. Studies have shown that, given a window side length of $\mathrm{N}$, a maximum particle displacement of N/4 is recommended to ensure a reasonable signal-to-noise ratio. The recommended minimum number of particles within each interrogation area is about 7 particles/interrogation area to ensure a good signal-to-noise ratio for cross correlation calculations [Keane, 1990]. 
Number of image pairs - The number of images can affect the accuracy of the calculated velocity components. Even though increased number of image pairs can invariably lead to improved signal-to-noise ratio and lower percentage errors in the measured values. This would incur a longer time spend on data acquisition and processing, and higher cost on the storage space. Typical allowable percentage error in the measured velocity components is 5-6 percent in fluid flow experiments. Using the following relation;

$$
\text { percent error in } \tau=\frac{\delta \tau}{\bar{\tau}} \times 100=\frac{\tau^{\prime}}{\bar{\tau} \sqrt{N}} \times 100 \quad 4-3
$$

where $\tau$ is the principle Reynolds shear stress, an estimated number of images required can be calculated. The percentage error for the Reynolds shear stress obtained at a point with maximum turbulence intensity is showed in Figure 4-32. From Figure 4-32, a percentage error of less than 3 percent in $\tau$ can be achieved at approximately 100 image pairs. However, the number of image pairs in these experiments was set at 250 , which gave an error of less than 2 percent. 


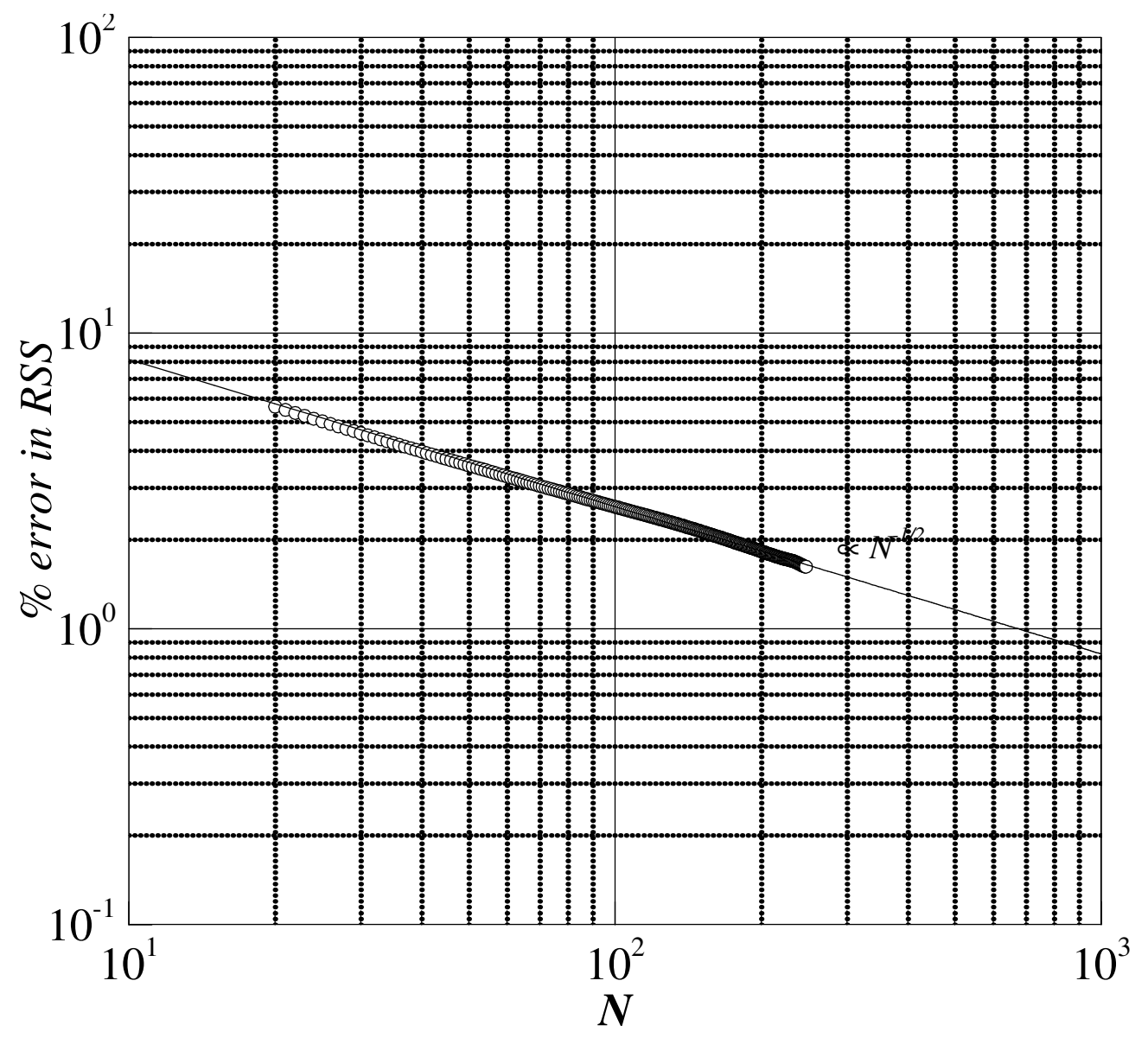

Figure 4-32 Percentage error in measuring the principle Reynolds shear stress at a point with maximum turbulence intensity

Resolution of CCD cameras - In these studies, two 1600 X 120012 bit CCD cameras (Model 1101MPRO, LaVision, Germany) were used. Each camera had a frame rate of 30 frames/sec and a CCD size of $7.4 \times 7.4 \mathrm{~mm}$. The number of vectors in the vector map is dependent upon the number of pixels in the CCD camera and the size of the interrogation area. The array of totally independent vectors, $N_{\mathrm{v}}$ is determined by the following equation: 


$$
N_{v}=\frac{\text { no. of pixels in CCD row }}{N_{\mathrm{int}}} \times \frac{\text { no. of pixels in CCD column }}{N_{\mathrm{int}}} \quad 4-4
$$

where $N_{\text {int }}$ is the side of the interrogation area in pixels.

In practice the number of independent vectors is greater than this because since information at the edges of the interrogation area was not used successive interrogation areas were overlapped to optimize the use of the information in the image map. Overlapping the interrogation regions by 50 percent of the side length is recommended. It is highly probable that if a particle is near the outer edge of an interrogation area it may be displaced into the neighboring interrogation area. Without overlapping these particles would not contribute to the calculation of a velocity vector. Therefore, overlapping interrogation areas can help maximize the use of the available information. With 50 percent overlapping of the interrogation areas the number of vectors obtained is:

$$
N_{v}=\frac{\text { no. of pixels in CCD row }}{(1-0.5) N_{\mathrm{int}}} \times \frac{\text { no. of pixels in CCD column }}{(1-0.5) N_{\mathrm{int}}} \quad 4-5
$$

The choice of $N_{\text {int }}$ is constrained by the conflicting constraints imposed by velocity dynamic range and spatial resolution considerations. Generally, an interrogation region side-length of 32 pixels provides a good compromise for cross-correlation. 


\subsubsection{3D PIV Setup}

This flow fields within and downstream of the polymeric heart valves were investigated using 3D PIV. 3D PIV allows the measurement of out-of-plane velocity information. Figure 4-33 shows the a typical 3D arrangement of PIV cameras with respect to the laser sheet.

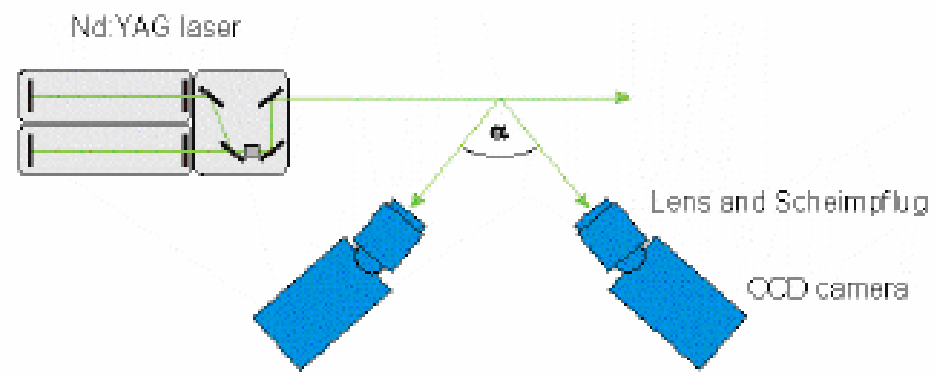

Figure 4-33 3D PIV camera configuration employed in this research

Scheimpflug rule - The two CCD cameras were arranged so that the same interrogation section in the flow was viewed from two different directions with respect to the light sheet. Both cameras incorporated a Scheimpflug mechanism at the back of the CCD. This was to increase the depth of view in both cameras as a result of the oblique viewing direction encountered in the 3D setup. In a conventional camera the object (film) plane, lens plane and subject plane are parallel to each other. However, in a Scheimpflug camera these planes intersect in a straight line as shown in Figure 4-34. In other words, the use of a scheimpflug mechanism allow the extension of the depth of view so that objects that are normally out-of-focus are brought into shape focus. 


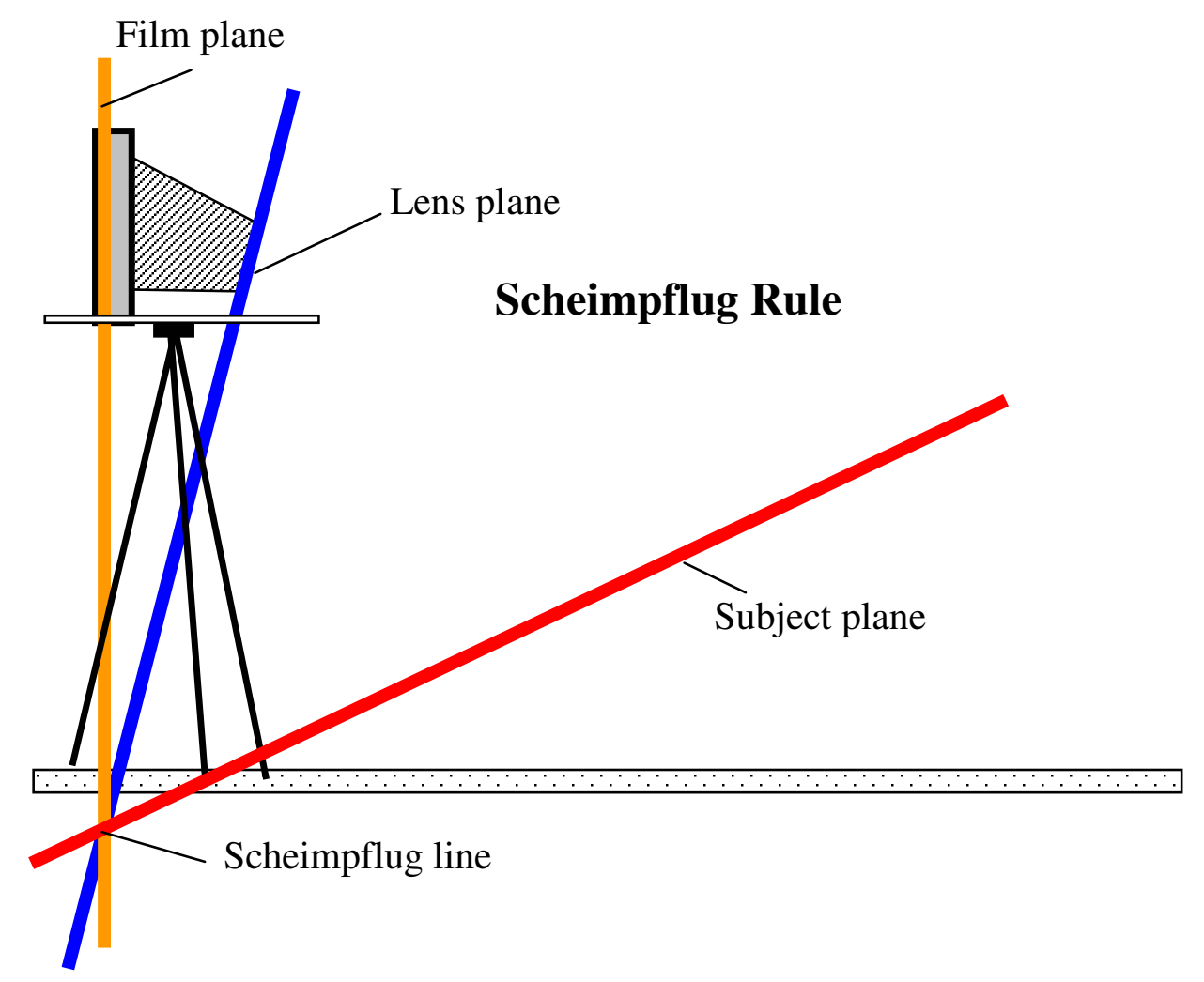

Figure 4-34 Schematic of scheimpflug rule. A scheimpflug mechanism is usually attached at the back of the CCD cameras to help increase the depth of the view so that more area could be brought into focus

Camera angles and lens - Studies have shown that the angle between the CCD cameras, $\alpha$, in a 3D PIV setup can influence the accuracy of the out-of-plane velocity measurement [Guezennec, 1994; Lawson, 1997]. Lawson et al demonstrated that the error of the outof-plane measurement can be minimized by using a camera angle between $20^{\circ}$ and $30^{\circ}$ with an $f$ number of 16 or higher. In the current experiments, $\alpha$ was set at $28-30^{\circ}$. The $f$ number refer to the aperture of the camera lens used. The aperture is the size of the lens opening, and changing the value of the aperture number effectively controls the amount of light reaching the film, or the CCD. The larger the $f$ number is the smaller the aperture 
is resulting in less light being allowed into the camera. The Nikon lenses used in these experiments had a maximum $f$ number of 32 and a minimum $f$ number of 2.8. Since both cameras had a different exposure time with the $1^{\text {st }}$ exposure time being much shorter than the $2^{\text {nd }}$, the $f$ numbers were set at 4 and 5.6 for the $1^{\text {st }}$ and $2^{\text {nd }}$ cameras, respectively.

Calibration - Correcting for off axis viewing is an essential part of stereoscopic 3D-PIV evaluation and should be done with great care. Typically, 3D PIV calibration was performed using non-opaque metal calibration plates, which were made up of an array of black and white dots with known vertical and horizontal spacing. The calibration was performed by taking preliminary images of the flow, and then, without shifting the distance between the cameras and the laser sheet or the angle between the cameras, the test section was replaced with the calibration target so that it was aligned with the laser sheet. Images of the target plate were captured, and skewing of the images were taken into account. This information was stored as the calibration set file and was used to process the preliminary flow data to output a final calibration set file. The final correction file was used for subsequent 3D-PIV data processing. See section 5.4.1 for detail experimental procedures of the 3D PIV studies. Figure 4-35 shows the calibration target used for the 3D PIV experiments. Each dot has diameter of $0.5 \mathrm{~mm}$ with horizontal and vertical spacing of $3 \mathrm{~mm}$ between each dot. The calibration target was created using Corel Draw software and printed on a piece of white paper and then pasted onto the back of a transparent $150 \times 95 \times 19 \mathrm{~mm}^{3}$ acrylic block . 


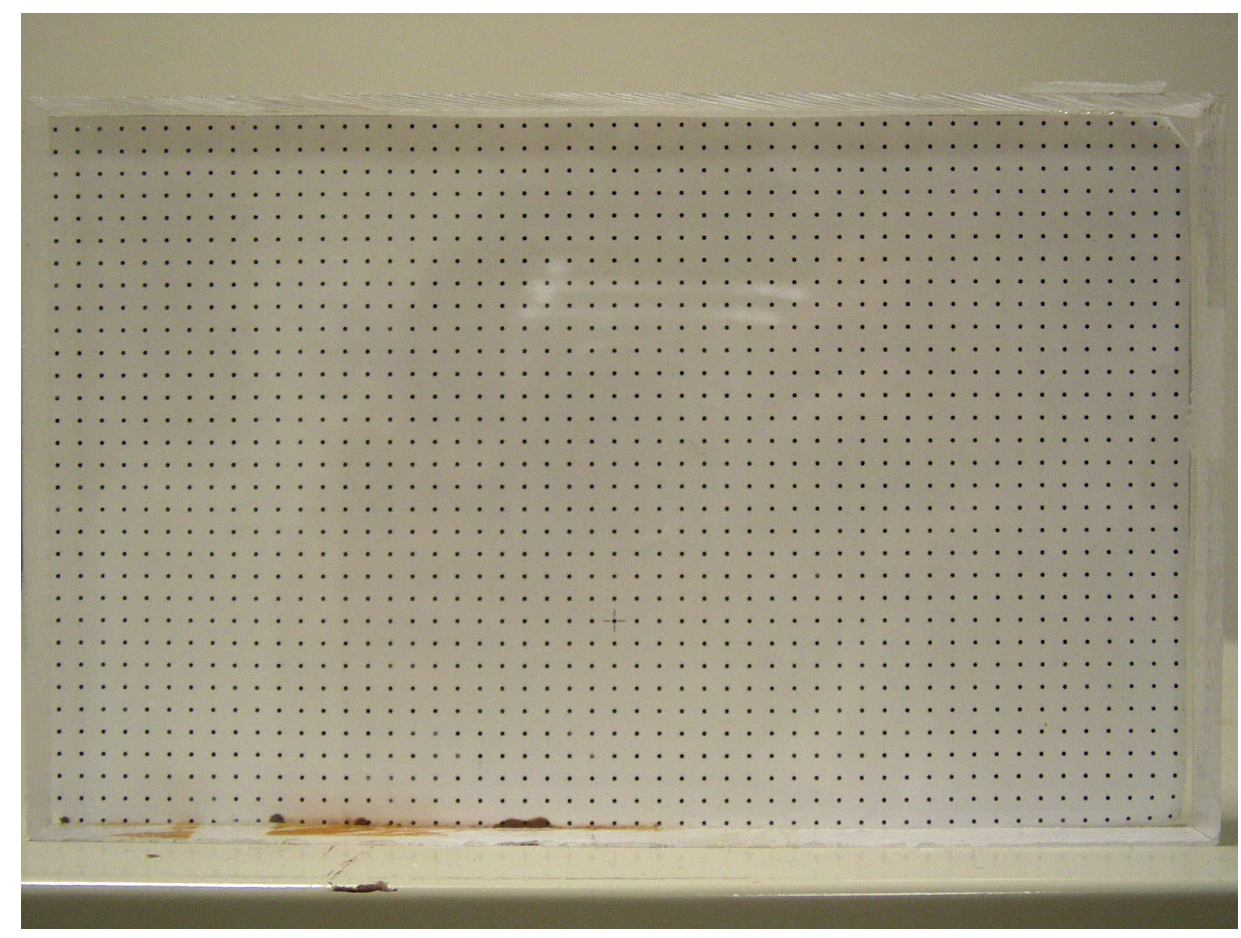

Figure 4-35 The calibration target used in the 3D PIV experiments. The thickness of the acrylic block should be similar to that of the test section to ensure accurate setup of the calibration file

Sequencing of laser pulses and camera shutters - Because flow field information was acquired throughout the cardiac cycle in these studies, a delay was required in the firing of the laser pulses and the synchronization of the opening and closing of the camera shutters. This synchronization was performed by the Programmable Timing Unit (PTU). A trigger signal at a frequency of $1.1667 \mathrm{~Hz}$ (equivalent to 70 beats/min) was sent from the pulse generator to the PTU for both the timing of the laser pulses and the camera shutters. A delay setting in the software controlled the timing of the velocity measurement for each time bin during the cardiac cycle. 


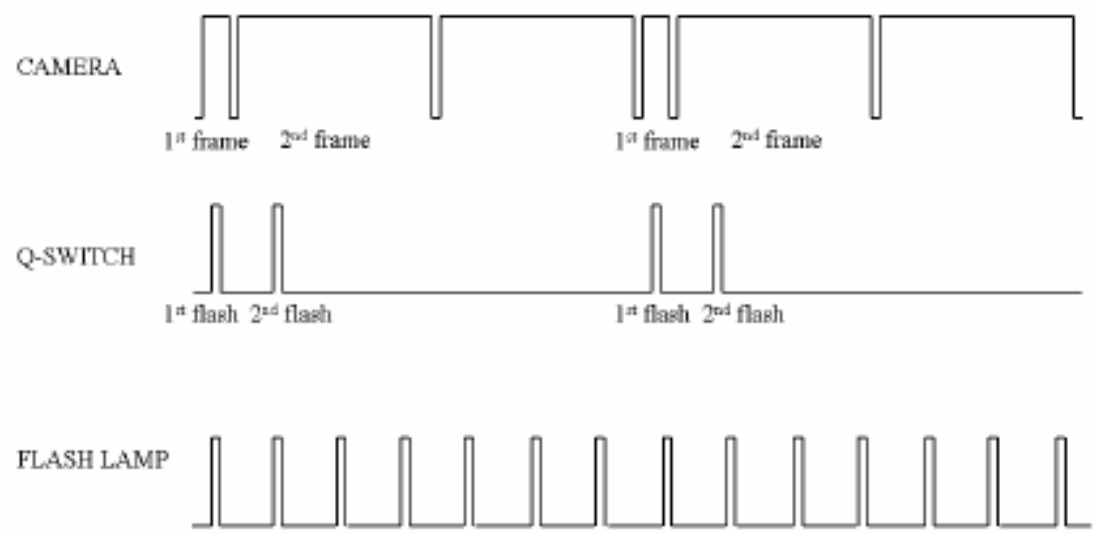

Figure 4-36 Timing sequence of a single camera. The CCD camera open longer during the second frame because of the delay in sending the first frame to the computer

Figure 4-36 shows the timing sequence for a single camera. The timing of the Q-switch coincided with the opening and closing of the camera shutter. The flash lamp firing frequency in this experiment was set at $30 \mathrm{~Hz}$. The open duration of the $2^{\text {nd }}$ frame of the camera was much longer than the $1^{\text {st }}$ due to the delay in transferring the $1^{\text {st }}$ image capture information to the computer. From Figure 4-36, it can be seen that the laser pulse separation was determined by the timing between the $1^{\text {st }}$ and $2^{\text {nd }}$ Q-switch pulse. The experimental protocol for 3D-PIV is discussed in greater detail in the following Chapter. 


\subsubsection{White Light Interferometry}

A white light interferometer was used to investigate the surface topography of the SJM bileaflet mechanical carbon valve and the Aortech polymeric heart valve (prototype B). The purpose of this study was to assess how different valve leaflet surface topography affects the hemodynamic performance of various valve types. The leaflets of the bileaflet mechanical valve are made of pyrolytic carbon known to be non-thrombogeneic and very durable, while the leaflets of the tri-leaflet polymeric heart valve are made of polyurethane material also known to be blood compatible.

Interferometry is the use of interference phenomena for measurement purposes, either for very small angles or for tiny distance increments (the displacement of two objects relative to one another). An interferometer is a device used to make such measurements. Though there are many different types and designs of interferometers, virtually all of them operate on the same basic principle. From a beam of light coming from a single source (a star, a laser, a lamp, etc.), two or more flat mirrors are used to split off different light beams. These beams are then combined so as to interfere with each other. What is looked for is alternating bands of light and dark, called fringes. Fringes are bright where the beams are constructively adding together and dark where they are canceling each other out.

What makes the interferometer such a precise measuring instrument is that these fringes are only one light-wavelength apart. In visible light (about $590 \mathrm{~nm}$ ), that corresponds to $1 / 43,000$ th of an inch. Any movement along the optical axis by either flat mirror causes 
the fringes to shift an equal amount in lockstep. The measurement of this movement is made by literally counting the number of fringes - each dimming and brightening of light - one wavelength at a time. Such a precise system is also incredibly sensitive so that any vibration, movement, thermal expansion, etc. is picked up as well.

Figure 4-37 shows a setup based on non-contact, three-dimensional, scanning white light and optical phase-shifting interferometry. Broadband light from a tungsten-halogen lamp is directed by a beam splitter to a Mirau [Bhushan, 1985] interferometric objective. The principle of the device, as illustrated in Figure 4-38, relies on placing a reflection reference mirror in the center of the objective lens and interposing a half mirror between the objective lens and the specimen. These components are arranged so that an interference pattern appears if the system is focused upon the specimen. The objective is mounted into a PZT translator and can be translated up to $100 \mu \mathrm{m}$ along the optical axis. The interferogram is imaged onto a video camera, and the video is digitized to 8-bit precision by a frame grabber during the PZT scan. The scan rate is set to produce roughly 90 degrees of phase shift between frames. Each frame was transferred to the host computer, which performed the acquisition and analysis functions and controlled the PZT scan initiation and rate. The vertical resolution can be as low as $0.1 \mathrm{~nm}$ and the horizontal resolution as low as 0.45 to $11.8 \mu \mathrm{m}$. 


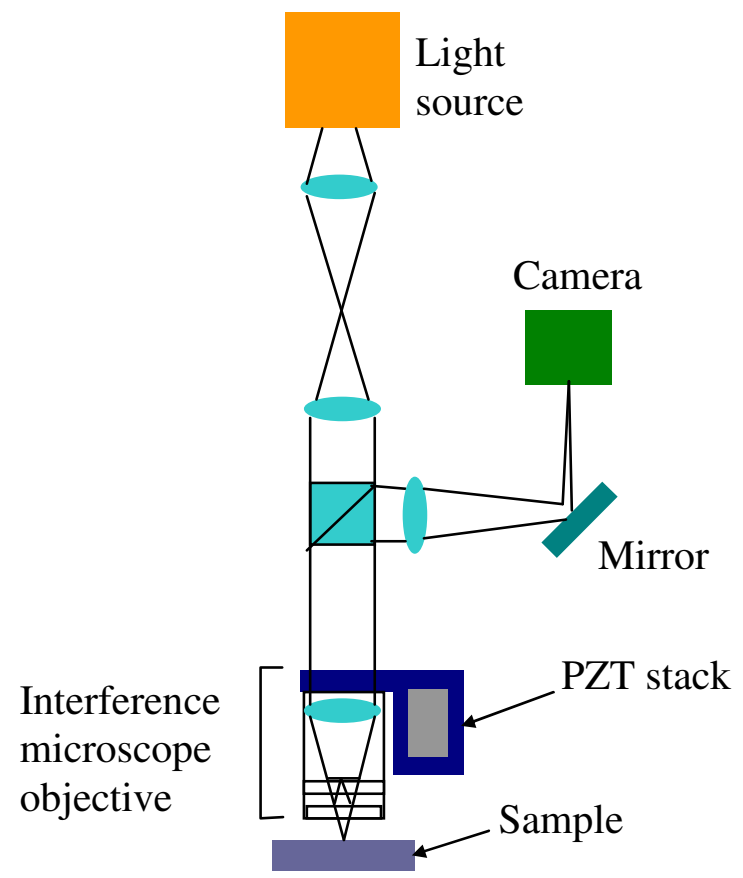

Figure 4-37 Setup for non-contact, three-dimensional, scanning white light and optical phase-shifting interferometry

\subsubsection{Principle of White Light Interferometer}

The terms "interferometry" and "interferometer" are both derived from the word interference. Interference is a phenomenon that occurs when one has waves of any kind sound waves, light waves, ocean waves, and seismic waves from earthquakes [Demtröder, 2003]. Whenever two waves come together at the same time and place, interference occurs. Resonance, beat frequencies, heterodyning, and dissonance are also interference phenomena. 
Interference Between Coincident Light Waves
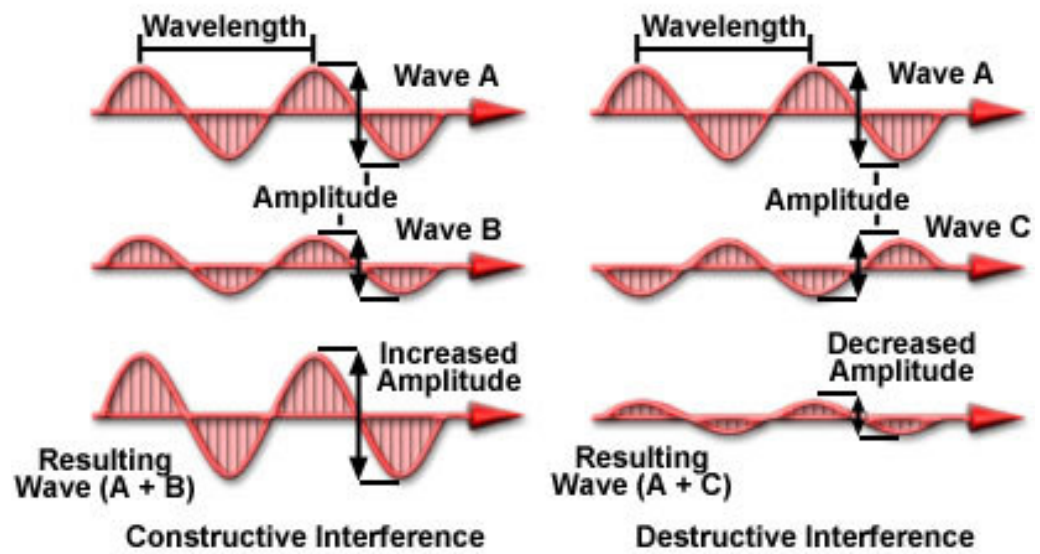

Destructive Interference

Thomas Young's Double Slit Experiment

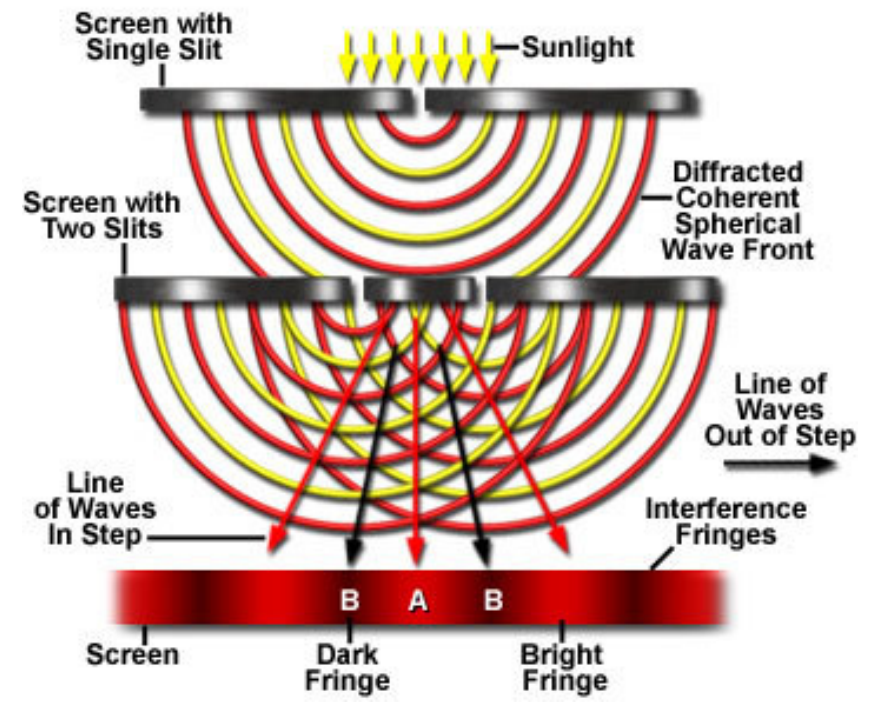

Figure 4-38 Interferences as a result of destructive and constructive wave interactions. Interference can be visualized as the addition of two waves. Depending upon the wave size (amplitude) and the degree to which they are in or out of step with each other (phase), the effects are either additive or subtractive

Most of the applications involving interferometry deal with the interference of two light waves. If both waves are in step or in phase, that is, the crest of both waves coincide, the two add together to form a single wave. This combined wave have a higher crest and deeper trough (or larger amplitude). In the case of light waves, two dimmer light beams 
add together to form a brighter beam - this is called constructive interference. On the other hand, destructive interference occurs when the two waves are out of step with each other, that is, the crest of one coincides with the trough of the other. Here, although the waves are still adding together, they cancel each other out. So, the amount of interference that occurs depends on both the amplitudes of the two waves and the degree to which their respective crests and troughs are in phase with each other. 


\section{CHAPTER 5: EXPERIMENTAL PROCEDURES/PROTOCOLS}

The previous chapter described the materials, methods and various flow loops used in these studies. This chapter is organized to give a description of each experimental procedure, including the experimental protocols for the hinge studies, the temperaturedependent study, the 3D PIV experiments, and the data reduction techniques.

\subsection{Static Leakage Flow Rate Experiments}

\subsubsection{Bileaflet Mechanical Heart Valves}

The static leakage loop described in section 4.4 was used to acquire the leakage rate through the CM $23 \mathrm{~mm}$, the SJM $27 \mathrm{~mm}$ and the two $27 \mathrm{~mm}$ prototype bileaflet clear housing valves. These prostheses were placed in the closed position with a pressure drop of $120 \mathrm{~mm} \mathrm{Hg}$ across the valves. In this study, the glycerin and water mixture was used as the working medium (see Table 4-2). An average of six readings were taken for each valve, with readings taken over a period of 30 seconds to a minute depending on the leakage rate. The valve was taken out of the loop and flushed with water after each reading to ensure that no debris or impurities accumulated inside the hinge region since the leakage rate has a tendency to decrease if the valve is not properly flushed.

\subsubsection{Polymeric Heart Valves With Temperature Effect}

The static leakage test setup for the polymeric valve was similar to that for the bileaflet valves (see section 4.4.2). The static leakage tests were performed at room $\left(20.5^{\circ} \mathrm{C}\right)$ and 
physiological temperatures $\left(37^{\circ} \mathrm{C}\right)$ for each valve with an average of six readings for each set. However, the leakage flow through these valves tended to oscillate if the check valve between the valve and collecting chamber was opened too quickly. Hence, to better quantify the leakage rate of each valve, an extra set of readings was done with the oscillation at both room and physiological temperatures. In total, four sets of measurements were performed for each valve: (1) two at room temperature, one with and one without oscillation and (2) two at physiological temperature, one with and one without oscillation. No flushing is necessary for these polymeric valve. Glycerin and water mixture was used as the working medium (Table 4-2).

\section{$\underline{5.2 \text { Flow Waveforms }}$}

\subsubsection{Mitral Flow And Pressure Waveforms}

CM $23 \mathbf{~ m m}$ - The specific physiologic conditions for investigating the CM valve were as follows: heart rate of 70 beats/min (corresponding to an $860 \mathrm{~ms}$ cardiac cycle time), cardiac output of $4.0 \mathrm{~L} / \mathrm{min}$, peak diastolic flow rate of $10.0 \mathrm{~L} / \mathrm{min}$, diastolic duration of $560 \mathrm{~ms}$, and peak left ventricular pressure of $120 \mathrm{mmHg}$. Figure 5-1 show the mitral flow and ventricular pressure waveforms.

\section{$27 \mathrm{~mm}$ clear valves (SJM $27 \mathrm{~mm}$ Standard and the two $27 \mathrm{~mm}$ prototype leakers) -} The specific physiologic conditions for investigating the three $27 \mathrm{~mm}$ clear valves were as follows: heart rate of 70 beats $/ \mathrm{min}$, cardiac output of $5.0 \mathrm{~L} / \mathrm{min}$, peak diastolic flow rate of $10.0 \mathrm{~L} / \mathrm{min}$, diastolic duration of $560 \mathrm{~ms}$, and peak left ventricular pressure of 120 mmHg (Figure 5-1). 


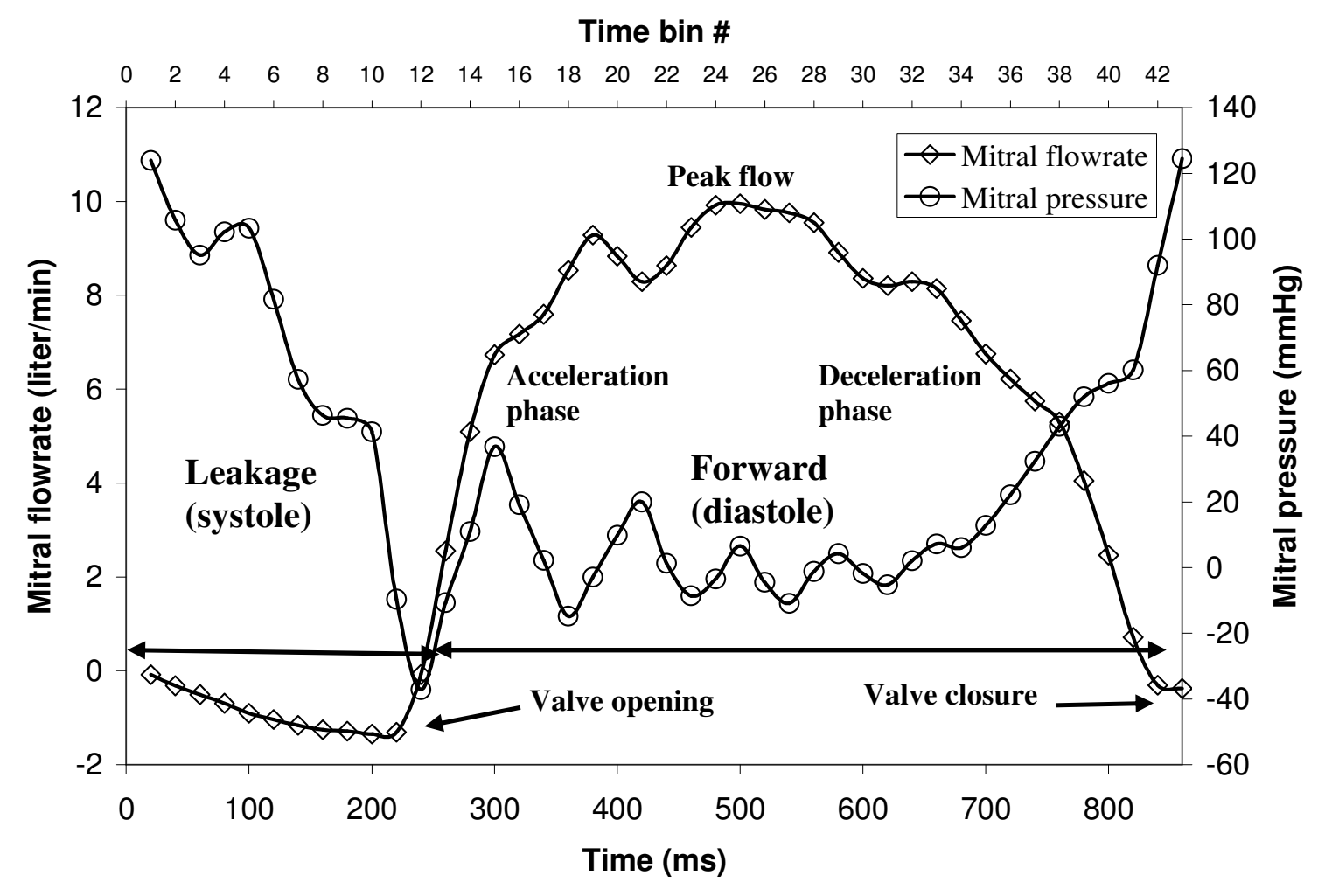

Figure 5-1 Mitral valve flowrate and pressure waveforms

\subsubsection{Aortic Flow And Pressure Waveforms}

Aortech $23 \mathrm{~mm}$ - The heart rate was set at 70 beats/min, with a cardiac output of 5.0 $\mathrm{L} / \mathrm{min}$, a peak systolic flow rate of $25.0 \mathrm{~L} / \mathrm{min}$, a mean aortic pressure of $90-100 \mathrm{mmHg}$ and a systolic flow phase duration of approximately 35 percent $(300 \mathrm{~ms})$ of the cardiac cycle time. Peak left aortic pressure was maintained at $120 \mathrm{mmHg}$. Figure 5-2 shows the aortic flow and pressure waveforms. 


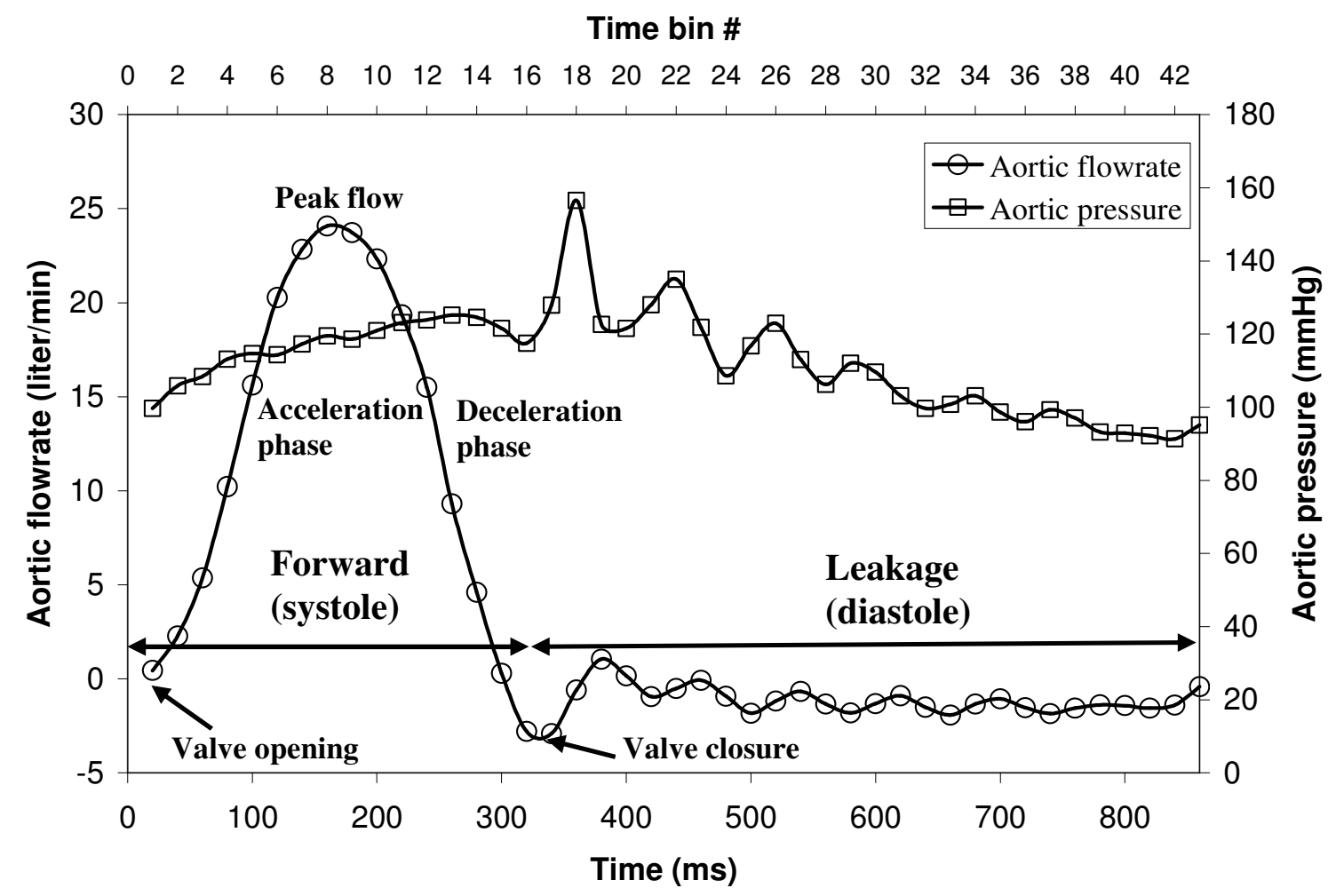

Figure 5-2 Aortic valve flowrate and pressure waveforms

\subsubsection{The Effect of Resistant and Compliant Units on The Waveforms}

The resistance of the mock loop was controlled by adjusting the clamp on the flexible 1" flexible hose. The effect of the resistance unit was to vary the average aortic pressure. For example, tightening the clamp elevated the pressure as shown in Figure 5-3. Increasing the resistance, besides reducing the flow rate in the system, also led to an unwanted result of increased 'ringing' or oscillation of the waveform during diastole. This is especially evident in the polymeric heart valves. However, an additional compliance unit downstream of the aortic valve reduced this unwanted 'ringing'. 


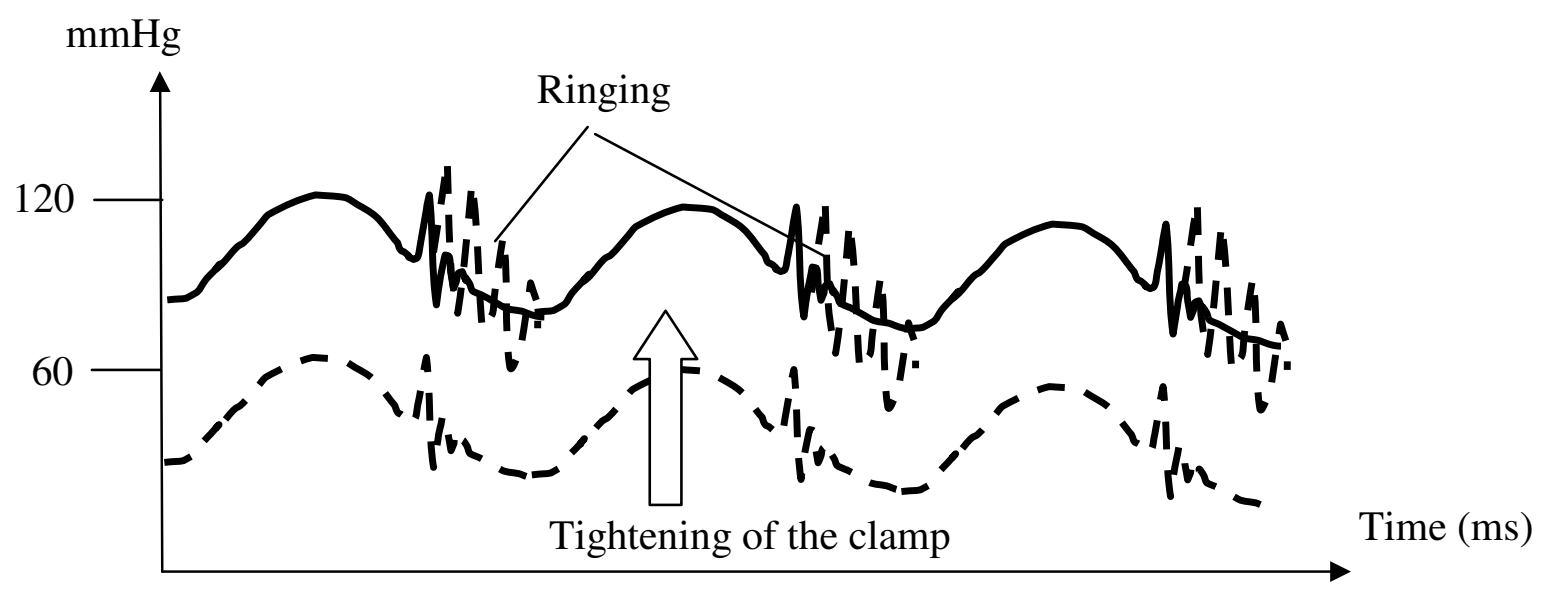

Figure 5-3 The effect of increasing resistant on the aortic pressure waveform

The compliance can also be used to decrease the average pressure in the aortic waveform, but it has a more dominant effect on the differential pressure gradient across the aortic valve. Figure 5-4 illustrates the effect of varying the compliance in the system. Reducing the solution level inside the compliance chamber reduces the differential pressure across the aortic valve.

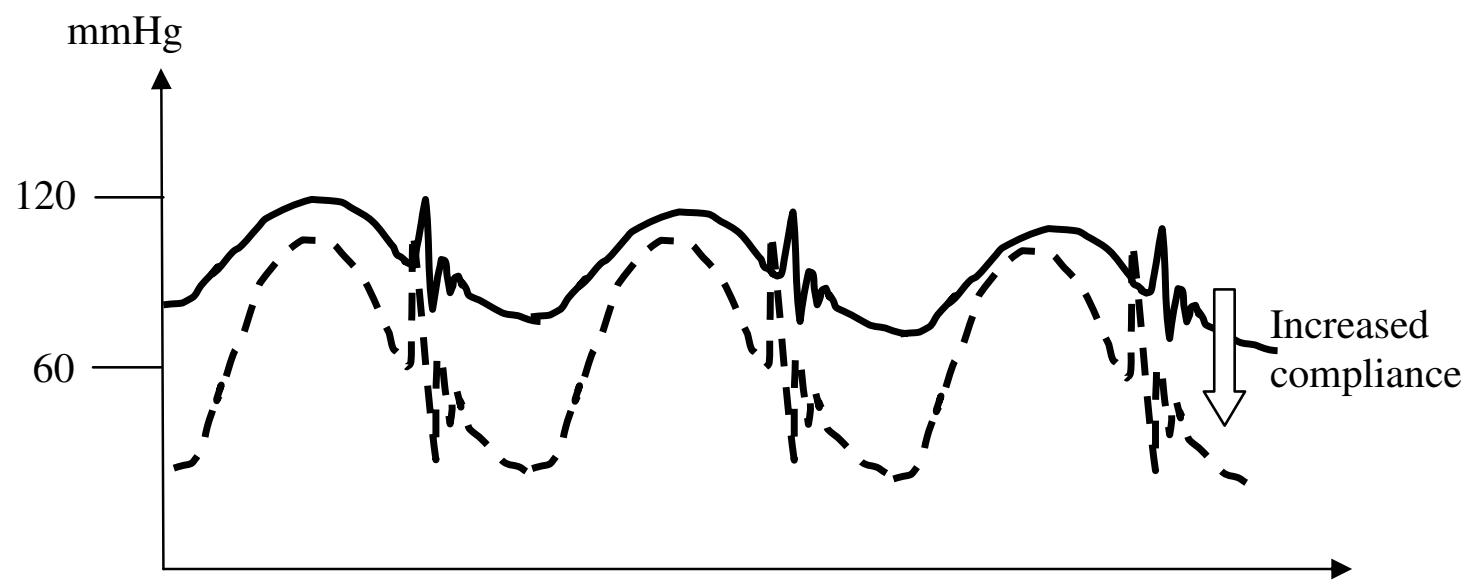

Time (ms)

Figure 5-4 The effect of increasing compliance on the aortic pressure waveform 
The mitral flow rate and pressure as well as the aortic flow waveform are easier to obtain compared to the aortic pressure waveform. More often than not, once a correct aortic pressure waveform is obtained, the remaining waveforms will also be in their physiological settings. However, as mentioned earlier, even though an additional compliance unit reduced the 'ringing' in the polymeric valve, it also increases the left ventricular pressure. This was the case for the polymeric valve prototype B and C, probably due to their thicker leaflets. In this case, an additional compliance unit between the mitral valve and the silicone bulb helped reduce the left ventricular pressure. Elevation of the reservoir level increased both the mitral and aortic flow rates at the expense of increasing the 'ringing' in the aortic pressure.

\subsection{Laser Doppler Velocimetry Experiments}

\subsubsection{Pulsatile Mitral Hinge Flow Experiments}

All hinge studies were performed using 2D LDV because the hinge region is near the surface of the valve housing making the measurement of third component impractical. In LDV, green and blue beams have the stronger power, thus ensuring a higher data rate. A single $2 \mathrm{D}$ transceiver probe was used to transmit the laser beams to the test section in backscattering mode. Figure 5-5 shows the 2D LDV configuration for these experiments with the probe mounted above the test section. Before commencement of the 2D LDV experiment, the flat level of the hinge had to be located and the hinge geometry mapped. A mixture of NaI, glycerin and water was used as the working medium Table 4-2. 


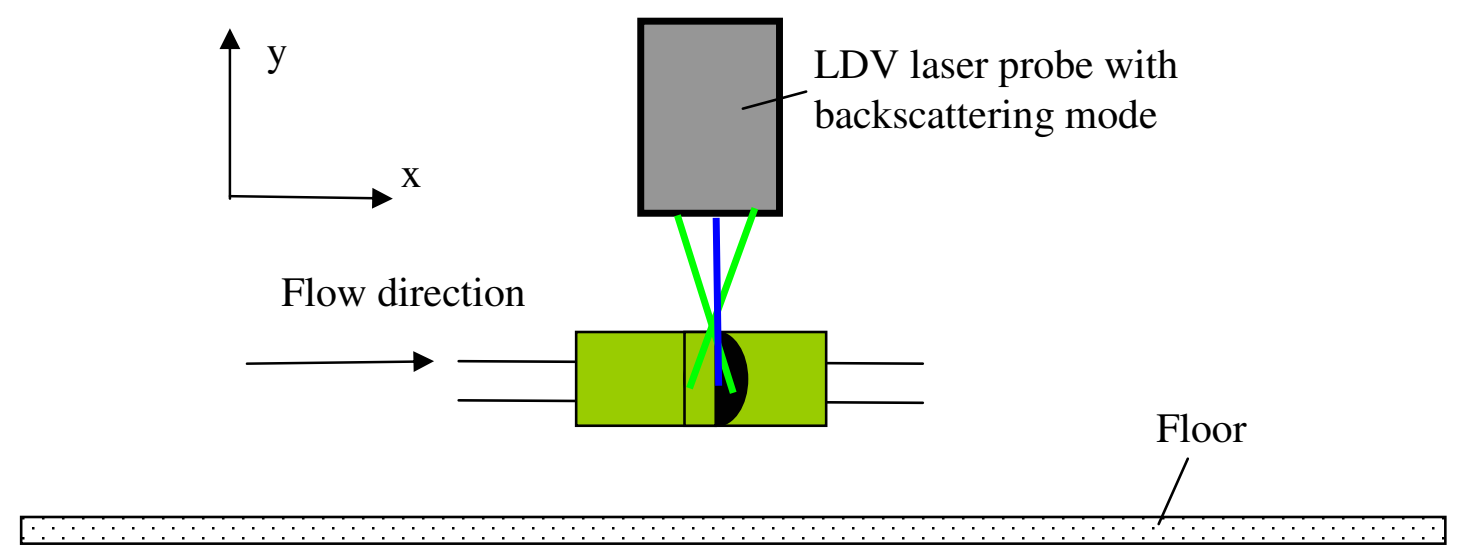

Figure 5-5 Position of 2D LDV probe with respect to the valve chamber

Marking the flat level in the hinge region -The hinge region in the valve housing is flat on the inner side. To locate the flat level in the hinge region, the laser probe volume was moved to the cross marked 1 as shown in Figure 5-6, and then lowered into the region of the main flow where the data rate is the highest $(\sim 100-400 \mathrm{~Hz})$. The probe was slowly moved up towards the hinge region until the data rate dropped to $<5 \mathrm{~Hz}$ with a display of a low frequency signal on an oscilloscope signifying the wall boundary. Noting the reading of the flat level on the dial gauge, the steps were repeated for the remaining crosses. The valve chamber was re-fastened to ensure that it was flat against the table and that the four dial gauges showed the same reading at the flat level before proceeding to next step of mapping the hinge region. 

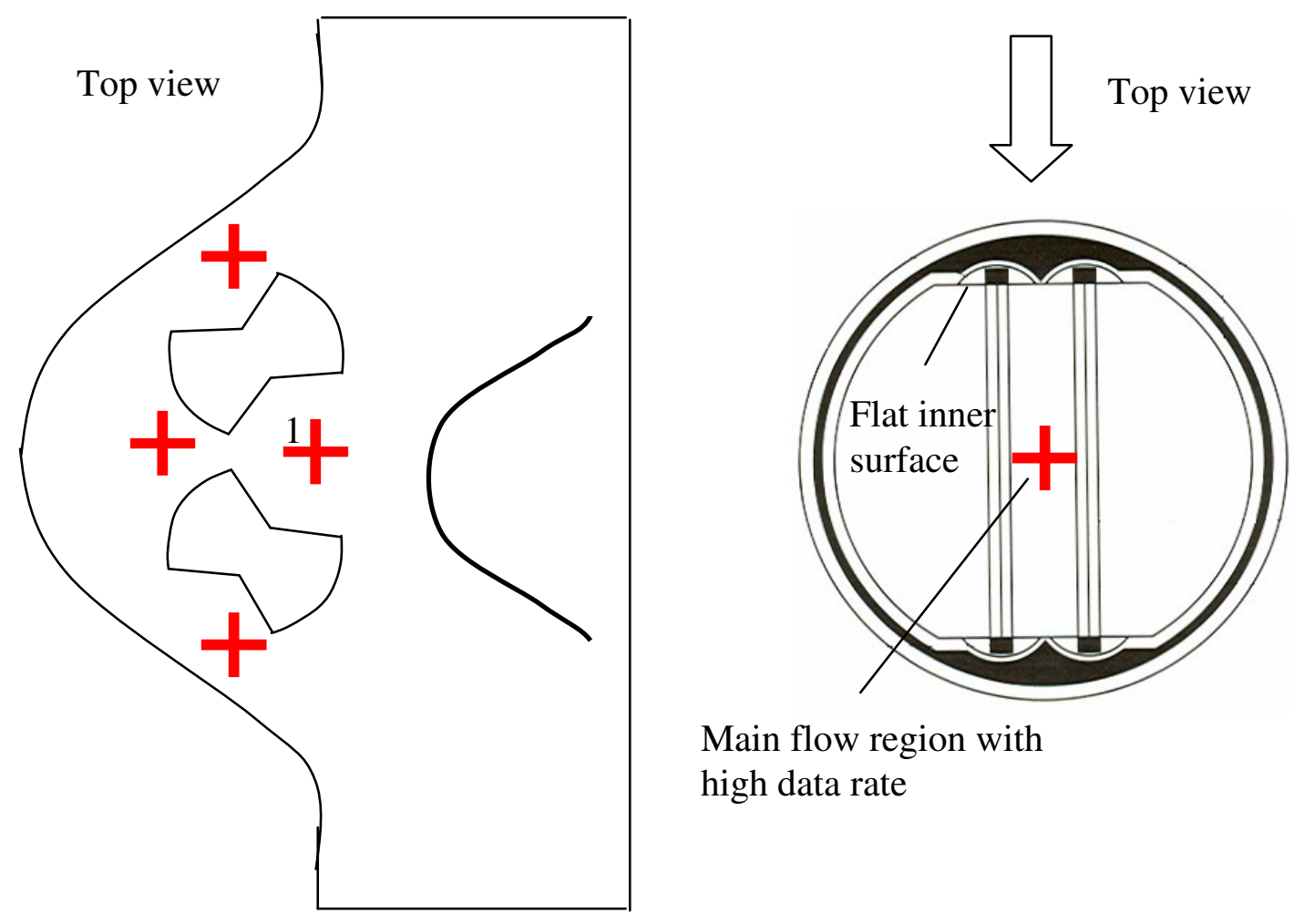

Main flow region with

high data rate

Figure 5-6 Positioning of the laser probe volume with respect to the location of the hinge region; red cross signify the various locations in which to place the probe volume to find the flat level at the hinge region

Mapping of the hinge region - Only one side of the hinge mechanism was mapped. The hinge geometry was first mapped visually at the primary flat level by moving the laser beams to the edge of the hinge until a bending of the beams was observed. Using the hinge boundary coordinates obtained in the first mapping as a reference, a subsequent, finer mapping of the hinge was done with the pulsatile loop running. In this mapping, a display of a low frequency signal on an oscilloscope signified the wall boundary. The hinge geometry was mapped by moving the probe volume across the hinge region as shown in Figure 5-7 beginning at the bottom of the geometry marked X. Hinge mapping 
is an approximate procedure to give an estimated outline of the hinge region hence it is not necessary to be overly precise with the mapping.
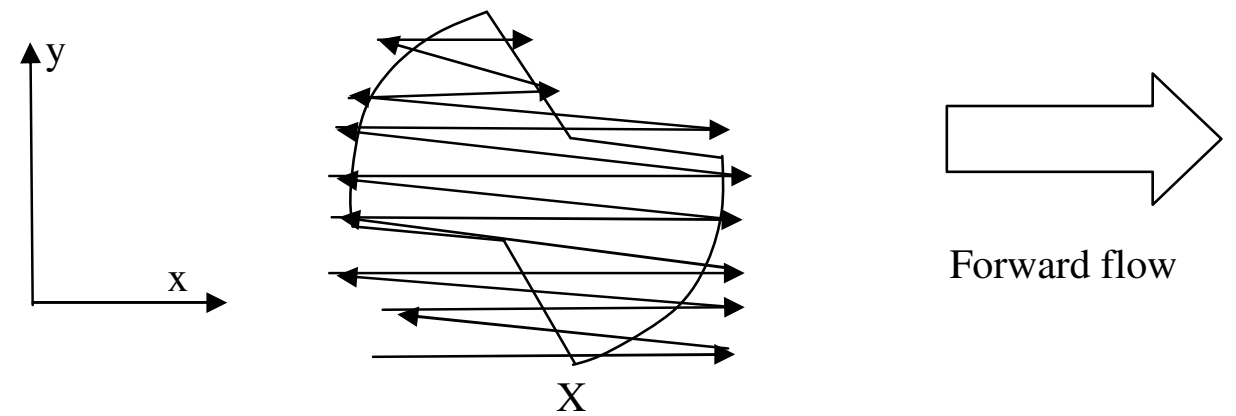

Forward flow

Figure 5-7 The edge of the hinge geometry was mapped by moving across the hinge in a predefined way to obtain an approximate co-ordinate of the hinge outline

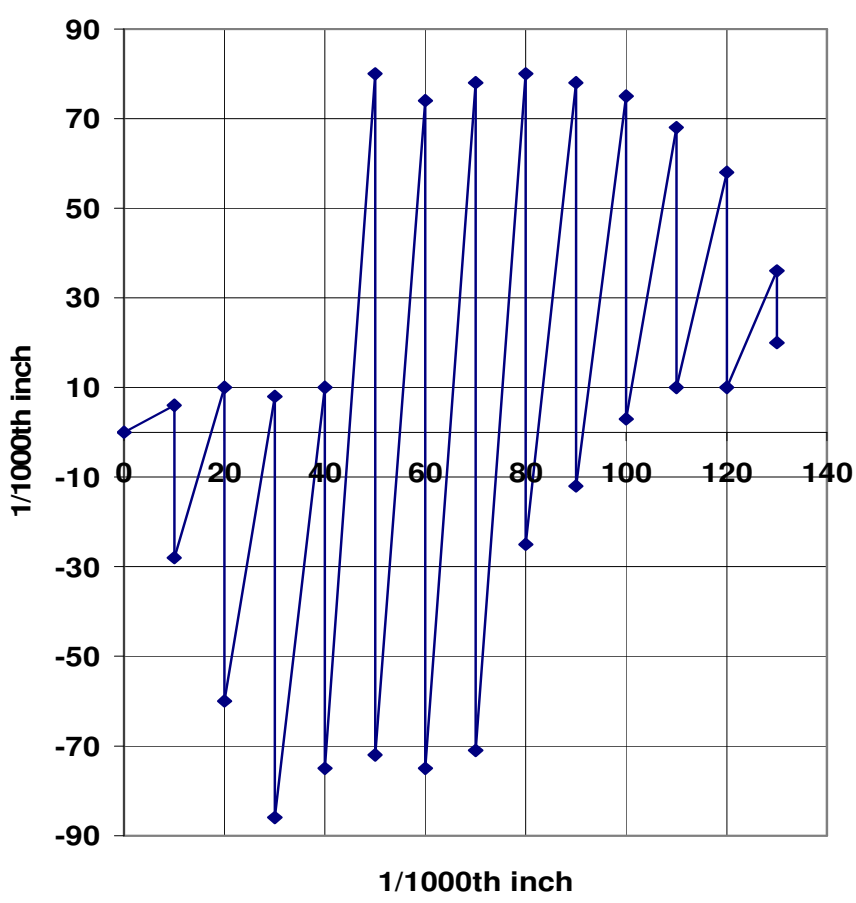

Figure 5-8 Approximated outline of the hinge geometry 
Figure 5-8 shows the mapping of the hinge at the flat level with an incremental resolution of $0.254 \mathrm{~mm}(0.01 \mathrm{inch})$ in both the $\mathrm{x}$ and $\mathrm{y}$ direction. The hinge outline provides an approximate location of the hinge region for subsequent LDV measurements. This procedure was performed for the CM $23 \mathrm{~mm}$ and the three prototype models of the SJM $27 \mathrm{~mm}$ Standard valves. Note that if the valve chamber is removed for cleaning the above protocol must be repeated. Also, it is important not to move the flow loop or the valve chamber midway through a particular measurement level inside the hinge.

\subsubsection{Measurement Sites Inside The Hinge Regions}

Figure 5-9 shows the beam crossing inside the hinge region. The size of the beam crossing is not proportional and is exaggerated relative to the size of the hinge pocket for illustration. The length of the probe volume in the major axis is approximately $140 \mu \mathrm{m}$ with a thickness of about $20 \mu \mathrm{m}$ inside the NaI solution. The minimum distance between the measurement levels inside the hinge pocket was $195 \mu \mathrm{m}$, and the spatial resolution across the hinge geometry was $0.127 \mathrm{~mm}$, or $127 \mu \mathrm{m}$. This setup was to ensure that the measurement locations do not overlap each other. 


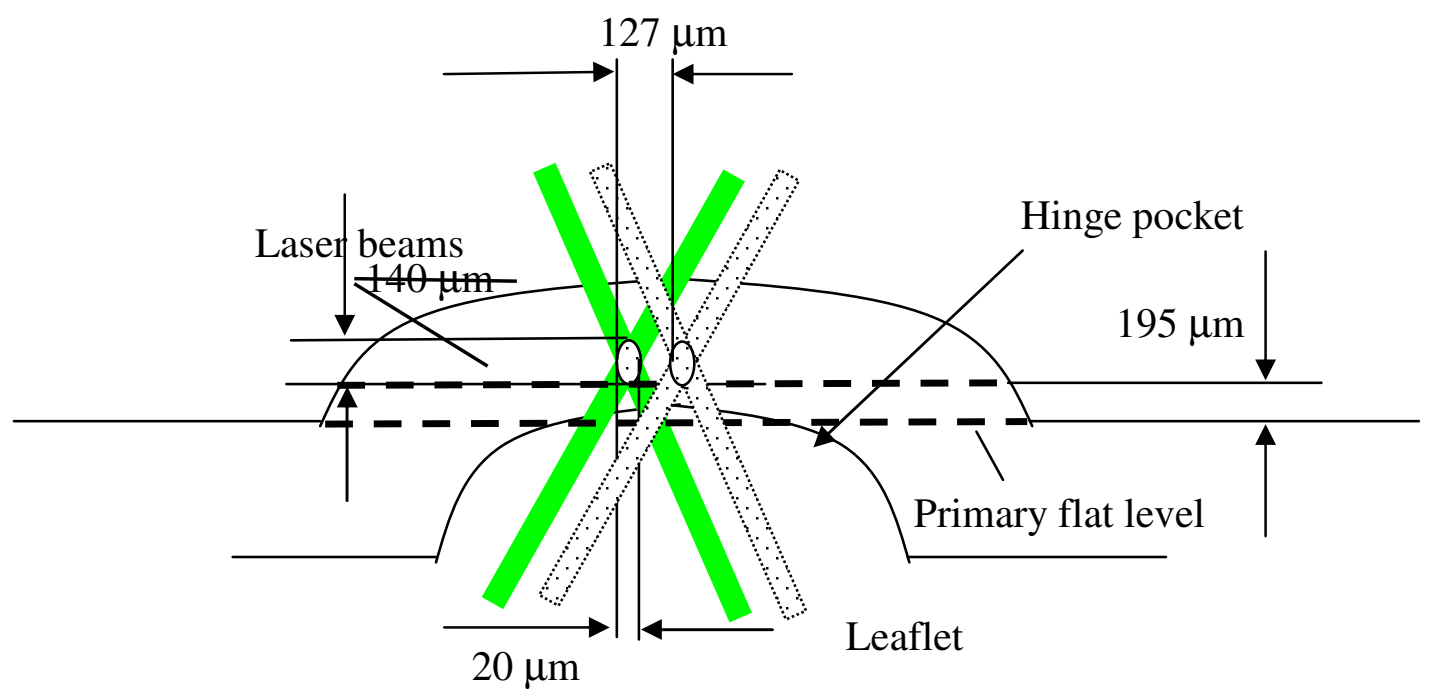

Figure 5-9 The size of the probe volume is smaller than the incremental resolution of the measurement

CM 23 mm - Within the CM 23 mm clear valve hinge, measurements were conducted at a total of 516 sites in the pulsatile flow with an incremental resolution of $0.254 \mathrm{~mm}(0.01$ inch) and $0.381 \mathrm{~mm}(0.015 \mathrm{in})$ in the $\mathrm{x}$ and $\mathrm{y}$ directions, respectively. Figure 5-10 shows the top view of the CM hinge region of the clear housing valve model and the coordinate system that was employed. Figure 5-11 shows the measurement locations inside and outside the hinge pocket of the four transparent valves. LDV measurements were performed on the following 4 planes as tabulated in Table 5-1. 


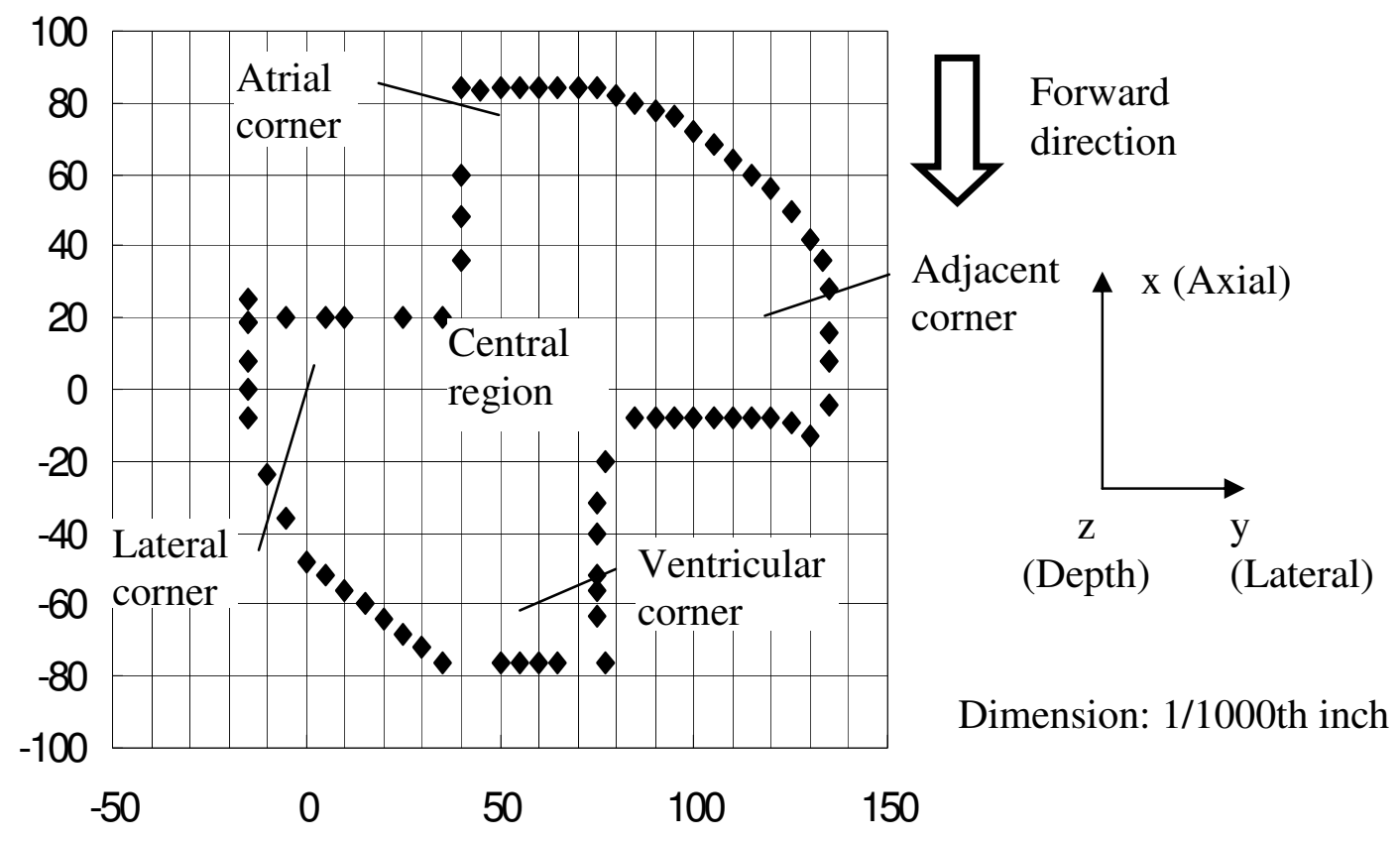

Figure 5-10 CM $23 \mathrm{~mm}$ hinge geometry as mapped by laser at primary flat level

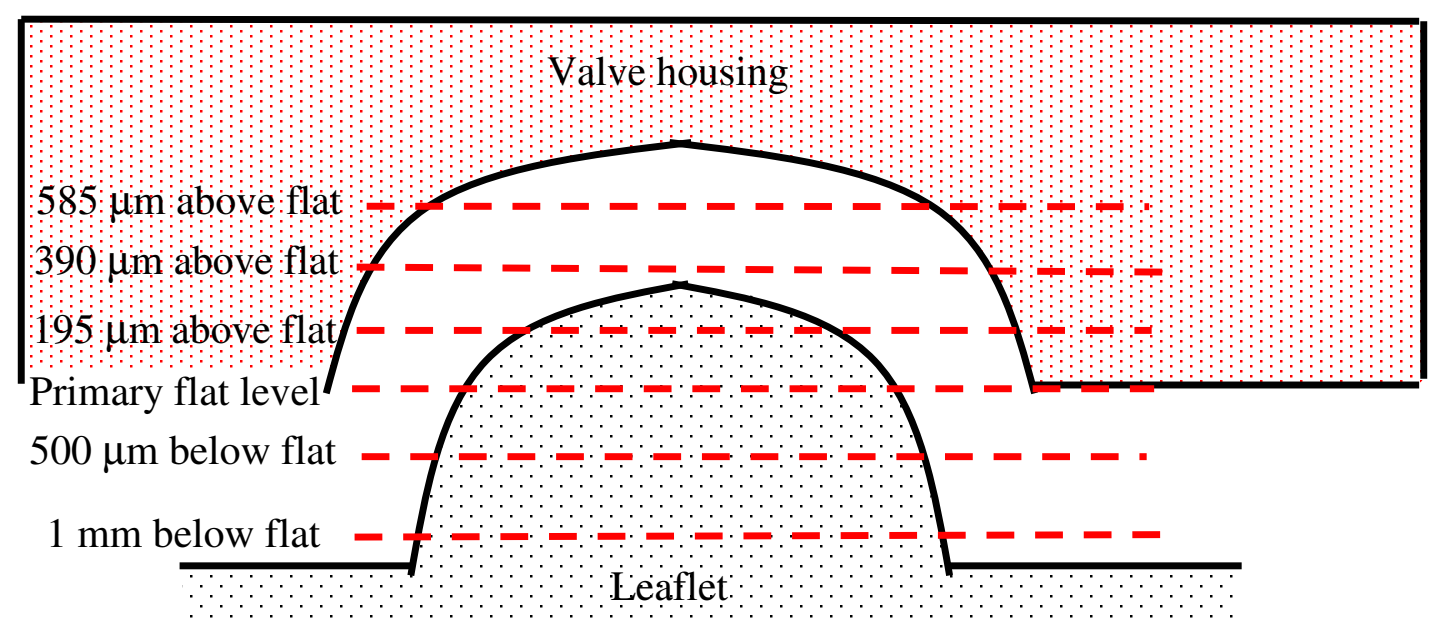

3 m m betow flat - -

Figure 5-11 The various measurements levels both inside and outside the hinge pocket of the four transparent bileaflet heart valves 
Table 5-1 Measurement levels inside the CM valve and the corresponding number of measurement locations

\begin{tabular}{c|cc}
\hline Valve Type & Measurement levels & $\begin{array}{c}\text { Number of measurement } \\
\text { locations }\end{array}$ \\
\hline CM 23mm & 1 mm below the primary flat & 92 \\
& Level with primary flat & 168 \\
& $190 \mu$ m depth into the hinge socket & 146 \\
& $390 \mu \mathrm{m}$ depth into the hinge socket & 110 \\
\hline
\end{tabular}

SJM 27 mm - Table 5-2 tabulates the measurement planes both inside and outside the hinge region of the three $27 \mathrm{~mm}$ clear housing valves. The measurements have an incremental resolution of $0.254 \mathrm{~mm}(0.01 \mathrm{inch})$ and $0.127 \mathrm{~mm}(0.005 \mathrm{in})$ in the $\mathrm{x}$ and $\mathrm{y}$ directions, respectively. Figure 5-12 shows the measurement locations at the flat level in the SJM 27 mm Standard valve. Figure 5-13 shows the top view of the hinge region of the three $27 \mathrm{~mm}$ bileaflet clear valves and the coordinate system that was employed. The optical access of the thumbnail region of the three $27 \mathrm{~mm}$ bileaflet clear valves was restricted by the interface between sections (2) and (3) of the valve holder (see Figure 4-12). The measurement locations at the thumbnail region were thus taken immediately downstream of the thumbnail recess at the same level as the primary flat (Figure 5-14). The measurements below the flat level and at the thumbnail region had an incremental resolution of $0.254 \mathrm{~mm}(0.01 \mathrm{inch})$ and $0.508 \mathrm{~mm}(0.02 \mathrm{in})$ in the $\mathrm{x}$ and $\mathrm{y}$ directions, respectively. 
Table 5-2 Various measurement levels in the three $27 \mathrm{~mm}$ transparent bileaflet valves and corresponding number of measurement locations

\begin{tabular}{|c|c|c|}
\hline Valve Types & Measurement levels & $\begin{array}{c}\text { Number of } \\
\text { measurement } \\
\text { locations }\end{array}$ \\
\hline \multirow{8}{*}{ SJM 27 mm Standard } & Thumbnail region & 155 \\
\hline & $3 \mathrm{~mm}$ below the primary flat level & 91 \\
\hline & $1 \mathrm{~mm}$ below the primary flat level & 201 \\
\hline & $500 \mu \mathrm{m}$ below the primary flat level & 199 \\
\hline & Level with primary flat & 252 \\
\hline & $195 \mu \mathrm{m}$ depth into the hinge pocket & 128 \\
\hline & $390 \mu \mathrm{m}$ depth into the hinge pocket & 17 \\
\hline & $585 \mu \mathrm{m}$ depth into the hinge pocket & 11 \\
\hline \multirow{7}{*}{$\begin{array}{c}27 \mathrm{~mm} \text { prototype low } \\
\text { leaker }\end{array}$} & Thumbnail region & 155 \\
\hline & $3 \mathrm{~mm}$ below the primary flat level & 98 \\
\hline & $1 \mathrm{~mm}$ below the primary flat level & 173 \\
\hline & $500 \mu \mathrm{m}$ below the primary flat level & 194 \\
\hline & Level with primary flat & 266 \\
\hline & $195 \mu \mathrm{m}$ depth into the hinge pocket & 151 \\
\hline & $390 \mu \mathrm{m}$ depth into the hinge pocket & 21 \\
\hline \multirow{8}{*}{$\begin{array}{l}27 \mathrm{~mm} \text { prototype high } \\
\text { leaker }\end{array}$} & Thumbnail region & 155 \\
\hline & $3 \mathrm{~mm}$ below the primary flat level & 106 \\
\hline & $1 \mathrm{~mm}$ below the primary flat level & 178 \\
\hline & $500 \mu \mathrm{m}$ below the primary flat level & 201 \\
\hline & Level with primary flat & 257 \\
\hline & $195 \mu \mathrm{m}$ depth into the hinge pocket & 142 \\
\hline & $390 \mu \mathrm{m}$ depth into the hinge pocket & 18 \\
\hline & $585 \mu \mathrm{m}$ depth into the hinge pocket & 14 \\
\hline
\end{tabular}




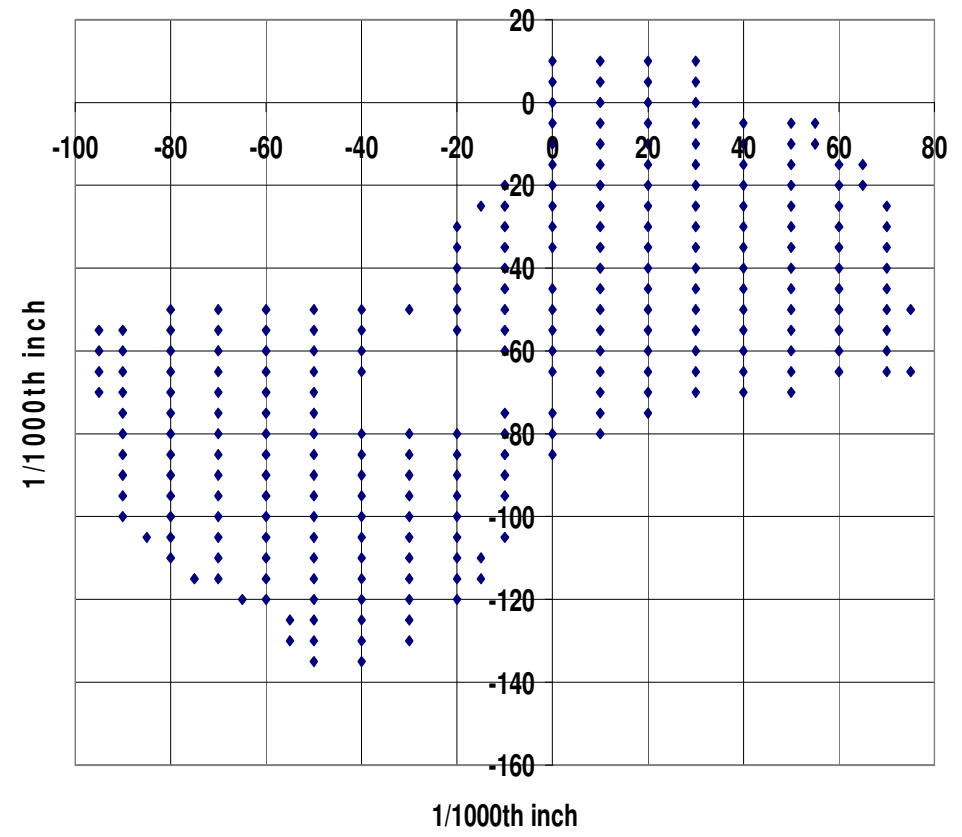

Figure 5-12 Measurement locations at the flat level of SJM $27 \mathrm{~mm}$ Standard
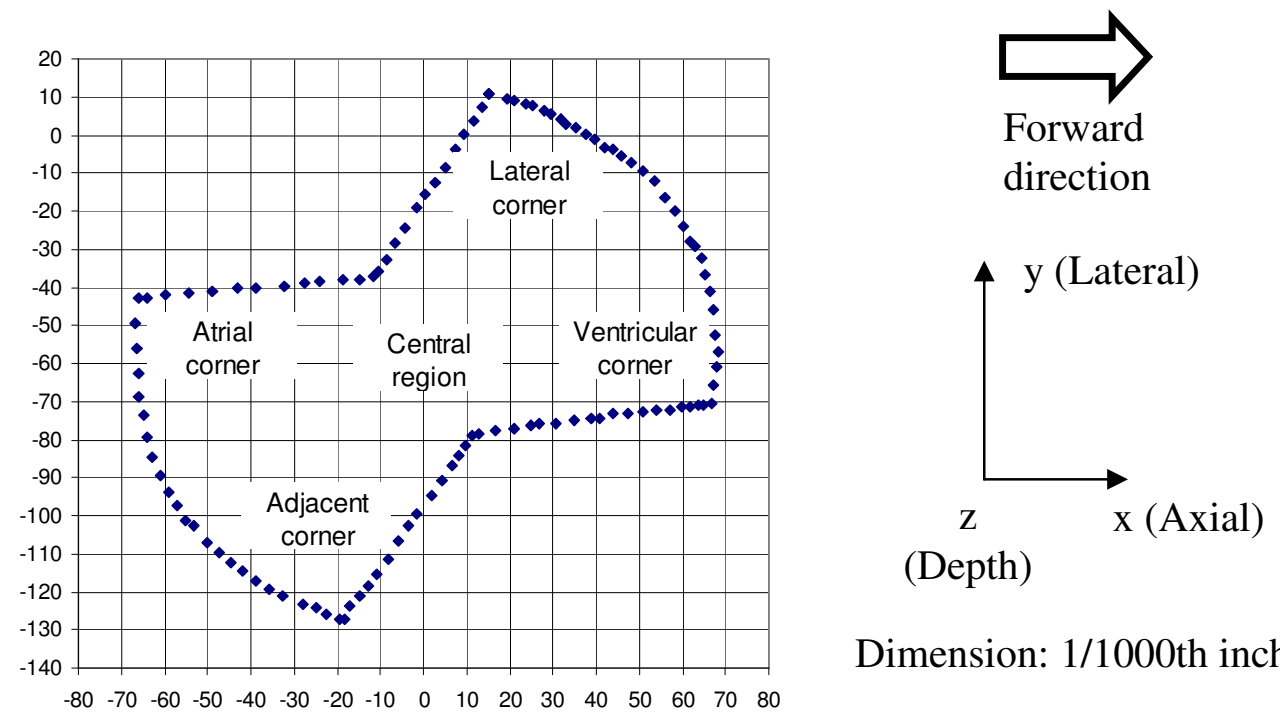

(Depth)

Dimension: 1/1000th inch

Figure 5-13 Hinge geometry of the three $27 \mathrm{~mm}$ transparent bileaflet clear valves as mapped by laser at primary flat level 


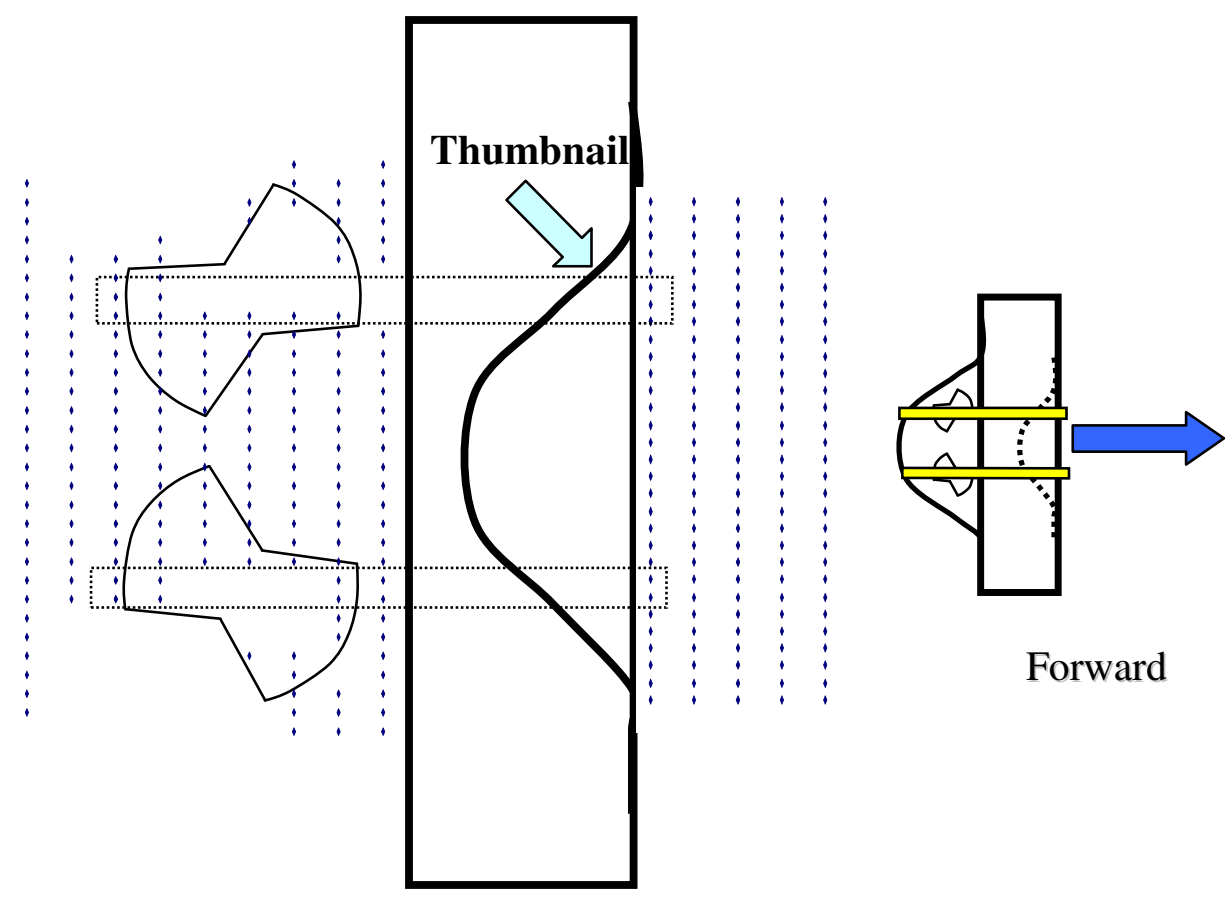

Figure 5-14 Measurement locations at the downstream of the thumbnail region and the below the flat level

\subsubsection{Pulsatile Aortic Flow Experiments}

2D LDV was performed on the three Aortech $23 \mathrm{~mm}$ polymeric valves. The setup was similar to that of the mitral hinge studies described earlier. Velocity measurement was acquired using one transceiver (green and blue beams) in backscattering mode. Figure 5-15 shows the LDV configuration of the polymeric heart valve experiments. Instead of placing the probe above the test section as in the hinge investigation, it was placed on the side of the valve chamber. Three measurement locations were acquired for each polymeric heart valves:

(1) Flow fields measurements downstream of the valves

(2) Flow field measurements inside the valves

(3) Flow fields measurements downstream of the stent 

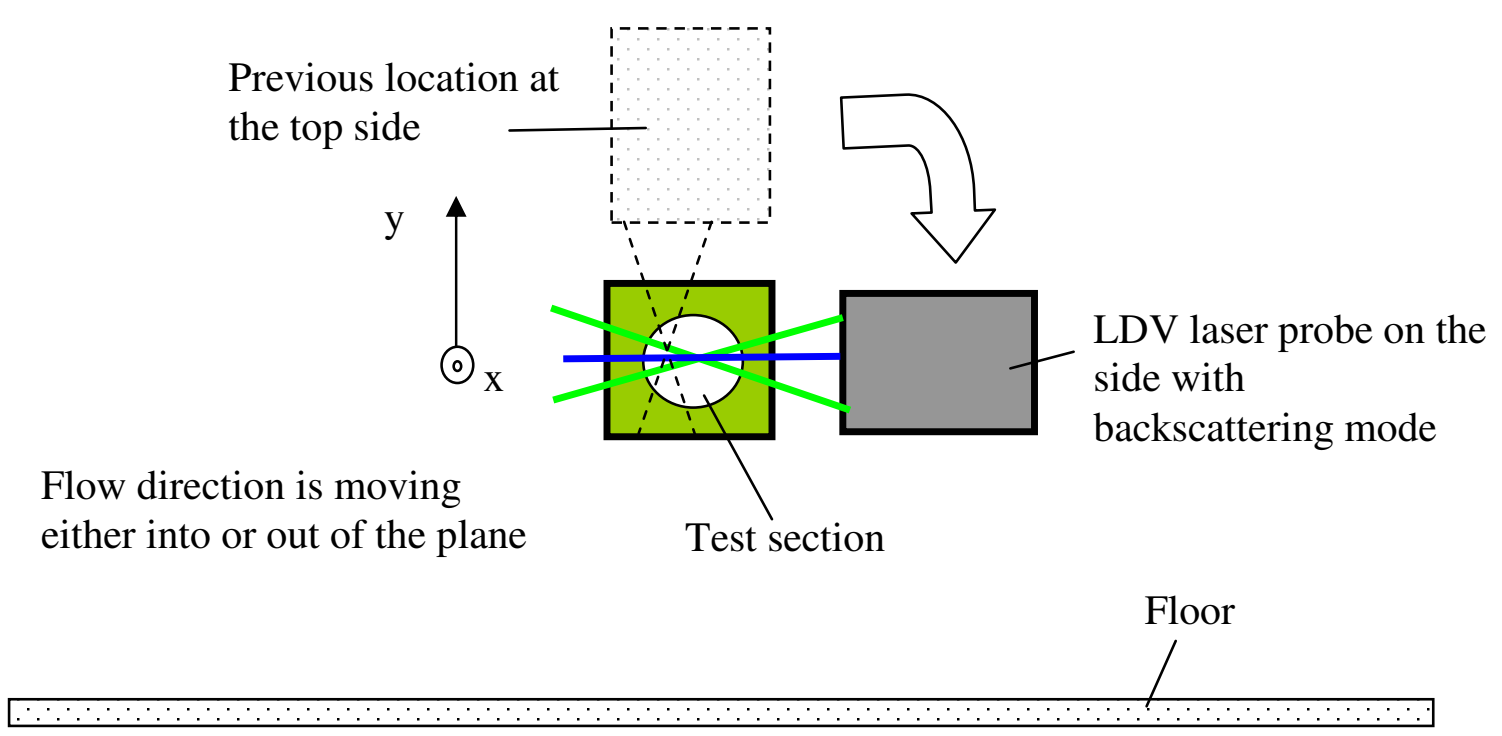

Figure 5-15 Position of the 2D LDV probe with respect to the valve chamber

Figure 5-17a shows the two orthogonal measurement planes downstream of the polymeric valves. Figure 5-17b shows the six measurement locations along each plane with distances referenced from the trailing edge of the leaflets. Figure 5-18 shows the locations of the measurement planes both inside the valve and immediately downstream of the stent. The stent inflow region is indicated as the region along the inner side of each stent. Table 5-3 tabulate the number of measurement locations at each measurement plane. 3D LDV was also employed to acquire measurement in the center plane in polymeric heart valve A. Figure 5-16 shows the position of the receiver and the two laser transmitter probes used for the acquiring the measurements. As discussed in section 4.8.3.1, the six color beams were aligned with each others to form a measurement probe volume. The scattered light from moving particle inside the probe volume was picked up by the receiver and then converted into velocity information by the computer. 


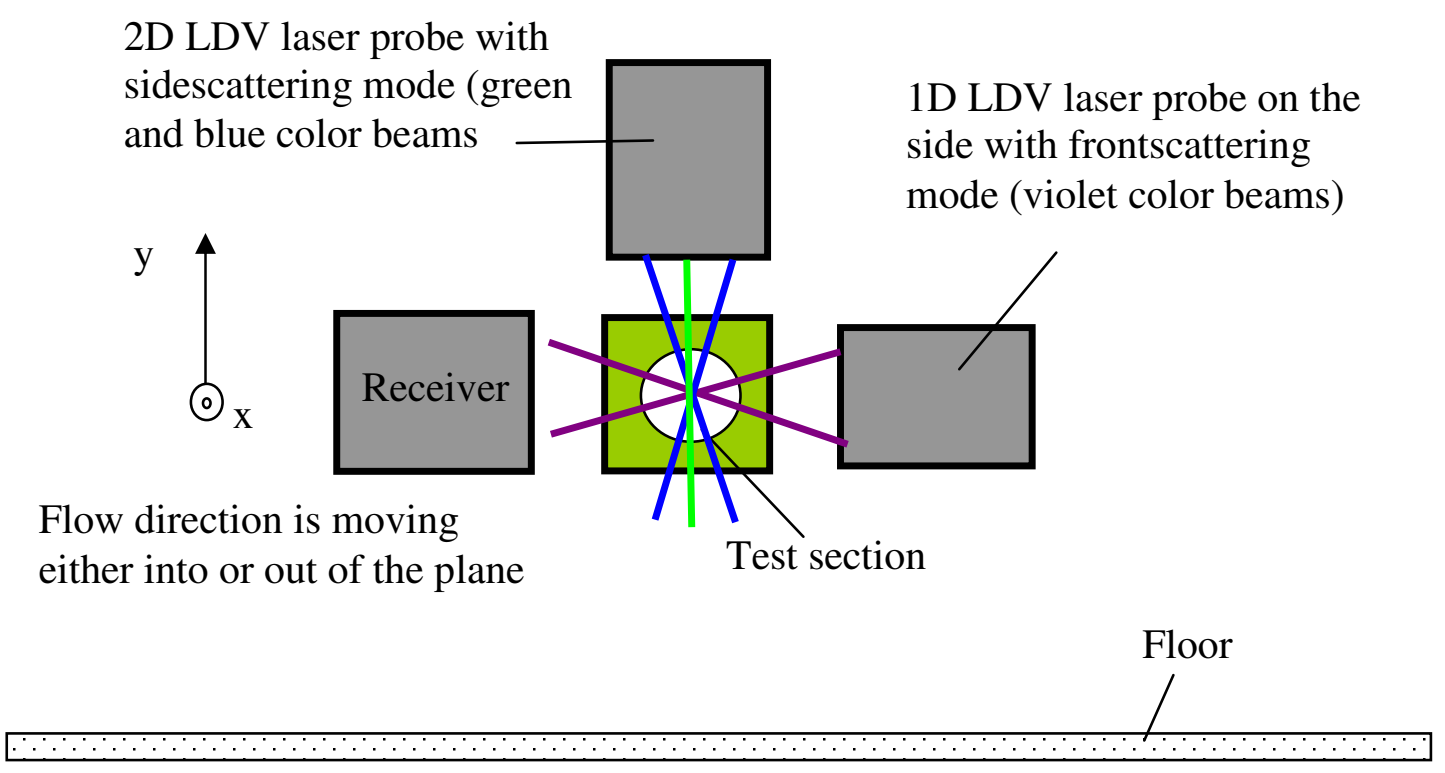

Figure 5-16 Position of the 2D LDV and 1D LDV probes with respect to the receiver and the test section. The violet beam is a much weaker beam compared to the green and blue laser beams, therefore the $1 \mathrm{D}$ violet laser probe is position across the receiver at a frontscattering mode to maximize the data rate

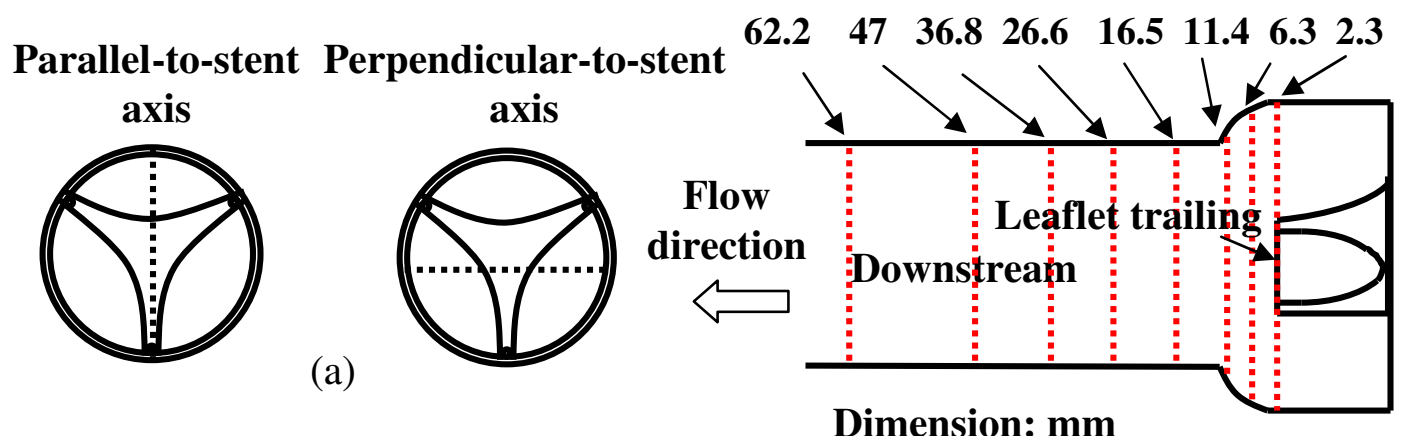

Figure 5-17 Measurement locations at the downstream of the polymeric heart valves 


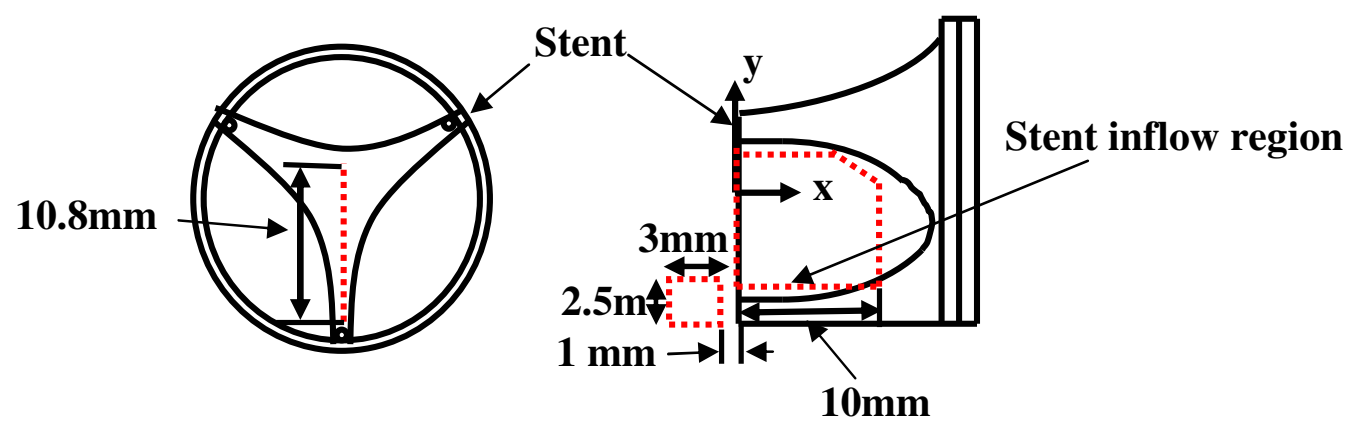

Figure 5-18 Measurement locations both inside and at the immediate downstream of the polymeric heart valve

Table 5-3 Measurement planes inside and proximal to the polymeric heart valve and corresponding number of measurement locations

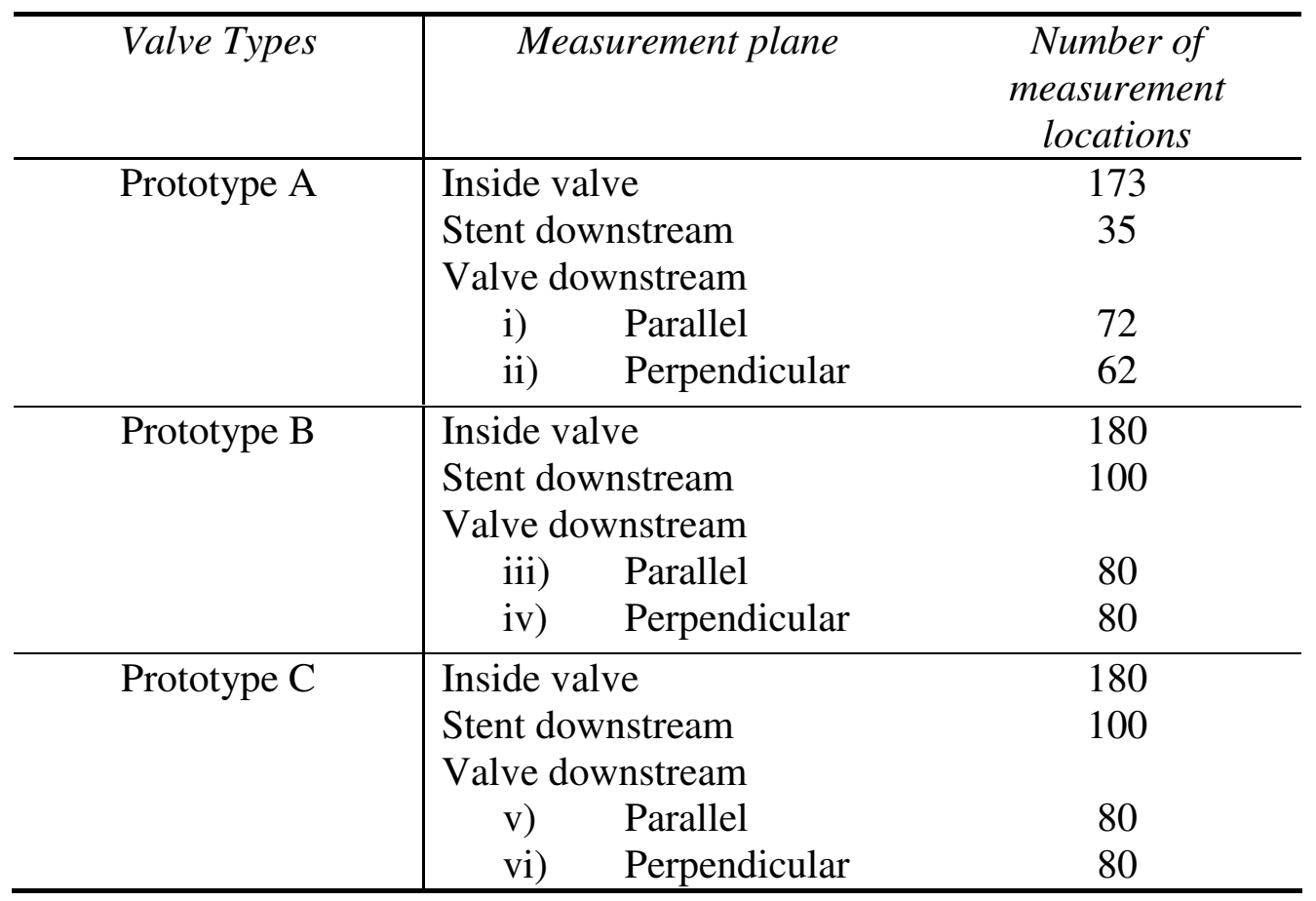




\subsection{Particle Imaging Velocimetry Experiments}

\subsubsection{Pulsatile Aortic Flow Experiments}

3D PIV was performed for the three Aortech $23 \mathrm{~mm}$ polymeric heart valves. Figure 4-17 and Figure 4-29 show, respectively, the flow loop and the 3D PIV system setup for this experiment. The external trigger signal was sent from the pulse generator to the PTU, which then synchronized the three output signals: (1) opening and closing of the camera shutter, (2) flash lamp and (3) Q-switch. The following steps were taken before the commencement of the calibration procedure:

1) the camera angle was set at approximately $30^{\circ}$,

2) lens aperture setting ( 4 for $1^{\text {st }}$ camera and 5.6 for the $2^{\text {nd }}$. Here the $1^{\text {st }}$ camera is defined as the camera with the first exposure of the flow fields)

3) Place $60 \mathrm{~mm}$ orange filter over the lenses.

4) Mix sufficient fluorescent particles in the solution (approximately $2-3 \mathrm{~mL}$ of fluorescent for about 12 liter of $\mathrm{NaI}$ solution)

5) Adjust the distance between the illuminated region in the test section and the laser lens to $500 \mathrm{~mm}$

6) Adjust the scheimpflug mechanism so that all dots on the target plate are in focus

\subsubsection{Calibration Procedure}

The first step in the calibration of 3D PIV is to take images of the calibration target and the preliminary flow field, and then output a preliminary set of calibration files. The second step, called self calibration, involves correcting the first calibration file from step 
1 and then using it to process the preliminary flow data, which also takes into account the correction for the skewing of the flow images.

Calibration Step 1 - The flow loop was started and about 50 image pairs of the flow field were captured. Without shifting the cameras or the laser, the test section was replaced with the calibration plate (Figure 5-19). The calibration target was mounted on a small traversing platform so that the length of the printed target was in line with the laser sheet (Figure 5-20) Care was taken to make sure that the cameras captured all of the calibration plate. The plate was translated by $0.6 \mathrm{~mm}$ from the cameras between exposures of the plate. In total, 8 exposures of the target plate were taken, four from each camera before and after the target translation. These images were correlated and adjusted for image skewing the target translation. These images were correlated and adjusted for image skewing (Figure 5-19). The software then output a calibration set file from these images.

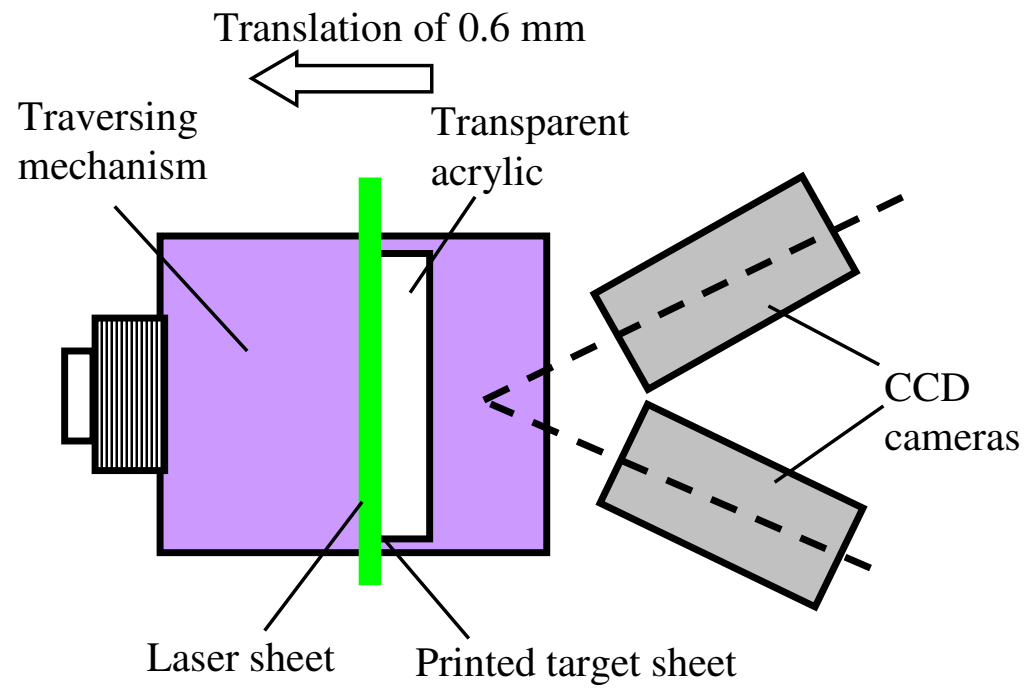

Figure 5-19 Translation of the calibration target during the calibration 


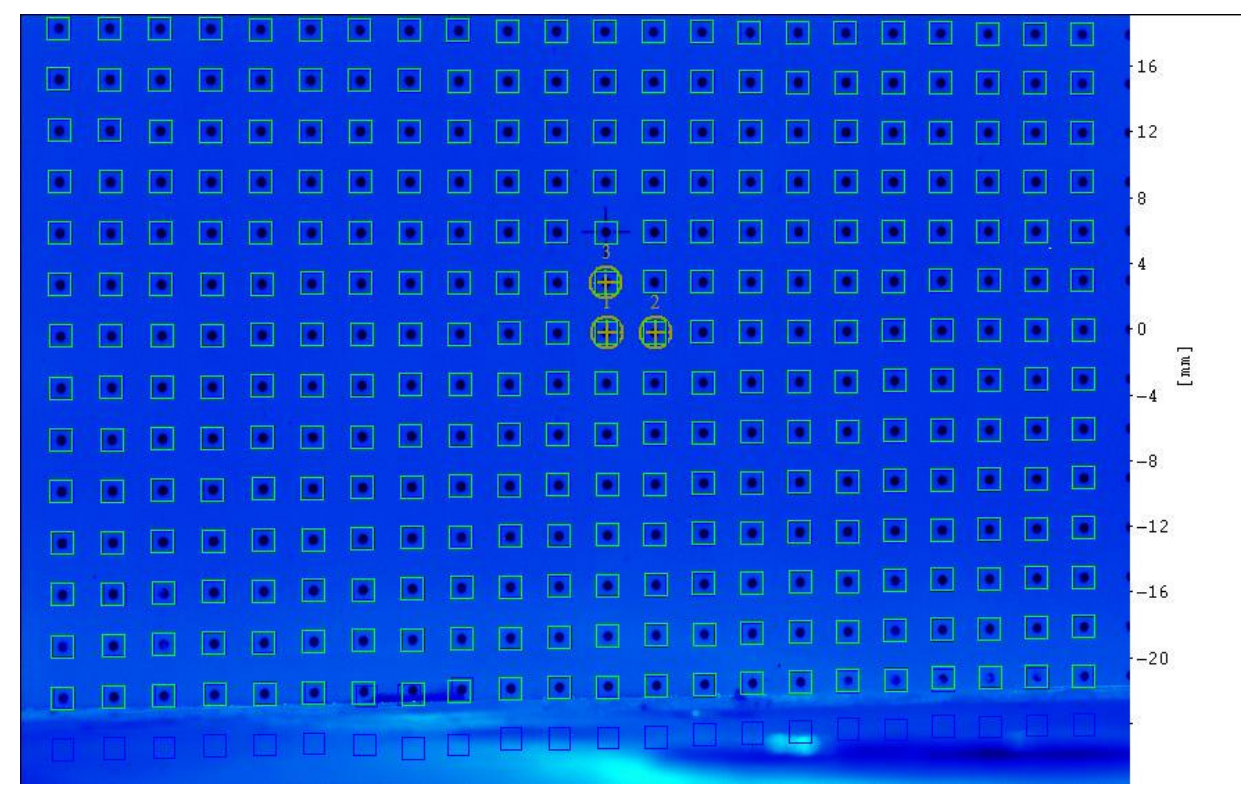

Figure 5-20 The mapping of the calibration target by Davis to calculate displacement function

Calibration Step 2 - The second step was performed by the Davis 7.0 software. The preliminary set of flow field data and the first calibration set file were loaded. Besides 'dewarping' the flow field image files as shown in Figure 5-21, the software also enables user input to help re-align the images. The software made use of the 'dewarp' and realigned image files to calculate the disparity map relative to the original images. The final mapping or calibration function file was checked for errors before being saved. All subsequent 3D PIV data used this corrected file for the calculation of velocity vectors (Figure 5-22). Figure 5-23 shows calculation of velocity vectors from the flow images. 


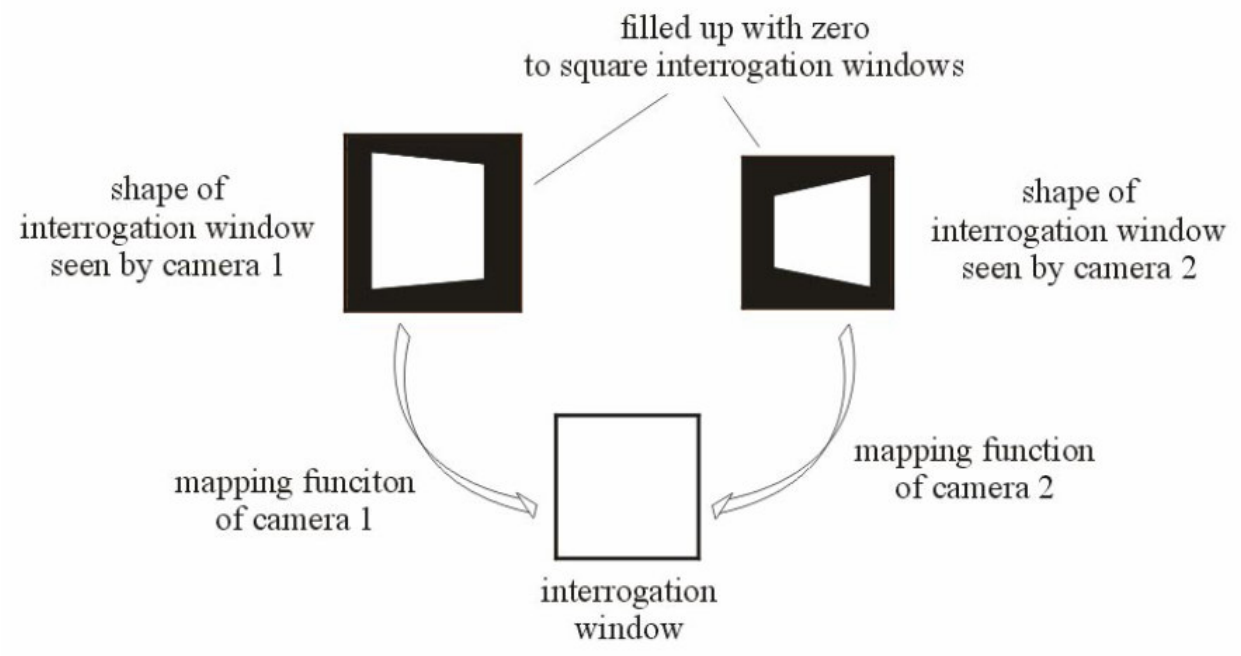

Figure 5-21 Mapping function is obtained from calibration target for each camera for the processing of velocity vectors

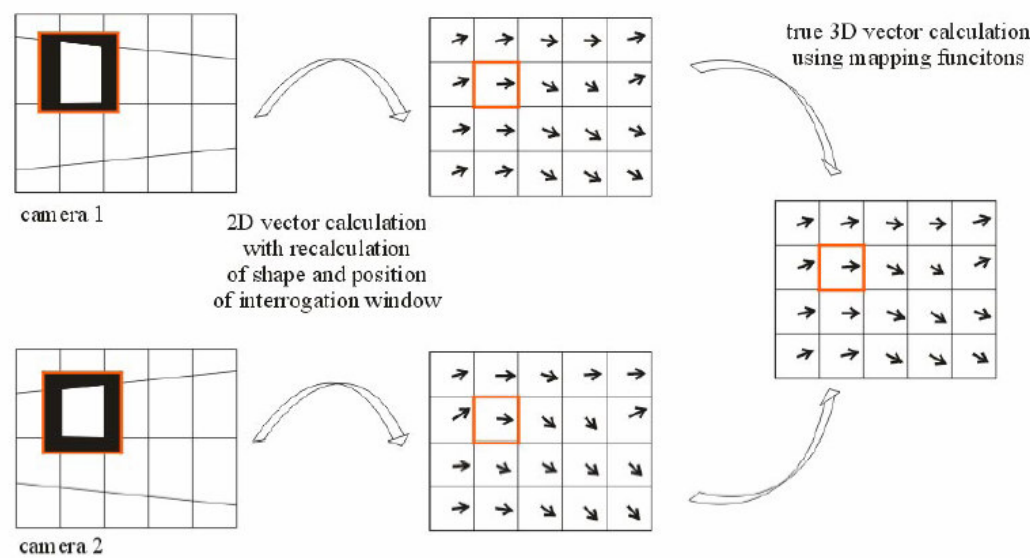

Figure 5-22 Vectors calculated from each camera are combined to produce 3D velocity map 


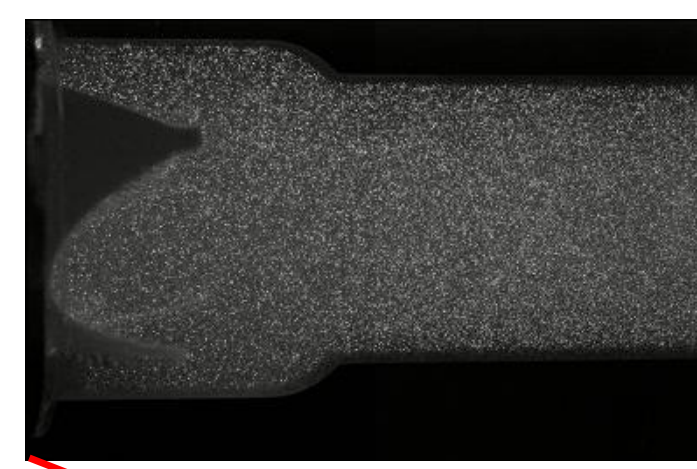

(a)

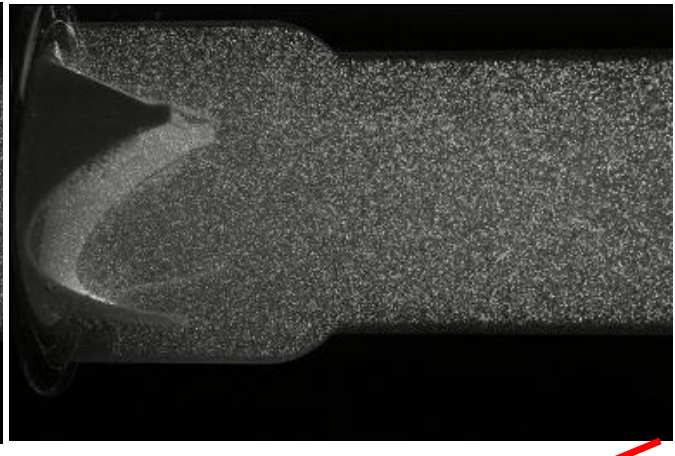

(b)

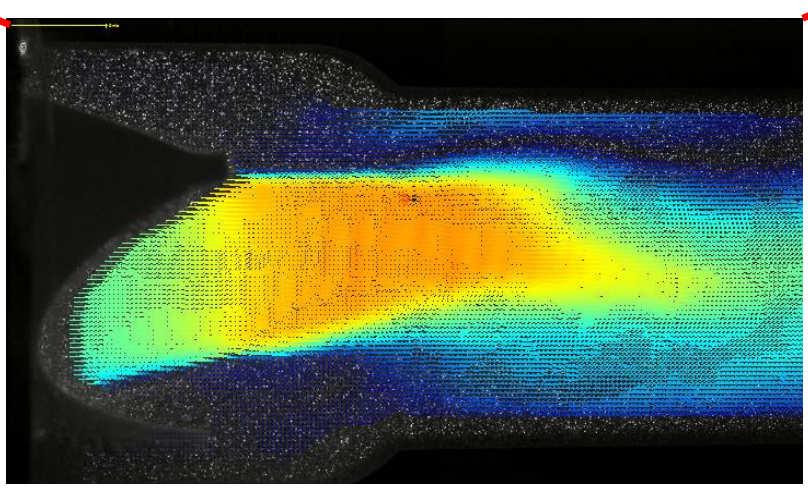

(c)

Figure 5-23 3D velocity vector map was obtained from the correlated images of the two CCD cameras. (a) The $1^{\text {st }}$ camera exposure, (b) the $2^{\text {nd }}$ camera exposure, and (c) the $3 \mathrm{D}$ velocity vectors from the two CCD cameras

Masking of raw images - A mask is used to prevent vector calculation in excluded regions of the particle image. Figure 5-24 shows a raw image of the polymeric valve before and after the masking. The mask contains information on the shape and position of arbitrary regions where vector calculation is either needed or not needed. The masking of a the raw image before processing was important in these experiments as it significantly reduced processing time. 


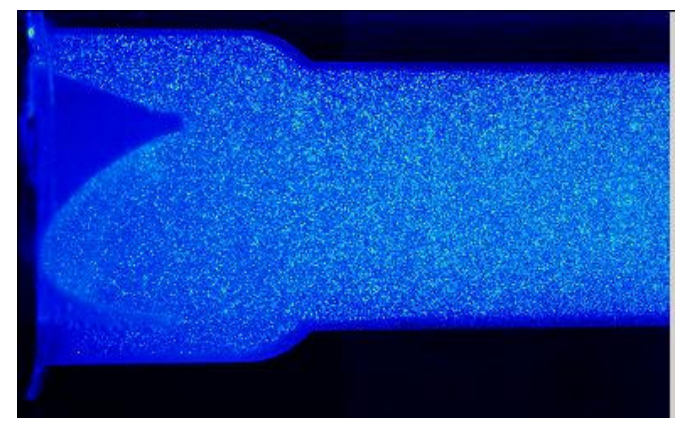

(a)

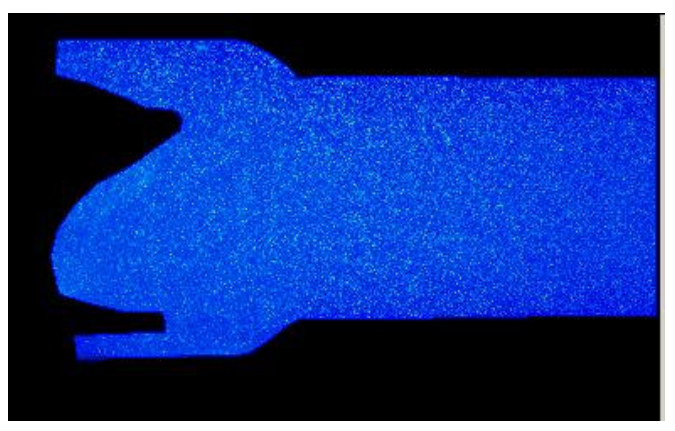

(b)

Figure 5-24 Application of masking to selectively process only the region of interest; (a) before masking, (b) after the application of masking

\subsubsection{Measurement Sites For Polymeric Valves}

The size of the interrogation area in 3D PIV was about $50 \times 65 \mathrm{~mm}^{2}$. Seven measurement planes were taken in the axis parallel to the stent. Figure 5-25 shows the spacing between each measurement plane, with the center of the valve as the reference plane. The distance between the laser sheet and the cameras was fixed throughout the measurements, and the translation between measurement planes was done by moving the valve chamber, which was marked with an equally spaced grid on either end of the chamber assembly. 250 image pairs were taken in each of the 43 time bins with time interval of $20 \mathrm{~ms}$. The Qswitch was set at $200 \mu$ s for both lasers. 

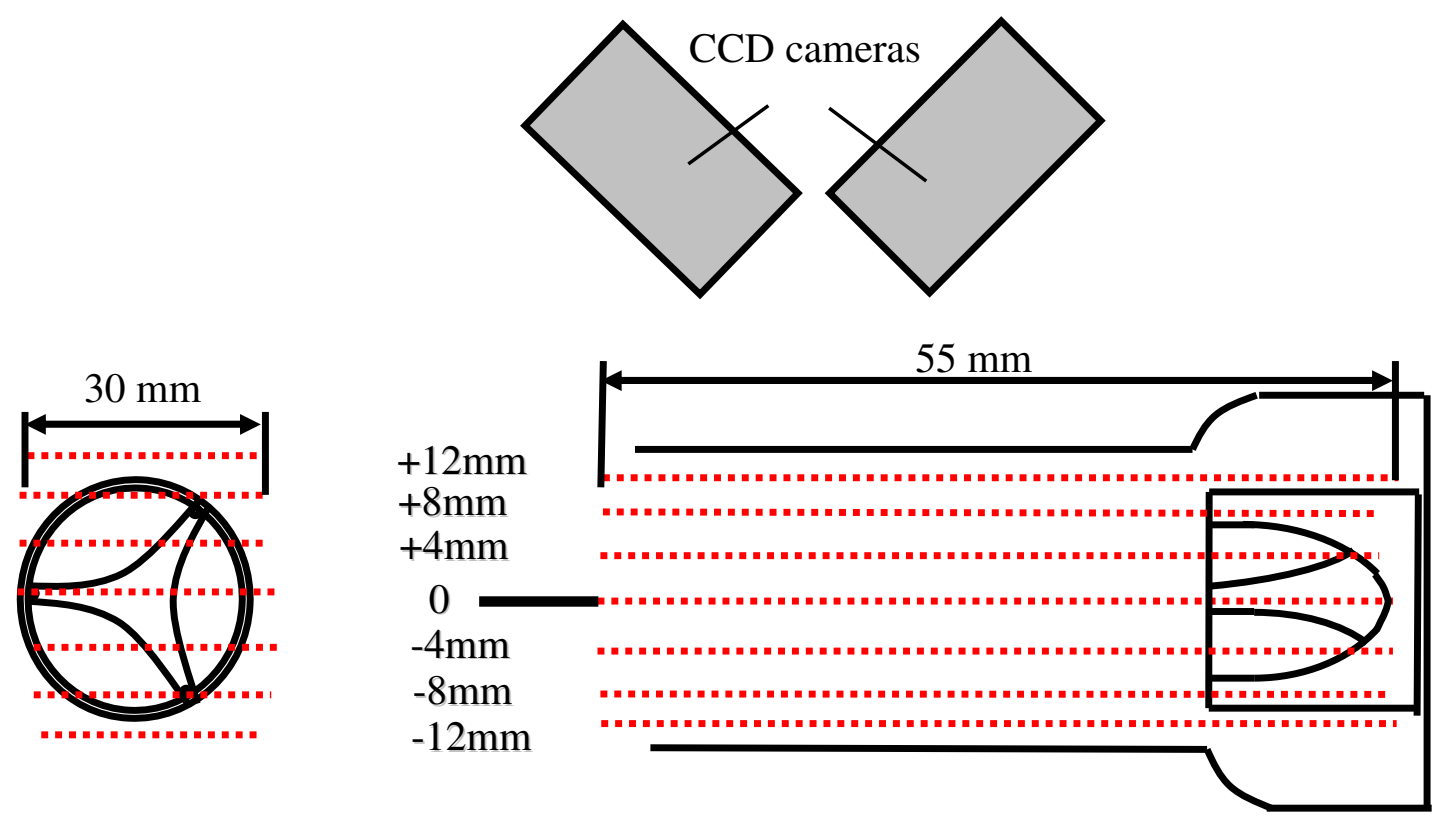

Figure 5-25 Measurement planes for the polymeric valves in the 3D PIV experiments

\subsubsection{Data Acquisition}

A time interval of $20 \mathrm{~ms}$ was advanced for every time bin measurement. This was set in the external signal dialog window in Davis 7.0. The frequency of the trigger signal from the pulse generator was $1.167 \mathrm{~Hz}$, which is equivalent to $70 \mathrm{beat} / \mathrm{min}$ and a period of 860 ms. Table 5-4 tabulates the approximate pulse separation for all the measurement planes in the three Aortech polymeric heart valves. 
Table 5-4 Pulse separation setting for the three polymeric heart valve

\begin{tabular}{|c|c|c|c|c|c|c|c|c|}
\hline & \multirow[b]{2}{*}{ Cardiac phases } & \multicolumn{7}{|c|}{ Pulse separation, $\Delta t$} \\
\hline & & 0 & +4 & +8 & +12 & -4 & -8 & -12 \\
\hline \multirow[t]{2}{*}{ Prototype A } & $\begin{array}{l}\text { Systolic phase } \\
\text { (time bin } 8-20 \text { ) }\end{array}$ & 75 & 75 & 75 & 150 & 100 & 75 & 100 \\
\hline & $\begin{array}{l}\text { Diastolic phase } \\
\text { (time bin 21-7) }\end{array}$ & 75 & 200 & 300 & 500 & 250 & 500 & 400 \\
\hline \multirow[t]{2}{*}{ Prototype B } & $\begin{array}{l}\text { Systolic phase } \\
\text { (time bin } 8-20 \text { ) }\end{array}$ & 100 & 75 & 75 & 100 & 75 & 75 & 75 \\
\hline & $\begin{array}{l}\text { Diastolic phase } \\
\text { (time bin 21-7) }\end{array}$ & 125 & 150 & 150 & 500 & 200 & 150 & 500 \\
\hline \multirow[t]{2}{*}{ Prototype C } & $\begin{array}{l}\text { Systolic phase } \\
\text { (time bin 8-20) }\end{array}$ & 75 & 75 & 75 & 100 & 75 & 75 & 100 \\
\hline & $\begin{array}{l}\text { Diastolic phase } \\
\text { (time bin 21-7) }\end{array}$ & 100 & 200 & 500 & 500 & 150 & 500 & 500 \\
\hline
\end{tabular}

\subsection{Temperature-Dependent Study}

The temperature-dependent study was performed on the $23 \mathrm{~mm}$ prototype A valve using the flow loop setup shown in Figure 4-18. The experiment was conducted at two temperatures: room $\left(21^{\circ}\right)$ and physiological $\left(37^{\circ}\right)$ temperatures. For the high temperature experiment, the glycerin/water solution was placed in the water bath to be heated to the physiological temperature of $37^{\circ}$ (the kinematic viscosity of the solution was $6.25 \mathrm{cSt}$ at room temperature and $3.5 \mathrm{cSt}$ at $37^{\circ}$ ). A thermometer was placed in the reservoir to monitor the temperature in the loop. The following steps describe the experimental procedure: 
1) Flow through the loop was initiated once the desired temperature was reached and stabilized.

2) The compliance and the resistance units were adjusted to obtain physiological flow and pressure waveforms

3) This flow loop was run for 30 to 60 mins until the temperature reached an equilibrium in the loop. At this time, water was added or drain off from the water bath as necessary to regulate the solution temperature in the reservoir.

4) Once the high speed cameras and the lighting were positioned properly, the high speed camera software was triggered to begin the capturing process, which lasted for 1 second

An average of six readings were taken for each temperature, together with the flow and pressure information. The leakage rate for each reading was calculated by integrating the area under the aortic flow curve. A detailed description of the Matlab codes for the processing of the high speed camera images can be found in Appendix A-E.

\section{$\underline{5.6 \text { Surface Topography Studies with Different Heart Valves }}$}

Surface metrology investigations were performed on the surfaces of the SJM $27 \mathrm{~mm}$ Standard mechanical bileaflet and Aortech $23 \mathrm{~mm}$ polymeric heart valves, i.e. on the inside (upstream) and outside (downstream) surfaces of these two valves. Two parameters were used to characterize the surface properties of these valves, Roughness average, $R_{\mathrm{a}}$, and the root-mean-square Roughness, $R_{\mathrm{q}}$. The former is universally recognized and the most used international parameter of roughness. It is the arithmetic 
mean of the departure of the roughness profile from the mean line. The latter describes the rms of $R_{\mathrm{a}}$.

$R_{a}=\frac{1}{L} \int_{0}^{L}|Y(x)| d x$

where $\mathrm{R}_{\mathrm{a}}=$ arithmetic average deviation from the mean line

$L$, the sampling length

$Y$, the ordinate of the profile

$$
R_{q}=\sqrt{\left[\frac{1}{L} \int_{0}^{L}(y(x))^{2} d x\right]}
$$

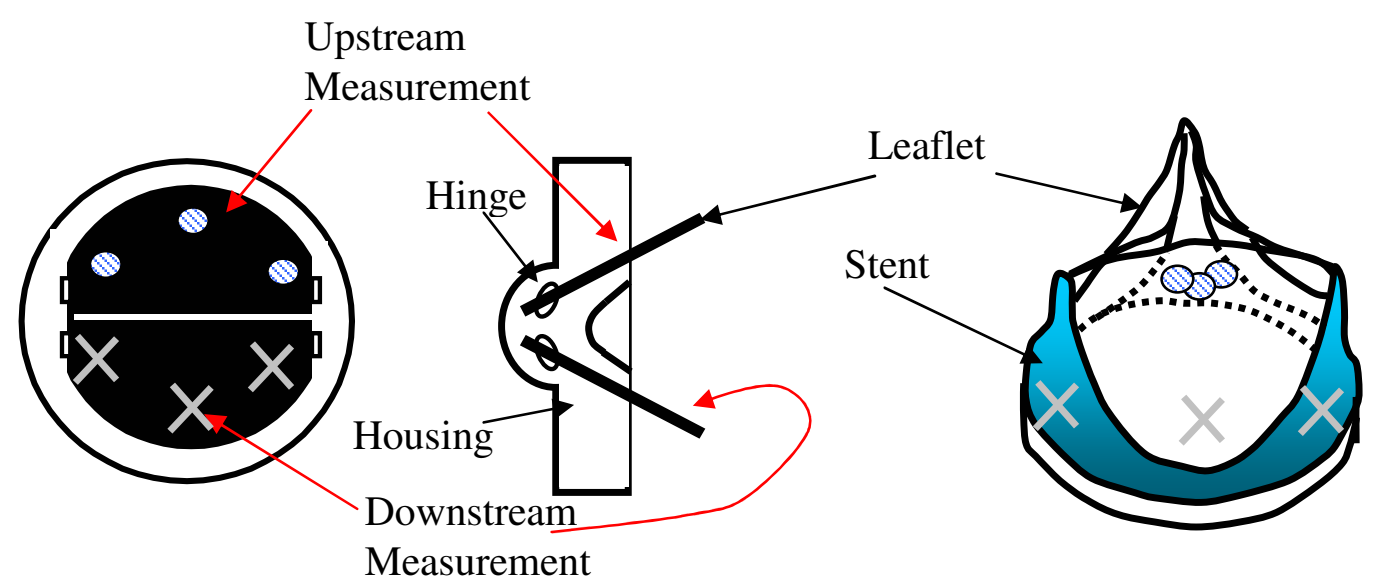

Figure 5-26 Surface measurement locations on prosthetic valves. Surface topography measurements were obtained on both sides (upstream and downstream facing surfaces) of the heart valves leaflets. The measurement location with respect to the upstream an downstream surfaces of the leaflet is specified by the cross (downstream) and the shaded circle (upstream)

In total three outer and three inner surface measurements were taken on the polymeric valve. The cross shows the measurement locations on the outer surface of the polymer 
valve while the shaded circle shows measurement locations at the inner surface of leaflet. The inner surface of the leaflet was folded and held down by a wire during the interferometry measurement. A piece of scotch tape rendered opaque by a black marker pen was affixed to the underside of the leaflet surface to prevent light from passing through since interferometry measurement cannot be made on a transparent surface. Only one of the outer surface measurement locations (cross) required the opaque tape because the remaining two have the opaque stent on the underside. For the bileaflet mechanical valves, three surface measurements were made on each side of the leaflet, i.e. upstream and downstream facing surfaces of the leaflet as shown in Figure 5-26. 


\section{CHAPTER 6: ANALYSIS}

\section{$\underline{6.1}$ Laser Doppler Velocimetry}

Measured 2D and 3D LDV velocities were computed off-line using transit-time weighted averaging to eliminate velocity bias. Velocity bias occurs when a larger or smaller average volume of fluid passes through the probe volume during periods when velocity is faster or slower than the mean [George, 1975]. Consequently, a histogram of individual velocity realizations is biased towards both ends of the velocity range.

Velocities were phase-averaged within $20 \mathrm{~ms}$ (since each cycle of $860 \mathrm{~ms}$ was divided into 43 time intervals, or bins) time windows for subsequent analysis. A total of approximately 21,500 measurements were made at a single location, and an average of approximately 500 measurements within each $20 \mathrm{~ms}$ time window were used for each reduction. The selection of this temporal resolution ensures a valid statistical result in each time window, and at the same time is short enough for the data set within it to be considered quasi-steady. The criterion for the number of measurements in a single time bin was discussed in Brandon Travis's PhD thesis [Travis, 2001c].

The maximum, or principal, values of the Reynolds shear stresses (RSS) were determined according to the formulation found in the literature [Healy, 1997; Hidgon, 1985]. The RSS values are of particular interest because they provide an indication of the magnitude 
of mechanical stresses (due to turbulence) that are experienced by the blood elements, such as red blood cells and platelets, when they travel through the heart valve.

The averaging procedure is summarized as follows. Within each time window, the axial velocity data was decomposed as follows:

$U=\bar{U}+u^{\prime}$

where $U$ is an instantaneous axial velocity measurement, $\bar{U}$ is the mean of all instantaneous $U$ measurements in the time window, and $u^{\prime}$ is the fluctuation about this mean value. The same procedure was performed for the instantaneous circumferential $(V)$ measurements. Then, the total mean velocity magnitude, $\left(\overline{U_{\text {mag }}}\right)$, in the time window was calculated as:

$$
\overline{U_{\text {mag }}}=\sqrt{(\bar{U})^{2}+(\bar{V})^{2}}
$$

With the mean and instantaneous terms both known, each fluctuating term was calculated along with the Reynolds shear stress, $\rho \overline{u^{\prime} v^{\prime}}$, and the two Reynolds normal stresses, $\rho \overline{u^{\prime} u^{\prime}}$ and $\rho \overline{v^{\prime} v^{\prime}}$. However, the peak Reynolds stresses occurring on a fluid element are defined by the principal Reynolds stresses at that location. For a two-component system, the two time-averaged principal normal stresses, $\overline{\sigma_{p 1}}$ and $\overline{\sigma_{p 2}}$, and the maximum time-averaged Reynolds shear stress, $\overline{\tau_{\max }}$, are given by the following equations:

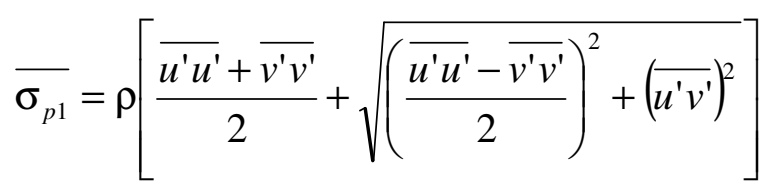




$$
\begin{aligned}
& \overline{\sigma_{p 2}}=\rho\left[\frac{\overline{u^{\prime} u^{\prime}}+\overline{v^{\prime} v^{\prime}}}{2}-\sqrt{\left(\frac{\overline{u^{\prime} u^{\prime}}-\overline{v^{\prime} v^{\prime}}}{2}\right)^{2}+\left(\overline{u^{\prime} v^{\prime}}\right)^{2}}\right] \quad \text { 6-4 } \\
& \overline{\tau_{\max }}=\frac{\overline{\sigma_{p 1}}-\overline{\sigma_{p 2}}}{2} \quad 6-5
\end{aligned}
$$

The Reynolds shear stress calculations obtained from equations 6-1, 6-2 and 6-3 pertain to the 2D LDV measurements. The following section describes the Reynolds shear stress calculations for the 3D LDV measurements. The determination of the three-dimensional stresses is a more involved process. Higdon et al. present the derivation of the equations used to calculate the principal stresses and direction cosines at a given point [Hidgon, 1985]. The three principal normal stresses are calculated as the eigenvalues of the Reynolds stress tensor. This is expressed mathematically as

$$
\left|\begin{array}{ccc}
\bar{\sigma}_{\mathrm{p}}^{\mathrm{n}}-\bar{\tau}_{11}^{\mathrm{n}} & -\bar{\tau}_{12}^{\mathrm{n}} & -\bar{\tau}_{13}^{\mathrm{n}} \\
-\bar{\tau}_{21}^{\mathrm{n}} & \bar{\sigma}_{\mathrm{p}}^{\mathrm{n}}-\bar{\tau}_{22}^{\mathrm{n}} & -\bar{\tau}_{23}^{\mathrm{n}} \\
-\bar{\tau}_{31}^{\mathrm{n}} & -\bar{\tau}_{32}^{\mathrm{n}} & \bar{\sigma}_{\mathrm{p}}^{\mathrm{n}}-\bar{\tau}_{33}^{\mathrm{n}}
\end{array}\right|=0
$$

Evaluating this determinant results in a cubic equation for $\bar{\sigma}_{p}^{n}$ :

$$
\left(\bar{\sigma}_{p}^{n}\right)^{3}-I_{1}^{n}\left(\bar{\sigma}_{p}^{n}\right)^{2}+I_{2}^{n}\left(\bar{\sigma}_{p}^{n}\right)-I_{3}^{n}=0 \quad 6-7
$$

with 


$$
\begin{aligned}
& I_{1}^{n}=\bar{\tau}_{11}^{n}+\bar{\tau}_{22}^{n}+\bar{\tau}_{33}^{n} \\
& I_{2}^{n}=\bar{\tau}_{11}^{n} \bar{\tau}_{22}^{n}+\bar{\tau}_{11}^{n} \bar{\tau}_{33}^{n}+\bar{\tau}_{22}^{n} \bar{\tau}_{33}^{n}-\bar{\tau}_{31}^{n} \bar{\tau}_{13}^{n}-\bar{\tau}_{32}^{n} \bar{\tau}_{23}^{n}-\bar{\tau}_{21}^{n} \bar{\tau}_{12}^{n} \\
& \begin{array}{c}
I_{3}^{n}=\bar{\tau}_{11}^{n} \bar{\tau}_{22}^{n} \bar{\tau}_{33}^{n}+\bar{\tau}_{12}^{n} \bar{\tau}_{23}^{n} \bar{\tau}_{31}^{n}+\bar{\tau}_{13}^{n} \bar{\tau}_{21}^{n} \bar{\tau}_{32}^{n}-\bar{\tau}_{31}^{n} \bar{\tau}_{22}^{n} \bar{\tau}_{13}^{n} \\
\quad \quad-\bar{\tau}_{32}^{n} \bar{\tau}_{23}^{n} \bar{\tau}_{11}^{n}-\bar{\tau}_{33}^{n} \bar{\tau}_{21}^{n} 1 \bar{\tau}_{12}^{n}
\end{array}
\end{aligned}
$$

The roots of equation are the principal normal stresses $\bar{\sigma}_{p 1}^{n}, \bar{\sigma}_{p 2}^{n}$, and $\bar{\sigma}_{p 3}^{n}$. Once the principal normal stresses have been determined, the three potential maximum shear stresses are calculated as

$$
\bar{\sigma}_{p i j}^{n}=\frac{1}{2}\left|\bar{\sigma}_{p i}^{n}-\bar{\sigma}_{p j}^{n}\right|
$$

These values are compared to determine the largest time-averaged shear stress, $\tau^{\mathrm{n}}{ }_{\max }$.

Accurate evaluation of turbulent stresses in pulsatile flows is complicated by cycle-tocycle variation in the mean periodic flow. Studies have shown that the accuracy of the phase averaging method as discussed previously can be easily affected by these variations, and, therefore, turbulent stress values may be overestimated [Liu, 2000; Tiederman, 1988; Travis, 2001c]. The phase averaging analysis is based on the assumption that mean periodic velocity is exactly repeatable from one cycle to the next, while cycle-resolved analysis treats each cycle as an independent part of the flow and assumes that the low frequency mean velocity $\bar{U}(t)$ and high frequency turbulence fluctuation $u^{\prime}(t)$ are not correlated in time and frequency domains. The formulation for cycle-resolved velocity has been well-documented [Tiederman, 1988]. The following describe a similar 
decomposition used by Brandon Travis [Travis, 2001c] to obtain the average velocity within a cycle.

$$
\left\langle\overline{u^{\prime} u^{\prime}}\right\rangle=\frac{1}{m x n} \sum_{i=1}^{m} \sum_{j=1}^{n}\left(u_{i j}^{\prime}-\bar{U}_{o o}\right)^{2}=\frac{1}{m x n} \sum_{i=1}^{m} \sum_{j=1}^{n}\left(\left(\bar{U}_{o j}-\bar{U}_{o o}\right)+\left(u_{i j}^{\prime}-\bar{U}_{o j}\right)\right)^{2}
$$

where $\bar{U}_{o o}=\frac{1}{m x n} \sum_{i=1}^{m} \sum_{j=1}^{n} u_{i j}^{\prime}$ is the mean velocity averaged over all cycle, and

$$
\bar{U}_{i o}=\frac{1}{n} \sum_{j=1}^{n} u_{i j}^{\prime} \text { is the mean velocity averaged over a single cycle. }
$$

After squaring the right hand side, Equation 6.8 becomes:

$$
\begin{aligned}
& \frac{1}{m x n} \sum_{i=1}^{m} \sum_{j=1}^{n}\left(u_{i j}^{\prime}-\bar{U}_{o o}\right)^{2}=\frac{1}{m x n} \sum_{i=1}^{m} \sum_{j=1}^{n}\left(\bar{U}_{i o}-\bar{U}_{o o}\right)^{2}+ \\
& \frac{1}{\operatorname{mxn}} \sum_{i=1}^{m} \sum_{j=1}^{n}\left(u_{i j}^{\prime}-\bar{U}_{i o}\right)^{2}+\frac{2}{m x n} \sum_{i=1}^{m} \sum_{j=1}^{n}\left(u_{i j}^{\prime}-\bar{U}_{i o}\right)\left(\bar{U}_{i o}-\bar{U}_{o o}\right)
\end{aligned}
$$

The last term of Equation 6.9 can be shown to be zero, as:

$$
\frac{1}{n} \sum_{j=1}^{n}\left(u_{i j}^{\prime}-\bar{U}_{i o}\right)=\bar{U}_{i o}-\bar{U}_{i o}=0 \quad 6-12
$$

Equation 6.9 can then be rewritten as:

$$
\frac{1}{m x n} \sum_{i=1}^{m} \sum_{j=1}^{n}\left(u_{i j}^{\prime}-\bar{U}_{o o}\right)^{2}=\frac{1}{m x n} \sum_{i=1}^{m} \sum_{j=1}^{n}\left(U_{i o}-\bar{U}_{o o}\right)^{2}+\frac{1}{m x n} \sum_{i=1}^{m} \sum_{j=1}^{n}\left(u_{i j}^{\prime}-\bar{U}_{i o}\right)^{2}
$$


The first term on the right side of Equation 6-11 represents cyclic variation in velocity measurements, and the second term represents variation in velocity measurements after cyclic variation is removed. A similar decomposition was performed to remove the cyclic component of the cross correlation between two velocity components:

$$
\begin{aligned}
\left\langle\overline{u^{\prime} v^{\prime}}\right\rangle & =\left(\frac{1}{m x n} \sum_{i=1}^{m} \sum_{j=1}^{n}\left(u_{i j}^{\prime}-\bar{U}_{o o}\right)\left(v_{i j}^{\prime}-\bar{V}_{o o}\right) G_{i j}\right)= \\
& \left(\frac{1}{m x n} \sum_{i=1}^{m} \sum_{j=1}^{n}\left(U_{i o}-\bar{U}_{o o}\right)\left(\bar{V}_{i o}-\bar{V}_{o o}\right) \bar{G}_{i o}\right)+\left(\frac{1}{m x n} \sum_{i=1}^{m} \sum_{j=1}^{n}\left(u_{i j}^{\prime}-\bar{U}_{i o}\right)\left(v_{i j}^{\prime}-\bar{V}_{i o}\right) G_{i j}\right)_{\text {filtered }}
\end{aligned}
$$

The Reynolds shear stress calculation is similar to those discussed earlier in this section.

Current studies obtained an average difference of 15.0 percent between the Reynolds shear stresses calculated by phase window averaging and cyclic averaging. The percent different is comparable to those obtained earlier by Brandon Travis [Travis, 2001c]. Therefore, all current LDV results are presented using the phase averaging method. A detail comparison of the two analysis method is presented in Appendix F.

\section{$\underline{6.2}$ Particle Image Velocimetry}

For stereo-PIV a correct calibration is an important prerequisite for measuring accurately the three velocity components. A major source of error in the stereo-PIV is the misalignment between the calibration plate and laser light sheet. However, this can be corrected by a self-calibration procedure, as described in section 5.4.1.1, that computes the cross-correlation and disparity map between images of camera 1 and 2 recorded at the 
same time. This section describes the self-callibration procedure and the data reduction of 3D PIV. A major parts of this section can be obtained from works by Lawson et al and Wieneke, B [Lawson, 1997; Wieneke, 2003]. The calibration procedure is divided into three major steps. The first two steps involved calibration of the system and the third the calculation of the three-dimensional displacement components. Figure 6-1 shows the flow chart for the sequence of steps in the 3D PIV experiments. Two functions were used to estimate the three components of particle displacement, namely the calibration coefficient functions and the displacement functions. The former was obtained from the $1^{\text {st }}$ and $2^{\text {nd }}$ steps, while the latter after performing the $3^{\text {rd }}$ step. 


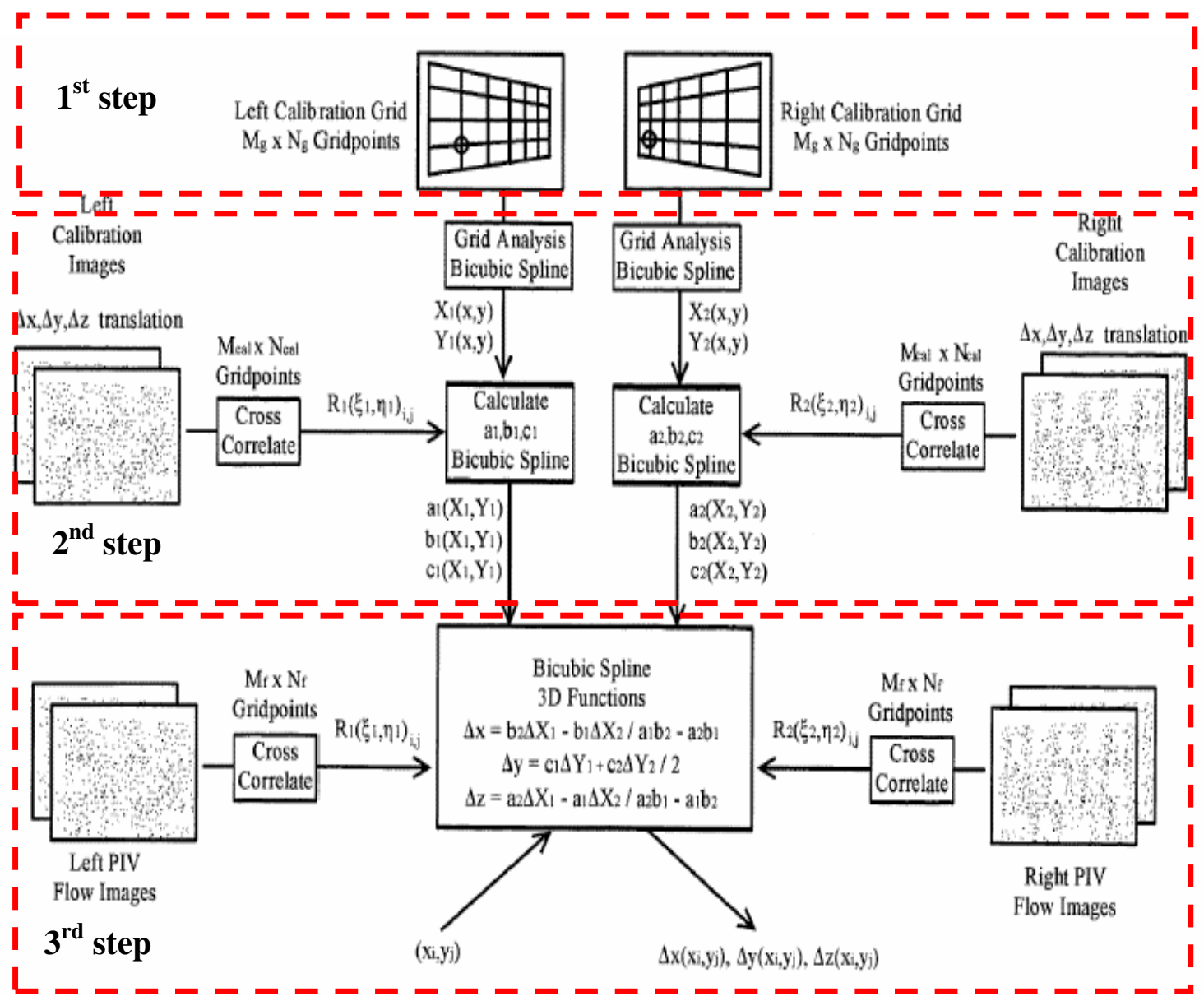

Figure 6-1 The step sequences for processing of vectors in 3D PIV experiments

In the first step, the calibration grid image date from the calibration plate were initially processed into the $X$ and $Y$ pixel coordinate function $X_{1}(x, y), X_{2}(x, y)$ and $Y_{1}(x, y), Y_{2}(x, y)$. These coordinate functions were used to derived the bicubic interpolation functions to be used in all subsequent image processing to reference any image pixel position $X_{1}, Y_{1}, X_{2}$, $Y_{2}$ into an actual space position $x$ and $y$. In the second step, to obtain the calibration coefficient functions, a preliminary pairs of flow images were correlated using cross correlation functions, 


$$
R_{1}(\xi, \eta)_{i, j}=\iint_{-\infty}^{+\infty} G_{1}\left(X_{1}^{c c}, Y_{1}^{c c}\right)_{i, j} \times H_{1}\left(X_{1}^{c c}-\xi, Y_{1}^{c c}-\eta\right)_{i, j} d X_{1}^{c c} d Y_{1}^{c c}
$$

and

$$
R_{2}(\xi, \eta)_{i, j}=\iint_{-\infty}^{+\infty} G_{2}\left(X_{2}^{c c}, Y_{2}^{c c}\right)_{i, j} \times H_{2}\left(X_{2}^{c c}-\xi, Y_{2}^{c c}-\eta\right)_{i, j} d X_{2}^{c c} d Y_{2}^{c c} \quad 6-16
$$

where $G_{1}\left(X_{1}^{c c}, Y_{1}^{c c}\right)_{i, j}, G_{2}\left(X_{2}^{c c}, Y_{2}^{c c}\right)_{i, j}$ and $H_{1}\left(X_{1}^{c c}, Y_{1}^{c c}\right)_{i, j}, H_{2}\left(X_{2}^{c c}, Y_{2}^{c c}\right)_{i, j}$ are the first and second exposure interrogation region images respectively and $R_{1}(\xi, \eta)_{i, j}, R_{2}(\xi, \eta)_{i, j}$ are the correlation outputs at gridepoint $(i, j)$. Before converting these data into the calibration functions, the correlation functions were initially converted to a pixel $\mathrm{mm}^{-1}$ quantity at each grid point $\left(X_{1}^{i}, Y_{1}^{j}\right)$ and $\left(X_{2}^{i}, Y_{2}^{j}\right)$ such that:

$$
\begin{aligned}
& a_{1}\left(X_{1}^{j}, Y_{1}^{j}\right)=\left.\frac{R_{1}(\xi, 0)_{i, j}, j}{\Delta x_{\text {cal }}}\right|^{\substack{i=1, M_{\text {cal }} \\
j=1, N_{\text {cal }}}} \\
& a_{2}\left(X_{2}^{j}, Y_{2}^{j}\right)=\left.\frac{R_{2}(\xi, 0)_{i, j}}{\Delta x_{c a l}}\right|^{\substack{i=1, M_{c a l} \\
j=1, N_{\text {cal }}}} \\
& b_{1}\left(X_{1}^{j}, Y_{1}^{j}\right)=\left.\frac{R_{1}\left(\xi_{, 0}, 0\right)_{i, j}}{\Delta z_{c a l}}\right|^{\substack{i=1, M_{c a l} \\
j=1, N_{c a l}}} \\
& b_{2}\left(X_{2}^{j}, Y_{2}^{j}\right)=\left.\frac{R_{2}\left(\xi_{, 0}\right)_{i, j}}{\Delta z_{\text {cal }}}\right|^{\substack{i=1, N_{\text {cal }} \\
j=1, N_{\text {cal }}}} \\
& c_{1}\left(X_{1}^{j}, Y_{1}^{j}\right)=\left.\frac{R_{1}\left(\xi_{, 0}\right)_{i, j}}{\Delta y_{\text {cal }}}\right|^{\substack{i=1, M_{\text {cal }} \\
j=1, N_{\text {cal }}}}
\end{aligned}
$$




$$
c_{2}\left(X_{2}^{j}, Y_{2}^{j}\right)=\left.\frac{R_{2}(\xi, 0)_{i, j}}{\Delta y_{c a l}}\right|^{\substack{i=1, M_{c a l} \\ j=1, N_{c a l}}}
$$

where $M_{c a l}, N_{c a l}$ is the grid size for the a, b and c coefficients and $\Delta x_{c a l}, \Delta y_{c a l}, \Delta z_{c a l}$ are the PIV preliminary calibration displacements in the $x, y$ and $z$ axes. Finally, these data were fed into a bicubic spline interpolation routine to produce the calibration coefficient functions $a_{1}\left(X_{1}, Y_{1}\right), a_{2}\left(X_{2}, Y_{2}\right), b_{1}\left(X_{1}, Y_{1}\right), b_{2}\left(X_{2}, Y_{2}\right), c_{1}\left(X_{1}, Y_{1}\right), c_{2}\left(X_{2}, Y_{2}\right)$, which permits the estimate of the $x, y, z$ calibration coefficients at any pixel position on the image. In the final step of the procedure, two sets of left and righ flow images were cross correlated and stored as the displacement arrays, $\Delta X_{1}\left(X_{1}^{i}, Y_{1}^{j}\right), \Delta Y_{1}\left(X_{1}^{i}, Y_{1}^{j}\right), \Delta X_{2}\left(X_{2}^{i}, Y_{2}^{j}\right)$, $\Delta X_{2}\left(X_{2}^{i}, Y_{2}^{j}\right)$ where

$$
\begin{aligned}
& \Delta X_{1}\left(X_{1}^{i}, Y_{1}^{j}\right)=\left.R_{1}(\xi, 0)\right|_{i, j} ^{\substack{i=1, M_{f} \\
j=1, N_{f}}} \\
& \Delta Y_{1}\left(X_{1}^{i}, Y_{1}^{j}\right)=\left.R_{1}(\xi, \eta)\right|_{i, j} ^{\substack{i=1, M_{f} \\
j=1, N_{f}}} \\
& \Delta X_{2}\left(X_{2}^{i}, Y_{2}^{j}\right)=\left.R_{2}(\xi, 0)\right|_{\substack{i=1, M_{f} \\
j=1, N_{f}}} ^{\substack{j, j \\
\text {. }}}
\end{aligned}
$$

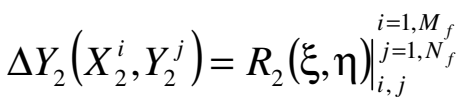

and $M_{f}, N_{f}$ are the flow grid sizes. These data were also fed into a bicubic spline interpolation to yield the displacement functions, which permited an estimate of the particle image displacement $X$ or $Y$ at any pixel position on the left and right images. 
Finally, from these displacement functions and the calibration coefficient functions, an estimate of the three compoenents of particle displacement can be made at any point inisde the calibrated flow area.

$$
\begin{aligned}
& \Delta x\left(x_{i}, y_{j}\right)=\frac{b_{2}\left(X_{2}^{i}, Y_{2}^{i}\right) \Delta X_{1}\left(X_{1}^{i}, Y_{1}^{j}\right)-b_{1}\left(X_{1}^{i}, Y_{1}^{j}\right) \Delta X_{2}\left(X_{2}^{i}, Y_{2}^{j}\right)}{a\left(X_{1}^{i}, Y_{1}^{j}\right) b_{2}\left(X_{2}^{i}, Y_{2}^{j}\right)-a_{2}\left(X_{2}^{i}, Y_{2}^{j}\right) b_{1}\left(X_{1}^{i}, Y_{1}^{j}\right)} \\
& \Delta y\left(x_{i}, y_{j}\right)=\frac{c_{1}\left(X_{1}^{i}, Y_{1}^{i}\right) \Delta Y_{1}\left(X_{1}^{i}, Y_{1}^{j}\right)+c_{2}\left(X_{2}^{i}, Y_{2}^{j}\right) \Delta Y_{2}\left(X_{2}^{i}, Y_{2}^{j}\right)}{2} \\
& \Delta z\left(x_{i}, y_{j}\right)=\frac{a_{2}\left(X_{2}^{i}, Y_{2}^{i}\right) \Delta X_{1}\left(X_{1}^{i}, Y_{1}^{j}\right)-a_{1}\left(X_{1}^{i}, Y_{1}^{j}\right) \Delta X_{2}\left(X_{2}^{i}, Y_{2}^{j}\right)}{a_{2}\left(X_{2}^{i}, Y_{2}^{j}\right) b_{1}\left(X_{1}^{i}, Y_{1}^{j}\right)-a_{1}\left(X_{1}^{i}, Y_{1}^{j}\right) b_{2}\left(X_{2}^{i}, Y_{2}^{j}\right)}
\end{aligned}
$$

where the image coordinates $\left(X_{1}^{i}, Y_{1}^{j}\right)$ and $\left(X_{2}^{i}, Y_{2}^{j}\right)$ are found from the $\mathrm{X}, \mathrm{Y}$ coordinate functions:

$$
\begin{array}{ll}
X_{1}^{i}=X_{1}\left(x_{i}, y_{i}\right) & 6-30 \\
Y_{1}^{i}=Y_{1}\left(x_{i}, y_{i}\right) & 6-31 \\
X_{2}^{i}=X_{2}\left(x_{i}, y_{i}\right) & 6-32 \\
Y_{2}^{i}=Y_{2}\left(x_{i}, y_{i}\right) & 6-33
\end{array}
$$

With known laser pulse separation ( $\Delta t)$ and the particle displacement information ( $\Delta x$, $\Delta y, \Delta z$ ), the velocity of the particles in three components can be obtained by

$$
\begin{aligned}
& u=\frac{\Delta x}{\Delta t} \\
& v=\frac{\Delta y}{\Delta t}
\end{aligned}
$$




$$
w=\frac{\Delta z}{\Delta t}
$$

The algorithm in DaVis makes use of the Central Difference Interrogation (CDI) method for the approximation of flow velocity. This velocity approximation is accurate on the of order of $\Delta \mathrm{t}^{2}$. The equation is written as:

$$
u(t)=\left.\frac{d x}{d t}\right|_{\tau=t}=\frac{x(t+\Delta t / 2)-x(t-\Delta t / 2)}{\Delta t}+\left.\frac{(\Delta t)^{2} d^{3} x}{24 \cdot d t^{3}}\right|_{\tau=t}+\ldots
$$

Studies have shown that the CDI algorithm is much more accurate that the traditional Forward Differencing Interogation (FDI) algorithm. In addition, CDI allows the velocity field to be calculated closer to flow boundaries as well as in a symmetric manner with respect to flow boundaries. Unlike the data reduction in LDV, the PIV velocity data were not binned. This is because the PIV data were obtained at the exact same instant in time in each cardiac cycle. Therefore the mean velocity obtained by averaging 250 cardiac cycles;

$$
\bar{U}=\frac{1}{m} \sum u
$$

where, $m$ is the number of cardiac cycle, $\bar{U}$ the mean velocity averaged over $m$ cycles, and $u$ is the instantaneous velocity. $\bar{U}$ pertain to a single velocity vector in a single 
measurement plane. To calculate the Reynolds shear stress from the 3D PIV results, each instantaneous velocity component is first decomposed into its mean $(\bar{U})$ and fluctuating components $\left(u^{\prime}\right)$ as shown in Equation 6-1. The principle normal stresses and timeaveraged Reynolds shear stresses calculated from Equations 6-3 and 6-4 are then incorporated into Equations 6-5 to 6-9 to obtain the three-dimension maximum Reynolds shear stress values. 


\section{CHAPTER 7: RESULTS}

This chapter presents the results from the individual experiments addressing each of the specific aims listed in Chapter 3. As described in Chapters 4 and 5, static leakage, LDV, and PIV experiments were performed in order to characterize hinge flow dynamics of bileaflet heart valves and the fluid dynamics performance of polymeric heart valves. These results are presented in the following hierarchy:

- Effect of hinge gap width tolerance on hinge flow dynamics (Mitral position)

- Static leakage rate experiments on the three $27 \mathrm{~mm}$ SJM prototype bileaflet heart valves and the CM $23 \mathrm{~mm}$ bileaflet heart valve

$\circ$ 2D LDV measurements in the hinge region of the three $27 \mathrm{~mm}$ bileaflet heart valves

- $\quad$ SJM 27 mm Standard bileaflet heart valve

- $27 \mathrm{~mm}$ prototype low leaker bileaflet heart valve

- $27 \mathrm{~mm}$ prototype high leaker bileaflet heart valve

- Effect of valve design on hinge flow dynamics (Mitral position)

- 2D LDV measurements in the hinge region of the CM $23 \mathrm{~mm}$ bileaflet heart valve

- Flow quantification in polymeric heart valves (Aortic position)

○ Temperature dependent leakage rate studies 
- Static leakage rate experiments at room $\left(20.5^{\circ} \mathrm{C}\right)$ and physiological $\left(37^{\circ} \mathrm{C}\right)$ temperatures

- Pulsatile leakage rate experiment with prototype A, Aortech 23 $\mathrm{mm}$ polymeric heart valve at room $\left(20.5^{\circ} \mathrm{C}\right)$ and physiological $\left(37^{\circ} \mathrm{C}\right)$ temperatures

- High-speed video and marker tracking to quantify three dimensional leaflet kinematics of prototype A, Aortech $23 \mathrm{~mm}$ polymeric heart valve

- 2D LDV measurements on the three prototype Aortech $23 \mathrm{~mm}$ polymeric heart valves

- prototype A, Aortech $23 \mathrm{~mm}$ polymeric heart valve

- prototype $\mathrm{B}$, Aortech $23 \mathrm{~mm}$ polymeric heart valve

- prototype $\mathrm{C}$, Aortech $23 \mathrm{~mm}$ polymeric heart valve

- 3D LDV center-plane measurement on prototype A, Aortech $23 \mathrm{~mm}$ polymeric heart valve

- prototype A, Aortech $23 \mathrm{~mm}$ polymeric heart valve

- 3D PIV measurements on the three prototype Aortech $23 \mathrm{~mm}$ polymeric heart valves

- prototype A, Aortech $23 \mathrm{~mm}$ polymeric heart valve

- prototype $\mathrm{B}$, Aortech $23 \mathrm{~mm}$ polymeric heart valve

- prototype $\mathrm{C}$, Aortech $23 \mathrm{~mm}$ polymeric heart valve

- Leaflet surface topography study

- prototype A, Aortech $23 \mathrm{~mm}$ polymeric heart valve

- SJM 27 mm Standard bileaflet heart valve 


\section{$\underline{7.1 \text { Effect Of Hinge Gap width Tolerance on Hinge Flow Dynamics (Mitral Position) }}$}

Static leakage rate measurements corresponding to a pressure drop of $120 \mathrm{~mm} \mathrm{Hg}$ were performed on each of the three $27 \mathrm{~mm}$ bileaflet heart valves and the CM $23 \mathrm{~mm}$ bileaflet heart valve in order to differentiate each of these valves based on their respective leakage performance. In order to study the effect of hinge gap with tolerance on hinge flow dynamics of the $27 \mathrm{~mm}$ bileaflet heartvalve, 2D LDV experiments were performed to measure the flow field in the hinge region. Details of these experiments are presented in Chapter 4 and 5. Following is a detailed presentation of the results from these experiments.

\subsubsection{Static Leakage Rate Experiments On The Three $27 \mathrm{~mm}$ Bileaflet Heart Valves And The CM 23 mm Bileaflet Heart Valve}

Figure 7-1 shows the leakage rate of the four clear housing valves. The highest and lowest leakage flow rates observed were from the $27 \mathrm{~mm}$ prototype high and low leaker valves, which had average leakage flow rates of $0.490 \mathrm{~L} / \mathrm{min}$ and $0.049 \mathrm{~L} / \mathrm{min}$, respectively. The leakage rate for SJM $27 \mathrm{~mm}$ Standard was between that of the

prototype high and low leakers at $0.351 \mathrm{~L} / \mathrm{min}$. CM $23 \mathrm{~mm}$ had a leakage rate of 0.486 $\mathrm{L} / \mathrm{min}$ and was comparable to that of the $27 \mathrm{~mm}$ prototype high leaker. See Appendix $\mathrm{G}$ for the tabulated leakage rate data for the clear housing valves. 


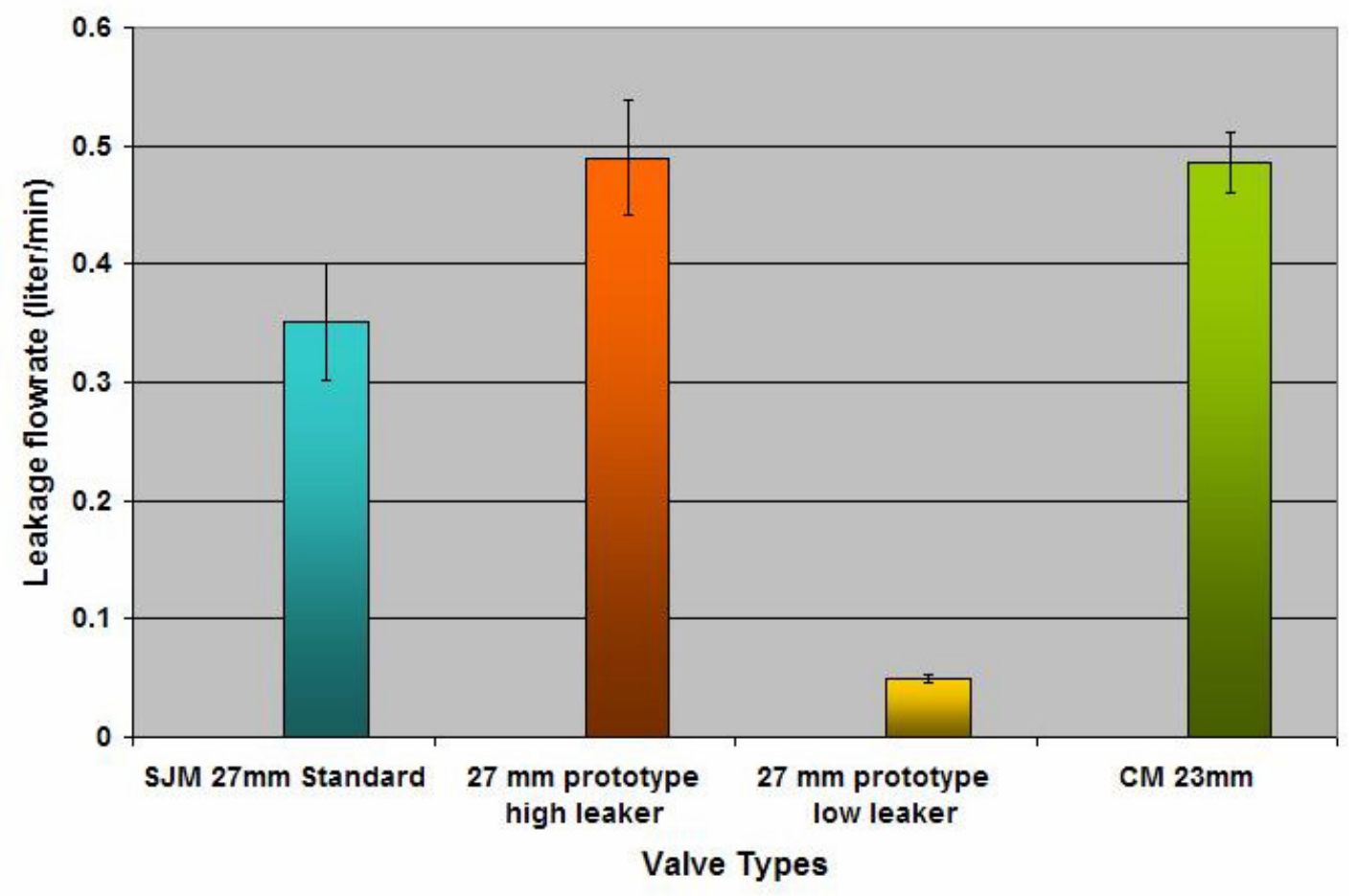

Figure 7-1 The leakage rate of the four clear valve models. The leakage rate of the SJM $\mathrm{mm}$ Standard valve was between that of the $27 \mathrm{~mm}$ prototype high and low leaker valves. The $27 \mathrm{~mm}$ prototype high leaker had similar leakage rate as that of the CM $23 \mathrm{~mm}$ bileaflet valve

\subsubsection{D LDV Mitral Hinge Studies With The Three Prototype $27 \mathrm{~mm}$ Bileaflet}

\section{Clear Housing Valves}

\subsubsection{SJM 27 mm Standard}

The results are presented in the form of color pictures, which illustrate the flow fields and Reynolds shear stress vectors plots at specific instances during the cardiac cycle. The measurement levels are presented in the following sequence: 1) primary flat; 2) levels above the primary flat; 3) levels below the primary flat; and 4) downstream of the thumbnail. For each measurement plane, the description of the flow during systole is presented first followed by that during diastole. The direction of the forward flow is from left to right (from the left atrium to left ventricle, Figure 5-13). All vector plots are color- 
coded by Velocity Magnitude (m/s) and Reynolds shear stress $\left(\right.$ dynes $\left./ \mathrm{cm}^{2}\right)$. Arrows point in the direction of the phase averaged mean velocity vectors and the length of the arrow is proportional to the velocity magnitude. Refer to Appendix $\mathrm{H}$ for the list of velocity magnitude $(\mathrm{m} / \mathrm{s})$ and Reynolds shear stress $\left(\right.$ dynes $\left./ \mathrm{cm}^{2}\right)$ animations. Terminology pertinent to the hinge design is shown in Figure 7-2 for orientation purposes. Figure 7-3 shows the leaflet positions at mid acceleration, peak diastole as well as valve closure and mid systole within the SJM $27 \mathrm{~mm}$ Standard clear valve hinge. These leaflet positions correspond to those in the images presented later. For clarity the leaflets are not shown on the color printouts of the velocity field.

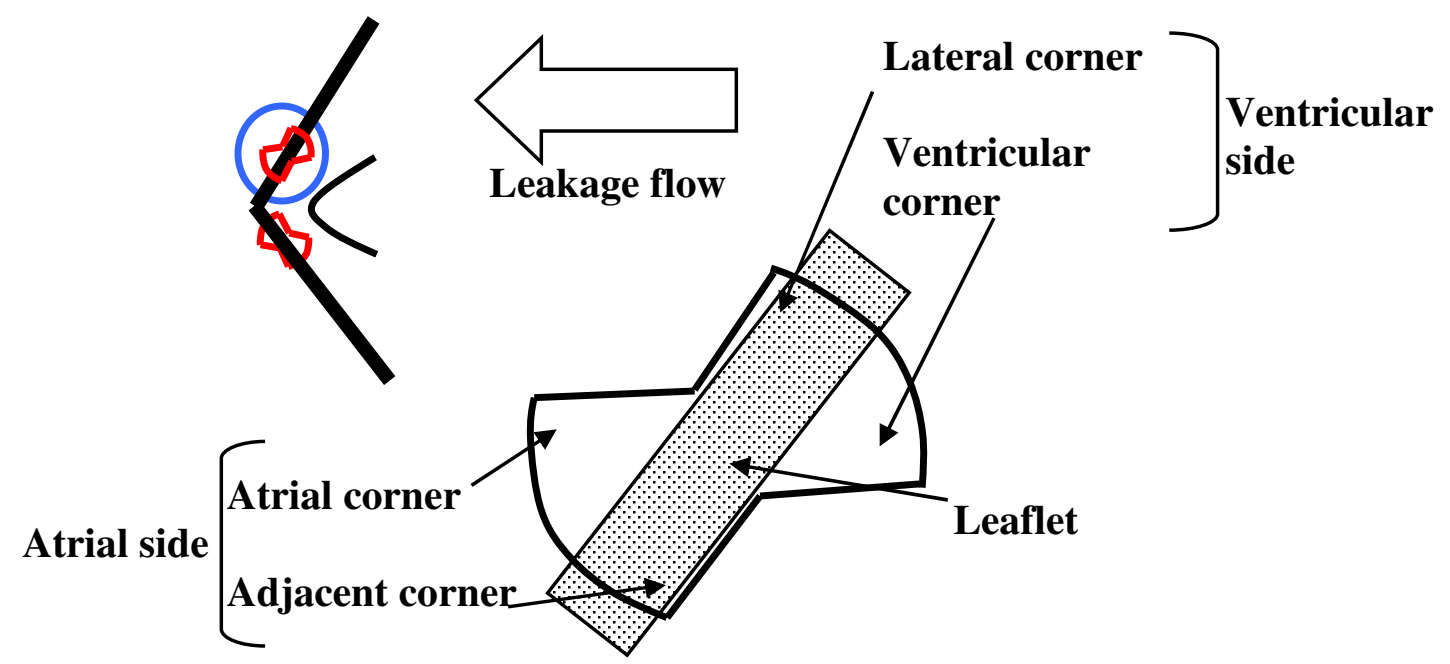

Figure 7-2 Pertinent terminology of SJM $27 \mathrm{~mm}$ Standard clear hinge design 

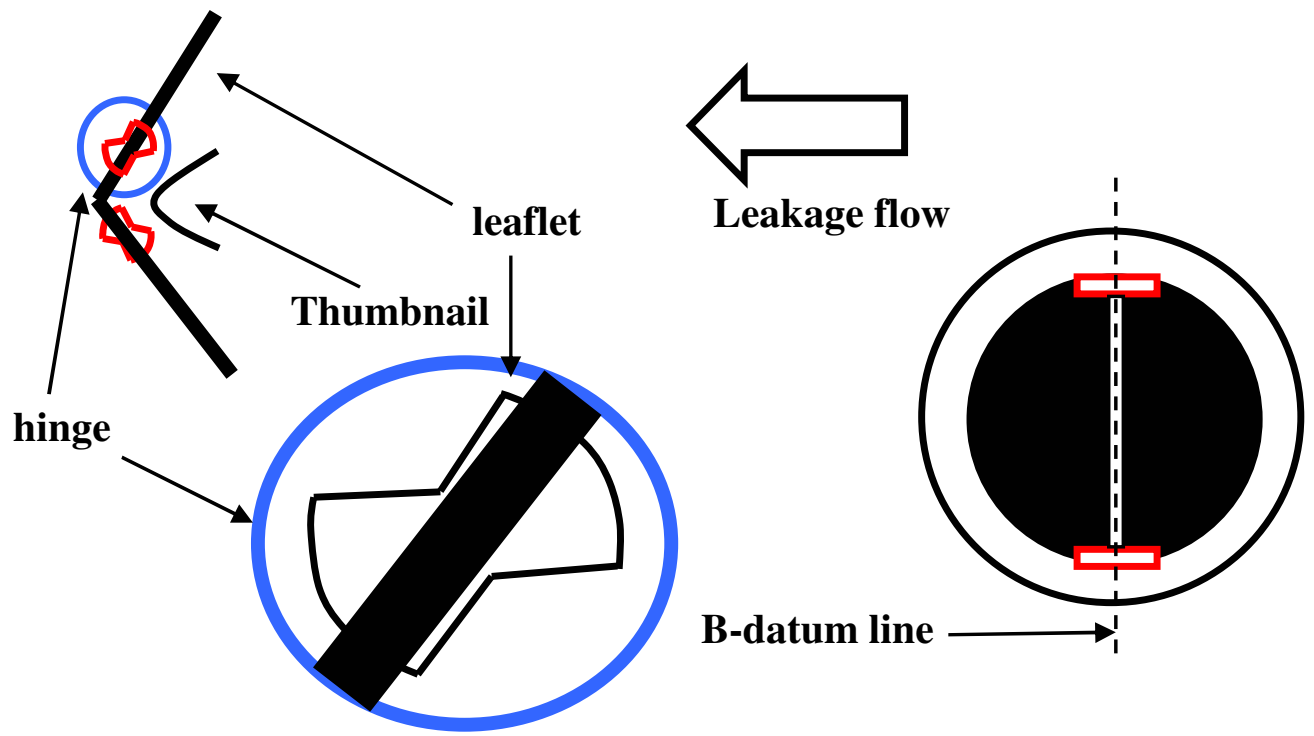

(a)
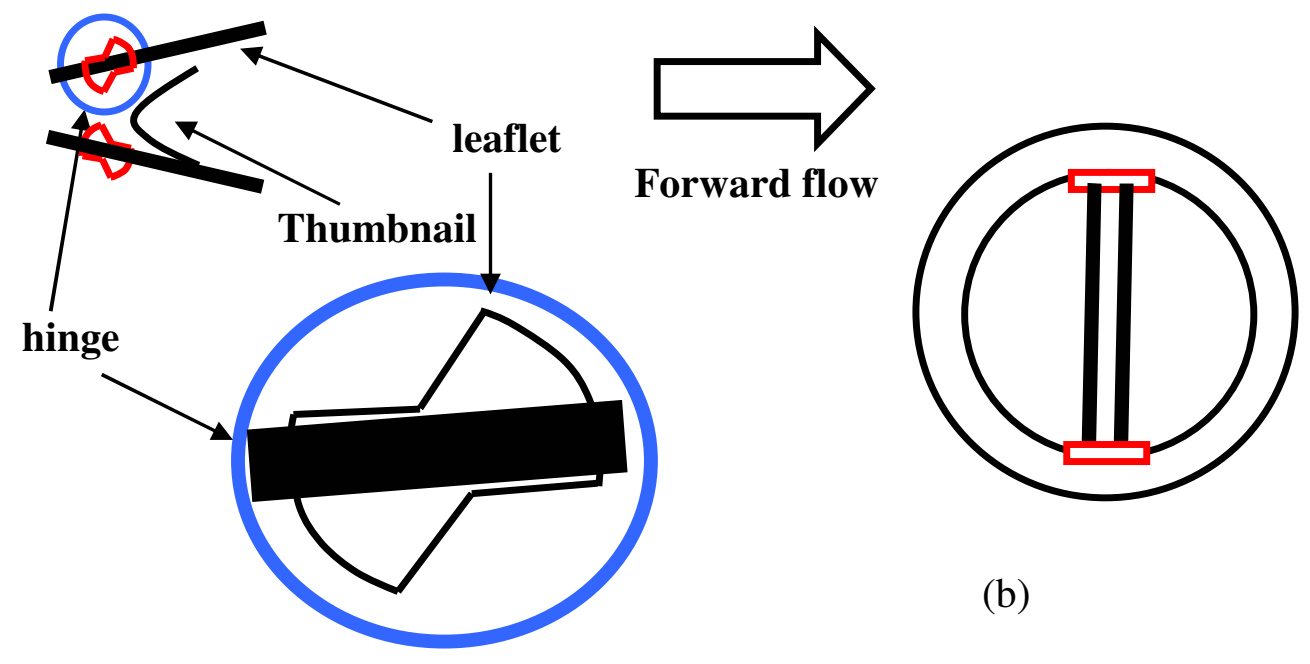

(b)

Figure 7-3 Leaflet position at (a) valve closure, mid systole, and (b) mid acceleration, peak diastole, within the SJM $27 \mathrm{~mm}$ Standard clear housing valve 


\subsection{Flat Level}

Animations reg_flat_vmag.avi and reg_flat_rss.avi show the velocity magnitude $(\mathrm{m} / \mathrm{s})$ and Reynolds shear stress (dynes $/ \mathrm{cm}^{2}$ ) vector plots at the flat level of the SJM $27 \mathrm{~mm}$ Standard clear valve throughout the cardiac cycle, respectively. Refer to Appendix H for the list of animations.

\section{Systole}

Figure 7-4 (time bin 1) shows the hinge flow fields at the onset of the systole. A developing leakage jet of $0.6 \mathrm{~m} / \mathrm{s}$ can be seen in the atrial corner of the hinge geometry. At the ventricular side the fluid $(0.4 \mathrm{~m} / \mathrm{s})$ was drawn towards both the lateral and the adjacent corners of the hinge. A thin band of flow was evident at the lower edge of the hinge geometry, and emerged as a leakage jet at the adjacent corner. Approaching mid systole (time bin 4, Figure 7-5), the leakage jet at the atrial corner reached a peak velocity of about $1.4 \mathrm{~m} / \mathrm{s}$, while the smaller leakage jet at the adjacent corner recorded a maximum velocity of $1.1 \mathrm{~m} / \mathrm{s}$. A region of low flow was also seen at the atrial corner nearer to the hinge wall. It was especially evident during mid systole when the leakage jet was strongest. The calculated shear stress level in the atrial corner jet had a range between 100 to 1,100 dynes $/ \mathrm{cm}^{2}$, while that in the adjacent jet fluctuated between 1,000 5,500 dynes $/ \mathrm{cm}^{2}$ (time bin 4, Figure 7-6). A smaller and much weaker leakage jet seen at the lateral corner had a velocity magnitude of $0.37 \mathrm{~m} / \mathrm{s}$ and a corresponding shear stress level of 300 dynes $/ \mathrm{cm}^{2}$. 


\section{$\underline{\text { Diastole }}$}

The velocity during the acceleration phase (time bin 15 , Figure 7-7) was generally less than $0.05 \mathrm{~m} / \mathrm{s}$ throughout the hinge geometry. This flow field distribution lasted for about $40-60 \mathrm{~ms}$ before the formation of the recirculation zone $(0.5 \mathrm{~m} / \mathrm{s})$ at the adjacent corner (time bin 27, Figure 7-8) at mid-diastole. Reynolds shear stress values were low throughout diastole except in the recirculation region, which had a Reynolds shear stress range of $10-250$ dynes $/ \mathrm{cm}^{2}$. The flow velocity and shear stress levels at the ventricular side of the hinge geometry were $0.05 \mathrm{~m} / \mathrm{s}$ and 10 dynes $/ \mathrm{cm}^{2}$, respectively.

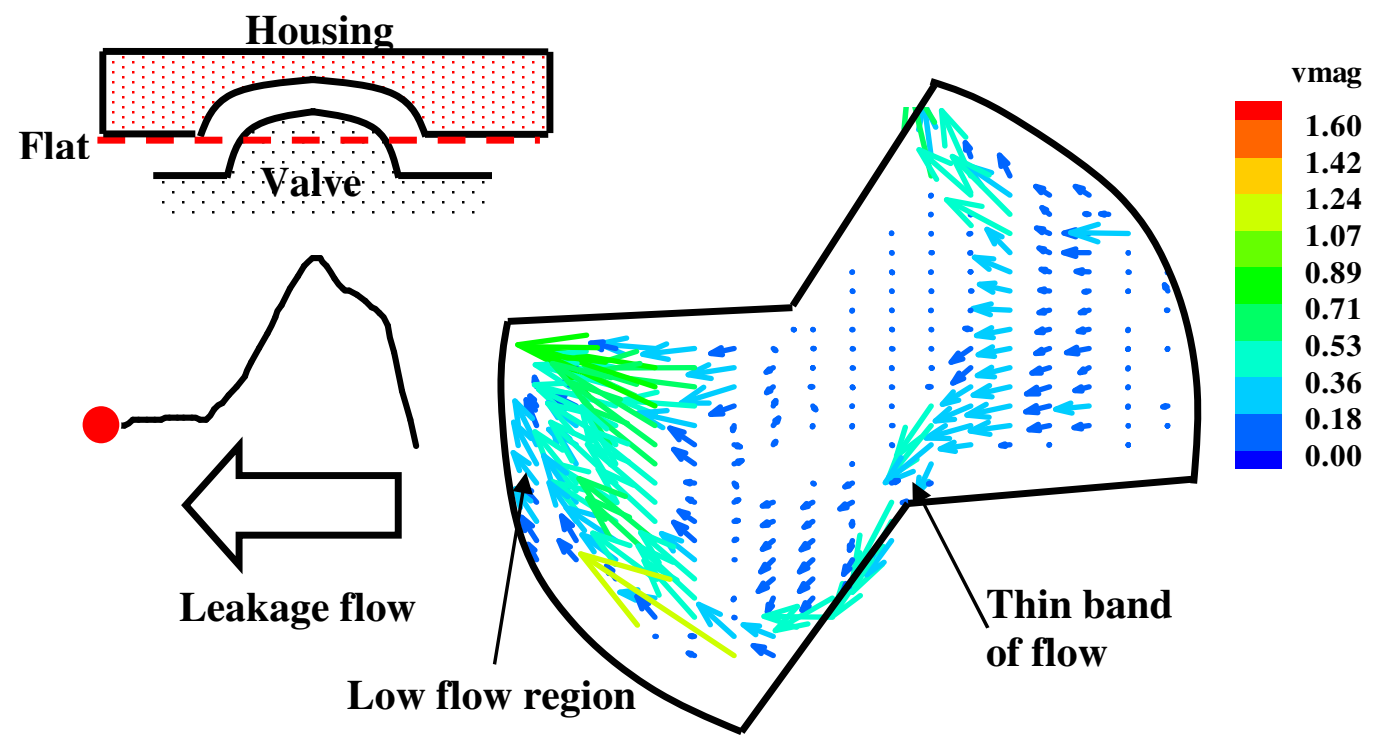

Figure 7-4 SJM $27 \mathrm{~mm}$ Standard mitral hinge flow field at early systole, time bin 1, flat level; leakage jets issuing from atrial, adjacent and lateral corners of the hinge geometry (avi, 680k, reg_flat_vmag.avi) 


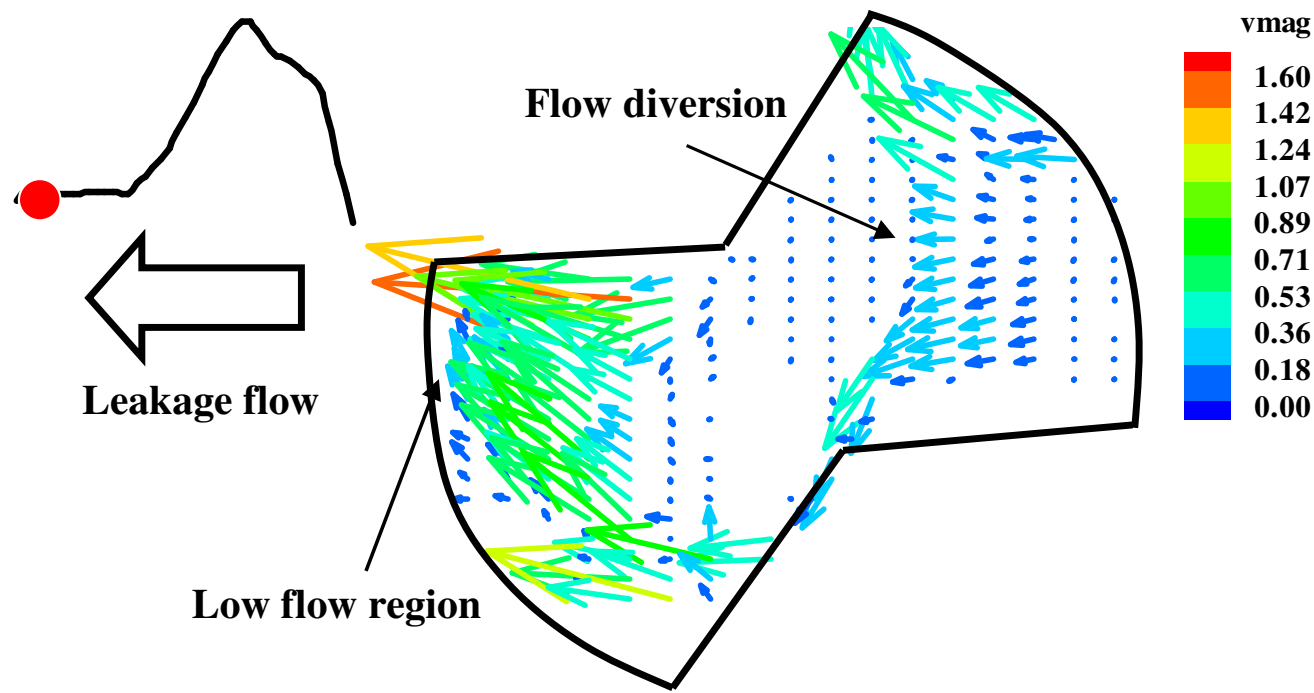

Figure 7-5 SJM $27 \mathrm{~mm}$ Standard mitral hinge flow field approaching mid systole, time bin 4, flat level; the diversion of flow by the leaflet ear at the ventricular side (avi, 680k, reg_flat_vmag.avi)
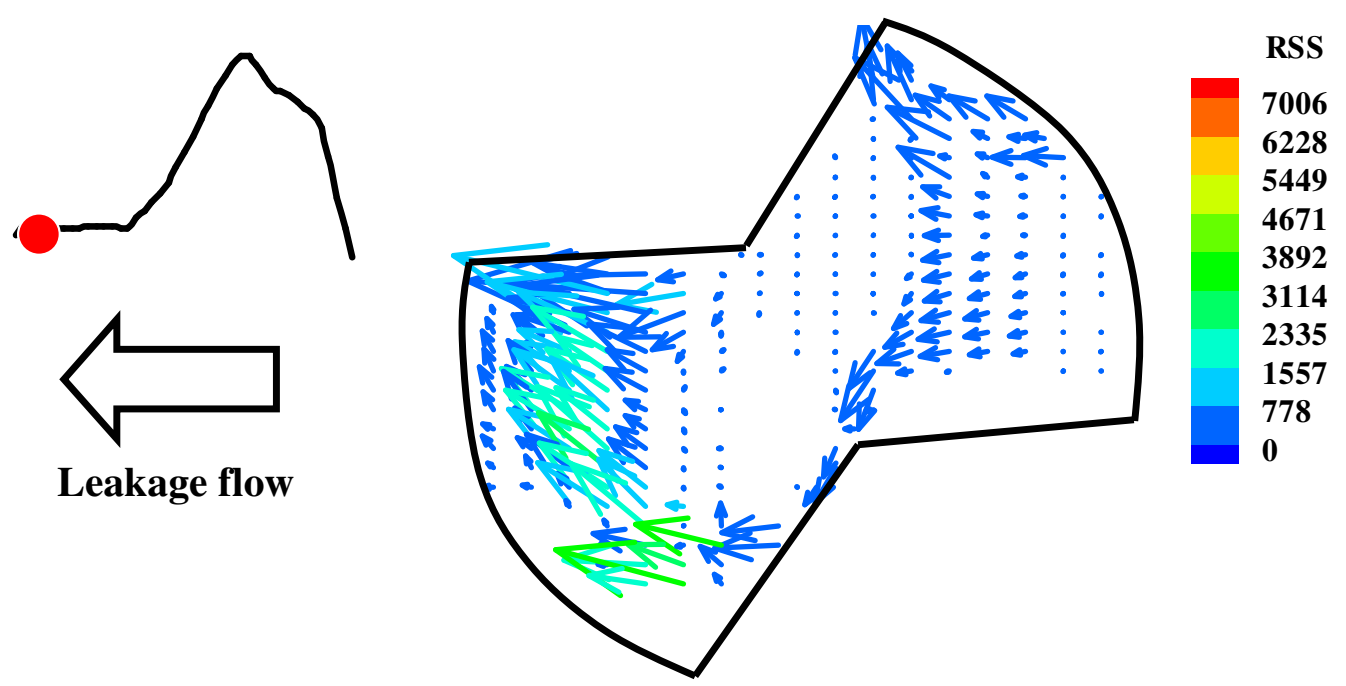

Figure 7-6 Reynolds shear stress distribution of the SJM $27 \mathrm{~mm}$ Standard at time bin 4, flat level; elevated Reynolds shear stress levels observed at the atrial and lateral corners of the hinge geometry (avi, 697k, reg_flat_rss.avi) 

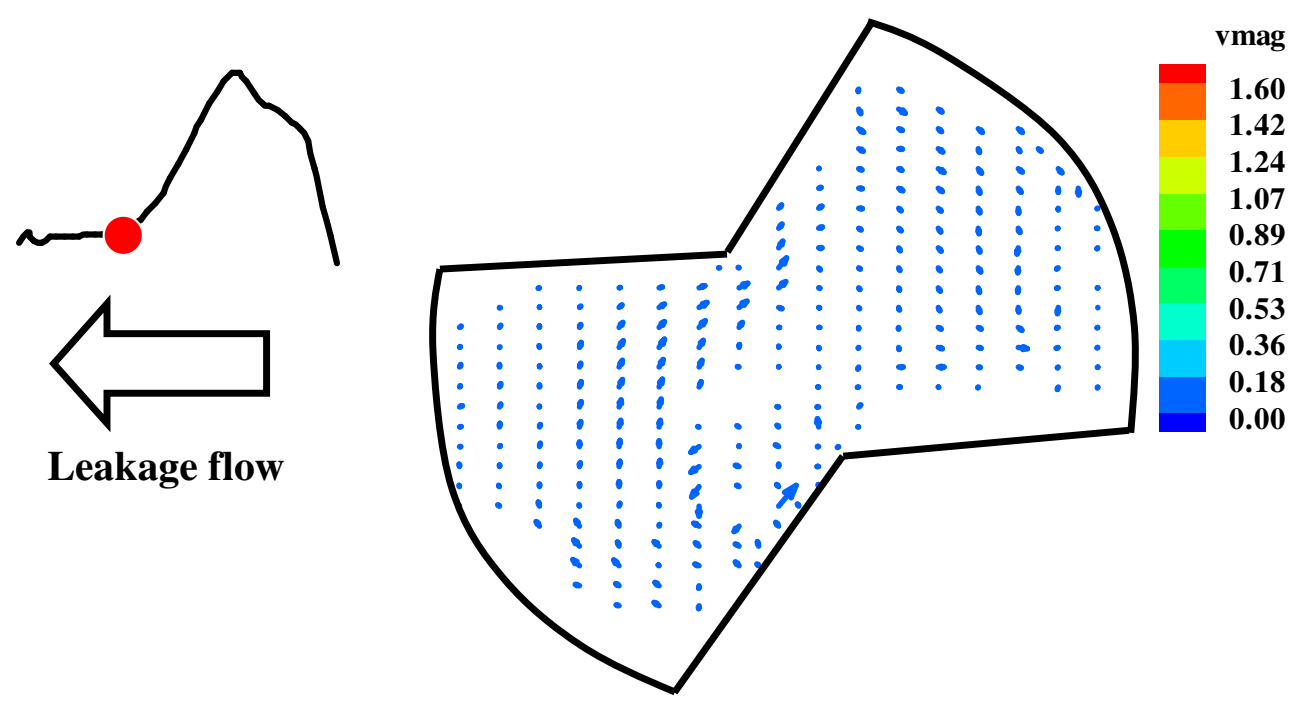

Figure 7-7 SJM $27 \mathrm{~mm}$ Standard mitral hinge flow field at early diastole, time bin 15, flat level; low flow persisted for 40-60 ms at start of diastole (avi, 680k, reg_flat_vmag.avi)
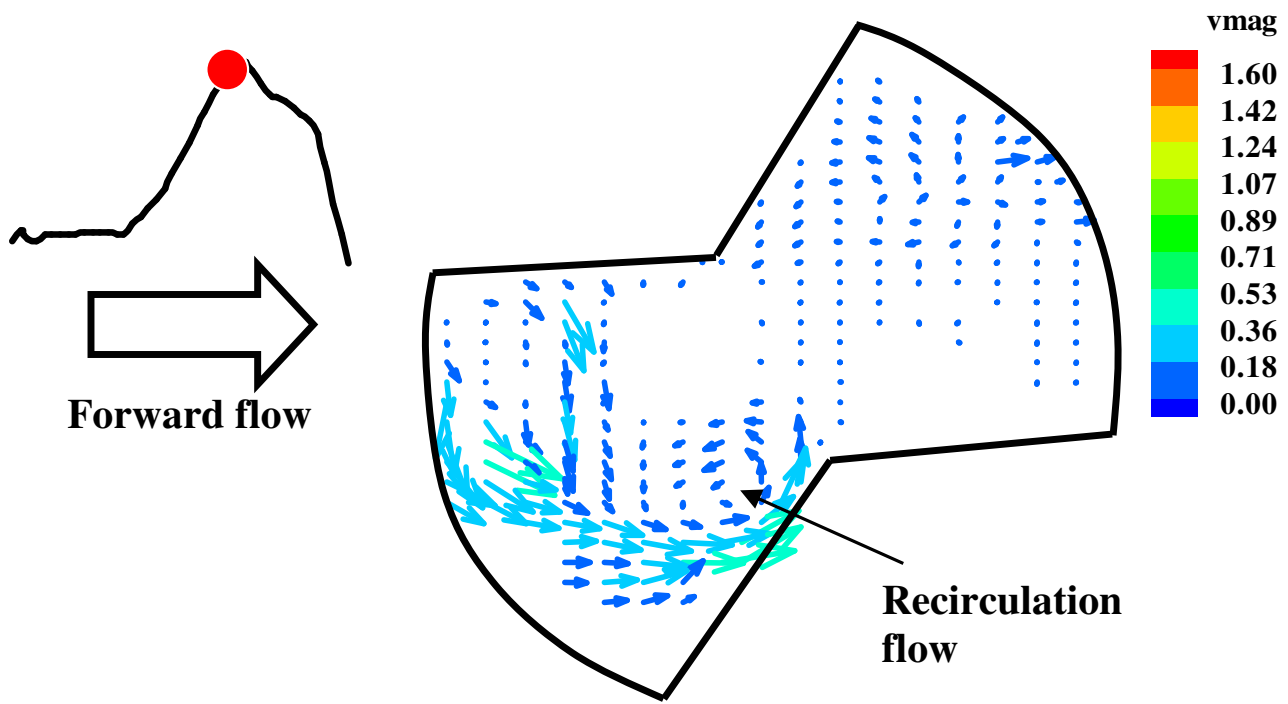

Figure 7-8 SJM $27 \mathrm{~mm}$ Standard mitral hinge flow field at mid diastole, time bin 27, flat level; recirculation flow appeared at the adjacent corner (avi, 680k, reg_flat_vmag.avi) 


\subsection{0 $\mu \mathrm{m}$ Above The Flat Level}

Animations reg_190um_above_flat_vmag.avi and reg_190um_above_flat_rss.avi show the velocity magnitude $(\mathrm{m} / \mathrm{s})$ and Reynolds shear stress $\left(\right.$ dynes $\left./ \mathrm{cm}^{2}\right)$ vector plots at the $190 \mu \mathrm{m}$ above the flat level of the SJM $27 \mathrm{~mm}$ Standard clear valve throughout the cardiac cycle, respectively. Refer to Appendix $\mathrm{H}$ for the list of animations.

\section{Systole}

Figure 7-9 (time bin 1) shows the flow patterns at $190 \mu \mathrm{m}$ above flat level at the beginning of systole. The flow trend was similar to that at the flat level. A large leakage jet with a velocity of approximately $1.3 \mathrm{~m} / \mathrm{s}$ was evident at the atrial corner, while a smaller jet of approximately $1.0 \mathrm{~m} / \mathrm{s}$ was seen at the adjacent corner. The leakage jet at the lateral corner seen at the flat level was not evident here. However, a similar flow diversion as a result of the leaflet ear was observed at the ventricular side, and a narrow band of flow $(0.6 \mathrm{~m} / \mathrm{s})$ between the lower edge of the leaflet ear and the edge of the hinge geometry. The flow velocity at the ventricular corner was low, $0.08 \mathrm{~m} / \mathrm{s}$, and did not change significantly during the systolic phase. Approaching mid systole (time bin 4, Figure 7-10), the leakage jet at the atrial corner reached a maximum velocity of $1.34 \mathrm{~m} / \mathrm{s}$, but abruptly reduced in size and velocity magnitude towards the ends of systole. The leakage jet at the adjacent corner attained a velocity of $0.9 \mathrm{~m} / \mathrm{s}$ at mid systole, and then increased in size (from 5 to 7 measurement locations) at end systole. Higher Reynolds shear stress levels were usually associated with the leakage jets on the atrial side, as well as the thin band of flow between the ear and the edge of the hinge geometry; the calculated Reynolds shear stress for the larger and smaller jets were 1,000 and 3,800 
dynes $/ \mathrm{cm}^{2}$, respectively, while the flow band had a range between 150 and 800 dynes $/ \mathrm{cm}^{2}$. The Reynolds shear stress at other parts of the hinge geometry were typically less than 100 dynes $/ \mathrm{cm}^{2}$. The highest Reynolds shear stress of 3,808 dynes $/ \mathrm{cm}^{2}$ was observed at the leakage jet in the adjacent corner during mid systole. The velocity was low $(<0.01 \mathrm{~m} / \mathrm{s})$ throughout the hinge geometry during the transition between systole and diastole (40-60 ms).

\section{$\underline{\text { Diastole }}$}

Figure 7-11 shows the flow pattern at mid-diastole (time bin 27) with the formation of a recirculation zone visible at the adjacent corner and a streak of flow at the atrial corner. Both regions recorded velocity magnitudes in the range between $0.09-0.16 \mathrm{~m} / \mathrm{s}$, and corresponding Reynolds shear stress values of less than 10 dynes $/ \mathrm{cm}^{2}$. The remaining region of the hinge had a low velocity of $0.02 \mathrm{~m} / \mathrm{s}$ and a corresponding Reynolds shear stress of less than $10 \mathrm{dynes} / \mathrm{cm}^{2}$, which persisted till the start of the systole. 


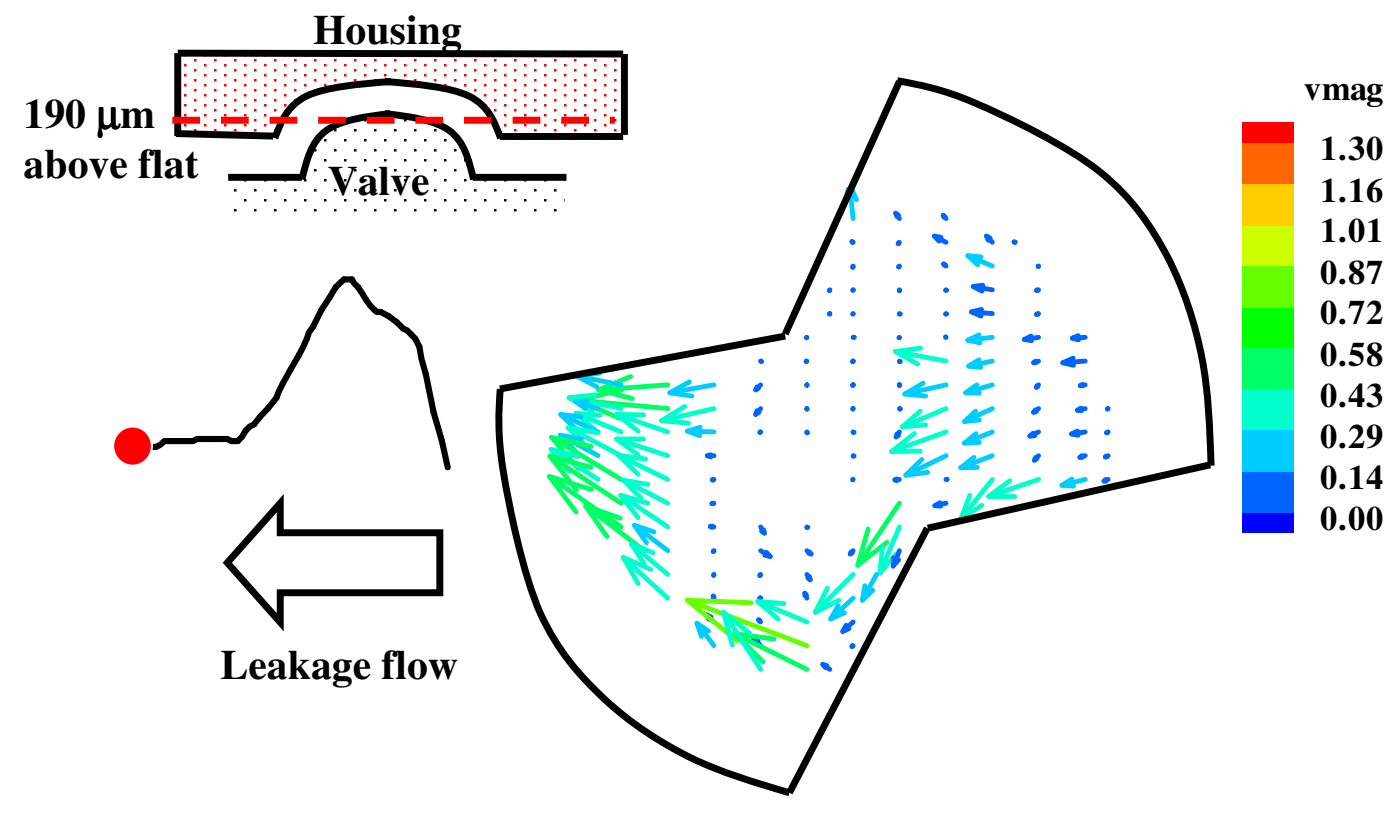

Figure 7-9 SJM $27 \mathrm{~mm}$ Standard mitral hinge flow field at early systole, time bin 1, 190 $\mu \mathrm{m}$ above flat level; leakage flow between the leaflet ear and the hinge wall (avi, 656k, reg_190um_above_flat_vmag.avi)
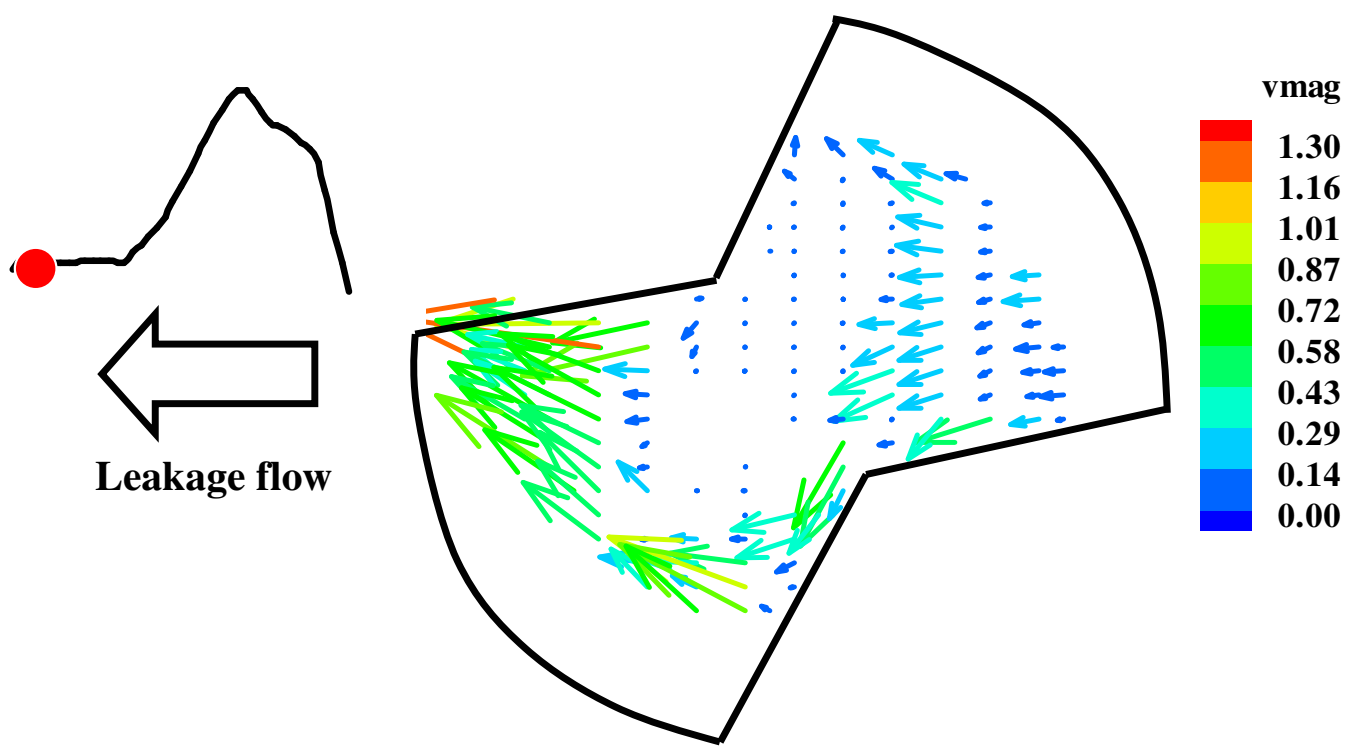

Figure 7-10 SJM $27 \mathrm{~mm}$ Standard mitral hinge flow field approaching mid systole, time bin 4, $190 \mu \mathrm{m}$ above flat level; strong leakage flow at the atrial corner (avi, 656k, reg_190um_above_flat_vmag.avi) 


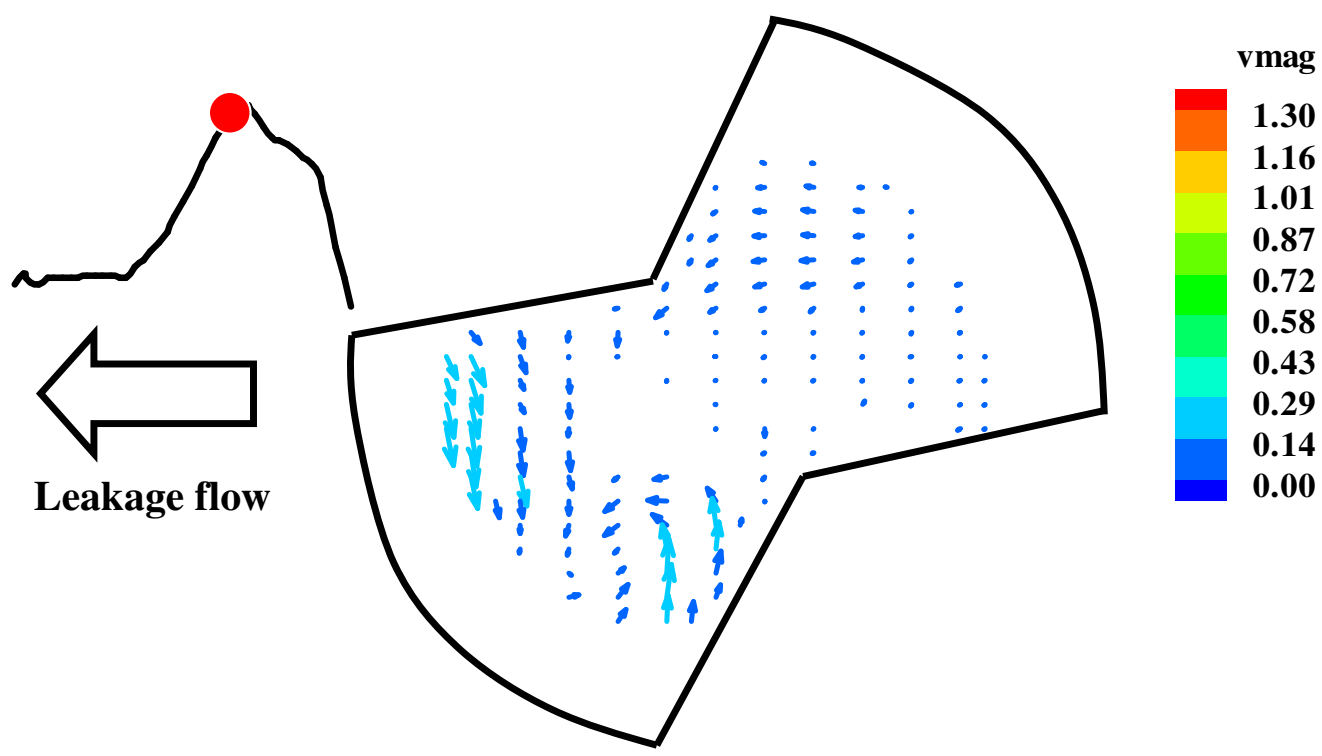

Figure 7-11 SJM $27 \mathrm{~mm}$ Standard mitral hinge flow field at mid diastole, time bin 27, $190 \mu \mathrm{m}$ above flat level; recirculation flow visible at the adjacent corner (avi, 656k, reg_190um_above_flat_vmag.avi)

\subsubsection{3 $390 \mu \mathrm{m}$ Above The Flat Level}

Animations reg_390um_above_flat_vmag.avi and reg_390um_above_flat_rss.avi show the velocity magnitude $(\mathrm{m} / \mathrm{s})$ and Reynolds shear stress $\left(\right.$ dynes $/ \mathrm{cm}^{2}$ ) vector plots at the $390 \mu \mathrm{m}$ above the flat level of the SJM $27 \mathrm{~mm}$ Standard clear valve throughout the cardiac cycle, respectively. Refer to Appendix H for the list of animations.

\section{Systole}

Figure 7-12 (time bin 2) shows the flow patterns at the beginning of the systole. The velocity magnitudes were low in the range between 0.02 and $0.7 \mathrm{~m} / \mathrm{s}$. A maximum leakage velocity of $0.76 \mathrm{~m} / \mathrm{s}$ and a corresponding Reynolds shear stress value of 1,261 dyne $/ \mathrm{cm}^{2}$ was recorded at the ventricular side during early systole. High leakage flow $(>0.14 \mathrm{~m} / \mathrm{s})$ were observed in locations along the ventricular edge of the hinge during mid 
systole, and persisted to end of systole. The Reynolds shear stress during systolic phase was generally less than 5 dynes $/ \mathrm{cm}^{2}$.

\section{Diastole}

The flow velocity during diastole (not shown) was typically less than $0.12 \mathrm{~m} / \mathrm{s}$, but a band of high velocity flow $(0.14 \mathrm{~m} / \mathrm{s})$ was observed along the atrial edge of the hinge wall. The calculated Reynolds shear stress values during diastole were less than 5 dynes $/ \mathrm{cm}^{2}$.

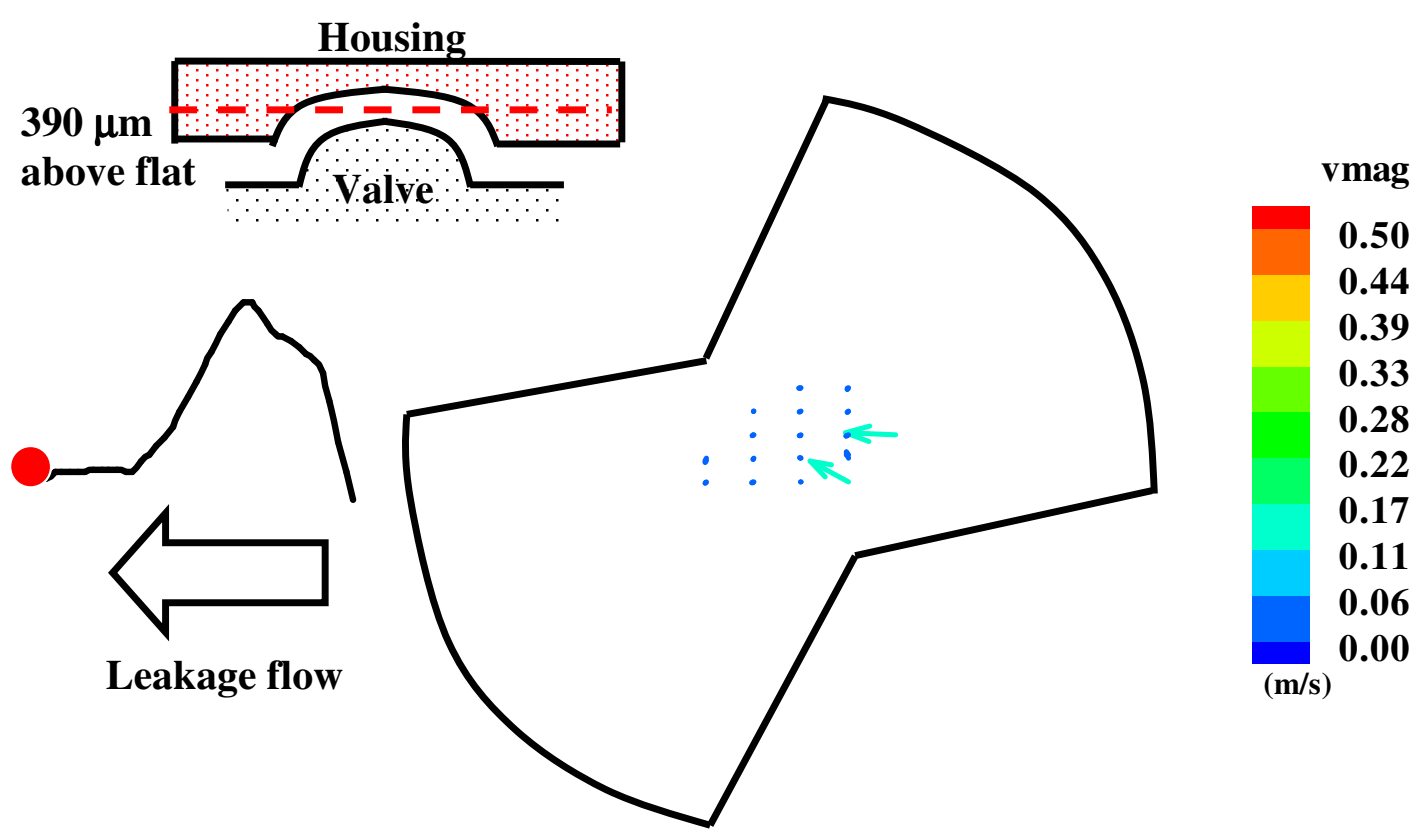

Figure 7-12 SJM $27 \mathrm{~mm}$ Standard mitral hinge flow field at early systole, time bin 2, 390 $\mu \mathrm{m}$ above flat level; low flow was evident throughout the cardiac cycle (avi, 603k, reg_390um_above_flat_vmag.avi) 


\subsection{5 $\mu \mathrm{m}$ Above Flat Level}

Animations reg_585um_above_flat_vmag.avi and reg_585um_above_flat_rss.avi show the velocity magnitude $(\mathrm{m} / \mathrm{s})$ and Reynolds shear stress $\left(\right.$ dynes $\left./ \mathrm{cm}^{2}\right)$ vector plots at the $585 \mu \mathrm{m}$ above the flat level of the SJM $27 \mathrm{~mm}$ Standard clear valve throughout the cardiac cycle, respectively. Refer to Appendix $\mathrm{H}$ for the list of animations.

Velocity magnitudes of less than $0.03 \mathrm{~m} / \mathrm{s}$ were observed at this level during both systole and diastole with low Reynolds stress levels of less than 10 dynes $/ \mathrm{cm}^{2}$. No discernable pulsatility of the velocity vectors was evident at this measurement level. Refer to reg_585um_above_flat_vmag.avi and reg_585um_above_flat_rss.avi for animations (Appendix H).

\subsection{0 $\mu \mathrm{m}$ Below The Flat Level}

Animations reg_500um_below_flat_vmag.avi and reg_500um_below_flat_rss.avi show the velocity magnitude $(\mathrm{m} / \mathrm{s})$ and Reynolds shear stress $\left(\right.$ dynes $\left./ \mathrm{cm}^{2}\right)$ vector plots at the $500 \mu \mathrm{m}$ below the flat level of the SJM $27 \mathrm{~mm}$ Standard clear valve throughout the cardiac cycle, respectively. Refer to Appendix $\mathrm{H}$ for the list of animations.

\section{Systole}

Figure 7-13 (time bin 1) shows the flow distribution at $500 \mu \mathrm{m}$ below the flat at the start of the systolic phase. A retrograde flow of $0.5 \mathrm{~m} / \mathrm{s}$ was seen between the two closing leaflets. A region of low flow was observed to be drawn towards the central flow as the leaflets close. Flow unsteadiness arises at valve closure in the vicinity of the closed 
leaflets. Three leakage jets became apparent at this time; two leakage jets emanating from the hinge pocket and a third lower velocity jet in the center region through the gap between the two leaflets, i.e. the B-datum line (see Figure 7-3). The leakage jets arising from the hinge pockets gain in strength towards mid systole and reach a peak velocity of approximately $2.1 \mathrm{~m} / \mathrm{s}$, while the retrograde flow at the center region attained a velocity of about $0.2 \mathrm{~m} / \mathrm{s}$ (time bin 4, Figure 7-14). On the other hand, the flow velocity outside these leakage jet was low at $0.06 \mathrm{~m} / \mathrm{s}$. Maximum Reynolds shear stress values of 2,590 dynes $/ \mathrm{cm}^{2}$ were observed at valve closure on the ventricular side of the hinge mechanism (time bin 4, Figure 7-15). Leakage jets emanating from the hinge pocket had Reynolds shear stress values between 1,000-2,000 dynes $/ \mathrm{cm}^{2}$. High Reynolds shear stress values were also observed in the central leakage jet in the range of $500-1,000$ dynes $/ \mathrm{cm}^{2}$ but decreased to about 300 to 800 dynes $/ \mathrm{cm}^{2}$ at mid systole.

\section{$\underline{\text { Diastole }}$}

Flow at the start of the diastole was characterized by a strong central jet of $0.5 \mathrm{~m} / \mathrm{s}$ with layer of shear flow $(0.13 \mathrm{~m} / \mathrm{s})$ between edge of the leaflet and the central jet. The layer of shear flow persisted throughout the diastole and was most apparent at peak diastole, where the velocity magnitude in the central jet reached $1.25 \mathrm{~m} / \mathrm{s}$. The lateral jets at middiastole recorded a velocity magnitude of $0.9 \mathrm{~m} / \mathrm{s}$ (time bin 27, Figure 7-16). Reynolds shear stress levels of about 100 dynes $/ \mathrm{cm}^{2}$ were observed along the layer of shear flow, but maximum Reynolds shear stress value of 1,000 dynes $/ \mathrm{cm}^{2}$ was seen inside the central forward flow jet at mid-diastole (time bin 27, Figure 7-17). 

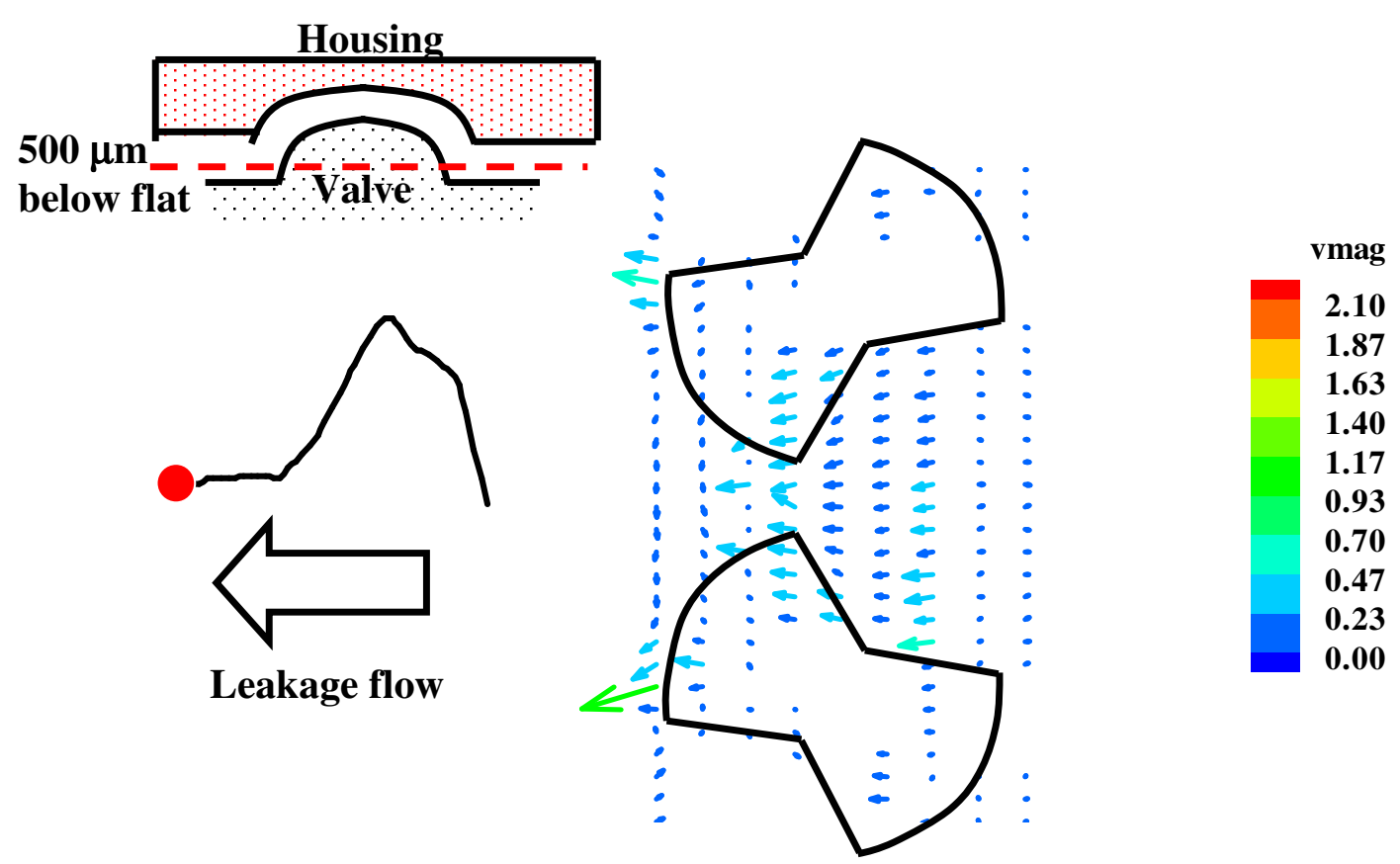

Figure 7-13 SJM $27 \mathrm{~mm}$ Standard mitral hinge flow field at early systole, time bin 1, 500 $\mu \mathrm{m}$ below flat level; low leakage flow evident at the hinge pockets and the between the closing leaflets (avi, 702k, reg_500um_below_flat_vmag.avi)

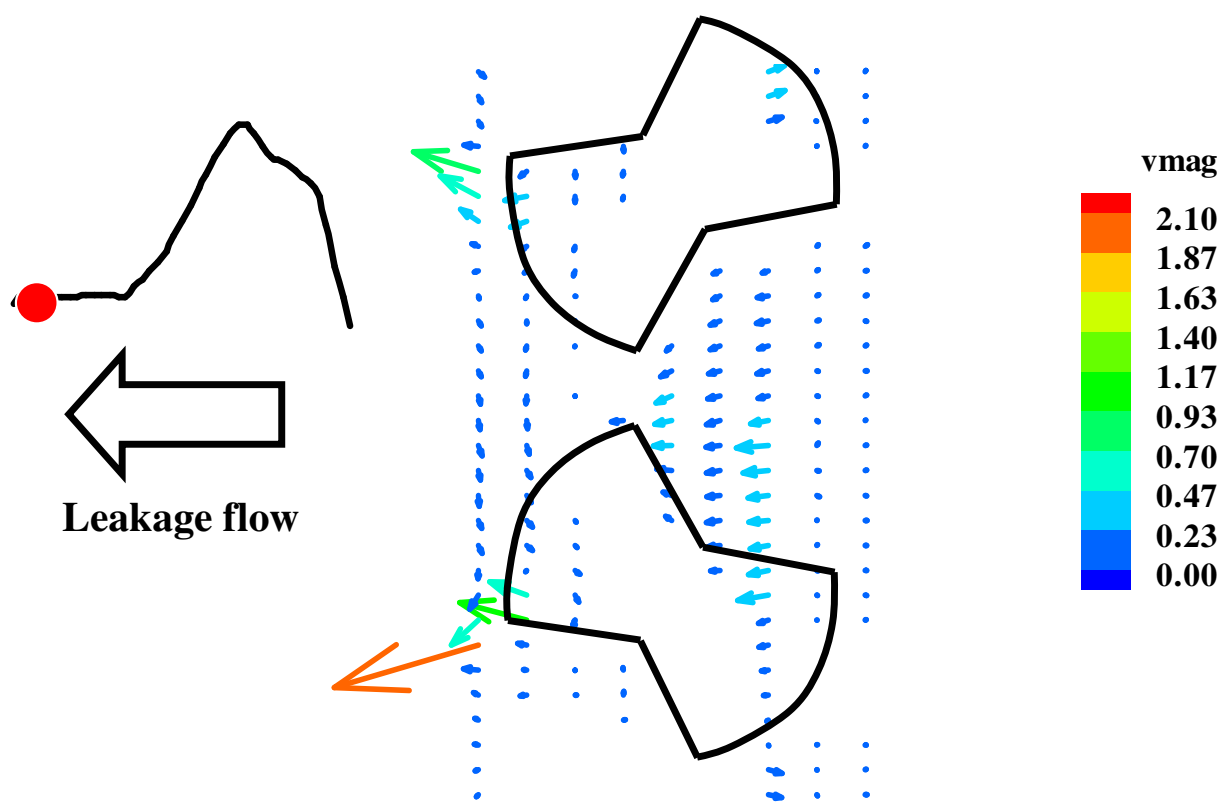

Figure 7-14 SJM $27 \mathrm{~mm}$ Standard mitral hinge flow field approaching mid systole, time bin 4, $500 \mu \mathrm{m}$ below flat level; the leakage jets emanating from the hinge regions (avi, 702k, reg_500um_below_flat_vmag.avi) 


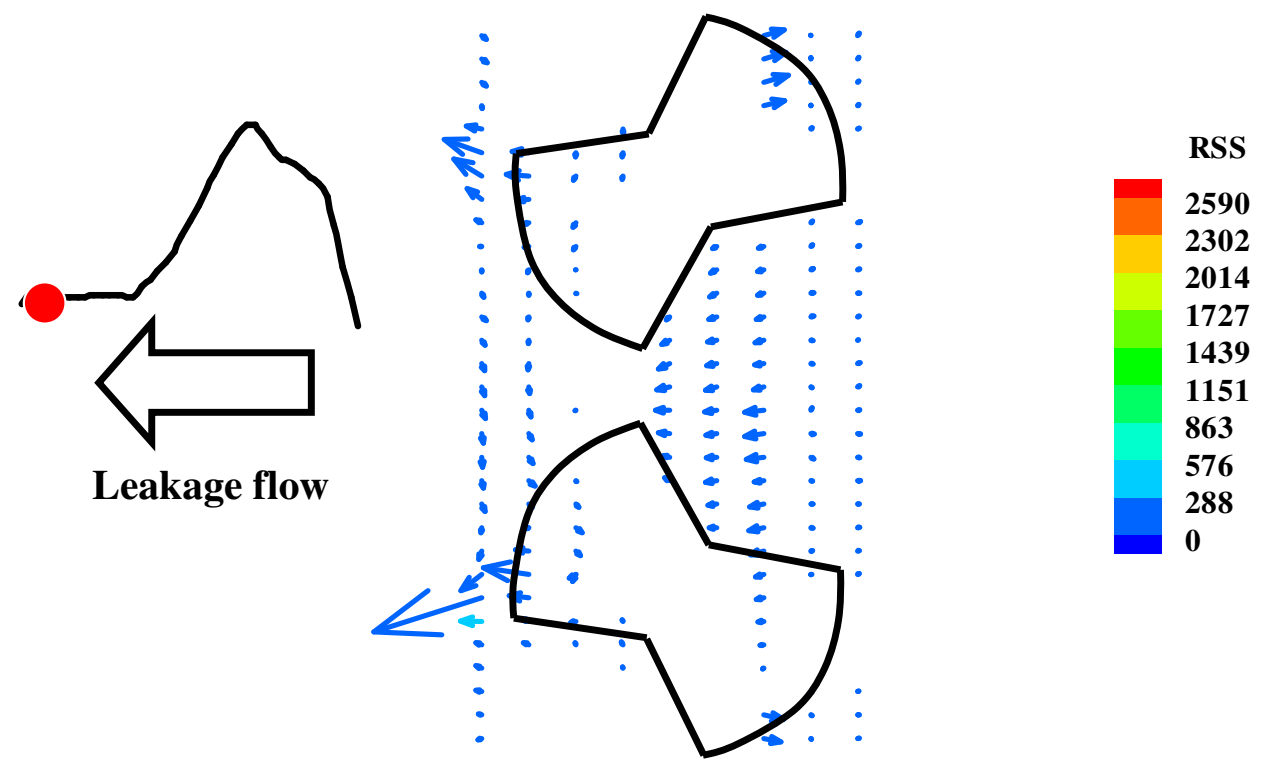

Figure 7-15 Reynolds shear stress distribution of the SJM $27 \mathrm{~mm}$ Standard at time bin 4, $500 \mu \mathrm{m}$ above flat level; elevated Reynolds shear stress levels coincided with regions of the leakage jets (avi, 708k, reg_500um_below_flat_rss.avi)
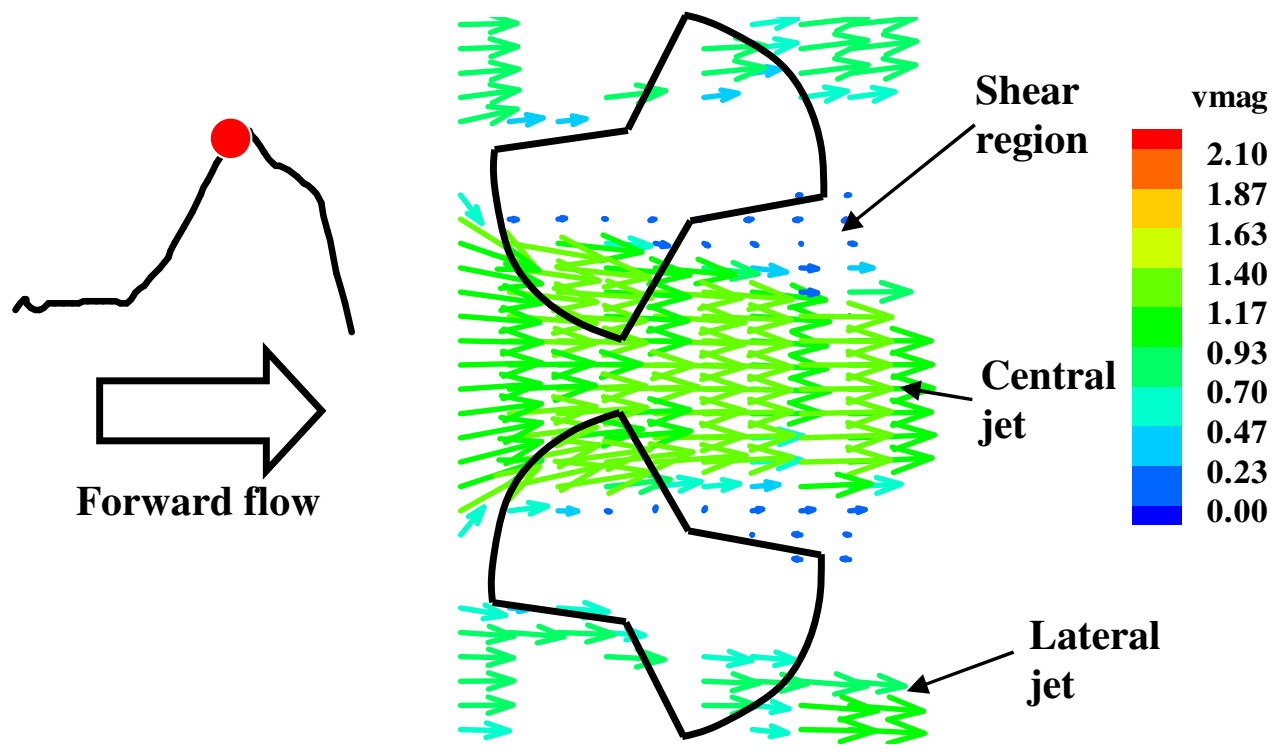

Figure 7-16 SJM $27 \mathrm{~mm}$ Standard mitral hinge flow field at mid diastole, time bin 27, $500 \mu \mathrm{m}$ below flat level; forward flow was characterized by one central jet and two lateral jets (avi, 702k, reg_500um_below_flat_vmag.avi) 

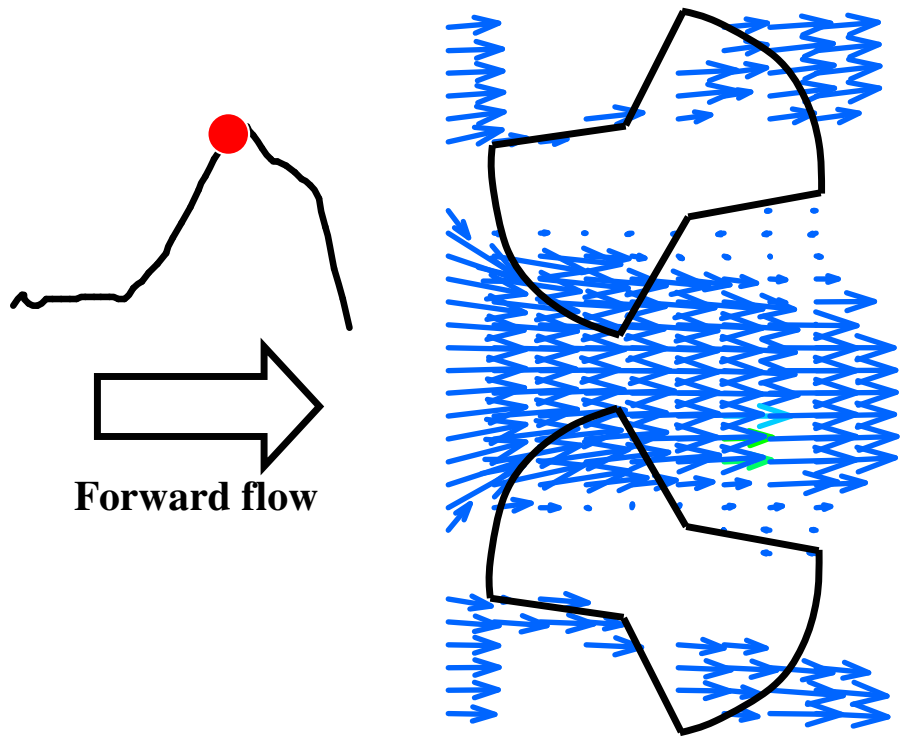

Figure 7-17 Reynolds shear stress distribution of the SJM $27 \mathrm{~mm}$ Standard at time bin 27, $500 \mu \mathrm{m}$ above flat level; Reynolds shear stress of 1,000 dynes $/ \mathrm{cm}^{2}$ was observed inside the central forward flow jet (avi, 708k, reg_500um_below_flat_rss.avi)

\subsubsection{6 $1 \mathrm{~mm}$ Below The Flat Level}

Animations reg_1mm_below_flat_vmag.avi and reg_1mm_below_flat_rss.avi show the velocity magnitude $(\mathrm{m} / \mathrm{s})$ and Reynolds shear stress $\left(\right.$ dynes $\left./ \mathrm{cm}^{2}\right)$ vector plots at the $1 \mathrm{~mm}$ below the flat level of the SJM $27 \mathrm{~mm}$ Standard clear valve throughout the cardiac cycle, respectively. Refer to Appendix $\mathrm{H}$ for the list of animations.

\section{Systole}

Flow patterns $1 \mathrm{~mm}$ below the flat level were similar to those at $500 \mu \mathrm{m}$ below the flat level, but with weaker leakage jets. Figure 7-18 (time bin 1) displays the flow vectors at the start of the systolic phase. Retrograde flows were drawn towards the central leakage flow. The same flow unsteadiness as seen at the $500 \mu \mathrm{m}$ below flat level appeared at valve closure in the vicinity of the leaflets. Only one weak leakage jet $(0.2 \mathrm{~m} / \mathrm{s})$ was seen 
issuing from the hinge pocket during systole in contrast to the three leakage jets observed at the $500 \mu \mathrm{m}$ above the flat level (time bin 4, Figure 7-19). High Reynolds shear stress values in the range of 100-1,000 dynes/ $/ \mathrm{cm}^{2}$ were observed at valve closure (time bin 43 , Figure 7-20), typically at the closing gap between the leaflets and also on the ventricular side of the hinge mechanism. The Reynolds shear stress values of the leakage jet from the hinge pocket were comparatively lower between $10-170$ dynes $/ \mathrm{cm}^{2}$.

\section{$\underline{\text { Diastole }}$}

At mid systole (time bin 27, Figure 7-21) when the leaflet was fully open, the central jet velocity reached a peak value of $1.39 \mathrm{~m} / \mathrm{s}$, while the lateral jets recorded a lower value of $0.9 \mathrm{~m} / \mathrm{s}$. Shear layers were also visible between the edge of the leaflet and the central jet.

The values of Reynolds shear stress in this shear flow region were higher at 50 dynes $/ \mathrm{cm}^{2}$ compared with that in the central jet of typically less than 10 dynes $/ \mathrm{cm}^{2}$. 

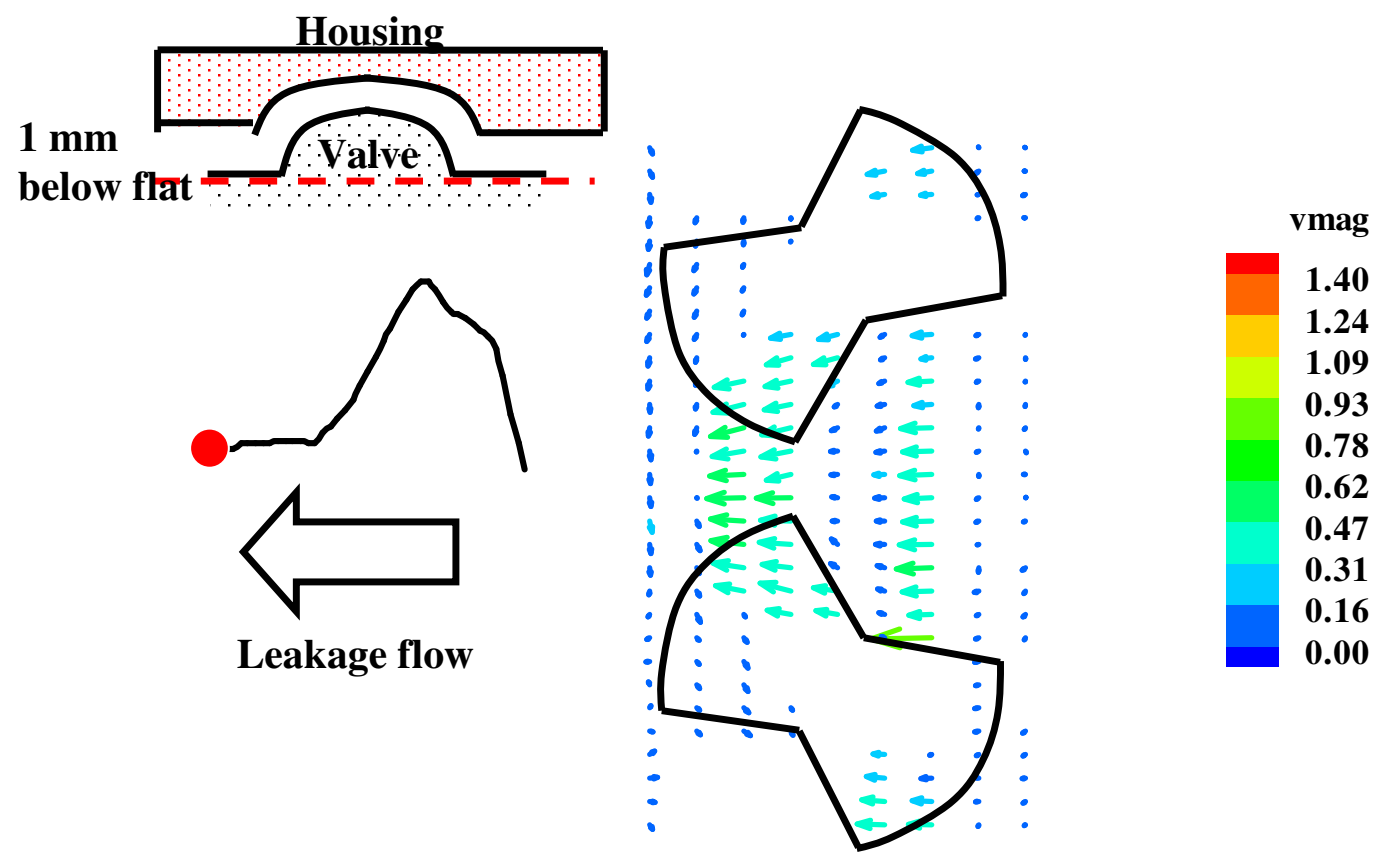

Figure 7-18 SJM $27 \mathrm{~mm}$ Standard mitral hinge flow field at early systole, time bin 1, 1 $\mathrm{mm}$ below flat level; leakage flow evident between the closing leaflets (avi, 691k, reg_1mm_below_flat_vmag.avi)

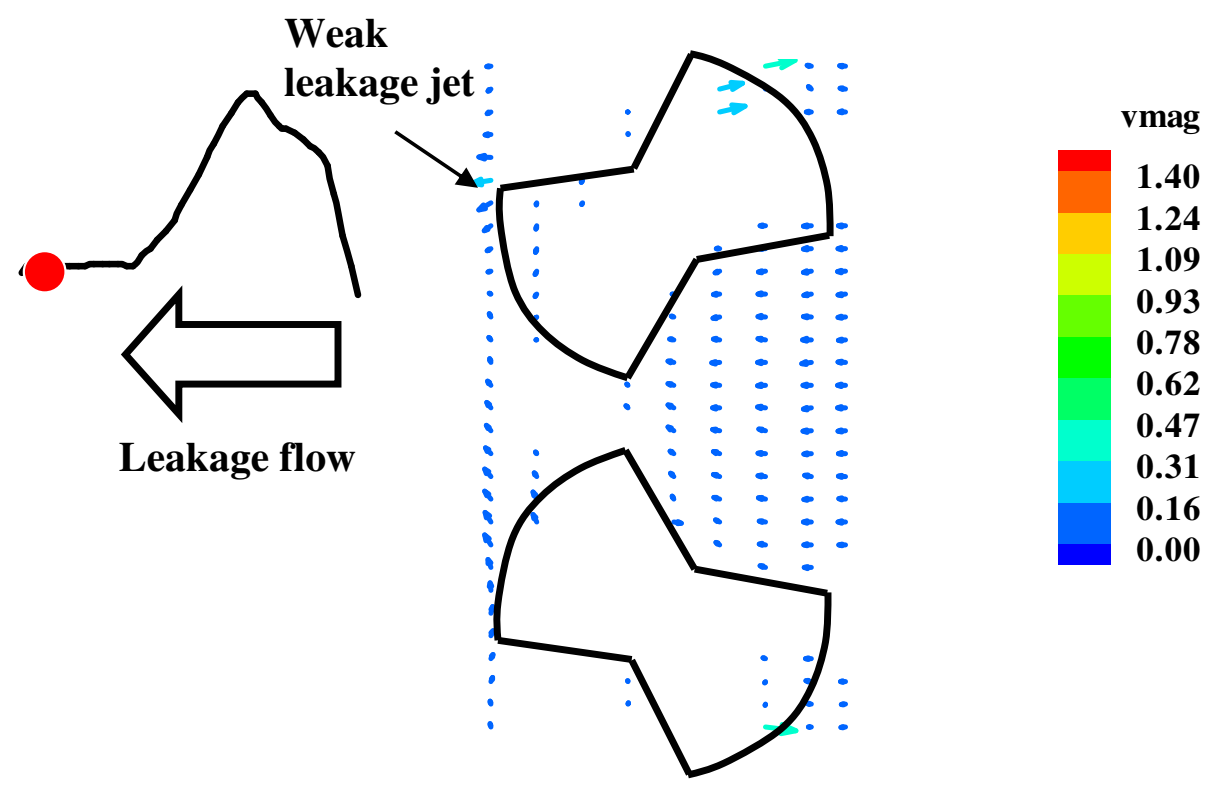

Figure 7-19 SJM 27 mm Standard mitral hinge flow field approaching mid systole, time bin 4, $1 \mathrm{~mm}$ below flat level; weak leakage jet was seen issuing from a single hinge pocket (avi, 691k, reg_1mm_below_flat_vmag.avi) 

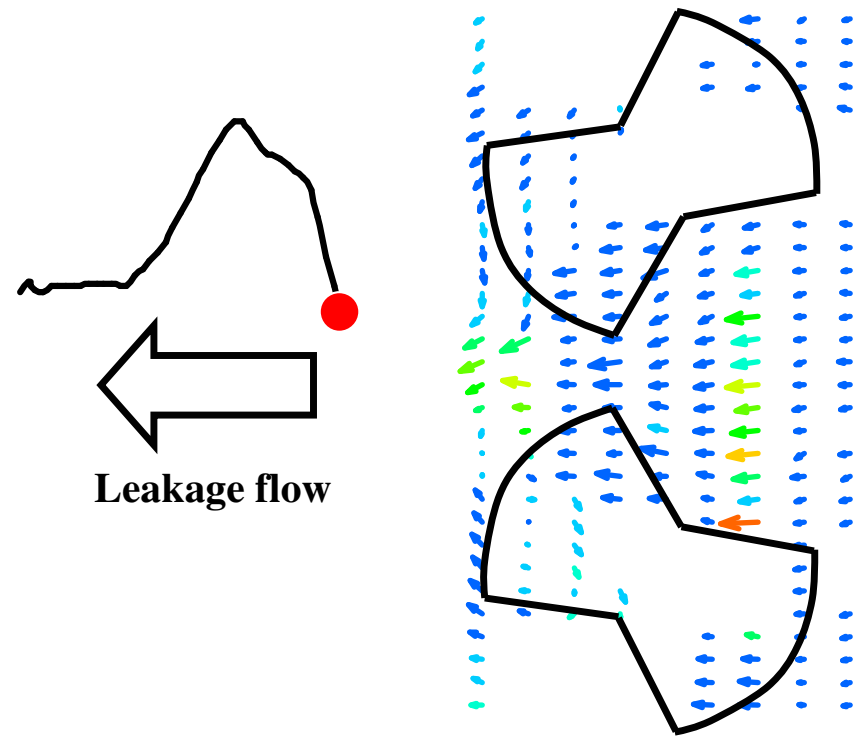

RSS

Figure 7-20 Reynolds shear stress distribution of the SJM $27 \mathrm{~mm}$ Standard at time bin 43, $1 \mathrm{~mm}$ above flat level; elevated Reynolds shear stress levels of 100-1,000 dynes $/ \mathrm{cm}^{2}$ were observed at valve closure (avi, 697k, reg_1mm_below_flat_rss.avi)
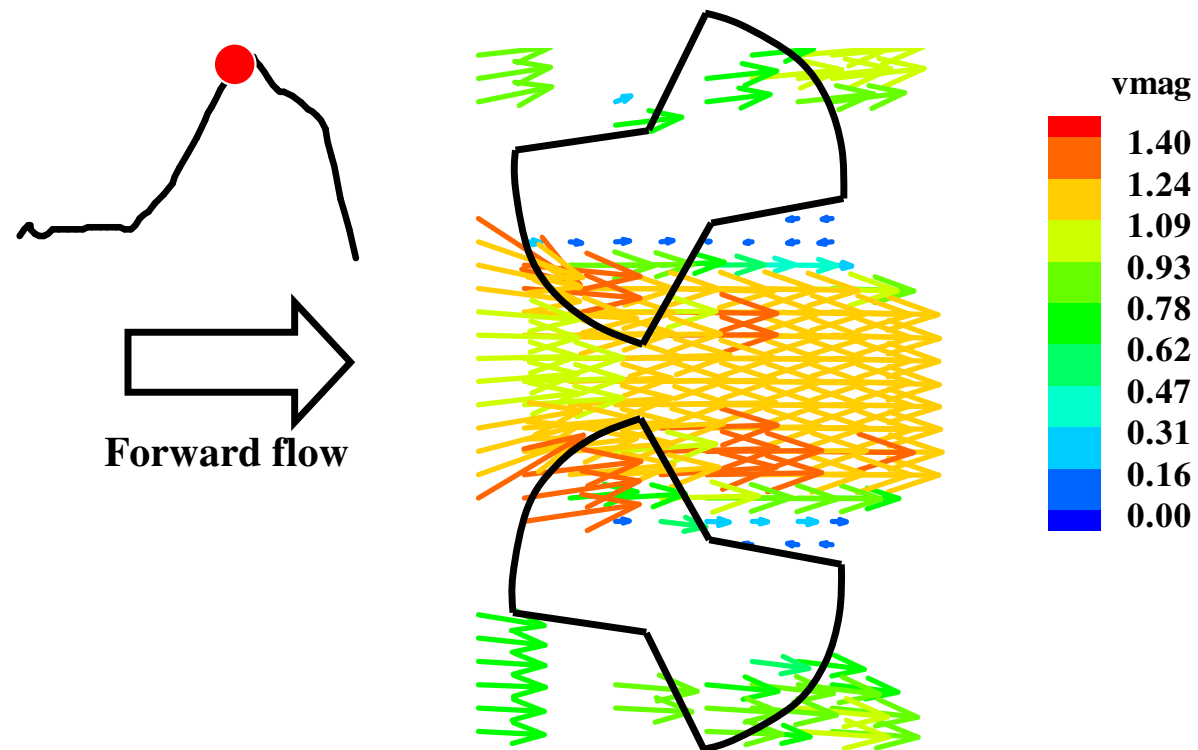

Figure 7-21 SJM $27 \mathrm{~mm}$ Standard mitral hinge flow field at mid diastole, time bin 27, 1 mm below flat level; elevated Reynolds shear stress observed between the central jet and the lateral jets (avi, 691k, reg_1mm_below_flat_vmag.avi) 


\subsubsection{7 $3 \mathrm{~mm}$ Below The Flat Level}

Animations reg_3mm_below_flat_vmag.avi and reg_3mm_below_flat_rss.avi show the velocity magnitude $(\mathrm{m} / \mathrm{s})$ and Reynolds shear stress $\left(\right.$ dynes $\left./ \mathrm{cm}^{2}\right)$ vector plots at the $3 \mathrm{~mm}$ below the flat level of the SJM $27 \mathrm{~mm}$ Standard clear valve throughout the cardiac cycle, respectively. Refer to Appendix $\mathrm{H}$ for the list of animations.

\section{$\underline{\text { Systole }}$}

Figure 7-22 (time bin 43) shows the flow profile at valve closure where disturbed flow was seen at the vicinity of the leaflets and a small central leakage jet $(0.3 \mathrm{~m} / \mathrm{s})$ emanating from the gap of the closed leaflets. Flow on the atrial side of the hinge was seen drawn towards the central leakage flow. Leakage jets from the hinge pocket were no longer visible at this measurement level. The recorded velocity at this measurement level was less than $0.06 \mathrm{~m} / \mathrm{s}$ through the remaining part of systole. Maximum Reynolds shear stress of 443 dynes $/ \mathrm{cm}^{2}$ was observed in the central leakage jet flow between the leaflets at valve closure. The surrounding fluid had Reynolds shear stress values ranging between $50-200$ dynes $/ \mathrm{cm}^{2}$.

\section{$\underline{\text { Diastole }}$}

Flow at mid-diastole was characterized by three forward jets (time bin 27, Figure 7-23). The central jet and the two lateral jets, respectively, reached velocity of $1.44 \mathrm{~m} / \mathrm{s}$ and 0.9 $\mathrm{m} / \mathrm{s}$ during mid-diastole. The shear layer region that was described in the $500 \mu \mathrm{m}$ and 1 $\mathrm{mm}$ below the flat levels was not present here. Reynolds shear stress values of 
approximately 55 dynes $/ \mathrm{cm}^{2}$ were seen in the central jet at the onset of the diastolic phase, but reduced to a value of less than 20 dynes $/ \mathrm{cm}^{2}$ by end-diastole.

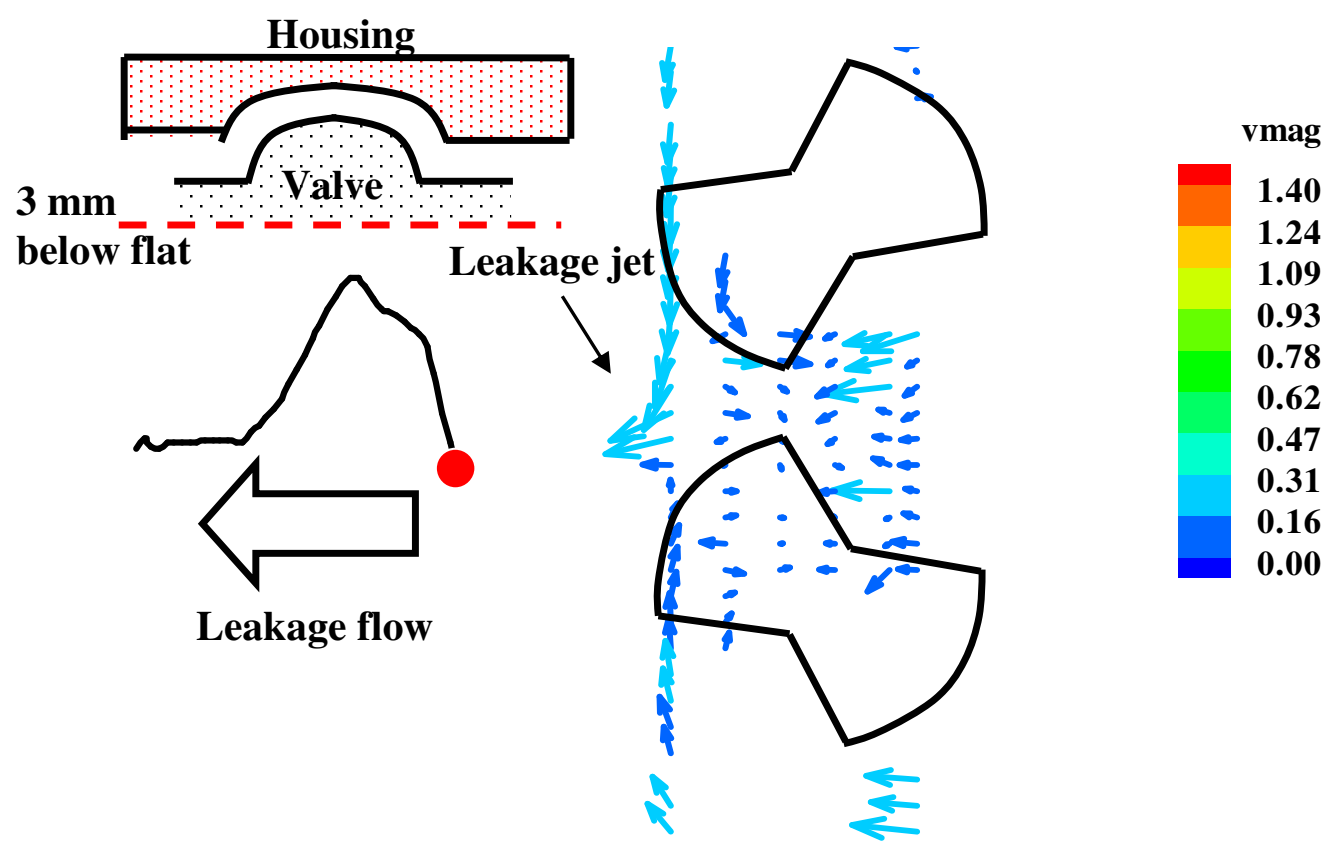

Figure 7-22 SJM $27 \mathrm{~mm}$ Standard mitral hinge flow field at early systole, time bin 43, 3 $\mathrm{mm}$ below flat level; disturbed flow at valve closure evident between the hinge mechanism (avi, 634k, reg_3mm_below_flat_vmag.avi) 

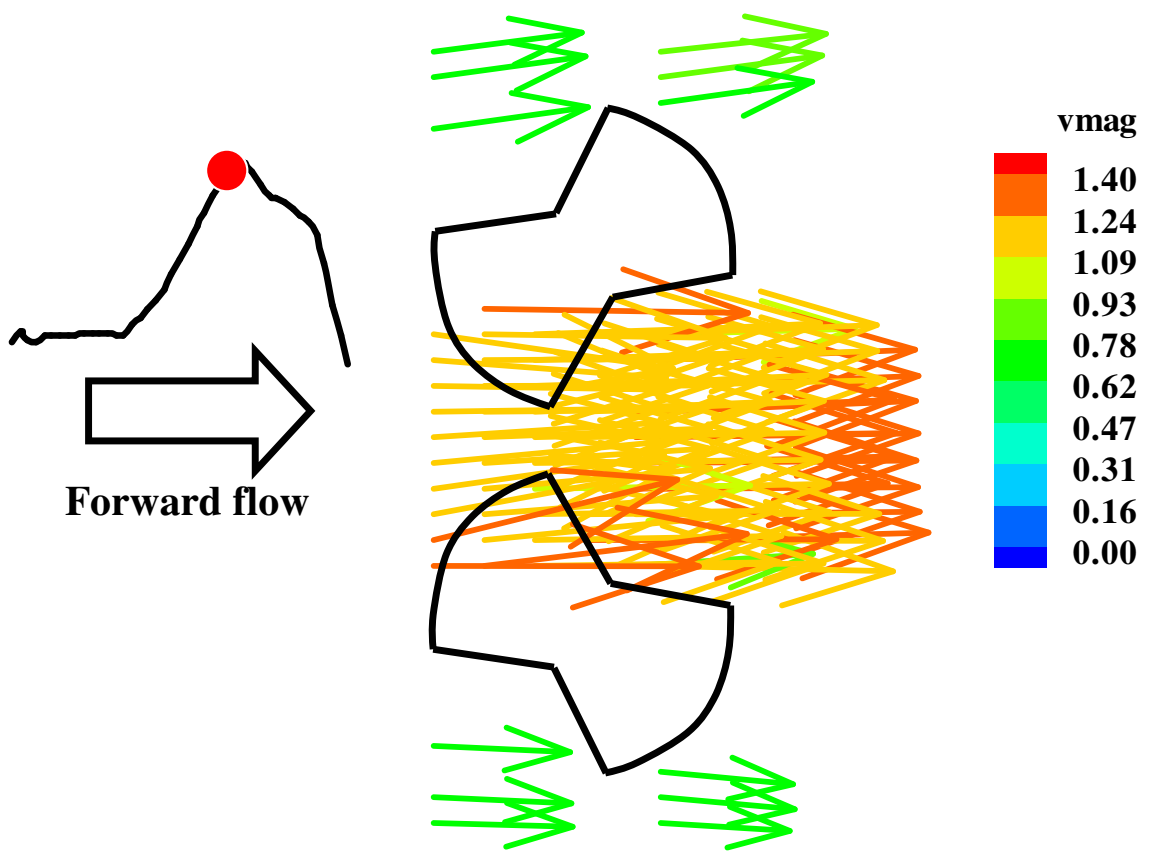

Figure 7-23 SJM $27 \mathrm{~mm}$ Standard mitral hinge flow field at mid diastole, time bin 27, 3 $\mathrm{mm}$ below flat level; three forward jets observed during diastole (avi, 634k, reg_3mm_below_flat_vmag.avi)

\subsection{Downstream of The Thumbnail Region}

Animations reg_thumbnail_vmag.avi and reg_thumbnail_rss.avi show the velocity magnitude $(\mathrm{m} / \mathrm{s})$ and Reynolds shear stress $\left(\right.$ dynes $\left./ \mathrm{cm}^{2}\right)$ vector plots at the downstream of the thumbnail region of the SJM $27 \mathrm{~mm}$ Standard clear valve throughout the cardiac cycle, respectively. Refer to Appendix $\mathrm{H}$ for the list of animations.

\section{Systole}

Figure 7-24 (time bin 1) displays the flow distribution downstream of the thumbnail region at the start of the systolic phase when the valve leaflets were closing. The flow patterns were relatively uniform throughout the measurement field with a velocity of approximately $0.14 \mathrm{~m} / \mathrm{s}$. The retrograde flow reduced to a velocity of $0.03 \mathrm{~m} / \mathrm{s}$ at mid 
systole and then decelerated further to velocity much lower than of $0.01 \mathrm{~m} / \mathrm{s}$ at the second half of the systolic phase before the onset of the diastolic phase. The reverse flow maintained a Reynolds shear stress value of approximately $10 \mathrm{dynes} / \mathrm{cm}^{2}$ at early systole before reducing to less than 5 dynes $/ \mathrm{cm}^{2}$ for the remaining part of the systolic phase.

\section{$\underline{\text { Diastole }}$}

Complex flow structures were observed during the diastolic phase. At the onset of diastole (time bin 18), a forward flow velocity of approximately $0.12 \mathrm{~m} / \mathrm{s}$ was recorded throughout the measurement level. As the flow progress to time bin 23 (accelerating phase, Figure 7-25), the lateral jets increased to a velocity magnitude of $0.4 \mathrm{~m} / \mathrm{s}$, while that in the center region decreased to a velocity of $0.03 \mathrm{~m} / \mathrm{s}$. As the flow approach the peak diastole, the velocity vectors in the stronger lateral jets reversed in direction. Reynolds shear stress of 100 dynes $/ \mathrm{cm}^{2}$ was measured at the shear layer between the central and lateral jets during diastole, while the Reynolds shear stress levels inside both the central and lateral jets were typically less than 50 dynes $/ \mathrm{cm}^{2}$. 

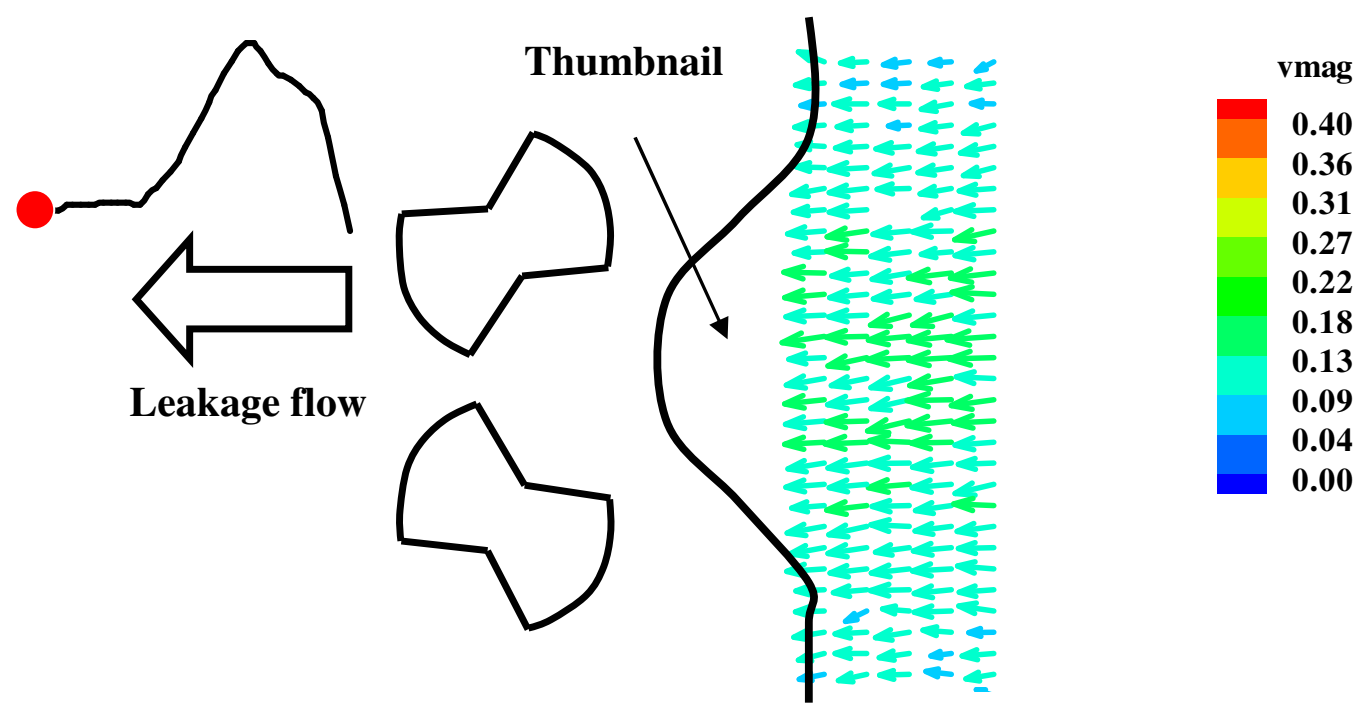

Figure 7-24 SJM $27 \mathrm{~mm}$ Standard mitral hinge flow field at early systole, time bin 1, downstream of thumbnail region, flat level; flow was relatively uniform during the $1^{\text {st }}$ part of systole (avi, 761k, reg_thumbnail_vmag.avi)

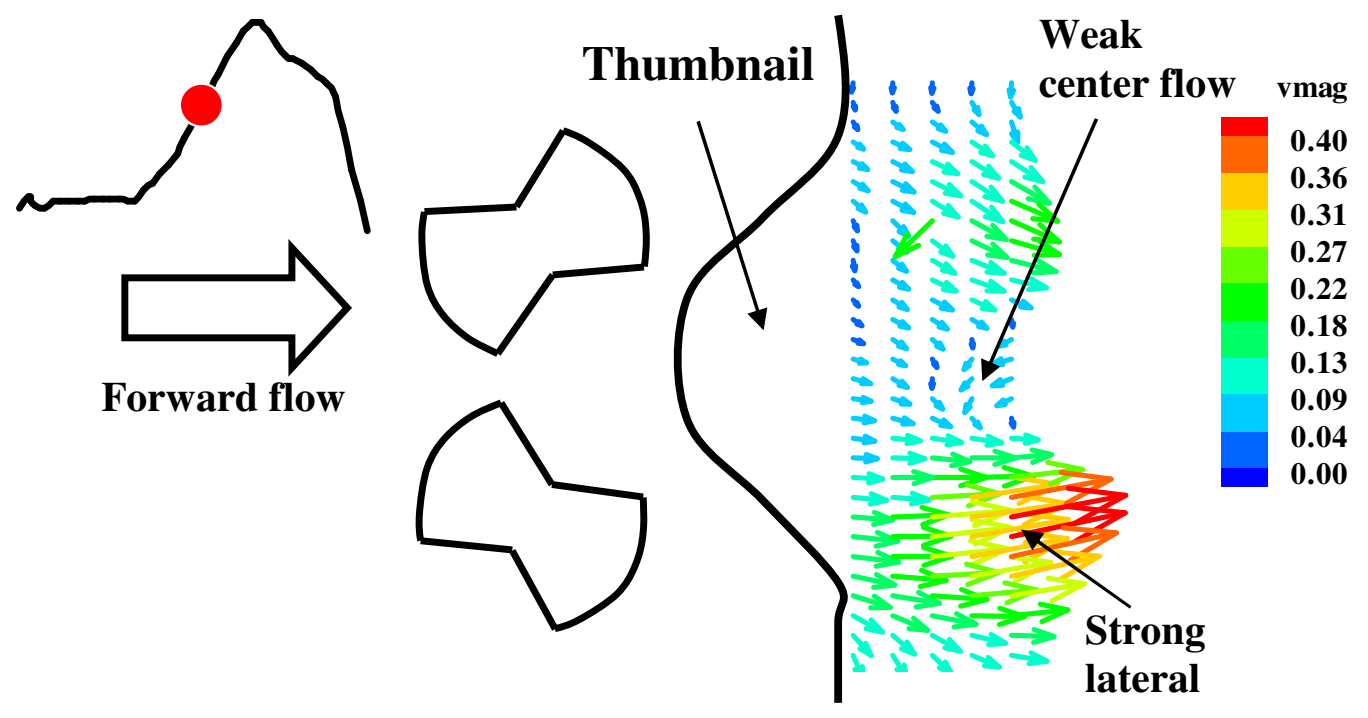

Figure 7-25 SJM $27 \mathrm{~mm}$ Standard mitral hinge flow field at accelerating phase, time bin 23, downstream of thumbnail region, flat level; this cardiac phase marked the beginning of the inversion of the lateral flows (avi, 761k, reg_thumbnail_vmag.avi) 


\subsubsection{27 mm Prototype Low Leaker}

The results are presented in the form of color pictures, which illustrate the flow fields and Reynolds shear stress vectors plots at specific instances during the cardiac cycle. The measurement levels are presented in the following sequence; 1) primary flat; 2) levels above the primary flat; 3) levels below the primary flat; and 4) downstream of the thumbnail. For each measurement plane, the description of the flow during systole is presented first followed by that during diastole. The direction of the forward flow is from left to right (from the left atrium to left ventricle, Figure 5-13). All vector plots are colorcoded by Velocity Magnitude (m/s) and Reynolds shear stress (dynes/cm²). Arrows point in the direction of the phase averaged mean velocity vectors and the length of the arrow is proportional to the velocity magnitude. Refer to Appendix $\mathrm{H}$ for the list of velocity magnitude $(\mathrm{m} / \mathrm{s})$ and Reynolds shear stress $\left(\right.$ dynes $\left./ \mathrm{cm}^{2}\right)$ animations. Terminology pertinent to the hinge design is shown in Figure 7-26 for orientation purposes. Refer to Figure 7-3 in section 7.1.2.1 for the position of the leaflet at mid acceleration, peak systole as well as valve closure and mid diastole within the $27 \mathrm{~mm}$ prototype low leaker clear valve hinge. These leaflet positions correspond to those in the images presented later. For clarity the leaflets are not shown on the color printouts of the velocity field. 


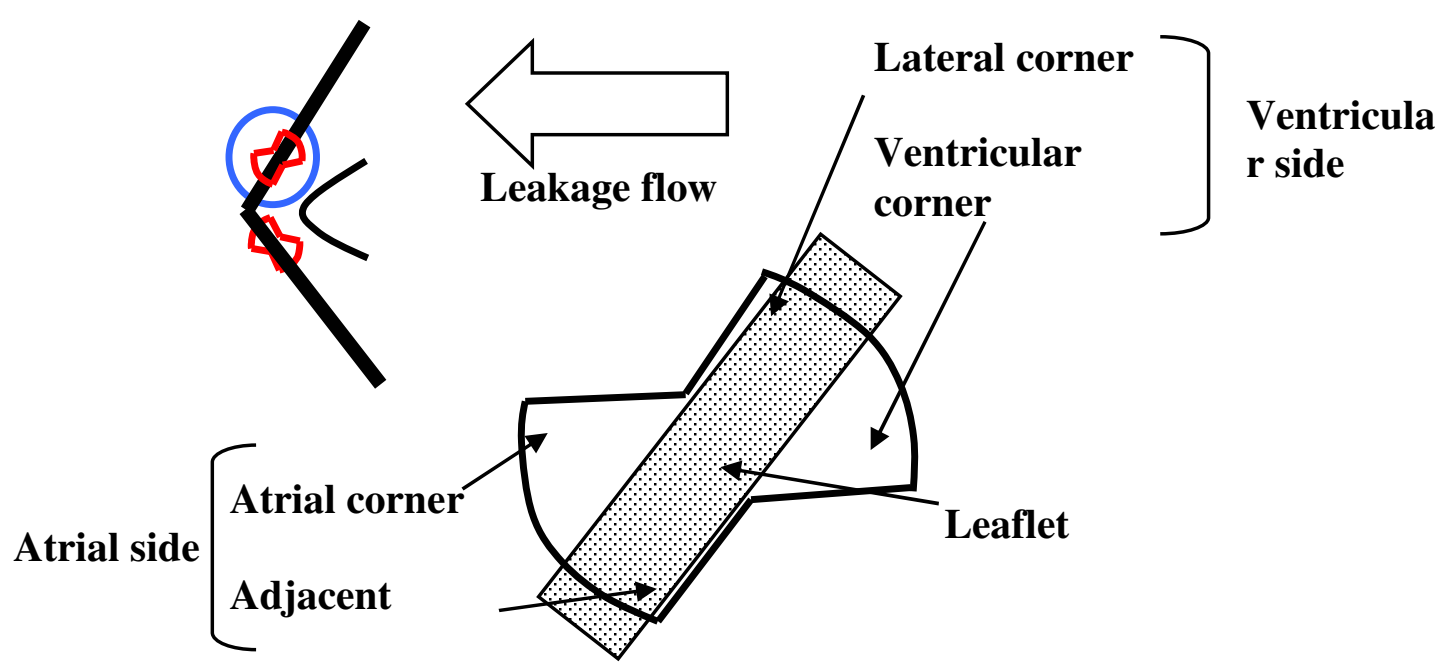

Figure 7-26 Pertinent terminology of the $27 \mathrm{~mm}$ prototype low and high leaker clear hinge designs

\subsection{Flat Level}

Animations low_flat_vmag.avi and low_flat_rss.avi show the velocity magnitude (m/s) and Reynolds shear stress (dynes $/ \mathrm{cm}^{2}$ ) vector plots at the flat level of the $27 \mathrm{~mm}$ prototype low leaker clear valve throughout the cardiac cycle, respectively. Refer to Appendix $\mathrm{H}$ for the list of animations.

\section{$\underline{\text { Systole }}$}

The flow profile at early systole (time bin 2, Figure 7-27) at the flat level in the prototype low leaker valve was characterized by a leakage jet at the atrial corner, and low flow at both the ventricular and the adjacent corners of the hinge geometry. The presence of the leaflet ear in the center of the geometry was visible at this measurement level and hinge flow on the ventricular side was accelerated through the gap between the ear and the edge of the hinge geometry into the atrial side. Similar flow diversion was also observed at the 
lateral corner. As the leakage flow progress towards mid systole, the leakage jet at the atrial corner increased to a magnitude of approximately $0.6 \mathrm{~m} / \mathrm{s}$ (time bin 4, Figure 7-28). A smaller but much higher velocity jet of more than $1.0 \mathrm{~m} / \mathrm{s}$ was identified at the adjacent corner at peak systole. Both jets then decelerated to about $0.4 \mathrm{~m} / \mathrm{s}$ toward late systole. The flow characteristics at the ventricular and lateral corners were similar throughout the systole with a velocity of around $0.05 \mathrm{~m} / \mathrm{s}$. A small region of low flow $(0.05 \mathrm{~m} / \mathrm{s})$ was observed at the atrial corner between the leakage jet and the edge of the hinge geometry and it persisted throughout the systolic phase. Maximum Reynolds shear stress of 5,633 dynes $/ \mathrm{cm}^{2}$ was observed in the leakage jet at the adjacent corner of the hinge region during mid systole (time bin 4, Figure 7-29). The shear stresses levels in the leakage jet at the adjacent corner $\left(1,000-3,000\right.$ dynes $\left./ \mathrm{cm}^{2}\right)$ were typically higher than those in the atrial corner $\left(500-900\right.$ dynes $\left./ \mathrm{cm}^{2}\right)$, even though the size of the former was much smaller. The shear stress levels in the ventricular and lateral corners were comparatively lower $(<$ 20 dynes $/ \mathrm{cm}^{2}$ ) than other regions, except for two measurement locations in the lateral corner, where the Reynolds shear stress values were $700-900$ dynes $/ \mathrm{cm}^{2}$. This isolated region of elevated shear stress coincided with the location of the flow diversion described earlier on.

\section{$\underline{\text { Diastole }}$}

The hinge flow during the acceleration phase (time bin 21, Figure 7-30) was characterized by forward flow at the adjacent and lateral corners of the hinge. The velocity magnitude at both regions increased to approximately $0.45 \mathrm{~m} / \mathrm{s}$ at mid-diastole (time bin 27, Figure 7-31). Also around mid-diastole, a region of recirculation became 
apparent at the adjacent corner with the eye of the recirculation lying next to the leaflet ear. Flow diversion was observed at the atrial corner as a result of the presence of the leaflet ear, which now rotated to an open position. Low flow occurred in the atrial and ventricular corners throughout the diastole, except for a small region of high flow at the atrial region next to the leaflet ear. The higher velocity flow in this region was directed by the ear into the recirculation region in the adjacent corner. For a $40 \mathrm{~ms}$ period between systole and diastole the flow velocity in the entire hinge was low at approximately 0.05 $\mathrm{m} / \mathrm{s}$. Reynolds shear stress values during diastole were in the range of $10-100 \mathrm{dynes} / \mathrm{cm}^{2}$. The calculated shear stress values in the recirculation zone were approximately 10 dynes $/ \mathrm{cm}^{2}$. The highest value $\left(100 \mathrm{dynes} / \mathrm{cm}^{2}\right)$ was observed at the adjacent corner between the recirculation zone and the edge of the hinge geometry.

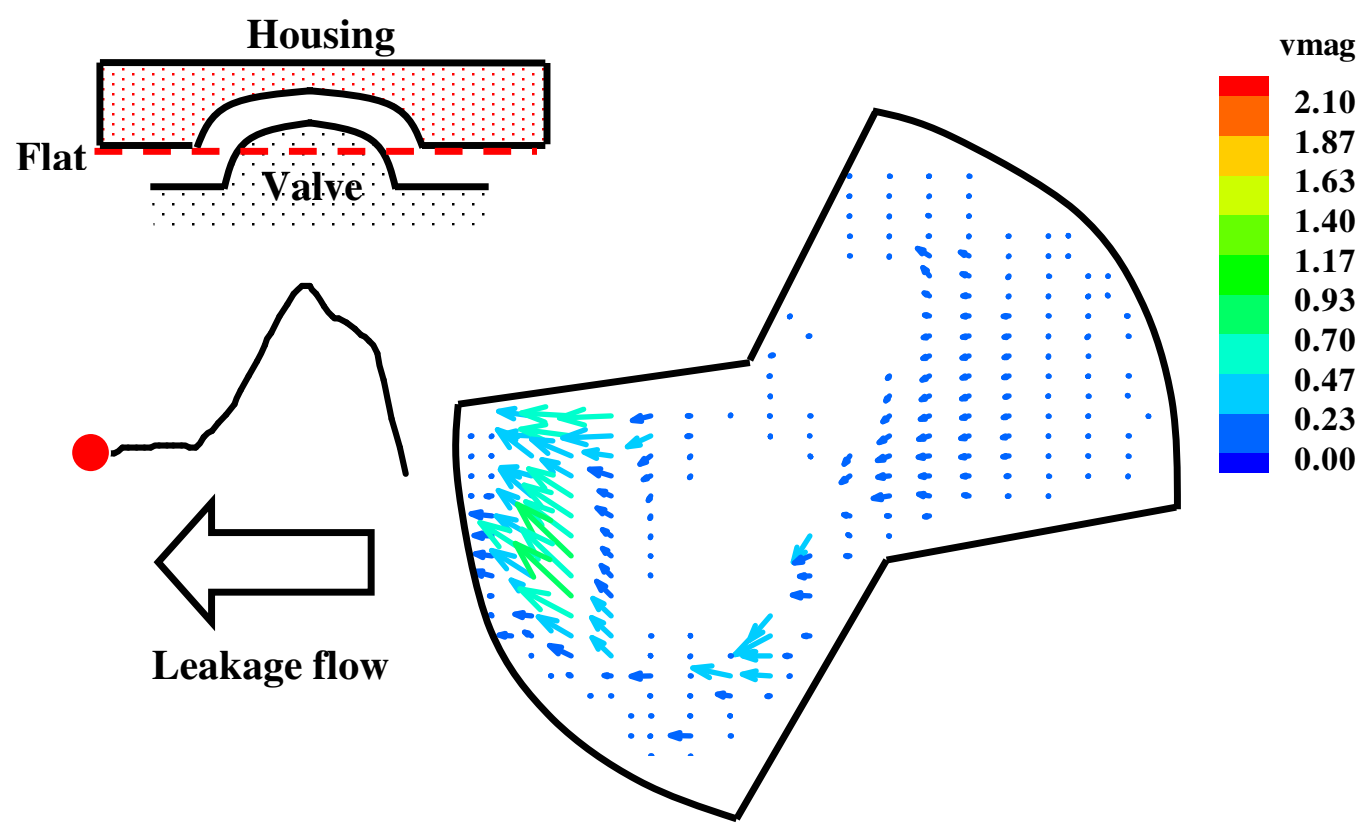

Figure 7-27 $27 \mathrm{~mm}$ prototype low leaker mitral hinge flow field at early systole, time bin 2, flat level; strong leakage jet appearing at the atrial corner of the hinge geometry (avi, $782 \mathrm{k}$, low_flat_vmag.avi) 


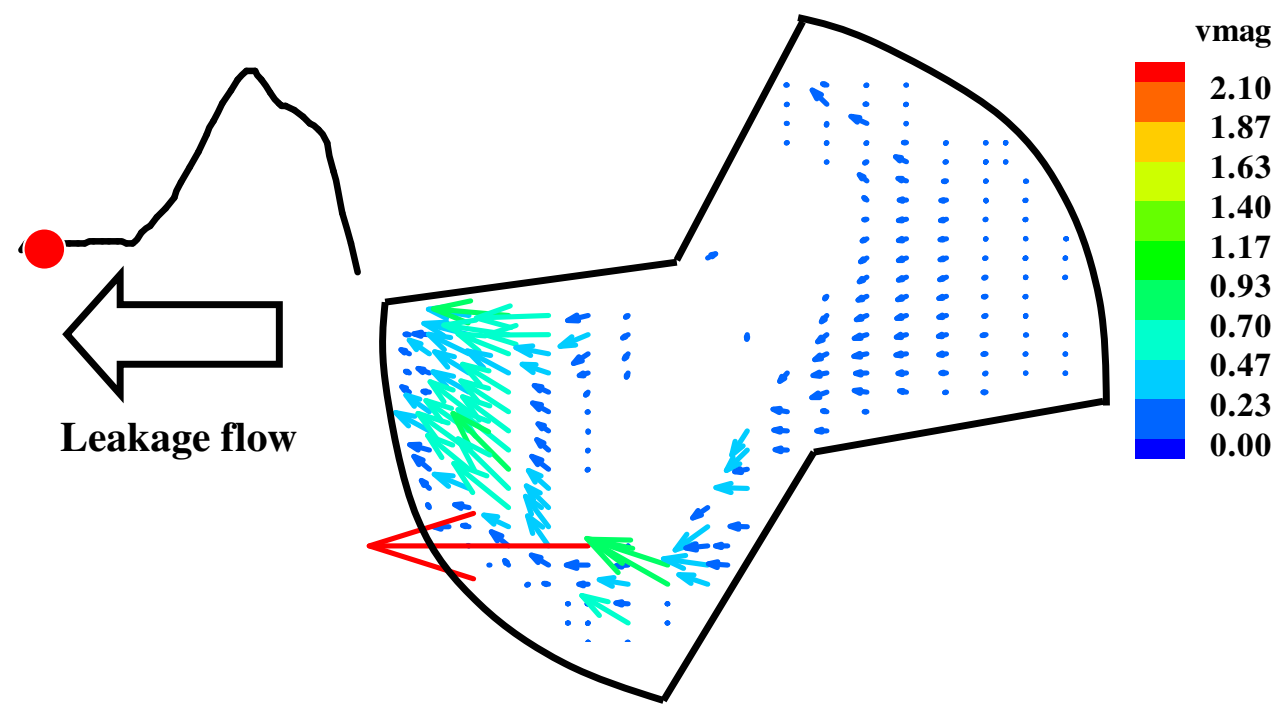

Figure 7-28 $27 \mathrm{~mm}$ prototype low leaker mitral hinge flow field approaching mid systole, time bin 4, flat level; second leakage jet became visible at the adjacent corner of the hinge geometry (avi, 782k, low_flat_vmag.avi)

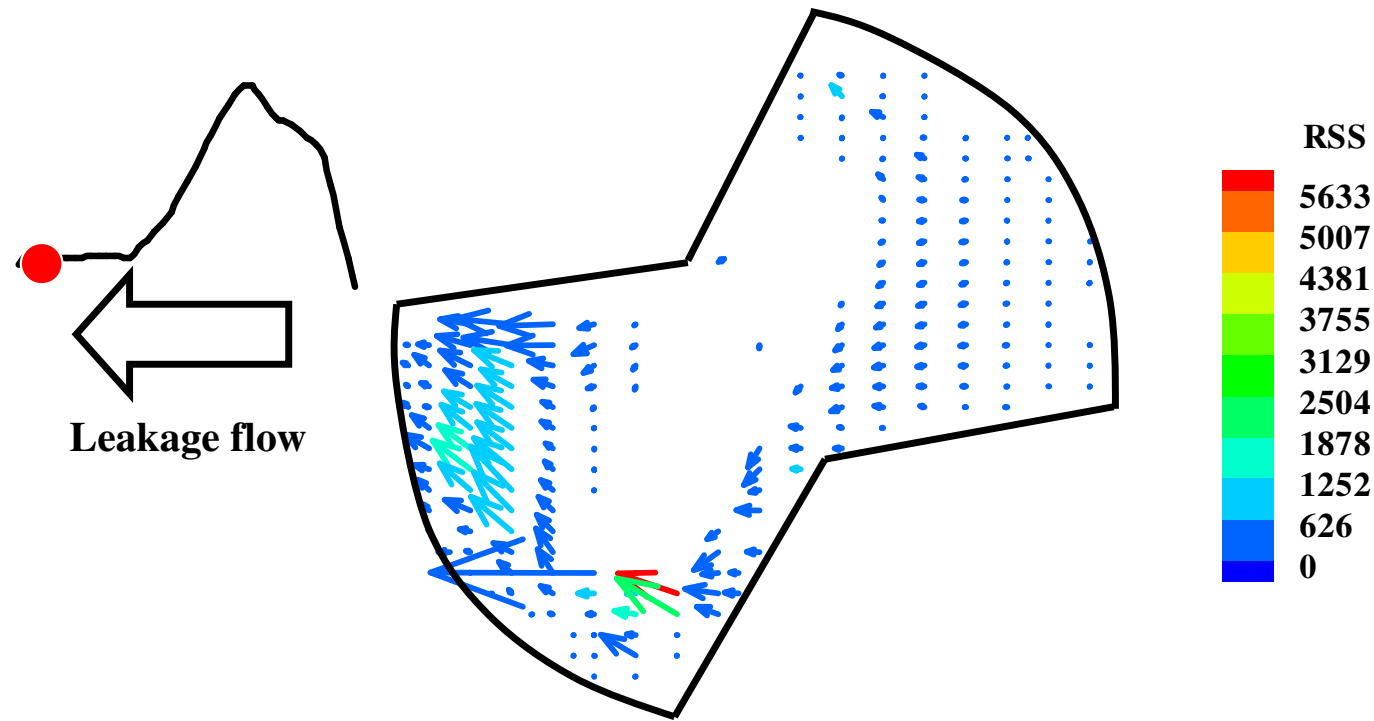

Figure 7-29 Reynolds shear stress distribution of the $27 \mathrm{~mm}$ prototype low leaker at time bin 4, flat level; elevated Reynolds shear stress levels observed at the atrial and lateral corners of the hinge geometry (avi, 740k, low_flat_rss.avi) 


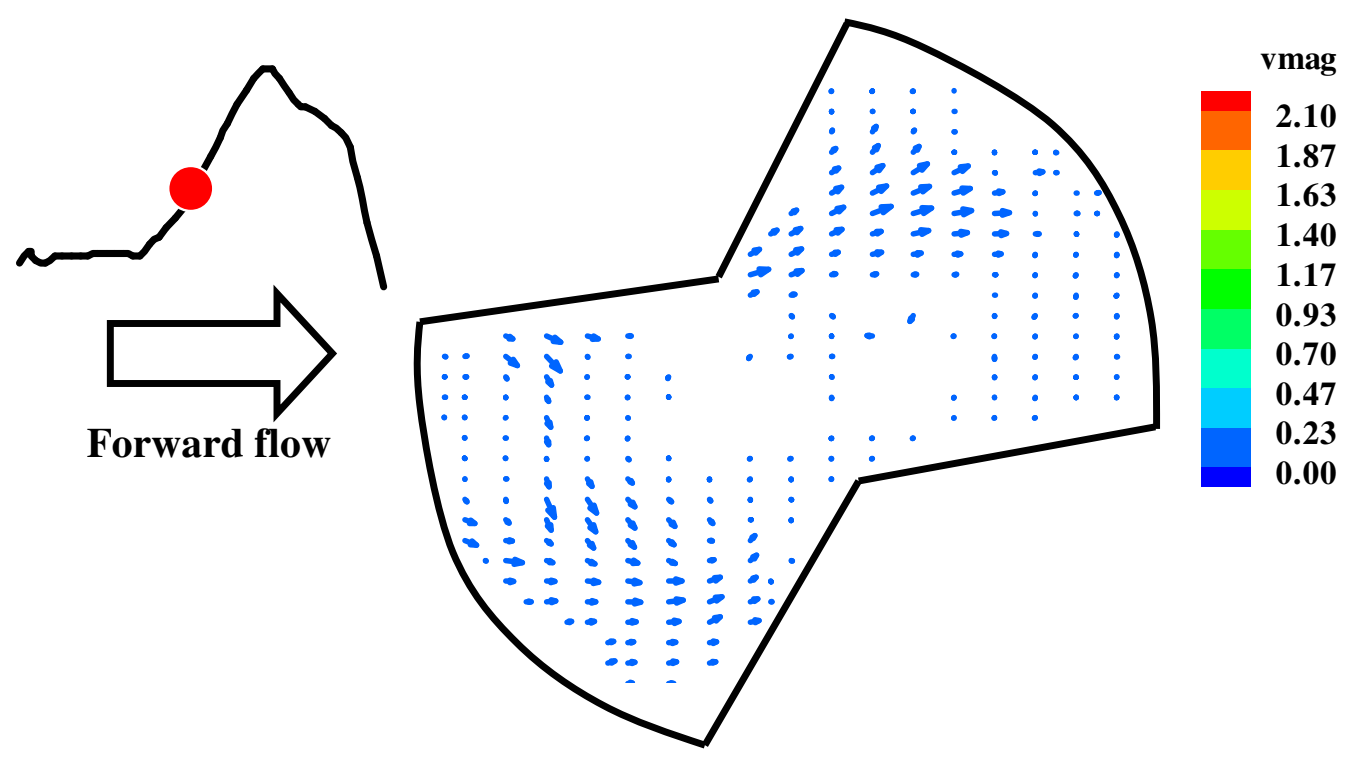

Figure 7-30 $27 \mathrm{~mm}$ prototype low leaker mitral hinge flow field at early diastole, time bin 21 , flat level; forward jets observed in both adjacent and lateral corner of the hinge during the acceleration phase (avi, 782k, low_flat_vmag.avi)

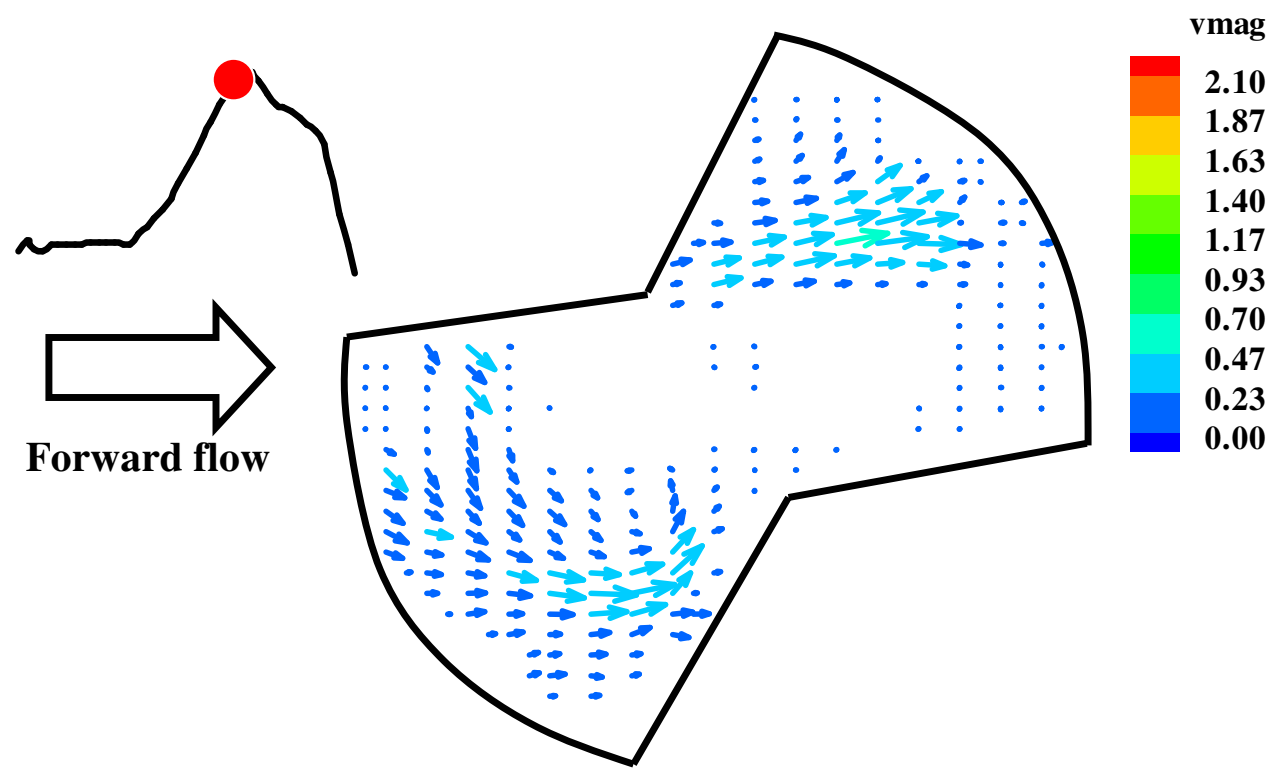

Figure 7-31 $27 \mathrm{~mm}$ prototype low leaker mitral hinge flow field at mid diastole, time bin 27, flat level; recirculation flow and a strong forward flow appeared at the adjacent and lateral corners, respectively (avi, 782k, low_flat_vmag.avi) 


\subsection{0 $\mu \mathrm{m}$ Above The Flat Level}

Animations low_190um_above_flat_vmag.avi and low_190um_above_flat_rss.avi show the velocity magnitude $(\mathrm{m} / \mathrm{s})$ and Reynolds shear stress $\left(\right.$ dynes $\left./ \mathrm{cm}^{2}\right)$ vector plots at the $190 \mu \mathrm{m}$ above the flat level of the $27 \mathrm{~mm}$ prototype low leaker clear valve throughout the cardiac cycle, respectively. Refer to Appendix $\mathrm{H}$ for the list of animations.

\section{Systole}

Figure 7-32 (time bin 2) shows the flow profile at the onset of systole at $190 \mu \mathrm{m}$ above the flat level. The flow patterns, in particular the flow diversion at the lateral corner and central part of the hinge, were similar to those observed at the flat level. Leakage jets were observed at the atrial and adjacent corners of the hinge geometry. A maximum velocity magnitude of $0.91 \mathrm{~m} / \mathrm{s}$ was recorded at the atrial corner toward mid systole (time bin 4, Figure 7-33), while a smaller leakage jet of approximately $1.03 \mathrm{~m} / \mathrm{s}$ was seen developed at the adjacent corner. The low flow region at the atrial corner that was observed at the flat level was not present at this level. The ventricular and lateral corners were typified by low flow of approximately $0.01-0.03 \mathrm{~m} / \mathrm{s}$ that persisted throughout systole. Maximum Reynolds shear stress of 4,146 dynes $/ \mathrm{cm}^{2}$ was observed in the leakage jet at the adjacent corner. The Reynolds shear stress values of the leakage jet at the adjacent corner were much higher $\left(1,500-4,000 \mathrm{dynes} / \mathrm{cm}^{2}\right)$ than that at the atrial corner (400-500 dynes/ $\mathrm{cm}^{2}$ ) (time bin 4, Figure 7-34). 


\section{Diastole}

Figure 7-35 shows the flow profile during mid-diastole (time bin 27). A strong forward flow $(0.4 \mathrm{~m} / \mathrm{s})$ was observed at the atrial side and was directed around the leaflet ear towards the recirculation zone at the adjacent corner. The recirculation region persisted throughout the diastole, but became weaker towards end diastole. The flow at the ventricular and lateral corners was low at $0.04 \mathrm{~m} / \mathrm{s}$ throughout diastole. About $40 \mathrm{~ms}$ elapse as the leaflet sweeps from the open to closed position. During this time, the flow velocity in the hinge was low at approximately $0.02 \mathrm{~m} / \mathrm{s}$. The maximum shear stress value of 24 dynes $/ \mathrm{cm}^{2}$ was recorded at the adjacent corner between the recirculation zone and the edge of the hinge geometry. The shear stress values during the diastole were low at around 1-20 dynes $/ \mathrm{cm}^{2}$.

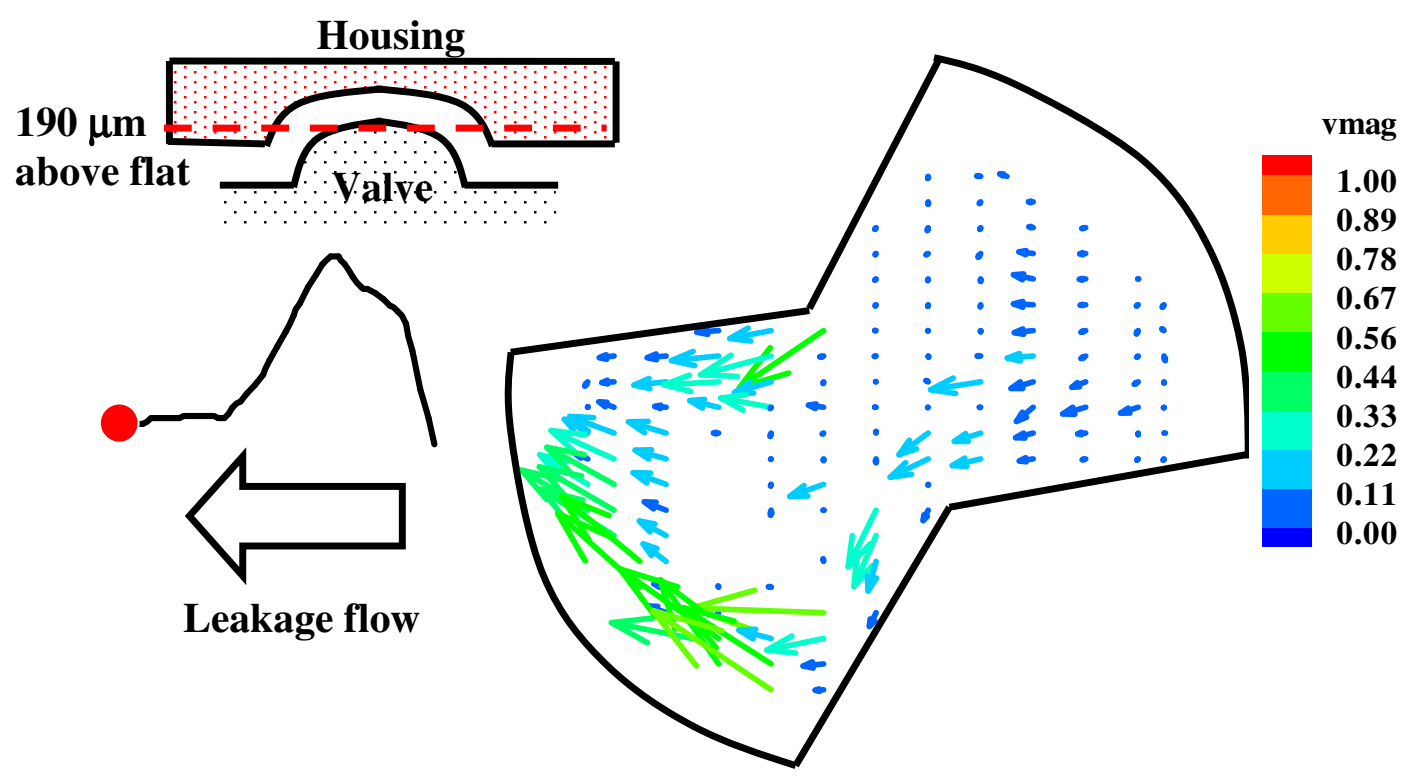

Figure 7-32 $27 \mathrm{~mm}$ prototype low leaker mitral hinge flow field at early systole, time bin 2, $190 \mu \mathrm{m}$ above flat level; leakage jets emanating from the atrial and adjacent corners (avi, 672k, low_190um_above_flat_vmag.avi) 


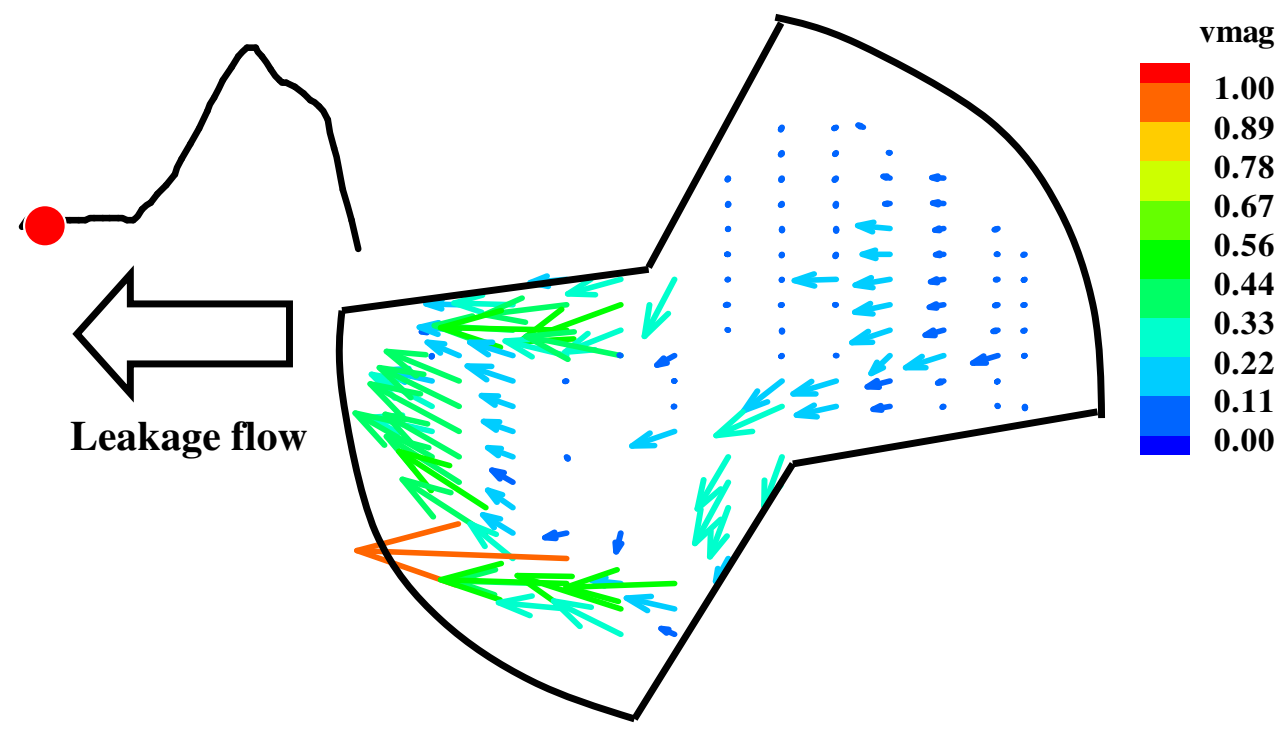

Figure 7-33 $27 \mathrm{~mm}$ prototype low leaker mitral hinge flow field approaching mid systole, time bin 4, $190 \mu \mathrm{m}$ above flat level (avi, 672k, low_190um_above_flat_vmag.avi)

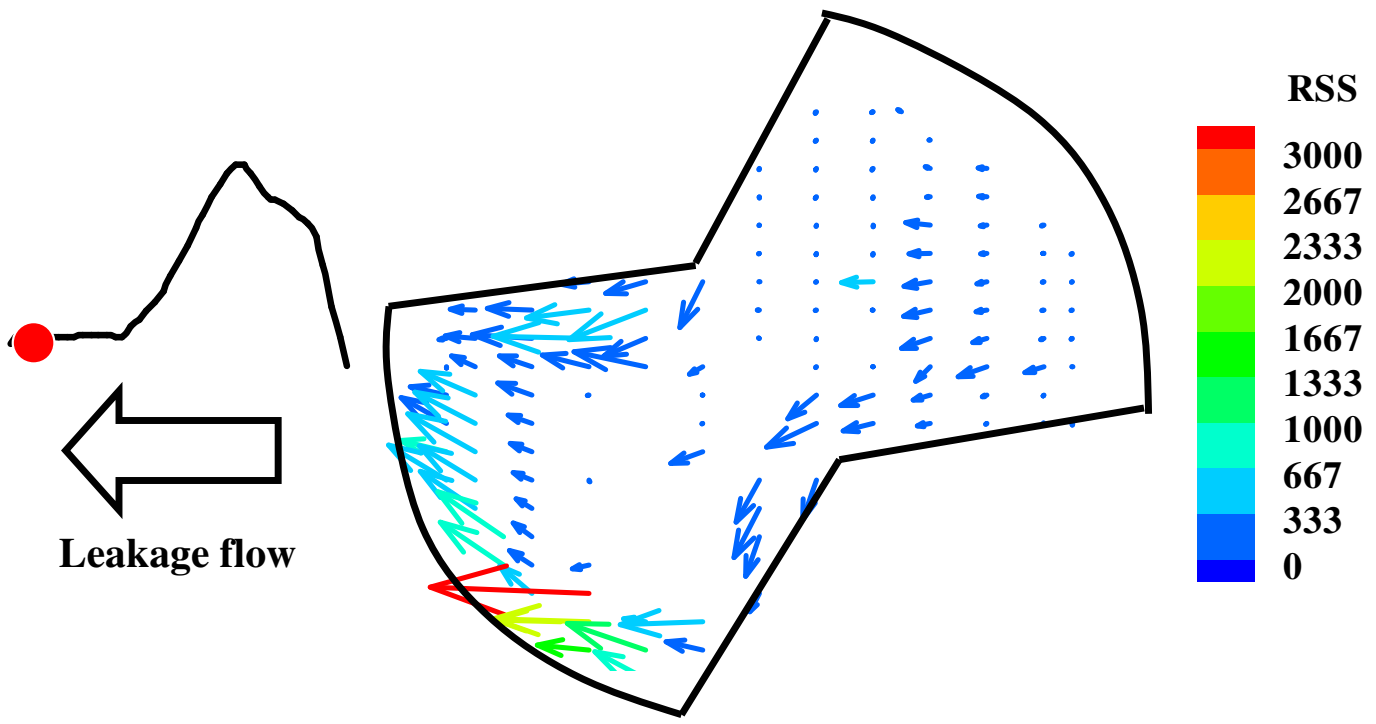

Figure 7-34 Reynolds shear stress distribution of the $27 \mathrm{~mm}$ prototype low leaker at time bin 4, $190 \mu \mathrm{m}$ above flat level; Reynolds shear stress levels in the adjacent corner were higher than those in the atrial corner (avi, 684k, low_190um_above_flat_rss.avi) 


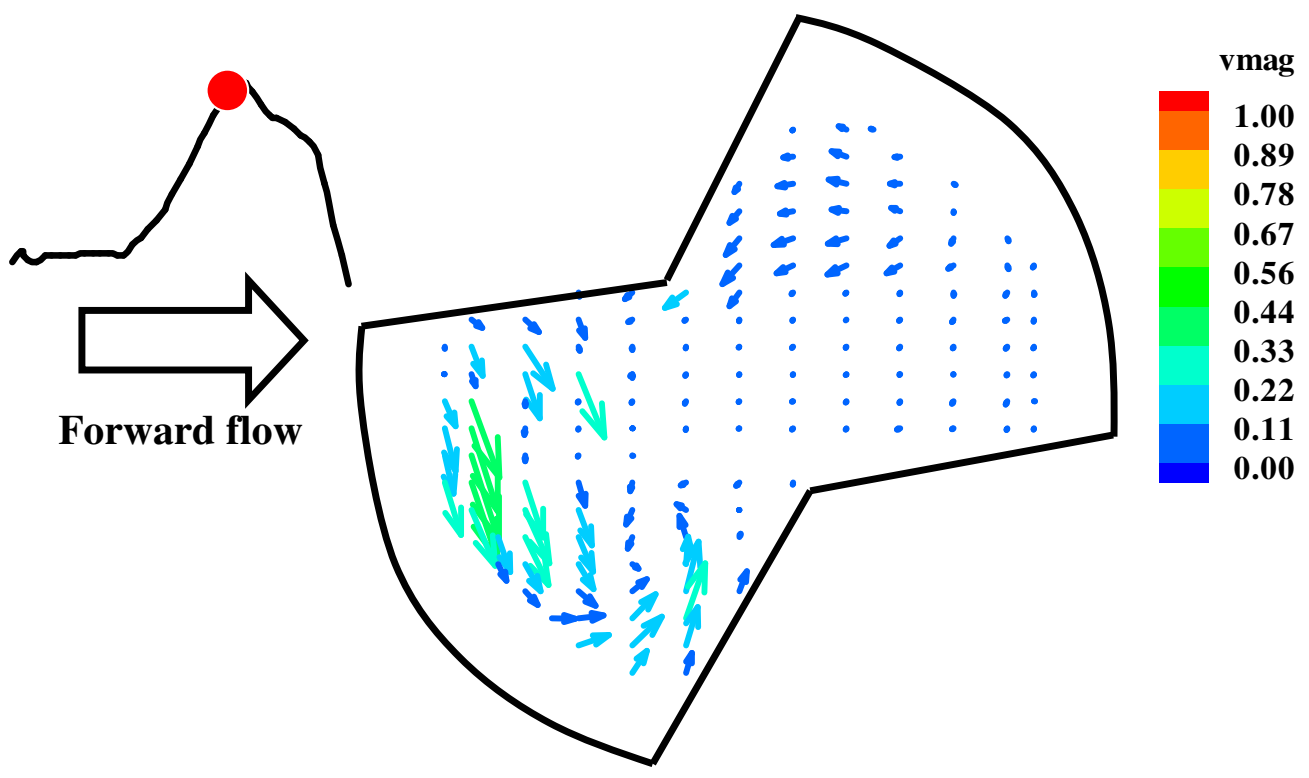

Figure 7-35 $27 \mathrm{~mm}$ prototype low leaker mitral hinge flow field at mid diastole, time bin 27, $190 \mu \mathrm{m}$ above flat level; recirculation visible at the adjacent corner (avi, 672k, low_190um_above_flat_vmag.avi)

\subsection{0 $\mu \mathrm{m}$ Above The Flat Level}

Animations low_390um_above_flat_vmag.avi and low_390um_above_flat_rss.avi show the velocity magnitude $(\mathrm{m} / \mathrm{s})$ and Reynolds shear stress $\left(\right.$ dynes $\left./ \mathrm{cm}^{2}\right)$ vector plots at the $390 \mu \mathrm{m}$ above the flat level of the $27 \mathrm{~mm}$ prototype low leaker clear valve throughout the cardiac cycle, respectively. Refer to Appendix $\mathrm{H}$ for the list of animations.

The velocity magnitude throughout systole was low in the range of $0.04-0.4 \mathrm{~m} / \mathrm{s}$, with a maximum velocity of $0.46 \mathrm{~m} / \mathrm{s}$ observed at the ventricular side of the hinge (time bin 5 , Figure 7-36). The Reynolds shear stress levels were less than 10 dynes $/ \mathrm{cm}^{2}$ during systole. Velocity magnitude during diastole was low at $0.04 \mathrm{~m} / \mathrm{s}$ with low Reynolds shear stress levels of less than 1.0 dynes $/ \mathrm{cm}^{2}$. 


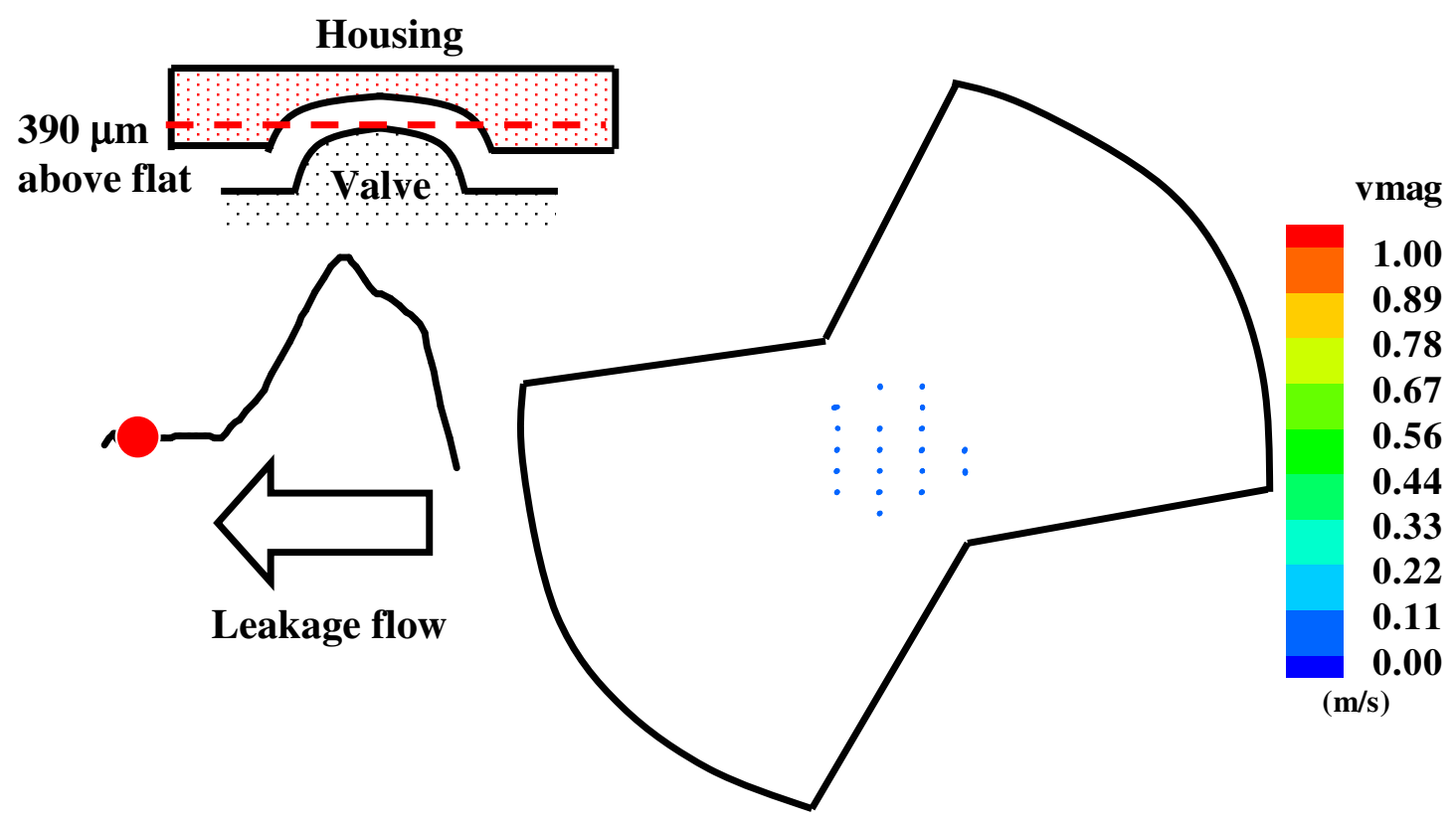

Figure 7-36 $27 \mathrm{~mm}$ prototype low leaker mitral hinge flow field approaching mid systole, time bin 5, $390 \mu \mathrm{m}$ above flat level; low flow was visible throughout the cardiac cycle (avi, 618k, low_390um_above_flat_vmag.avi)

\subsection{0 $\mu \mathrm{m}$ Below The Flat Level}

Animations low_500um_below_flat_vmag.avi and low_500um_below_flat_rss.avi show the velocity magnitude $(\mathrm{m} / \mathrm{s})$ and Reynolds shear stress $\left(\right.$ dynes $\left./ \mathrm{cm}^{2}\right)$ vector plots at the $500 \mu \mathrm{m}$ below the flat level of the $27 \mathrm{~mm}$ prototype low leaker clear valve throughout the cardiac cycle, respectively. Refer to Appendix $\mathrm{H}$ for the list of animations.

\section{$\underline{\text { Systole }}$}

Figure 7-37 (time bin 42) shows the beginning of the systole where flow on the ventricular side of the valve was flowing toward the atrial side $(0.26 \mathrm{~m} / \mathrm{s})$, while flow in the center between the two leaflets was still moving in the forward direction $(0.35 \mathrm{~m} / \mathrm{s})$. A stream of retrograde flow $(0.3 \mathrm{~m} / \mathrm{s})$ was evident between the two hinge mechanisms as 
the leaflets swept to the closed position. This reverse flow collided with a band of low velocity flow observed in the center region between the leaflets. Flow at time bin 1 was seen accelerating towards the closing gap giving rise to retrograde flow of $0.5 \mathrm{~m} / \mathrm{s}$ between the leaflets with a corresponding Reynolds shear stress of 400 dynes $/ \mathrm{cm}^{2}$. The retrograde flow was abruptly terminated as the leaflets shut, leading to an area of unsteady flow $(<0.1 \mathrm{~m} / \mathrm{s})$ in the proximity of the hinge mechanisms and elevated shear stress levels of 1,000 dynes $/ \mathrm{cm}^{2}$. A central leakage jet of $0.2 \mathrm{~m} / \mathrm{s}$, and corresponding shear stress levels of 500 dynes $/ \mathrm{cm}^{2}$, was still visible between the closed leaflets and persisted throughout systole. Leakage jets of $0.7 \mathrm{~m} / \mathrm{s}$ appeared to emanate from the hinge geometry during the leakage phase and gradually gain in strength towards mid systole (time bin 4, Figure 7-38). A maximum Reynolds shear stress of 1,437 dynes $/ \mathrm{cm}^{2}$ was recorded in these leakage jets at time bin 4 (Figure 7-39). End systole was characterized by low flow velocity of $0.03 \mathrm{~m} / \mathrm{s}$ throughout the measurement level, and this persisted for about $80 \mathrm{~ms}$ before the noticeable central jet appeared between the leaflets in early diastole.

\section{$\underline{\text { Diastole }}$}

The flow profile at the start of the diastole, when the leaflets swing open, was characterized by a central jet and two lateral jets. Both the central and lateral jets then reached a velocity of approximately $1.0 \mathrm{~m} / \mathrm{s}$ at peak diastole (time bin 27, Figure 7-40). Maximum Reynolds shear stress levels of 400 dynes $/ \mathrm{cm}^{2}$ was recorded in the shear flow region between the central jet and the edge of the leaflets at mid-diastole. The Reynolds shear stress levels in the central and lateral jets were generally less than 100 dynes $/ \mathrm{cm}^{2}$ at 
the onset of the diastole and then reduced significantly to less than 10 dynes $/ \mathrm{cm}^{2}$ at middiastole.

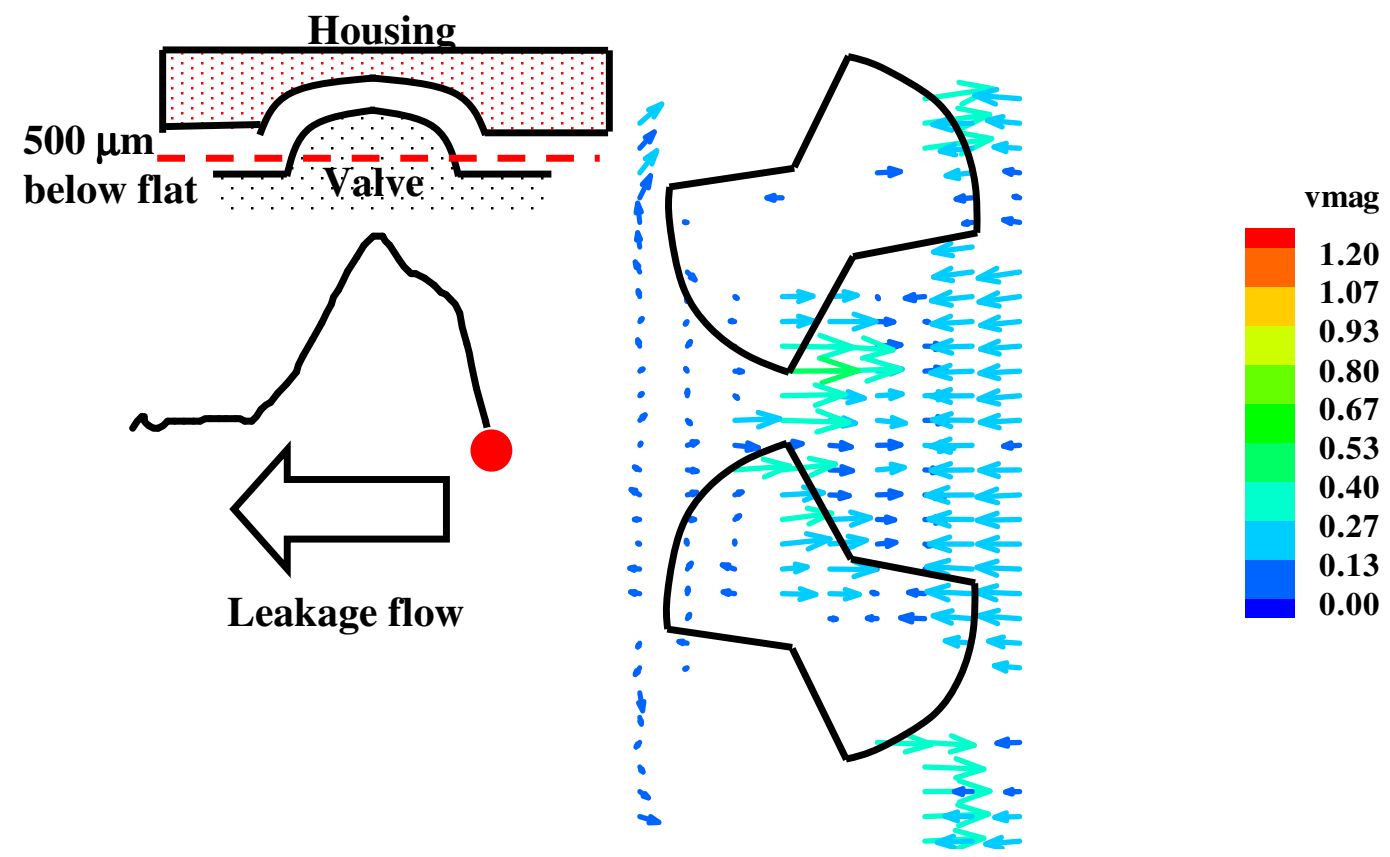

Figure 7-37 $27 \mathrm{~mm}$ prototype low leaker mitral hinge flow field at early systole, time bin $43,500 \mu \mathrm{m}$ below flat level; flow mixing occurred at between hinge mechanism at valve closure (avi, 712k, low_500um_below_flat_vmag.avi)
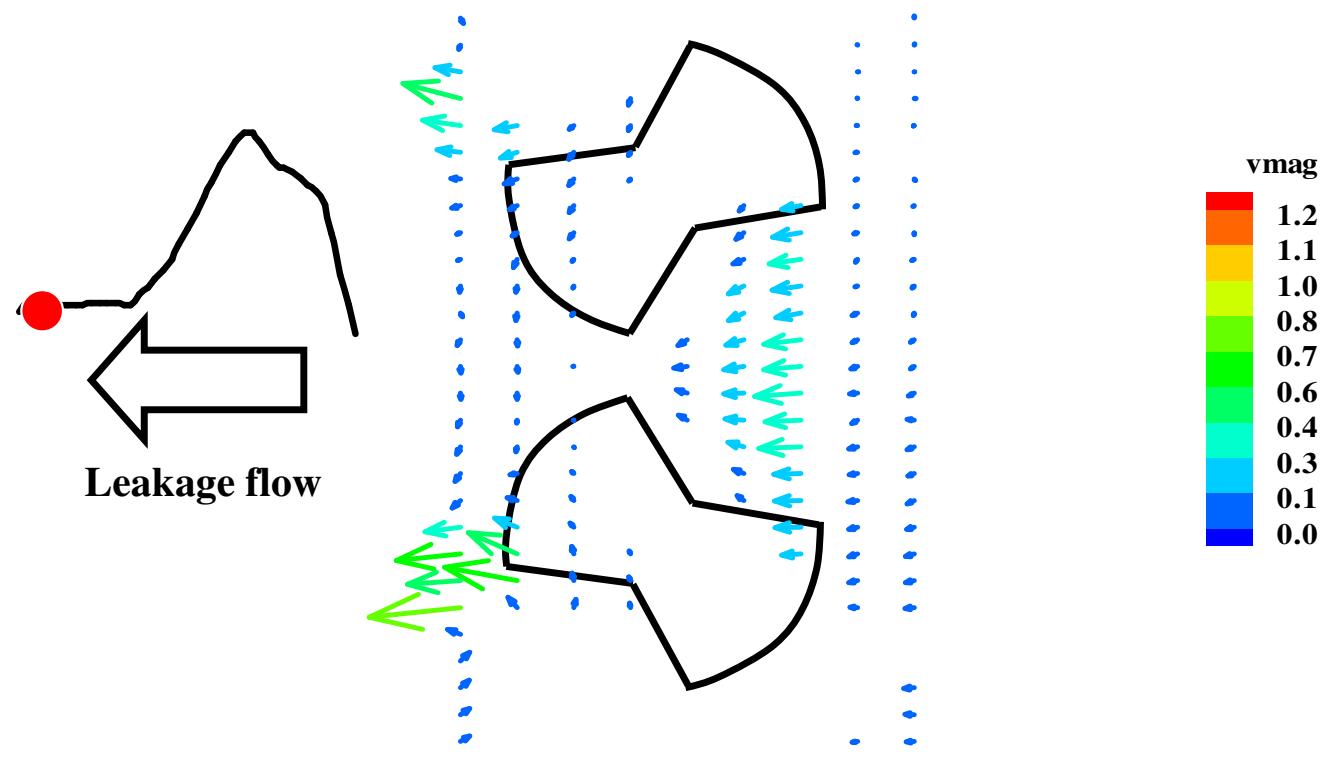

Figure 7-38 $27 \mathrm{~mm}$ prototype low leaker mitral hinge flow field approaching mid systole, time bin 4, $500 \mu \mathrm{m}$ below flat level; two prominent leakage jets issuing from the hinge pockets (avi, 712k, low_500um_below_flat_vmag.avi) 

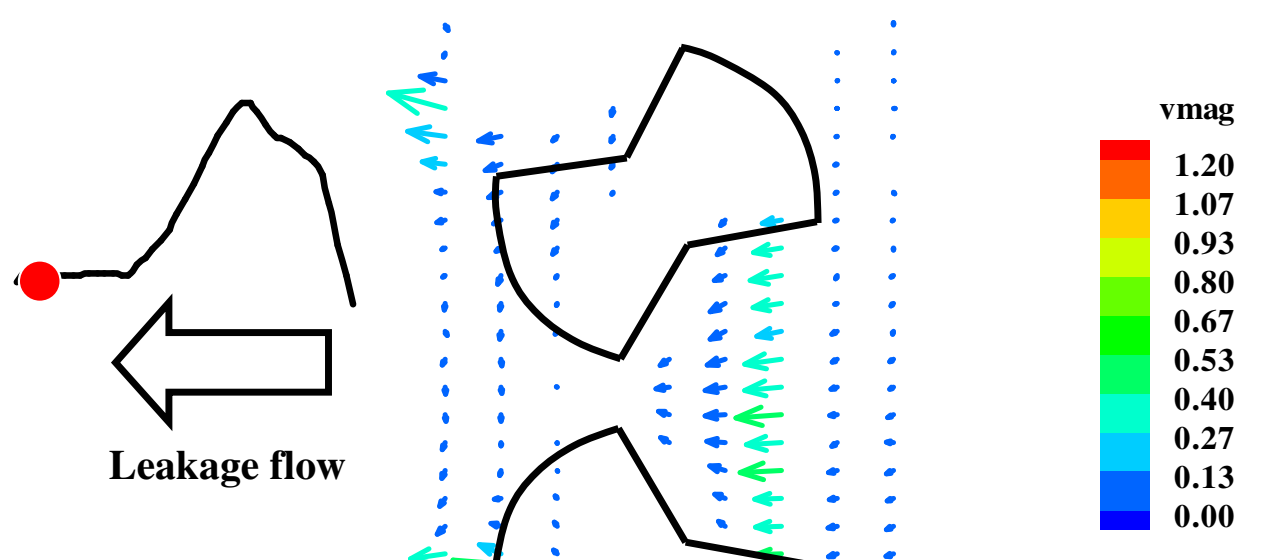

Figure 7-39 Reynolds shear stress distribution of the $27 \mathrm{~mm}$ prototype low leaker at time bin 4, $500 \mu \mathrm{m}$ below flat level; elevated Reynolds shear stress levels observed at the leakage jets emanating from the hinge region and the ventricular side of the hinge mechanism (avi, 708k, low_500um_below_flat_rss.avi)
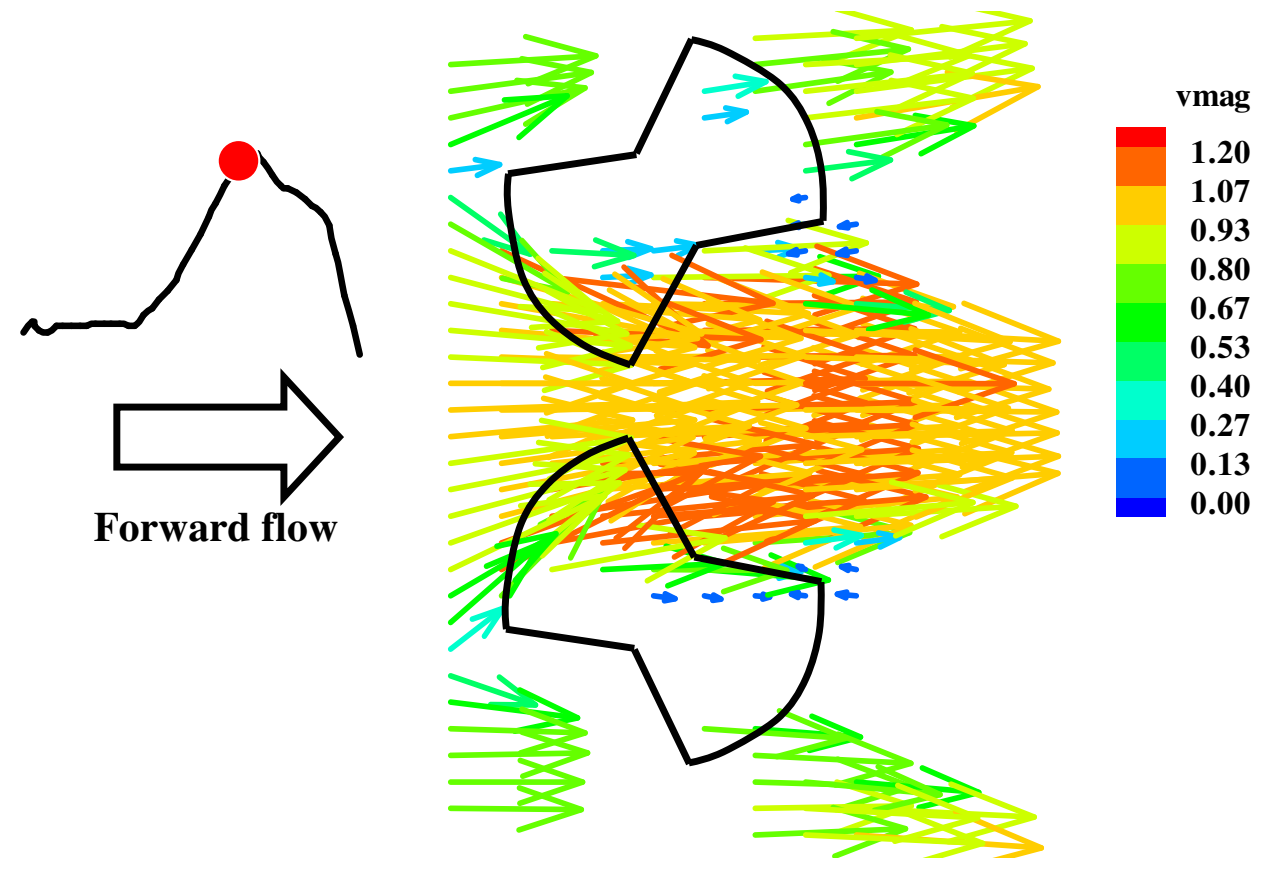

Figure 7-40 $27 \mathrm{~mm}$ prototype low leaker mitral hinge flow field at peak diastole, time bin $27,500 \mu \mathrm{m}$ below flat level; the opened leaflets split the forward flow into three separate forward jets (avi, 712k, low_500um_below_flat_vmag.avi) 


\subsubsection{5 $1 \mathrm{~mm}$ Below The Flat Level}

Animations low_1mm_below_flat_vmag.avi and low_1mm_below_flat_rss.avi show the velocity magnitude $(\mathrm{m} / \mathrm{s})$ and Reynolds shear stress $\left(\right.$ dynes $\left./ \mathrm{cm}^{2}\right)$ vector plots at the $1 \mathrm{~mm}$ below the flat level of the $27 \mathrm{~mm}$ prototype low leaker clear valve throughout the cardiac cycle, respectively. Refer to Appendix $\mathrm{H}$ for the list of animations.

\section{$\underline{\text { Systole }}$}

The flow field distributions at $1 \mathrm{~mm}$ below the flat level were similar to those at $500 \mu \mathrm{m}$ below the flat. The transition between systole and diastole was characterized by a mix of reverse flow from the ventricular side and forward flow from the atrial side at the center of the two leaflets. Figure 7-41 (time bin 1) shows the flow fields distribution at the $1 \mathrm{~mm}$ below flat level during valve closure. Retrograde flow appeared briefly between the closing leaflets during valve closure and reduced in magnitude from $0.6 \mathrm{~m} / \mathrm{s}$ to $0.2 \mathrm{~m} / \mathrm{s}$ when the leaflets fully shut. Lateral flow from the top side can be seen flowing towards the closed leaflets. Leakage jets emanating from one hinge pocket became apparent

during the systole (time bin 4, Figure 7-42). Maximum Reynolds shear stress was recorded at one of the leakage jets emanating from the hinge pockets. The shear stress levels during systole were less than 50 dynes $/ \mathrm{cm}^{2}$.

\section{$\underline{\text { Diastole }}$}

Three forward jets were observed during the diastolic phase. A velocity of $1.3 \mathrm{~m} / \mathrm{s}$ was measured in both the central and lateral jets at peak diastole, while that at the shear region was low at $0.05 \mathrm{~m} / \mathrm{s}$ (time bin 27, Figure 7-43). The corresponding Reynolds shear stress 
levels in the three forward flow jets was less than 50 dynes $/ \mathrm{cm}^{2}$, which was much lower than that $\left(200\right.$ dynes $\left./ \mathrm{cm}^{2}\right)$ measured in the shear layer region.

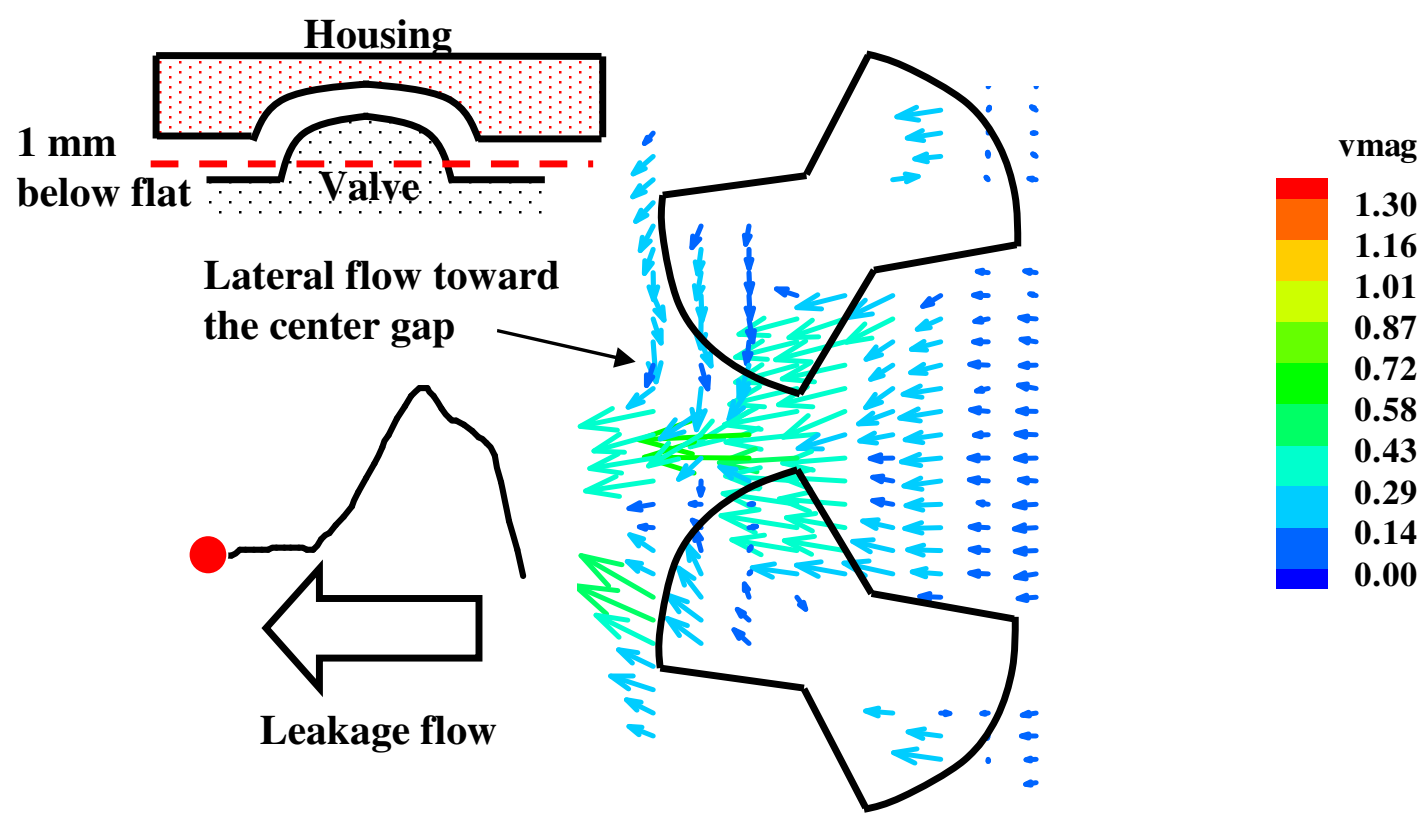

Figure 7-41 $27 \mathrm{~mm}$ prototype low leaker mitral hinge flow field at early systole, time bin 1, $1 \mathrm{~mm}$ below flat level; leakage flow visible at the gap between closing leaflets (avi, 703k, low_1mm_below_flat_vmag.avi) 


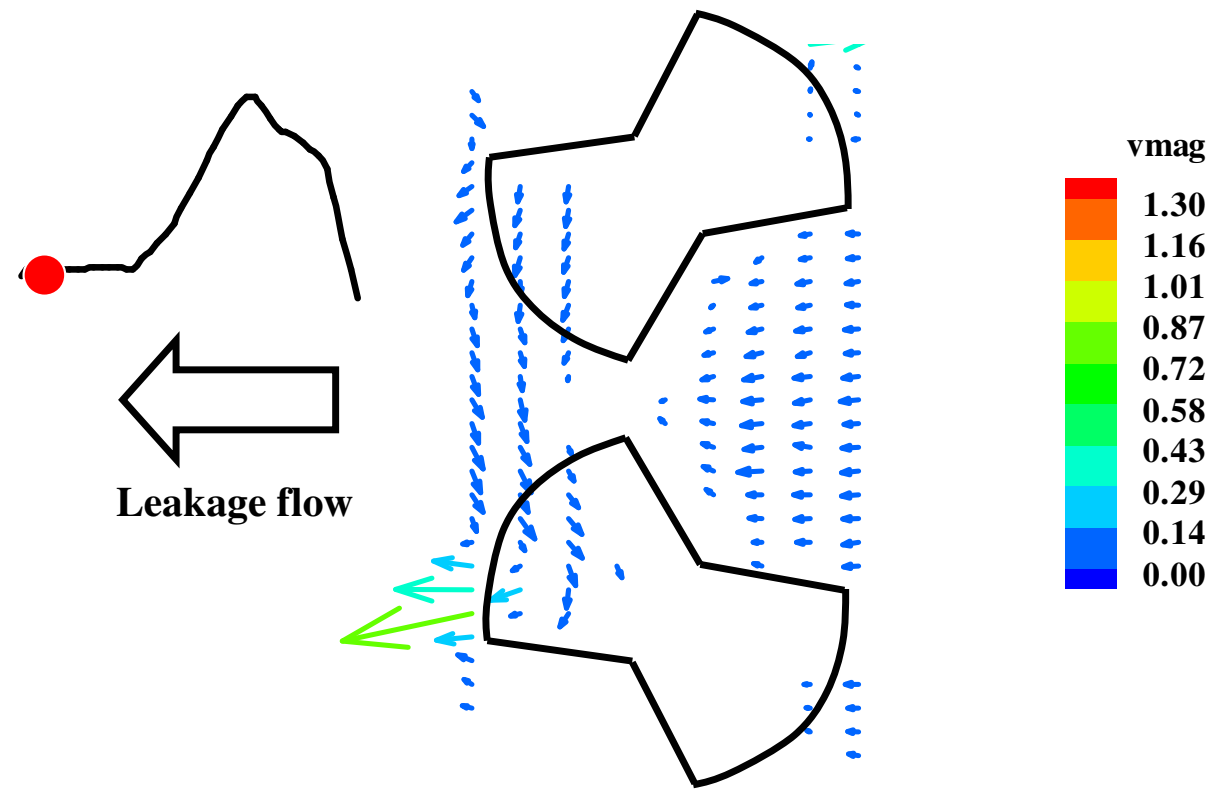

Figure 7-42 $27 \mathrm{~mm}$ prototype low leaker mitral hinge flow field toward mid systole, time bin 4, $1 \mathrm{~mm}$ below flat level; single leakage issuing from hinge pocket (avi, 703k, low_1mm_below_flat_vmag.avi)
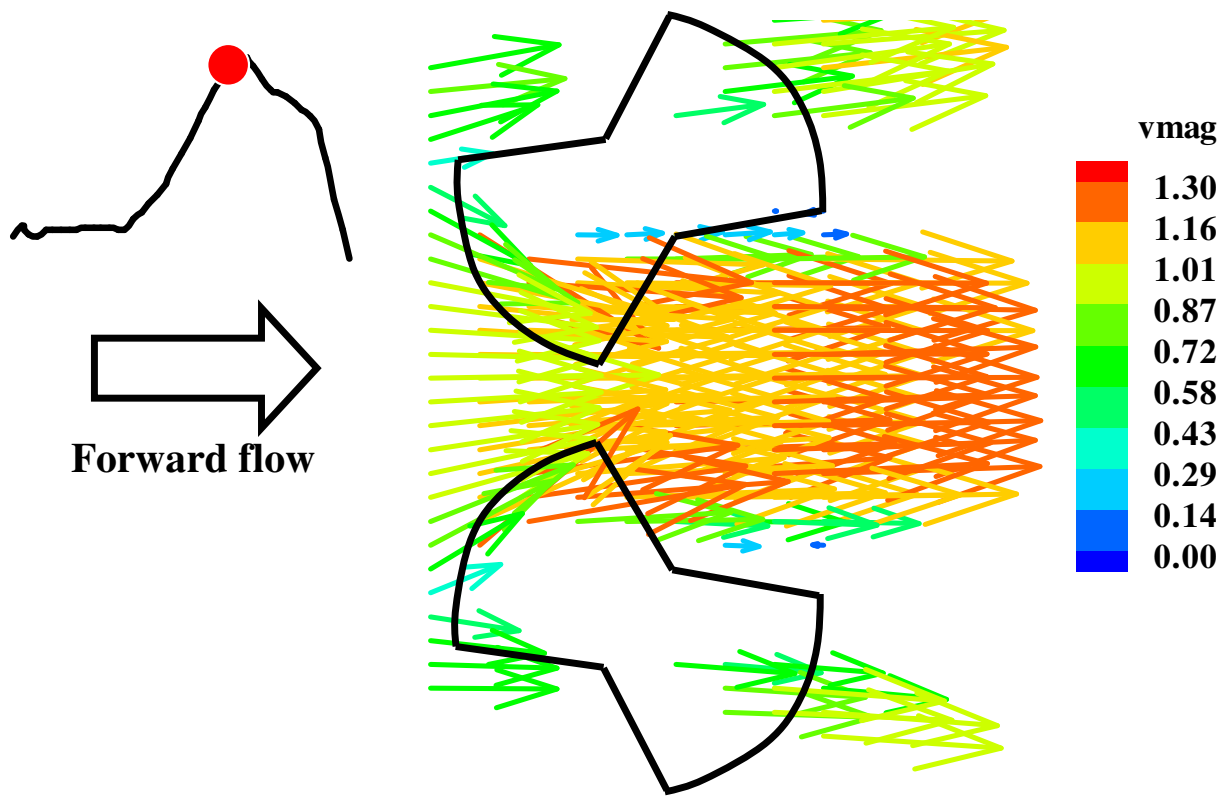

Figure 7-43 $27 \mathrm{~mm}$ prototype low leaker mitral hinge flow field at mid diastole, time bin $27,1 \mathrm{~mm}$ below flat level; the velocity of the central stream reached $1.3 \mathrm{~m} / \mathrm{s}$ at mid diastole, while that at the shear region was low at $0.05 \mathrm{~m} / \mathrm{s}$ (avi, $703 \mathrm{k}$, low_1mm_below_flat_vmag.avi) 


\subsubsection{6 $3 \mathrm{~mm}$ Below The Flat Level}

Animations low_3mm_below_flat_vmag.avi and low_3mm_below_flat_rss.avi show the velocity magnitude $(\mathrm{m} / \mathrm{s})$ and Reynolds shear stress $\left(\right.$ dynes $\left./ \mathrm{cm}^{2}\right)$ vector plots at the $3 \mathrm{~mm}$ below the flat level of the $27 \mathrm{~mm}$ prototype low leaker clear valve throughout the cardiac cycle, respectively. Refer to Appendix $\mathrm{H}$ for the list of animations.

\section{Systole}

Figure 7-44 (time bin 42) shows the flow field at valve closure. Unsteady flow and a small retrograde flow were observed, respectively, in the middle of the hinge mechanism and the closing leaflet gap. Towards mid systole, the velocity of the small retrograde flow at the middle of the hinge mechanism reduced abruptly from $0.4 \mathrm{~m} / \mathrm{s}$ to less than $0.01 \mathrm{~m} / \mathrm{s}$. No retrograde flow was evident in the measurement level during mid and late systole. Maximum Reynolds shear stress of 633 dynes $/ \mathrm{cm}^{2}$ was recorded in the retrograde flow at valve closure. The flow velocity at this measurement level after valve closure was typically less than $0.01 \mathrm{~m} / \mathrm{s}$ with Reynolds shear stress levels of approximately 7 dynes $/ \mathrm{cm}^{2}$.

\section{$\underline{\text { Diastole }}$}

Three forward jets were observed during the diastolic phase. Peak velocities of $1.35 \mathrm{~m} / \mathrm{s}$ and $0.91 \mathrm{~m} / \mathrm{s}$ were measured at the central jet and lateral jets, respectively, during middiastole. Shear flow region between the central jet and the leaflets was not evident at this measurement level (time bin 27, Figure 7-45). The Reynolds shear stress levels inside 
both the central and lateral jets during diastole were low ranging between 10 and 50 dynes $/ \mathrm{cm}^{2}$.
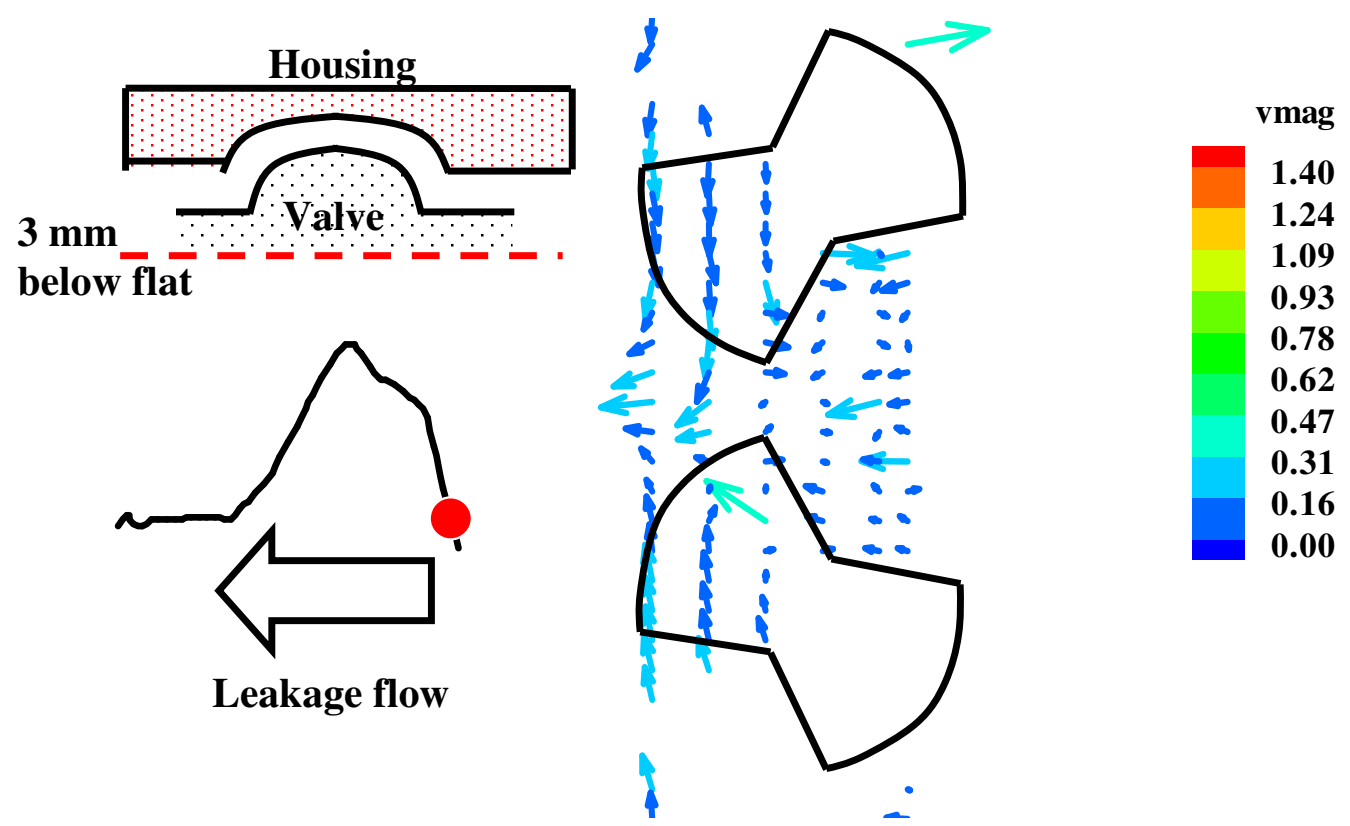

Figure 7-44 $27 \mathrm{~mm}$ prototype low leaker mitral hinge flow field at early systole, time bin 42, $3 \mathrm{~mm}$ below flat level; disturb flow at valve closure (avi, 643k, low_3mm_below_flat_vmag.avi)
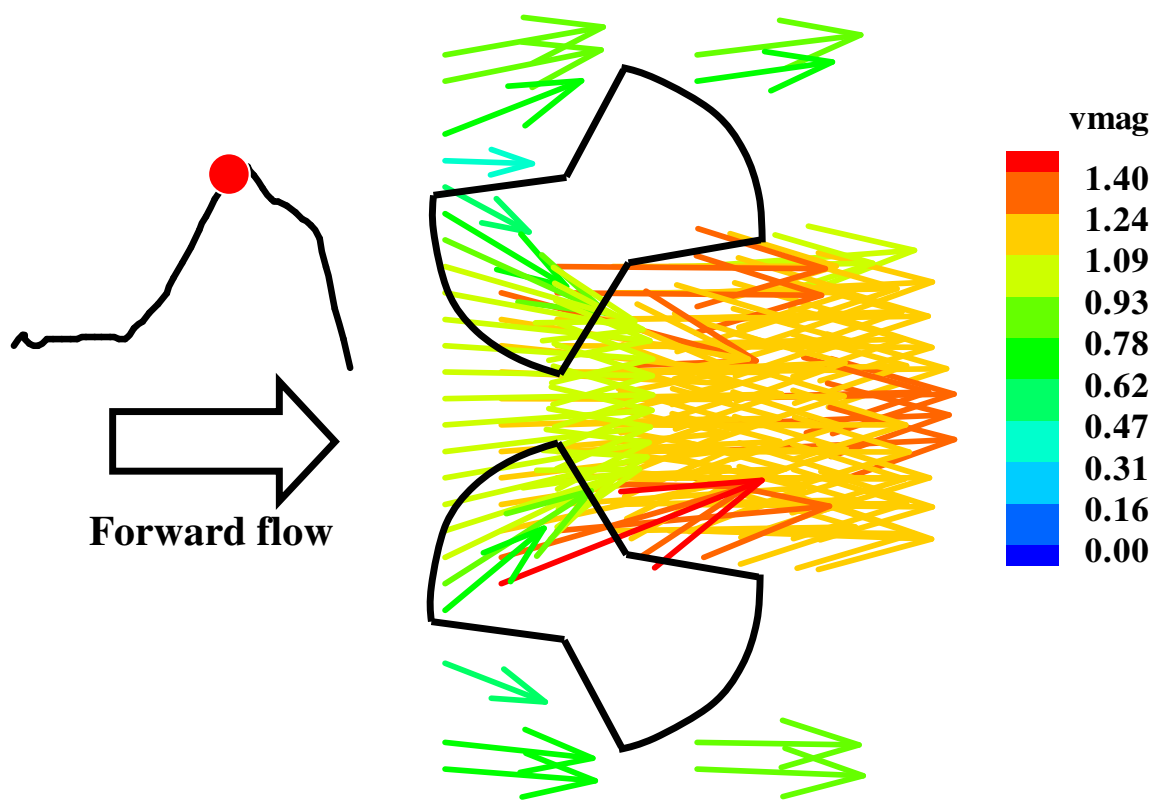

Figure 7-45 $27 \mathrm{~mm}$ prototype low leaker mitral hinge flow field at mid diastole, time bin $27,3 \mathrm{~mm}$ below flat level; maximum forward velocity of $1.35 \mathrm{~m} / \mathrm{s}$ was measured at the central flow stream at mid diastole (avi, 643k, low_3mm_below_flat_vmag.avi) 


\subsection{Downstream of The Thumbnail Region}

Animations low_thumbnail_vmag.avi and low_thumbnail_rss.avi show the velocity magnitude $(\mathrm{m} / \mathrm{s})$ and Reynolds shear stress $\left(\right.$ dynes $\left./ \mathrm{cm}^{2}\right)$ vector plots at the downstream of the thumbnail region of the $27 \mathrm{~mm}$ prototype low leaker clear valve throughout the cardiac cycle, respectively. Refer to Appendix $\mathrm{H}$ for the list of animations.

\section{$\underline{\text { Systole }}$}

Figure 7-46 time bin 1) shows the flow distribution at the start of the systole. Retrograde flow of approximately $0.25 \mathrm{~m} / \mathrm{s}$ was seen immediately downstream of the thumbnail region. The reverse flow then reduced to a velocity of $0.06 \mathrm{~m} / \mathrm{s}$ at peak systole with a corresponding Reynolds shear stress of less than 15 dynes $/ \mathrm{cm}^{2}$.

\section{$\underline{\text { Diastole }}$}

The acceleration phase at time bin 23 (Figure 7-47) was characterized by complex flow structures with a center low flow region $(0.1 \mathrm{~ms})$ flanked by stronger lateral forward flows $(0.35 \mathrm{~m} / \mathrm{s})$. As the cycle progresses towards peak diastole, the velocity vectors in the stronger lateral jets reversed in direction, giving rise to a shear layer between the central and lateral jets. A maximum Reynolds shear stress of 100 dynes $/ \mathrm{cm}^{2}$ was recorded at the shear layer, while shear stress levels of less than $10 \mathrm{dynes} / \mathrm{cm}^{2}$ was measured inside both the central and lateral jets. 


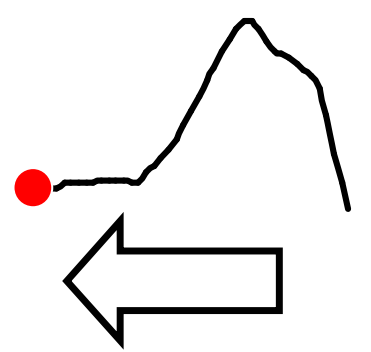

Leakage flow
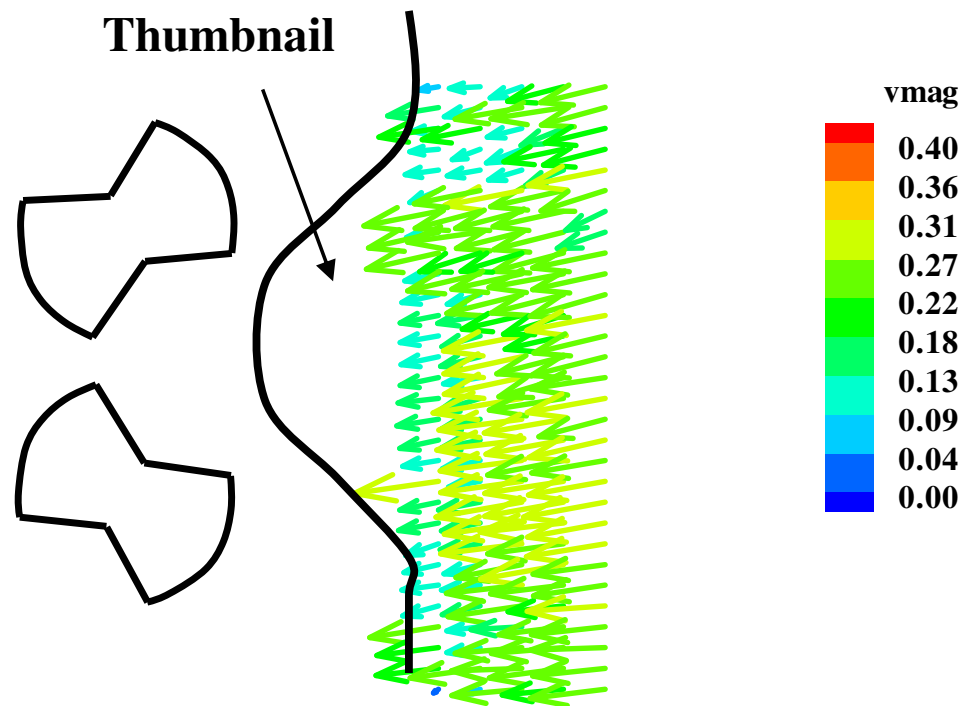

Figure 7-46 $27 \mathrm{~mm}$ prototype low leaker mitral hinge flow field at early systole, time bin 1, downstream of thumbnail region, flat level; disturbed flow at the lower part of the measurement field (avi, 733k, low_thumbnail_vmag.avi)

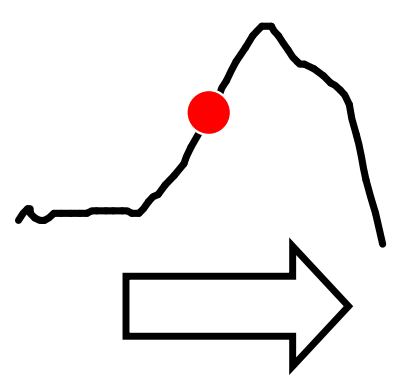

Forward flow

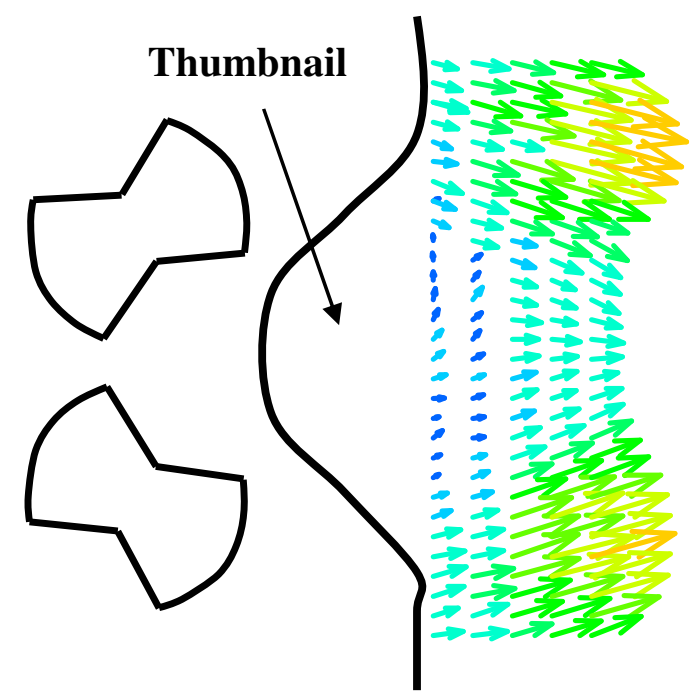

Figure 7-47 $27 \mathrm{~mm}$ prototype low leaker mitral hinge flow field approaching mid diastole, time bin 23, downstream of thumbnail region, flat level; inversion of the lateral jets into leakage flow (avi, 733k, low_thumbnail_vmag.avi) 


\subsubsection{27 mm Prototype High Leaker}

The results are presented in the form of color pictures, which illustrate the flow fields and Reynolds shear stress vectors plots at specific instances during the cardiac cycle. The measurement levels are presented in the following sequence; 1) primary flat; 2) levels above the primary flat; 3) levels below the primary flat; and 4) downstream of the thumbnail. For each measurement plane, the description of the flow during systole is presented first followed by that during diastole. The direction of the forward flow is from left to right (from the left atrium to left ventricle, Figure 5-13). All vector plots are colorcoded by Velocity Magnitude $(\mathrm{m} / \mathrm{s})$ and Reynolds shear stress $\left(\right.$ dynes $/ \mathrm{cm}^{2}$ ). Arrows point in the direction of the phase averaged mean velocity vectors and the length of the arrow is proportional to the velocity magnitude. Refer to Appendix $\mathrm{H}$ for the list of velocity magnitude $(\mathrm{m} / \mathrm{s})$ and Reynolds shear stress $\left(\right.$ dynes $\left./ \mathrm{cm}^{2}\right)$ animations. Terminology pertinent to the hinge design is shown in Figure 7-26 for orientation purposes. Refer to Figure 7-3 in section 7.1.2.1 for the position of the leaflet at mid acceleration, peak systole as well as valve closure and mid diastole within the $27 \mathrm{~mm}$ prototype high leaker clear valve hinge. These leaflet positions correspond to those in the images presented later. For clarity the leaflets are not shown on the color printouts of the velocity field.

\subsection{Flat Level}

Animations high_flat_vmag.avi and high_flat_rss.avi show the velocity magnitude $(\mathrm{m} / \mathrm{s})$ and Reynolds shear stress (dynes $/ \mathrm{cm}^{2}$ ) vector plots at the flat level of the $27 \mathrm{~mm}$ prototype high leaker clear valve throughout the cardiac cycle, respectively. Refer to Appendix $\mathrm{H}$ for the list of animations. 


\section{Systole}

Figure 7-48 (time bin 1) shows the flow field distribution at the early stage of the systole. The flow patterns at the flat level were similar to those measured in the SJM $27 \mathrm{~mm}$ Standard and $27 \mathrm{~mm}$ prototype low leaker valves, except for higher velocity magnitude and larger leakage jets in both the atrial and adjacent corners. The two leakage jets appear to merge into a single leakage jet occupying a larger part of the atrial side. On the ventricular side, the flow diversion was more pronounced compared to the SJM $27 \mathrm{~mm}$ Standard and $27 \mathrm{~mm}$ prototype low leaker valves. Approaching mid systole at time bin 4 (Figure 7-49) the atrial corner jet reached a maximum velocity of $1.86 \mathrm{~m} / \mathrm{s}$, while the peak velocities in the adjacent and lateral corners jets were 1.57 and $0.96 \mathrm{~m} / \mathrm{s}$, respectively. At end systole, the leakage jets at the atrial and lateral corners had reduced in size and their velocity decelerated to less than $0.4 \mathrm{~m} / \mathrm{s}$, while the leakage jet observed in the adjacent corner reduced to about $0.8 \mathrm{~m} / \mathrm{s}$. Throughout systole a region of low flow $(<0.2 \mathrm{~m} / \mathrm{s})$ was observed along the atrial side hinge wall. The Reynolds shear stress reached 4,000 dynes $/ \mathrm{cm}^{2}$ in the adjacent jet, while in both the atrial and lateral jets, the peak Reynolds shear stress recorded were 3 to 5 times smaller at 1,440 and 700 dynes $/ \mathrm{cm}^{2}$, respectively (time bin 4 , Figure 7-50). The Reynolds shear stress levels recorded on the ventricular corner were less than $10 \mathrm{dynes} / \mathrm{cm}^{2}$.

\section{Diastole}

The flat level velocities in the prototype high leaker valve during diastole were lower than those in the SJM $27 \mathrm{~mm}$ Standard and $27 \mathrm{~mm}$ prototype low leaker valve. Flow velocities throughout the hinge during diastole were typically lower than $0.04 \mathrm{~m} / \mathrm{s}$, except 
in the recirculation zone at the adjacent corner, which reach a maximum forward flow velocity of $0.2 \mathrm{~m} / \mathrm{s}$ (time bin 27, Figure 7-51). The Reynolds shear stress levels throughout the hinge during diastole were typically less than 10 dynes $/ \mathrm{cm}^{2}$.

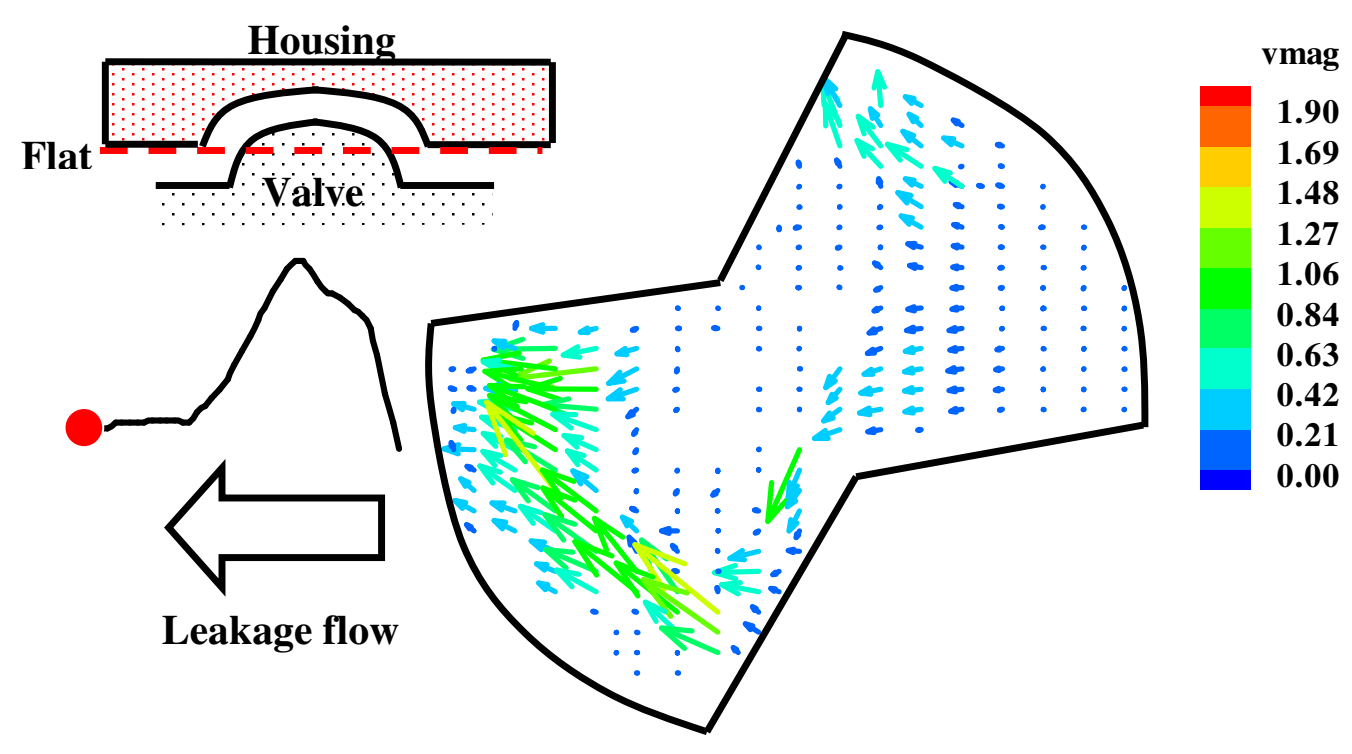

Figure 7-48 $27 \mathrm{~mm}$ prototype high leaker mitral hinge flow field at early systole, time bin 1, flat level; leakage jets at both the atrial and adjacent corner appeared to merge into a single jet occupying a large part of the atrial side (avi, 719k, high_flat_vmag.avi) 


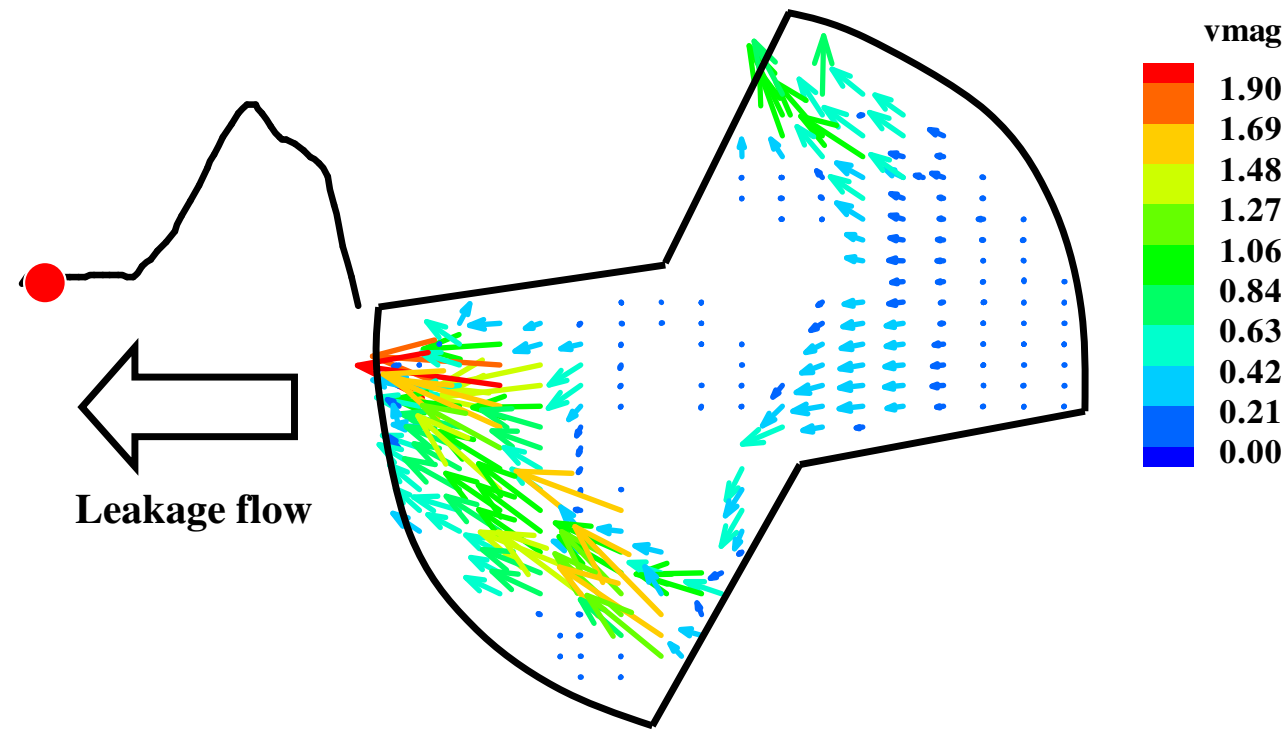

Figure 7-49 $27 \mathrm{~mm}$ prototype high leaker mitral hinge flow field approaching mid systole, time bin 4, flat level; the leakage jets at atrial and adjacent corners appeared to merge into a larger jet (avi, 719k, high_flat_vmag.avi)

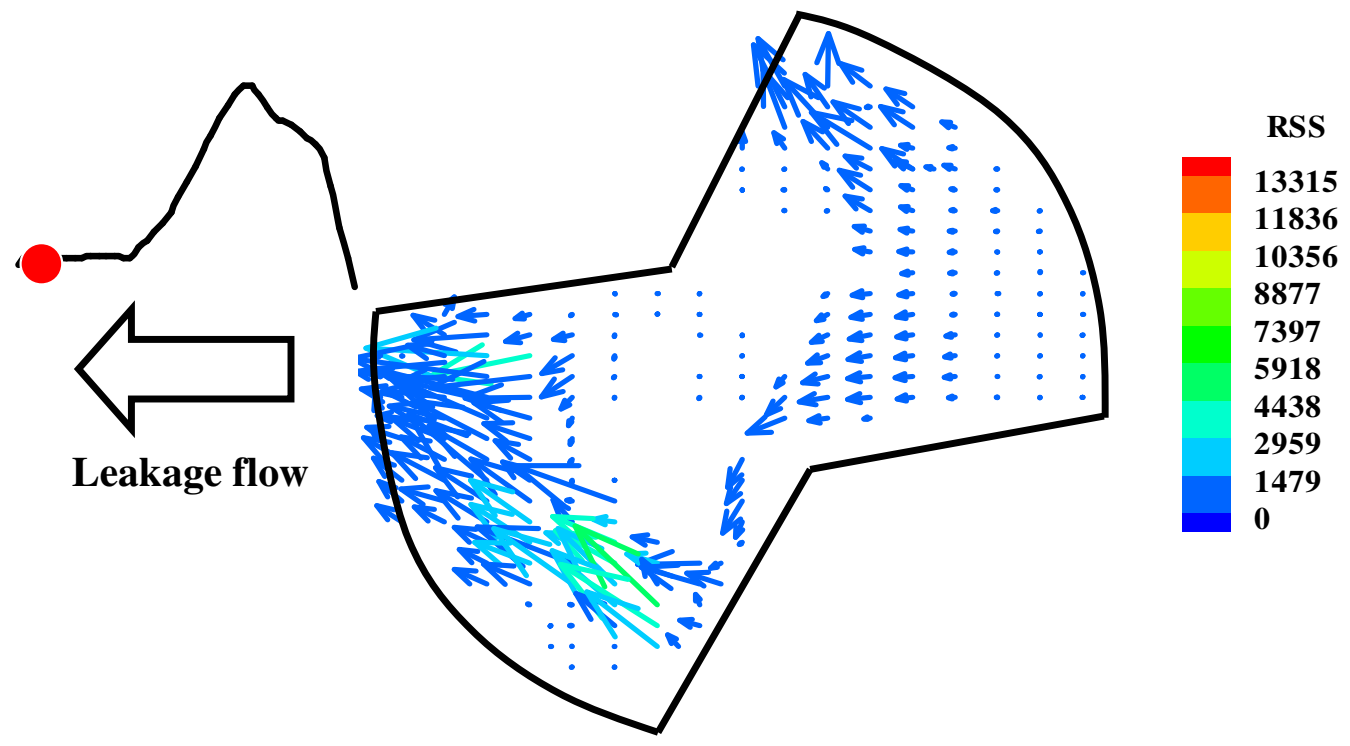

Figure 7-50 Reynolds shear stress distribution of the $27 \mathrm{~mm}$ prototype high leaker at time bin 4, flat level; elevated Reynolds shear stress levels observed at the atrial and lateral corners of the hinge geometry (avi, 734k, high_flat_rss.avi) 

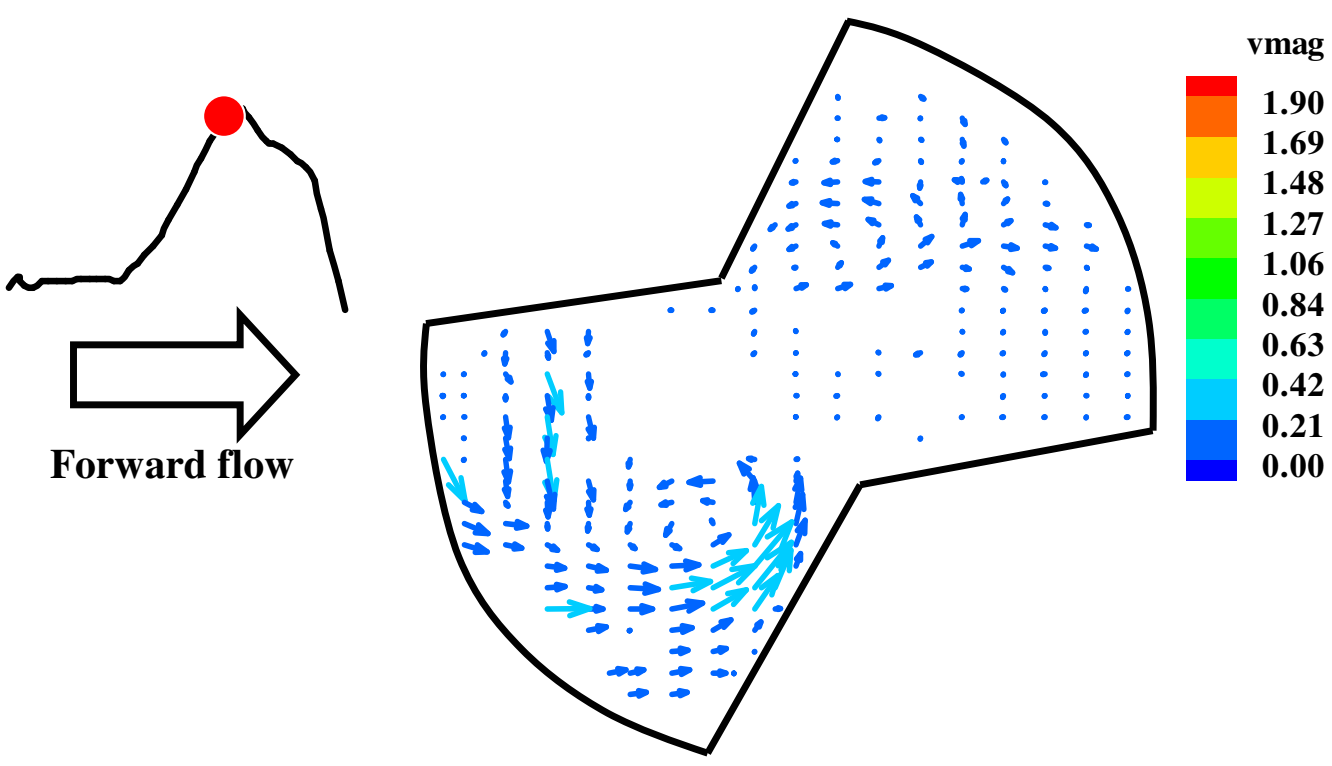

Figure 7-51 $27 \mathrm{~mm}$ prototype high leaker mitral hinge flow field at mid diastole, time bin 27, flat level; recirculation flow appeared at the adjacent corner of hinge geometry (avi, 719k, high_flat_vmag.avi)

\subsection{0 $\mu \mathrm{m}$ Above The Flat Level}

Animations high_190um_above_flat_vmag.avi and high_190um_above_flat_rss.avi show the velocity magnitude $(\mathrm{m} / \mathrm{s})$ and Reynolds shear stress $\left(\right.$ dynes $\left./ \mathrm{cm}^{2}\right)$ vector plots at the $190 \mu \mathrm{m}$ above the flat level of the $27 \mathrm{~mm}$ prototype high leaker clear valve throughout the cardiac cycle, respectively. Refer to Appendix $\mathrm{H}$ for the list of animations.

\section{$\underline{\text { Systole }}$}

Figure 7-52 (time bin 1) shows the flow patterns at $190 \mu \mathrm{m}$ above the flat level during early systole. Leakage jets were seen in the hinge in three different regions: the atrial corner close to the edge of the leaflet ear, the adjacent corner, and at the ventricular corner next to the leaflet. An area of low flow $(<0.2 \mathrm{~m} / \mathrm{s})$ was seen along the atrial corner wall. The observed flow pattern was similar to that seen at the flat level, and also in both 
the flat and $190 \mu \mathrm{m}$ above the flat levels in the SJM $27 \mathrm{~mm}$ Standard and $27 \mathrm{~mm}$ prototype low leaker valves. The leakage flow in the ventricular side reached a maximum velocity of $1.95 \mathrm{~m} / \mathrm{s}$ towards mid systole (time bin 4 , Figure 7-53), while those at the atrial and adjacent corners fluctuated between 0.3 and $1.2 \mathrm{~m} / \mathrm{s}$. The observed flow profile persisted till late systole before abruptly decreased to less than $0.1 \mathrm{~m} / \mathrm{s}$ at the beginning of diastole. A stream of leakage flow, not seen in other valves at the same measurement level, was observed during late systole between the leaflet ear and the hinge wall. This leakage flow had a velocity of $0.3 \mathrm{~m} / \mathrm{s}$ and a corresponding maximum Reynolds shear stress value of 7,625 dynes $/ \mathrm{cm}^{2}$. The Reynolds shear stress levels of the leakage jets at the atrial and adjacent corners lies between the range of 1,000 to $7,500 \mathrm{dynes} / \mathrm{cm}^{2}$, while those in the ventricular and lateral corners were lower and ranged between 500 and 2,500 dynes/cm² (time bin 4, Figure 7-54)

\section{$\underline{\text { Diastole }}$}

The flow velocity during diastole was typically less than $0.02 \mathrm{~m} / \mathrm{s}$ throughout the entire hinge region and the corresponding Reynolds shear stress were less than 15 dynes $/ \mathrm{cm}^{2}$ (time bin 27, Figure 7-55). A recirculation region was observed at the adjacent corner at mid diastole, and persisted throughout the diastole. 


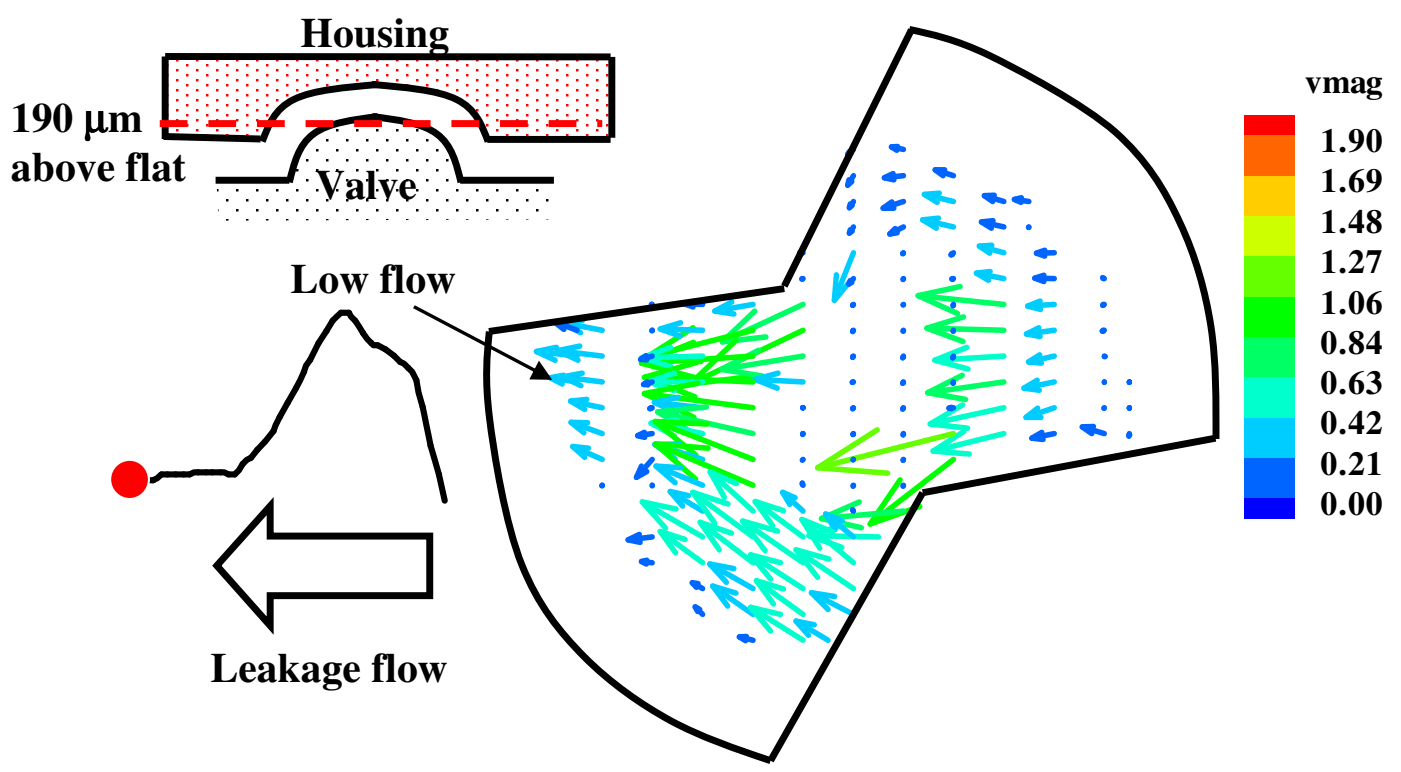

Figure 7-52 $27 \mathrm{~mm}$ prototype high leaker mitral hinge flow field at early systole, time bin $1,190 \mu \mathrm{m}$ above flat level; low flow of less than $0.2 \mathrm{~m} / \mathrm{s}$ observed close to the atrial corner (avi, 694k, high_190um_above_flat_vmag.avi)

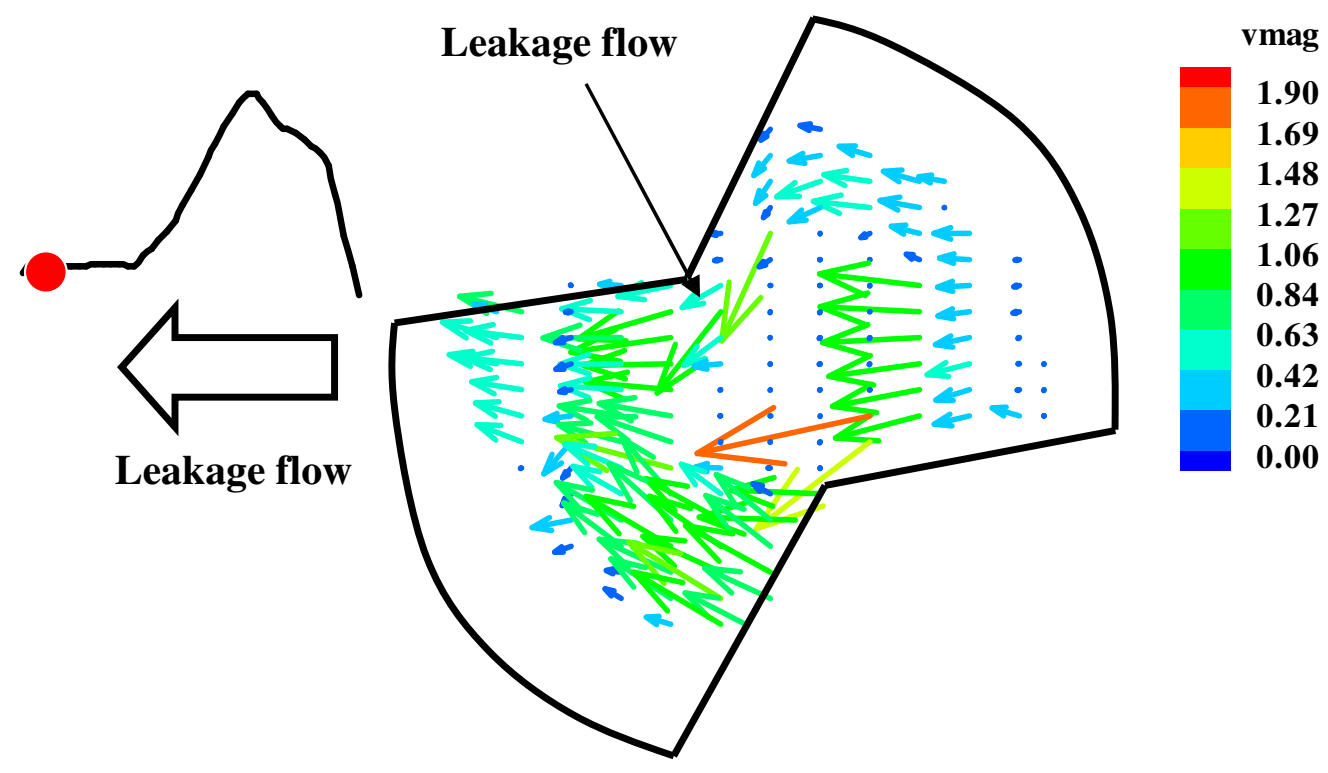

Figure 7-53 $27 \mathrm{~mm}$ prototype high leaker mitral hinge flow field toward mid systole, time bin 4, $190 \mu \mathrm{m}$ above flat level; leakage flow appeared to flow along the edge of the leaflet ear (avi, 694k, high_190um_above_flat_vmag.avi) 


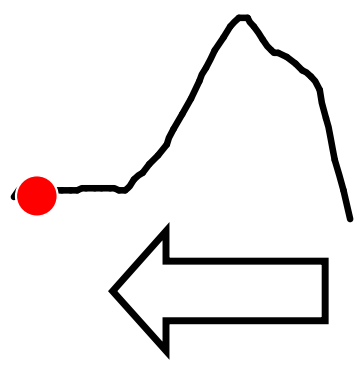

Leakage flow

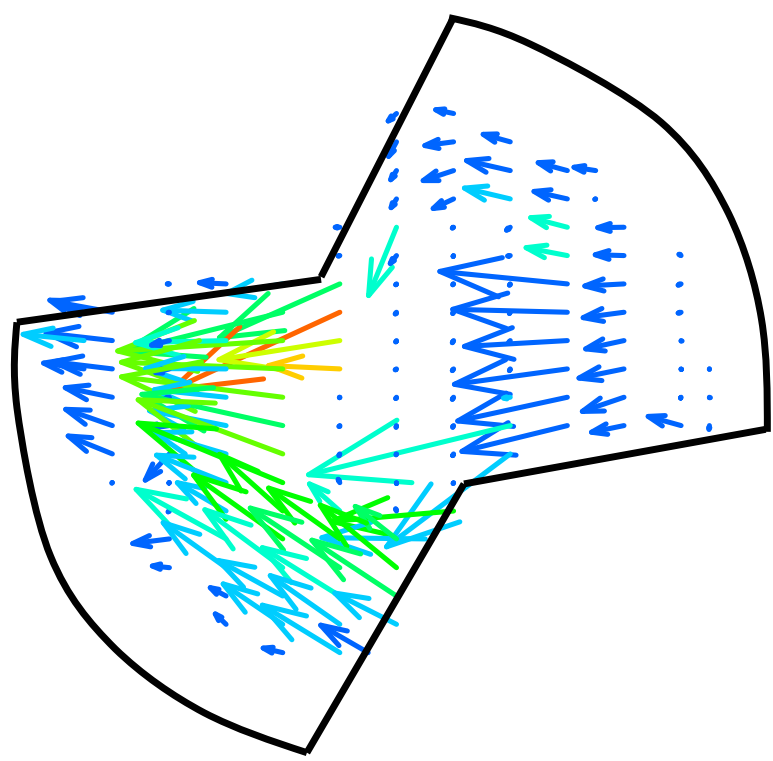

RSS

3000

2667

2333

2000

1667

1333

1000

667

333

0

Figure 7-54 Reynolds shear stress distribution of the $27 \mathrm{~mm}$ prototype high leaker at time bin 4, $190 \mu \mathrm{m}$ above level; elevated Reynolds shear stress levels of at least 500 dynes $/ \mathrm{cm}^{2}$ observed throughout at the hinge region (avi, 710k, high_190um_above_flat_rss.avi)

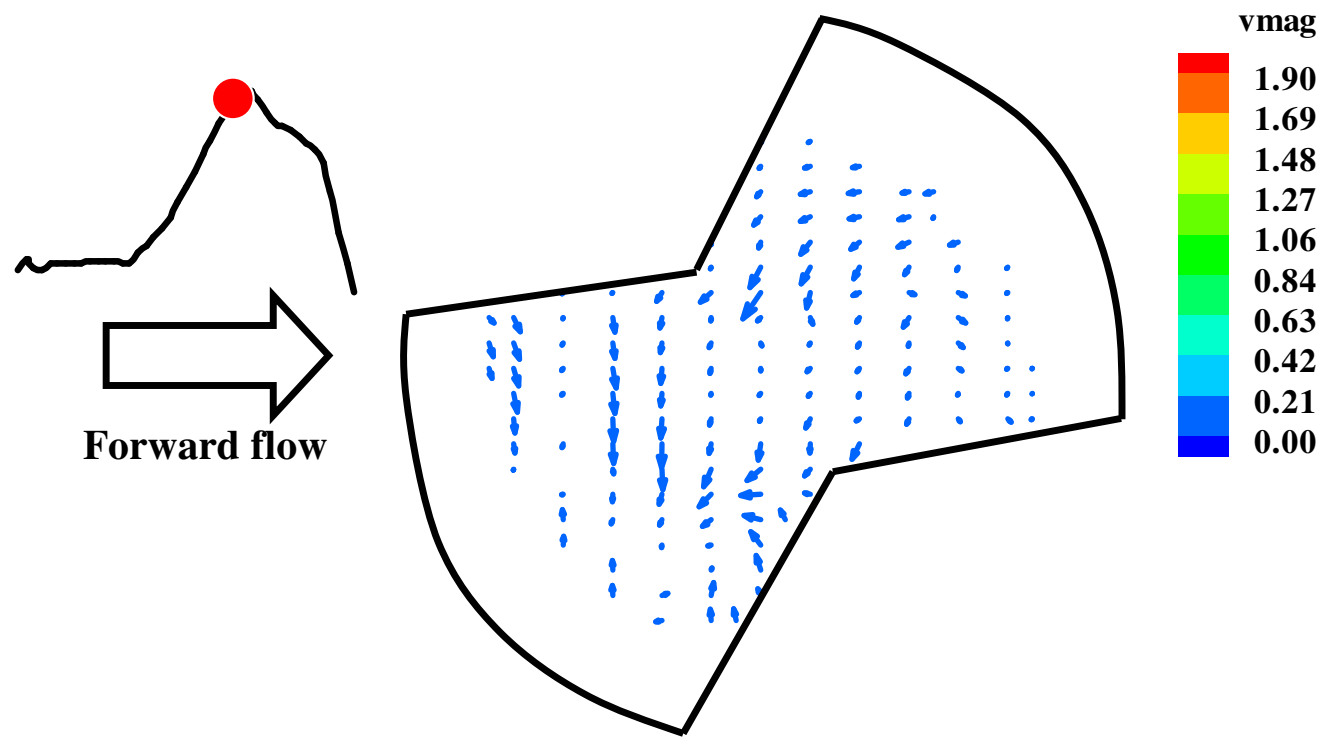

Figure 7-55 $27 \mathrm{~mm}$ prototype high leaker mitral hinge flow field at mid diastole, time bin 27, $190 \mu \mathrm{m}$ above flat level; low recirculation flow observed at the adjacent corner (avi, 694k, high_190um_above_flat_vmag.avi) 


\subsubsection{3 $390 \mu \mathrm{m}$ Above The Flat Level}

Animations high_390um_above_flat_vmag.avi and high_390um_above_flat_rss.avi show the velocity magnitude $(\mathrm{m} / \mathrm{s})$ and Reynolds shear stress $\left(\right.$ dynes $\left./ \mathrm{cm}^{2}\right)$ vector plots at the $390 \mu \mathrm{m}$ above the flat level of the $27 \mathrm{~mm}$ prototype high leaker clear valve throughout the cardiac cycle, respectively. Refer to Appendix $\mathrm{H}$ for the list of animations.

Figure 7-56 (time bin 9) shows the flow distribution during mid systole. The flow velocities during systole in the hinge region were typically less than $0.01 \mathrm{~m} / \mathrm{s}$ with corresponding Reynolds shear stress levels of less than 5 dynes $/ \mathrm{cm}^{2}$, except for the leakage flow observed at the ventricular side. The leakage jet reached a maximum velocity of $0.85 \mathrm{~m} / \mathrm{s}$ and a corresponding maximum Reynolds shear stress of 1,147 dynes $/ \mathrm{cm}^{2}$ at mid systole. Flow velocities during diastole were less than $0.02 \mathrm{~m} / \mathrm{s}$ with Reynolds shear stress level close to zero.

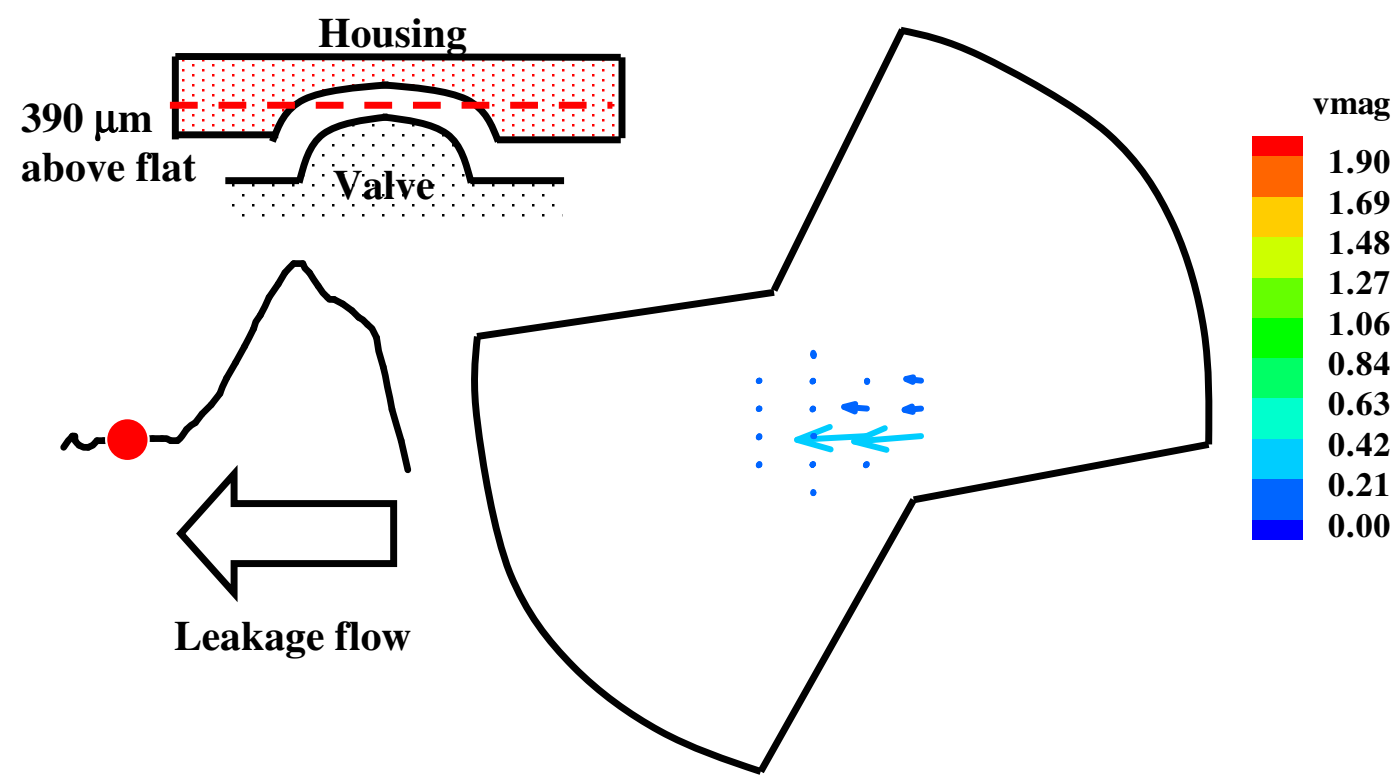

Figure 7-56 $27 \mathrm{~mm}$ prototype high leaker mitral hinge flow field at mid systole, time bin 9, $390 \mu \mathrm{m}$ above flat level (avi, 615k, high_390um_above_flat_vmag.avi) 


\subsection{5 $\mu \mathrm{m}$ Above The Flat Level}

Animations high_585um_above_flat_vmag.avi and high_585um_above_flat_rss.avi show the velocity magnitude $(\mathrm{m} / \mathrm{s})$ and Reynolds shear stress $\left(\right.$ dynes $\left./ \mathrm{cm}^{2}\right)$ vector plots at the $585 \mu \mathrm{m}$ above the flat level of the $27 \mathrm{~mm}$ prototype high leaker clear valve throughout the cardiac cycle, respectively e. Refer to Appendix $\mathrm{H}$ for the list of animations.

The velocities in the hinge region were typically less than $0.06 \mathrm{~m} / \mathrm{s}$ throughout the cardiac cycle, with a corresponding Reynolds shear stress of less than 5 dynes $/ \mathrm{cm}^{2}$. The exception was a single measurement location near the atrial side of the hinge geometry, which reached a Reynolds shear stress of 115 dynes $/ \mathrm{cm}^{2}$ during late systole.

\subsection{0 $\mu \mathrm{m}$ Below The Flat Level}

Animations high_500um_below_flat_vmag.avi and high_500um_below_flat_rss.avi show the velocity magnitude $(\mathrm{m} / \mathrm{s})$ and Reynolds shear stress $\left(\mathrm{dynes} / \mathrm{cm}^{2}\right)$ vector plots at the $500 \mu \mathrm{m}$ below the flat level of the $27 \mathrm{~mm}$ prototype high leaker clear valve throughout the cardiac cycle, respectively. Refer to Appendix $\mathrm{H}$ for the list of animations.

\section{Systole}

Figure 7-57 (time bin 43) shows the flow profile at $500 \mu \mathrm{m}$ below the flat level at valve closure. Leakage flow can be seen flowing through the gap between the leaflets. Three jets were visible at time bin 4 immediately before mid systole (Figure 7-58), after valve closure. Two leakage jets were observed to emanate from the hinge region and one between the closed leaflets. The leakage jets emanating from the hinge regions reached a 
peak velocity of $1.0 \mathrm{~m} / \mathrm{s}$ at mid systole with a corresponding Reynolds shear stress level of $1,320 \mathrm{dynes} / \mathrm{cm}^{2}$. The central leakage flow reached a velocity of approximately 0.25 $\mathrm{m} / \mathrm{s}$ with a Reynolds shear stress value of approximately 100 dynes $/ \mathrm{cm}^{2}$ (time bin 4, Figure 7-59). Flow velocities on the ventricular side were typically low at $0.06 \mathrm{~m} / \mathrm{s}$ with Reynolds shear stress levels of less than 5 dynes $/ \mathrm{cm}^{2}$.

\section{$\underline{\text { Diastole }}$}

A small central jet of $0.2 \mathrm{~m} / \mathrm{s}$ was observed between the opening leaflets at early diastole (time bin 20). The central jet then increased in both size and velocity towards mid diastole, drawing in the surrounding fluid. The central jet reached a peak velocity of 1.3 $\mathrm{m} / \mathrm{s}$ at peak systole (time bin 27 , Figure 7-60). Region of high velocity gradient was seen between the central jet and the lateral jets, and persisted through the end of diastole. The calculated Reynolds shear stress in this shear layer region was 250 dyne $/ \mathrm{cm}^{2}$, while those in both the central and the lateral jets were 100 and 30 dynes $/ \mathrm{cm}^{2}$, respectively. 


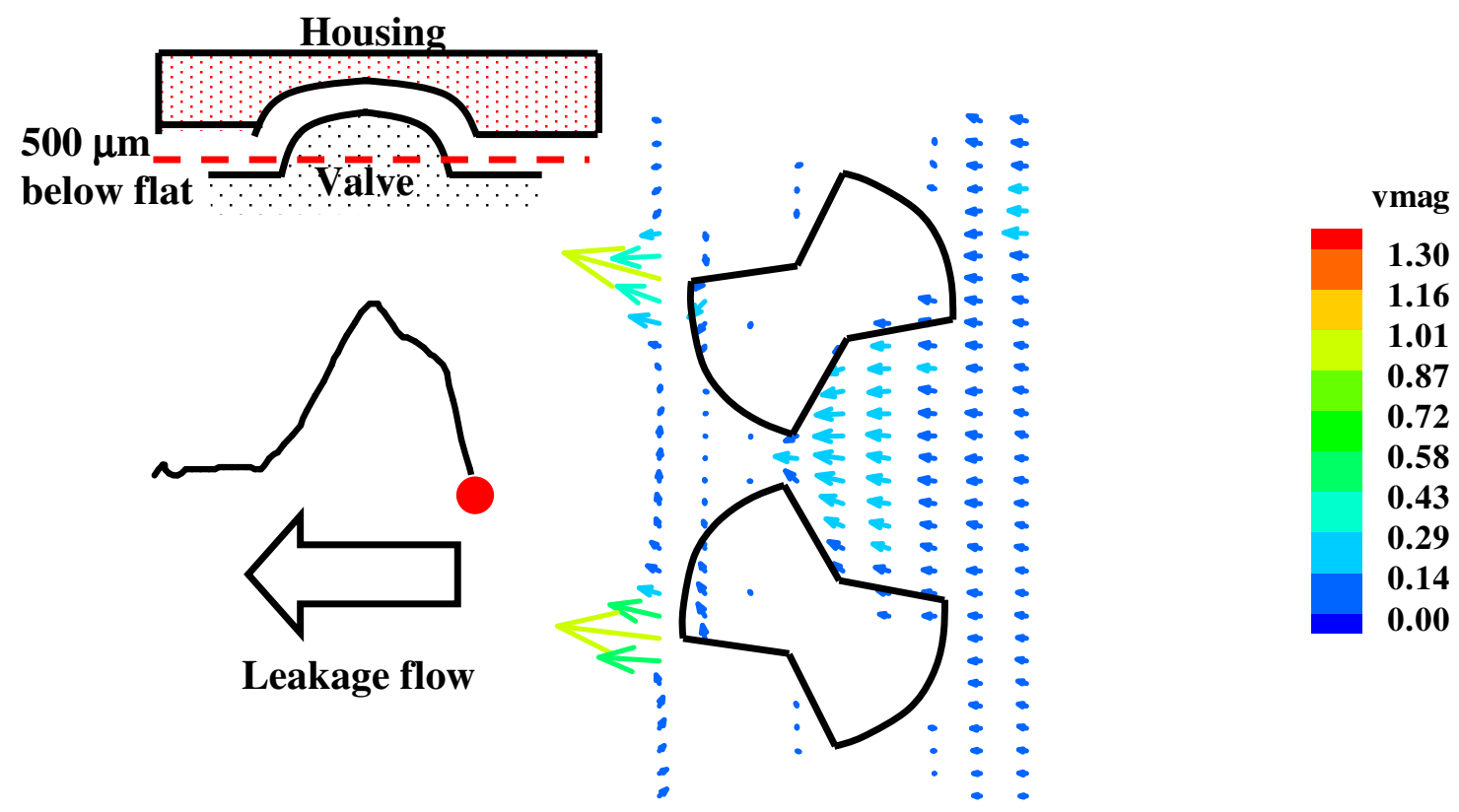

Figure 7-57 $27 \mathrm{~mm}$ prototype high leaker mitral hinge flow field at early systole, time bin 43, $500 \mu \mathrm{m}$ below flat level; strong leakage jets emanating from the hinge pockets and between the leaflet gap (avi, 714k, high_500um_below_flat_vmag.avi)

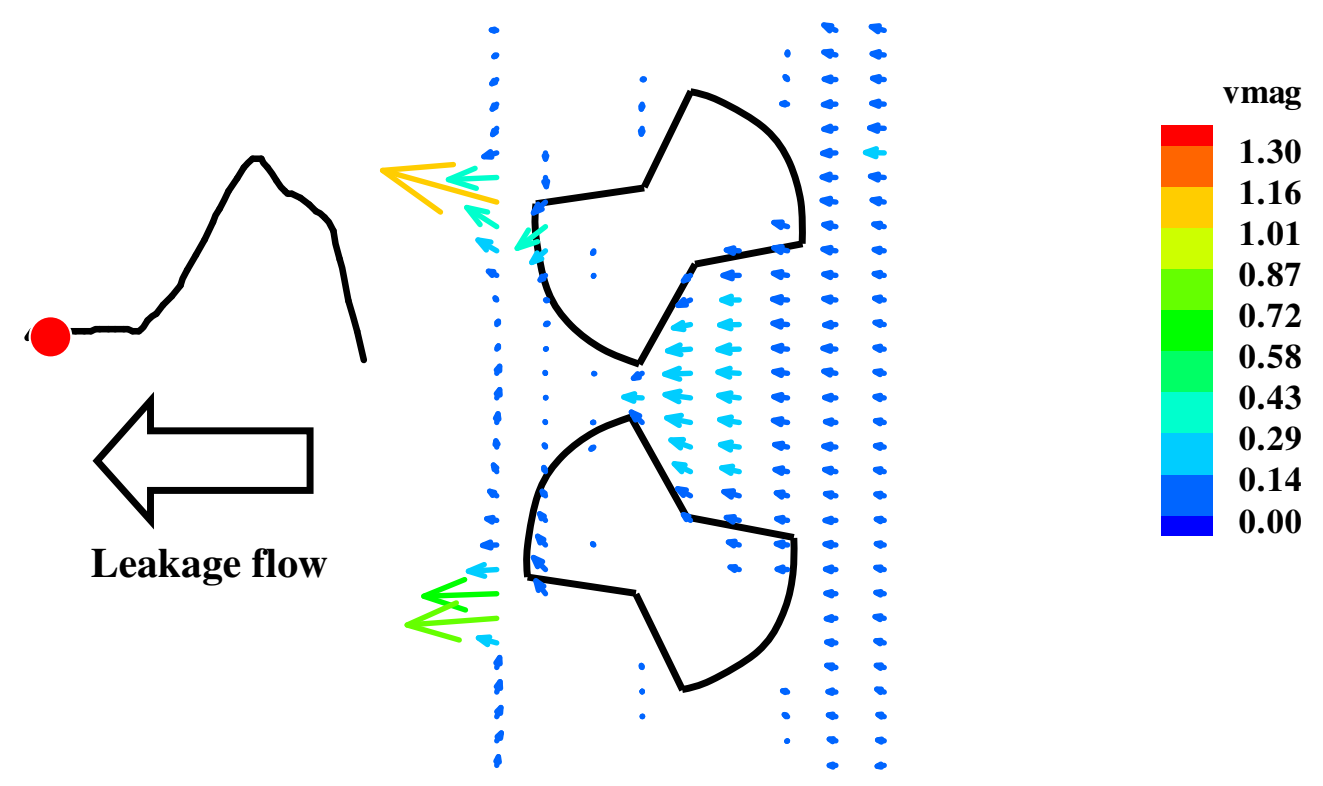

Figure 7-58 $27 \mathrm{~mm}$ prototype high leaker mitral hinge flow field approaching mid systole, time bin 4, $500 \mu \mathrm{m}$ below flat level; the leakage jet issuing from the hinge accelerated to velocity of $1.0 \mathrm{~m} / \mathrm{s}$ at mid systole (avi, 714k, high_500um_below_flat_vmag.avi) 


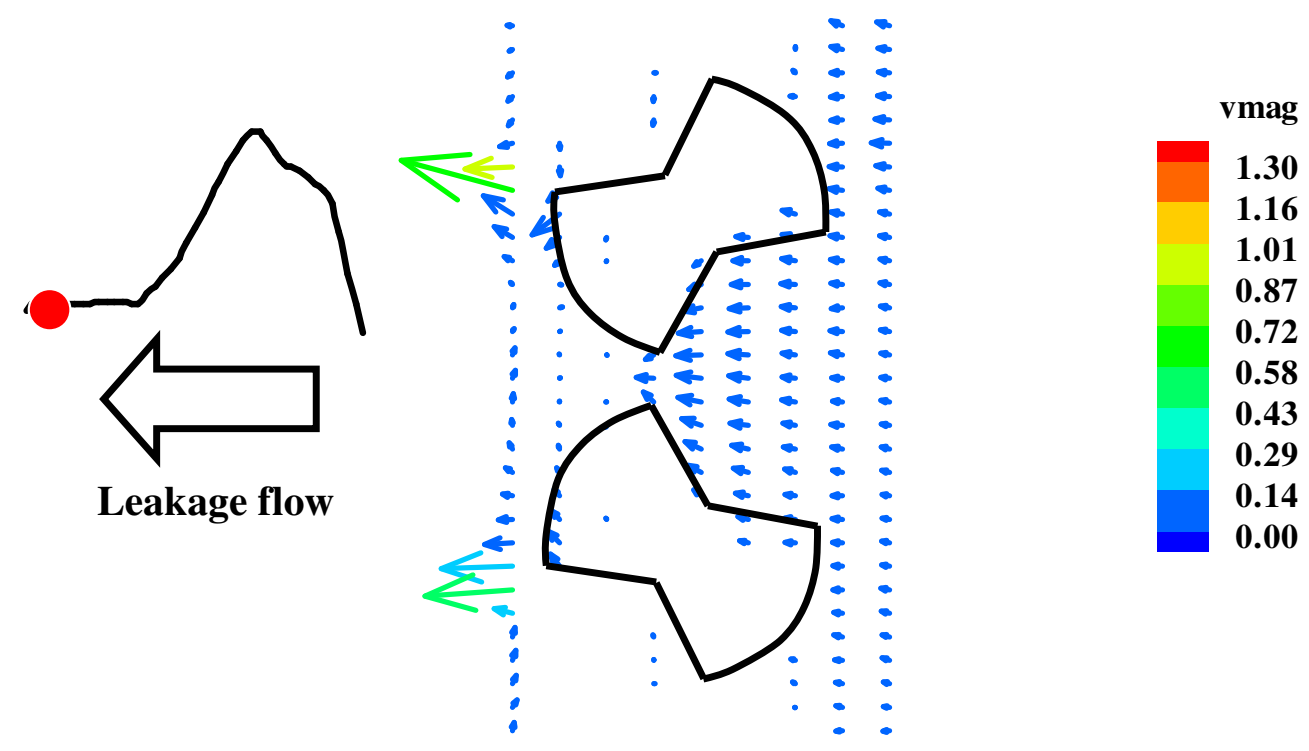

Figure 7-59 Reynolds shear stress distribution of the $27 \mathrm{~mm}$ prototype high leaker at time bin 4, $500 \mu \mathrm{m}$ below flat level; elevated Reynolds shear stress levels observed at the leakage jets issuing from the hinge regions (avi, 722k, high_500um_below_flat_rss.avi)
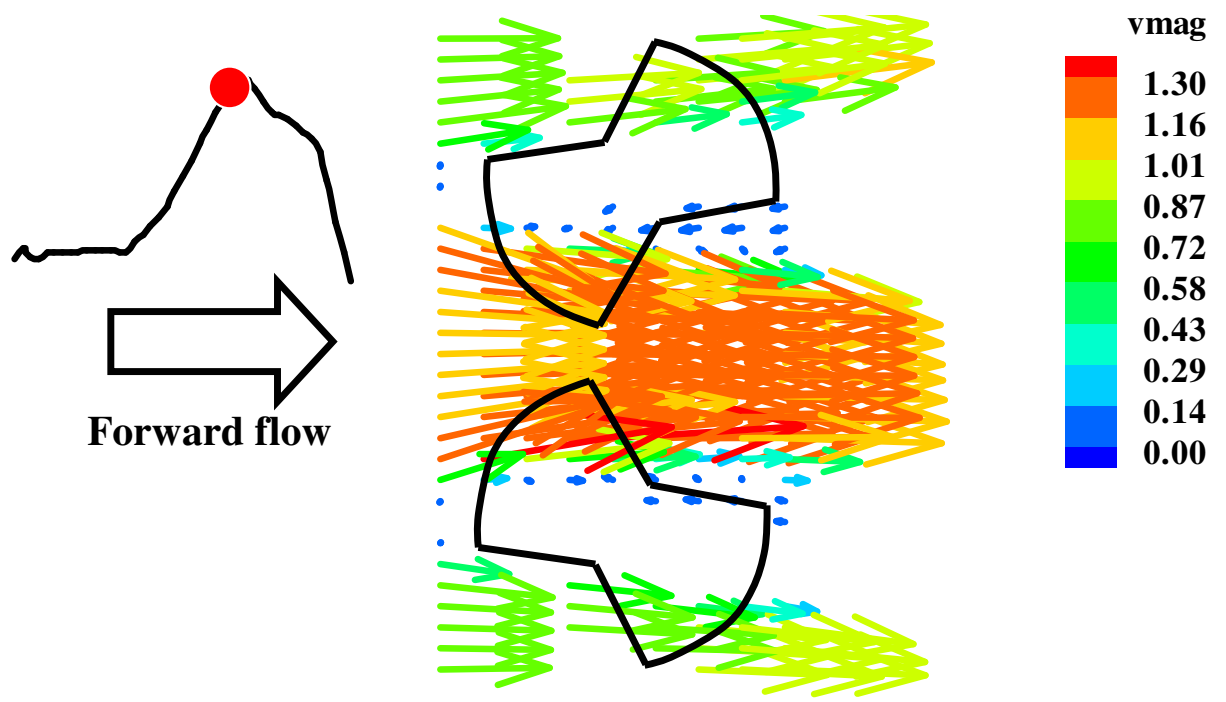

Figure 7-60 $27 \mathrm{~mm}$ prototype high leaker mitral hinge flow field at mid diastole, time bin 27, $500 \mu \mathrm{m}$ below flat level; three forward jets observed during diastole with maximum velocity $1.3 \mathrm{~m} / \mathrm{s}$ in the central jet (avi, 714k, high_500um_below_flat_vmag.avi) 


\subsubsection{6 $1 \mathrm{~mm}$ Below The Flat Level}

Animations high_1mm_below_flat_vmag.avi and high_1mm_below_flat_rss.avi show the velocity magnitude $(\mathrm{m} / \mathrm{s})$ and Reynolds shear stress $\left(\right.$ dynes $\left./ \mathrm{cm}^{2}\right)$ vector plots at the 1 $\mathrm{mm}$ below the flat level of the $27 \mathrm{~mm}$ prototype high leaker clear valve throughout the cardiac cycle, respectively. Refer to Appendix $\mathrm{H}$ for the list of animations.

\section{Systole}

The flow field velocities throughout systole in measurement plane were generally below $0.05 \mathrm{~m} / \mathrm{s}$, except for the leakage jets from the hinge, which reached a peak velocity of 0.2 m/s during mid systole (time bin 9, Figure 7-61). The calculated Reynolds shear stress levels in the central and hinge jets were 200 and 100 dynes $/ \mathrm{cm}^{2}$, respectively.

\section{$\underline{\text { Diastole }}$}

The flow fields at $1 \mathrm{~mm}$ below the flat level during valve opening were similar to those seen at the $500 \mu \mathrm{m}$ below the flat level. Velocities of $0.6 \mathrm{~m} / \mathrm{s}$ and $1 \mathrm{~m} / \mathrm{s}$ were recorded during early diastole inside the central and lateral jets, respectively. At peak systole, the central and the lateral flows reached a velocity magnitude of $1.4 \mathrm{~m} / \mathrm{s}$ and $1.0 \mathrm{~m} / \mathrm{s}$, respectively (time bin 27, Figure 7-62). A peak Reynolds shear stress of 340 dynes $/ \mathrm{cm}^{2}$ was recorded in the region between the central jet and the edge of the leaflets, while the Reynolds shear stress levels inside the central and lateral jets were typically below 100 dynes/cm ${ }^{2}$. 


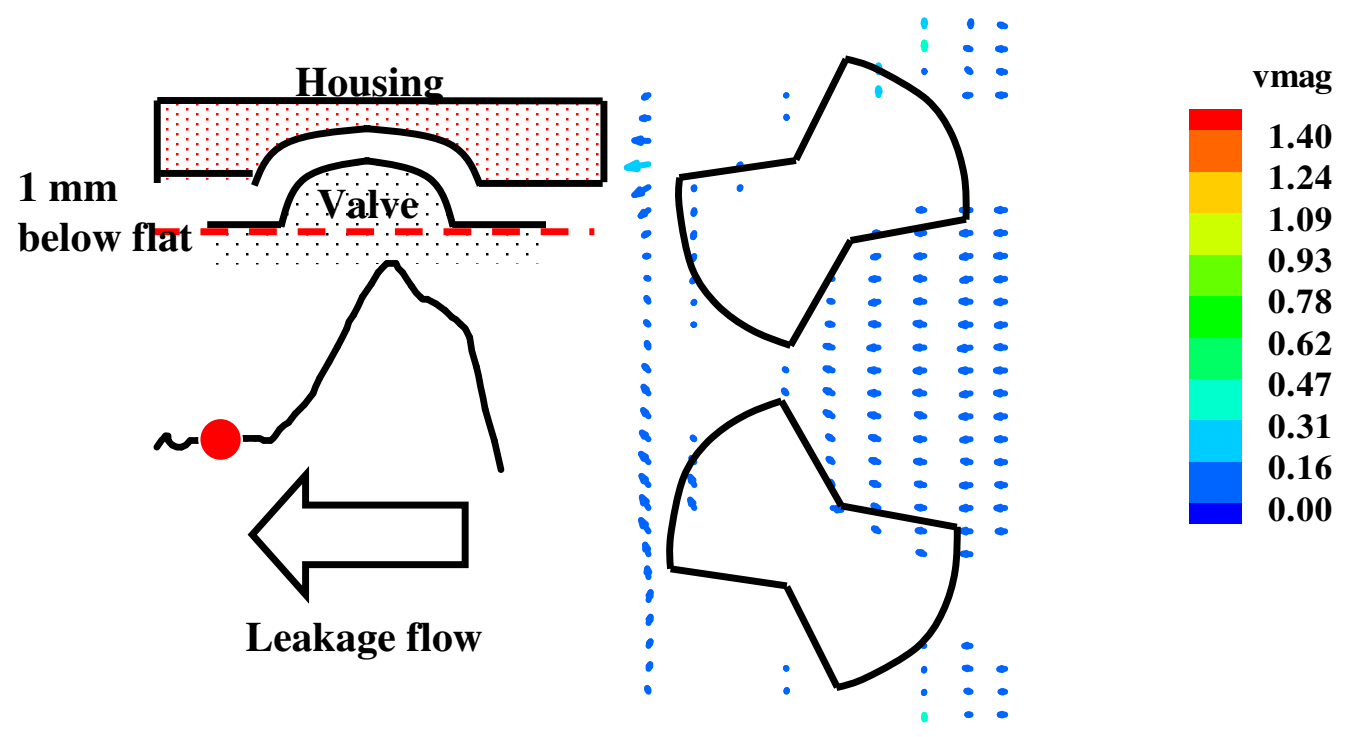

Figure 7-61 $27 \mathrm{~mm}$ prototype high leaker mitral hinge flow field at mid systole, time bin $9,1 \mathrm{~mm}$ below flat level; leakage jet of $0.2 \mathrm{~m} / \mathrm{s}$ was observed issuing from the hinge (avi, 703k, high_1mm_below_flat_vmag.avi)
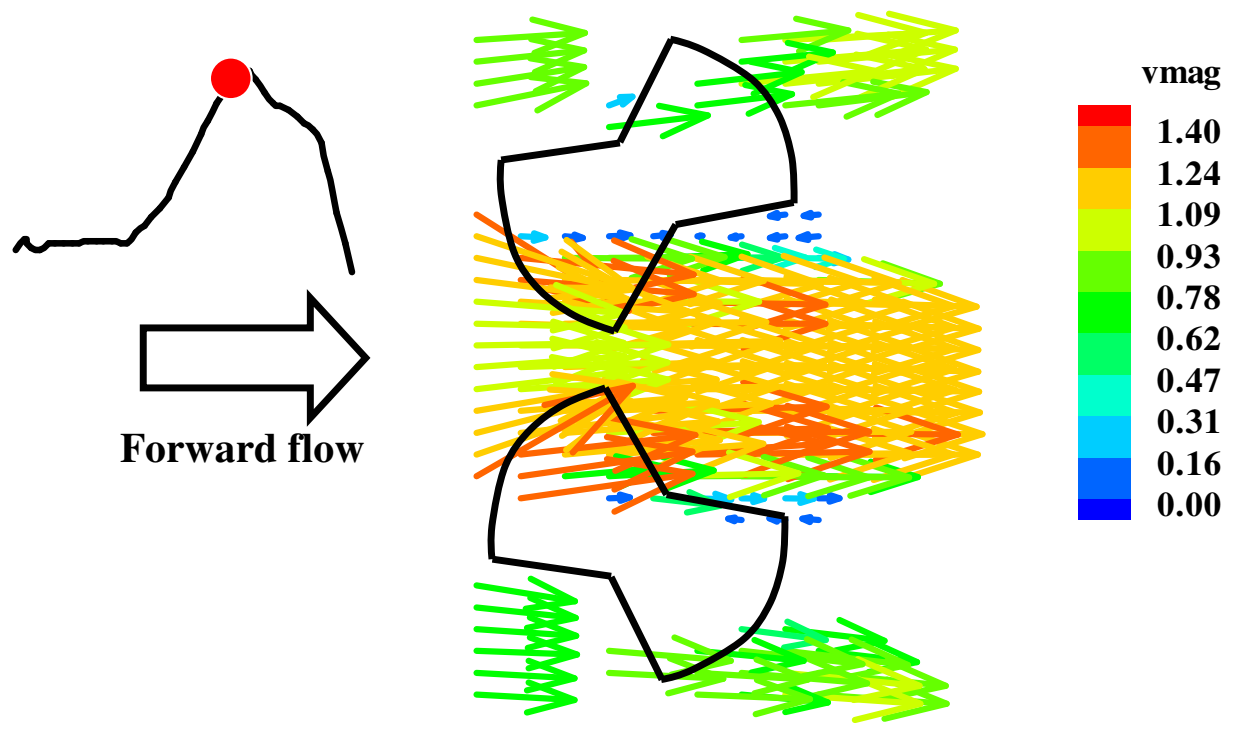

Figure 7-62 $27 \mathrm{~mm}$ prototype high leaker mitral hinge flow field at mid diastole, time bin 29, $1 \mathrm{~mm}$ below flat level; three forward jets were observed during systole with a maximum velocity of $1.4 \mathrm{~m} / \mathrm{s}$ recorded in the central jet (avi, 703k, high_1mm_below_flat_vmag.avi) 


\subsubsection{7 $3 \mathrm{~mm}$ Below The Flat Level}

Animations high_3mm_below_flat_vmag.avi and high_3mm_below_flat_rss.avi show the velocity magnitude $(\mathrm{m} / \mathrm{s})$ and Reynolds shear stress $\left(\right.$ dynes $\left./ \mathrm{cm}^{2}\right)$ vector plots at the 3 $\mathrm{mm}$ below the flat level of the $27 \mathrm{~mm}$ prototype high leaker clear valve throughout the cardiac cycle, respectively. Refer to Appendix $\mathrm{H}$ for the list of animations.

\section{Systole}

Figure 7-63 (time bin 43) shows the beginning of the systolic phase at $3 \mathrm{~mm}$ below the flat level. Flow at the atrial side of the closed leaflets was drawn towards a small central leakage jet $(0.54 \mathrm{~m} / \mathrm{s})$ between the closed leaflets. Leakage jets from the hinge pocket observed at $500 \mu \mathrm{m}$ and $1 \mathrm{~mm}$ below the flat levels were not visible at this level. Flow velocity magnitudes less than $0.05 \mathrm{~m} / \mathrm{s}$ were recorded during mid and late systole. A maximum Reynolds shear stress level of 446 dynes $/ \mathrm{cm}^{2}$ was recorded at valve closure in the central leakage jet between the closed leaflets. However, the Reynolds shear stress values throughout the investigated region during systole were generally less than 20 dynes $/ \mathrm{cm}^{2}$.

\section{Diastole}

Three forward flow jets were observed during diastole. Peak velocities of $1.4 \mathrm{~m} / \mathrm{s}$ and 1.0 $\mathrm{m} / \mathrm{s}$ were recorded at mid diastole in the central and the lateral jets, respectively (time bin 27, Figure 7-64). The Reynolds shear stress levels throughout the investigated region were less than 10 dynes $/ \mathrm{cm}^{2}$. 


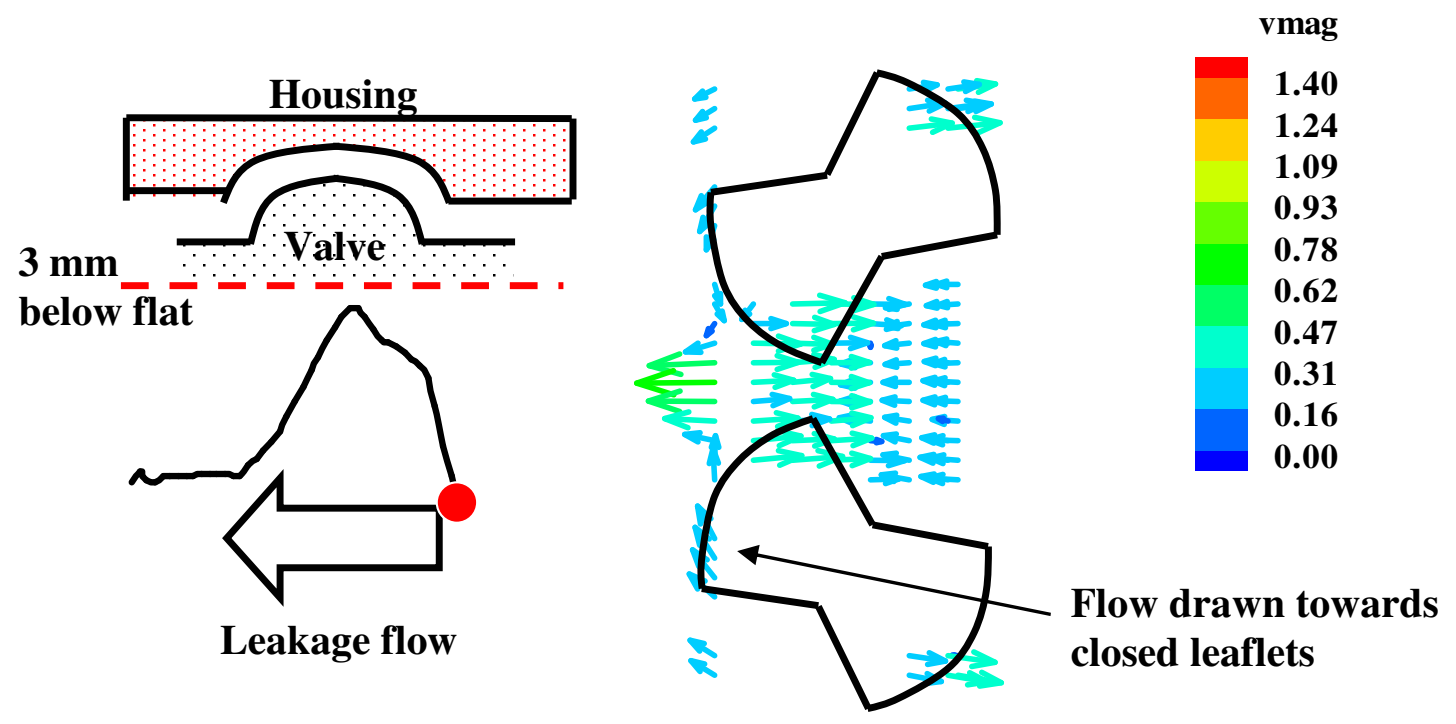

Figure 7-63 $27 \mathrm{~mm}$ prototype high leaker mitral hinge flow field at early systole, time bin 43, $3 \mathrm{~mm}$ below flat level; flow at the atrial side of the closed leaflets was drawn towards a small central leakage jet between the closed leaflets (avi, 666k, high_3mm_below_flat_vmag.avi)
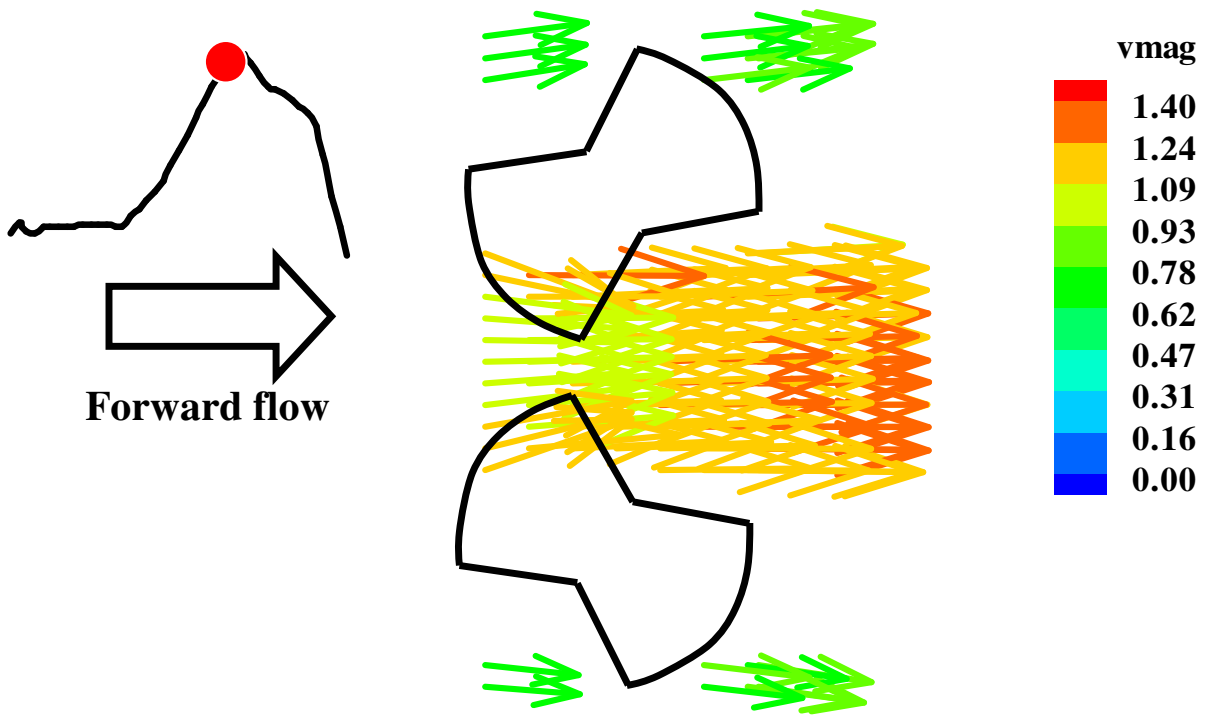

Figure 7-64 $27 \mathrm{~mm}$ prototype high leaker mitral hinge flow field at mid diastole, time bin $27,3 \mathrm{~mm}$ below flat level; three forward jets were observed during systole with a peak velocity of $1.4 \mathrm{~m} / \mathrm{s}$ measured in the central forward jet (avi, 666k, high_3mm_below_flat_vmag.avi) 


\subsection{Downstream of The Thumbnail Region}

Animations high_thumbnail_vmag.avi and high_thumbnail_rss.avi show the velocity magnitude $(\mathrm{m} / \mathrm{s})$ and Reynolds shear stress $\left(\right.$ dynes $\left./ \mathrm{cm}^{2}\right)$ vector plots at the downstream of the thumbnail region of the $27 \mathrm{~mm}$ prototype high leaker clear valve throughout the cardiac cycle, respectively. Refer to Appendix $\mathrm{H}$ for the list of animations.

\section{$\underline{\text { Systole }}$}

Complex flow structures similar to those in SJM $27 \mathrm{~mm}$ Standard and $27 \mathrm{~mm}$ prototype low leaker valves were observed during systole. Figure 7-65 (time bin 38) shows the flow distribution at the start of the systole. Leakage flow of approximately $0.2 \mathrm{~m} / \mathrm{s}$ was seen immediately downstream of the thumbnail region. The velocity of this leakage jet reduced to approximately $0.05 \mathrm{~m} / \mathrm{s}$ at late systole with a corresponding Reynolds shear stress of less than 10 dynes $/ \mathrm{cm}^{2}$.

\section{Diastole}

A velocity of $0.15 \mathrm{~m} / \mathrm{s}$ was measured in the forward flow downstream of the thumbnail at the onset of the diastolic phase (time bin 18). During the acceleration phase at time bin 23 (Figure 7-66), the flow velocity in the central forward flow region decreased to less than $0.08 \mathrm{~m} / \mathrm{s}$, while that in the lateral regions increased to more than $0.4 \mathrm{~m} / \mathrm{s}$. The subsequent flow development observed at this measurement level was similar to those in the SJM 27 $\mathrm{mm}$ Standard and $27 \mathrm{~mm}$ prototype low leaker valves. Flow reversal was observed in the stronger lateral flows, giving rise to Reynolds shear stress levels of more than 100 
dynes $/ \mathrm{cm}^{2}$ between the central and the lateral jets. The Reynolds shear stress at other measurement locations during diastole was less than 5 dynes $/ \mathrm{cm}^{2}$.

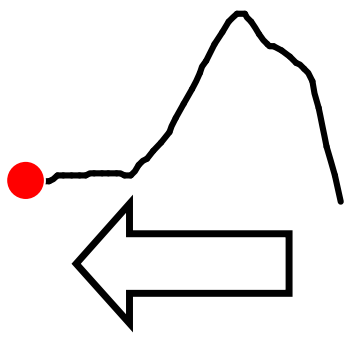

Leakage flow
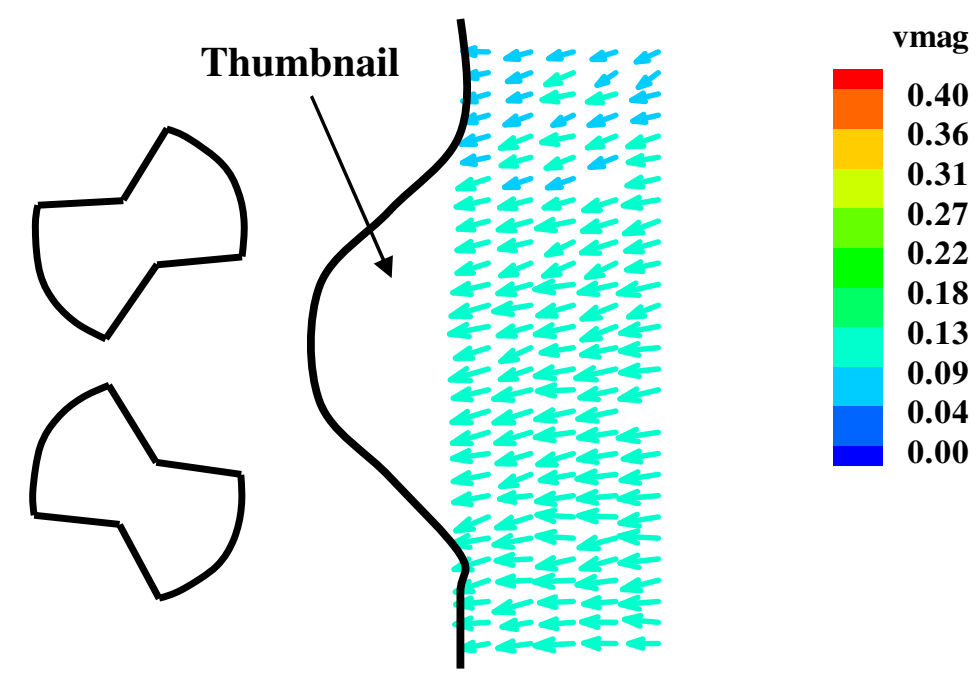

Figure 7-65 $27 \mathrm{~mm}$ prototype high leaker mitral hinge flow field at early systole, time bin 1, downstream of thumbnail region, flat level; uniform retrograde flow of $0.2 \mathrm{~m} / \mathrm{s}$ was observed immediately downstream of the thumbnail (avi, 716k, high_thumbnail_vmag.avi)

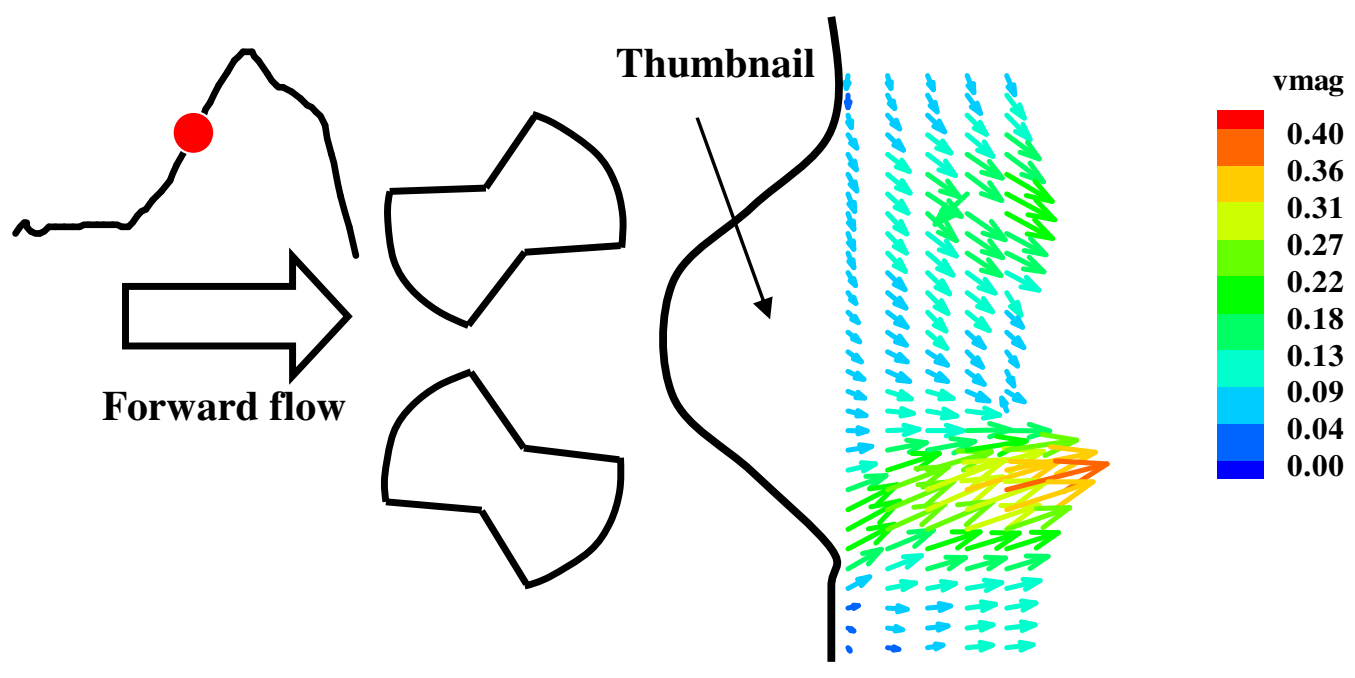

Figure 7-66 $27 \mathrm{~mm}$ prototype high leaker mitral hinge flow field approaching mid diastole, time bin 23, downstream of thumbnail region, flat level; lateral flow increased in magnitude during the acceleration phase (avi, 716k, high_thumbnail_vmag.avi) 


\subsection{Effect of Valve Design on Hinge Flow Dynamics (Mitral Position)}

In order to study the effect of valve design on hinge flow dynamics, 2D LDV experiments were performed to measure the flow field in the hinge region of the CM 23 mm bileaflet heart valve. Details of these experiments are presented in Chapter 4 and 5 . Following is a detailed presentation of the results from these experiments.

\subsubsection{D LDV Mitral Hinge Studies With The CM $23 \mathrm{~mm}$ Bileaflet Clear Housing} Valves

\subsubsection{CM $23 \mathrm{~mm}$}

The results are presented using color images to illustrate the flow fields and Reynolds shear stress distributions at specific instances during the cardiac cycle. They are presented in the following order: 1) primary flat; 2) levels above the primary flat; and 3) levels below the primary flat. . For each measurement plane, the description of the flow during systole is presented first followed by that during diastole. The direction of the forward flow is from top to bottom (from the left atrium to left ventricle, Figure 5-10). All vector plots are color-coded by Velocity Magnitude $(\mathrm{m} / \mathrm{s})$ and Reynolds shear stress $\left(\right.$ dynes $\left./ \mathrm{cm}^{2}\right)$. Arrows point in the direction of the phase-averaged mean velocity vector and the length of the arrow is proportional to the velocity magnitude. Refer to Figure 5-1 for the mitral flow and pressure waveforms, and Appendix $\mathrm{H}$ for the list of velocity magnitude $(\mathrm{m} / \mathrm{s})$ and Reynolds shear stress $\left(\right.$ dynes $\left./ \mathrm{cm}^{2}\right)$ animations. Terminology pertinent to the hinge design is shown in Figure 7-67 for orientation purposes. Figure 7-68 shows the leaflet positions at mid acceleration, peak diastole as well as valve closure and mid systole within the CM $23 \mathrm{~mm}$ clear valve hinge. These leaflet positions 
correspond to those in the images presented later. For clarity the leaflets are not shown on the color printouts of the velocity field.

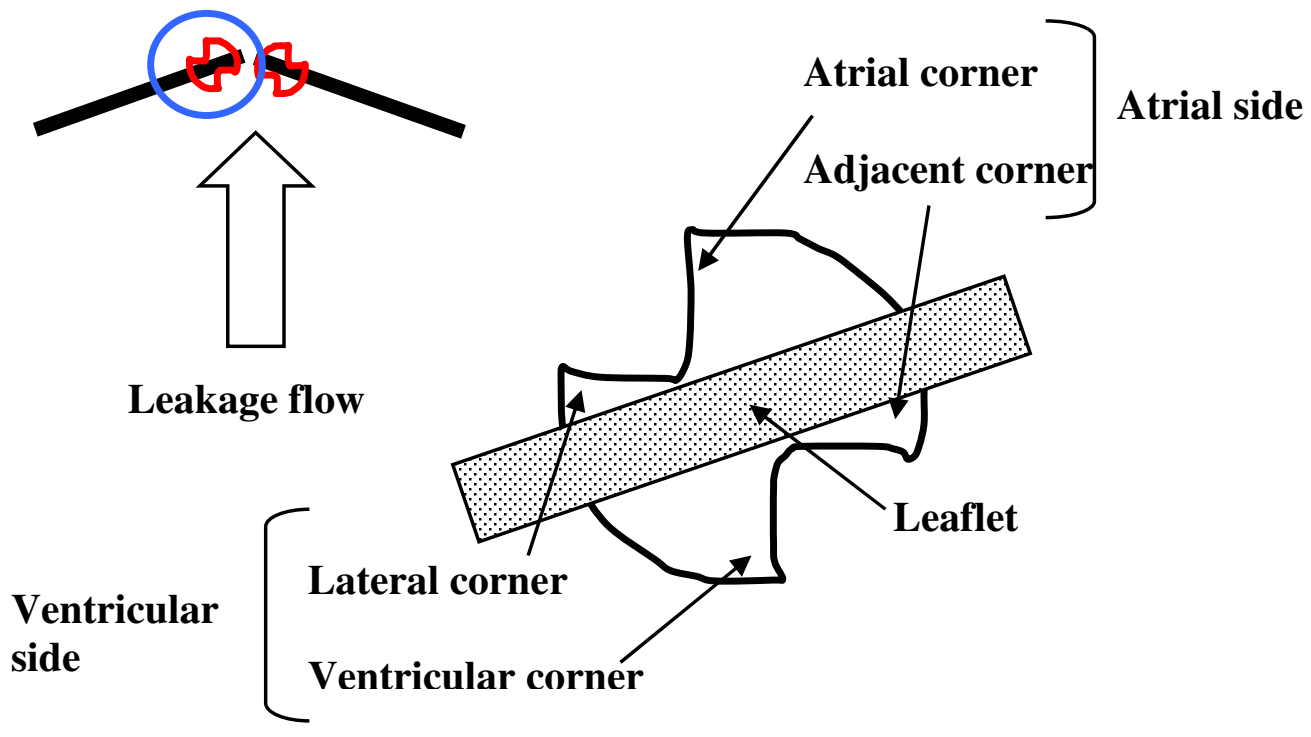

Figure 7-67 Pertinent terminology of CM $23 \mathrm{~mm}$ clear hinge design 

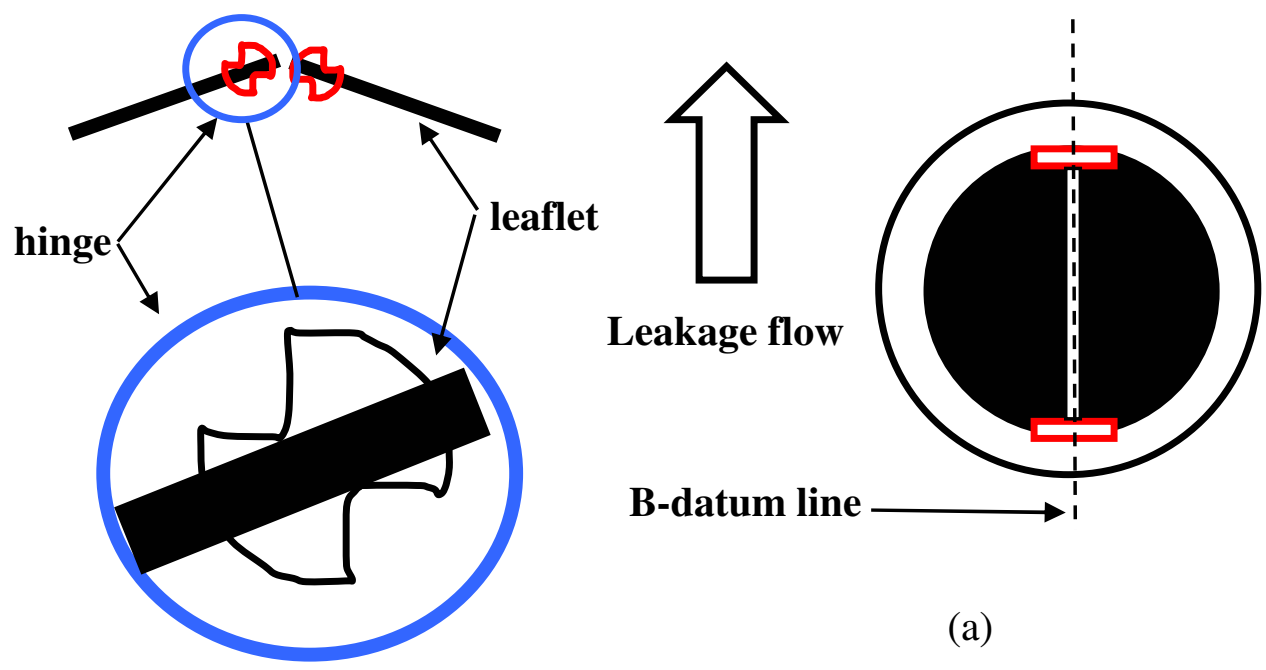

(a)
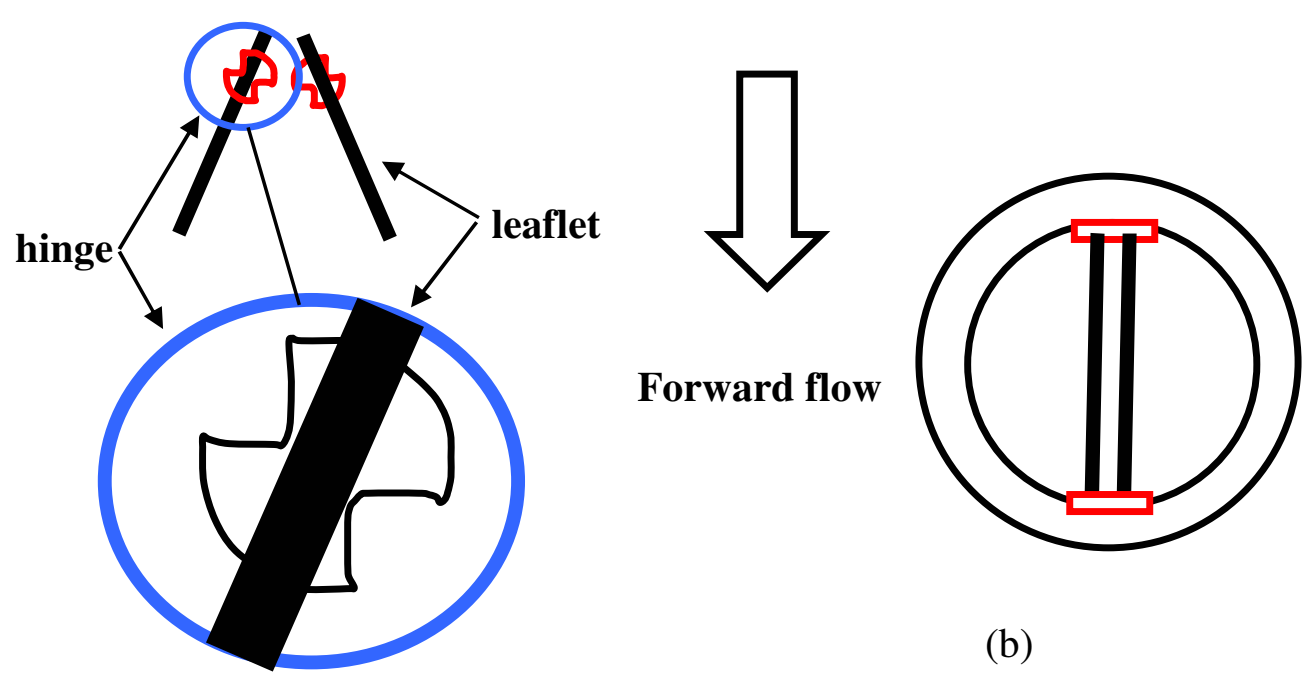

(b)

Figure 7-68 Leaflet position at (a) valve closure, mid systole, and (b) mid acceleration, peak diastole, within the CM $23 \mathrm{~mm}$ clear housing valve 


\subsection{Flat Level}

Animations CM_flat_vmag.avi and CM_flat_rss.avi show the velocity magnitude (m/s) and Reynolds shear stress (dynes $/ \mathrm{cm}^{2}$ ) vector plots at the flat level of the CM $23 \mathrm{~mm}$ clear valve throughout the cardiac cycle, respectively. Refer to Appendix $\mathrm{H}$ for the list of animations.

\section{Systole}

Figure 7-69 (time bin 2) shows onset of leakage flow, when the leakage jet was observed in the atrial corner of the hinge. Flow was drawn towards the atrial side of the hinge by the backward motion of the leaflet leading to a maximum velocity of $3.17 \mathrm{~m} / \mathrm{s}$ in this region and a corresponding Reynolds shear stress of 5,510 dynes $/ \mathrm{cm}^{2}$ (time bin 2, Figure 7-70). Another area of high velocity was in the lateral corner of the hinge, where the fluid reached a velocity of approximately $2 \mathrm{~m} / \mathrm{s}$. A small leakage jet was also seen at the adjacent corner; it recorded a peak velocity of $1.2 \mathrm{~m} / \mathrm{s}$ and a corresponding Reynolds shear stress of 5,054 dynes $/ \mathrm{cm}^{2}$. The velocity in the central region of the hinge was relatively low, in the range of $0.2-0.4 \mathrm{~m} / \mathrm{s}$. The Reynolds shear stress magnitudes in the atrial and adjacent corners of the hinge reached a maximum value of approximately 3,000 dynes $/ \mathrm{cm}^{2}$. The Reynolds shear stresses measured near the lateral corner of the hinge were relatively low, about 300 dynes $/ \mathrm{cm}^{2}$. The flow patterns approaching mid systole in Figure 7-71 (time bin 4) were similar to those at early systole (time bin 1). The leakage jet observed at time bin 4 had decelerated to a velocity of approximately $2.4 \mathrm{~m} / \mathrm{s}$. Here a mixture of forward and leakage flows were observed - flow in the atrial corner of the hinge was predominantly leakage, while that in the central region of the hinge was a 
combination of forward and leakage flows. The peak velocity of the leakage flow through the pivot eventually decreased in magnitude to approximately $1 \mathrm{~m} / \mathrm{s}$. This reduction in velocity extended to the whole hinge region where the average velocity observed was on the order of $0.01 \mathrm{~m} / \mathrm{s}$. Lack of measurements at both the lateral and ventricular corners of the hinge were due to low data rates in these regions. This could be due to out-of-plane (parallel to z-axis) movement of the fluid particles at these corners.

\section{$\underline{\text { Diastole }}$}

At early diastole (time bin 13, Figure 7-72), the flow was predominantly directed forward, with a maximum velocity of $0.54 \mathrm{~m} / \mathrm{s}$ occurring in the lateral corner of the hinge. The velocity magnitude was approximately $0.2-0.3 \mathrm{~m} / \mathrm{s}$, which is much lower than that seen

during leakage flow. Similar flow profiles and magnitudes can be seen at peak diastole (time bins 23, Figure 7-73) where velocity within the hinge region was more homogenous (low velocity gradient) at approximately $0.2-0.3 \mathrm{~m} / \mathrm{s}$. In both time bins the average value of the Reynolds shear stress at most sites was approximately 200 dynes $/ \mathrm{cm}^{2}$. The lack of measurements in the central region could be attributed to the obstruction caused by the leaflet ear. 


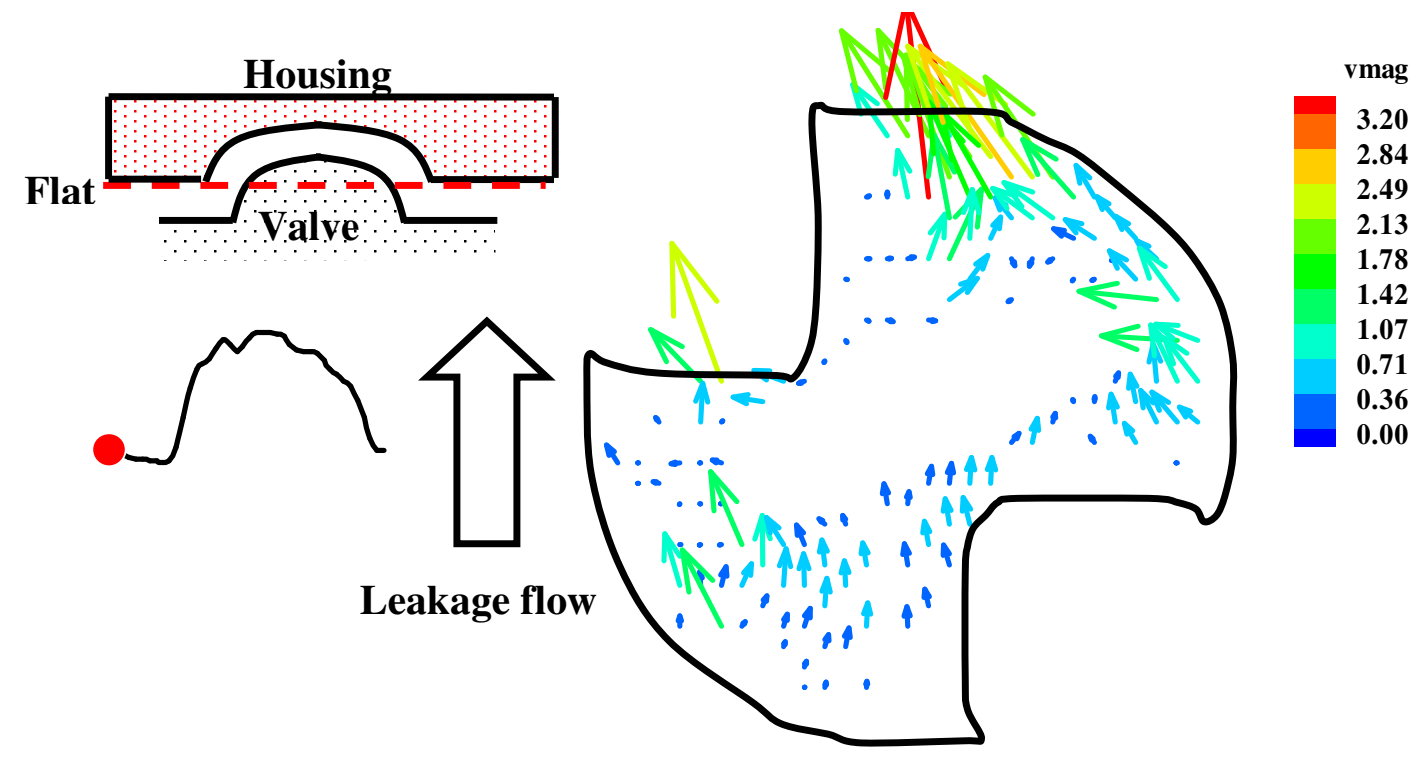

Figure 7-69 CM $23 \mathrm{~mm}$ mitral hinge flow field at early systole, time bin 2, flat level; leakage jet emanating from the atrial and lateral corners of the hinge geometry (avi, 656k, CM_flat_vmag.avi)

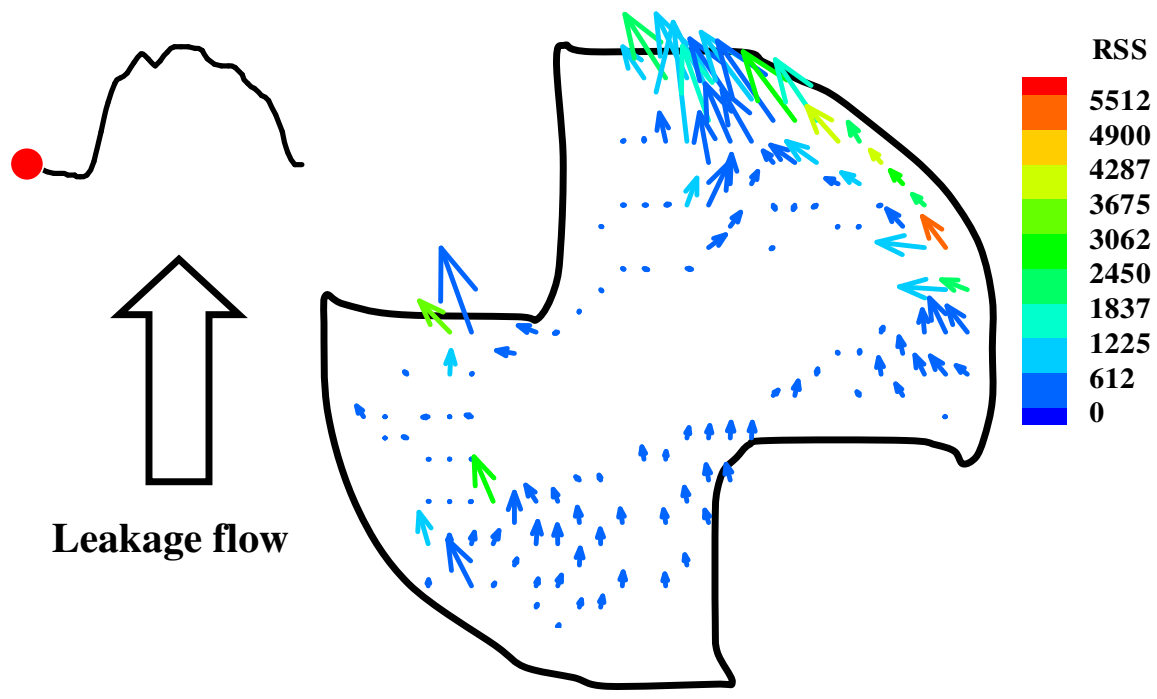

Figure 7-70 Reynolds shear stress distribution of the CM $23 \mathrm{~mm}$ at early systole, time bin 2, flat level; elevated Reynolds shear stress levels observed at the atrial and lateral corners of the hinge geometry (avi, 673k, CM_flat_rss.avi) 

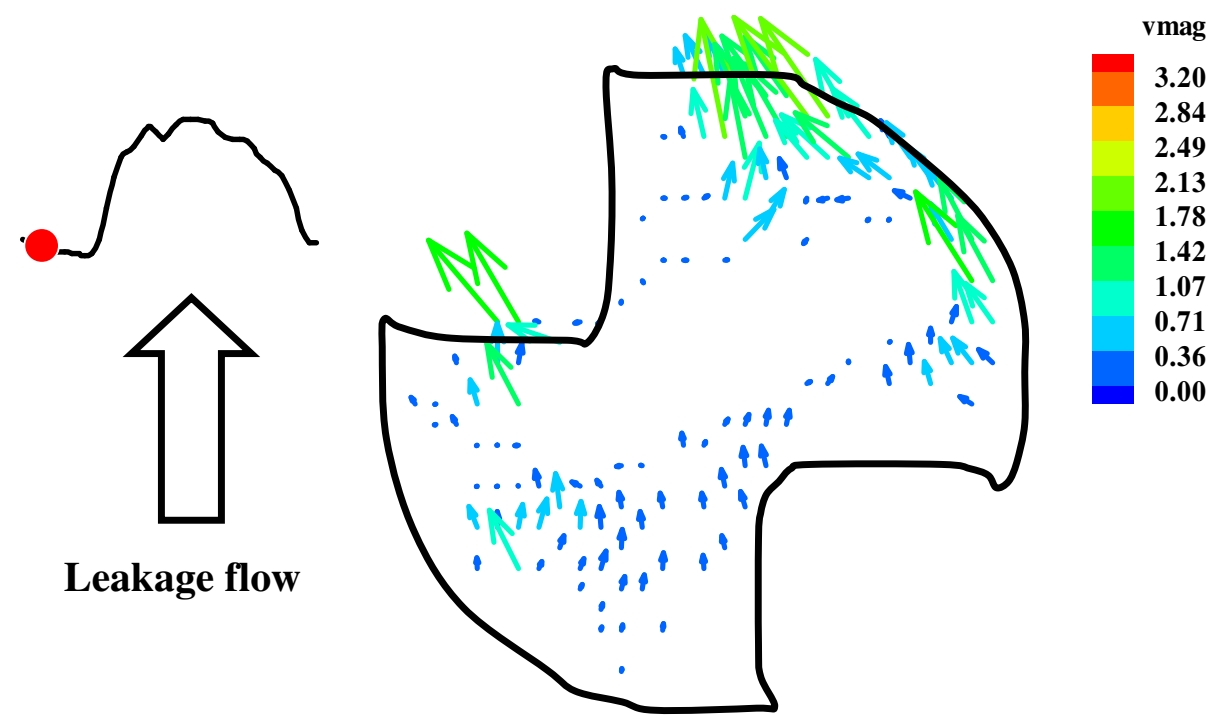

Figure 7-71 CM $23 \mathrm{~mm}$ mitral hinge flow field approaching mid systole, time bin 4, flat level; the leakage jets were still apparent at the two locations (avi, 656k, CM_flat_vmag.avi)

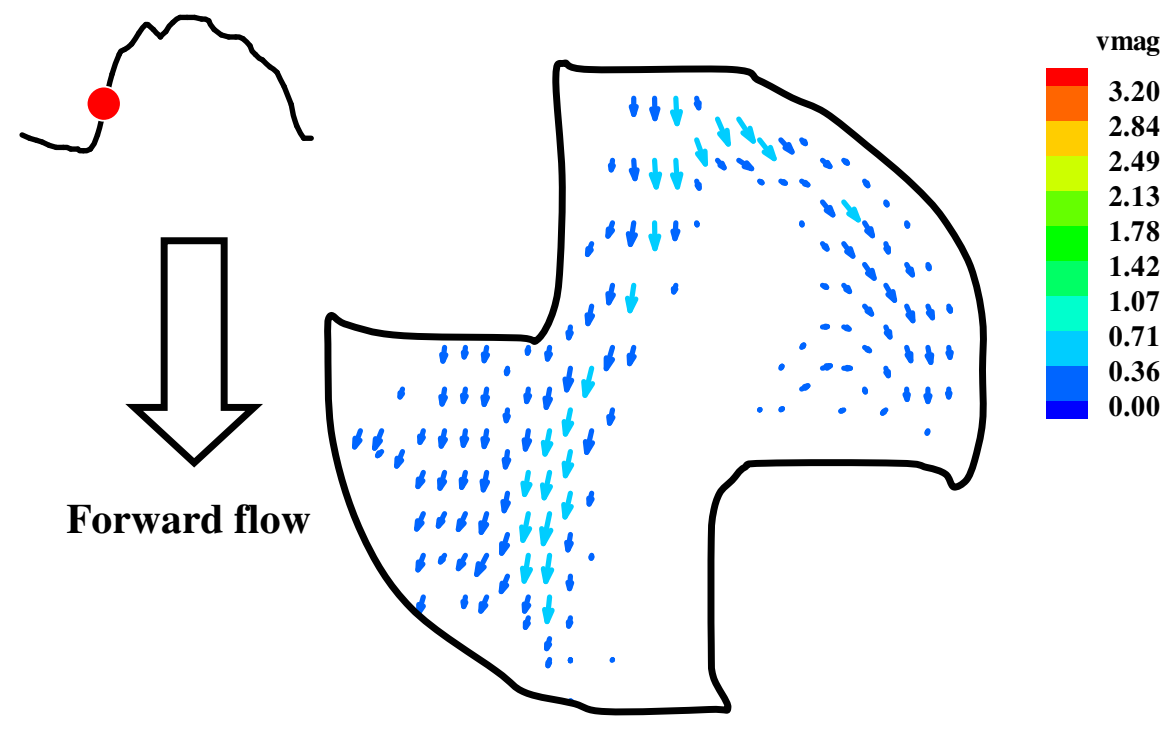

Figure 7-72 CM $23 \mathrm{~mm}$ mitral hinge flow field at early diastole, time bin 13, flat level. The flow is predominantly in the forward direction with flow velocities much lower than those observed during systole (avi, 656k, CM_flat_vmag.avi) 


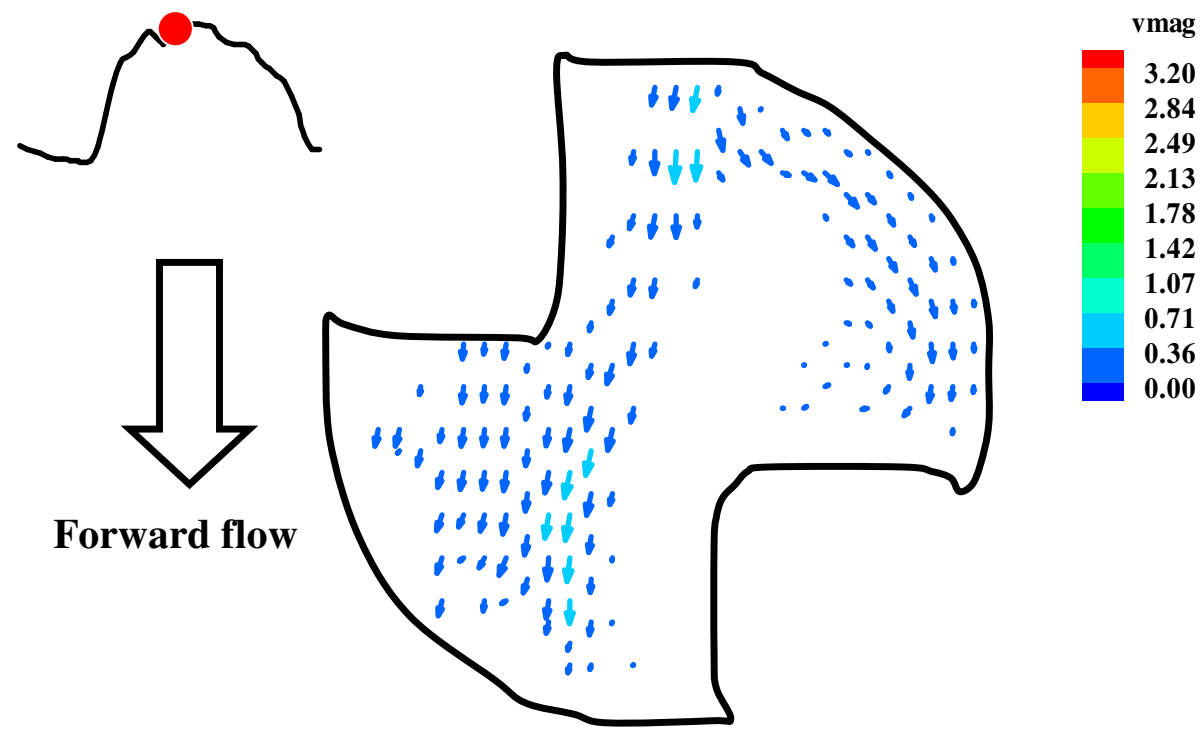

Figure 7-73 CM $23 \mathrm{~mm}$ mitral hinge flow field at peak diastole, time bin 23, flat level; recirculation zone visible at the adjacent corner (avi, 656k, CM_flat_vmag.avi)

\subsection{0 $\mathrm{\mu m}$ Above The Flat}

Animations CM_190um_above_flat_vmag.avi and CM_190um_above_flat_rss.avi show the velocity magnitude $(\mathrm{m} / \mathrm{s})$ and Reynolds shear stress $\left(\right.$ dynes $\left./ \mathrm{cm}^{2}\right)$ vector plots at the $190 \mu \mathrm{m}$ above the flat level of the CM $23 \mathrm{~mm}$ clear valve throughout the cardiac cycle, respectively. Refer to Appendix $\mathrm{H}$ for the list of animations.

\section{Systole}

At the onset of leakage flow above the flat (time bin 2, Figure 7-74), two distinct jets can be observed at both the atrial and lateral parts of the hinge. A peak velocity of $2.9 \mathrm{~m} / \mathrm{s}$ and a corresponding maximum Reynolds shear stress of 5,640 dynes $/ \mathrm{cm}^{2}$ were measured at the atrial corner of the hinge, while a peak velocity of $2 \mathrm{~m} / \mathrm{s}$ was attained in the lateral corner (Figure 7-75. These regions of leakage flow may lead to the entrainment of the 
fluid surrounding the hinge. For the remaining parts of the hinge, the velocity field was of relatively low magnitude, approximately $0.05 \mathrm{~m} / \mathrm{s}$. Isolated regions of high velocity fluid separated by low velocity regimes may result in extremely high turbulent shear stresses at their interface, in the atrial and lateral corners. The maximum Reynolds shear stress at time bin 1 was approximately 3,000 dynes $/ \mathrm{cm}^{2}$ in the atrial corner of the hinge. Another region of high turbulent shear stress $\left(3,000\right.$ dynes $\left./ \mathrm{cm}^{2}\right)$ was recorded along the lateral boundary of the hinge. High Reynolds shear stress regions corresponded with those of high velocity gradients. At mid systole (time bin 5, Figure 7-76), a reduction in velocity magnitude was observed for the two leakage jets. The peak velocity $(2 \mathrm{~m} / \mathrm{s})$ in the lateral corner of the hinge was higher than that at the atrial corner $(0.9 \mathrm{~m} / \mathrm{s})$. A region of forward flow $(0.3 \mathrm{~m} / \mathrm{s})$ at the adjacent corner of the hinge also began to form at mid systole. While the leakage jets continued to decelerate at time bin 5, the forward flow region at the adjacent corner of the hinge continued to increase in size.

\section{$\underline{\text { Diastole }}$}

Early diastole (time bin 15, Figure 7-77) was characterized by a prominent forward flow region at the adjacent corner of the hinge. This region began to form around time bin 5 and continued to increase in magnitude towards the end systolic and beginning of the diastolic phases. This increase extended into peak diastole (time bin 22, Figure 7-78), where it reached a peak velocity of $0.77 \mathrm{~m} / \mathrm{s}$. The central region of the hinge where no measurements were obtained increased in size compared to that seen at the flat level indicating that the leaflet's pivot has extended further up into this part of the hinge socket and blocked off most of the measurement sites. Towards the end of the systolic phase, the 
values of Reynolds shear stress at most locations decreased to 500 dynes $/ \mathrm{cm}^{2}$. This decrease continued well into early and mid diastole (time bins 15 and 22).

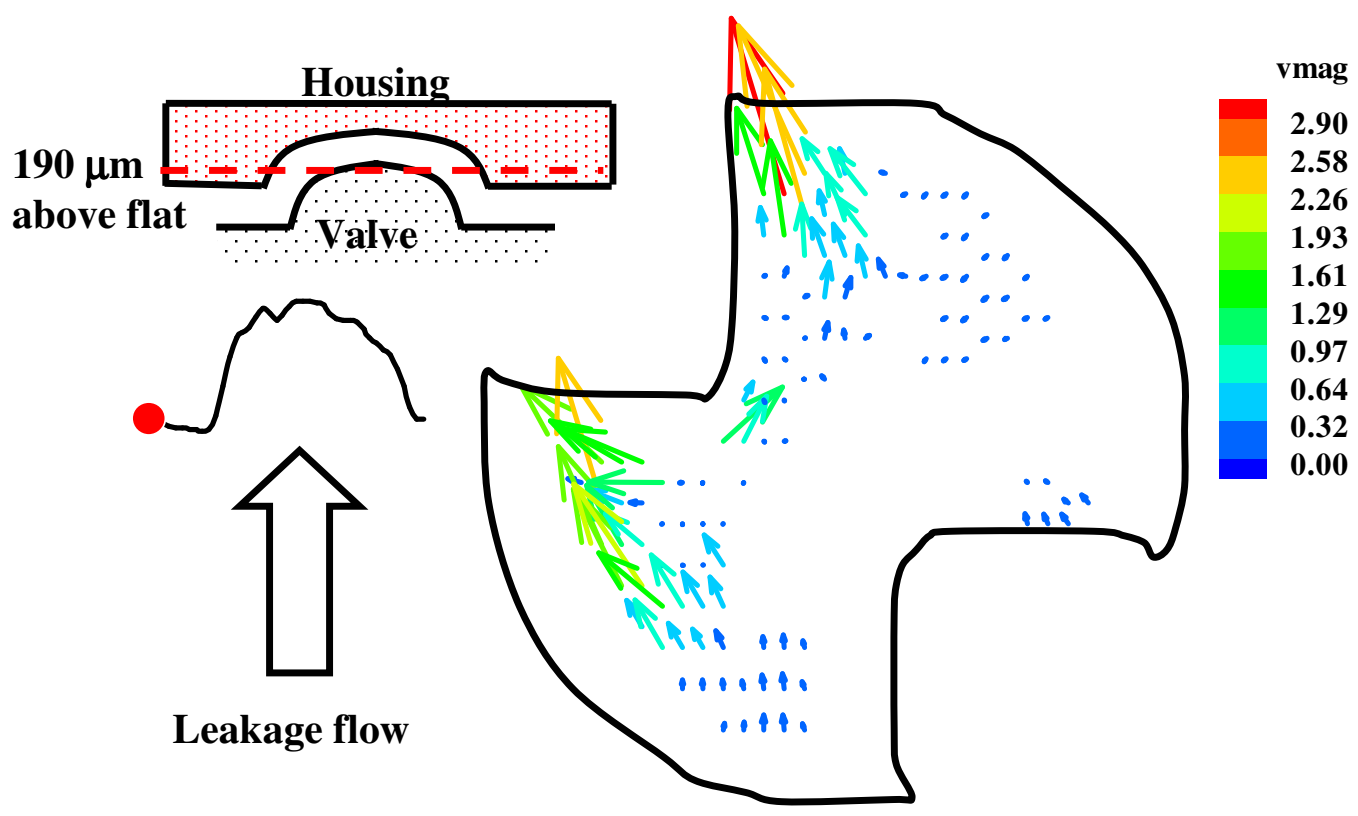

Figure 7-74 CM $23 \mathrm{~mm}$ mitral hinge flow field at early systole, time bin 2, $190 \mu \mathrm{m}$ above flat level; leakage jets visible at the atrial and lateral corners of the hinge (avi, 713k, CM_190um_above_flat_vmag.avi)

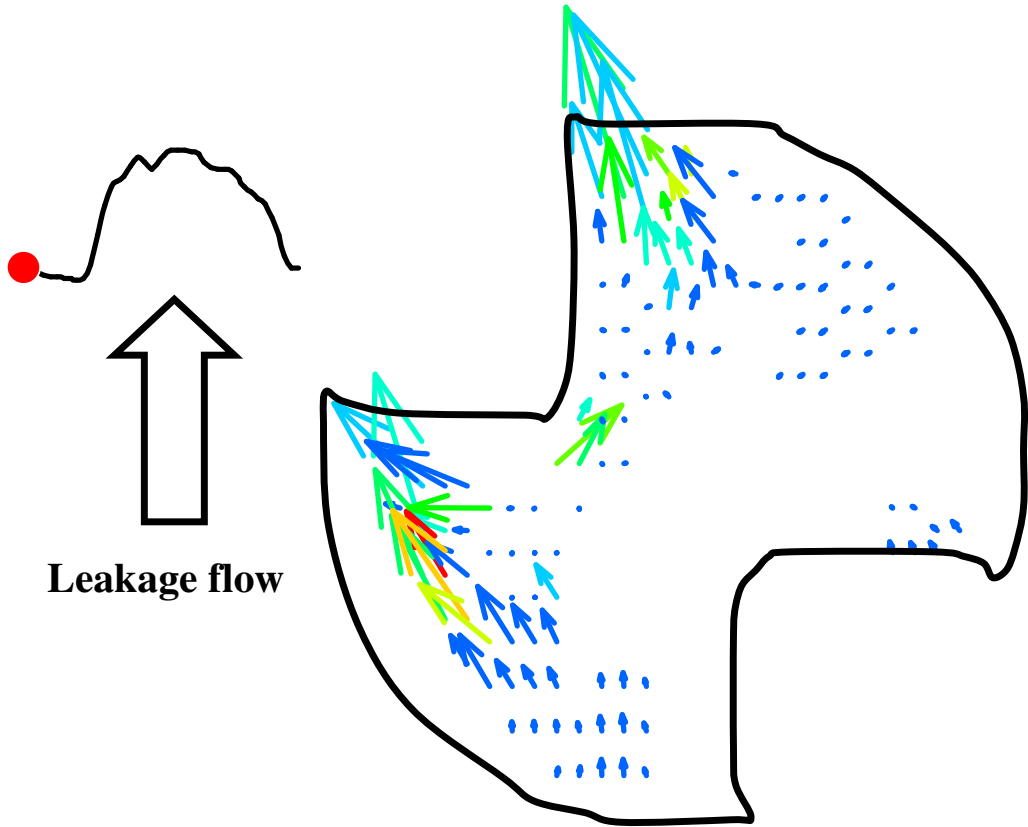

RSS

4000

3556

3111

2667

2222

1778

1333

889

444

0

Figure 7-75 Reynolds shear stress distribution of the CM $23 \mathrm{~mm}$ at early systole, time bin 2, $190 \mu \mathrm{m}$ above flat level; elevated Reynolds shear stress levels observed at the atrial and lateral corners of the hinge (avi, 729k, CM_190um_above_flat_rss.avi) 


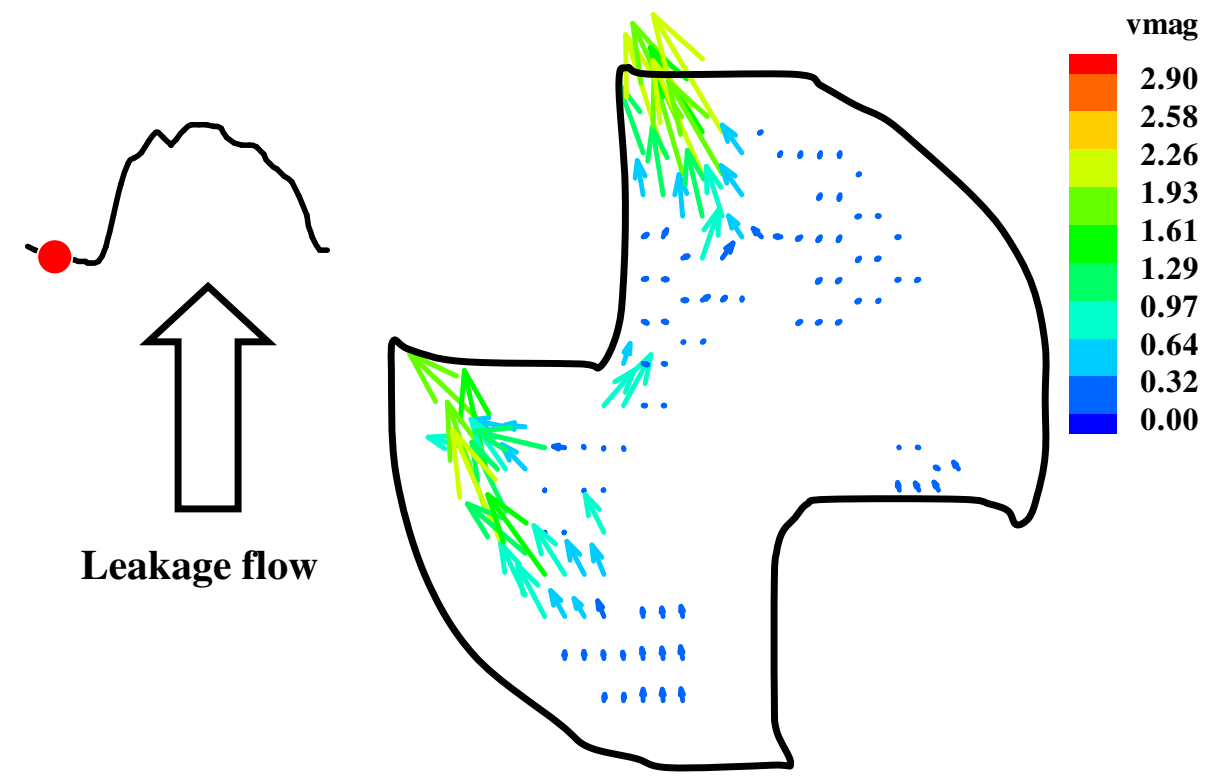

Figure 7-76 CM $23 \mathrm{~mm}$ mitral hinge flow field at mid systole, time bin 5, $190 \mu \mathrm{m}$ above flat level. The leakage velocities of the two leakage jets were lower than those measured during early systole (avi, 713k, CM_190um_above_flat_vmag.avi)

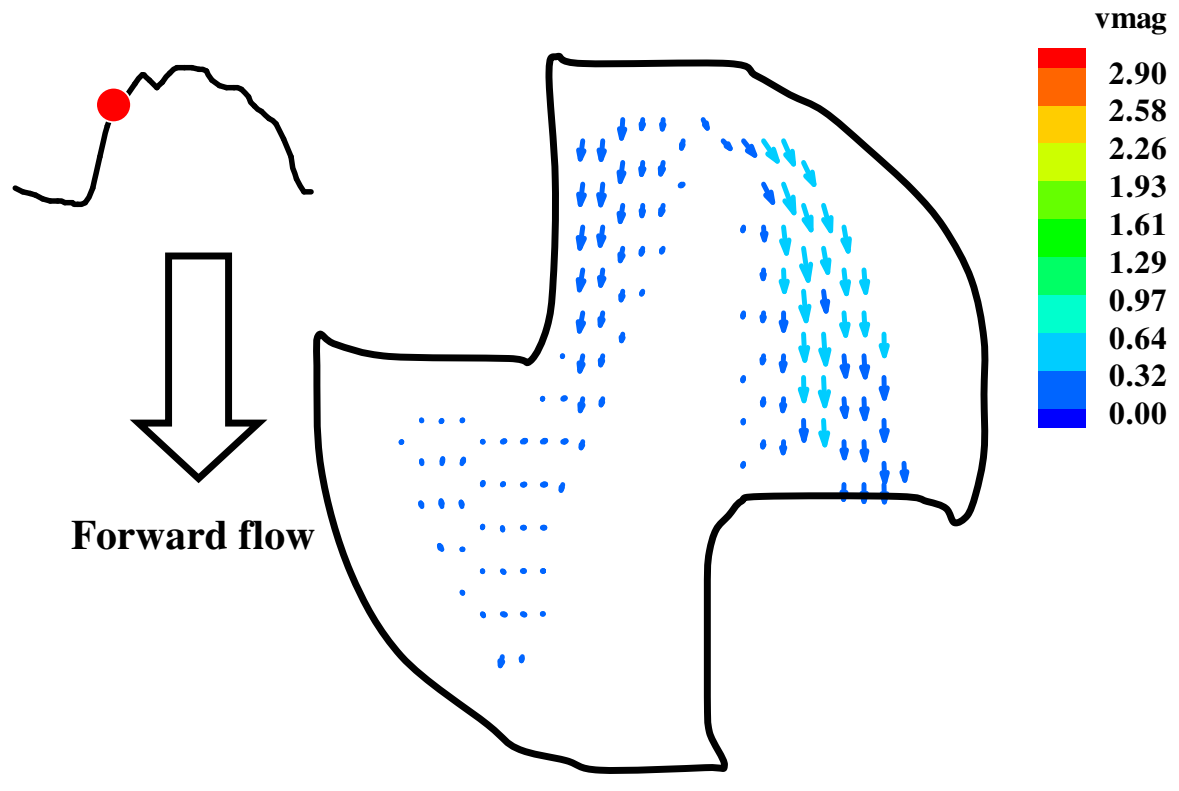

Figure 7-77 CM $23 \mathrm{~mm}$ mitral hinge flow field at early diastole, time bin 15, $190 \mu \mathrm{m}$ above flat level; no recirculation is visible at the adjacent corner (avi, 713k, CM_190um_above_flat_vmag.avi) 


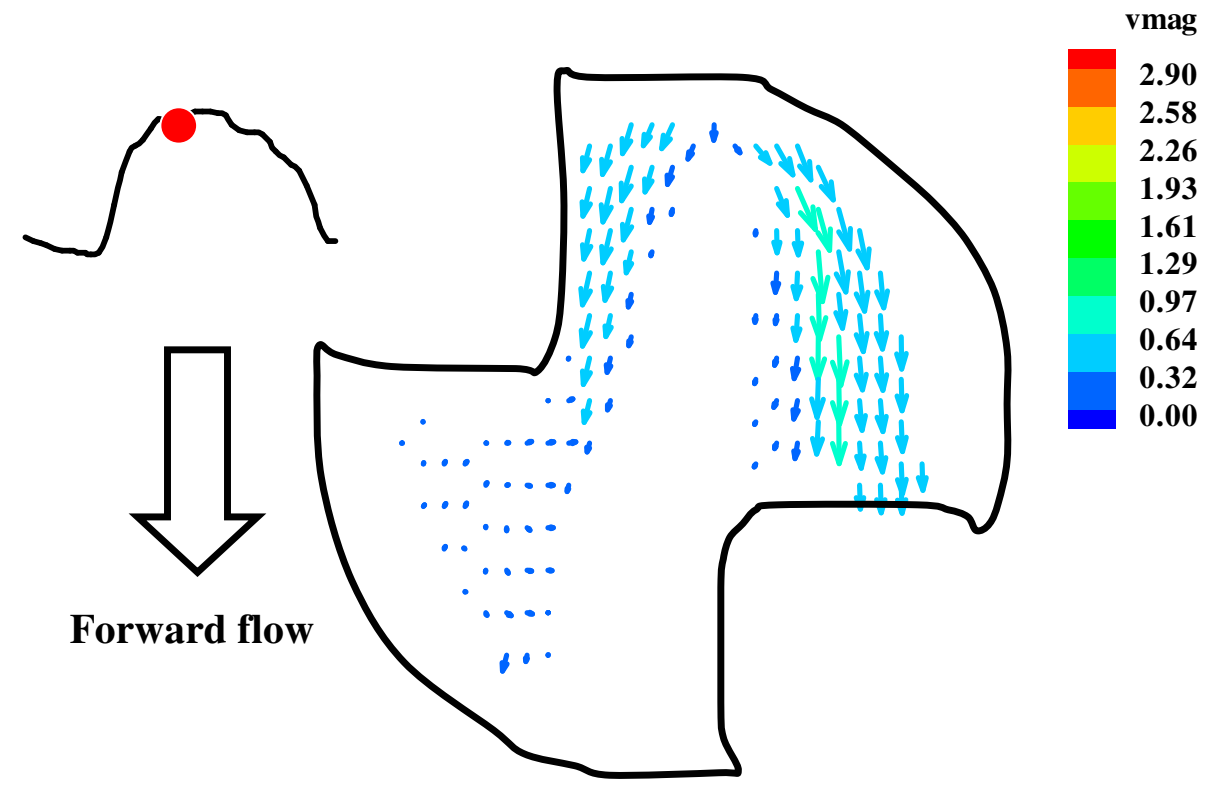

Figure 7-78 CM $23 \mathrm{~mm}$ mitral hinge flow field at mid diastole, time bin 22, $190 \mathrm{um}$ above flat level. A maximum forward velocity of $0.77 \mathrm{~m} / \mathrm{s}$ was measured at the adjacent corner of the hinge (avi, 713k, CM_190um_above_flat_vmag.avi)

\subsection{0 $\mu \mathrm{m}$ Above The Flat}

Animations CM_390um_above_flat_vmag.avi and CM_390um_above_flat_rss.avi show the velocity magnitude $(\mathrm{m} / \mathrm{s})$ and Reynolds shear stress $\left(\right.$ dynes $\left./ \mathrm{cm}^{2}\right)$ vector plots at the $390 \mu \mathrm{m}$ above the flat level of the CM $23 \mathrm{~mm}$ clear valve throughout the cardiac cycle, respectively. Refer to Appendix $\mathrm{H}$ for the list of animations.

\section{Systole}

At onset of leakage flow (time bin 43, Figure 7-79), a narrow stream of leakage jet following the hinge wall boundary can be observed. It was especially pronounced at the atrial corner of the hinge. The leakage jet at this region was connected to other regions (the flat and the $190 \mu \mathrm{m}$ above levels) of leakage flow along the hinge boundary. 
Measurements at this level were not obstructed by the leaflet pivot. Leakage velocity magnitudes of $1 \mathrm{~m} / \mathrm{s}$ were seen at the atrial corner of the hinge. Another region of high leakage flow of $1.5 \mathrm{~m} / \mathrm{s}$ was observed at the ventricular corner of the hinge. The existence of such a high flow region could be explained by the fact that the fluid flowing into the atrial corner of the hinge was directed over the top of the leaflet pivot. Although measurements were not obstructed by the pivot, the velocities remained relatively low throughout most of the central part of the hinge. Towards mid systole (time bin 3, Figure 7-80), the magnitude of the leakage jet reached a peak velocity of $2.52 \mathrm{~m} / \mathrm{s}$ and a corresponding Reynolds shear stress of $1,500 \mathrm{dynes} / \mathrm{cm}^{2}$ in the lateral corner (time bin 3, Figure 7-81). The velocity at the central region of the hinge remained relatively low, showing that the flow dynamics in that region were still very much affected by the pivot. At time bin 43, a peak Reynolds shear stress of 2,000 dynes $/ \mathrm{cm}^{2}$ was observed at both the atrial and ventricular boundaries of the hinge. The Reynolds shear stress increased at time bin 3 reaching a value of 2,500 dynes $/ \mathrm{cm}^{2}$. At time bin 3, higher Reynolds shear stresses were observed in the upper boundary of the hinge. Toward mid diastole (time bin 16, Figure 7-82), the reduced leakage flow was replaced by a relatively low peak forward flow of $0.3 \mathrm{~m} / \mathrm{s}$ at the atrial corner of the hinge. The values of Reynolds shear stress at most sites had decreased to approximately 500 dynes $/ \mathrm{cm}^{2}$. 


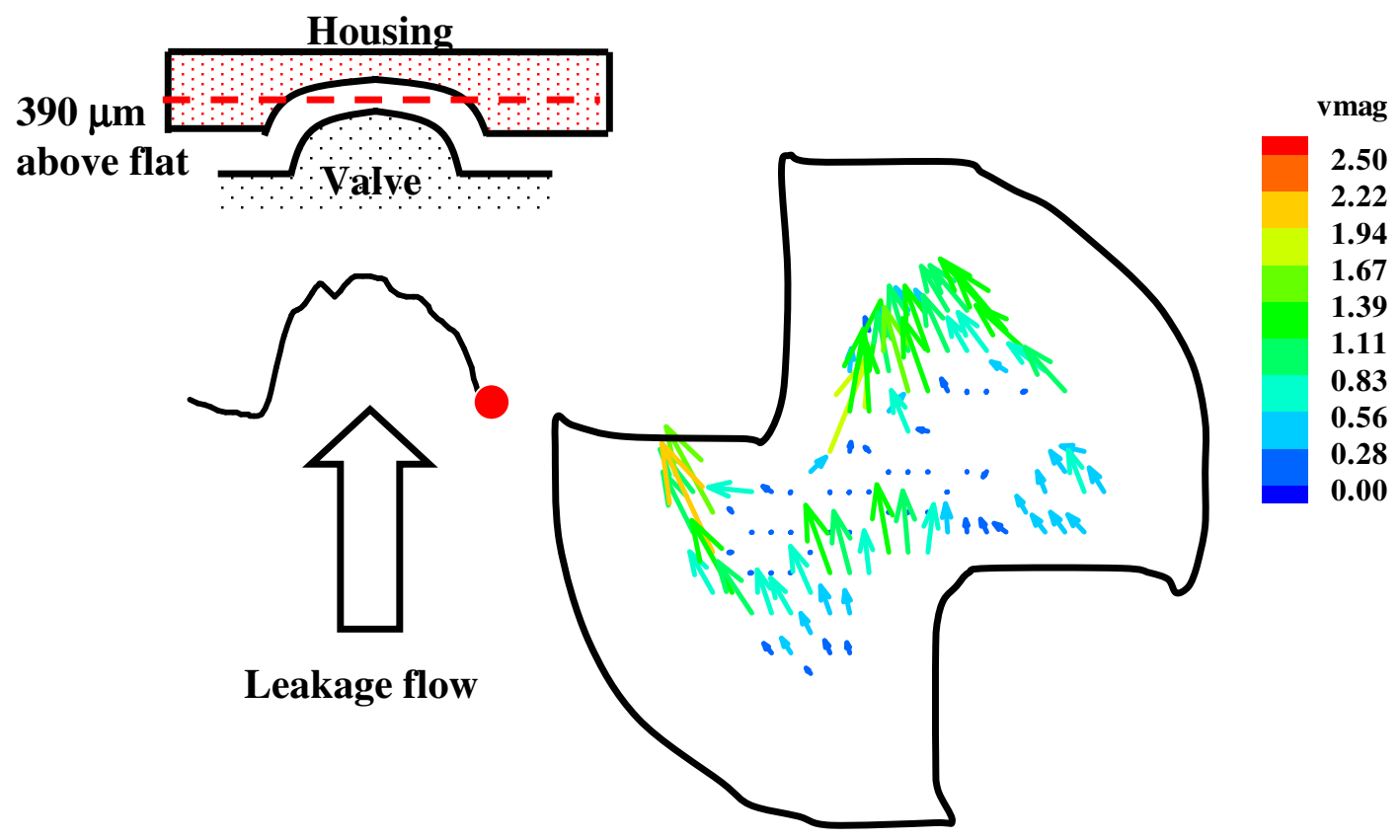

Figure 7-79 CM $23 \mathrm{~mm}$ mitral hinge flow field at early systole, time bin 43, $390 \mu \mathrm{m}$ above flat level; leakage flow appeared along the outer edge of the hinge geometry (avi, 628k, CM_390um_above_flat_vmag.avi)
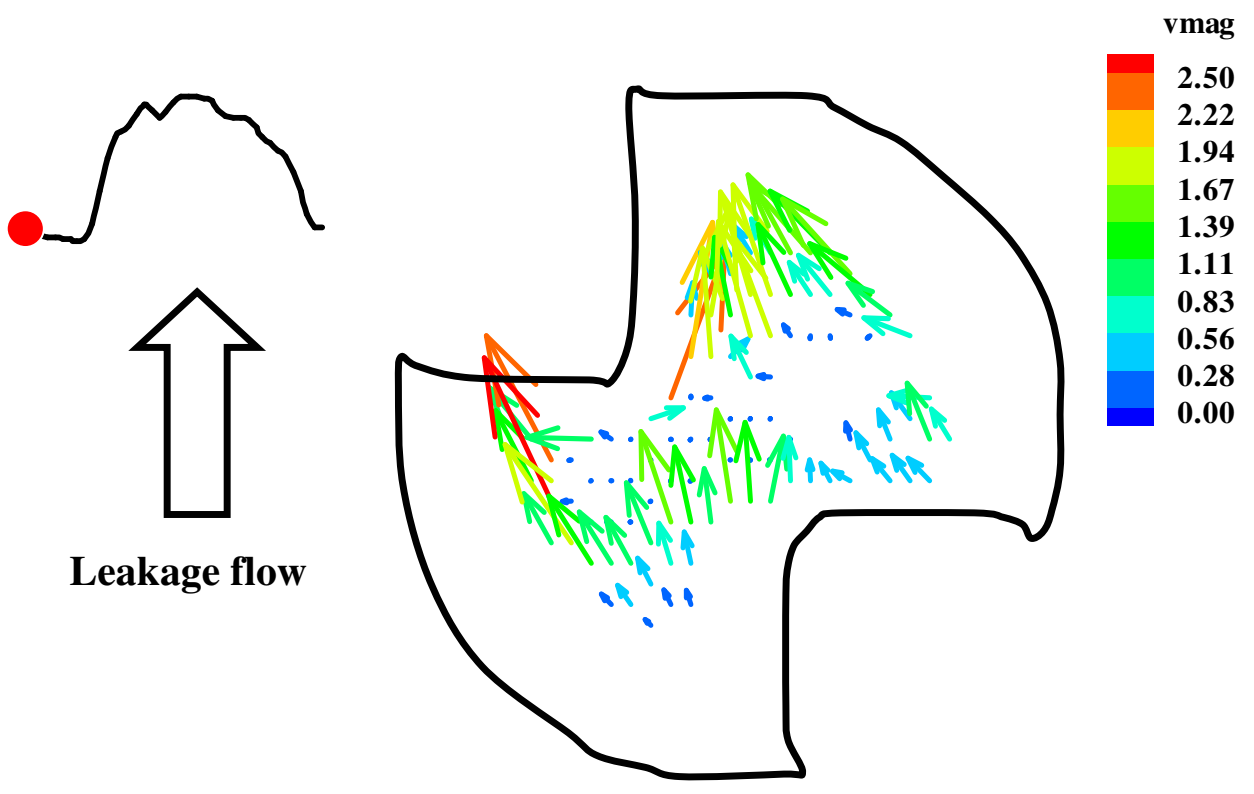

Figure 7-80 CM $23 \mathrm{~mm}$ mitral hinge flow field approaching mid systole, time bin 3, 390 $\mu \mathrm{m}$ above flat level. Peak velocity of $2.52 \mathrm{~m} / \mathrm{s}$ was observed at the lateral corner with low flow at the central region(avi, 628k, CM_390um_above_flat_vmag.avi) 

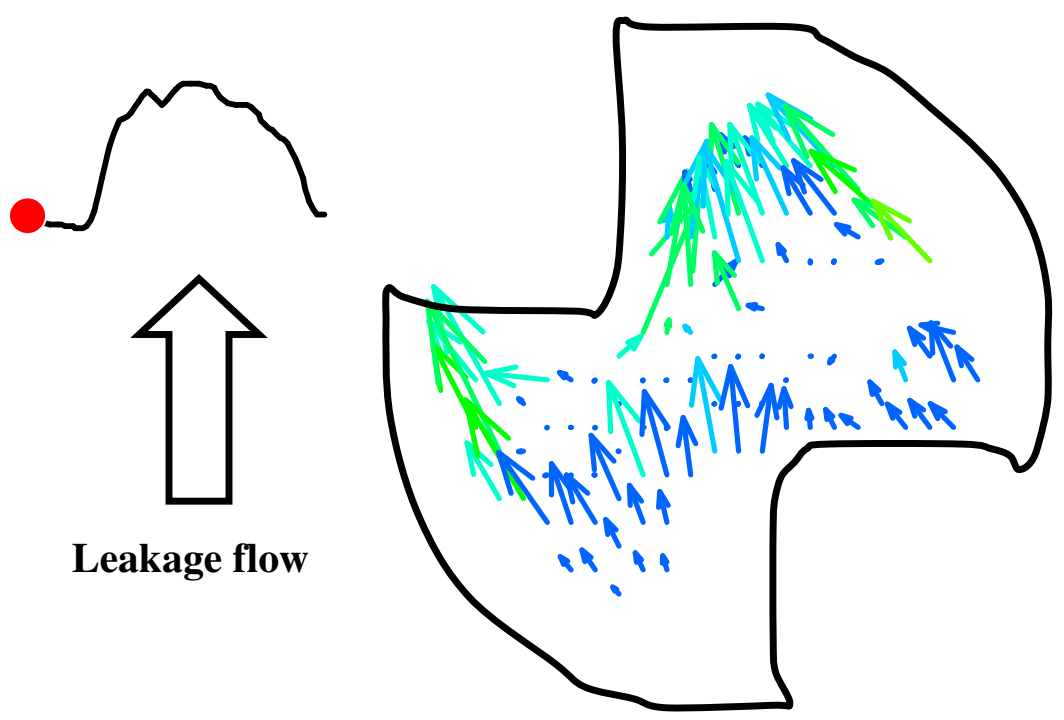

Figure 7-81 Reynolds shear stress distribution of the CM $23 \mathrm{~mm}$ at early systole, time bin 3, $390 \mu \mathrm{m}$ above flat level; elevated Reynolds shear stress levels were recorded in the atrial and lateral corners (avi, 644k, CM_390um_above_flat_rss.avi)

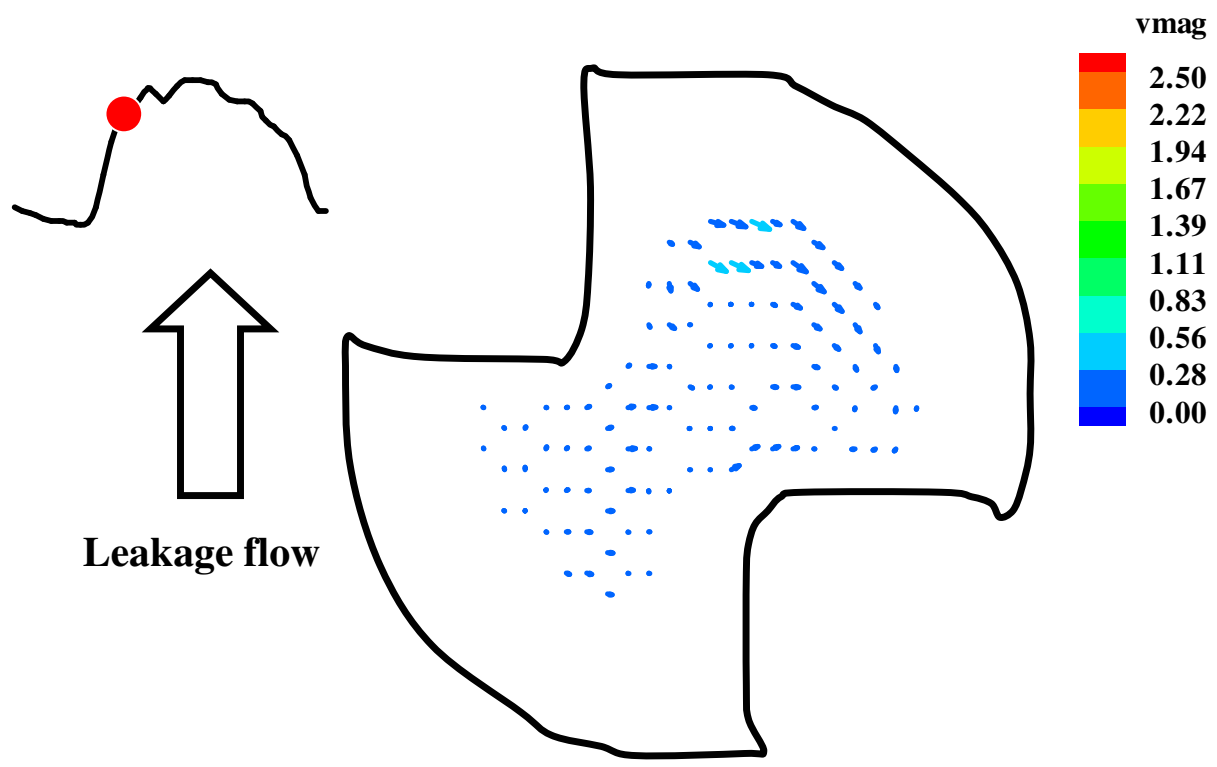

Figure 7-82 CM $23 \mathrm{~mm}$ mitral hinge flow field approaching mid diastole, time bin 16, $390 \mu \mathrm{m}$ above flat level. Low flow of less than $0.3 \mathrm{~m} / \mathrm{s}$ was observed throughout the hinge (avi, 628k, CM_390um_above_flat_vmag.avi) 


\subsection{1mm Below The Flat Level}

Animations CM_1mm below_flat_vmag.avi and CM_1mm below_flat_rss.avi show the velocity magnitude $(\mathrm{m} / \mathrm{s})$ and Reynolds shear stress $\left(\right.$ dynes $\left./ \mathrm{cm}^{2}\right)$ vector plots at the $1 \mathrm{~mm}$ below the flat level of the CM $23 \mathrm{~mm}$ clear valve throughout the cardiac cycle, respectively. Refer to Appendix $\mathrm{H}$ for the list of animations.

It can be seen from Figure 7-83, Figure 7-84, Figure 7-85 that the direction and magnitude of the flow vectors below the flat level were the result of the position of the leaflets rather than that of the hinge location. Toward mid diastole (time bin 14, Figure 7-83), a region of forward flow began to form at the center between the two hinge sockets. The maximum velocity observed was approximately $0.8 \mathrm{~m} / \mathrm{s}$ on the atrial side of the hinge region, while two lateral jets of approximately $0.5 \mathrm{~m} / \mathrm{s}$ were seen directed over to the sides of the hinge. An increase in magnitude of the central jet can be observed at time bin 23 (Figure 7-84) where it reached a maximum velocity of $1.0 \mathrm{~m} / \mathrm{s}$. However, as the flow approached the end of the systolic phase and into the start of the diastolic phase (time bin 12) the energy of the central jet dissipated and reached a low velocity of 0.25 $\mathrm{m} / \mathrm{s}$. At the beginning of the systolic phase (time bin 43, Figure 7-85), a leakage flow of approximately $0.2 \mathrm{~m} / \mathrm{s}$ can be seen surrounding the hinge. A central jet with a peak velocity of $0.5 \mathrm{~m} / \mathrm{s}$ formed in the gap between the two leaflets near the atrial corner of the hinge. The highest Reynolds shear stress of 543 dynes $/ \mathrm{cm}^{2}$ was obtained during the diastolic phase (time bin 1, Figure 7-86). Two regions of high stress were observed; one inside the central leakage jet and another at the right lateral corner. The Reynolds shear stress of the leakage flow had an average value of about 300 dynes $/ \mathrm{cm}^{2}$. 


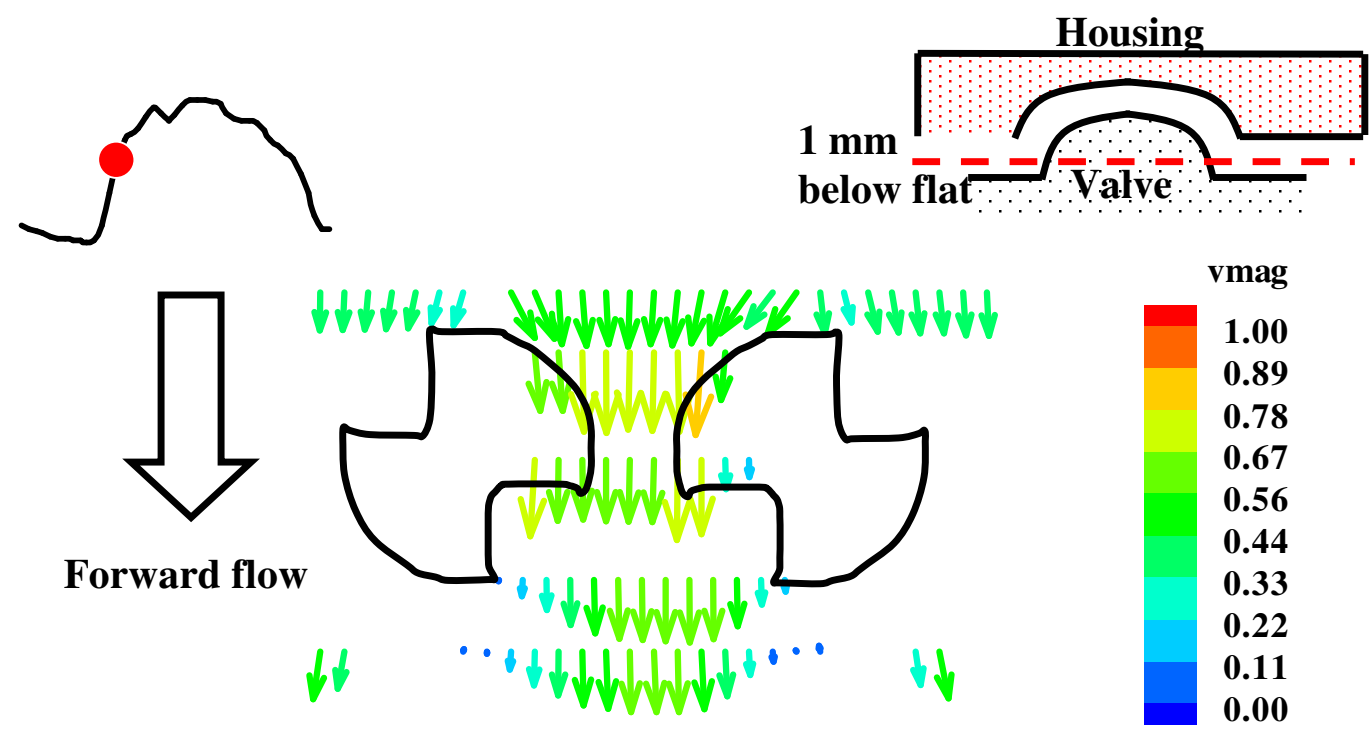

Figure 7-83 CM $23 \mathrm{~mm}$ mitral hinge flow field approaching mid diastole, time bin 14, 1 $\mathrm{mm}$ below flat level; the influence of the leaflets is apparent at the below flat measurement levels (avi, 1,030k, CM_1mm below_flat_vmag.avi)
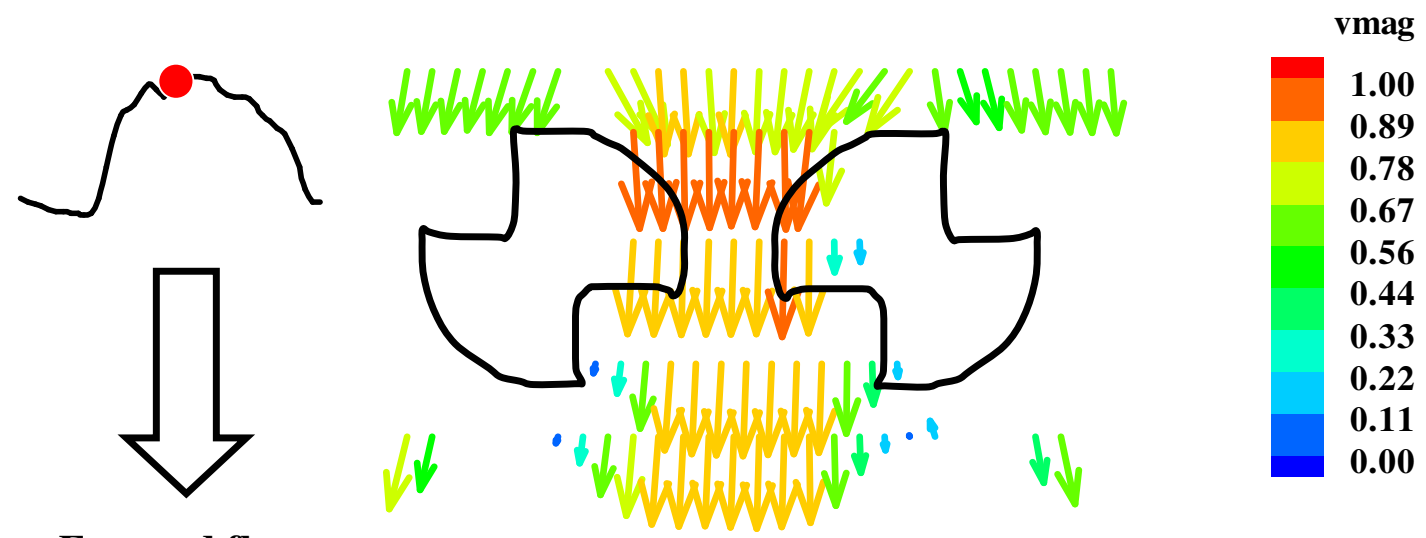

Forward flow

Figure 7-84 CM $23 \mathrm{~mm}$ mitral hinge flow field at peak diastole, time bin $23,1 \mathrm{~mm}$ below flat level; acceleration of the central and two laterals jets is observed (avi, 1,030k, CM_1mm below_flat_vmag.avi) 

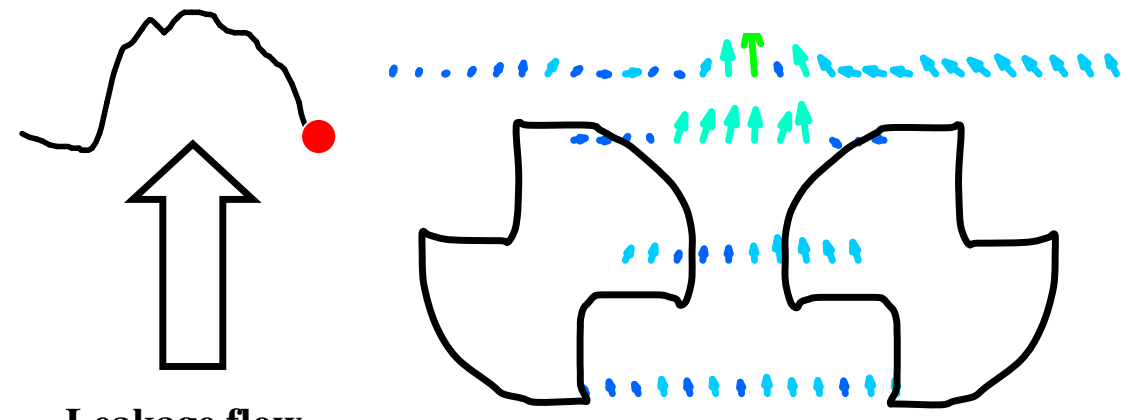

Leakage flow

Figure 7-85 CM $23 \mathrm{~mm}$ mitral hinge flow field at early systole, time bin 43, $1 \mathrm{~mm}$ below flat level; leakage jet appeared at the gap between the closing leaflets (avi, 1,030k, CM_1mm below_flat_vmag.avi)

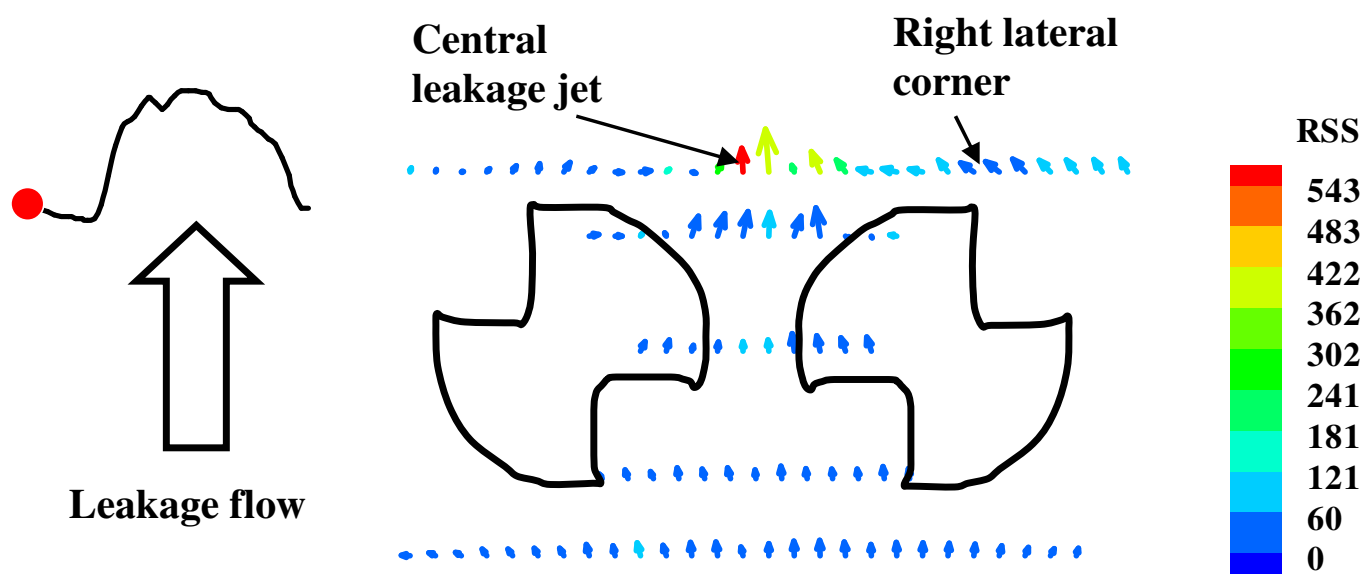

Figure 7-86 Reynolds shear stress distribution of the CM $23 \mathrm{~mm}$ at early systole, time bin 1, $1 \mathrm{~mm}$ below flat level; elevated Reynolds shear stress levels between gap of the closing leaflets (avi, 1,015k, CM_1mm below_flat_rss.avi) 


\subsection{Flow Quantification in Polymeric Heart Valves in the Aortic Position}

The temperature dependent leakage studies comprise of the static leakage rate measurements performed on the three Aortech $23 \mathrm{~mm}$ polymeric heart valves and a pulsatile leakage experiment conducted with prototype A. These studies were conducted at both room $\left(20.5^{\circ} \mathrm{C}\right)$ and physiological $\left(37^{\circ} \mathrm{C}\right)$ temperatures in order to study the effect of temperature on the static and pulsatile leakage performance. In addition, high-speed video and marker tracking experiments were conducted at the room $\left(20.5^{\circ} \mathrm{C}\right)$ and physiological $\left(37^{\circ} \mathrm{C}\right)$ temperatures to investigate the temperature-dependent leaflet kinematics of the polymeric valve prototype A.

In order to characterize the fluid dynamic performance of the three polymeric heart valves, 2D LDV, 3D LDV, and 3D PIV experiments were conducted inside and downstream of the heart valves. To characterize the surface roughness of the leaflets a surface topography experiment was performed on the leaflets of the polymeric valve prototype A and the SJM $27 \mathrm{~mm}$ Standard bileaflet heart valve. Details of these experiments are presented in Chapters 4 and 5. Following is a detailed presentation of the results from these experiments.

\subsubsection{Temperature Dependent Leakage Rate Studies}

\subsubsection{Static Leakage Rate Experiments On The Three Aortech 23 mm Polymeric Heart Valves}

Figure 7-87 shows the leakage flow rate for the three polymeric heart valves. Prototype C had a higher leakage rate of approximately $3.5 \mathrm{~L} / \mathrm{min}$ compared with that of 
approximately $1.0 \mathrm{~L} / \mathrm{min}$ from both prototypes $\mathrm{A}$ and $\mathrm{B}$, for all conditions. The leakage rates at room temperature were compared to those at physiological temperature using a non parametric Mann-Whitney test (data not normally distributed). The test showed that the leakage rates at room temperature were significantly higher than those at physiological temperature $(\mathrm{p}<0.05)$. For the test condition with oscillation, the leakage rates for prototypes $\mathrm{B}$ and $\mathrm{C}$ at room temperature were higher than those measured at physiological temperature, while the reverse was true for prototype A.

The leakage rates of prototypes $\mathrm{A}$ and $\mathrm{B}$ at room temperature without oscillation were comparable to the those under oscillation condition at both room and physiological temperatures (Figure 7-87). For prototype C, the highest and lowest leakage rate were observed at room temperature without oscillation and at physiological temperature with oscillation (Figure 7-87), respectively. See Appendix G for the tabulated leakage rate data for these polymeric valves.

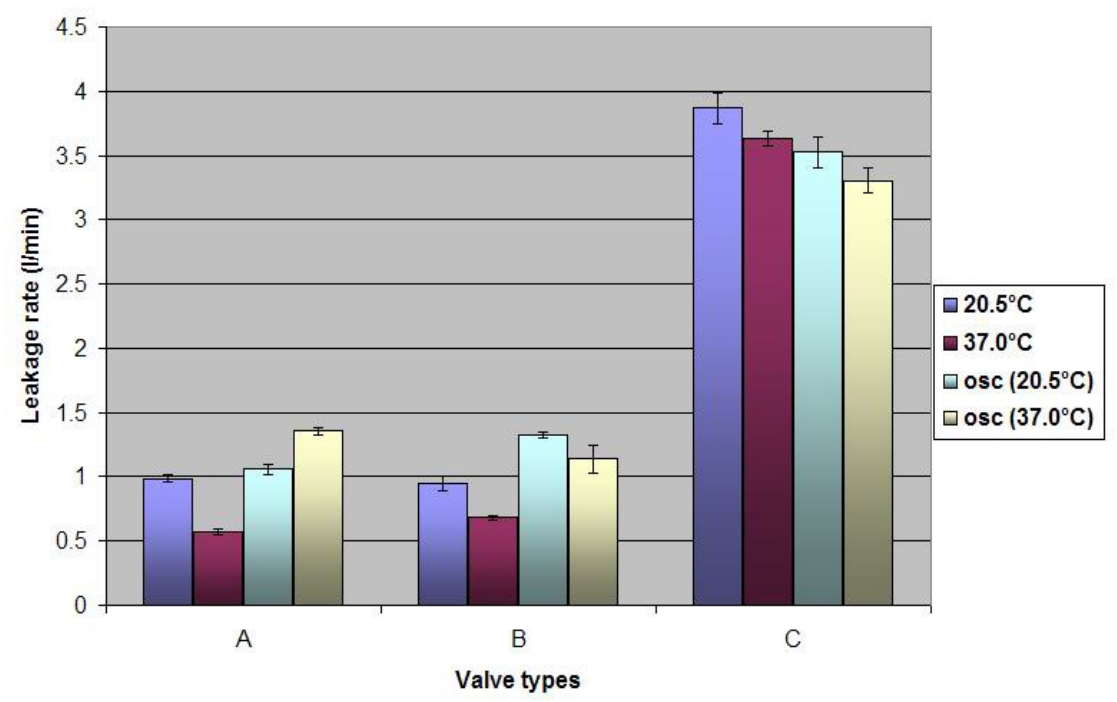

Figure 7-87 The leakage rate of the three polymeric heart valves was tested at both room $\left(20.5^{\circ} \mathrm{C}\right)$ and physiological $\left(37.0^{\circ} \mathrm{C}\right)$ temperatures. An additional set of leakage testing was performed with the oscillation (osc) of the valves to take into account the actual dynamics of the valve under pulsatile settings 


\subsubsection{Pulsatile Leakage Rate Experiments On The Prototype Polymeric Valve A}

Figure 7-88 compares the prototype A aortic flow waveforms between the room $\left(20.5^{\circ} \mathrm{C}\right)$ and physiological $\left(37.0^{\circ} \mathrm{C}\right)$ temperatures. For each temperature, the aortic waveform was averaged over a total of 20 readings, and the leakage volume rate was calculated by integrating the flow rate during the leakage phase. The leakage rate of the valve at room and physiological temperatures were $0.37 \pm 0.06 \mathrm{liter} / \mathrm{min}$ and $0.39 \pm 0.05$ liter $/ \mathrm{min}$, respectively $(\mathrm{p}>0.05)$.

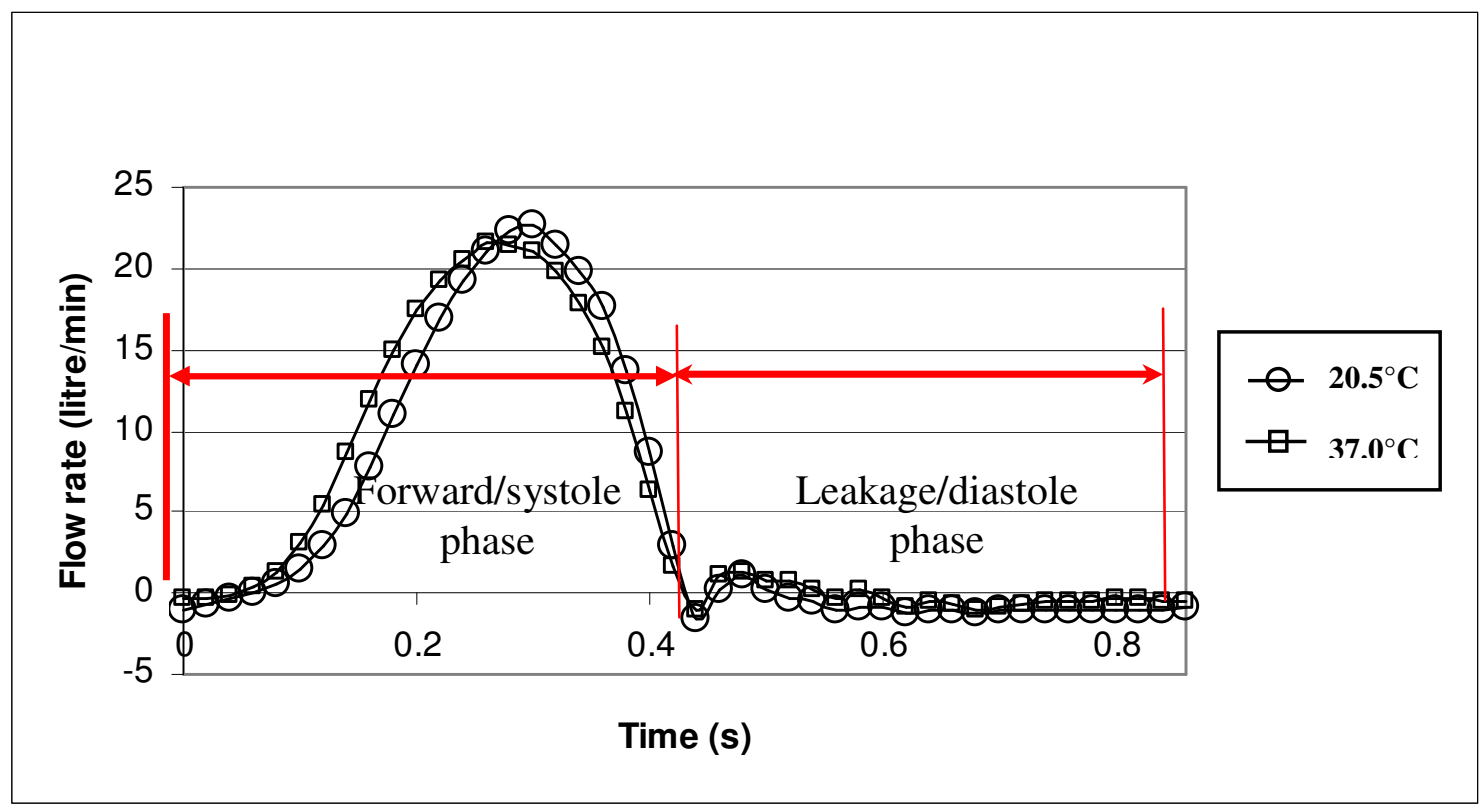

Figure 7-88 Comparison of the leakage flow rate for the prototype polymeric valve A between the room $\left(20.5^{\circ} \mathrm{C}\right)$ and physiological $\left(37.0^{\circ} \mathrm{C}\right)$ temperatures in the aortic position

\subsubsection{D High-Speed Video and Marker Experiments}

Table 7-1 shows the recorded peak velocity of the individual markers observed on the valve leaflet at different temperatures. Two set of experiments were performed at each temperature. Marker \#6 coincided with the edge of the leaflet and marker \#13 the high center region (as defined in section 4.2.1) of the leaflet (see section 4.6.2 for the 
individual position of the markers on the leaflet). Figure 7-89 and Figure 7-90 show the velocity magnitude of marker \#13 located in the high central region of the valve at room and physiological temperatures, respectively. Autotracking of the markers showed that the peak velocity occurred during the opening and closing phases, which coincided with the first and second peaks as shown in the figures. These figures also show oscillation of the marker position during the diastolic period.

Table 7-1 Tabulated peak velocity of the marker point during the opening and closing phases at both the room $\left(20.5^{\circ} \mathrm{C}\right)$ and physiological $\left(37.0^{\circ} \mathrm{C}\right)$ temperatures. The number in the parenthesis refer to the position of the marker

\begin{tabular}{|c|c|c|c|c|}
\hline & \multicolumn{4}{|c|}{ Fluid Temperature $\left({ }^{\circ} \mathrm{C}\right)$} \\
\hline & $\begin{array}{c}1^{\text {st }} \text { experiment } \\
20.5^{\circ} \mathrm{C} \\
\end{array}$ & $\begin{array}{c}2^{\text {nd }} \text { experiment } \\
20.5^{\circ} \mathrm{C} \\
\end{array}$ & $\begin{array}{c}1^{\mathrm{st}} \text { experiment } \\
37.0^{\circ} \mathrm{C}\end{array}$ & $\begin{array}{c}2^{\text {nd }} \text { experiment } \\
37.0^{\circ} \mathrm{C} \\
\end{array}$ \\
\hline $\begin{array}{c}\text { Peak Opening } \\
\text { Velocity } \\
\text { (marker \#) }\end{array}$ & $\begin{array}{c}0.94 \mathrm{~m} / \mathrm{s} \\
(6)\end{array}$ & $\begin{array}{c}1.28 \mathrm{~m} / \mathrm{s} \\
(6)\end{array}$ & $\begin{array}{c}1.07 \mathrm{~m} / \mathrm{s} \\
(6)\end{array}$ & $\begin{array}{c}1.92 \mathrm{~m} / \mathrm{s} \\
(5)\end{array}$ \\
\hline $\begin{array}{c}\text { Peak Closing } \\
\text { Velocity } \\
\text { ( marker \#) }\end{array}$ & $\begin{array}{c}0.94 \mathrm{~m} / \mathrm{s} \\
(13)\end{array}$ & $\begin{array}{c}1.38 \mathrm{~m} / \mathrm{s} \\
(13)\end{array}$ & $\begin{array}{c}1.09 \mathrm{~m} / \mathrm{s} \\
(13)\end{array}$ & $\begin{array}{c}1.34 \mathrm{~m} / \mathrm{s} \\
(13)\end{array}$ \\
\hline
\end{tabular}




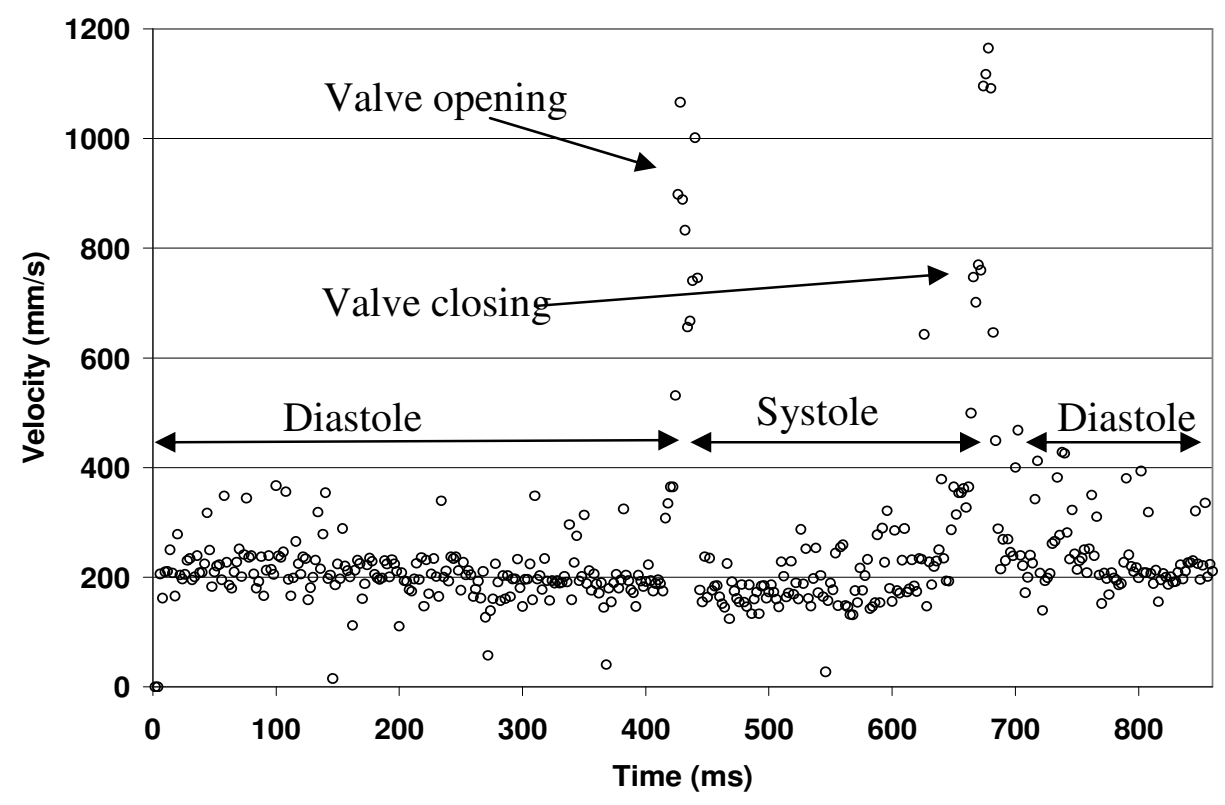

Figure 7-89 Velocity of marker \# 13 at the high central region of the leaflet of prototype polymeric valve A tested at a temperature of $20.5^{\circ} \mathrm{C}$. The graph shows two peaks, which corresponded to the valve opening and closing phases

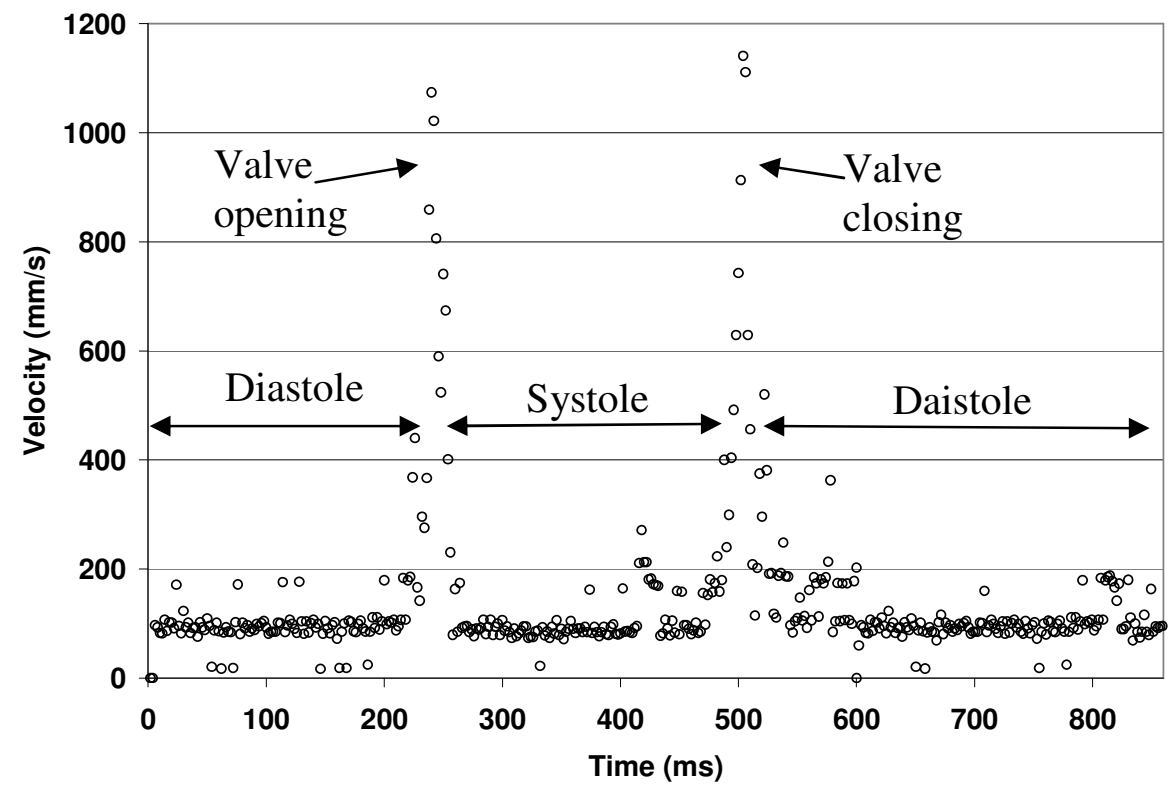

Figure 7-90 Velocity of marker \# 13 at high central region of the leaflet of prototype polymeric valve A tested at a temperature of $37{ }^{\circ} \mathrm{C}$. The two peaks corresponded to the valve opening and closing phases 
$3 \mathrm{D}$ reconstruction of the leaflet motion in both $20.5^{\circ} \mathrm{C}$ and $37^{\circ} \mathrm{C}$ demonstrated similar leaflet opening and closing dynamic, therefore only the results from the $20.5^{\circ} \mathrm{C}$ condition is presented (refer to Appendix $\mathbf{J}$ for the leaflet motion results at $37^{\circ} \mathrm{C}$ ). Figure $7-91$, also illustrated in 3D_reconstructed_leaflet_motion_room_temp.avi (Appendix H), shows the opening and closing pattern of the polymeric valve leaflet at room temperature $\left(20.5^{\circ} \mathrm{C}\right)$. Typically, the highest opening and closing velocities were recorded at markers \#6 and \#13 located at the edge and the high central region of the leaflet, respectively. During the opening phase when the leaflet begins to open from the rear of the leaflet towards the edge (Figure 7-91a-b), the highest velocity tended to be on the edge of the leaflet (marker \# 6), while in the closing phase, the highest velocity was at high central region of the leaflet (marker \# 13). It can be seen from Figure 7-91c that a crest was formed when the valve was fully open. Oscillation of the leaflet can be observed when the valve closed and persisted throughout the entire phase of diastole. 


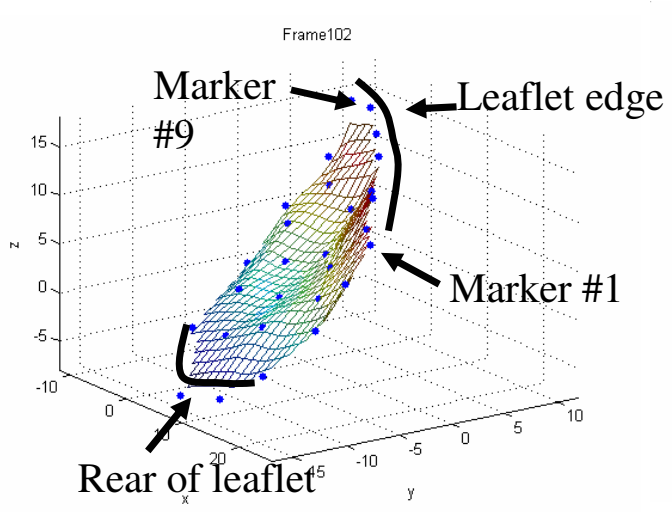

(a)

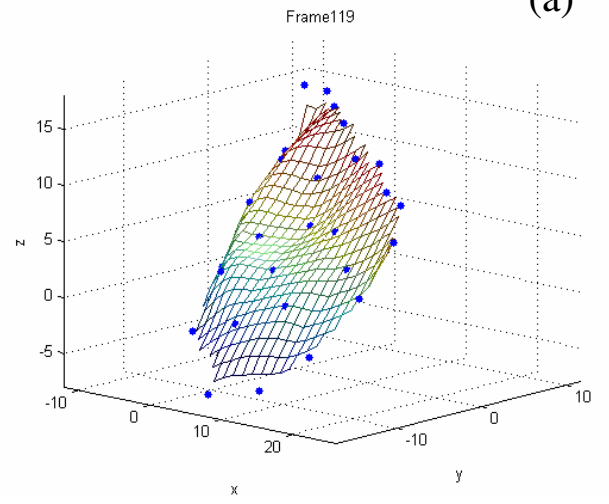

(b)

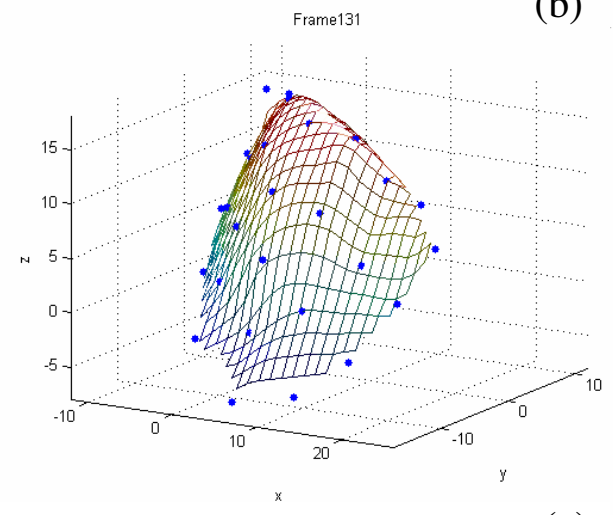

(c)

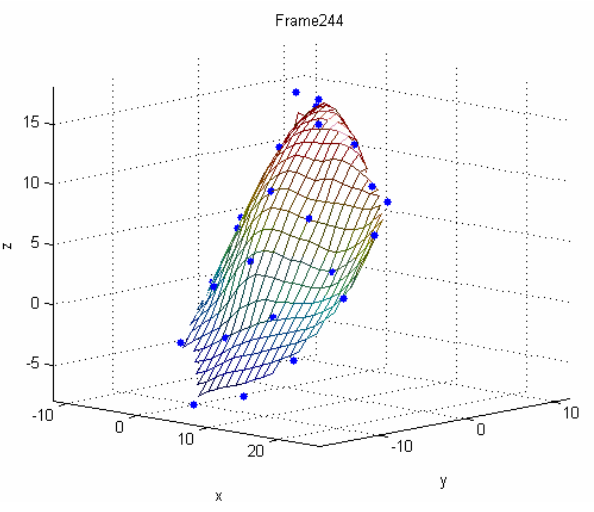

(d)

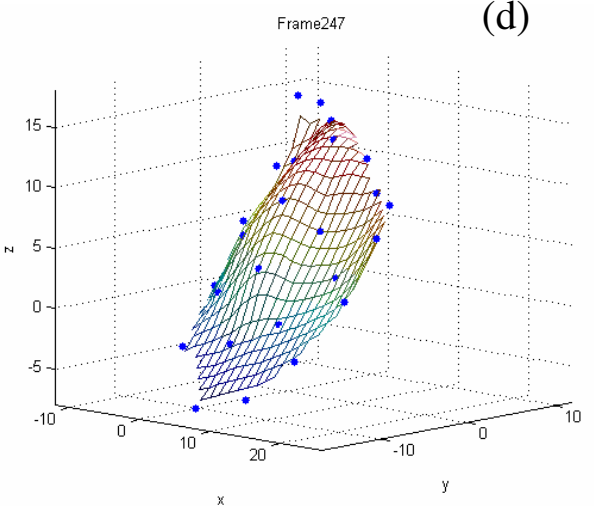

(e)

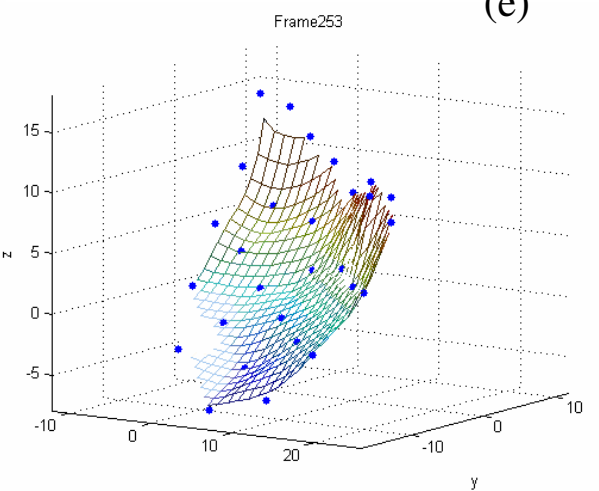

(f)

Figure 7-91 The motion of the leaflet through a single cardiac cycle (temperature $20.5^{\circ} \mathrm{C}$ ). (a) The beginning of the opening phase; (b) Leaflet at the verge of opening; (c) Leaflet at fully opened position; (d) The beginning of the closing phase; (e) The closing phase; and (f) Leaflet at fully closed position (wmv, 3,764k, 3D_reconstructed_leaflet_motion_room_temp.wmv) 
Figure 7-92, also illustrated in animation high_speed_camera_upstream_room_temp.avi (Appendix H), shows the dynamics of the valve leaflet through a single cardiac cycle under the $20.5^{\circ} \mathrm{C}$ condition (refer to Appendix $\mathrm{J}$ for the leaflet motion results at $37^{\circ} \mathrm{C}$ ). During opening, the top leaflet (seen at the top right corner) opened 26 ms earlier than the other two leaflets (Figure 7-92b). The center of the leaflet edge was seen to open first, causing a wrinkle on either side of the leaflet near the stent and a slight crest at the central region of the valve. As the cycle continued, the wrinkle gradually unfolded so that the entire leaflet took on an arch shape (Figure 7-92c). A deep channel was still visible at the commissure region during the fully open phase, and this was a result of the leaflet being attached to the stent (Figure 7-92c).

During valve closure, the leaflets closed at a slower velocity than during the opening phase. The edges of the leaflets first come in contact with each other near the commissure region forming wrinkles along the leaflet edge (Figure 7-92d). At this time, the crest was still visible on the leaflet at the high central region. A small gap channel at the commisural region was visible throughout the diastole (Figure 7-92e). The valve oscillated or 'bounced' in the fully closed position, which caused the small leakage gap at the coaptation zone to vary in size during diastole (Figure 7-92f). 


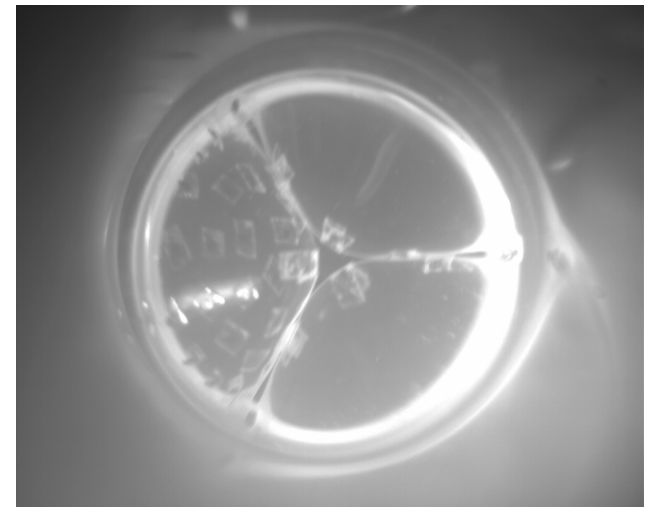

(a)

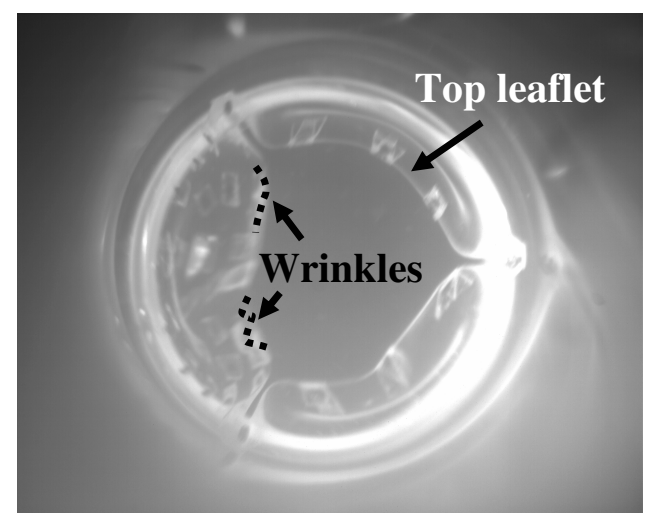

(b)

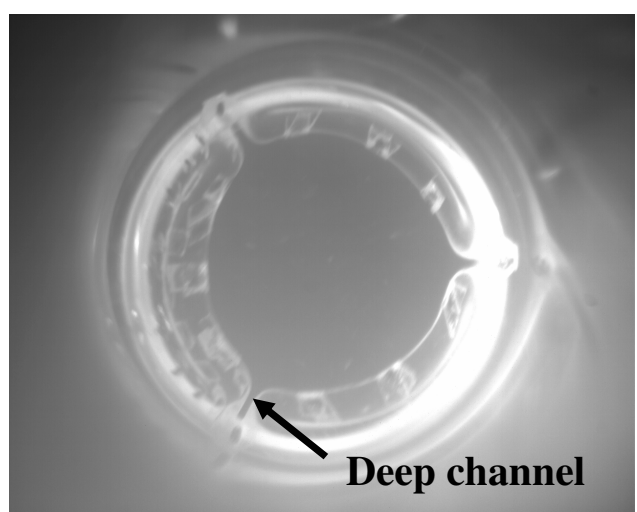

(c)

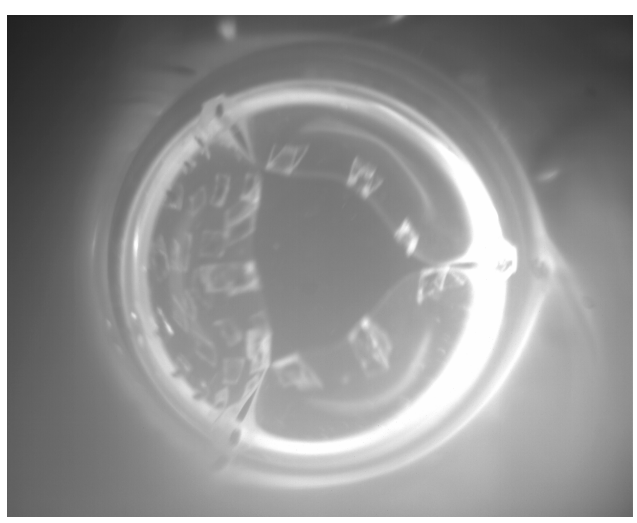

(d)

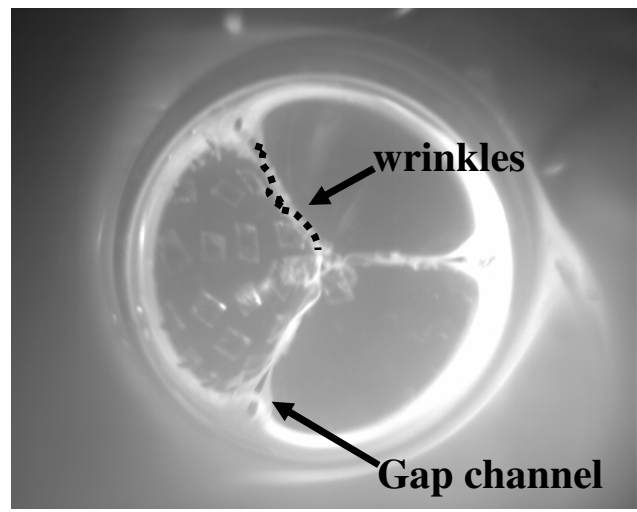

(e)

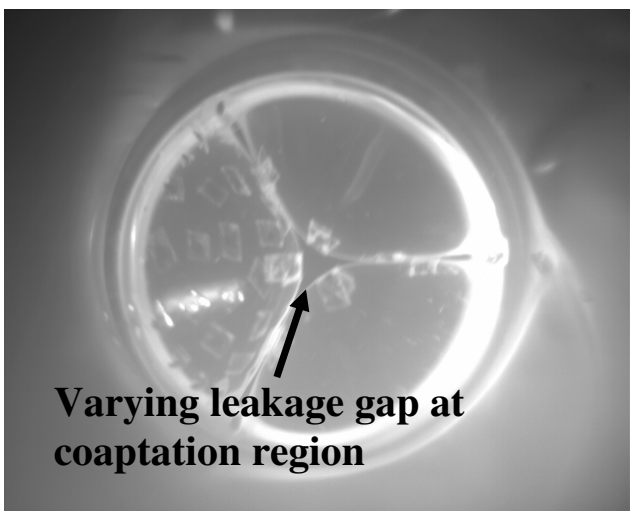

(f)

Figure 7-92 Upstream high speed camera image of the valve through the dogleg (temperature $-20.5^{\circ} \mathrm{C}$ ). (a) The beginning of opening phase; (b) valve opening; (c) valve when full open; (d) valve closing; (e) valve when closed; and e) oscillation of valve leaflet during diastolic phase. (wmv, 1,850k, animation high_speed_camera_upstream_room_temp.wmv) 


\subsubsection{D LDV Measurements On The Three Prototype Aortech 23 mm Polymeric Heart Valves}

The results presented in this section are based on phase averaged velocity fields. The flow characteristics of prototype A are presented first followed by those of the prototypes B and C. For each prototype valve, the results are presented in the following order: 1) downstream flow distribution; 2) measurements immediately downstream of the stent; and 3) measurements within the polymeric heart valve. For each measurement plane, the description of the flow during systole is presented first followed by that during diastole. All contour plots are appropriately color-coded by Velocity Magnitude $(\mathrm{m} / \mathrm{s})$ or Reynolds shear stress (dynes $/ \mathrm{cm}^{2}$ ). Refer to Figure 5-17 and Figure 5-18 for the position of various measurement planes. In these figures the direction of the forward flow is from right to left (from the left ventricle into the aorta). Arrows point in the direction of the phase averaged mean velocity vectors and the length of the arrow is proportional to the velocity magnitude. Refer to Figure 5-2 for the aortic flow and pressure waveforms, and Appendix $\mathrm{H}$ for the velocity magnitude $(\mathrm{m} / \mathrm{s})$ and Reynolds shear stress $\left(\right.$ dynes $/ \mathrm{cm}^{2}$ ) animations. Figure 7-93 shows the terminology pertinent to the following description of the polymeric heart valve results. 

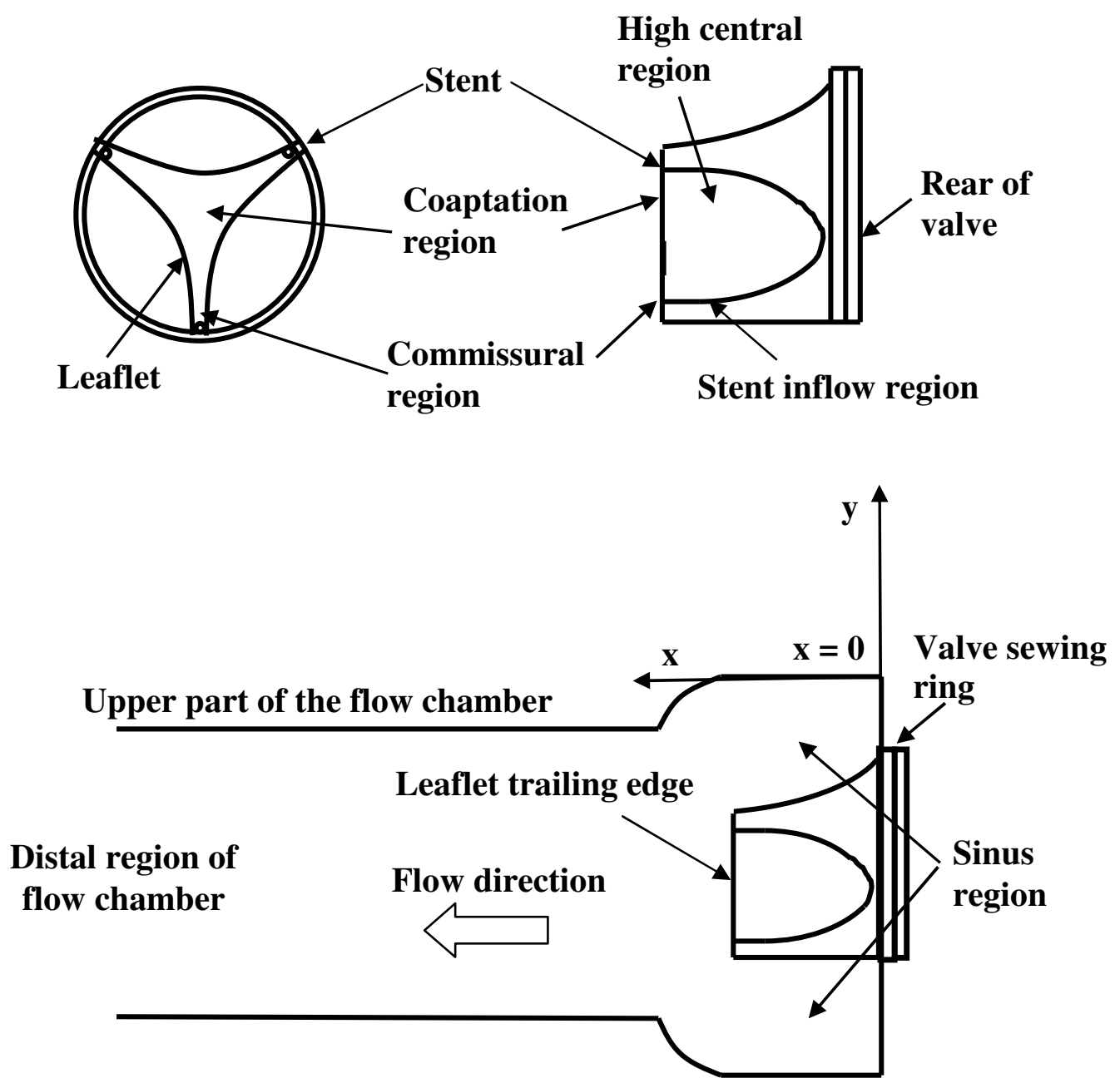

Figure 7-93 Pertinent terminology for the three Aortech $23 \mathrm{~mm}$ trileaflet polymeric heart valves. All downstream measurements were made with reference from the valve sewing ring, which is marked $\mathrm{x}=0$

\subsubsection{Closed Commissure Valve With 80 um Leaflet Thickness (Prototype A)}

\subsection{Flow Fields Downstream of the Valve}

Animations A_parallel_downstream_vmag.avi and A_parallel_downstream_rss.avi show the velocity magnitude $(\mathrm{m} / \mathrm{s})$ and Reynolds shear stress $\left(\right.$ dynes $\left./ \mathrm{cm}^{2}\right)$ contour plots of the flow fields in the plane parallel to the stent axis (see Figure 5-17), respectively. Similarly, animations A_perpend_downstream_vmag.avi and A_perpend_downstream_rss.avi show 
the velocity magnitude $(\mathrm{m} / \mathrm{s})$ and Reynolds shear stress $\left(\right.$ dynes $\left./ \mathrm{cm}^{2}\right)$ contour plots of the flow fields in the plane perpendicular to the stent axis (see Figure 5-17), respectively. Refer to Appendix $\mathrm{H}$ for the list of animations.

\section{$\underline{\text { Systole }}$}

The flow patterns along the parallel-to-stent axis measurement plane were similar to those measured along the perpendicular-to-stent axis. Hence only flow fields along the parallel-to-stent axis are shown. Figure 7-94 shows the flow field downstream of prototype A along the parallel-axis during the acceleration phase (time bin 5). A central orifice jet with a velocity of $1.8 \mathrm{~m} / \mathrm{s}$ was seen exiting from the valve orifice. Flow separation occurred at the trailing edge of the valve leaflet with reattachment points 75 $\mathrm{mm}$ and $60 \mathrm{~mm}$ downstream from the edge of the valve sewing ring in the upper and lower parts of the flow chamber, respectively. Recirculation zones were observed along the edge of the central orifice jet with vortices inside the sinus region. Figure 7-95 shows the flow field downstream of prototype A during peak systole (time bin 9). The peak systolic velocity of $2.8 \mathrm{~m} / \mathrm{s}$ occurred in the contraction region, or vena contracta, of the central orifice jet at the end of the sinus region 21.3 to $26.4 \mathrm{~mm}$ downstream from the edge of the valve sewing ring. This vena contracta phenomenon resulting from jet flow through an orifice is depicted schematically in Figure 7-95b. The central jet had a diameter of approximately $12 \mathrm{~mm}$. The location of the flow re-attachment point in the upper part of the flow chamber at peak systole extended beyond the measurement plane, while that in the lower part of the flow chamber maintained at approximately $60 \mathrm{~mm}$ downstream from the edge of the valve sewing ring. The flow inside the recirculation 
zone and the vortices inside the sinus region were more pronounced at peak systole than those seen in the acceleration phase. During deceleration (time bin 14), the velocity in the central orifice jet decreased from $2.8 \mathrm{~m} / \mathrm{s}$ to $1.25 \mathrm{~m} / \mathrm{s}$. The recirculation region around the central orifice jet reached a maximum velocity of $0.25 \mathrm{~m} / \mathrm{s}$ along the wall of the flow chamber.

A maximum Reynolds shear stress of approximately 8,300 dynes $/ \mathrm{cm}^{2}$ was measured in the central orifice jet during peak systole and its location coincided with the vena contracta (time bin 9, Figure 7-96). Elevated Reynolds shear stress levels ranging between 700-1700 dynes $/ \mathrm{cm}^{2}$ were also observed along the edge of the central orifice jet and in location $75 \mathrm{~mm}$ downstream from the valve sewing ring during peak systole.

\section{$\underline{\text { Diastole }}$}

During the diastolic phase (time bin 21), the fluid downstream of the valve was essentially stationary with average flow velocities of less than $0.04 \mathrm{~m} / \mathrm{s}$ and Reynolds shear stress levels lower than 10 dynes/cm² (Figure 7-97). 


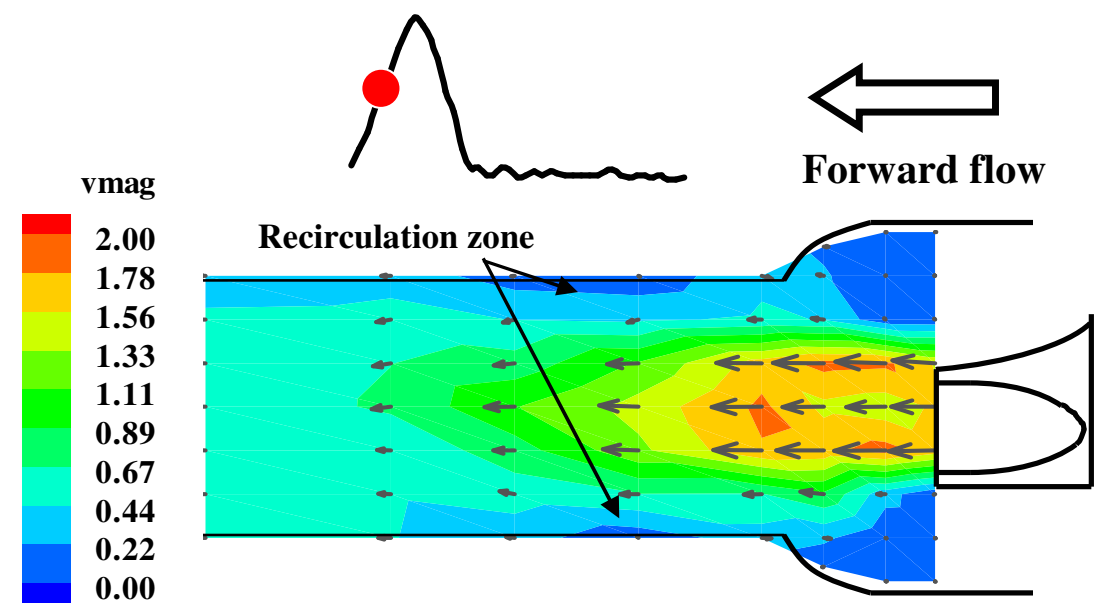

Figure 7-94 Velocity fields downstream of prototype $A$ at acceleration phase, time bin 5, center plane; a developing central orifice jet of $1.8 \mathrm{~m} / \mathrm{s}$ was seen issuing from the orifice of the polymeric valve with formation of recirculation zone between the edge of the valve and $75 \mathrm{~mm}$ downstream from the edge of the valve sewing ring (avi, 586k, A_parallel_downstream_vmag.avi)

(a)
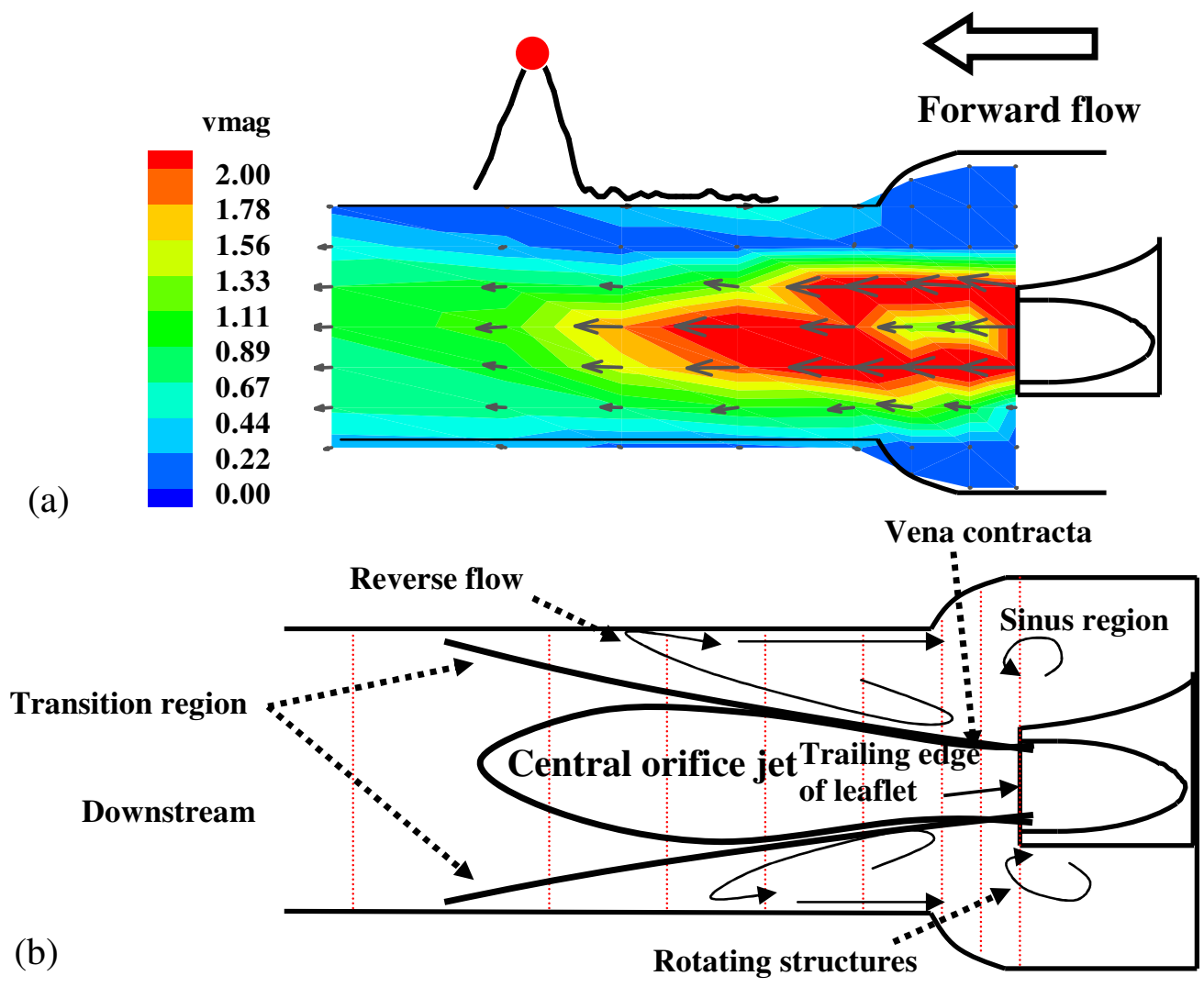

Figure 7-95 Velocity fields downstream of prototype A at peak systole, time bin 9, center plane; (a) Velocity vectors acquired along the parallel-axis plane at peak systole, (b) Schematic of the flow field 


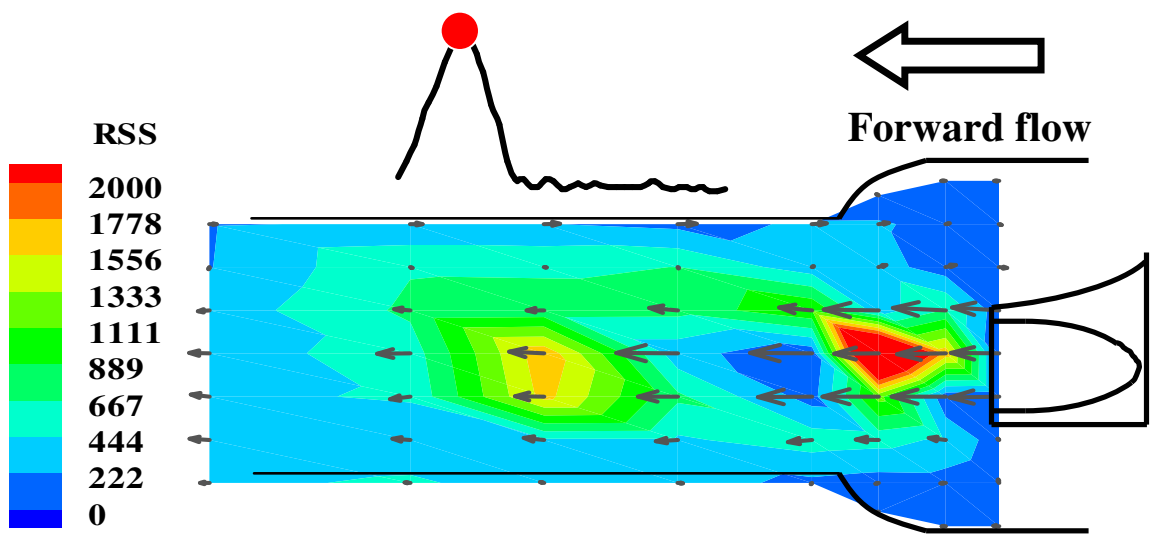

Figure 7-96 Reynolds shear stress distribution of prototype A at peak systole, time bin 9, center plane; elevated Reynolds shear stress levels were observed at the region of flow contraction, edge of the central orifice jet and $60 \mathrm{~mm}$ downstream from the edge of the valve (avi, 600k, A_parallel_downstream_rss.avi)

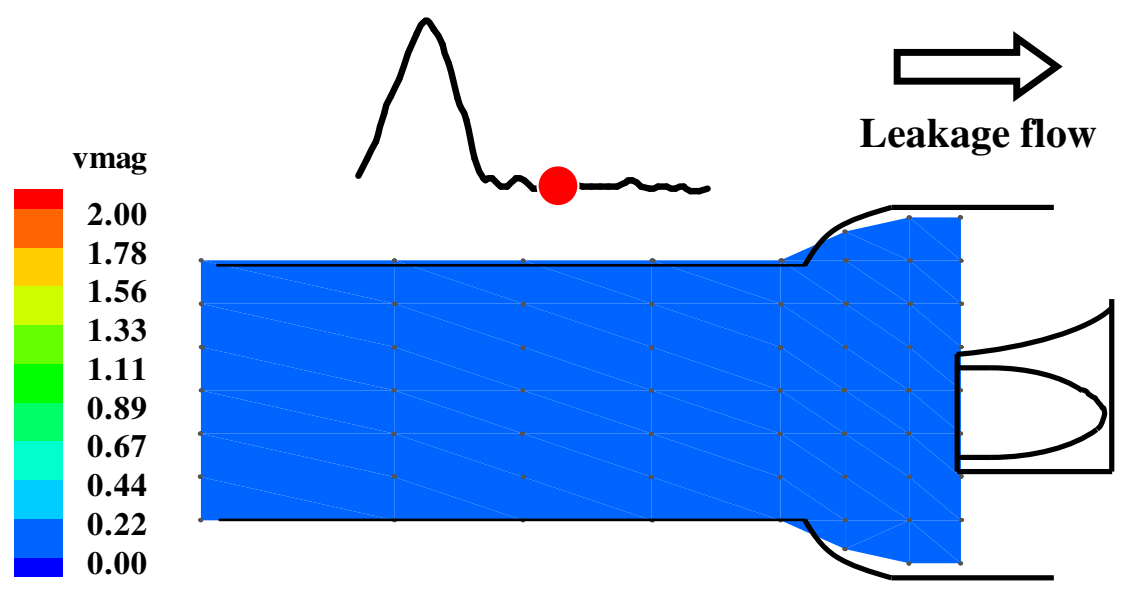

Figure 7-97 Velocity fields downstream of prototype A at mid diastole, time bin 21, center plane; the flow field during diastole was low at around $0.04 \mathrm{~m} / \mathrm{s}$ (avi, 586k, A_parallel_downstream_vmag.avi)

\subsection{Flow Downstream of the Stent}

Animations A_stent_downstream_vmag.avi and A_stent_downstream_rss.avi show the velocity magnitude $(\mathrm{m} / \mathrm{s})$ and Reynolds shear stress $\left(\right.$ dynes $\left./ \mathrm{cm}^{2}\right)$ contour plots of the flow 
field downstream of stent of the polymeric prototype A valve throughout the cardiac cycle, respectively. Refer to Appendix $\mathrm{H}$ for the list of animations.

\section{Systole}

Detailed flow field measurements were acquired immediately downstream of the stent as the flow features of this region were not fully resolved in the downstream flow measurements presented in section 7.3.3.1.1. Figure 7-98 shows the flow fields in the region downstream of the (prototype A) stent during the acceleration phase (time bin 5). A forward jet with a velocity of $0.87 \mathrm{~m} / \mathrm{s}$ was seen issuing from the commissural region coinciding with the location of the deep channel shown in Figure 7-92c. At peak systole (time bin 9), the forward jet accelerated to a maximum velocity of $0.98 \mathrm{~m} / \mathrm{s}$ (Figure 7-99). In contrast, the velocity of the flow surrounding the forward jet was typically less than $0.05 \mathrm{~m} / \mathrm{s}$ indicating flow separation. During deceleration (time bin 14), the forward jet issuing from the deep channel reduced from $0.87 \mathrm{~m} / \mathrm{s}$ to $0.49 \mathrm{~m} / \mathrm{s}$. The flow velocity in the surrounding fluid at this time phase was lower than $0.05 \mathrm{~m} / \mathrm{s}$. A maximum Reynolds shear stress of $1,260 \mathrm{dynes} / \mathrm{cm}^{2}$ was observed at the tip of the stent during peak systole (time bin 9, Figure 7-100), while the shear stress levels farther from the stent were only on the order of 30 to 100 dynes $/ \mathrm{cm}^{2}$.

\section{Diastole}

During valve closure, regions of unsteady high velocity flow were observed near the stent. Figure 7-101 shows the flow field in the region downstream of the stent during mid diastole (time bin 21). A leakage jet that persisted throughout diastole was observed at 
the stent region with velocities oscillating between $0.54 \mathrm{~m} / \mathrm{s}$ and $0.14 \mathrm{~m} / \mathrm{s}$. The location of the leakage jet coincided with the location of the stent post when the valve was closed. The Reynolds shear stress levels downstream of the stent tip during diastole were typically below 25 dynes $/ \mathrm{cm}^{2}$, except for occasional elevated shear stresses of more than 200 dynes $/ \mathrm{cm}^{2}$ at the stent tip.

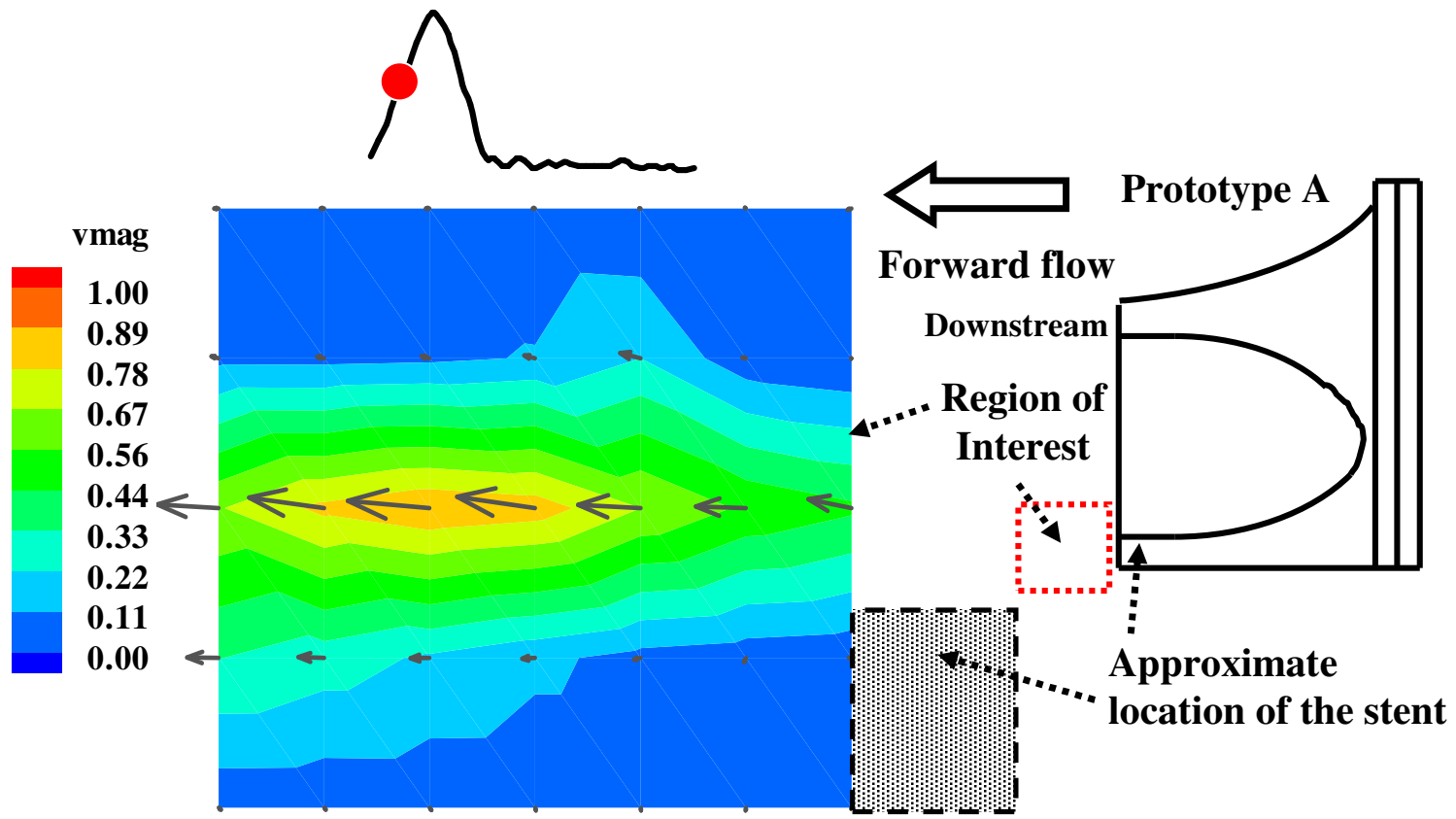

Figure 7-98 Velocity fields downstream of the stent of prototype A during acceleration phase, time bin 5; high forward jet of $0.87 \mathrm{~m} / \mathrm{s}$ was observed to emanate from the deep channel at the commissural region of the prototype A (avi, 589k, A_stent_downstream_vmag.avi) 

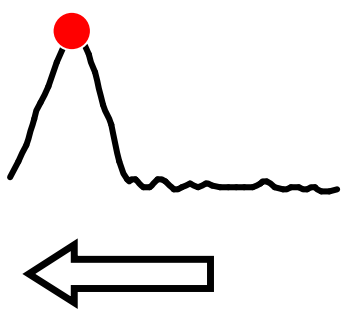

Forward flow

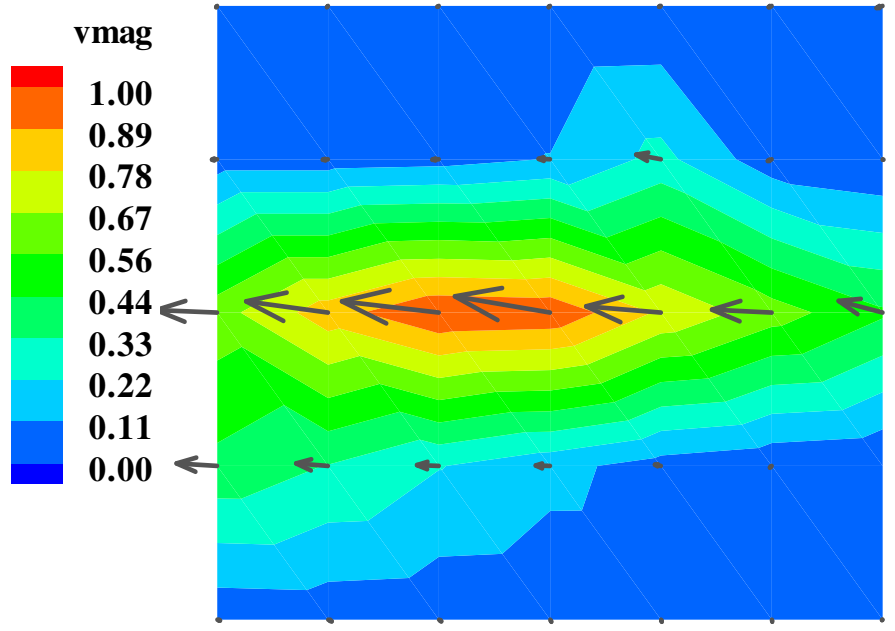

Figure 7-99 Velocity fields downstream of the stent of prototype A during peak systole, time bin 9; high velocity jet emanates from the gap channel at the commissural region (avi, 589k, A_stent_downstream_vmag.avi)

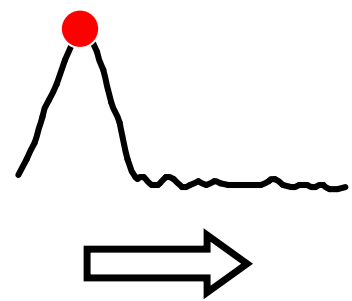

Leakage flow

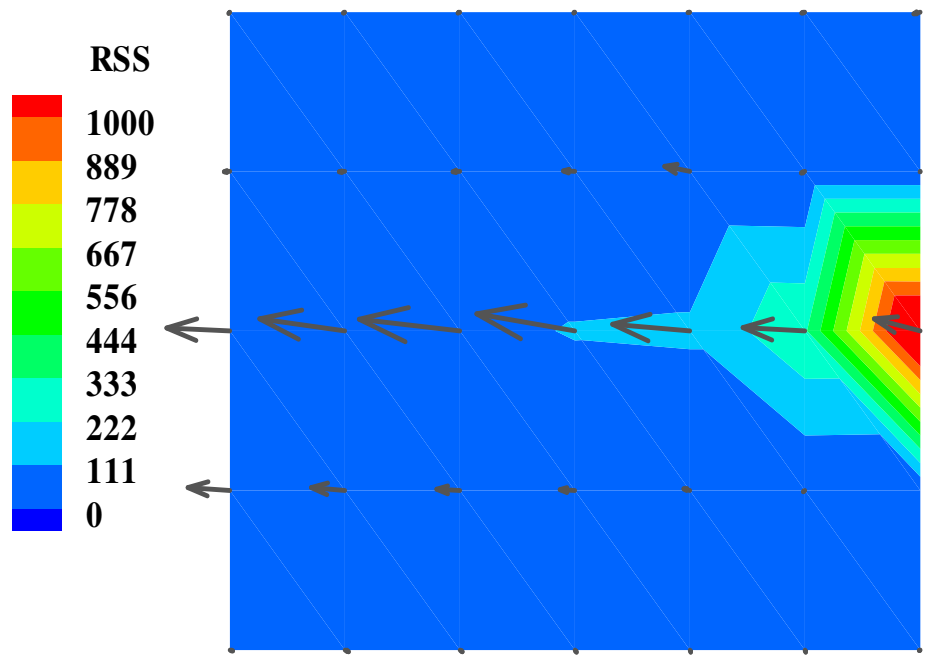

Figure 7-100 Reynolds shear stress distribution of prototype A during peak systole, time bin 9; elevated Reynolds shear stress of more than 1,000 dynes $/ \mathrm{cm}^{2}$ was observed at the stent tip, which coincided with the location of the deep channel as observed in Figure 7-92c (avi, 601k, A_stent_downstream_rss.avi) 


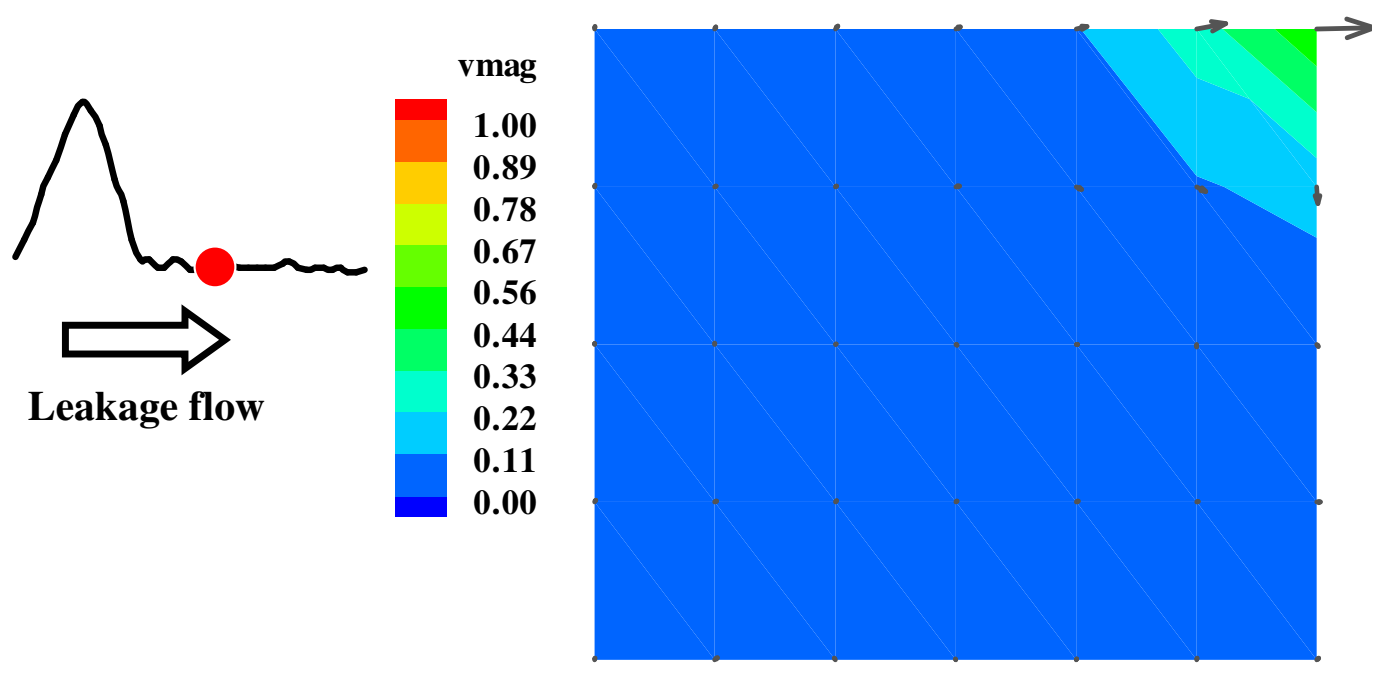

Figure 7-101 Velocity fields downstream of the stent of prototype A during mid diastole, time bin 21; the leakage jet has a flow velocity of approximately $0.7 \mathrm{~m} / \mathrm{s}$ (avi, 589k, A_stent_downstream_vmag.avi)

\subsection{Flow Inside the Valve}

Animations A_inside_valve_vmag.avi and A_inside_valve_rss.avi show the velocity magnitude $(\mathrm{m} / \mathrm{s})$ and Reynolds shear stress $\left(\right.$ dynes $\left./ \mathrm{cm}^{2}\right)$ contour plots of the flow field inside the polymeric prototype A valve throughout the cardiac cycle, respectively. Refer to Appendix $\mathrm{H}$ for the list of animations.

\section{Systole}

Figure 7-102 shows the velocity fields inside prototype A during the acceleration phase (time bin 5). An elevated velocity of approximately $2.0 \mathrm{~m} / \mathrm{s}$ was measured along the trailing edge of the polymeric valve leaflet. A region of lower velocity flow $(<0.5 \mathrm{~m} / \mathrm{s})$ was observed along the stent inflow region upstream of the stagnation zone in the 
commissural region. The low flow along the stent inflow region was most evident during both the acceleration and deceleration phases of the cardiac cycle.

Figure 7-103 shows the flow field of prototype A during peak systole (time bin 9). The velocity accelerated to a maximum of $2.6 \mathrm{~m} / \mathrm{s}$ at the trailing edge of the valve leaflet. The low flow region observed in the previous time phase was no longer present; however, the stagnation area was still evident at the commissural region. The flow pattern at this time phase appeared to direct the forward flow near the inflow stent upwards over the stagnation area at the leaflet trailing edge. During the deceleration phase (time bin 14), the flow velocity along the trailing edge of the valve decrease abruptly from $2.6 \mathrm{~m} / \mathrm{s}$ to $0.4 \mathrm{~m} / \mathrm{s}$, while the flow velocity in the surrounding fluid was typically less than $0.2 \mathrm{~m} / \mathrm{s}$.

The maximum Reynolds shear stress of 9,000 dynes $/ \mathrm{cm}^{2}$ occurred along the trailing edge of the leaflet at the top side of the commissural region during peak systole flow (time bin 9, Figure 7-104). Examination of the shear stress distribution during systole indicated that high Reynolds shear stress values tended to occur near the edge of the valve and ranged between 2,000 and 9,000 dynes $/ \mathrm{cm}^{2}$. The shear stress levels in the stagnation region during systole were less than 5 dynes $/ \mathrm{cm}^{2}$ while those in the low flow region along the stent averaged 100 dynes $/ \mathrm{cm}^{2}$, during both the acceleration and deceleration phases.

\section{$\underline{\text { Diastole }}$}

Figure 7-105 shows the magnitude and location of the leakage flow within the valve during diastole (time bin 21). The peak leakage velocity of $0.5 \mathrm{~m} / \mathrm{s}$ was observed directly 
upstream (relative to the forward flow direction) of the coaptation region, 3 to $4 \mathrm{~mm}$ from the leaflet edge. The flow inside the prototype A was unsteady at valve closure, but these fluctuations persisted for only 140 to $160 \mathrm{~ms}$. The leakage flow also oscillated in both magnitude and location during early diastole, but became stable in location $220 \mathrm{~ms}$ after valve closure. Flows inside the valve were directed toward the stent post and the high central region (time bin 21, Figure 7-106). This split flow phenomenon persisted throughout diastole. The Reynolds shear stress levels during diastole were typically around 60 dynes $/ \mathrm{cm}^{2}$.

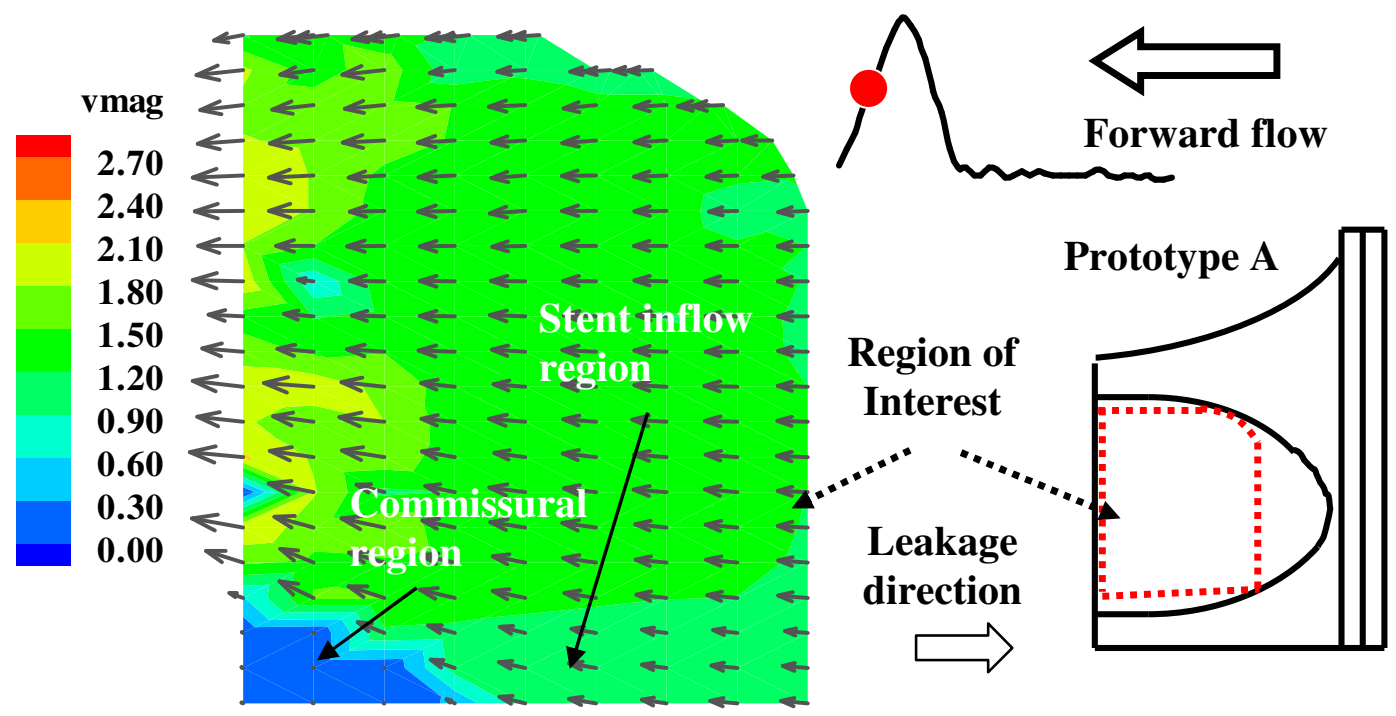

Figure 7-102 Velocity fields inside prototype A during acceleration phase, time bin 5; high velocity of approximately $2.0 \mathrm{~m} / \mathrm{s}$ was observed along the trailing edge of the leaflet. An area of stagnation occurred at the commissural region with a region of lower flow (1.0 $\mathrm{m} / \mathrm{s}$ ) along the stent inflow region (avi, 586k, A_inside_valve_vmag.avi) 


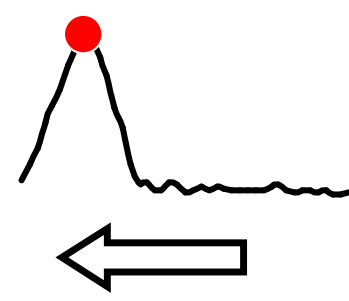

Forward flow

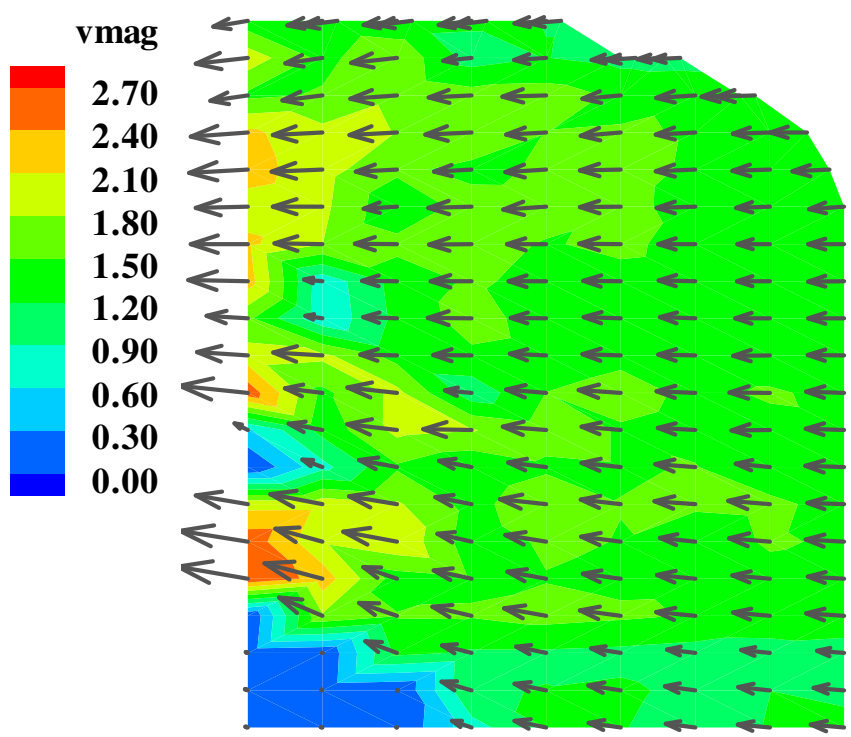

Figure 7-103 Velocity fields inside prototype A at peak systole, time bin 9; a peak forward velocity of $2.6 \mathrm{~m} / \mathrm{s}$ was observed at the edge of the leaflet above the commissural region (avi, 586k, A_inside_valve_vmag.avi)
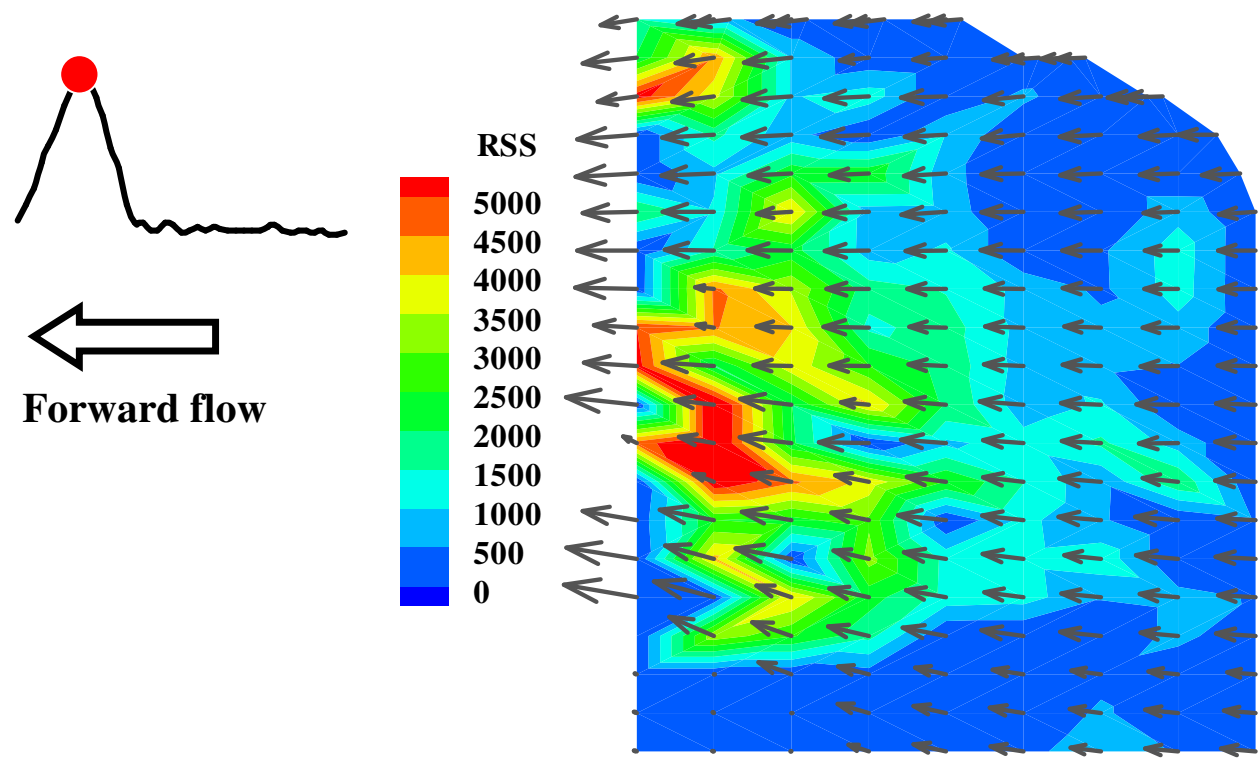

Figure 7-104 Reynolds shear stress distribution of prototype A at peak systole, time bin 9; maximum Reynolds shear stress of more than 5,500 dynes $/ \mathrm{cm}^{2}$ occurred at the trailing edge of the valve. The Reynolds shear stress levels further upstream inside the valve range between 600 to 4,000 dynes $/ \mathrm{cm}^{2}$. The shear stress levels at the stagnation area were less than 5 dynes $/ \mathrm{cm}^{2}$ (avi, 610k, A_inside_valve_rss.avi) 


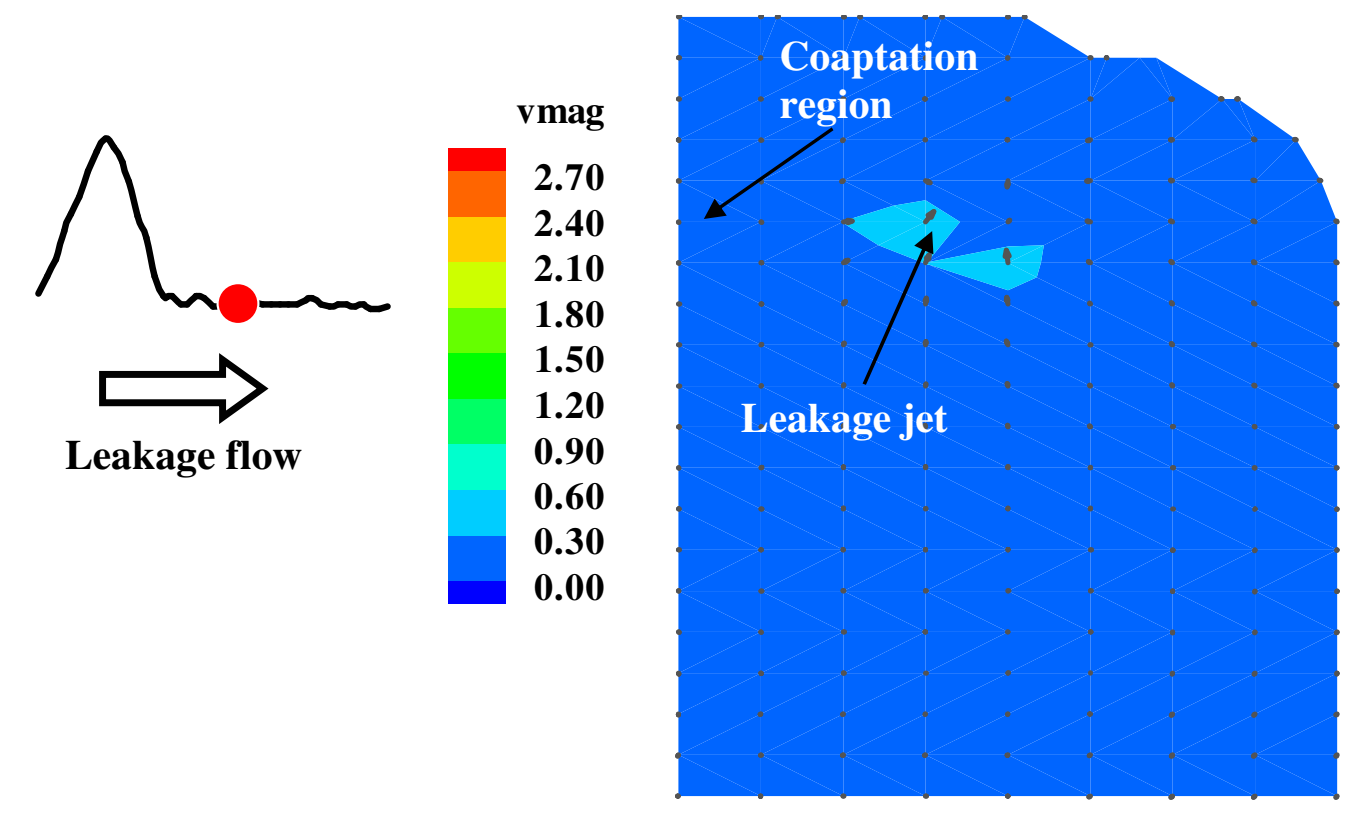

Figure 7-105 Velocity fields inside prototype A during mid diastole, time bin 21; the leakage jet was 3-4 mm from the coaptation region (avi, 586k, A_inside_valve_vmag.avi)

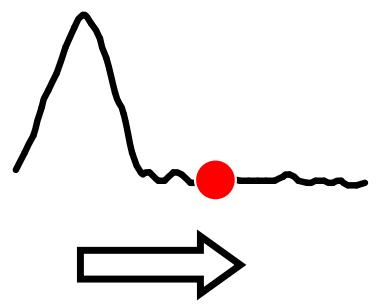

Leakage flow

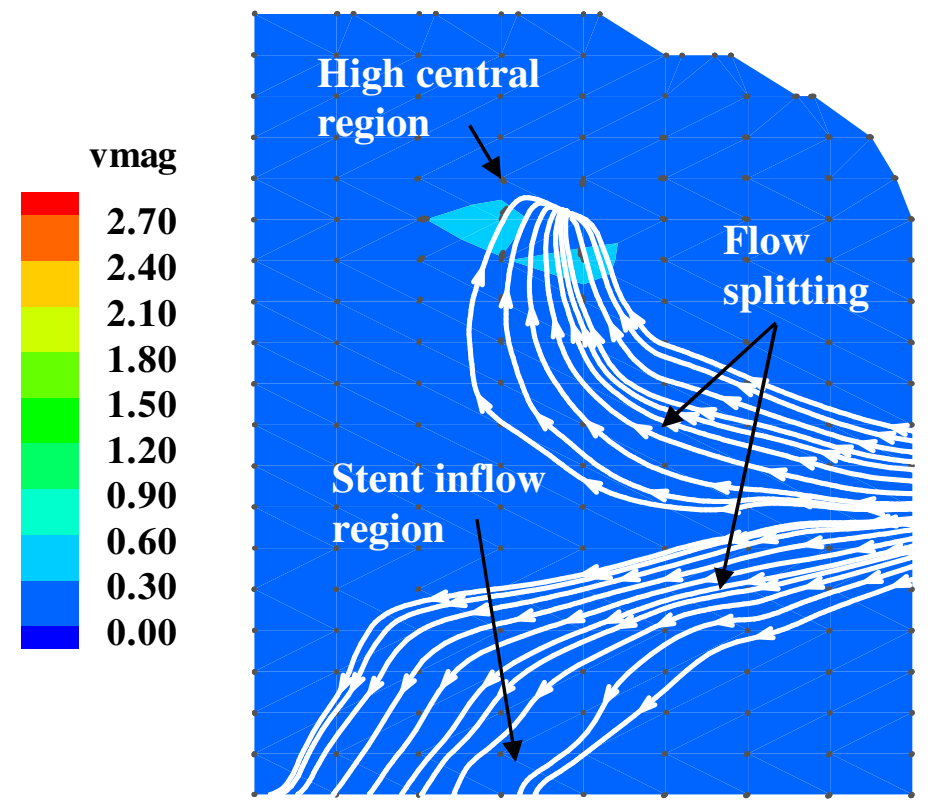

Figure 7-106 Velocity fields inside prototype A during mid diastole, time bin 21; flow during diastole was directed toward the stent inflow region and the high central region inside the valve (avi, 586k, A_inside_valve_vmag.avi) 


\subsubsection{Open Commissure Valve With $120 \mu$ Leaflet Thickness (Prototype B)}

\subsection{Flow Fields Downstream of the Valve}

Animations B_parallel_downstream_vmag.avi and B_parallel_downstream_rss.avi show the velocity magnitude $(\mathrm{m} / \mathrm{s})$ and Reynolds shear stress $\left(\right.$ dynes $\left./ \mathrm{cm}^{2}\right)$ contour plots of the flow fields in the plane parallel to the stent axis (see Figure 5-17), respectively. Similarly, animations B_perpend_downstream_vmag.avi and B_perpend_downstream_rss.avi show the velocity magnitude $(\mathrm{m} / \mathrm{s})$ and Reynolds shear stress $\left(\right.$ dynes $\left./ \mathrm{cm}^{2}\right)$ contour plots of the flow fields in the plane perpendicular to the stent axis (see Figure 5-17), respectively. Refer to Appendix $\mathrm{H}$ for the list of animations.

\section{$\underline{\text { Systole }}$}

The flow fields downstream of prototype B during both systole and diastole, were similar to those described above for prototype A. Thus, only the flow field at the parallel-to-stent axis of prototype B is presented. Figure 7-107 shows the flow field downstream of prototype B along the parallel-to-stent axis during the acceleration phase (time bin 5). A central orifice jet with a velocity of $1.5 \mathrm{~m} / \mathrm{s}$ was observed emanating from the valve orifice. Flow separation was observed at the trailing edge of the valve leaflet, while reattachment points were observed $50 \mathrm{~mm}$ downstream from the valve sewing ring in both the upper and lower parts of the flow chamber. At peak systole (time bin 9) the flow in the central orifice jet increased to a maximum flow velocity of $2.2 \mathrm{~m} / \mathrm{s}$, which was lower than that found in prototype A (Figure 7-108). However the location of the peak velocity was the same for both valves. The location of the flow re-attachment point in 
both the upper and lower parts of the flow chamber at peak systole remained at approximately $50 \mathrm{~mm}$ downstream from the valve sewing ring. The central jet had a diameter of approximately $16 \mathrm{~mm}$. The flow inside the recirculation zone was more pronounced than that in the previous time phase increasing from $0.15 \mathrm{~m} / \mathrm{s}$ to $0.4 \mathrm{~m} / \mathrm{s}$. Similar vortex structures were observed inside the sinus region with a velocity of $0.5 \mathrm{~m} / \mathrm{s}$. During deceleration (time phase 14), the velocity of the central jet reduced from $2.2 \mathrm{~m} / \mathrm{s}$ to approximately $1.2 \mathrm{~m} / \mathrm{s}$, while the recorded flow velocity inside the sinus region was typically less than $0.07 \mathrm{~m} / \mathrm{s}$. Figure 7-109 shows the Reynolds shear stress distribution of the flow field downstream of prototype B at peak systole (time bin 9). A maximum Reynolds shear stress of 1,400 dynes $/ \mathrm{cm}^{2}$ was calculated along the edge of the central orifice jet during peak systole. Elevated shear stresses of more than $300 \mathrm{dynes} / \mathrm{cm}^{2}$ were observed more than $70 \mathrm{~mm}$ from the edge of the valve.

\section{$\underline{\text { Diastole }}$}

The flow downstream of the trailing edge of the valve leaflet during diastole (time bin 21) was essentially stationary with velocities less than $0.1 \mathrm{~m} / \mathrm{s}$ (Figure 7-110). However, a retrograde flow of $0.25 \mathrm{~m} / \mathrm{s}$ was seen at the trailing edge of the leaflet that persisted throughout diastole. Reynolds shear stress levels at all investigated region (during diastole) were approximately 100 dynes $/ \mathrm{cm}^{2}$. 


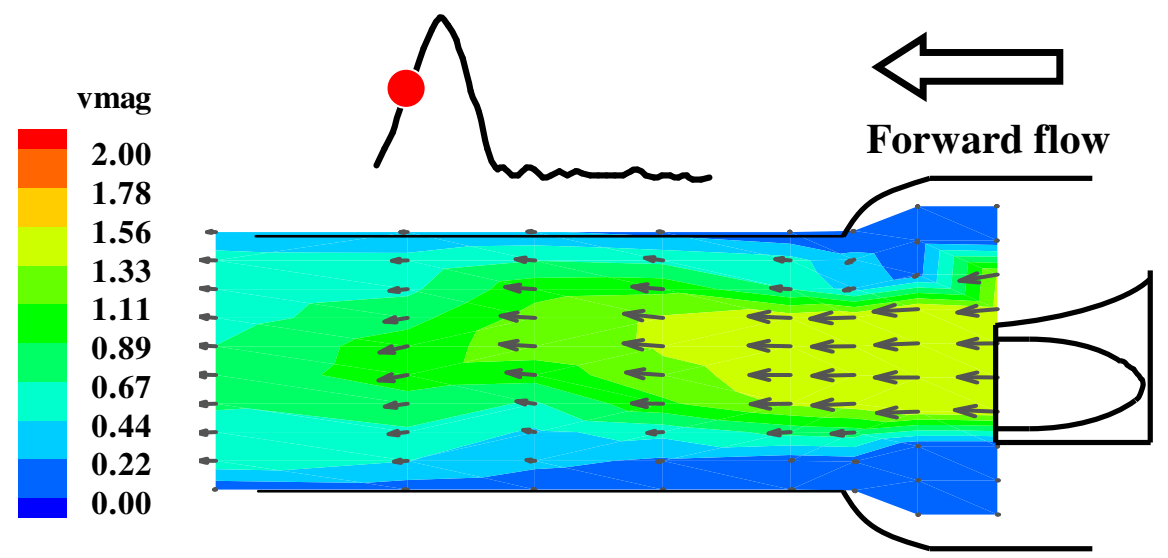

Figure 7-107 Velocity fields downstream of prototype B at acceleration phase, time bin 5, center plane; a developing central orifice jet of $1.5 \mathrm{~m} / \mathrm{s}$ was seen issuing from the orifice of the polymeric valve with formation of a recirculation zone between the edge of the valve and $75 \mathrm{~mm}$ downstream from the valve sewing ring (avi, 586k, B_parallel_downstream_vmag.avi)

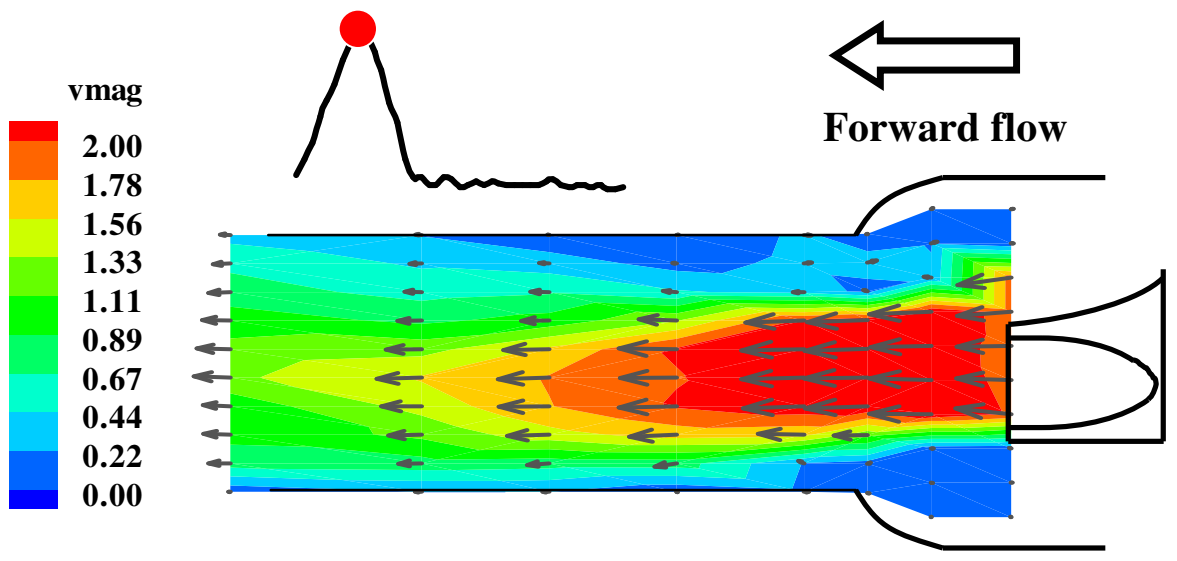

Figure 7-108 Velocity fields downstream of prototype B at peak systole, time bin 9, center plane; a maximum velocity of $2.2 \mathrm{~m} / \mathrm{s}$ was acquired along the parallel-axis plane at peak systole (avi, 586k, B_parallel_downstream_vmag.avi) 


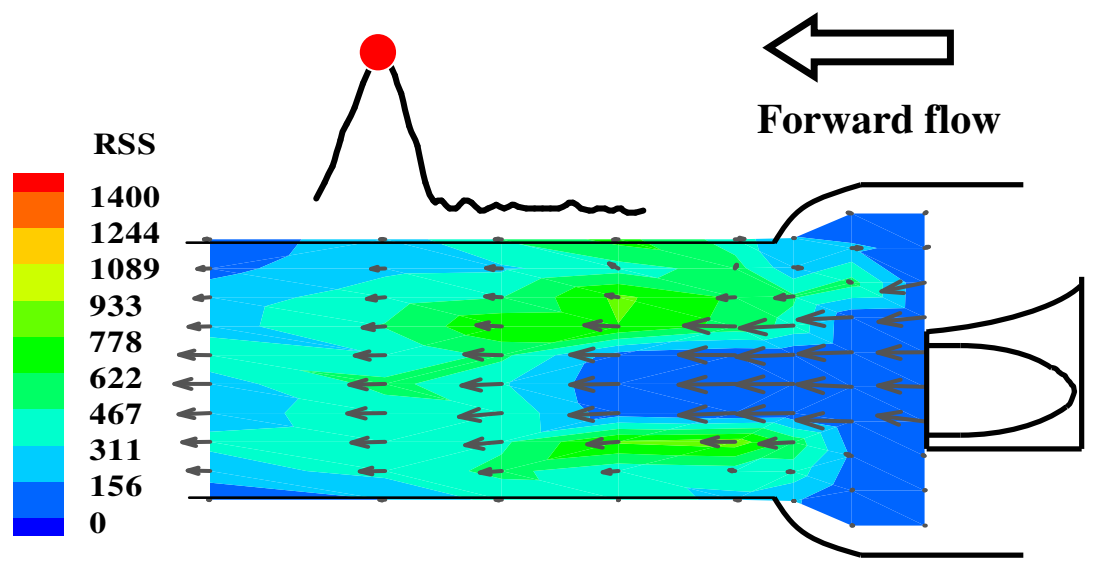

Figure 7-109 Reynolds shear stress distribution of prototype B at peak systole, time bin 9, center plane; elevated Reynolds shear stress levels of more than 1,000 dynes $/ \mathrm{cm}^{2}$ were observed at edge of the central orifice jet and $60 \mathrm{~mm}$ downstream from the edge of the valve (avi, 604k, B_parallel_downstream_rss.avi)

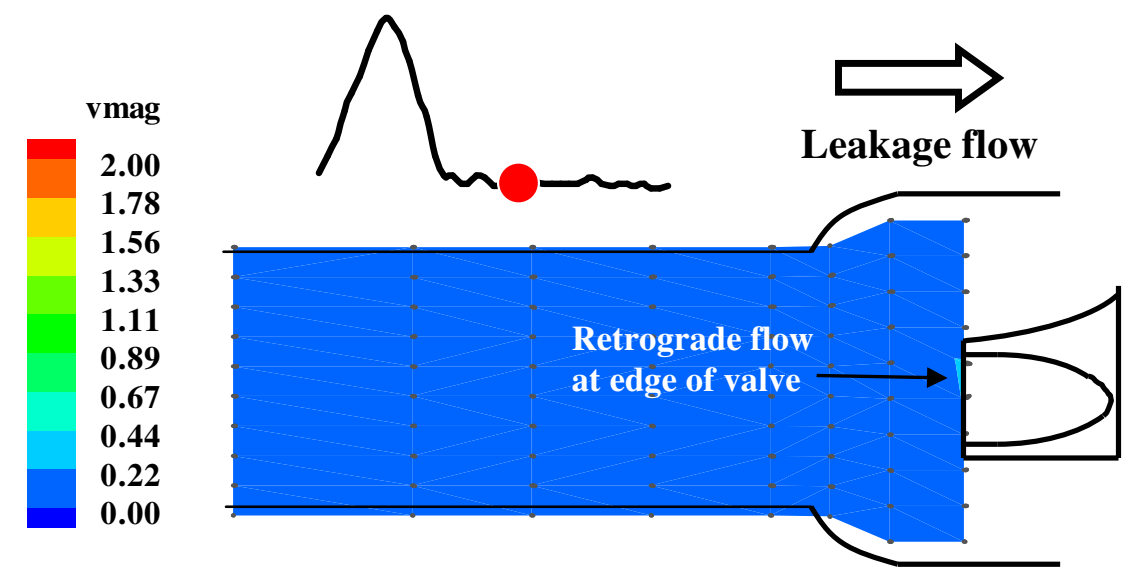

Figure 7-110 Velocity fields downstream of prototype B at mid diastole, time bin 21, center plane; the flow field during diastole was lesser than $0.1 \mathrm{~m} / \mathrm{s}$. A retrograde flow of $0.25 \mathrm{~m} / \mathrm{s}$ was observed at the trailing edge of the leaflet throughout diastole (avi, 586k, B_parallel_downstream_vmag.avi)

\subsection{Flow Downstream of the Stent}

Animations B_stent_downstream_vmag.avi and B_stent_downstream_rss.avi show the velocity magnitude $(\mathrm{m} / \mathrm{s})$ and Reynolds shear stress $\left(\right.$ dynes $\left./ \mathrm{cm}^{2}\right)$ contour plots of the flow 
field downstream of stent of the polymeric prototype B valve throughout the cardiac cycle, respectively. Refer to Appendix $\mathrm{H}$ for the list of animations.

\section{Systole}

Figure 7-111 shows the flow fields in the region downstream of the prototype B stent during the acceleration phase (time bin 5). A forward flow of $1.9 \mathrm{~m} / \mathrm{s}$ was observed issuing from the valve orifice. Figure 7-112 shows the flow distribution downstream of the stent during peak systole. The forward flow jet increased to a maximum velocity of $2.3 \mathrm{~m} / \mathrm{s}$ at peak systole immediately downstream of the stent. Examination of Figure 7-112 during the forward flow period shows three distinct flow regions: a region of high velocity forward flow $(>2.0 \mathrm{~m} / \mathrm{s})$ coming through the central valve orifice, a region of low velocity $(<0.1 \mathrm{~m} / \mathrm{s})$ flow below the stent tip, and a transition region $(1.0 \mathrm{~m} / \mathrm{s})$ between the high and low velocity flows. At the end of systole, the flow in the high velocity region slowed down to approximately $0.5 \mathrm{~m} / \mathrm{s}$, but the general features of the

three flow regimes persisted. A maximum Reynolds shear stress of 6,000 dynes $/ \mathrm{cm}^{2}$ was observed immediately downstream of the stent tip during peak systole (time bin 9, Figure 7-113). The location of the maximum shear stress did not correspond with that of the highest velocity, but rather occurred in the transition region between the high and low velocity flows.

\section{$\underline{\text { Diastole }}$}

During early diastole, the flow downstream of the stent was unsteady with leakage flow beginning near the commissural region. The leakage flow, shown $100 \mathrm{~ms}$ after valve 
closure in Figure 7-114 (time bin 21), persisted throughout diastole with velocities between 0.3 and $0.5 \mathrm{~m} / \mathrm{s}$. The Reynolds shear stress levels throughout diastole were only 50 dynes $/ \mathrm{cm}^{2}$.

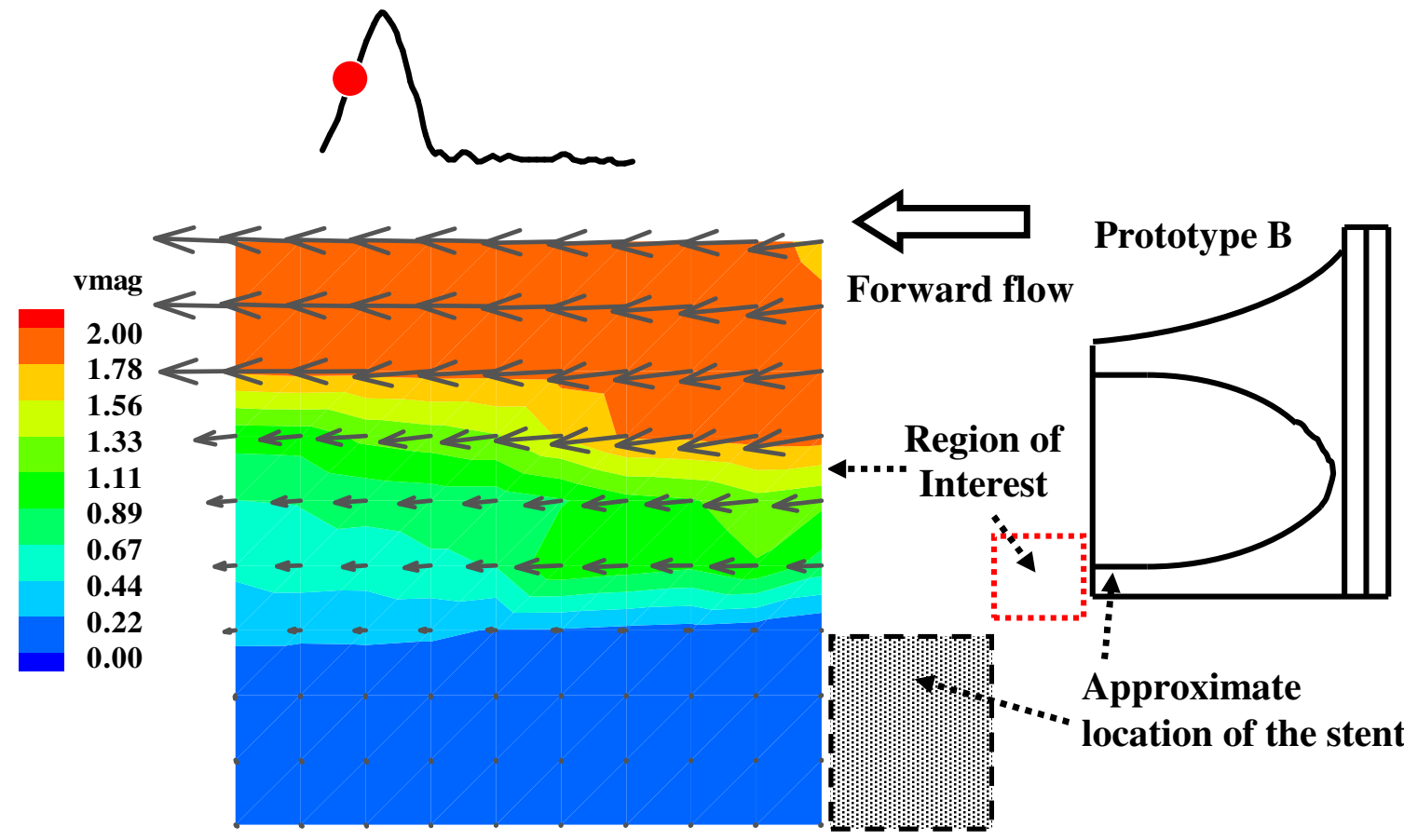

Figure 7-111 Velocity fields downstream of the stent of prototype B during acceleration phase, time bin 5; high forward flow of $1.9 \mathrm{~m} / \mathrm{s}$ was seen emanating from the commissural region of the prototype B (avi, 613k, B_stent_downstream_vmag.avi) 

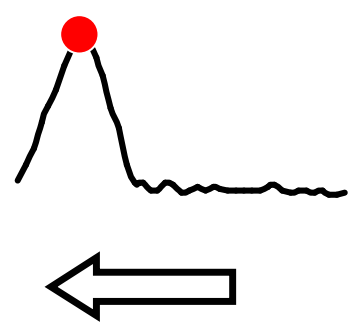

Forward flow

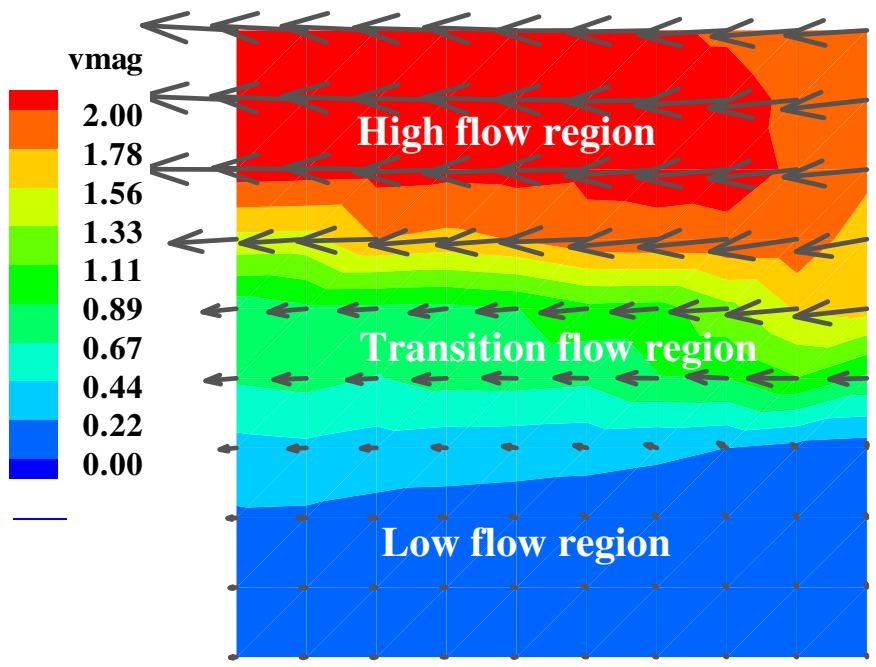

Figure 7-112 Velocity fields downstream of the stent of prototype B during peak systole, time bin 9; maximum velocity of $2.3 \mathrm{~m} / \mathrm{s}$ was observed issuing from the orifice of the valve. Three distinct flow regions were observed throughout the systole (avi, 613k, B_stent_downstream_vmag.avi)

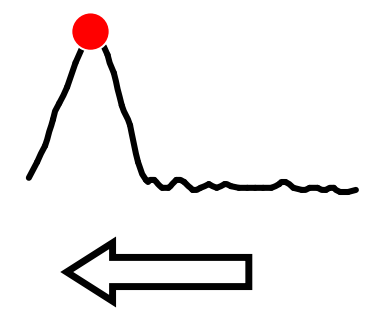

Forward flow

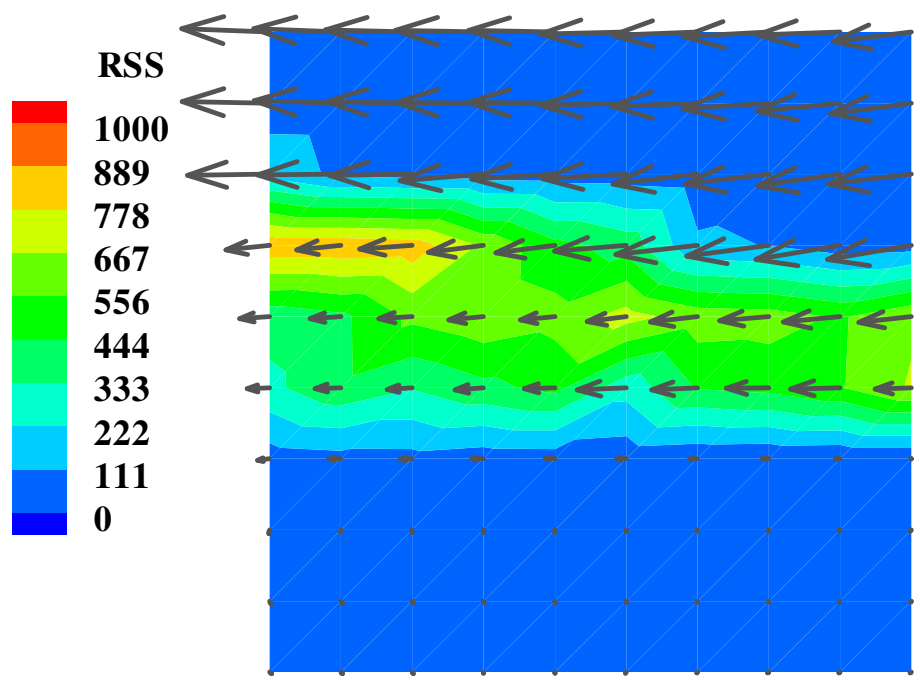

Figure 7-113 Reynolds shear stress distribution of prototype B during peak systole, time bin 9; elevated Reynolds shear stress of more than 1,000 dynes $/ \mathrm{cm}^{2}$ was observed at transition flow between the high and low flow regions (avi, 618k, B_stent_downstream_rss.avi) 


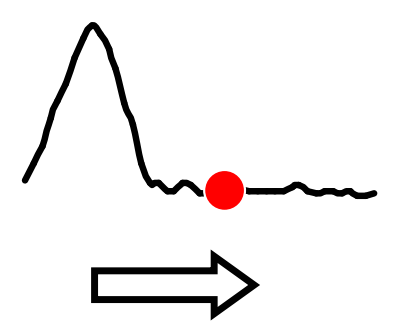

Leakage flow

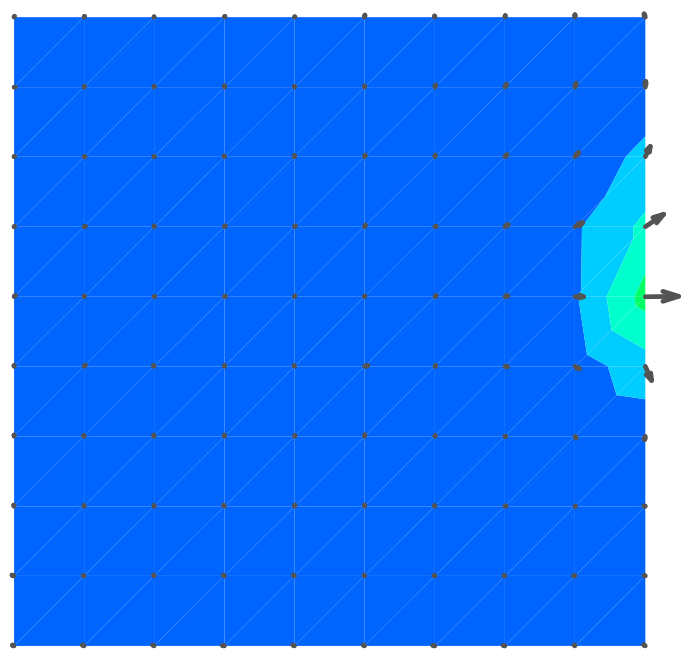

Figure 7-114 Velocity fields downstream of the stent of prototype B during mid diastole, time bin 21; leakage flow was observed through the channel gap (see Figure 7-92e for the definition) at the commissure region (avi, 613k, B_stent_downstream_vmag.avi)

\subsection{Flow Inside the Valve}

Animations B_inside_valve_vmag.avi and B_inside_valve_rss.avi show the velocity magnitude $(\mathrm{m} / \mathrm{s})$ and Reynolds shear stress $\left(\right.$ dynes $\left./ \mathrm{cm}^{2}\right)$ contour plots of the flow field inside the polymeric prototype B valve throughout the cardiac cycle, respectively. Refer to Appendix $\mathrm{H}$ for the list of animations.

\section{$\underline{\text { Systole }}$}

Figure 7-115 shows the velocity fields inside prototype B during the acceleration phase (time bin 5). A velocity of approximately $2.0 \mathrm{~m} / \mathrm{s}$ was observed along the trailing edge of the valve. The flow profile at this time phase inside prototype B was similar to that of prototype A. However, the stagnation region located at the commissure of prototype A (Figure 7-102) is not observed in prototype B. 
Figure 7-116 shows the flow fields inside prototype B at peak systole (time bin 9) where a maximum forward flow velocity of $2.1 \mathrm{~m} / \mathrm{s}$ was recorded at the edge of the leaflet. The flow field at peak systole was relatively uniform and the low flow region that was shown in Figure 7-102 for prototype A was not evident. The flow velocity along the leaflet railing edge during the deceleration phase (time bin 14) was approximately $0.54 \mathrm{~m} / \mathrm{s}$, while those nearer to the rear of the valve was less than $0.30 \mathrm{~m} / \mathrm{s}$. The Reynolds shear stress values at all investigated locations inside the valve were less than $100 \mathrm{dynes} / \mathrm{cm}^{2}$ during systole (time bin 9, Figure 7-117).

\section{$\underline{\text { Diastole }}$}

After valve closure, the flow inside the valve was unsteady for 100 to $120 \mathrm{~ms}$. A leakage jet appeared inside the valve approximately $160 \mathrm{~ms}$ after valve closure, oscillating in strength and position for 60 to $80 \mathrm{~ms}$ before stabilizing. A leakage jet was observed 320 $\mathrm{ms}$ after valve closure and it appeared to originate approximately $2 \mathrm{~mm}$ upstream from the trailing edge of the leaflet and was significantly larger than the leakage jet observed in prototype A. The velocity of the leakage jet immediately following stabilization was $2.0 \mathrm{~m} / \mathrm{s}$. As the cardiac cycle progressed, the jet gradually increased in both speed and size, reaching a maximum velocity of $3.6 \mathrm{~m} / \mathrm{s}$ approximately $360 \mathrm{~ms}$ after valve closure (time bin 38, Figure 7-118). At this point, the leakage jet extended $5 \mathrm{~mm}$ from the leaflet edge, occupying almost half of the measurement plane. Flow patterns inside the prototype B was seen directed toward both the coaptation and commissural regions of the valve during diastole (similar flow patterns observed in prototype A, Figure 7-106). This flow splitting had a velocity of approximately $0.20 \mathrm{~m} / \mathrm{s}$. The magnitude of the Reynolds shear 
stress in the leakage jet during diastole ranged between 2,000 dynes $/ \mathrm{cm}^{2} 220 \mathrm{~ms}$ after valve closure and 13,000 dynes $/ \mathrm{cm}^{2} 360 \mathrm{~ms}$ after valve closure. The Reynolds shear stress values at other measurement locations inside the valve were generally less than 50 dynes $/ \mathrm{cm}^{2}$ during diastole (time bin 38, Figure 7-119).

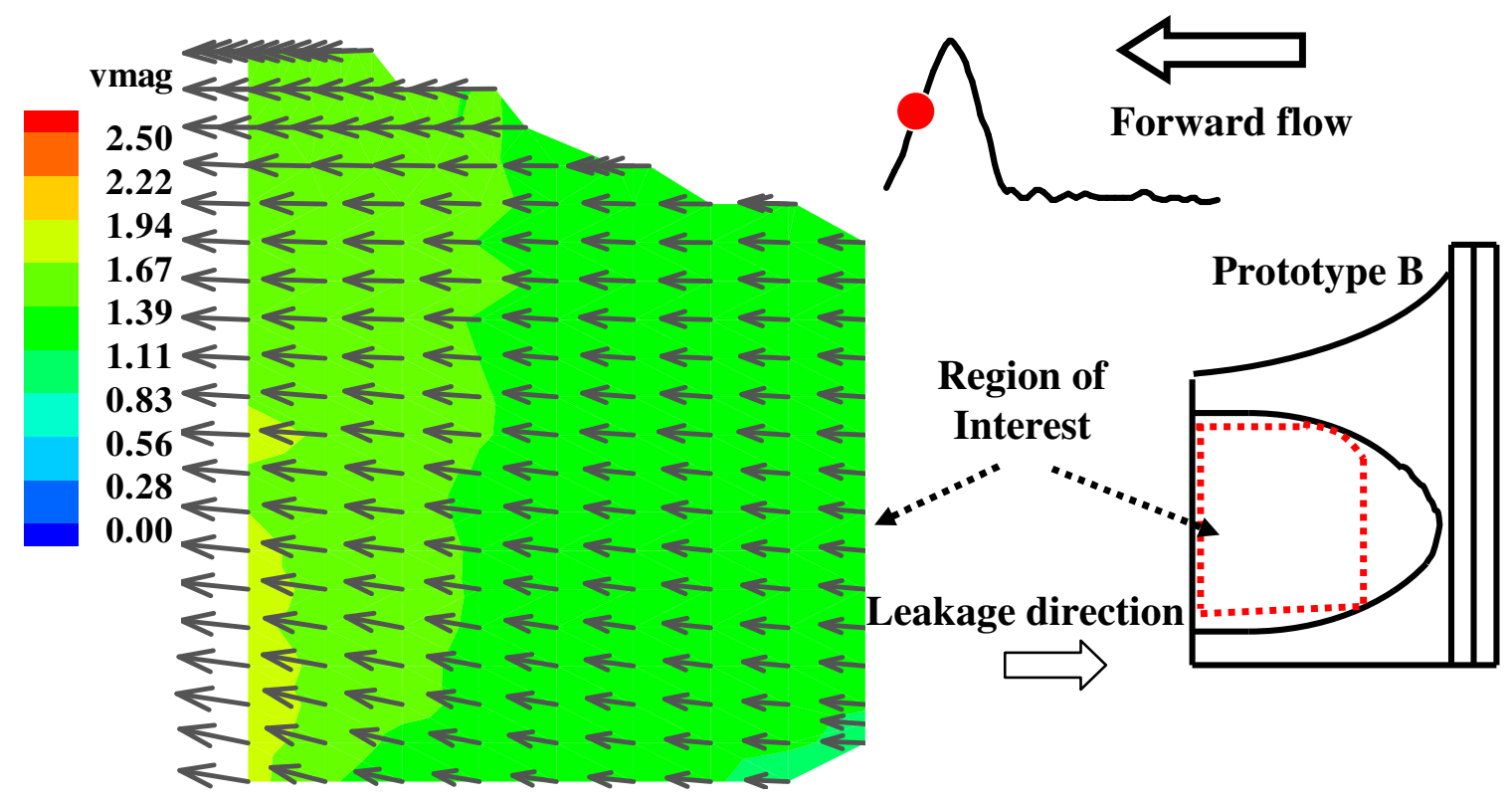

Figure 7-115 Velocity fields inside prototype B during acceleration phase, time bin 5; high velocity of approximately $2.0 \mathrm{~m} / \mathrm{s}$ was observed along the trailing edge of the leaflet. No area of stagnation was observed in commissural region of prototype B (avi, 594k, B_inside_valve_vmag.avi) 

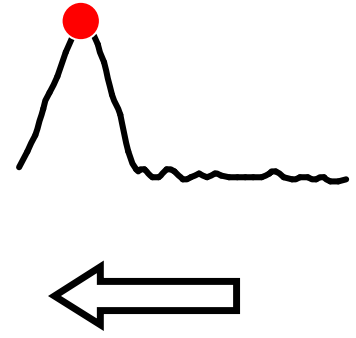

Forward flow

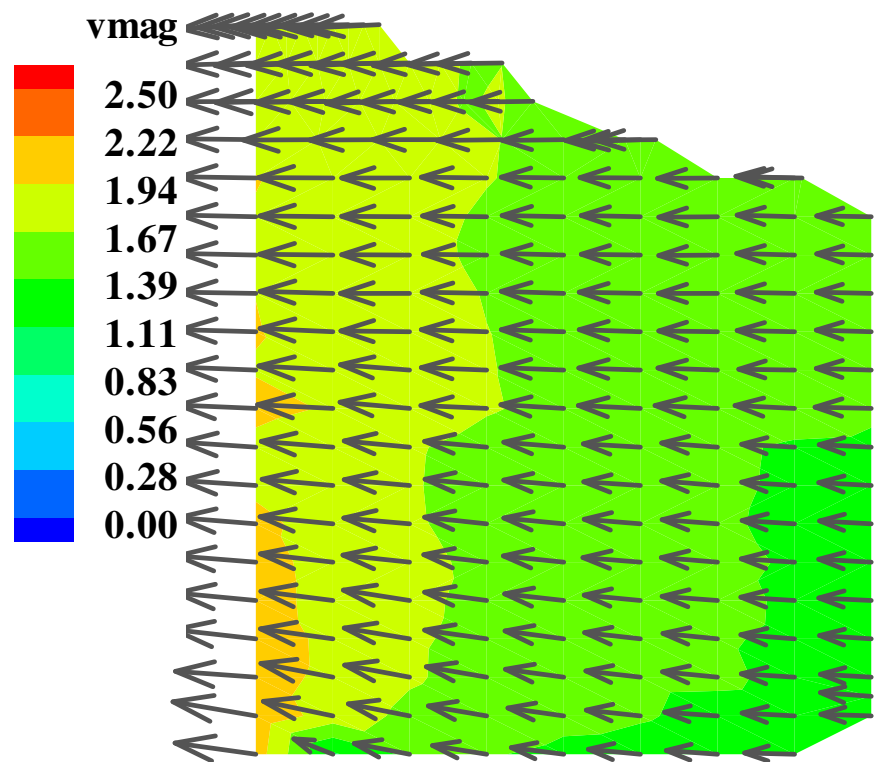

Figure 7-116 Velocity fields inside prototype B during peak systole, time bin 9; flow velocity closer to the trailing edge of the valve was typically higher than that inside the valve (avi, 594k, B_inside_valve_vmag.avi)
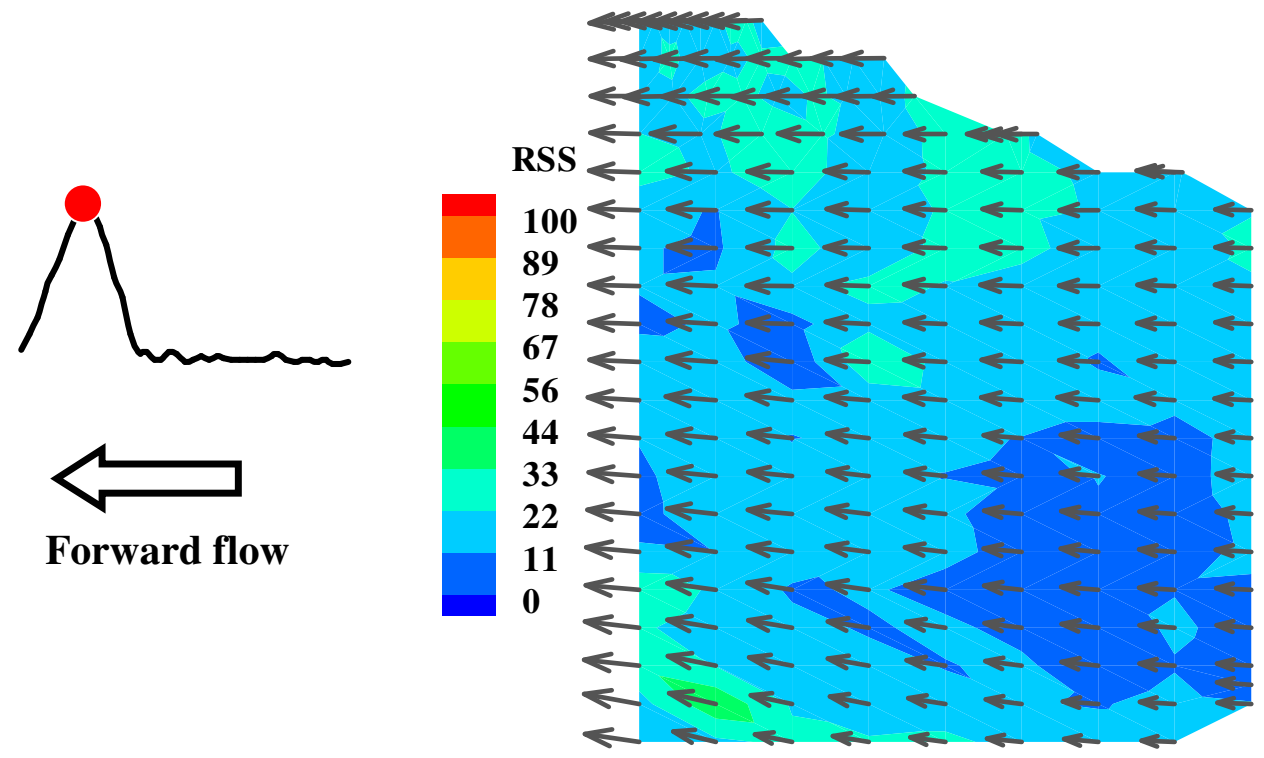

Figure 7-117 Reynolds shear stress distribution of prototype B during peak systole, time bin 9; Reynolds shear stress level during systole was typically less than 100 dynes $/ \mathrm{cm}^{2}$ (avi, 592k, B_inside_valve_rss.avi) 


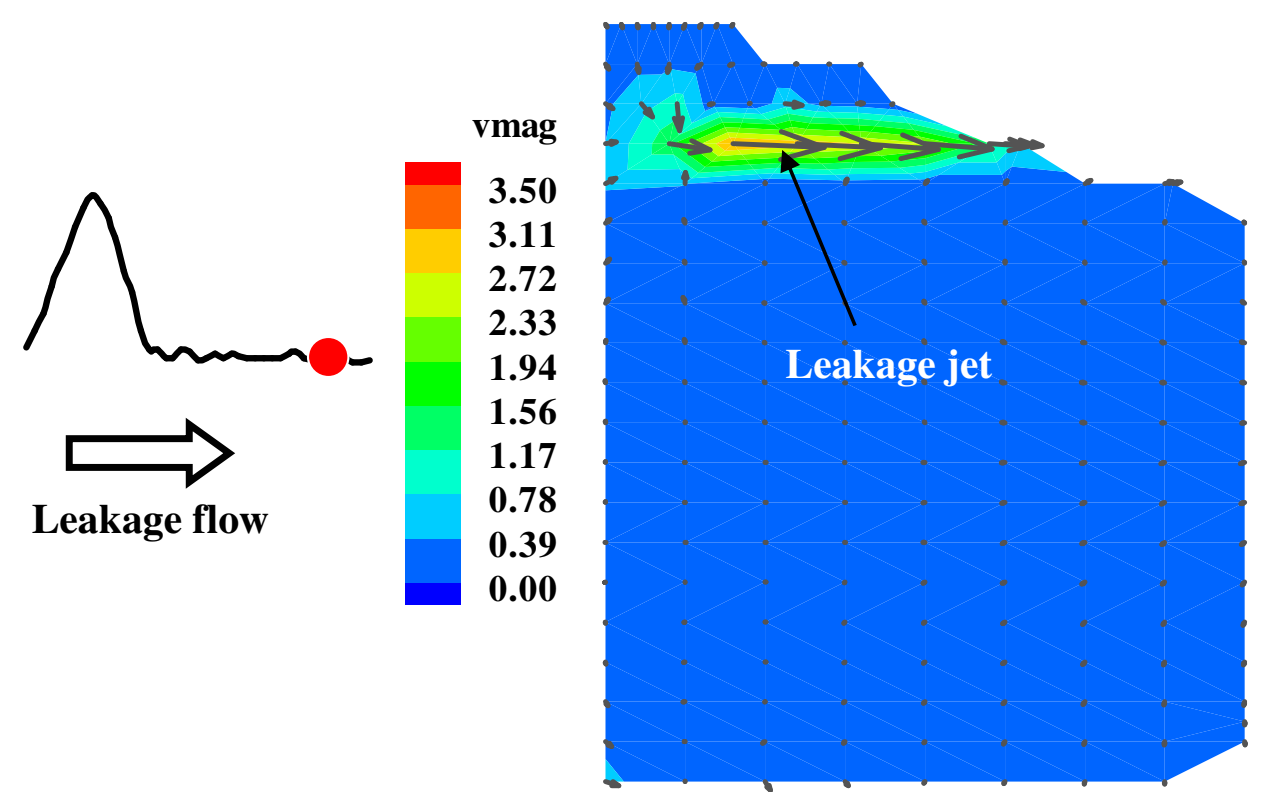

Figure 7-118 Velocity fields inside prototype B during late diastole, time bin 38; a leakage jet of more than $3.5 \mathrm{~m} / \mathrm{s}$ was observed $2 \mathrm{~mm}$ upstream from the trailing edge of the leaflet and extended $5 \mathrm{~mm}$ inside the valve (avi, 594k, B_inside_valve_vmag.avi)
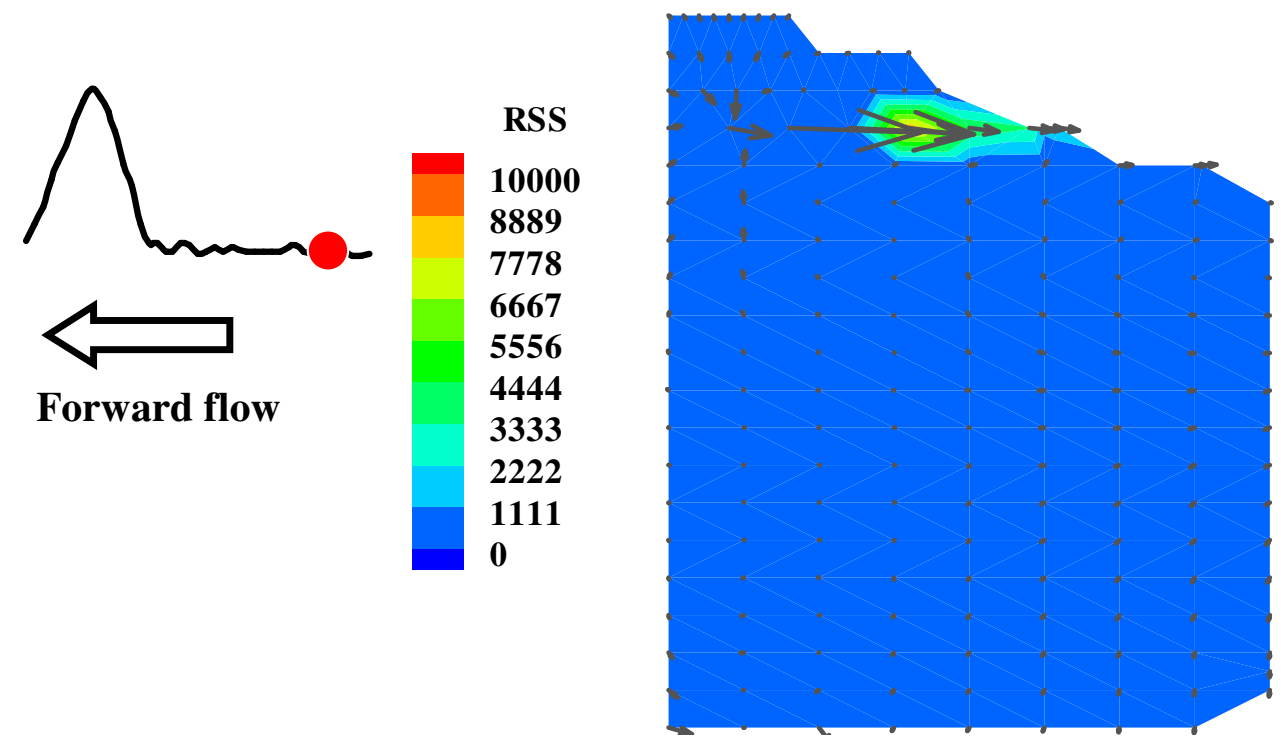

Figure 7-119 Reynolds shear stress distribution of prototype B during late diastole, time bin 38; maximum Reynolds shear stress of more than 10,000 dynes $/ \mathrm{cm}^{2}$ occurred at the leakage jet inside the valve during diastole. The Reynolds shear stress levels at other measurement locations inside the valve were less than 50 dynes $/ \mathrm{cm}^{2} \mathrm{c}(\mathrm{avi}, 592 \mathrm{k}$, B_inside_valve_rss.avi) 


\subsubsection{Semi-Open Commissure Valve With 120 um Leaflet Thickness (Prototype C)}

\subsection{Flow Fields Downstream of the Valve}

Animations C_parallel_downstream_vmag.avi and C_parallel_downstream_rss.avi show the velocity magnitude $(\mathrm{m} / \mathrm{s})$ and Reynolds shear stress $\left(\right.$ dynes $\left./ \mathrm{cm}^{2}\right)$ contour plots of the flow fields in the plane parallel to the stent axis (see Figure 5-17), respectively. Similarly, animations C_perpend_downstream_vmag.avi and C_perpend_downstream_rss.avi show the velocity magnitude $(\mathrm{m} / \mathrm{s})$ and Reynolds shear stress $\left(\right.$ dynes $\left./ \mathrm{cm}^{2}\right)$ contour plots of the flow fields in the plane perpendicular to the stent axis (see Figure 5-17), respectively. Refer to Appendix $\mathrm{H}$ for the list of animations.

\section{Systole}

The flow patterns along the parallel-to-stent axis measurement plane were similar to those measured along the perpendicular-to-stent axis plane. Hence only flow fields along the parallel-to-stent axis plane are shown. The flow profiles for prototype $\mathrm{C}$ were similar to those observed in the prototypes $\mathrm{A}$ and $\mathrm{B}$. Figure 7-120 shows the flow field downstream of prototype $\mathrm{C}$ along the parallel-to-stent axis during the acceleration phase (time bin 5). The developing central orifice jet reached a velocity of $1.5 \mathrm{~m} / \mathrm{s}$ during the acceleration phase. Flow separation was observed at the trailing edge of the valve, while the reattachment points occurred approximately $40 \mathrm{~mm}$ downstream from the valve sewing ring in the upper and lower parts of the flow chamber. At peak systole (time bin 9, Figure 7-121), the central orifice jet increased to a maximum velocity of $2.2 \mathrm{~m} / \mathrm{s} 22 \mathrm{~mm}$ from the edge of the valve sewing ring. Simultaneously, the region of the recirculation 
zone expanded farther downstream with the re-attachment points occurring at $40 \mathrm{~mm}$ and $75 \mathrm{~mm}$ downstream from the valve sewing ring in the upper and lower parts of the flow chamber, respectively. The jet had a diameter of approximately $18 \mathrm{~mm}$. Vortex structures similar to those seen in prototypes A and B were also evident in the sinus region of prototype C. The recirculation generated a flow velocity of $0.3 \mathrm{~m} / \mathrm{s}$ along the wall of the valve chamber. During the deceleration phase (time bin 14), the size of the central orifice jet contracted and its flow velocity decreased from $2.2 \mathrm{~m} / \mathrm{s}$ to $0.8 \mathrm{~m} / \mathrm{s}$. The flow velocity inside the sinus region at this time phase was less than $0.05 \mathrm{~m} / \mathrm{s}$.

Maximum Reynolds shear stress value of 1,252 dynes $/ \mathrm{cm}^{2}$ occurred in the central orifice flow during peak systole $25 \mathrm{~mm}$ downstream from the valve sewing ring (time bin 9 , Figure 7-122). Reynolds shear stresses in the range of $100-400$ dynes $/ \mathrm{cm}^{2}$ were typically observed in the region distal to the flow chamber and at the edge of the central orifice flow during systole.

\section{Diastole}

Flow velocities during diastole (time bin 21) were less than $0.06 \mathrm{~m} / \mathrm{s}$ throughout the measurement plane, with the exception of the flow immediately downstream of the trailing edge of the leaflet at which intermittent retrograde flow with velocities up to 0.4 m/s were observed (Figure 7-123). Maximum Reynolds shear stress of 100 dynes $/ \mathrm{cm}^{2}$ was observed in the retrograde flow at the trailing edge of the leaflet during diastole. In contrast, the Reynolds shear stress levels at other measurement locations were typically less than 20 dynes $/ \mathrm{cm}^{2}$. 


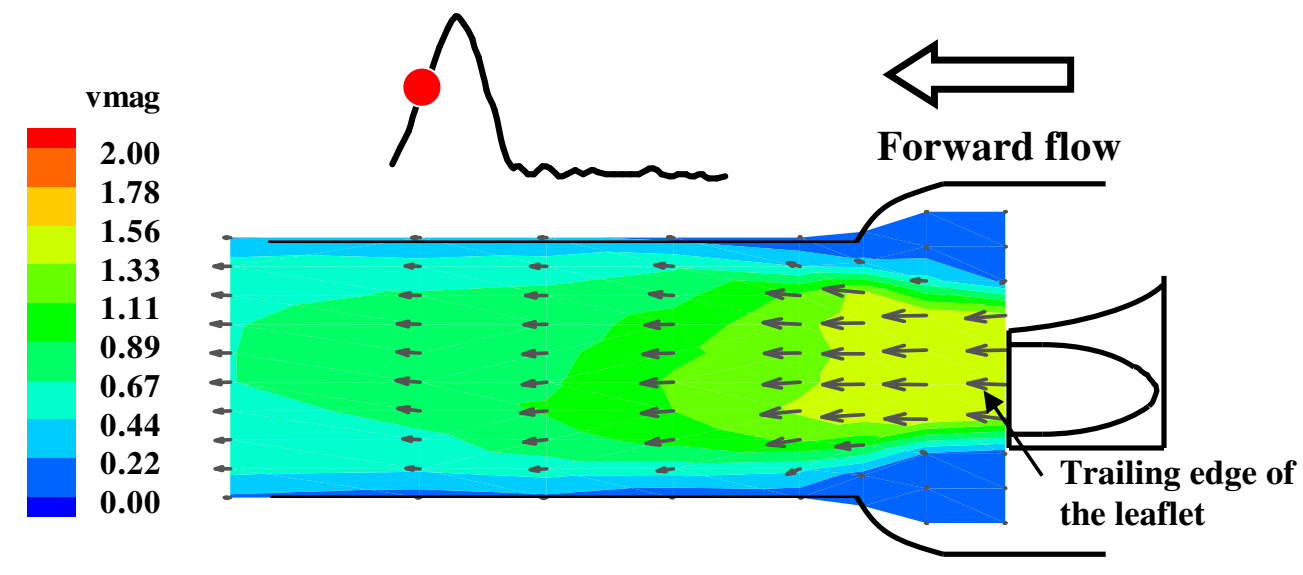

Figure 7-120 Velocity fields downstream of prototype $C$ at acceleration phase, time bin 5, center plane; a developing central orifice jet of $1.5 \mathrm{~m} / \mathrm{s}$ was seen issuing from the orifice of the polymeric valve (avi, 586k, C_parallel_downstream_vmag.avi)

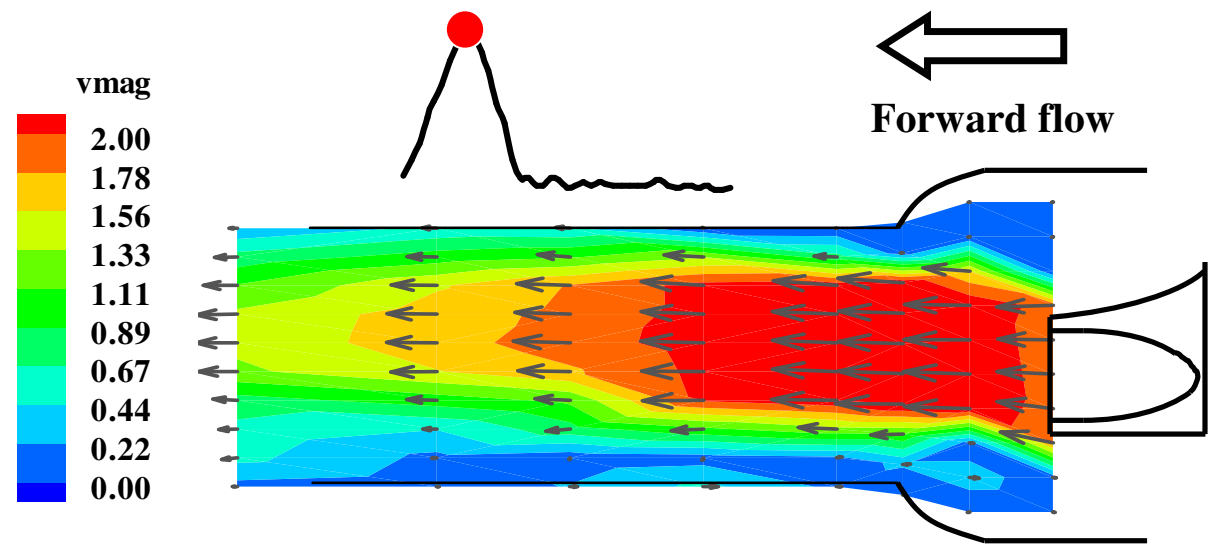

Figure 7-121 Velocity fields downstream of prototype $\mathrm{C}$ at peak systole, time bin 9, center plane; a peak velocity of $2.1 \mathrm{~m} / \mathrm{s}$ was measured in the central orifice jet (avi, 586k, C_parallel_downstream_vmag.avi) 


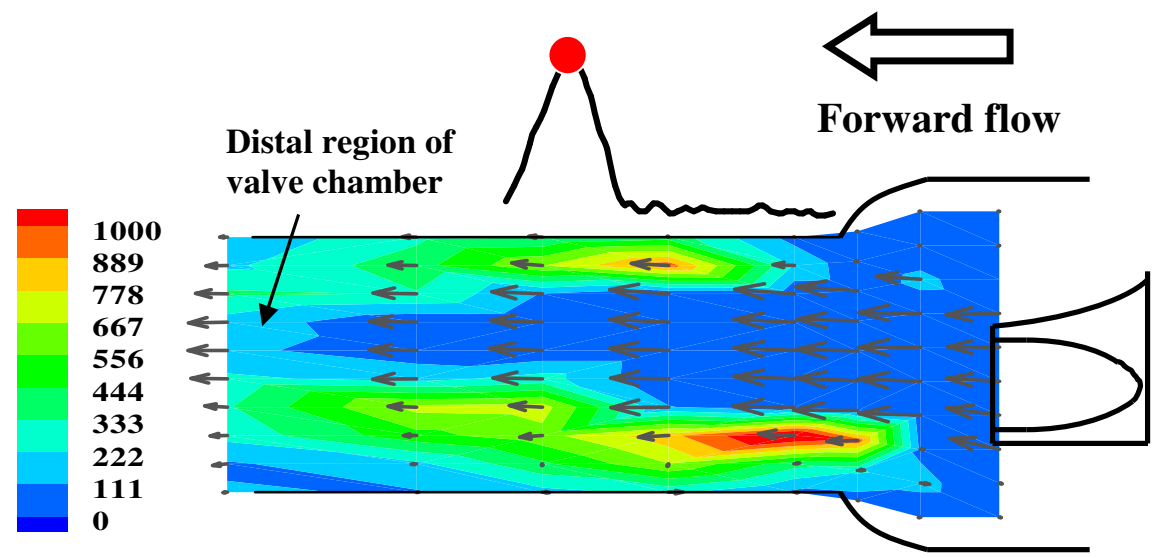

Figure 7-122 Reynolds shear stress distribution of prototype $C$ at peak systole, time bin 9, center plane; elevated Reynolds shear stress levels of more than 1,000 dynes $/ \mathrm{cm}^{2}$ were observed at edge of the central orifice jet and $60 \mathrm{~mm}$ downstream from the edge of the valve (avi, 599k, C_parallel_downstream_rss.avi)

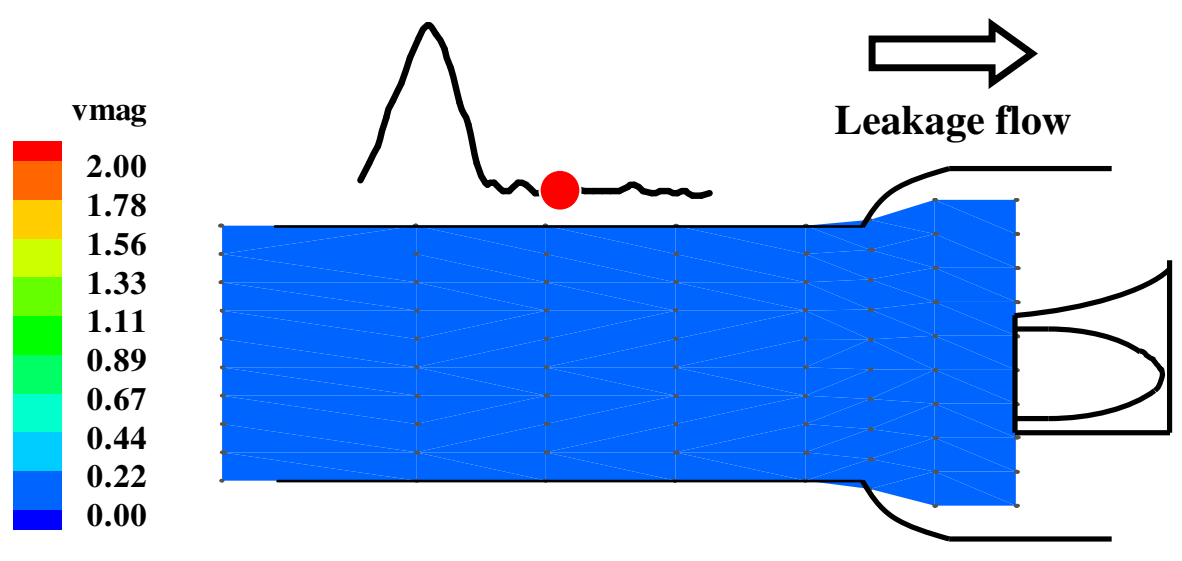

Figure 7-123 Velocity fields downstream of prototype $\mathrm{C}$ at mid diastole, time bin 21, center plane; the flow field during diastole was low at around $0.04 \mathrm{~m} / \mathrm{s}$ (avi, 586k, C_parallel_downstream_vmag.avi)

\subsection{Flow Downstream of the Stent}

Animations C_stent_downstream_vmag.avi and C_stent_downstream_rss.avi show the velocity magnitude $(\mathrm{m} / \mathrm{s})$ and Reynolds shear stress $\left(\right.$ dynes $\left./ \mathrm{cm}^{2}\right)$ contour plots of the flow 
field downstream of stent of the polymeric prototype $\mathrm{C}$ valve throughout the cardiac cycle, respectively. Refer to Appendix $\mathrm{H}$ for the list of animations.

\section{Systole}

Figure 7-124 shows the flow fields downstream of the stent during the acceleration phase (time bin 5). Two high velocity jets of $1.5 \mathrm{~m} / \mathrm{s}$ were seen issuing from the commissural region of prototype $C$ : one at the top right corner of Figure 7-124, with a smaller and weaker jet beneath it. At peak systole (time bin 9, Figure 7-125), the upper jet reached a velocity of $2.0 \mathrm{~m} / \mathrm{s}$, while the lower jet a velocity of $1.0 \mathrm{~m} / \mathrm{s}$. The velocities at other measurement locations were less than $0.03 \mathrm{~m} / \mathrm{s}$. During deceleration phase (time bin 14), the upper jet reduced to a flow velocity of $0.38 \mathrm{~m} / \mathrm{s}$, while the lower jet to a velocity of $0.16 \mathrm{~m} / \mathrm{s}$. The flow velocity in the surrounding fluid was less than $0.04 \mathrm{~m} / \mathrm{s}$.

Maximum Reynolds shear stress values of 1,050 dynes $/ \mathrm{cm}^{2}$ were observed in the smaller forward flow jet while the Reynolds shear stress values in the larger forward flow jet were less than 130 dynes $/ \mathrm{cm}^{2}$ (time bin 9 , Figure $7-126$ ). This flow pattern gave rise to two regions of flow separation thus creating two shear layers: one between the two jets and another just below the smaller jet. The Reynolds shear stress in these shear layers ranged from 100 to 300 dynes $/ \mathrm{cm}^{2}$ during systole.

\section{$\underline{\text { Diastole }}$}

The velocity downstream of the stent decelerated to less than $0.01 \mathrm{~m} / \mathrm{s}$ at the instant of valve closure. A leakage jet of approximately $0.6 \mathrm{~m} / \mathrm{s}$ was observed at the stent tip $20 \mathrm{~ms}$ 
after valve closure and persisted throughout diastole (time bin 21, Figure 7-127). A smaller leakage jet of $0.4 \mathrm{~m} / \mathrm{s}$ was seen below the top leakage jet $100 \mathrm{~ms}$ after valve closure. The location of the smaller leakage jet coincided with the position of a gap channel (see Figure 7-92e for the location of gap channel during diastole) formed in the commissural region during diastole, while the location of the larger leakage jet corresponded to leakage flow along the edge of the valve. Similar leakage jets were also observed in prototype A during diastole. The Reynolds shear stress values during diastole were less than 50 dynes $/ \mathrm{cm}^{2}$, except at the gap channel where the two leakage jets were observed. The recorded Reynolds shear stress values in these leakage jets ranged between 100 to 400 dynes $/ \mathrm{cm}^{2}$ throughout diastole.

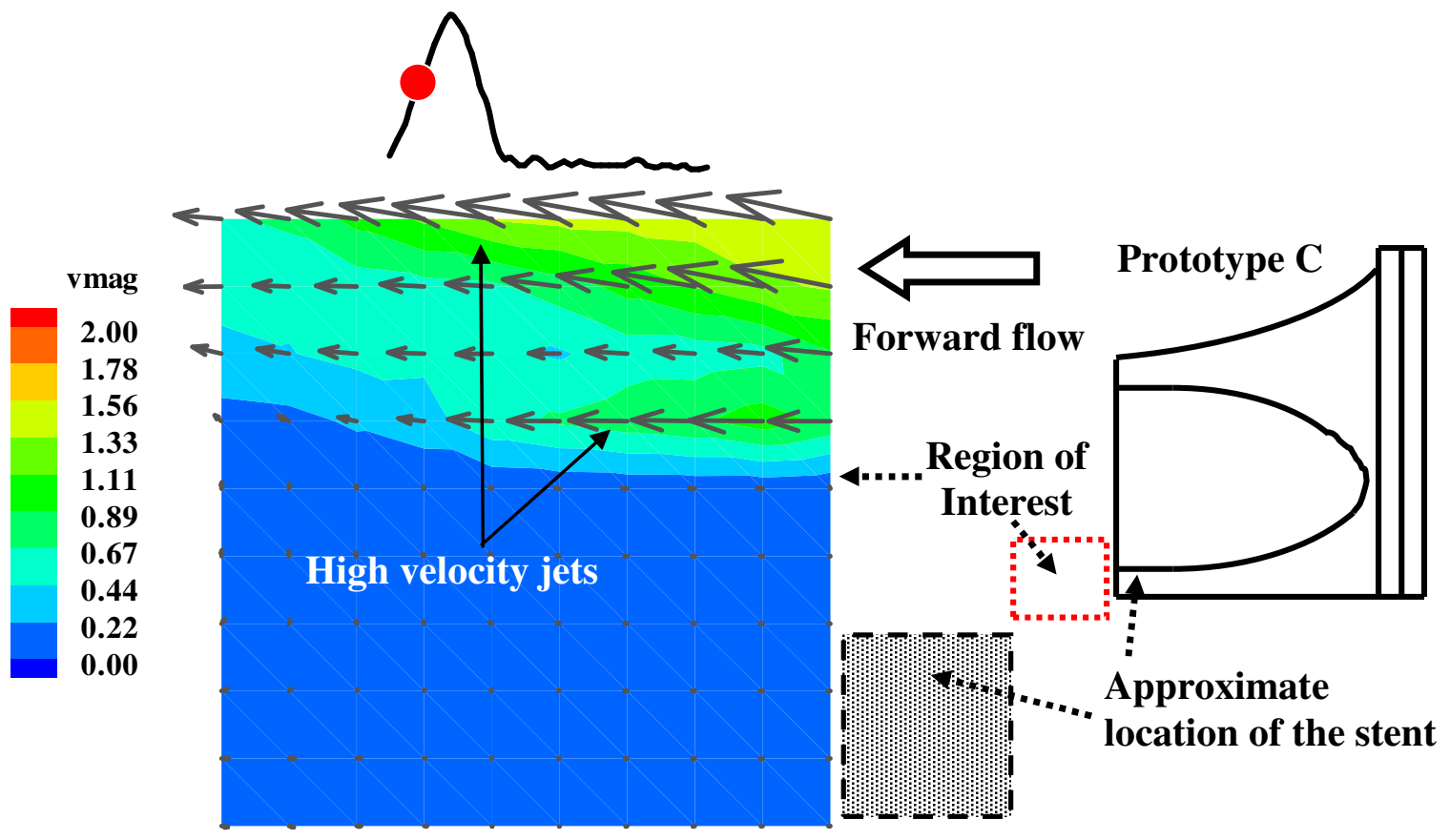

Figure 7-124 Velocity fields downstream of the stent of prototype $\mathrm{C}$ during acceleration phase, time bin 5 ; two forward jets $(1.59 \mathrm{~m} / \mathrm{s})$ were seen issuing from the commissural region during the acceleration phase (avi, 605k, C_stent_downstream_vmag.avi) 


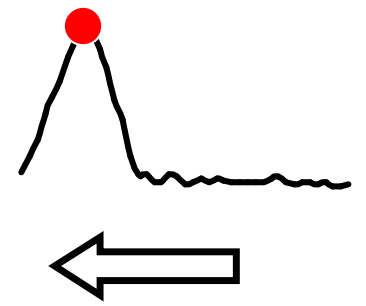

Forward flow

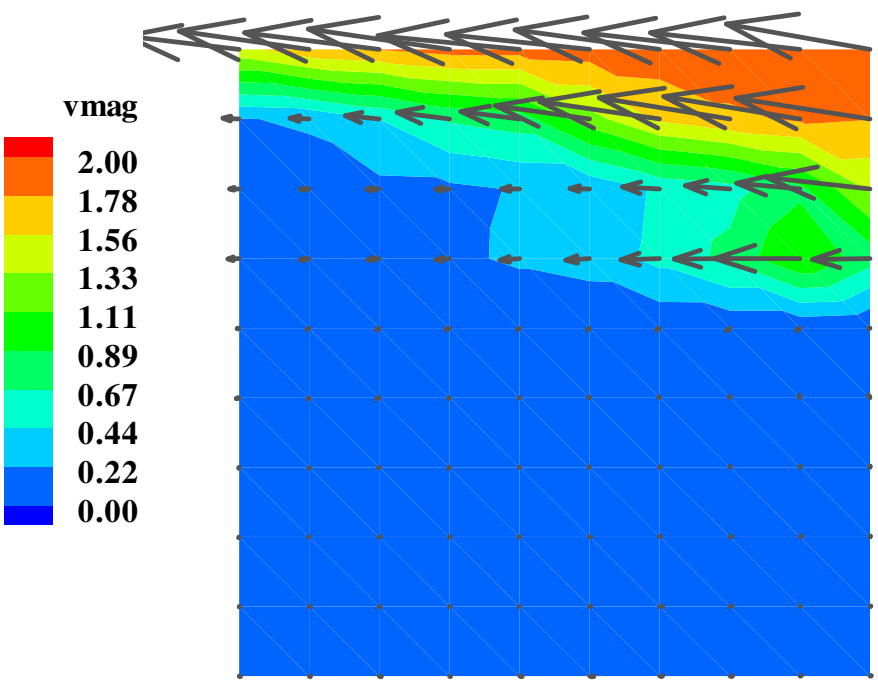

Figure 7-125 Velocity fields downstream of the stent of prototype $\mathrm{C}$ during peak systole, time bin 9; high velocity jet of $1.9 \mathrm{~m} / \mathrm{s}$ was seen emanating from the commissural region at peak systole (avi, 605k, C_stent_downstream_vmag.avi)

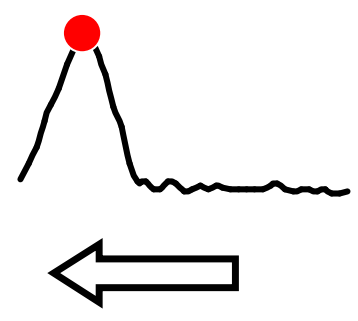

Forward flow

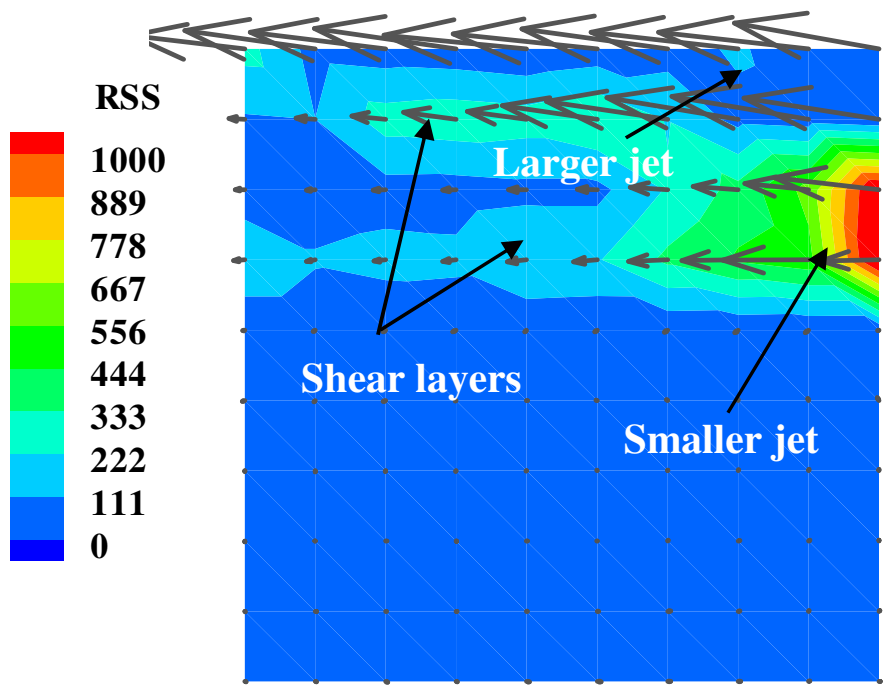

Figure 7-126 Reynolds shear stress distribution of prototype $C$ during peak systole, time bin 9; elevated Reynolds shear stress of more than 1,000 dynes $/ \mathrm{cm}^{2}$ was observed in the smaller forward jet, while the upper jet recorded a Reynolds shear stress of less than 130 dynes $/ \mathrm{cm}^{2}$ (avi, 619k, C_stent_downstream_rss.avi) 


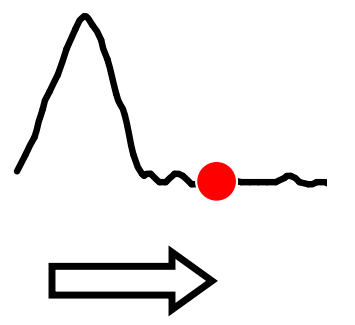

Leakage flow

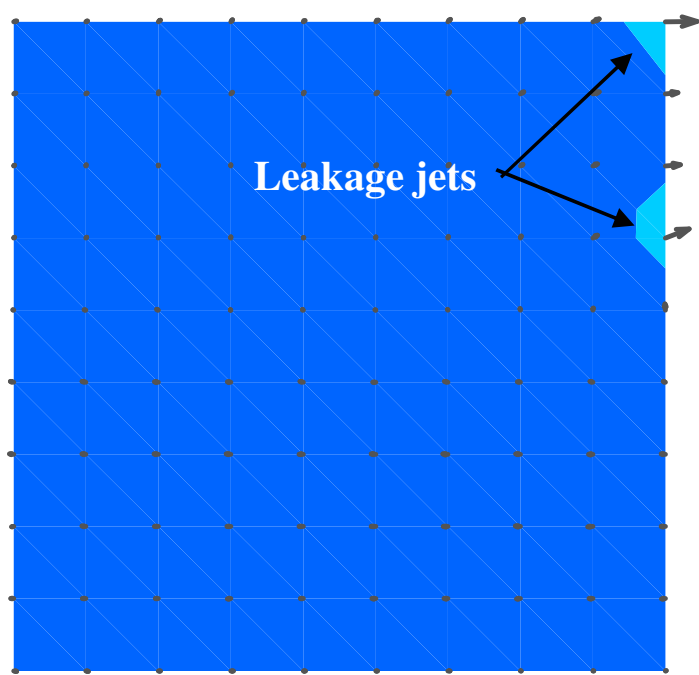

Figure 7-127 Velocity fields downstream of the stent of prototype $\mathrm{C}$ during mid diastole, time bin 21; leakage jets of approximately $0.61 \mathrm{~m} / \mathrm{s}$ were seen at the commissural region during mid diastole (avi, 605k, C_stent_downstream_vmag.avi)

\subsection{Flow Inside the Valve}

Animations C_inside_valve_vmag.avi and C_inside_valve_rss.avi show the velocity magnitude $(\mathrm{m} / \mathrm{s})$ and Reynolds shear stress $\left(\right.$ dynes $\left./ \mathrm{cm}^{2}\right)$ contour plots of the flow field inside the polymeric prototype $\mathrm{C}$ valve throughout the cardiac cycle, respectively. Refer to Appendix $\mathrm{H}$ for the list of animations.

\section{Systole}

Figure 7-128 shows the flow distribution inside prototype $\mathrm{C}$ during the acceleration phase (time bin 5). A velocity of approximately $1.4 \mathrm{~m} / \mathrm{s}$ was observed along the trailing edge of the valve leaflet. The flow pattern at this time phase inside prototype $\mathrm{C}$ was similar to that of prototype B. At peak systole (time bin 9), the flow along the edge of the valve reached a maximum velocity of $2.0 \mathrm{~m} / \mathrm{s}$ (Figure 7-129). The flow field at this time phase was relatively uniform inside the valve $(>1.4 \mathrm{~m} / \mathrm{s})$ and the low flow along the stent inflow 
region as illustrated in Figure 7-102 for prototype A was not evident. During the deceleration phase (time bin 14), the flow velocity along the leaflet trailing edge decreased from $2.0 \mathrm{~m} / \mathrm{s}$ to $0.48 \mathrm{~m} / \mathrm{s}$. The Reynolds shear stress values during systole inside the valve were typically less than $100 \mathrm{dynes} / \mathrm{cm}^{2}$.

\section{Diastole}

Flow inside the valve was unsteady during diastole with intermittent leakage flow ranging between 0.5 and $2.0 \mathrm{~m} / \mathrm{s}$ along the trailing edge of the valve, at the high central region of the valve, and also near the commissural region of prototype $\mathrm{C}$ (time bin 23, Figure 7-130). In contrast to prototypes $\mathrm{A}$ and $\mathrm{B}$, a well defined leakage jet was not observed at the high central region of prototype $\mathrm{C}$ during diastole because the retrograde flow along the trailing edge of the leaflet tended to mix with the leakage jet at the high central region. These retrograde flows persisted throughout the diastole. Flow splitting was observed inside the valve throughout diastole. The flow was directed toward both the stent inflow and toward the high central regions.

The average Reynolds shear stress levels inside the valve reached 300 dynes $/ \mathrm{cm}^{2}$ at valve closure as a result of the disturbed flow inside the valve (time bin 23, Figure 7-131). The Reynolds shear stress values of the leakage jets at the high central and the commissural regions were approximately 1,000 dynes $/ \mathrm{cm}^{2}$, while those measured along the leaflet's trailing edge ranged between $1,000-3,000 \mathrm{dynes} / \mathrm{cm}^{2}$. 


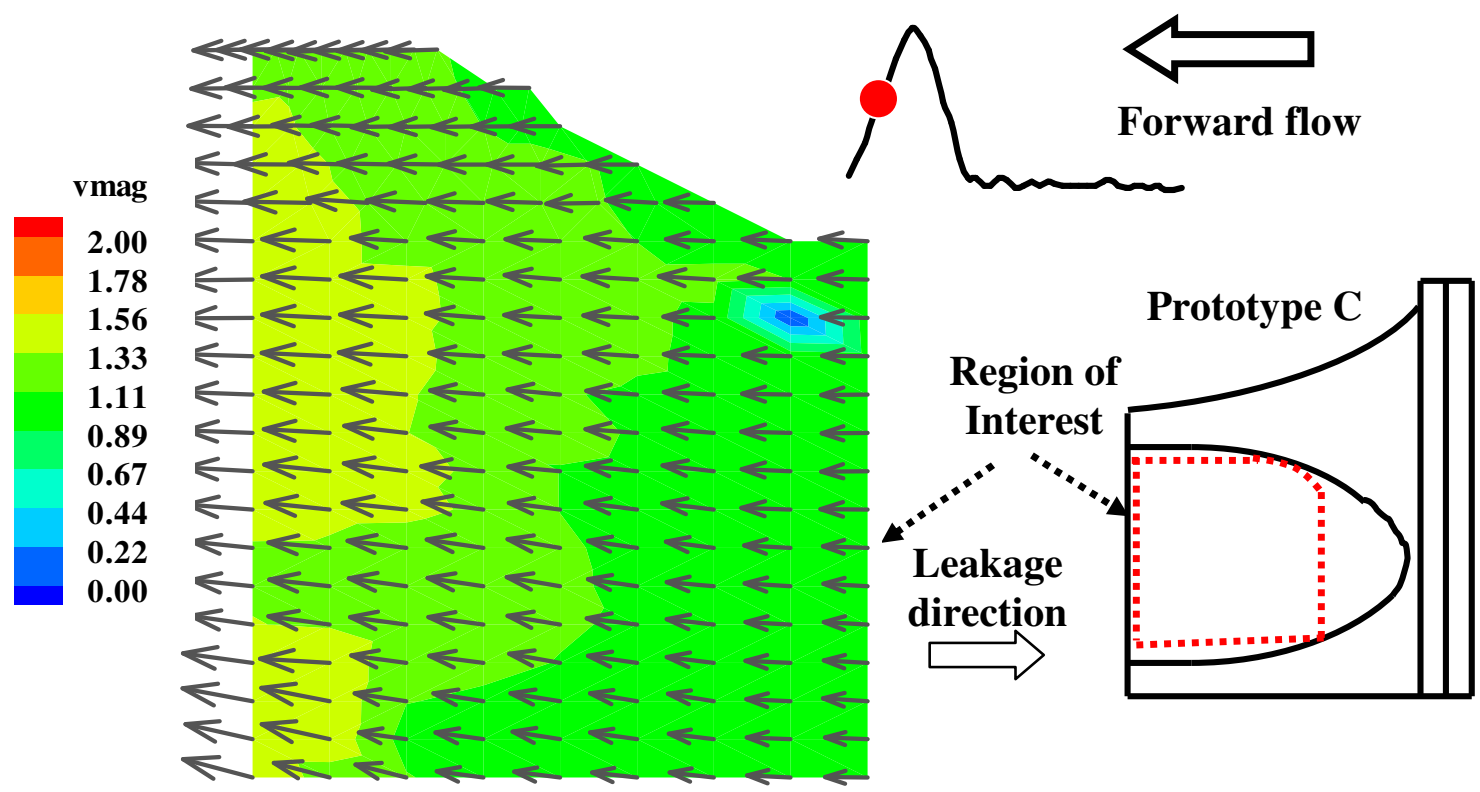

Figure 7-128 Velocity fields inside prototype $\mathrm{C}$ during acceleration phase, time bin 5; high velocity of approximately $1.4 \mathrm{~m} / \mathrm{s}$ was observed along the trailing edge of the leaflet. No area of stagnation was observed in commissural region (avi, 684k, C_inside_valve_vmag.avi)

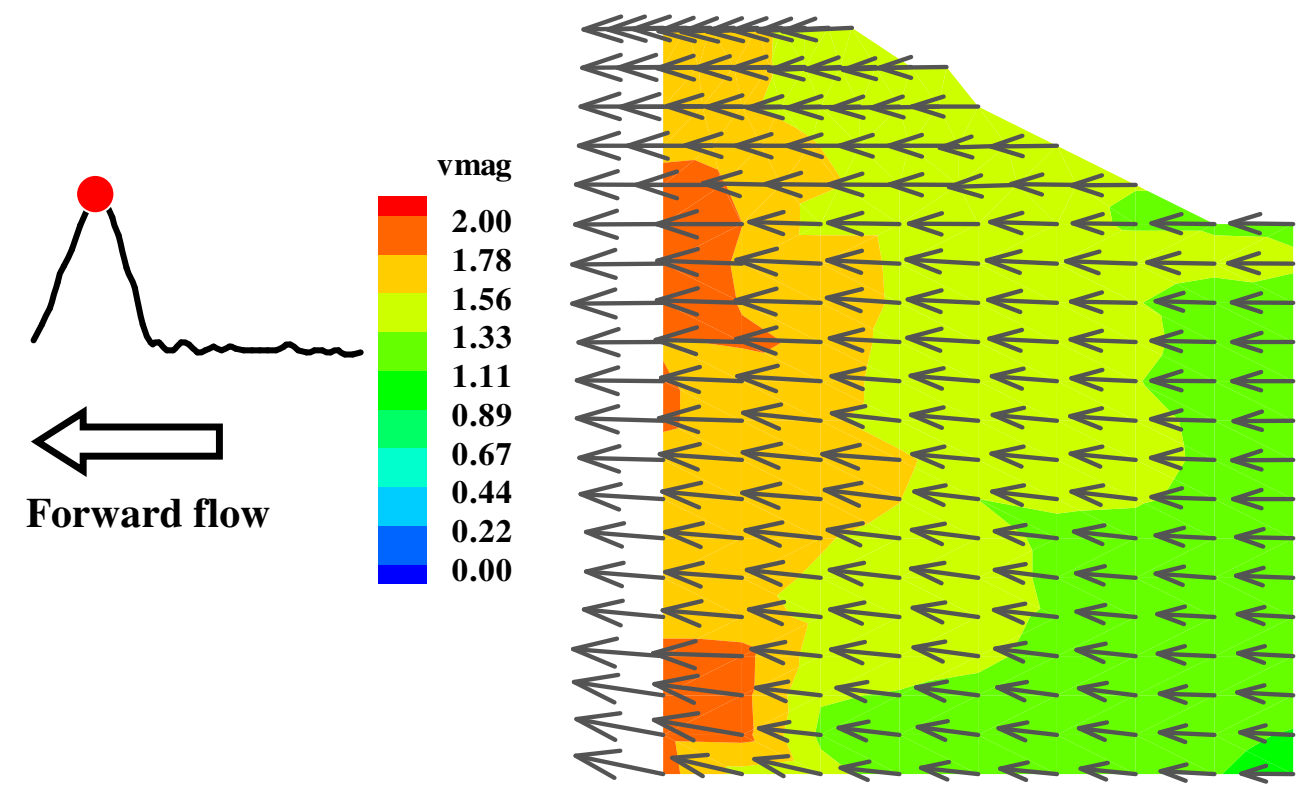

Figure 7-129 Velocity fields inside prototype $\mathrm{C}$ at peak systole, time bin 9; a maximum velocity of $2.1 \mathrm{~m} / \mathrm{s}$ was recorded along the edge of the valve during peak systole (avi, 684k, C_inside_valve_vmag.avi) 


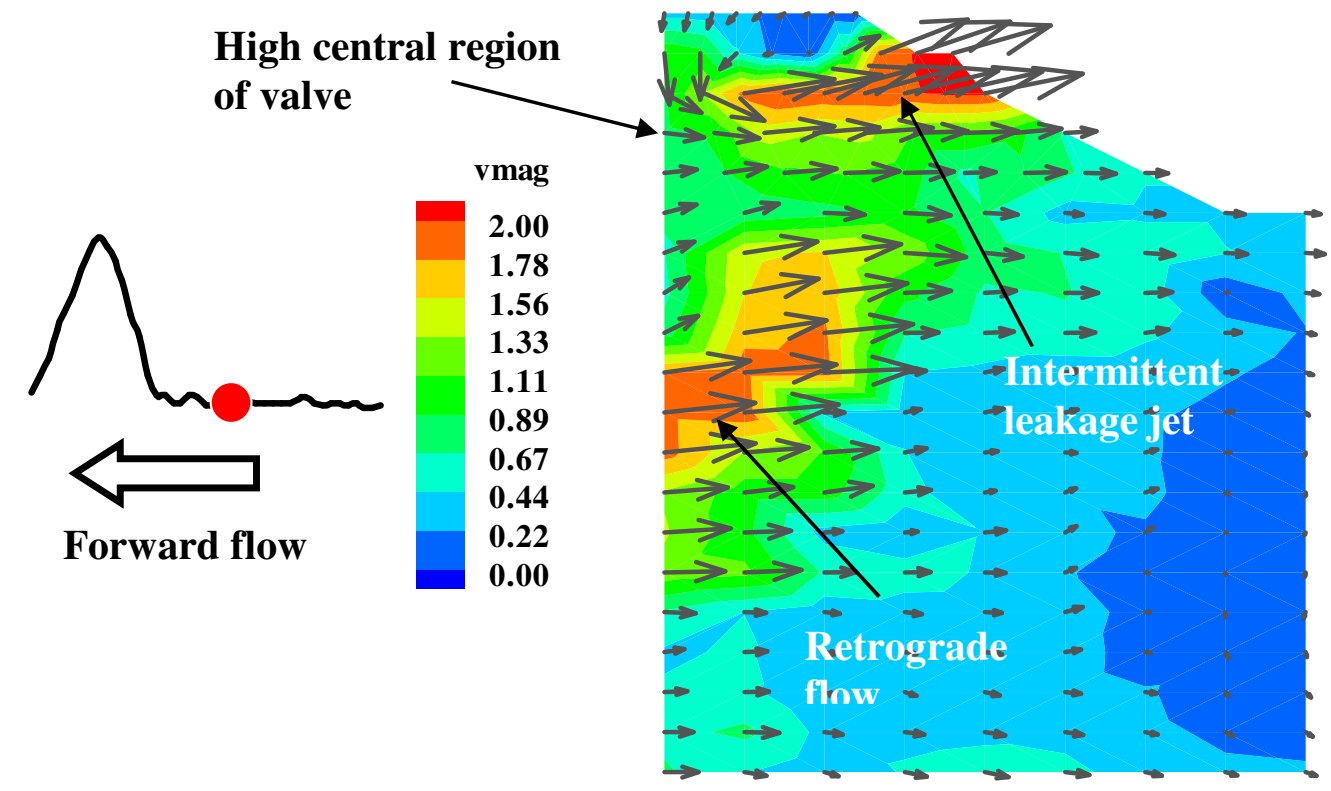

Figure 7-130 Velocity fields inside prototype $\mathrm{C}$ during diastole, time bin 23; retrograde flow of approximately $1.8 \mathrm{~m} / \mathrm{s}$ appeared along leaflet trailing edge, while a leakage jet of $2.0 \mathrm{~m} / \mathrm{s}$ appeared at the high central region of the valve (avi, 684k, C_inside_valve_vmag.avi)
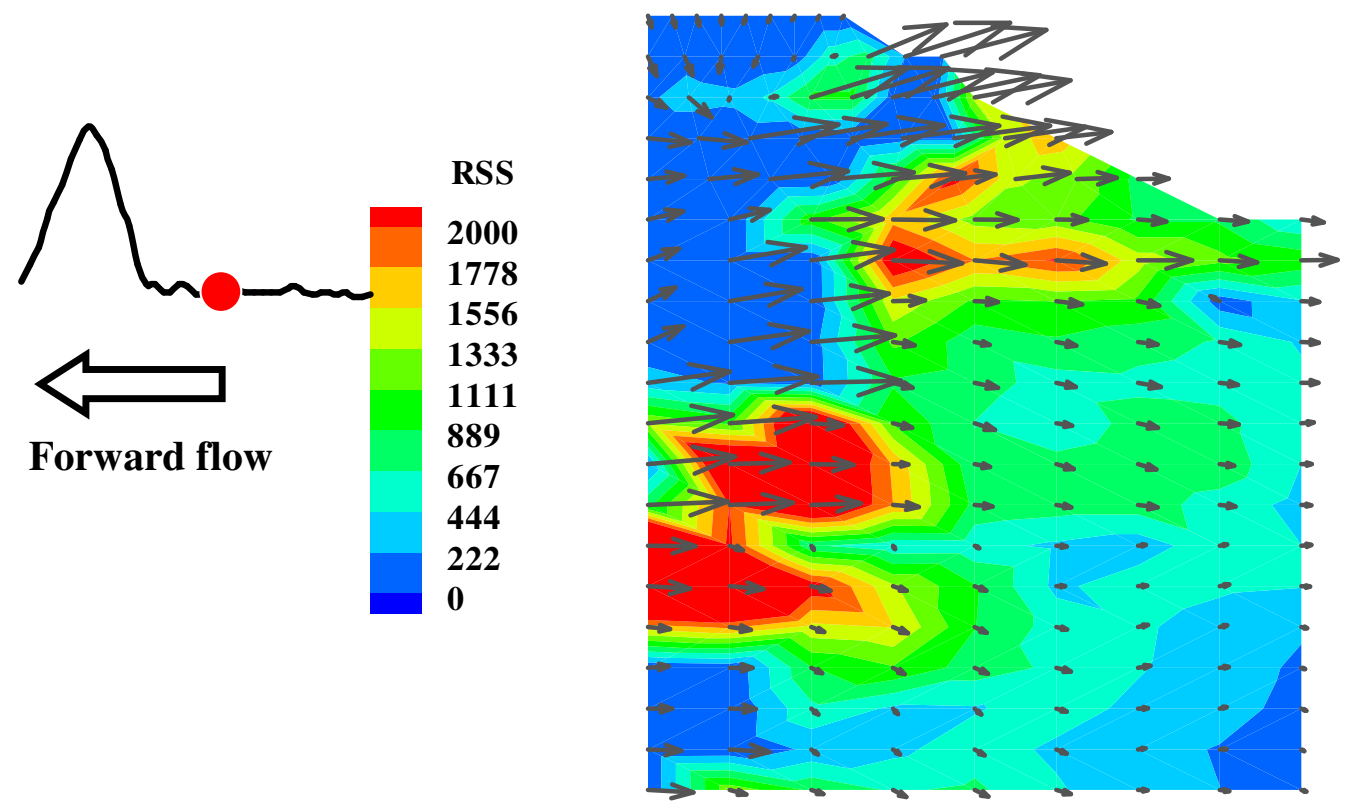

Figure 7-131 Reynolds shear stress distribution of prototype $\mathrm{C}$ during diastole, time bin 23; elevated Reynolds shear stress of more than 1,000 dynes $/ \mathrm{cm}^{2}$ occurred along the edge of the valve during diastole (avi, 706k, C_inside_valve_rss.avi) 


\subsubsection{D LDV Measurements On The Prototype Polymeric Valve A}

The results presented in this section are based on phase averaged velocity fields. The results to be presented are those from the parallel-axis measurement plane of prototype A. The description of the flow fields begin with those in the systolic phase followed by those in the diastolic phase. All contour plots are color-coded by Velocity Magnitude $(\mathrm{m} / \mathrm{s})$ or

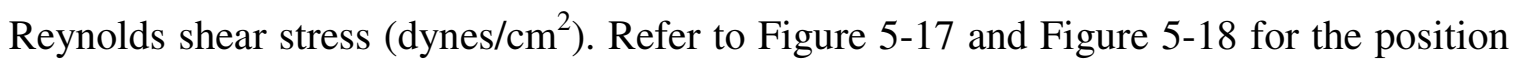
of various measurement planes. In these figures the direction of the forward flow is from right to left (from left ventricle into the aorta). Arrows point in the direction of the phase averaged mean velocity vectors and the length of the arrow is proportional to the velocity magnitude. Refer to Figure 5-2 for the aortic flow and pressure waveforms, and Appendix $\mathrm{H}$ for the velocity magnitude $(\mathrm{m} / \mathrm{s})$ and Reynolds shear stress $\left(\right.$ dynes $/ \mathrm{cm}^{2}$ ) animations. Figure 7-93 shows the terminology pertinent to the following description of the polymeric heart valve results.

\subsubsection{Closed Commissure Valve With 80 um Leaflet Thickness (Prototype A)}

7.3.4.1.1 Flow Fields Downstream of the Valve

Animations $\quad$ A_parallel_downstream_3DLDV_vmag and A_parallel_downstream_3DLDV_rss.avi the velocity magnitude (m/s) and Reynolds shear stress $\left(\right.$ dynes $\left./ \mathrm{cm}^{2}\right)$ contour plots of the flow fields of the parallel-to-stent axis measurement plane downstream of the polymeric prototype A valve throughout the cardiac cycle, respectively. Refer to Appendix $\mathrm{H}$ for the list of animations. 


\section{Systole}

Figure 7-132 shows the flow field downstream of prototype A along the parallel-to-stent axis during the acceleration phase (time bin 5). The flow patterns were similar to those observed in the three polymeric prototype valves in the 2D LDV studies. A velocity of $1.25 \mathrm{~m} / \mathrm{s}$ was recorded in the central orifice jet during the acceleration phase. Flow separation and re-attachment occurred at the trailing edge of the leaflet and $75 \mathrm{~mm}$ downstream from the valve sewing ring, respectively. Flow along the chamber wall was in the forward direction, and had a velocity of approximately $0.1 \mathrm{~m} / \mathrm{s}$. A vortex was observed in the top sinus region. At peak systole (time bin 9), the central jet, which had a diameter of about $12 \mathrm{~mm}$, accelerated to a velocity of $2.0 \mathrm{~m} / \mathrm{s}$ at a location $21 \mathrm{~mm}$ from the valve sewing ring, coinciding with the contraction of the central jet (Figure 7-133). The central jet decelerated to a velocity of approximately $0.7 \mathrm{~m} / \mathrm{s}$ at a distance $60 \mathrm{~mm}$ downstream from the valve sewing ring. Flow separation was observed at the trailing edge of the leaflet, while reattachment points at approximately $65 \mathrm{~mm}$ and $45 \mathrm{~mm}$ downstream from the valve sewing ring in the upper and lower parts of the flow chamber, respectively. A velocity of $0.2 \mathrm{~m} / \mathrm{s}$ was observed inside the recirculation along the chamber wall flowing upstream into the sinus region. During the deceleration phase (time bin 14), the flow velocity of the central jet decreased from $2.0 \mathrm{~m} / \mathrm{s}$ to $0.5 \mathrm{~m} / \mathrm{s}$, and the recirculation zone $(<0.2 \mathrm{~m} / \mathrm{s})$ along the wall expanded into the center of the flow channel (Figure 7-134).

A maximum Reynolds shear stress of 1,555 dynes $/ \mathrm{cm}^{2}$ was measured at the edge of the central orifice jet during peak systole (time bin 9, Figure 7-135). The Reynolds shear 
stress levels at the distal region of the flow chamber ranged between 200 to 700 dynes $/ \mathrm{cm}^{2}$, while those inside the sinus region and the central orifice jet were typically less than 50 dynes $/ \mathrm{cm}^{2}$. During the deceleration phase, elevated Reynolds shear stress levels ranged between 400 to 700 dynes $/ \mathrm{cm}^{2}$ was recorded along the edge of the central orifice jet (Figure 7-136).

\section{Diastole}

Retrograde flow velocity of $0.4 \mathrm{~m} / \mathrm{s}$ was observed downstream of the leaflet trailing edge at valve closure before reducing to approximately $0.08 \mathrm{~m} / \mathrm{s}$ at early diastole. Figure 7-137 (time bin 21) shows the flow field downstream of prototype A along the parallel-axis during mid diastole. The flow velocity downstream from the leaflet trailing edge and inside the sinus region were less than 0.08 and $0.05 \mathrm{~m} / \mathrm{s}$, respectively. The observed flow pattern persisted throughout the diastole. No retrograde flow was observed along the trailing edge of the leaflet. The Reynolds shear stress levels during diastole was less than 100 dynes $/ \mathrm{cm}^{2}$ throughout the investigated region, and persisted through the diastole.

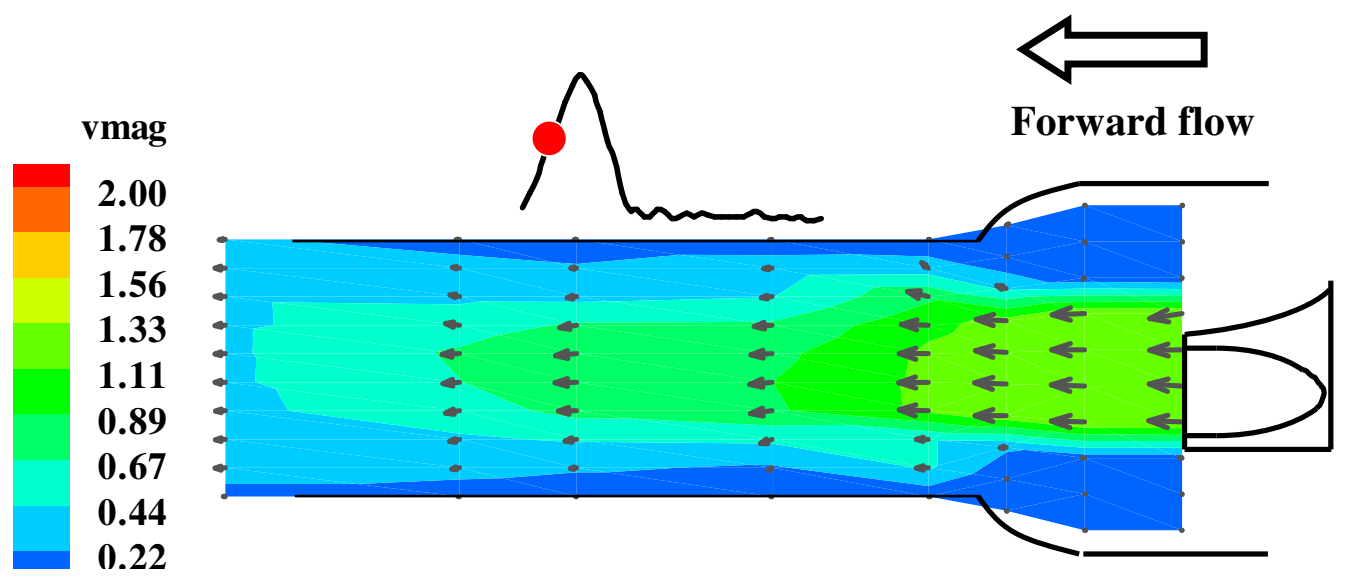

Figure 7-132 3D LDV velocity fields downstream of prototype A at acceleration phase, time bin 5, center plane; a developing central orifice jet of $1.3 \mathrm{~m} /$. A vortex was observed inside the top sinus region (avi, 454k, A_parallel_downstream_3DLDV_vmag.avi) 


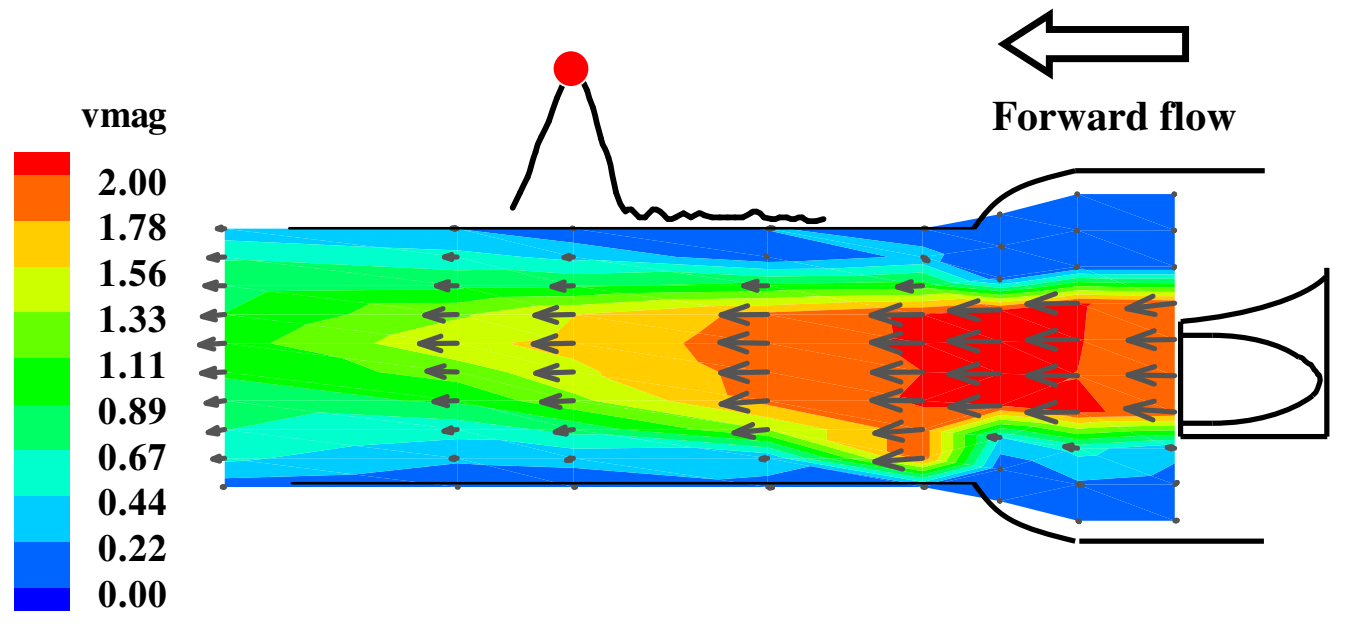

Figure 7-133 3D LDV Velocity fields downstream of prototype A at peak systole, time bin 9, center plane; flow velocity of $2.0 \mathrm{~m} / \mathrm{s}$ was observed inside the central orifice jet at location coinciding with that of the flow constriction observed $21 \mathrm{~mm}$ downstream from the valve sewing ring (avi, 454k, A_parallel_downstream_3DLDV_vmag.avi)

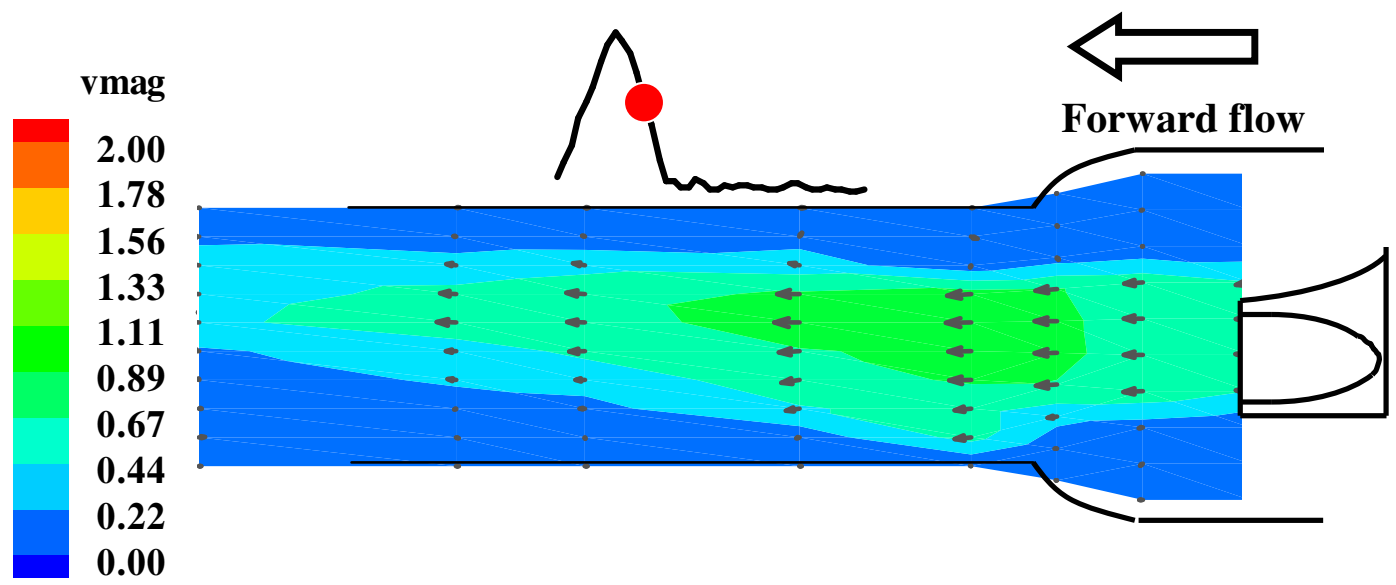

Figure 7-134 3D LDV Velocity fields downstream of prototype A at deceleration phase, time bin 14, center plane; flow velocity in the central orifice jet reduced from 2.0 to 0.5 $\mathrm{m} / \mathrm{s}$. The recirculation zone expanded into the center of the flow chamber (avi, $454 \mathrm{k}$, A_parallel_downstream_3DLDV_vmag.avi) 


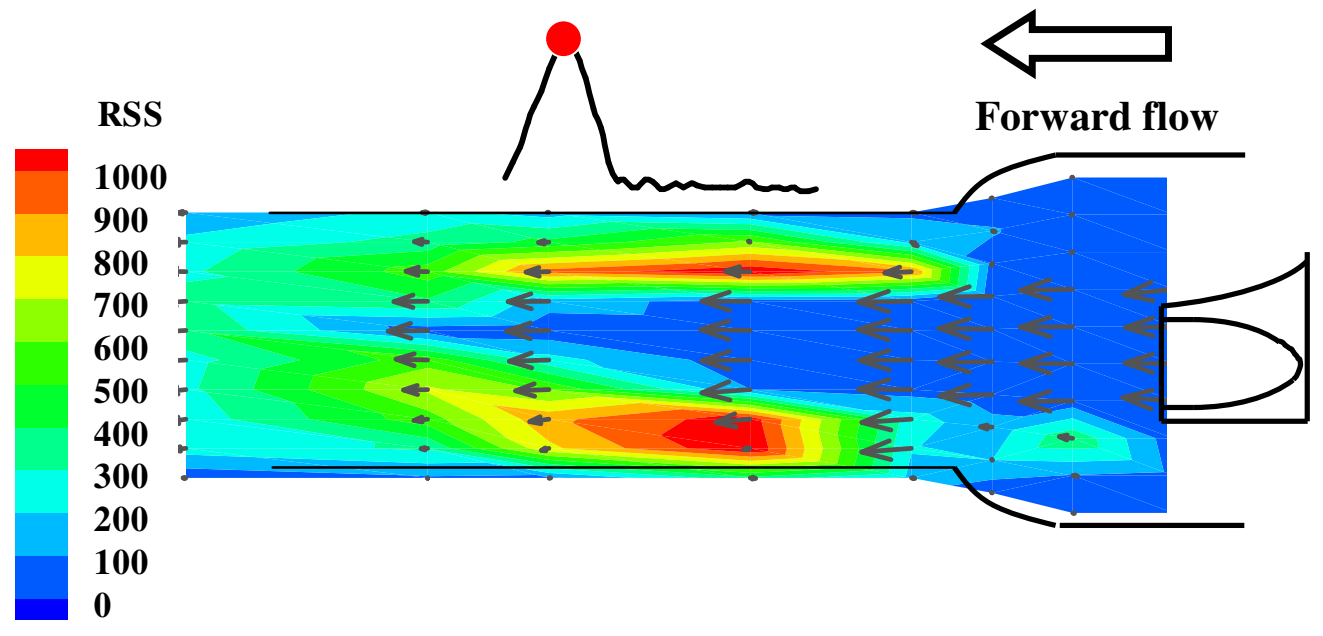

Figure 7-135 3D LDV Reynolds shear stress distribution downstream of prototype A at peak systole, time bin 9, center plane; elevated Reynolds shear stress levels were observed along the edge of the central orifice jet and at the distal region of the flow chamber (avi, 468k, A_parallel_downstream_3DLDV_rss.avi)

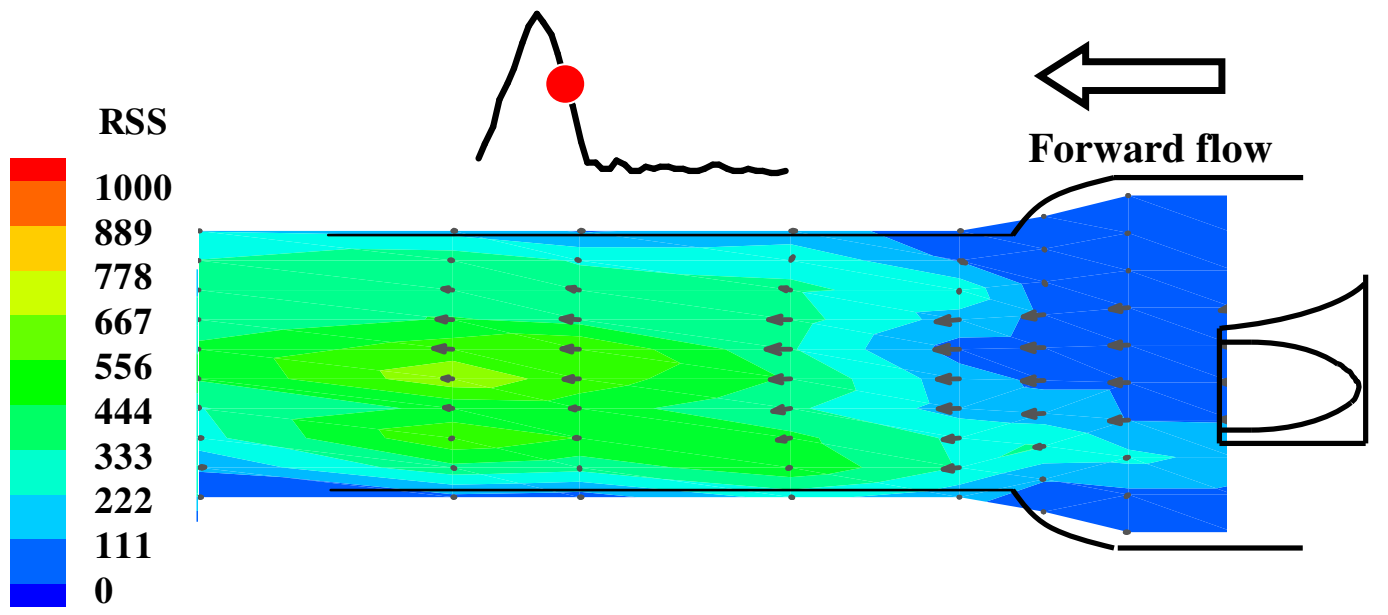

Figure 7-136 3D LDV Reynolds shear stress distribution downstream of prototype A at deceleration phase, time bin 14, center plane; elevated Reynolds shear stress levels were observed at the distal region of the flow chamber (avi, 468k, A_parallel_downstream_3DLDV_rss.avi) 


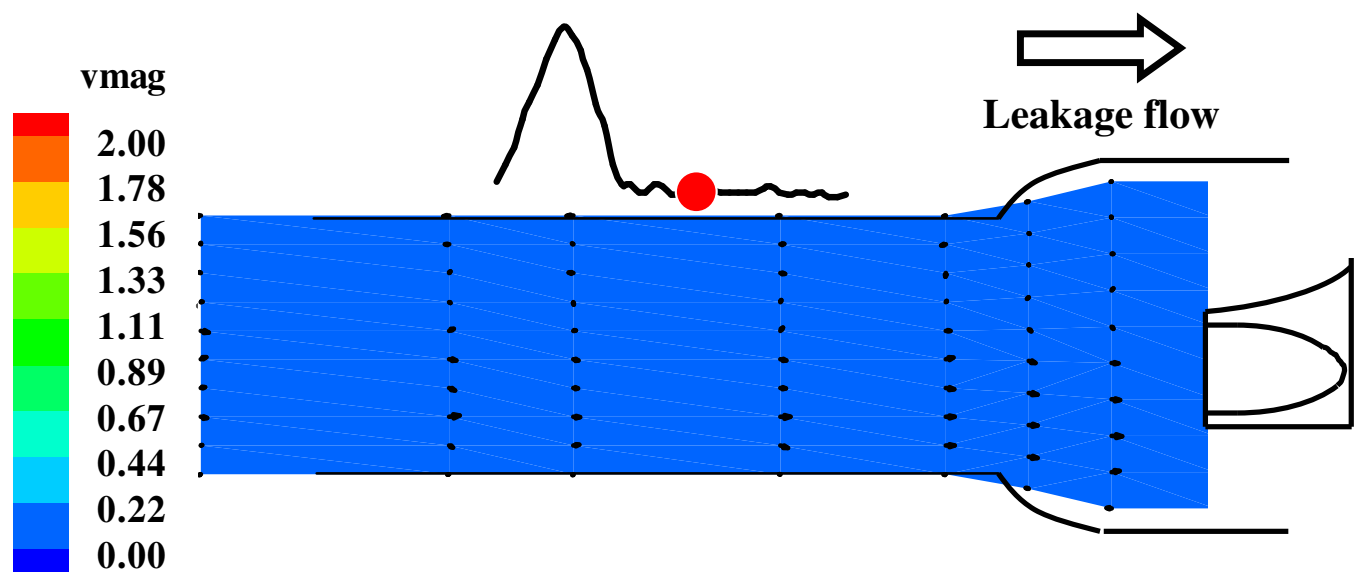

Figure 7-137 3D LDV velocity fields downstream of prototype A at mid diastole, time bin 21, center plane; the flow field during diastole was less than $0.08 \mathrm{~m} / \mathrm{s}$ (avi, 454k, A_parallel_downstream_3DLDV_vmag.avi)

\subsubsection{D PIV Measurements On The Three Prototype Aortech $23 \mathrm{~mm}$ Polymeric}

\section{Heart Valves}

The results presented are based on phase averaged velocity fields. The flow characteristics of prototype A are presented first followed by those of prototypes B and C. For each prototype valve, the results are presented in the following order: 1) center plane; 2) $\pm 4 \mathrm{~mm}$ planes; 3) $\pm 8 \mathrm{~mm}$ planes, 4) $\pm 12 \mathrm{~mm}$ planes. For each measurement plane, the description of the flow during systole is presented first, followed by that during diastole. For each time phase the description of the flow field downstream from the trailing edge of the leaflet is presented first followed by that inside the valve (all downstream description of the flow fields are referenced from the edge of the sewing ring as defined in Figure 7-138). All contour plots are appropriately color-coded by Velocity Magnitude $(\mathrm{m} / \mathrm{s})$ and Reynolds shear stress $\left(\right.$ dynes $\left./ \mathrm{cm}^{2}\right)$. Refer to Figure 5-25 for the position of various measurement planes. The direction of the forward flow is from right to left (from the left ventricle into the aorta). Refer to Figure 5-2 for the aortic flow and pressure 
waveforms, and Appendix $\mathrm{H}$ for the velocity magnitude $(\mathrm{m} / \mathrm{s})$ and Reynolds shear stress (dynes $/ \mathrm{cm}^{2}$ ) animations. Figure 7-138 shows the terminology pertinent to the following description of the polymeric heart valve results.
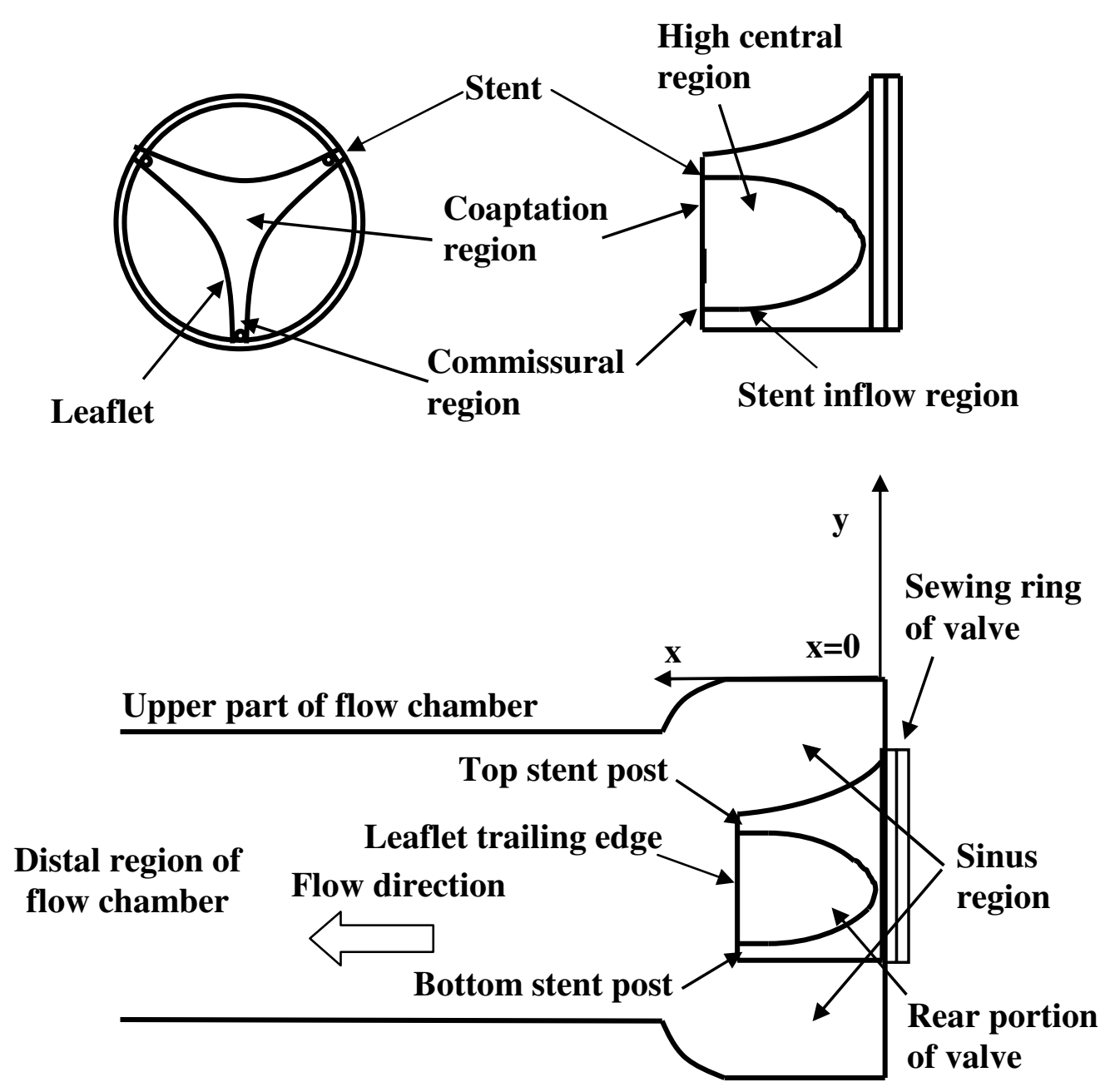

Figure 7-138 Pertinent terminology for the three Aortech $23 \mathrm{~mm}$ trileaflet polymeric heart valves. All downstream measurements were made with reference from the valve sewing ring, which is marked $\mathrm{x}=0$ 


\subsubsection{Closed Commissure Valve With 80 um Leaflet Thickness (Prototype A)}

\subsection{Center Plane}

Animations A_center_plane_vmag.avi and A_center_plane_rss.avi show the velocity magnitude $(\mathrm{m} / \mathrm{s})$ and Reynolds shear stress $\left(\right.$ dynes $\left./ \mathrm{cm}^{2}\right)$ contour plots for the center measurement plane of prototype A, throughout the cardiac cycle, respectively. Animation A_isosurface_vmag.avi shows the 3D reconstructed iso-surface of the velocity magnitude of the prototype A, throughout the cardiac cycle. Refer to Appendix $\mathrm{H}$ for the list of animations.

\section{Systole}

Figure 7-139 shows the center plane flow field downstream of prototype A during the acceleration phase (time bin 5). A central orifice jet of $1.0 \mathrm{~m} / \mathrm{s}$ was observed issuing from the orifice of the valve with the formation of a vortex ring along the edge of the central jet. The central orifice flow reached a maximum velocity magnitude of $2.4 \mathrm{~m} / \mathrm{s}$ at peak systole (time bin 9, Figure 7-140) and its location coincided with that of the vena contracta observed approximately $20 \mathrm{~mm}$ downstream from the valve sewing ring. The central orifice jet had a diameter of approximately $13 \mathrm{~mm}$. The velocity vector plot in Figure 7-140 shows that the central orifice jet exiting from the valve orifice had a flat profile, which became parabolic approximately $45 \mathrm{~mm}$ downstream from the valve sewing ring. Figure 7-141 displays the iso-surface of the velocity magnitude downstream of valve $\mathrm{A}$ at peak systole (time bin 9). The iso-surface of the velocity magnitude was characterized by a three lobe flow profile, which became more evident $55 \mathrm{~mm}$ downstream from the valve sewing ring. It appeared that each lobe on the velocity iso- 
surface coincided with a region of high velocity flow issuing from the commissural region. Flow separation was observed at the trailing edge of the leaflet. Two reattachments points were observed downstream from the valve sewing ring: one in the upper part of the flow chamber had extended beyond the measurement plane, while another in the lower part of the flow chamber was $55 \mathrm{~mm}$ downstream of the valve sewing ring. Velocity of $0.6 \mathrm{~m} / \mathrm{s}$ was seen inside the recirculation zone. Flow in the sinus region was generally less than $0.02 \mathrm{~m} / \mathrm{s}$ with occasional vortex structures appearing briefly during the acceleration and deceleration phases. During the deceleration phase (time bin 14), the velocity in the central orifice jet decelerated from $2.4 \mathrm{~m} / \mathrm{s}$ to $1.1 \mathrm{~m} / \mathrm{s}$. The velocity in the recirculation zone in the upper part of flow chamber reduced from 0.6 $\mathrm{m} / \mathrm{s}$ to $0.4 \mathrm{~m} / \mathrm{s}$. Flow patterns inside the valve during systole were similar to those observed in the LDV studies. A maximum velocity of $2.0 \mathrm{~m} / \mathrm{s}$ was measured at the trailing edge of the leaflet at peak systole with low velocity flow of $0.5 \mathrm{~m} / \mathrm{s}$ along the stent inflow region of the valve throughout systole.

Figure 7-142 shows the Reynolds shear stress distribution for prototype A at peak systole (time bin 9). Elevated Reynolds shear stress levels of more than 3,000 dynes $/ \mathrm{cm}^{2}$ were observed in the following regions: the shear layer region between the central orifice jet and the surrounding fluid; at the trailing edge of the leaflet; and in the region of turbulence $55 \mathrm{~mm}$ downstream of the valve sewing ring. 


\section{Diastole}

The velocity downstream from the trailing edge of the leaflet during diastole was typically less than $0.02 \mathrm{~m} / \mathrm{s}$, except for the flow inside the recirculation zone $(<0.4 \mathrm{~m} / \mathrm{s})$ observed along the wall of the valve chamber during early diastole. The velocity inside the recirculation zone decreased to less than $0.02 \mathrm{~m} / \mathrm{s}$ during late diastole. Inside the valve a leakage jet with a velocity of $1.63 \mathrm{~m} / \mathrm{s}$ was observed at the coaptation region during mid-diastole (time bin 21, Figure 7-143). Leakage flow was also seen briefly near the commissural region along the stent post during early diastole. Flow inside the valve was directed toward the stent post and the high central region (time bin 21, Figure 7-144). This split flow phenomenon persisted throughout diastole becoming more noticeable when the leakage jets in the coaptation and commissural regions appeared. Elevated Reynolds shear stress of 5,000 dynes $/ \mathrm{cm}^{2}$ was observed in the leakage jet at the high central region of the valve. In contrast, the Reynolds shear stress levels in the flow field downstream of the leaflet trailing edge were typically less 100 dynes $/ \mathrm{cm}^{2}$ (Figure 7-145) 


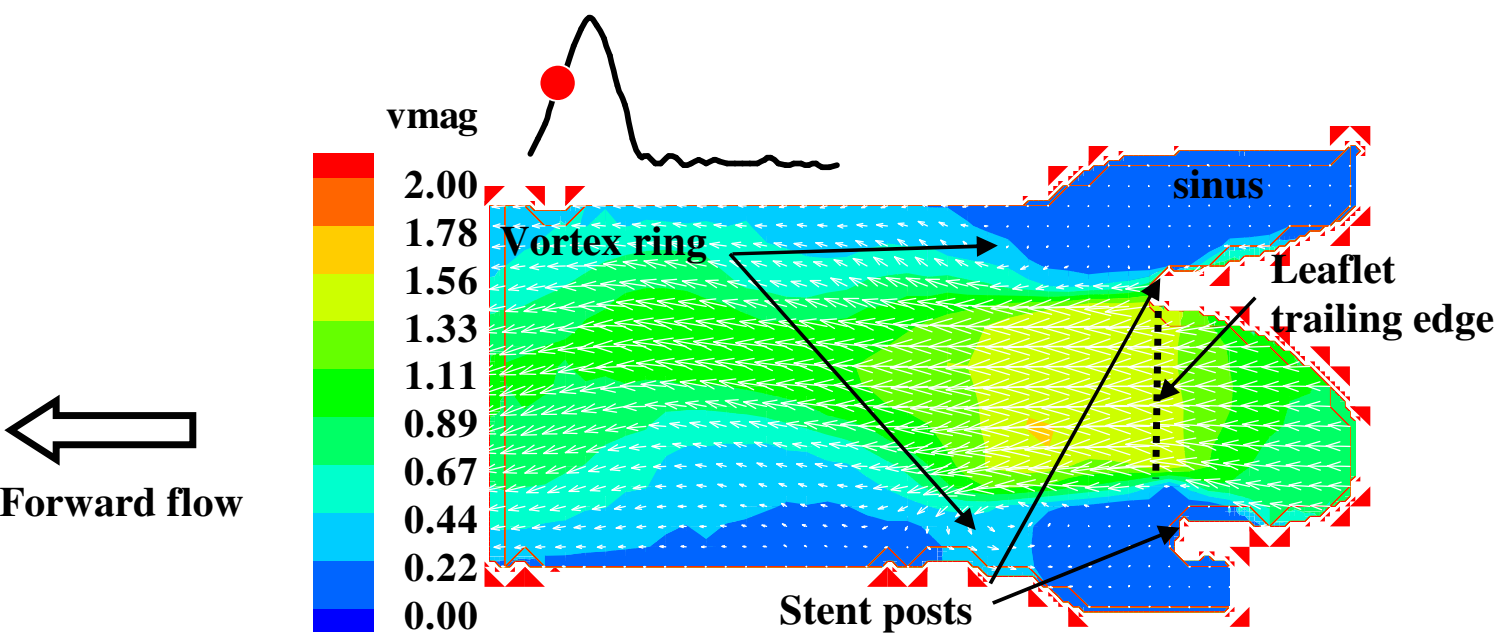

Figure 7-139 Velocity fields at the center plane of prototype A during acceleration phase, time bin 5; vortex ring was observed at the edge of the central orifice jet (avi, 615k, A_center_plane_vmag.avi)

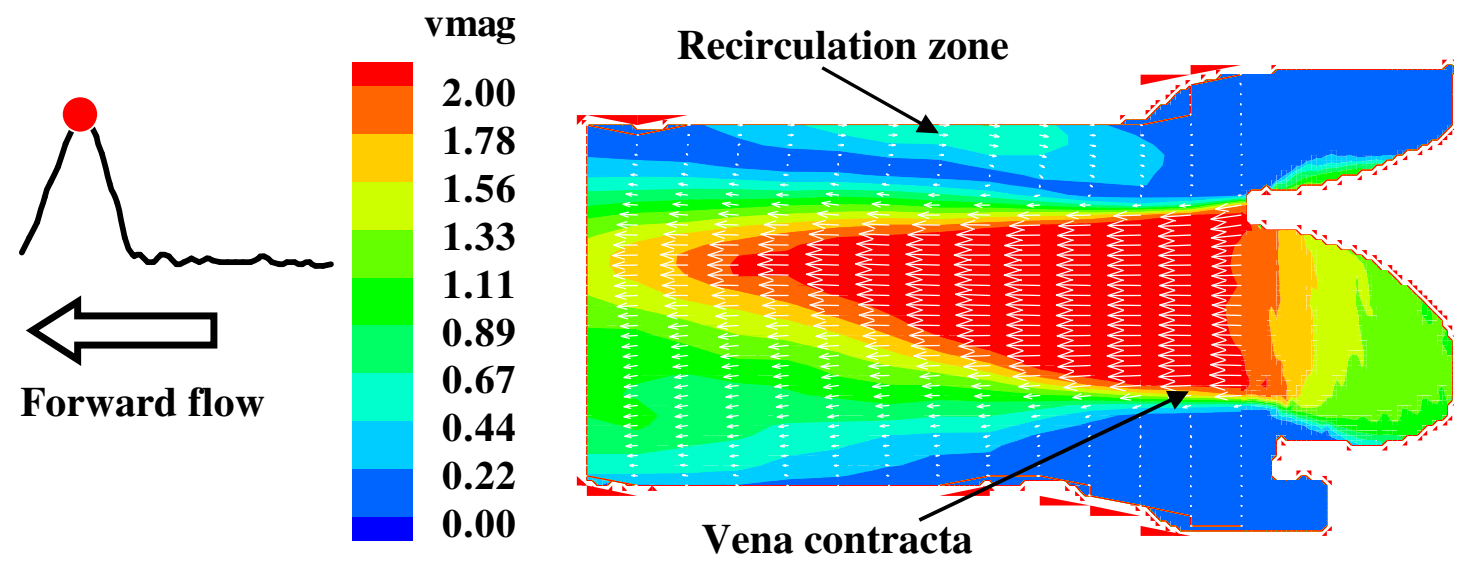

Figure 7-140 Velocity fields at the center plane of prototype A during peak systole, time bin 9; velocity central orifice jet of $2.3 \mathrm{~m} / \mathrm{s}$ was observed issuing from the valve orifice with flow separation occurring at the trailing edge of the leaflet. Plug flow profile was evident at the immediate downstream of leaflet trailing edge and became more parabolic at the distal end of the flow chamber (avi, 615k, A_center_plane_vmag.avi) 

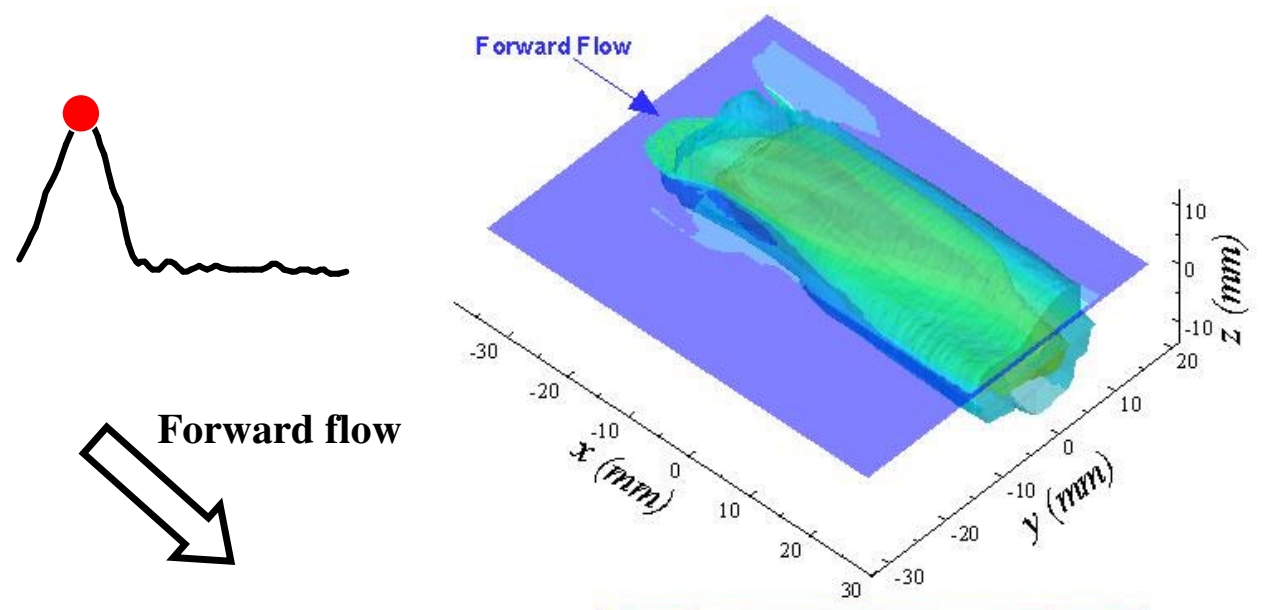

vmag $(\mathrm{m} / \mathrm{s}): \quad \begin{array}{llllllllll}0.0 & 0.3 & 0.5 & 0.8 & 1.0 & 1.3 & 1.5 & 1.8 & 2.0\end{array}$

Figure 7-141 Iso-surface of velocity magnitude of prototype A at peak systole, time bin 9; the flow profile at peak systole had a three-lobe configuration (avi, 915k, A_isosurface_vmag.avi)

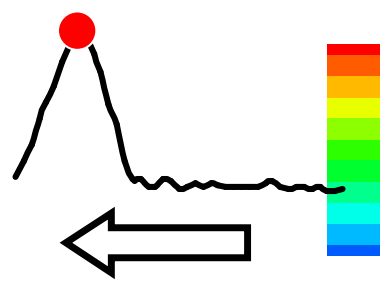

3000

2400

1800

1200

600

Forward flow

\section{Distal region of flow}

\section{chamber}

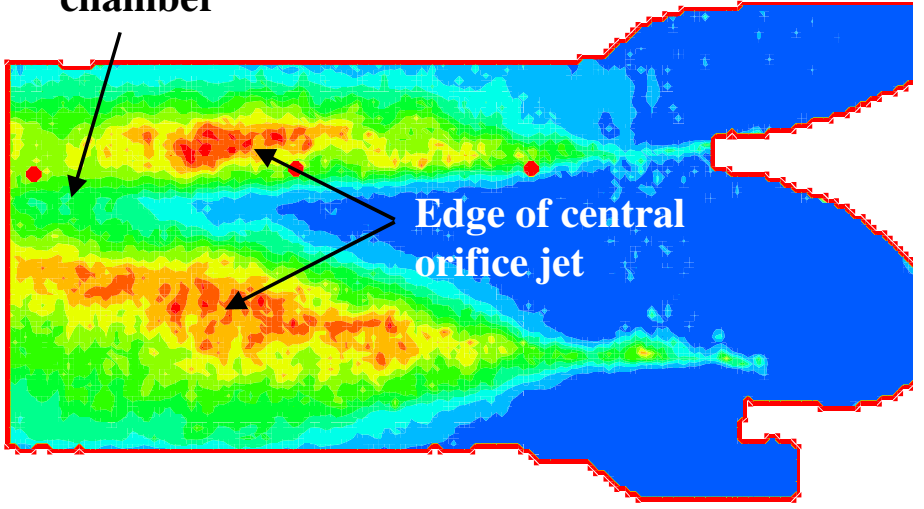

Figure 7-142 Reynolds shear stress contour plot of the center plane of prototype A during peak systole, time bin 9; high Reynolds shear stress values coincided with regions of high velocity gradient typically observed at the edge of the central orifice jet and at the distal part of the flow chamber (avi, 821k, A_center_plane_rss.avi) 


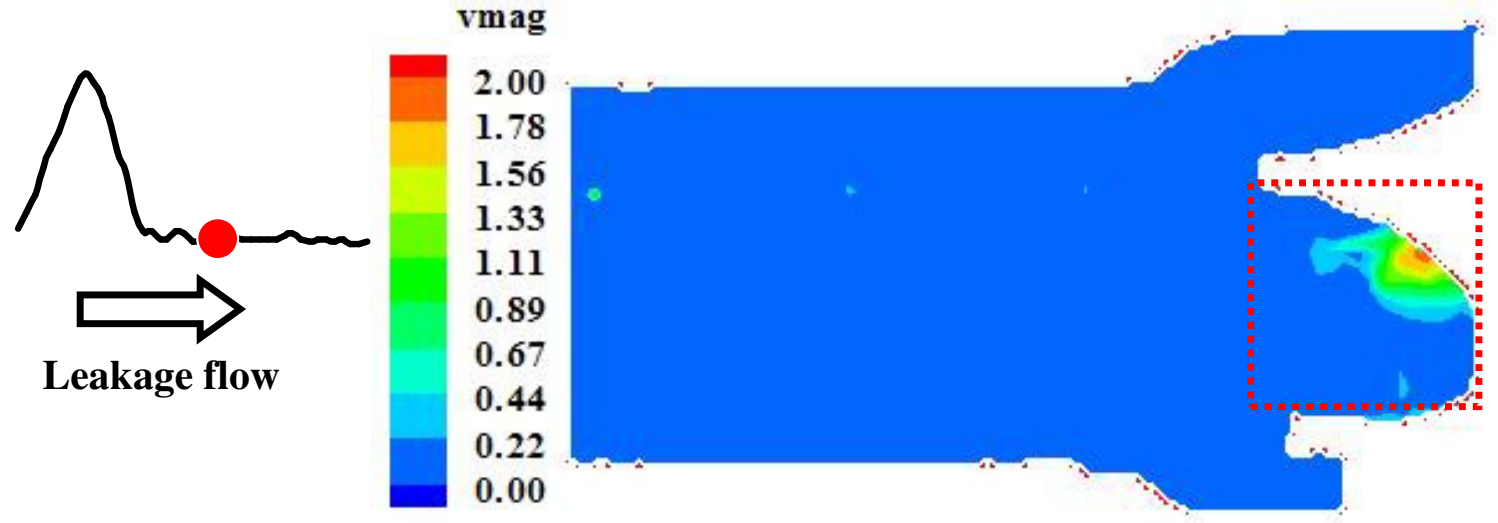

Figure 7-143 Velocity fields at the center plane of prototype A during mid diastole, time bin 21; leakage jet appeared at the coaptation region of the valve. See Figure 7-144 for the flow fields inside the enlarged red dotted box (avi, 615k, A_center_plane_vmag.avi)

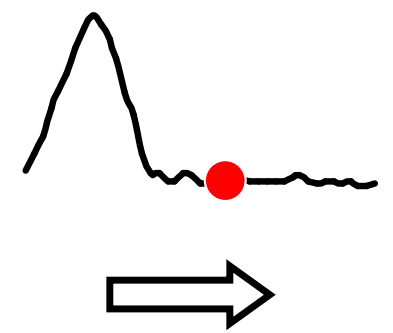

Leakage flow

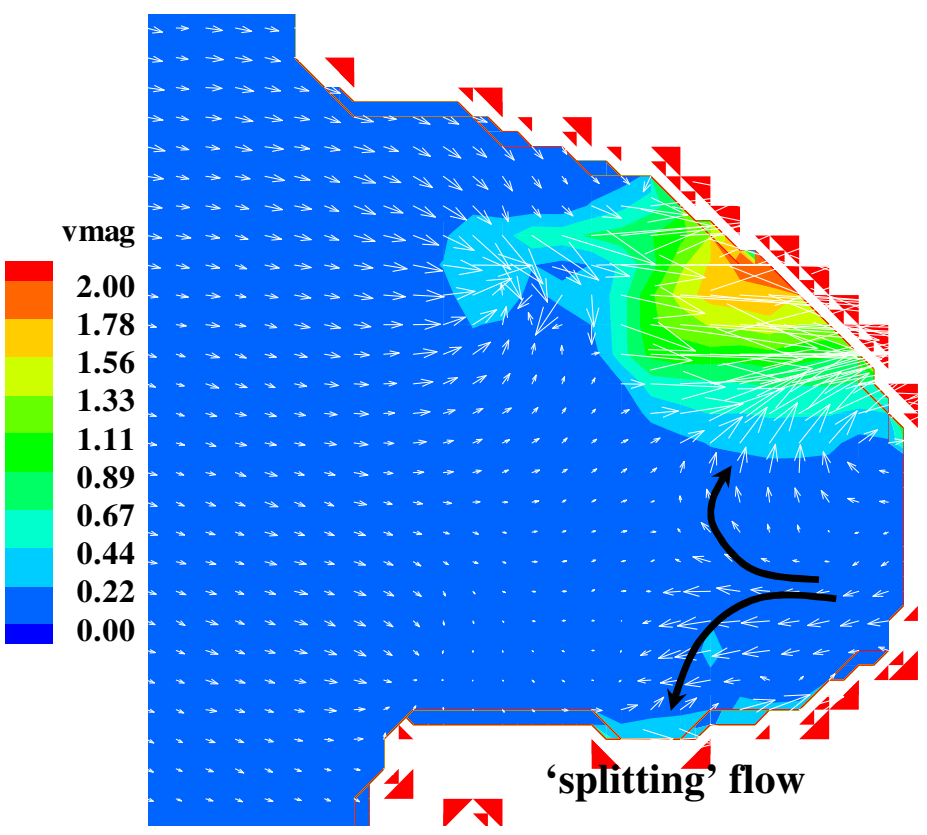

Figure 7-144 Velocity fields inside prototype A during mid diastole, time bin 21, center plane; split flow phenomenon evident inside the valve; one directed toward the lower stent and another toward the leakage jet at the high central region 


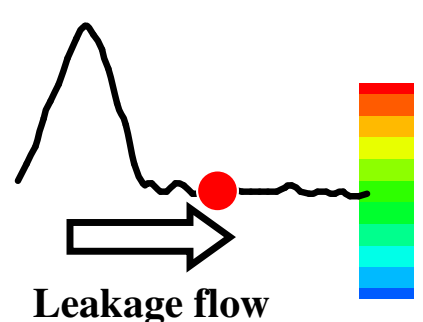

RSS

3000

2400

1800

1200

600

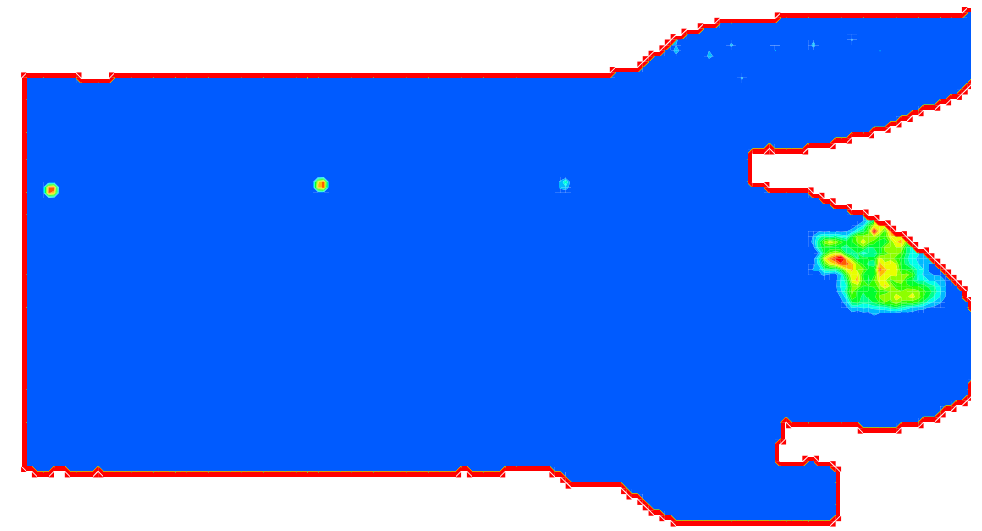

Figure 7-145 Reynolds shear stress contour plot of the center plane of prototype A during mid diastole, time bin 21; elevated Reynolds shear stress of $5,000 \mathrm{dynes} / \mathrm{cm}^{2}$ was observed in the leakage jet at the high central region of the valve (avi, 821, A_center_plane_rss.avi)

\subsubsection{2 $\pm 4 m m$ Plane}

Animations A_+4mm_plane_vmag.avi (A_-4mm_plane_vmag.avi) and A_+4mm_plane_rss.avi (A_-4mm_plane_rss.avi) show the velocity magnitude $(\mathrm{m} / \mathrm{s})$ and Reynolds shear stress $\left(\right.$ dynes $/ \mathrm{cm}^{2}$ ) contour plots for the two $4 \mathrm{~mm}$ offset measurement planes of prototype A throughout the cardiac cycle, respectively. Refer to Appendix $\mathrm{H}$ for the list of animations. The flow patterns in the two offset measurement planes were similar, hence only flow fields of one offset measurement plane are shown.

\section{Systole}

Figure 7-146 shows the $+4 \mathrm{~mm}$ offset plane downstream from the leaflet trailing edge of prototype A during the acceleration phase (time bin 5). A velocity of $1.5 \mathrm{~m} / \mathrm{s}$ was observed inside the central orifice jet with a vortex ring forming along the edge of the jet. At peak systole (time bin 14), the central jet accelerated to a maximum velocity of 2.4 
$\mathrm{m} / \mathrm{s}$ (Figure 7-147). The central jet had a diameter of approximately $12 \mathrm{~mm}$. Flow separation was observed at the leaflet trailing edge, while the reattachment points were observed $48 \mathrm{~mm}$ and $27 \mathrm{~mm}$ downstream of the valve sewing ring in the upper and lower parts of the flow chamber, respectively. The velocity in the recirculation zone was approximately $0.3 \mathrm{~m} / \mathrm{s}$ and no vortices were visible inside the sinus region. The velocity in the central orifice jet during the deceleration phase (time bin 14) reached approximately $1.3 \mathrm{~m} / \mathrm{s}$, while the flow inside the recirculation zone in the upper part of the flow chamber reached a velocity of $0.3 \mathrm{~m} / \mathrm{s}$. Flow patterns inside the valve during systole were similar to those in the center plane. Maximum flow velocity of $2.0 \mathrm{~m} / \mathrm{s}$ was observed along the trailing edge of the leaflet peak systole. The flow along the stent inflow region was approximately $0.5 \mathrm{~m} / \mathrm{s}$. Elevated Reynolds shear stress values of approximately 3,000 dynes $/ \mathrm{cm}^{2}$ were observed at two locations during peak systole: along the edge of the central orifice jet and in the distal region of the flow chamber (time bin 9, Figure 7-148).

\section{Diastole}

The velocity downstream from the leaflet trailing edge was low $(<0.01 \mathrm{~m} / \mathrm{s})$ throughout diastole (time bin 21, Figure 7-149). Flow velocity inside the sinus region was less than $0.01 \mathrm{~m} / \mathrm{s}$ with no visible vortices. The flow pattern inside the valve during diastole was more complex compared to that outside the valve. A leakage jet of approximately $0.3 \mathrm{~m} / \mathrm{s}$ was seen inside the valve near the top stent post close to the commissural region throughout diastole. Unlike the center plane, no split flow was observed inside the valve during diastole except for the appearance of occasional vortex structures near the top 
stent post. The Reynolds shear stress levels outside the valve were typically less than 100 dynes $/ \mathrm{cm}^{2}$. However, Reynolds shear stress values of more than 1,000 dynes $/ \mathrm{cm}^{2}$ were observed in the leakage jet inside the valve (Figure 7-150).

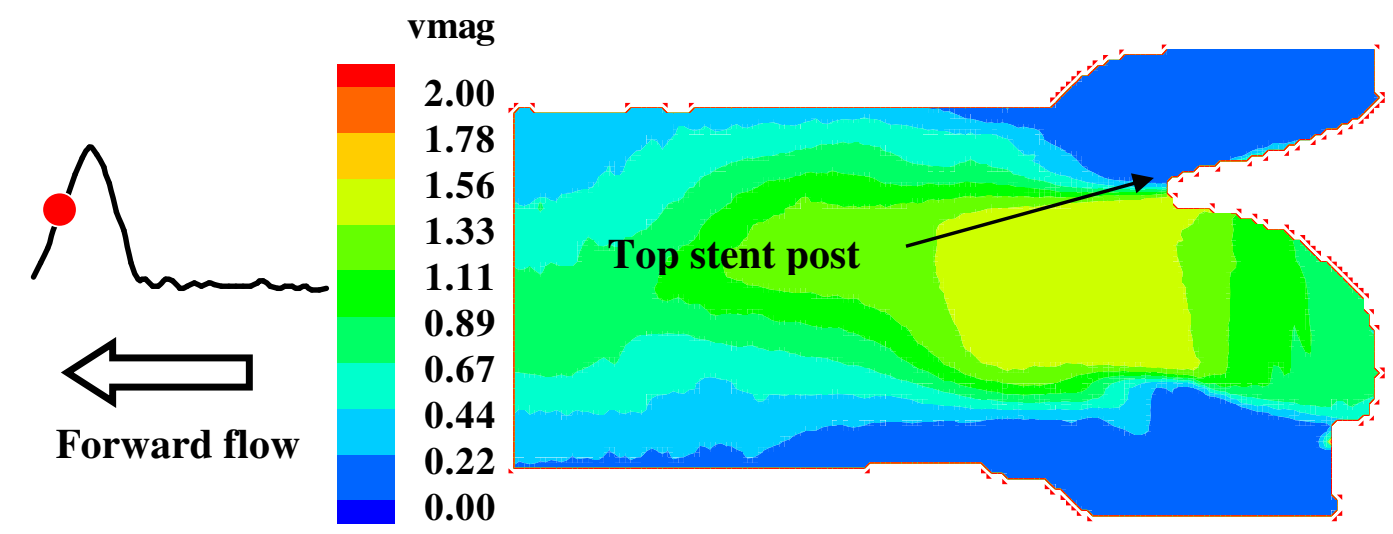

Figure 7-146 Velocity fields at the $+4 \mathrm{~mm}$ offset plane of prototype A acceleration phase, time bin 5 ; velocity of $1.5 \mathrm{~m} / \mathrm{s}$ was measured inside the central orifice jet (avi, 623k, A_+4mm_plane_vmag.avi)

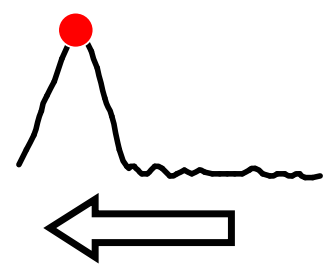

Forward flow vmag

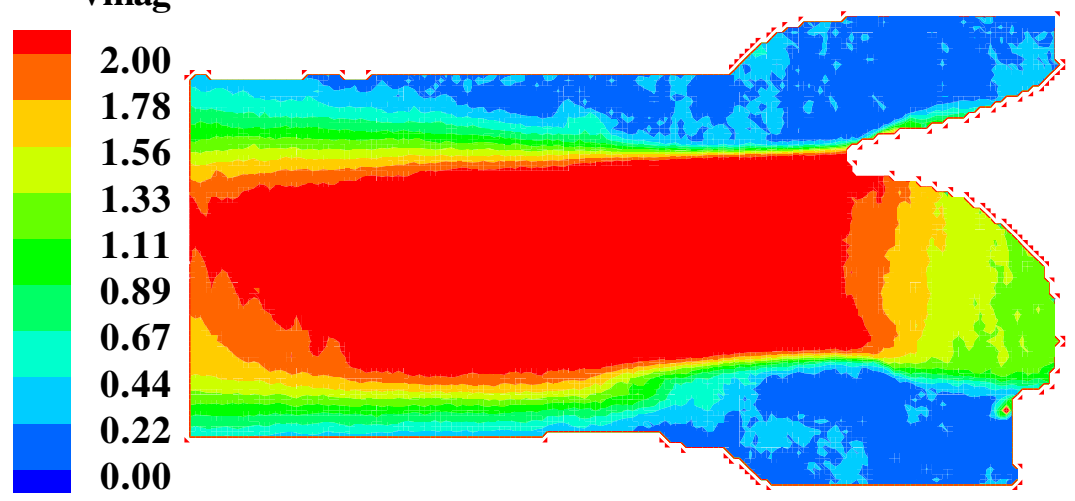

Figure 7-147 Velocity fields at the $+4 \mathrm{~mm}$ offset plane of prototype A during peak systole, time bin 9; a maximum velocity of $2.4 \mathrm{~m} / \mathrm{s}$ was observed inside the central orifice jet at a location coinciding with the contraction of flow $20 \mathrm{~mm}$ from the valve sewing ring (avi, 623k, A_+4mm_plane_vmag.avi) 


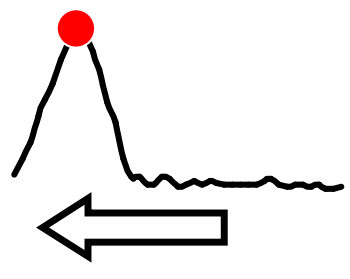

Forward flow

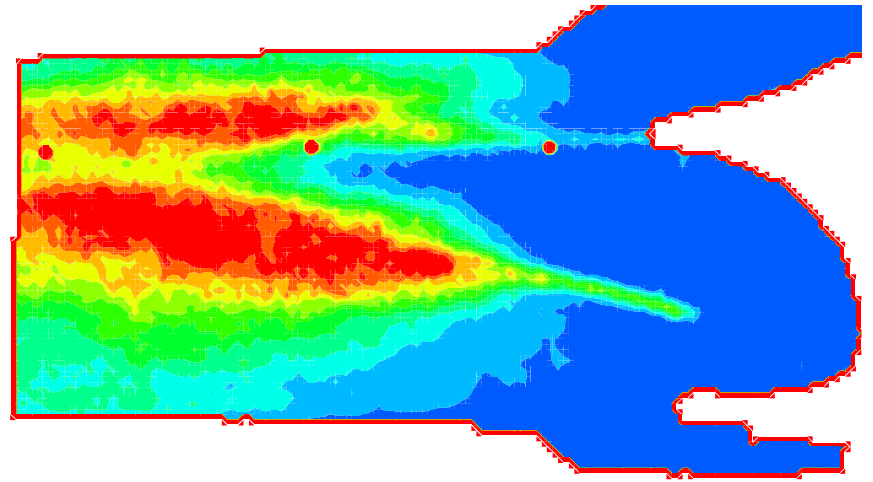

Figure 7-148 Reynolds shear stress contour plot of the $+4 \mathrm{~mm}$ offset plane of prototype A during peak systole, time bin 9; high Reynolds shear stress values coincided with regions of high velocity gradient typically observed at the edge of the central orifice jet and at the distal part of the flow chamber (avi, 652k, A_+4mm_plane_rss.avi)
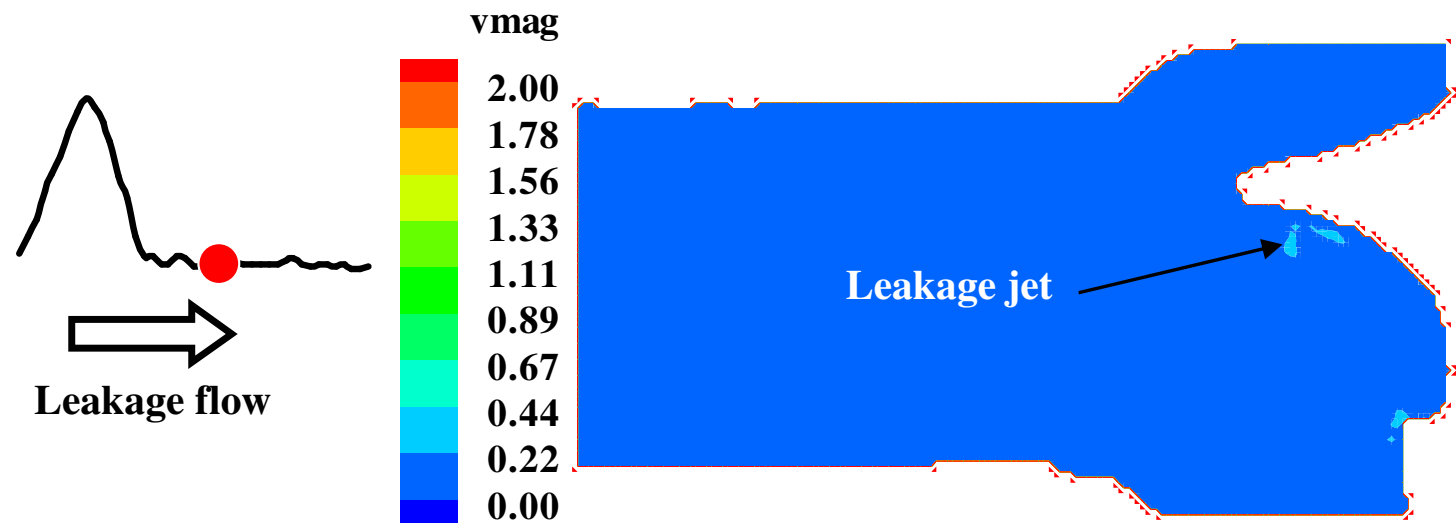

Figure 7-149 Velocity fields at the $+4 \mathrm{~mm}$ offset plane of prototype A during mid diastole, time bin 21; leakage jet appeared at the near the coaptation region of the valve (avi, 623k, A_+4mm_plane_vmag.avi) 


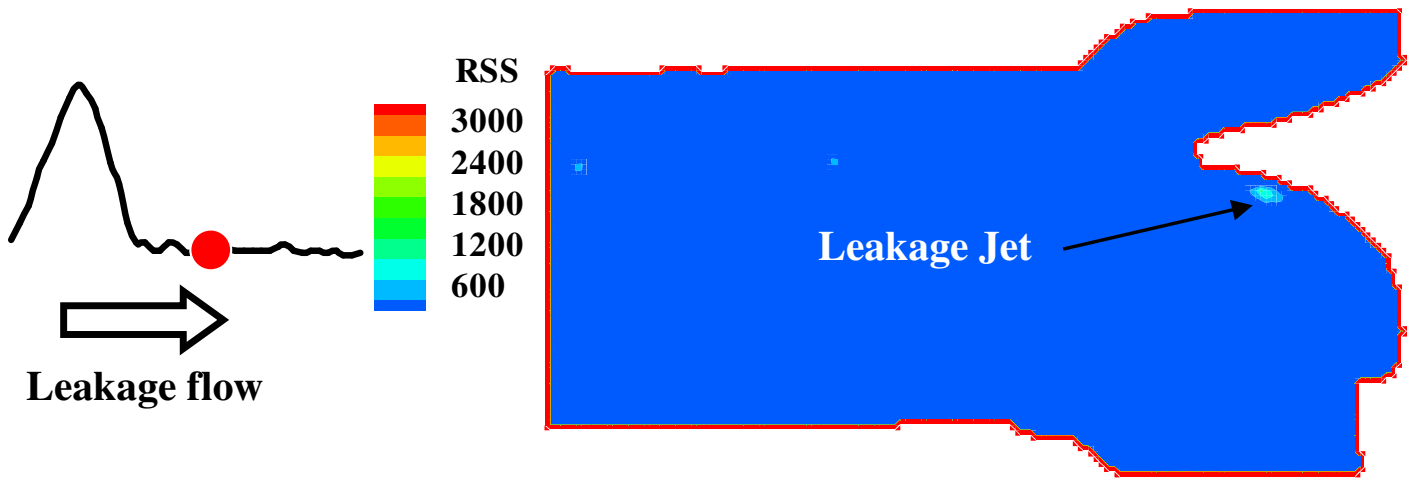

Figure 7-150 Reynolds shear stress contour plot of the $+4 \mathrm{~mm}$ offset plane of prototype A during mid diastole, time bin 21; elevated Reynolds shear stress of more than 1,000 dynes $/ \mathrm{cm}^{2}$ was observed in the leakage jet inside the valve (avi, 652k, A_+4mm_plane_rss.avi)

\subsubsection{3 $\pm 8 m m$ Plane}

Animations A_+8mm_plane_vmag.avi (A_-8mm_plane_vmag.avi) and A_+8mm_plane_rss.avi (A_-8mm_plane_rss.avi) show the velocity magnitude (m/s) and Reynolds shear stress $\left(\right.$ dynes $/ \mathrm{cm}^{2}$ ) contour plots for the two $8 \mathrm{~mm}$ offset measurement planes of prototype A throughout the cardiac cycle, respectively. Refer to Appendix $\mathrm{H}$ for the list of animations. The flow patterns in the two offset measurement planes were similar, hence only flow fields of one offset measurement plane are shown.

\section{Systole}

Figure 7-151 shows the flow field for $+8 \mathrm{~mm}$ offset plane of prototype A during the acceleration phase (time bin 5). The central orifice jet at the $+8 \mathrm{~mm}$ offset plane appeared to emanate from the commissural region of the valve (refer to Figure 5-25 for the position of $8 \mathrm{~mm}$ offset measurement planes). A velocity of $0.8 \mathrm{~m} / \mathrm{s}$ was observed in the central orifice jet in the middle of the chamber approximately $25 \mathrm{~mm}$ downstream from the valve 
sewing ring. At peak systole (time bin 9, Figure 7-152), the flow in the central orifice jet reached a maximum velocity of $2.1 \mathrm{~m} / \mathrm{s}$. The velocity of the central orifice jet decelerated to approximately $1.6 \mathrm{~m} / \mathrm{s} 55 \mathrm{~mm}$ downstream from the valve sewing ring at which point it mixed with surrounding fluid. The flow separation occurred at the trailing edge of the leaflet, while the reattachment points occurred $27 \mathrm{~mm}$ and $50 \mathrm{~mm}$ downstream from the valve sewing ring in the upper and lower parts of the flow chamber, respectively. The velocity inside the valve reached a maximum value of $2.0 \mathrm{~m} / \mathrm{s}$ near the trailing edge of the leaflet at peak systole, while the flow inside the valve closer to the rear of the valve reached a maximum velocity of $1.2 \mathrm{~m} / \mathrm{s}$. During the deceleration phase (time bin 14 ), the velocity of the central orifice jet decelerated to $0.7 \mathrm{~m} / \mathrm{s}$, while the flow inside the valve to a velocity of $0.8 \mathrm{~m} / \mathrm{s}$. Reynolds shear stress values ranging between 1,000 to 3,000 dynes $/ \mathrm{cm}^{2}$ were observed $35 \mathrm{~mm}$ downstream from the valve sewing ring at peak systole. The Reynolds shear stress levels inside the valve along the trailing edge of the leaflet were approximately 800 dynes $/ \mathrm{cm}^{2}$ (time bin 9, Figure 7-153). The Reynolds shear stress inside the sinus region was close to zero during systole.

\section{$\underline{\text { Diastole }}$}

Figure 7-154 shows the flow field of the $+8 \mathrm{~mm}$ offset plane of prototype A during mid diastole (time bin 21). The flow velocity downstream from the trailing edge of the leaflet during diastole was typically less than $0.01 \mathrm{~m} / \mathrm{s}$. No vortices were observed in the sinus region, which had velocities of typically less than $0.01 \mathrm{~m} / \mathrm{s}$. A leakage jet with a velocity of $0.3 \mathrm{~m} / \mathrm{s}$ persisted throughout diastole near the top stent post inside the valve close to the commissural region. The Reynolds shear stress levels throughout the investigated 
region during diastole were typically close to zero, except for the leakage jet inside the valve, which had a Reynolds shear stress value of approximately 300 dynes $/ \mathrm{cm}^{2}$.

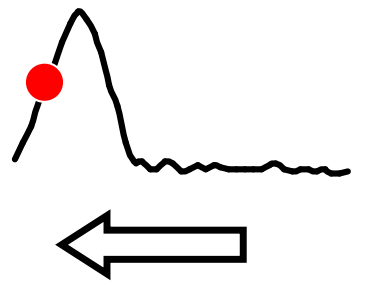

Forward flow

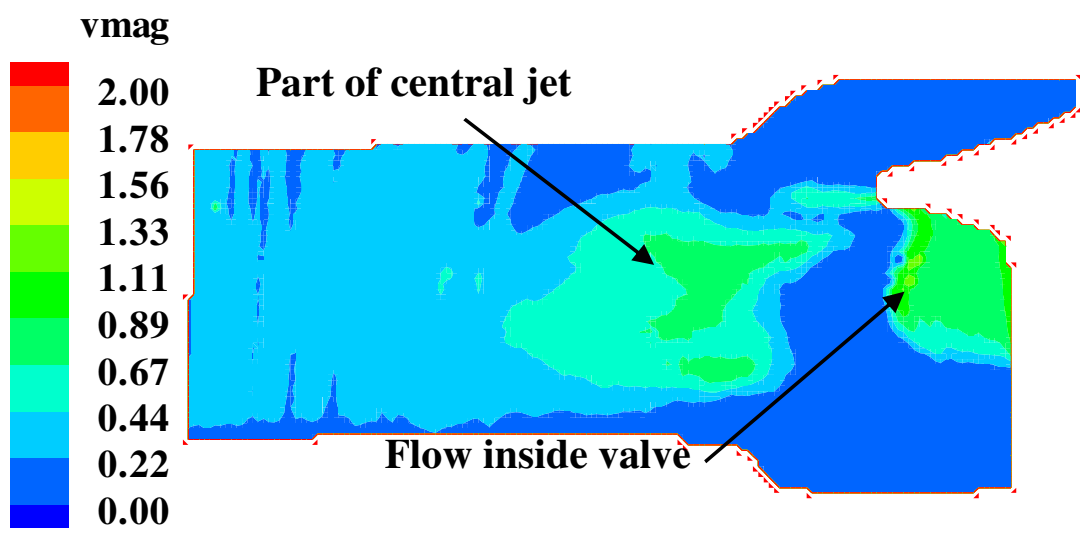

Figure 7-151 Velocity fields at the $+8 \mathrm{~mm}$ offset plane of prototype A during the acceleration phase, time bin 5; part of the central orifice jet downstream of the valve and that inside the valve were visible at this offset plane (avi, 602k, A_+8mm_plane_vmag.avi)

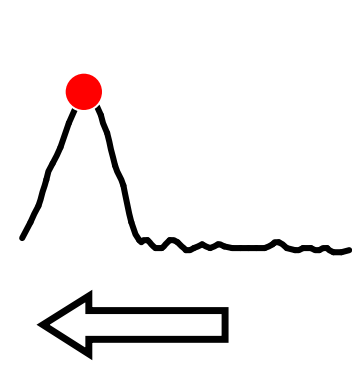

Forward flow

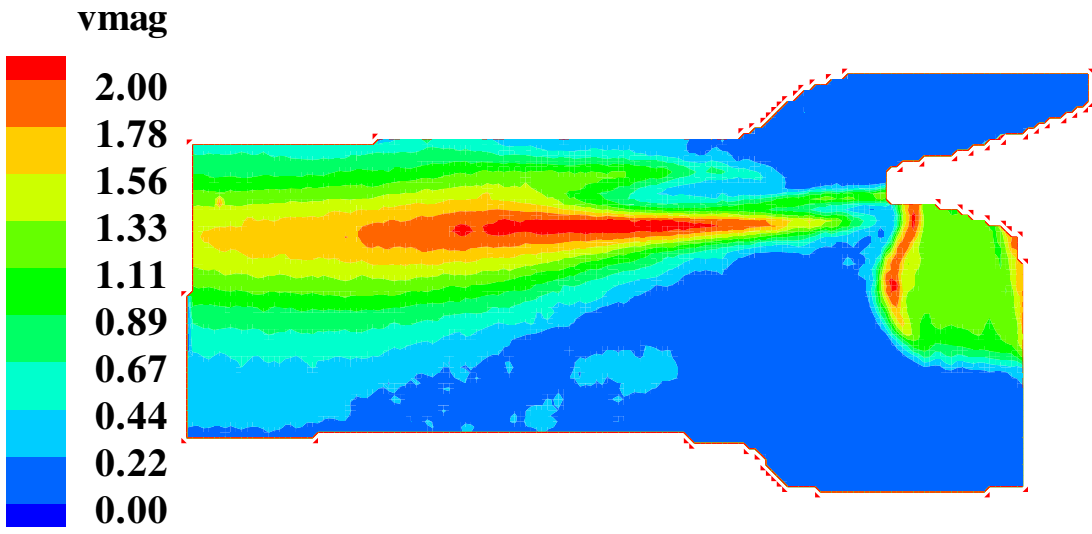

Figure 7-152 Velocity fields at the $+8 \mathrm{~mm}$ offset plane of prototype A during peak systole, time bin 9; the central orifice jet reached the velocity of $2.1 \mathrm{~m} / \mathrm{s}$ at peak systole. Elevated velocity of $2.0 \mathrm{~m} / \mathrm{s}$ was also observed inside the valve near the trailing edge of the leaflet (avi, 602k, A_+8mm_plane_vmag.avi) 


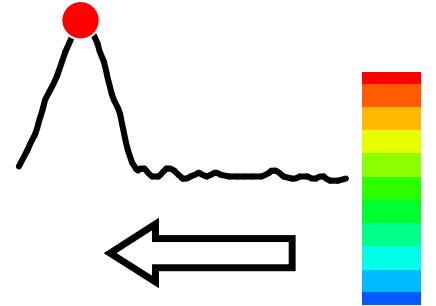

Forward flow
RSS
3000
2400
1800
1200
600

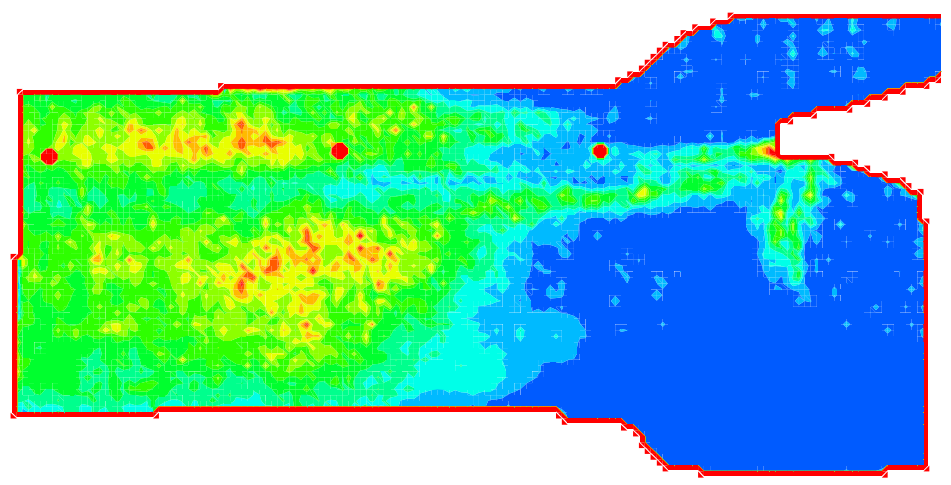

Figure 7-153 Reynolds shear stress contour plot of the $+8 \mathrm{~mm}$ offset plane of prototype A during peak systole, time bin 9; high Reynolds shear stress values coincided with regions of high velocity gradient typically observed at the edge of the central orifice jet (avi, 666k, A_+8mm_plane_rss.avi)

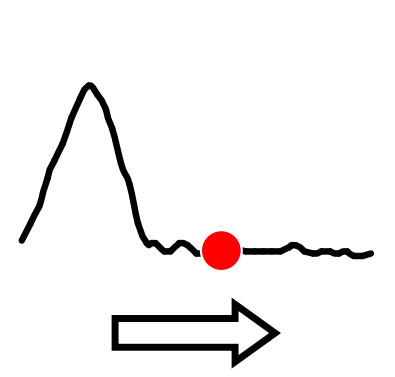

Leakage flow

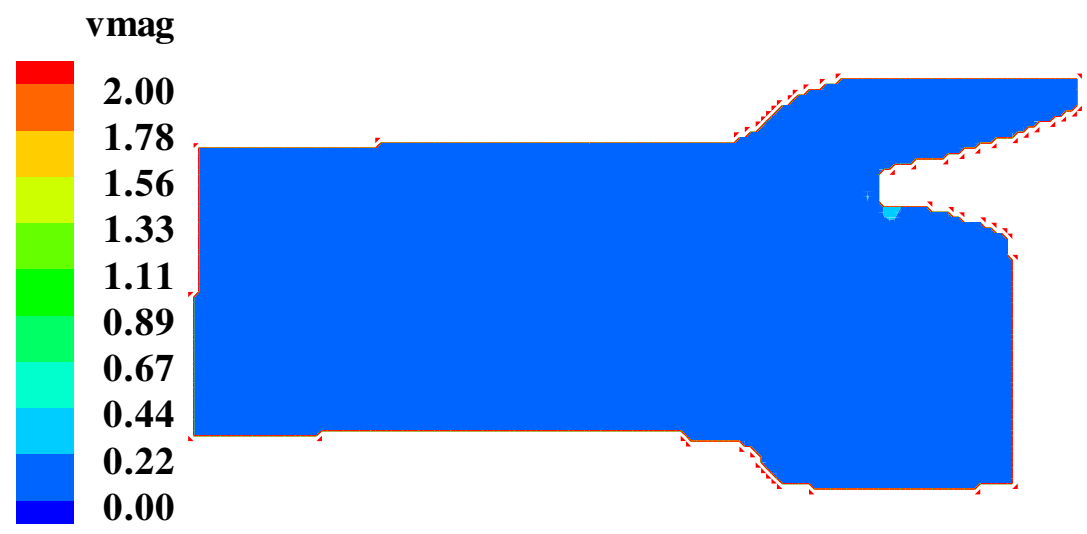

Figure 7-154 Velocity fields at the $+8 \mathrm{~mm}$ offset plane of prototype A during mid diastole, time bin 21; a small leakage jet of $0.3 \mathrm{~m} / \mathrm{s}$ was observed near the top stent post of the valve and persisted throughout the diastole (avi, 602k, A_+8mm_plane_vmag.avi)

\subsubsection{4 $\pm 12 \mathrm{~mm}$ Plane}

Animations A_+12mm_plane_vmag.avi (A_-12mm_plane_vmag.avi) and A_+12mm_plane_rss.avi (A_-12mm_plane_rss.avi) show the velocity magnitude (m/s) and Reynolds shear stress (dynes $/ \mathrm{cm}^{2}$ ) contour plots for the $12 \mathrm{~mm}$ offset measurement planes of prototype A throughout the cardiac cycle, respectively. Refer to Appendix $\mathrm{H}$ for 
the list of animations. The flow patterns in the two offset measurement planes were similar, hence only flow fields of one offset measurement plane are shown.

\section{Systole}

Figure 7-155 shows the flow field for the $+12 \mathrm{~mm}$ offset plane of prototype A during the acceleration phase (time bin 5). The central orifice jet was not visible at this measurement plane during the acceleration phase. The $+12 \mathrm{~mm}$ offset measurement plane captured flow characteristics outside the valve in the sinus region but not inside the valve. The velocity throughout the measurement plane was less than $0.03 \mathrm{~m} / \mathrm{s}$ except in the region 45 $\mathrm{mm}$ downstream from the valve sewing ring, which had a velocity of $0.3 \mathrm{~m} / \mathrm{s}$. Figure 7-156 shows the flow field characteristics of the $+12 \mathrm{~mm}$ offset plane at peak systole (time bin 14). During this time phase, a vortex structure was formed between the forward central orifice jet $(0.6 \mathrm{~m} / \mathrm{s})$ and a reverse flow region $(0.5 \mathrm{~m} / \mathrm{s}) 35 \mathrm{~mm}$ from the valve sewing ring. This vortex structure persisted into the deceleration phase. No vortices were seen in the sinus region during systole. The flow patterns during the deceleration phase (time bin 14) were similar to those observed at peak systole; however, the velocity in the forward central orifice jet had decelerated to $0.2 \mathrm{~m} / \mathrm{s}$, while the reverse flow to a velocity of $0.3 \mathrm{~m} / \mathrm{s}$. Reynolds shear stress levels ranging between 1,000 to 3,000 dynes $/ \mathrm{cm}^{2}$ were observed $45 \mathrm{~mm}$ downstream from the valve sewing ring during peak systole (time bin 9 , Figure 7-157). This location is characterized by turbulence mixing between the central jet and the surrounding fluid. 


\section{$\underline{\text { Diastole }}$}

Figure 7-158 (time bin 21) shows the flow field for the $+12 \mathrm{~mm}$ offset plane of prototype A during mid diastole (time bin 21). The velocity throughout the measurement plane was typically less than $0.04 \mathrm{~m} / \mathrm{s}$. A vortex was observed at the edge of the sinus region persisting throughout diastole. The Reynolds shear stress levels of the measurement plane during diastole were typically less than 40 dynes $/ \mathrm{cm}^{2}$.

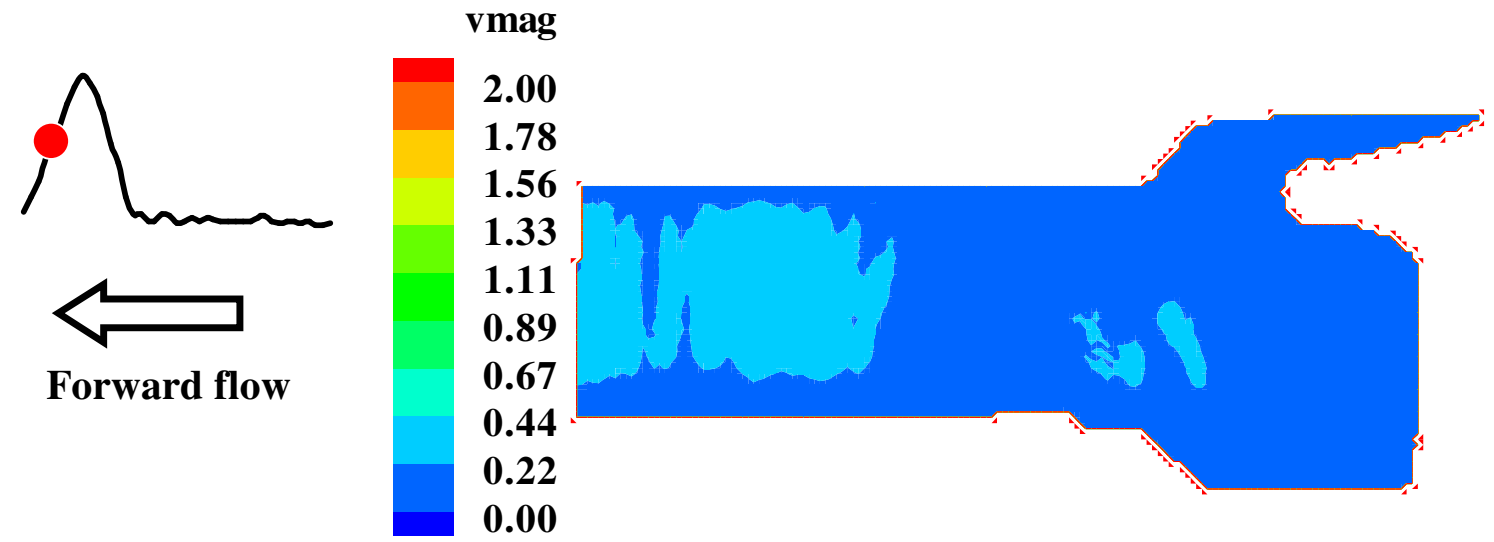

Figure 7-155 Velocity fields at the $+12 \mathrm{~mm}$ offset plane of prototype A during the acceleration phase, time bin 5; flow velocity downstream from the leaflet trailing edge was typically less than $0.03 \mathrm{~m} / \mathrm{s}$ during the acceleration phase (avi, 569k, A_+12mm_plane_vmag.avi) 


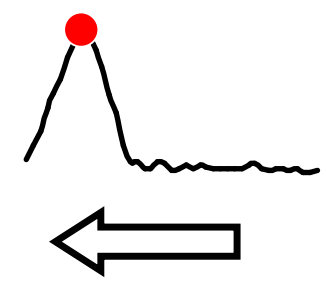

Forward flow

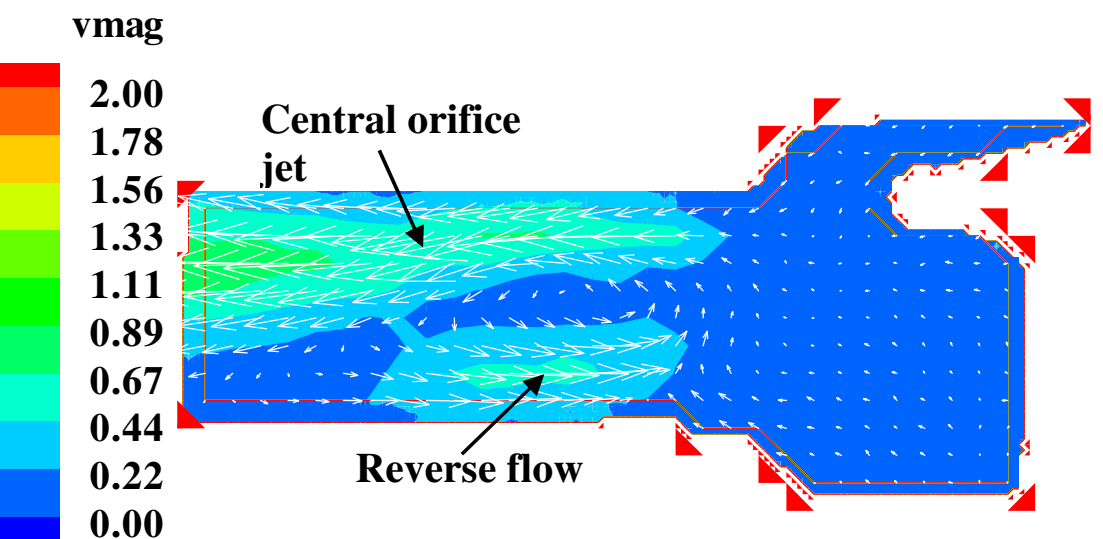

0.00

Figure 7-156 Velocity fields at the $+12 \mathrm{~mm}$ offset plane of prototype A during peak systole, time bin 9; the central orifice jet of $0.75 \mathrm{~m} / \mathrm{s}$ was observed at location $35 \mathrm{~mm}$ downstream from the valve sewing ring (avi, 569k, A_+12mm_plane_vmag.avi)

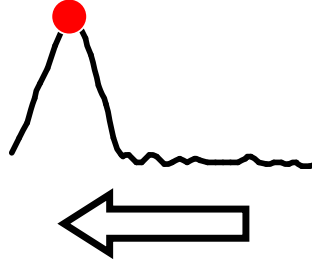

Forward flow
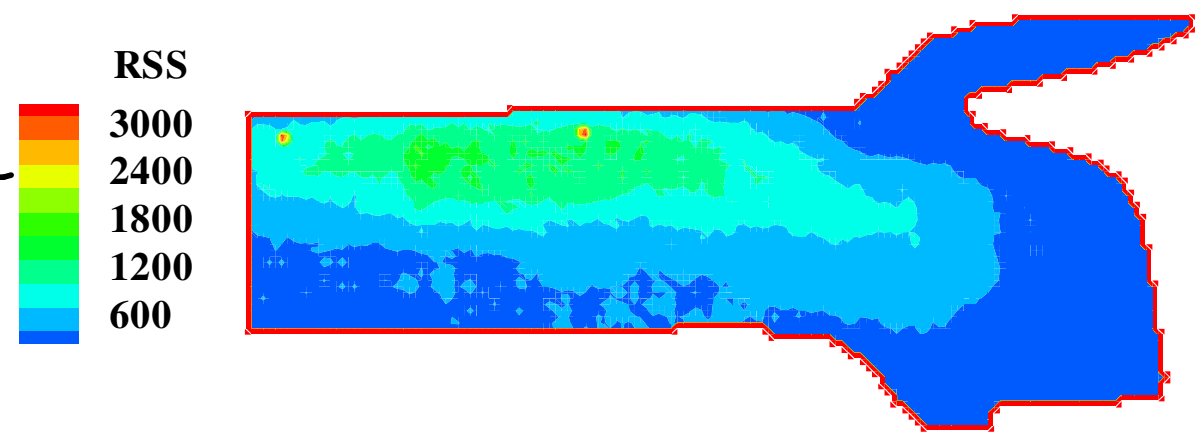

Figure 7-157 Reynolds shear stress contour plot of the $+12 \mathrm{~mm}$ offset plane of prototype A peak systole, time bin 9; elevated Reynolds shear stress was observed $45 \mathrm{~mm}$ downstream of the valve sewing ring at the location where the central orifice jet mix with the surrounding flow (avi, 663k, A_+12mm_plane_rss.avi) 


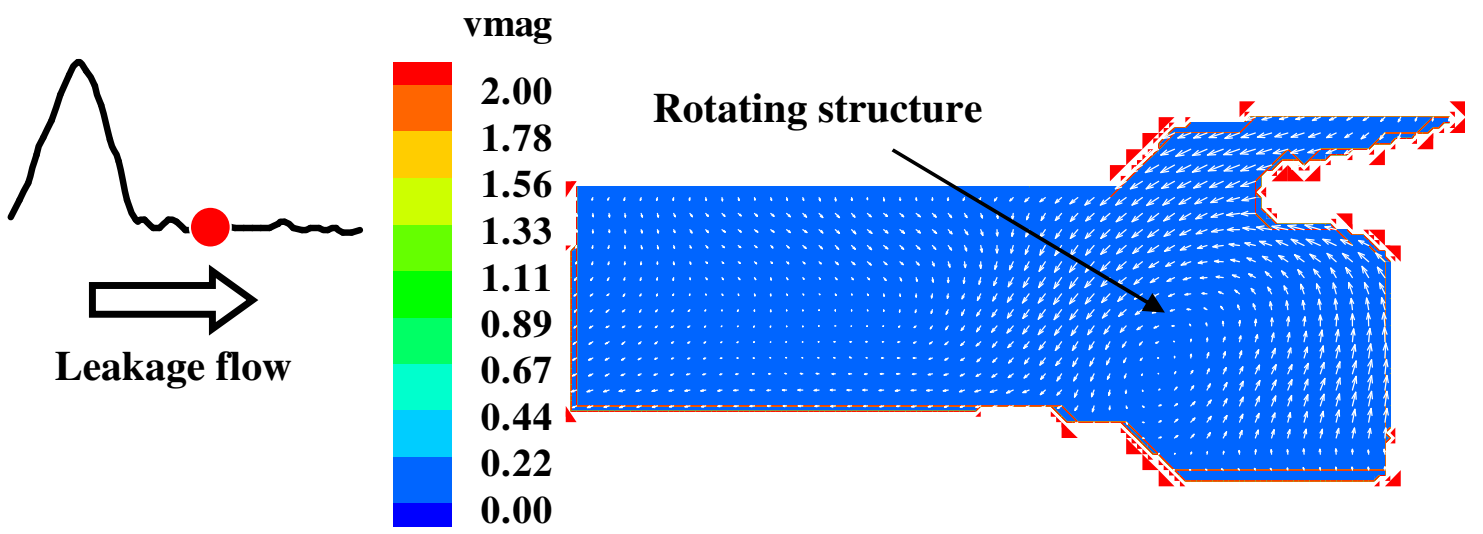

Figure 7-158 Velocity fields at the $+12 \mathrm{~mm}$ offset plane of prototype A during mid diastole, time bin 21; vortex structure was observed inside the edge of the sinus region throughout the diastole (avi, 569k, A_+12mm_plane_vmag.avi)

\subsubsection{Open Commissure Valve With $120 \mu$ Leaflet Thickness (Prototype B)}

\subsection{Center Plane}

Animations B_center_plane_vmag.avi and B_center_plane_rss.avi show the velocity magnitude $(\mathrm{m} / \mathrm{s})$ and Reynolds shear stress $\left(\right.$ dynes $\left./ \mathrm{cm}^{2}\right)$ contour plots for the center measurement plane of prototype B throughout the cardiac cycle, respectively. Animation B_isosurface_vmag.avi shows the 3D reconstructed iso-surface of the velocity magnitude of prototype B throughout the cardiac cycle. Refer to Appendix $\mathrm{H}$ for the list of animations. Refer to Figure 7-138 for the terminology pertinent to the following description of the prototype $B$ results.

\section{$\underline{\text { Systole }}$}

Figure 7-159 shows the center plane flow field downstream of prototype B during the acceleration phase (time bin 5). The flow characteristics during the accelerating phase were similar to those seen in prototype A. A velocity of $1.5 \mathrm{~m} / \mathrm{s}$ was observed inside the 
central orifice jet with the formation of a vortex ring along the edge of the central jet. At peak systole (time bin 9), the central orifice jet reached a maximum velocity of $2.1 \mathrm{~m} / \mathrm{s}$ (Figure 7-160). The central jet had a diameter of about $16 \mathrm{~mm}$. A vena contracta was visible at a location $20 \mathrm{~mm}$ downstream from the valve sewing ring. The velocity flow profile of the central orifice jet in prototype B was similar to that seen in prototype A. The central jet exiting from the valve had a flat profile, which became parabolic at a distance $50 \mathrm{~mm}$ from the valve sewing ring, at which point the central jet velocity decelerated to approximately $1.6 \mathrm{~m} / \mathrm{s}$. Figure $7-161$ shows the iso-surface of the velocity magnitude downstream of valve B at peak systole (time bin 9). Prototype B displayed the same three lobe flow profile that was observed with prototype A; however, prototype B had a slightly lower forward flow jet than prototype A. Flow separation was observed at the trailing edge of the leaflet, while the reattachment points occurred $53 \mathrm{~mm}$ and $58 \mathrm{~mm}$ downstream from the valve sewing ring in the upper and lower parts of the flow chamber, respectively. A velocity of $0.5 \mathrm{~m} / \mathrm{s}$ was seen inside the recirculation zone. Vortex structures were not observed in the sinus region, which had a velocity of generally less than $0.07 \mathrm{~m} / \mathrm{s}$ during systole. During the deceleration phase (time bin 14), the velocity in the central orifice jet decelerated from $2.1 \mathrm{~m} / \mathrm{s}$ to $1.0 \mathrm{~m} / \mathrm{s}$. The velocity of the recirculation zone in the upper part of flow chamber decelerated from $0.5 \mathrm{~m} / \mathrm{s}$ to $0.4 \mathrm{~m} / \mathrm{s}$. The flow patterns inside prototype B during systole were similar to those observed in prototype A. An elevated velocity of more than $2.0 \mathrm{~m} / \mathrm{s}$ was typically observed along the trailing edge of the leaflet at peak systole, while the velocity along the stent inflow region was $0.5 \mathrm{~m} / \mathrm{s}$. 
The Reynolds shear stress distribution observed in prototype B at peak systole was similar to that observed in prototype A (time bin 9, Figure 7-162). Reynolds shear stress values ranging between 500 and 3,000 dynes $/ \mathrm{cm}^{2}$ were observed along the edge of the central orifice jet and at the commissure region during systole.

\section{Diastole}

Figure 7-163 shows the center plane flow field downstream of prototype B during mid diastole (time bin 21). The flow velocity downstream of the leaflet trailing edge was typically less than $0.04 \mathrm{~m} / \mathrm{s}$. The general flow features inside prototype B during diastole were similar to those observed in prototype A. A leakage jet of $2.0 \mathrm{~m} / \mathrm{s}$ was observed at the coaptation region inside the valve during valve closure that persisted throughout diastole fluctuating between 0.6 and $2.0 \mathrm{~m} / \mathrm{s}$ as a result of the valve's oscillation. Flow inside prototype $\mathrm{B}$ was directed toward both the coaptation and commissural regions of the valve during diastole with a velocity of approximately $0.10 \mathrm{~m} / \mathrm{s}$. A leakage jet of 0.73 $\mathrm{m} / \mathrm{s}$, not seen in prototype A, was occasionally observed at the commissural region of the valve. Reynolds shear stress values of more than 5,000 dynes $/ \mathrm{cm}^{2}$ were observed in the proximity of the leakage jet at the coaptation region as well as at the commissural region inside the valve (Figure 7-164). 

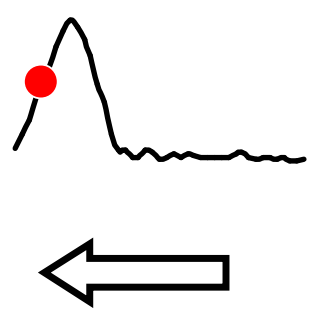

Forward flow vmag

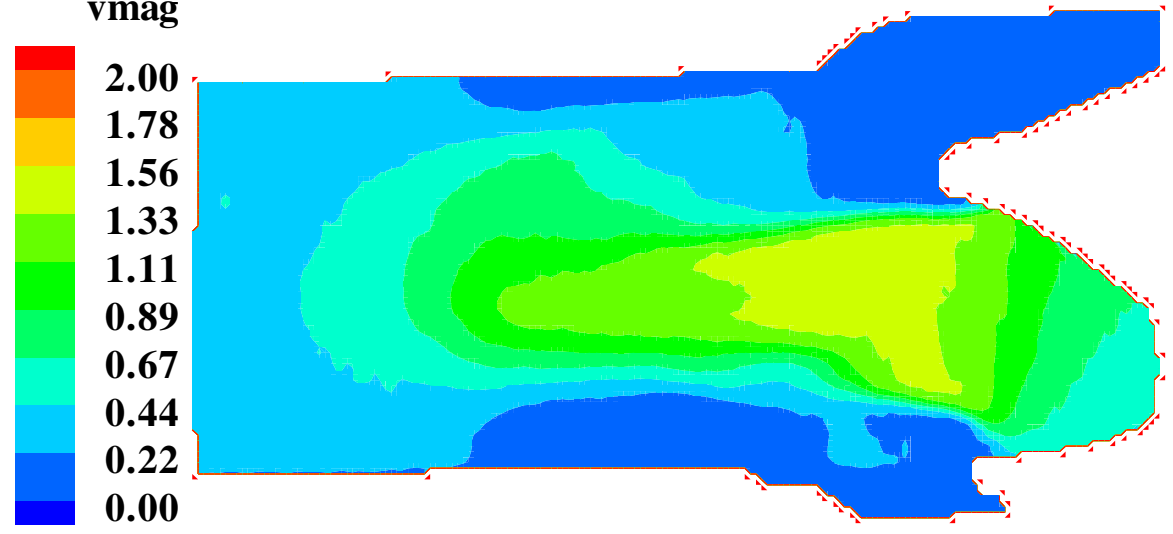

Figure 7-159 Velocity fields at the center plane of prototype B during acceleration phase, time bin 5; central orifice jet of $1.5 \mathrm{~m} / \mathrm{s}$ was observed emanating from the valve orifice. Vortex ring was seen along the edge of the central jet (avi, 594k, B_center_plane_vmag.avi)

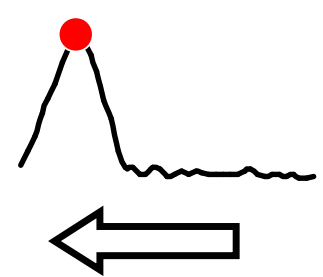

Forward flow

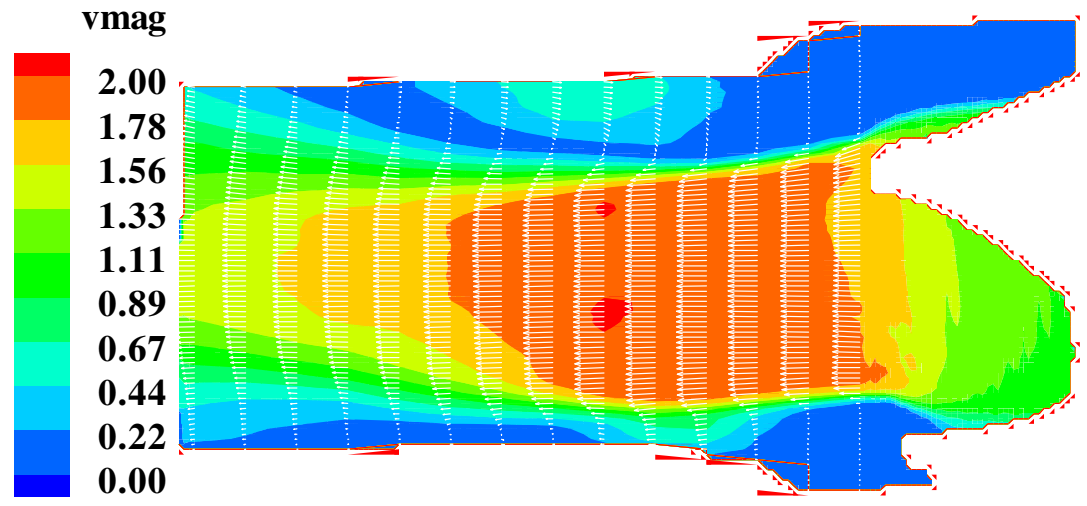

Figure 7-160 Velocity fields at the center plane of prototype B during peak systole, time bin 9; recirculation flow observed at the upper region of the chamber wall (avi, 594k, B_center_plane_vmag.avi) 

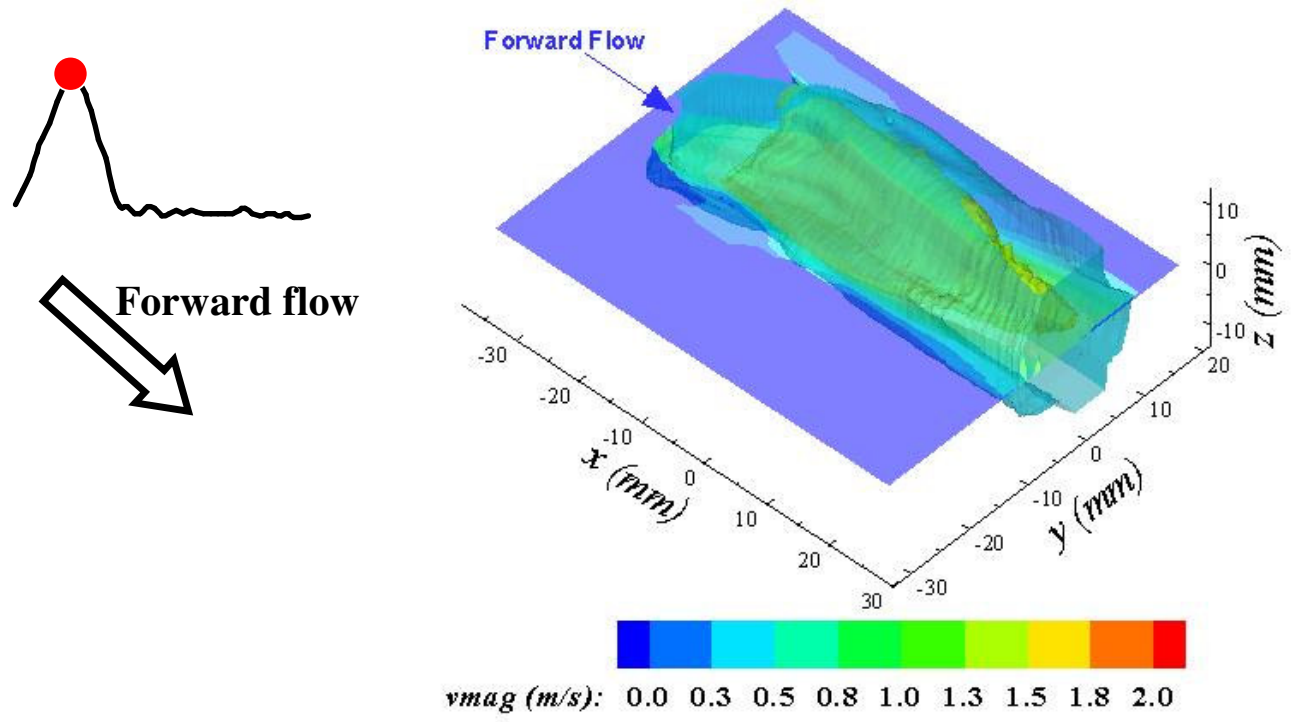

Figure 7-161 Iso-surface of velocity magnitude of prototype B at peak systole, time bin 9 . The iso-surface of velocity had a three lobe configuration (avi, 945k, B_isosurface_vmag.avi)

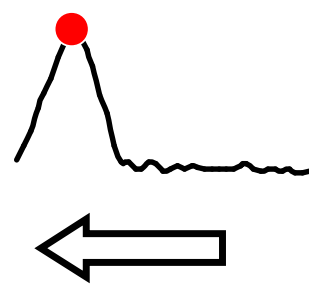

Forward flow
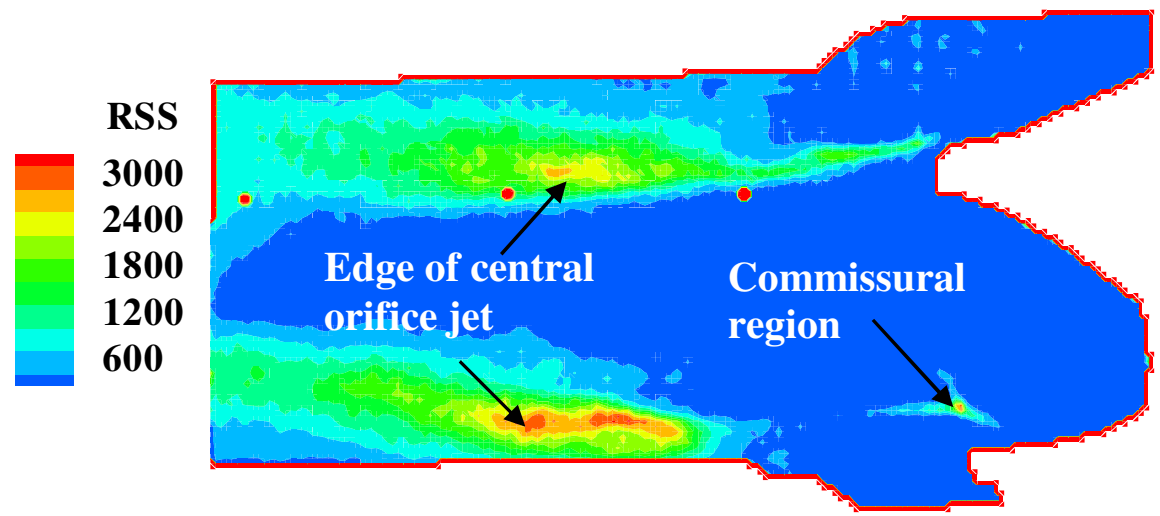

Figure 7-162 Reynolds shear stress contour plot of the center plane of prototype B during peak systole, time bin 9; high Reynolds shear stress values observed along the edge of the central orifice jet and at the commissural region inside the valve (avi, 728k, B_center_plane_rss.avi) 


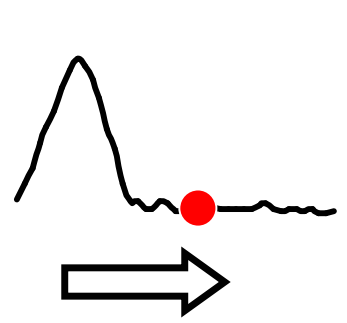

Leakage flow

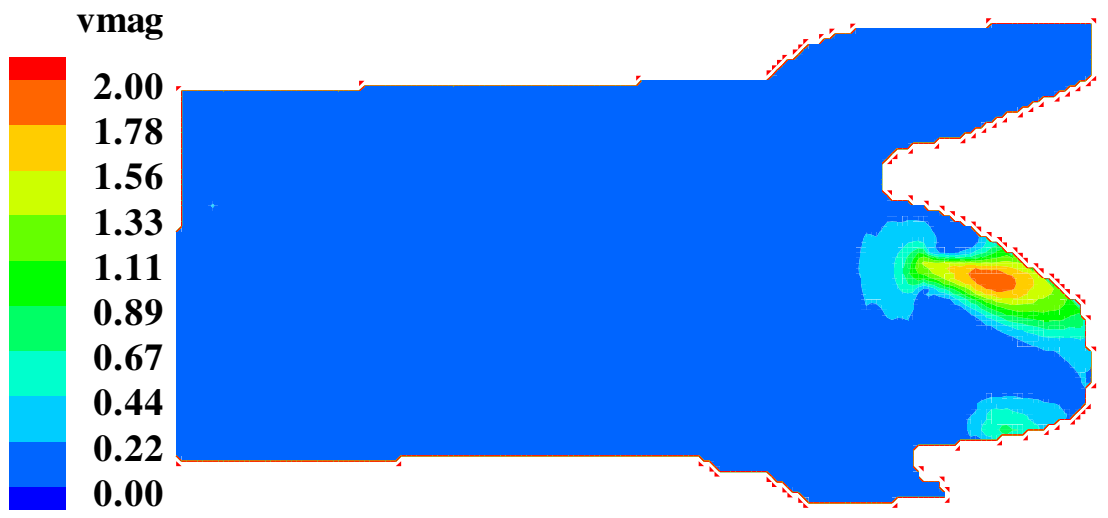

Figure 7-163 Velocity fields at the center plane of prototype B during mid diastole, time bin 21; leakage jets observed at the both the coaptation and commissural regions of the valve (avi, 594k, B_center_plane_vmag.avi)
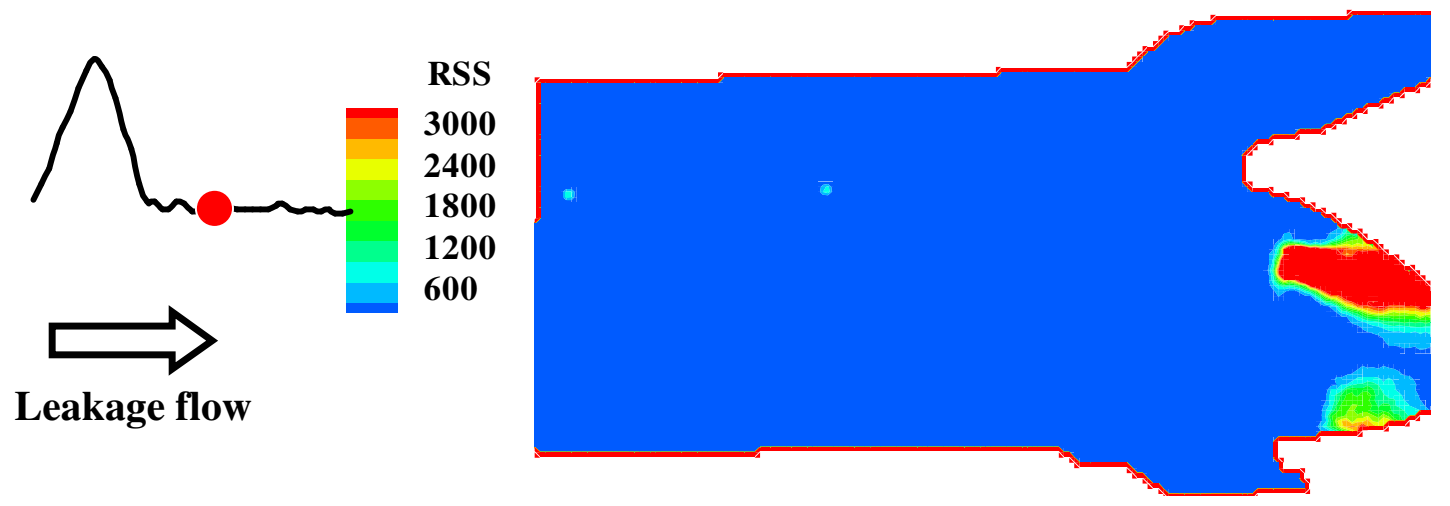

Figure 7-164 Reynolds shear stress contour plot of the center plane of prototype B during mid diastole, time bin 21; elevated Reynolds shear stress levels of more than 5,000 dynes $/ \mathrm{cm}^{2}$ were observed in the leakage jets at the coaptation region and the commissural region (avi, 728k, B_center_plane_rss.avi)

\subsubsection{2 $\pm 4 \mathrm{~mm}$ Plane}

Animations $\quad$ B_+4mm_plane_vmag.avi (B_-4mm_plane_vmag.avi) and B_+4mm_plane_rss.avi (B_-4mm_plane_rss.avi) show the velocity magnitude $(\mathrm{m} / \mathrm{s})$ and Reynolds shear stress $\left(\right.$ dynes $/ \mathrm{cm}^{2}$ ) contour plots for the $4 \mathrm{~mm}$ offset measurement planes 
of prototype B throughout the cardiac cycle, respectively. Refer to Appendix $\mathrm{H}$ for the list of animations. The flow patterns in the two offset measurement planes were similar, hence only flow fields of one offset measurement plane are shown.

\section{Systole}

Figure 7-165 shows the $+4 \mathrm{~mm}$ offset plane downstream from the leaflet trailing edge of prototype B during the acceleration phase (time bin 5). A velocity of $1.4 \mathrm{~m} / \mathrm{s}$ was reached inside the central orifice jet and a vortex ring was observed along the edge of the central jet. The separation and re-attachment points were observed, respectively, at the trailing edge of the leaflet and $45 \mathrm{~mm}$ downstream from the valve sewing ring. The flow inside the recirculation zone reached a velocity of approximately $0.13 \mathrm{~m} / \mathrm{s}$. At peak systole, the central orifice jet accelerated to a velocity of $2.1 \mathrm{~m} / \mathrm{s}$ (time bin 9, Figure 7-166). The central orifice jet had a diameter of approximately $13 \mathrm{~mm}$. The re-attachment point in the upper part of the flow chamber maintained at $45 \mathrm{~mm}$ downstream from the valve sewing ring, while that in the lower part of the flow chamber appeared to have shifted farther downstream beyond the measurement plane. The flow velocity inside the recirculation zone fluctuated between 0.08 and $0.14 \mathrm{~m} / \mathrm{s}$. No vortices were visible inside the sinus region. During the deceleration phase (time bin 14), the velocity in the central orifice jet decelerated to $1.0 \mathrm{~m} / \mathrm{s}$ and the flow in the recirculation region to a velocity of $0.3 \mathrm{~m} / \mathrm{s}$. Flow inside the valve was similar to that observed in the center plane during systole. A maximum velocity of $2.0 \mathrm{~m} / \mathrm{s}$ was reached along the trailing edge of the leaflet at peak systole. The flow along the stent inflow region had a velocity of approximately $0.5 \mathrm{~m} / \mathrm{s}$. 
Figure 7-167 shows the Reynolds shear stress distribution for the $4 \mathrm{~mm}$ offset plane of prototype B at peak systole (time bin 9). Reynolds shear stress values ranging from 600 to 2,200 dynes $/ \mathrm{cm}^{2}$ were recorded along the edge of the central orifice jet, at the commissural region, and along the trailing edge of the valve. The Reynolds shear stresses at the sinus region and inside the valve were typically less than 50 dynes $/ \mathrm{cm}^{2}$.

\section{$\underline{\text { Diastole }}$}

Figure 7-168 shows the flow field for the $+4 \mathrm{~mm}$ offset plane of prototype B during mid diastole (time 20). The velocity downstream from the leaflet trailing edge was typically less than $0.07 \mathrm{~m} / \mathrm{s}$. The flow inside the sinus region was less than $0.04 \mathrm{~m} / \mathrm{s}$ with no visible vortex structures. Inside the valve a leakage jet with a velocity of approximately $0.5 \mathrm{~m} / \mathrm{s}$ was visible near the coaptation region of the valve during valve closure. The leakage jet accelerated to a velocity of approximately $0.95 \mathrm{~m} / \mathrm{s}$ at mid diastole (time bin 20, Figure 7-169). Two vortex structures were visible inside the valve: one at the stent near the rear of the valve and another at the top stent post next to the leakage jet. The vortex near the leakage jet had a velocity of approximately $0.95 \mathrm{~m} / \mathrm{s}$, while that at the rear of the valve had a velocity of $0.03 \mathrm{~m} / \mathrm{s}$. These two vortices lasted into late diastole dissipating just prior to the opening of the valve. No flow splitting was seen in the $4 \mathrm{~mm}$ offset plane.

Elevated Reynolds shear stress values $\left(>1,900\right.$ dynes $\left./ \mathrm{cm}^{2}\right)$ were seen mainly in the leakage jet during diastole. The Reynolds shear stresses inside the two vortices were between 80 to 200 dynes $/ \mathrm{cm}^{2}$ (Figure 7-170). 


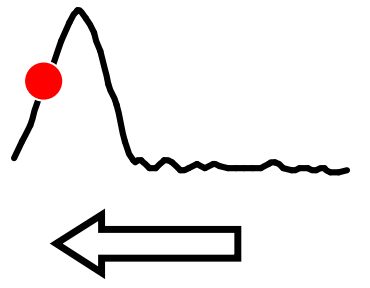

Forward flow

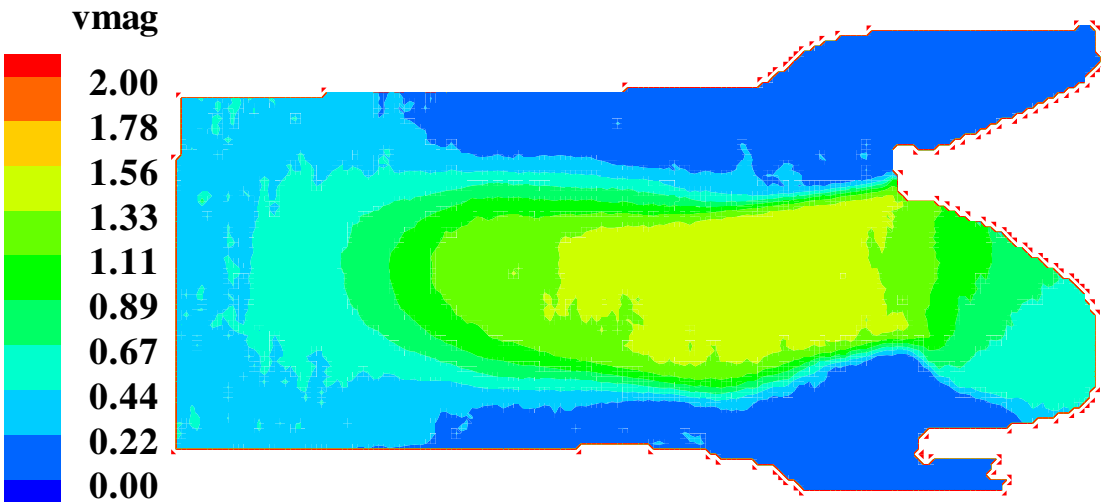

Figure 7-165 Velocity fields at the $+4 \mathrm{~mm}$ offset plane of prototype $\mathrm{B}$ acceleration phase, time bin 5; velocity of $1.4 \mathrm{~m} / \mathrm{s}$ was measured inside the central orifice jet (avi, $602 \mathrm{k}$, B_+4mm_plane_vmag.avi)

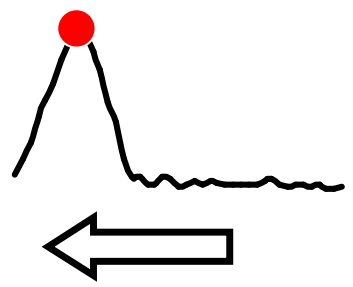

Forward flow

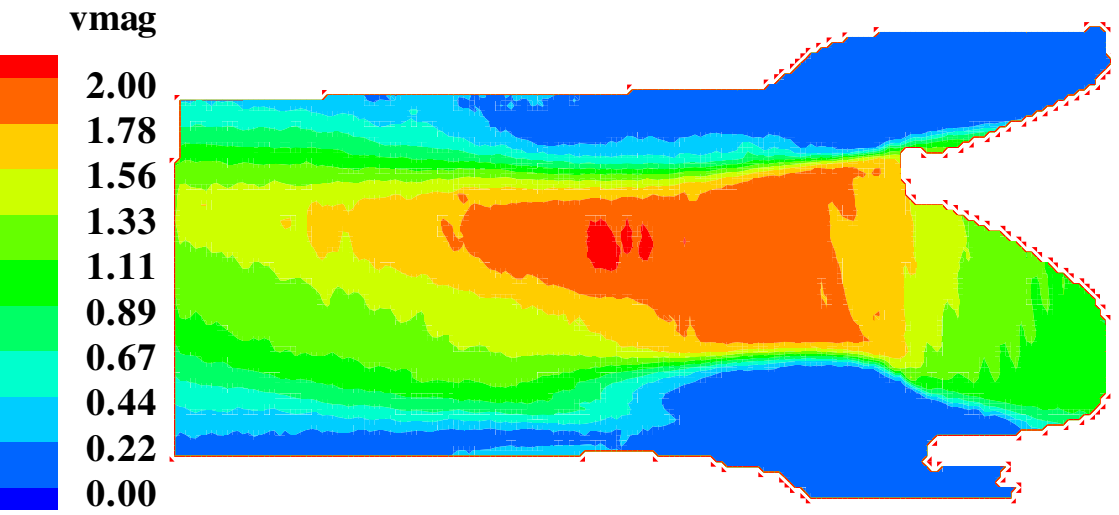

Figure 7-166 Velocity fields at the $+4 \mathrm{~mm}$ offset plane of prototype B during peak systole, time bin 9; maximum flow velocity of $2.1 \mathrm{~m} / \mathrm{s}$ was reached inside the central orifice jet at peak systole (avi, 602k, B_+4mm_plane_vmag.avi) 

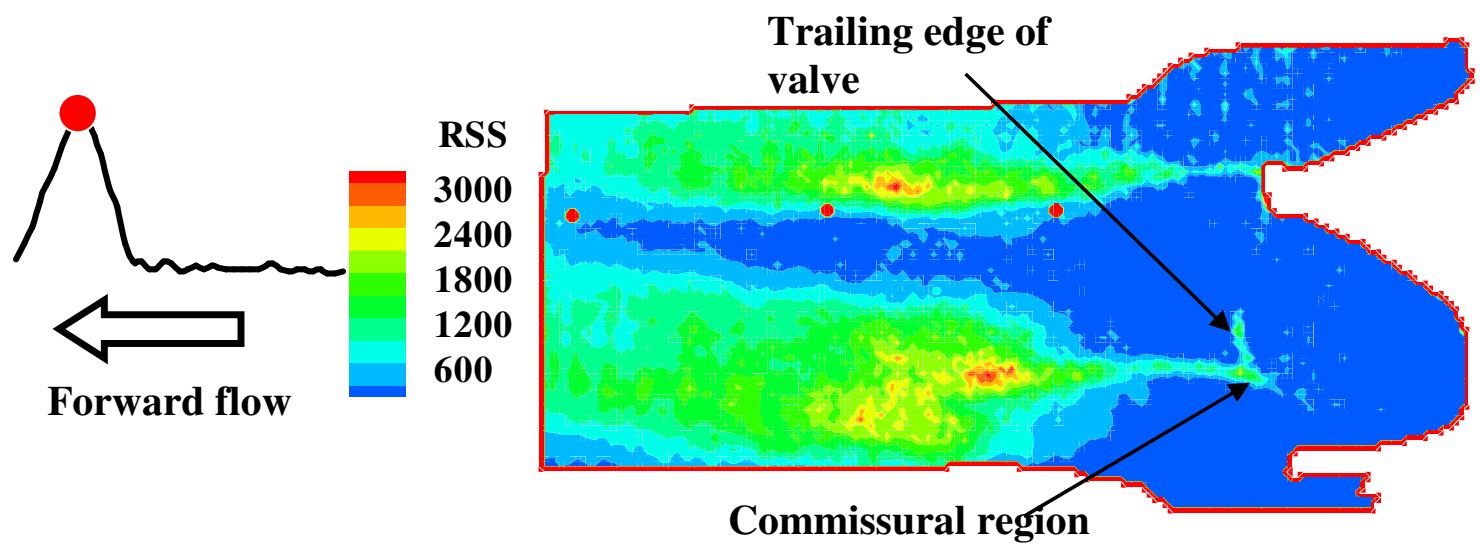

Figure 7-167 Reynolds shear stress contour plot of the $+4 \mathrm{~mm}$ offset plane of prototype B during peak systole, time bin 9; high Reynolds shear stress values observed along the edge of the central orifice jet, the trailing edge of the leaflet, and at the commissural region of the valve (avi, 818k, B_+4mm_plane_rss.avi)

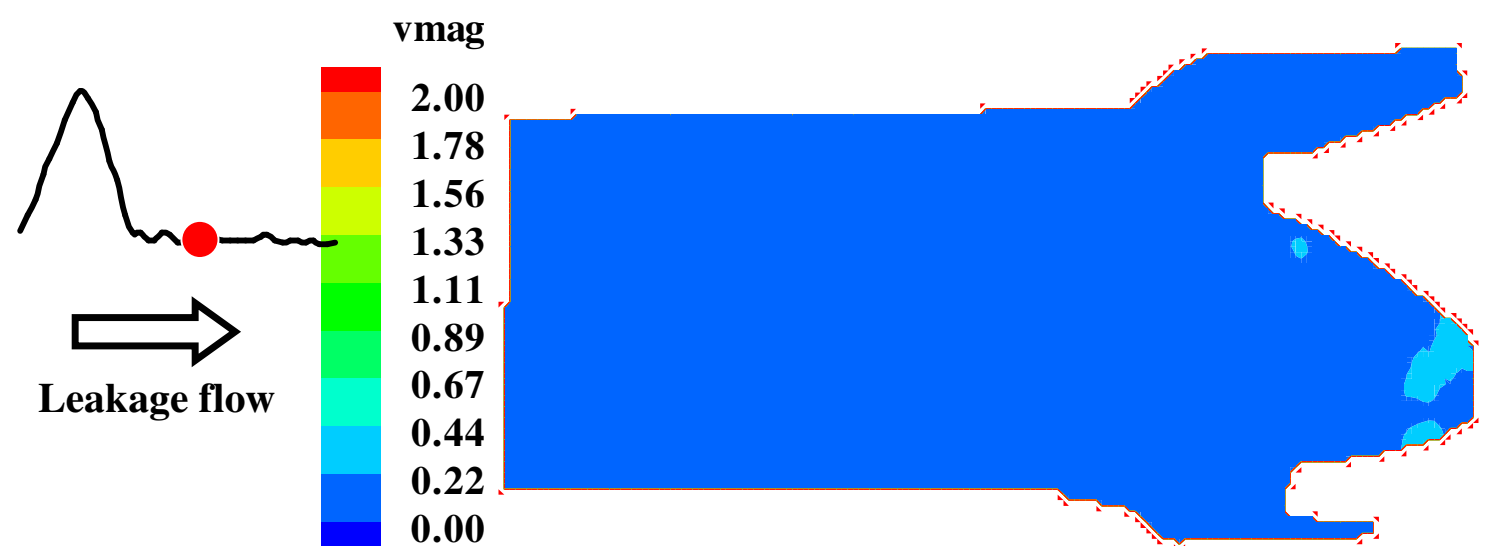

Figure 7-168 Velocity fields at the $+4 \mathrm{~mm}$ offset plane of prototype B during mid diastole, time bin 20; leakage jet of $0.95 \mathrm{~m} / \mathrm{s}$ appeared at the near the coaptation region of the valve (avi, 602k, B_+4mm_plane_vmag.avi) 


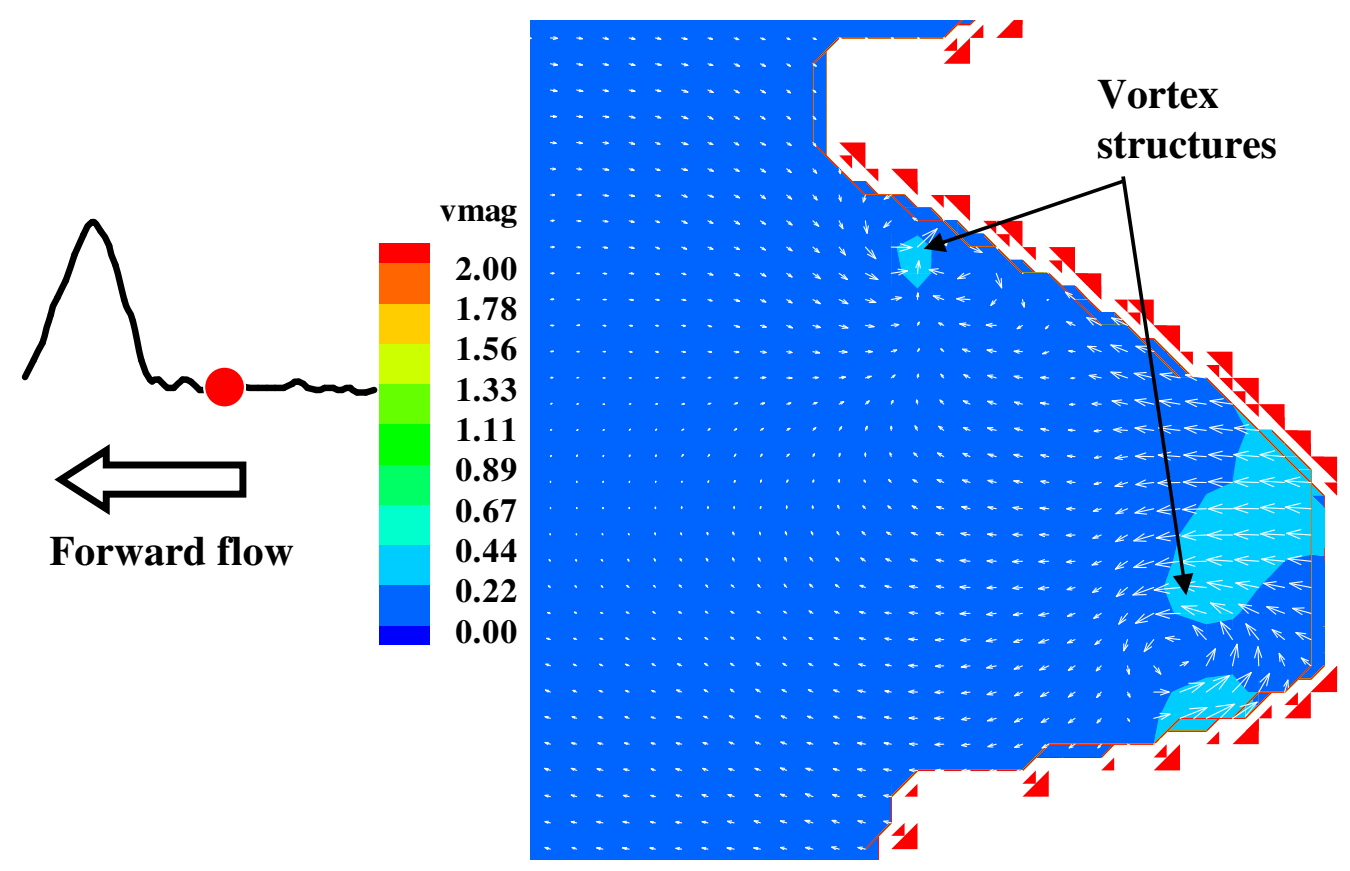

Figure 7-169 Velocity vectors inside prototype B during mid-diastole, time bin 20, +4 $\mathrm{mm}$ offset plane; vortex structures were observed at two location inside the valve; one near the top stent post next to the leakage jet and another near the rear of the valve

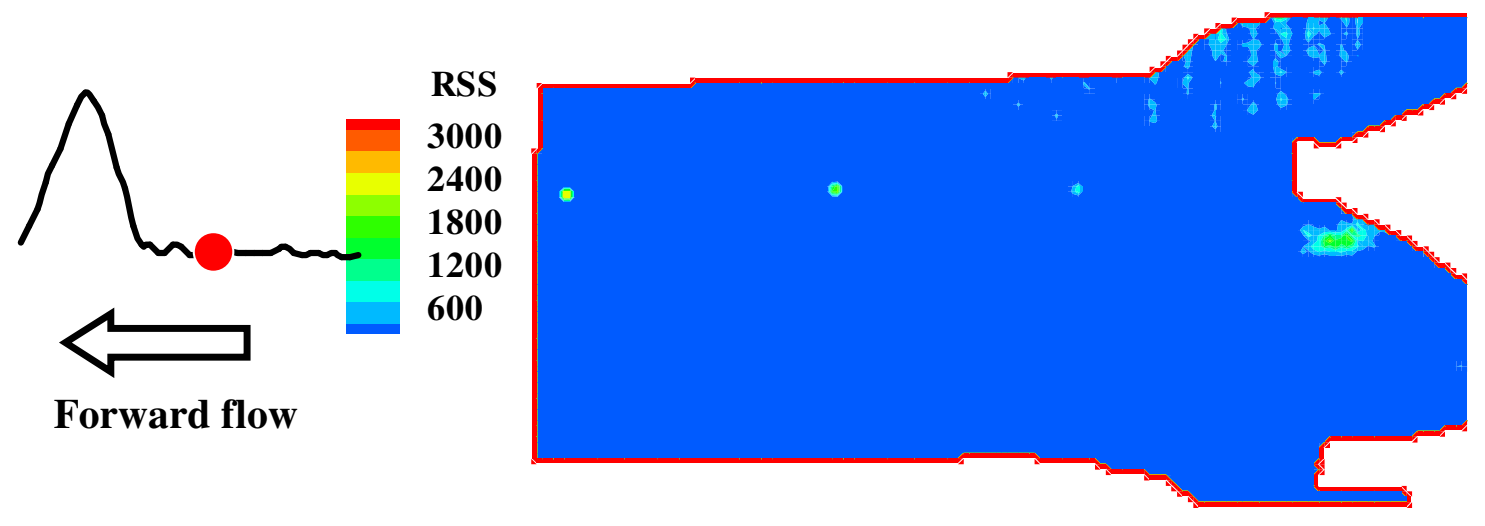

Figure 7-170 Reynolds shear stress contour plot of the $+4 \mathrm{~mm}$ offset plane of prototype B during mid-diastole, time bin 20; elevated Reynolds shear stress levels of more than 1,900 dyne $/ \mathrm{cm}^{2}$ were observed in the leakage jet at the high central region of the valve (avi, 602k, B_+4mm_plane_vmag.avi) 


\subsubsection{3 $\pm 8 \mathrm{~mm}$ Plane}

Animations $\quad$ B_+8mm_plane_vmag.avi (B_-8mm_plane_vmag.avi) and B_+8mm_plane_rss.avi (B_-8mm_plane_rss.avi) show the velocity magnitude $(\mathrm{m} / \mathrm{s})$ and Reynolds shear stress $\left(\right.$ dynes $/ \mathrm{cm}^{2}$ ) contour plots for the $8 \mathrm{~mm}$ offset measurement planes of prototype B throughout the cardiac cycle, respectively. Refer to Appendix $\mathrm{H}$ for the list of animations. The flow patterns in the two offset measurement planes were similar, hence only flow fields of one offset measurement plane are shown.

\section{Systole}

Figure 7-171 shows the flow field for the $+8 \mathrm{~mm}$ offset plane of prototype $\mathrm{B}$ during the acceleration phase (time bin 5). A central orifice jet of approximately $1.5 \mathrm{~m} / \mathrm{s}$ was observed emanating from the valve orifice near the commissural region. A vortex was seen briefly along the upper edge of the central jet, with velocity of $0.05 \mathrm{~m} / \mathrm{s}$. At peak systole (time bin 9, Figure 7-172), the central jet reached a maximum velocity of $2.1 \mathrm{~m} / \mathrm{s}$ $30 \mathrm{~mm}$ downstream from the valve sewing ring. The central jet velocity then decelerated to $1.5 \mathrm{~m} / \mathrm{s} 50 \mathrm{~mm}$ downstream from the valve sewing ring when it mixed with the surrounding fluid $(<0.4 \mathrm{~m} / \mathrm{s})$. Flow separation occurred at he leaflet trailing edge, while the re-attachment points appeared to have occurred beyond the measurement plane. A region of reverse flow with a velocity of $0.6 \mathrm{~m} / \mathrm{s}$ was evident at the lower edge of the central orifice jet giving rise to the formation of two vortices: one between the central forward jet and the reverse flow and another below the reverse flow. The two vortices had velocities of approximately $0.6 \mathrm{~m} / \mathrm{s}$ and persisted through the latter part of the systole. The flow patterns during the deceleration phase were similar to those observed at peak 
systole, however, both the central forward jet and the reverse flow decelerated to a velocity of approximately $1.0 \mathrm{~m} / \mathrm{s}$. The flow inside the valve during systole was in the forward direction with a maximum velocity of $1.6 \mathrm{~m} / \mathrm{s}$ recorded at the leaflet trailing edge at peak systole. Figure 7-173 shows the Reynolds shear stress distribution for the $+8 \mathrm{~mm}$ offset plane of prototype B at peak systole (time bin 9). Elevated Reynolds shear stress values between $600-3,000$ dynes $/ \mathrm{cm}^{2}$ were confined mainly to the edge of the central orifice jet and the region between the reverse flow and the central jet. The Reynolds shear stress inside the sinus region was less than 50 dynes $/ \mathrm{cm}^{2}$.

\section{$\underline{\text { Diastole }}$}

Figure 7-174 shows the flow field for the $+8 \mathrm{~mm}$ offset plane of prototype B during mid diastole (time bin 21). The velocity downstream from the trailing edge of the leaflet during diastole was typically less than $0.07 \mathrm{~m} / \mathrm{s}$. The flow velocity inside the sinus region was less than $0.03 \mathrm{~m} / \mathrm{s}$ with a vortex seen occasionally near the rear of the valve. Flow inside the valve mainly consisted of a leakage jet near the commissural region with a velocity of approximately $0.6 \mathrm{~m} / \mathrm{s}$ that persisted throughout diastole. The maximum Reynolds shear stress of 2,000 dynes $/ \mathrm{cm}^{2}$ was recorded inside the leakage jet during diastole. The Reynolds shear stress levels downstream from the trailing edge of the leaflet and in the sinus region were typically less than $30 \mathrm{dynes} / \mathrm{cm}^{2}$. 


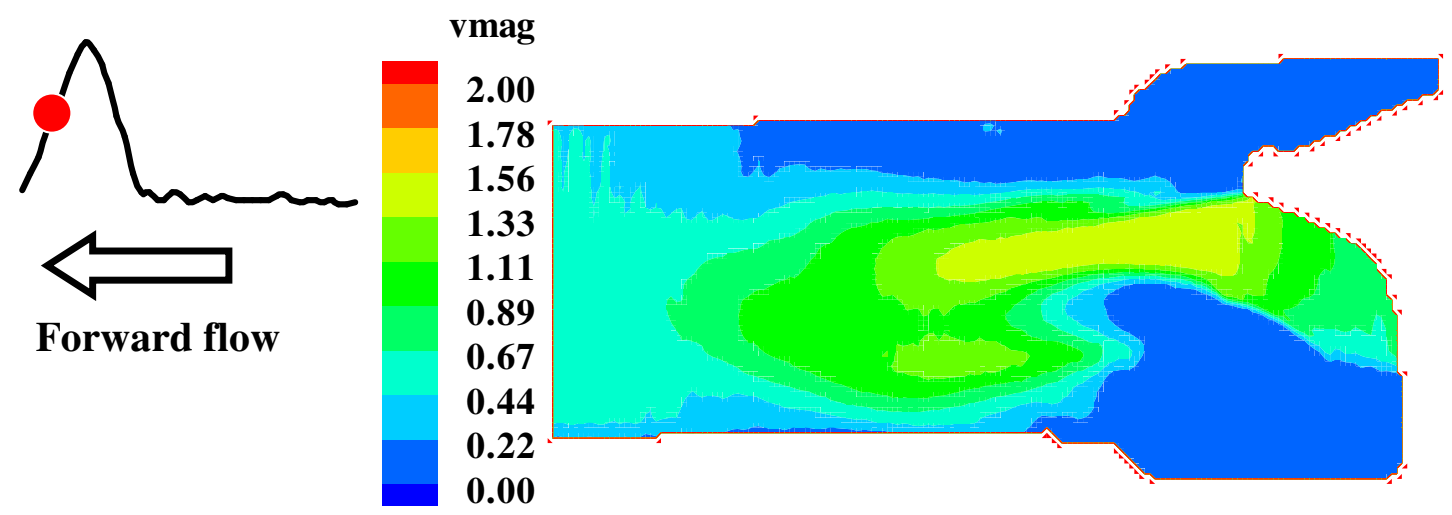

Figure 7-171 Velocity fields at the $+8 \mathrm{~mm}$ offset plane of prototype $\mathrm{B}$ during the acceleration phase, time bin 5; a velocity of $1.5 \mathrm{~m} / \mathrm{s}$ was observed inside the central orifice jet (avi, 614k, B_+8mm_plane_vmag.avi)

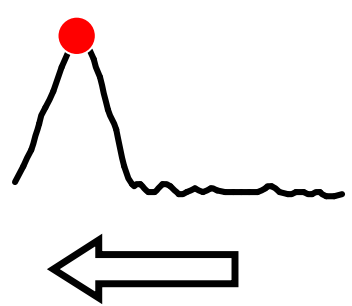

Forward flow

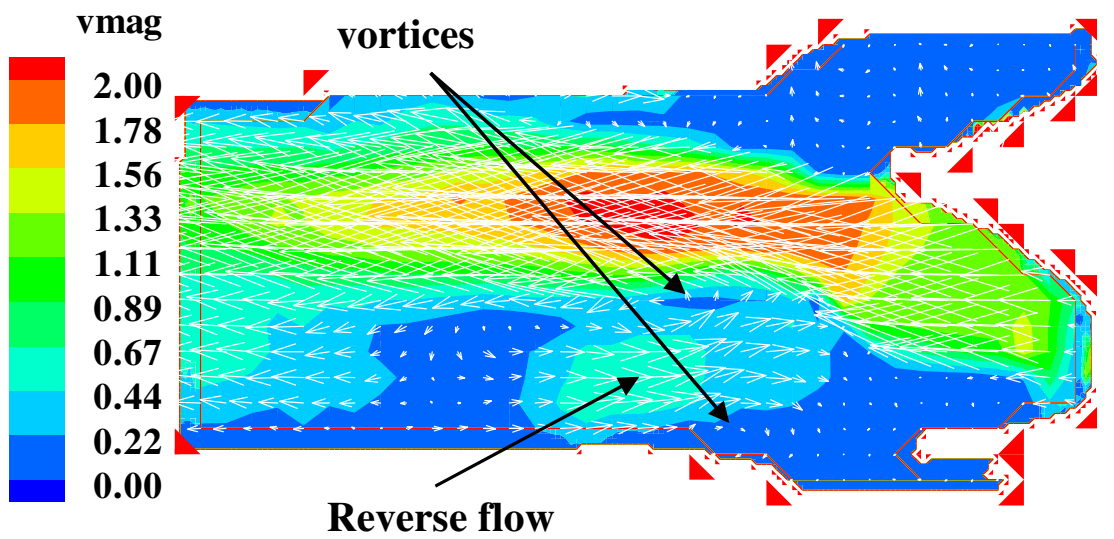

Figure 7-172 Velocity fields at the $+8 \mathrm{~mm}$ offset plane of prototype B during peak systole, time bin 9; a region of reverse flow of $0.6 \mathrm{~m} / \mathrm{s}$ was evident at the lower edge of the central orifice jet (avi, 614k, B_+8mm_plane_vmag.avi) 

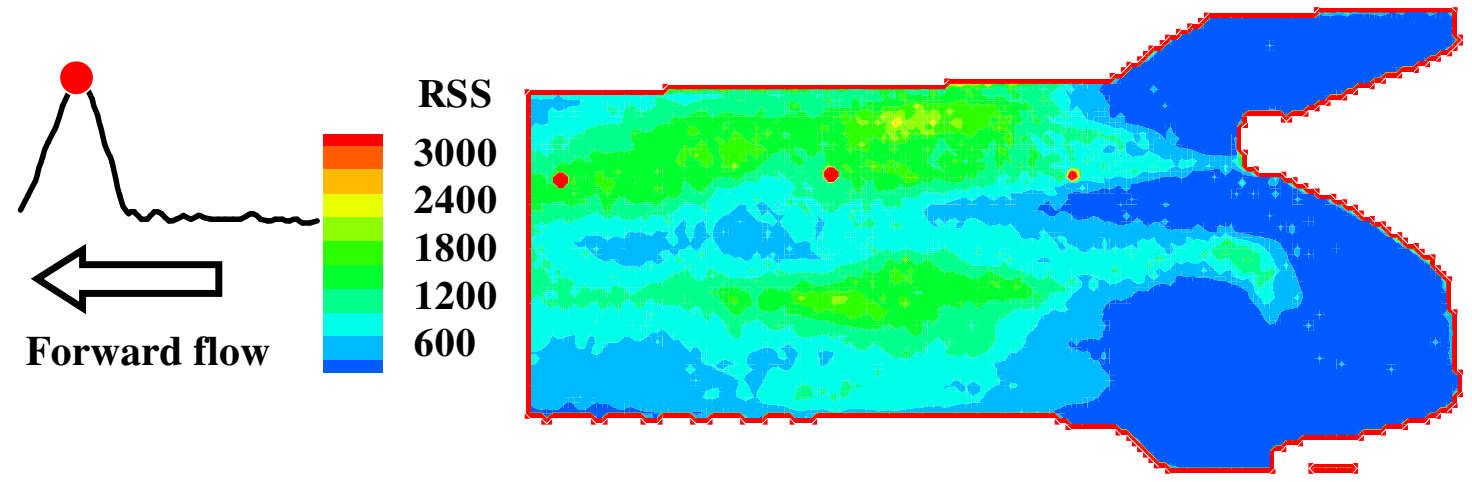

Figure 7-173 Reynolds shear stress contour plot of the $+8 \mathrm{~mm}$ offset plane of prototype B during peak systole, time bin 9; high Reynolds shear stress values coincided with regions of high velocity gradient typically observed along the edge of the central orifice jet (avi, 629k, B_+8mm_plane_rss.avi)

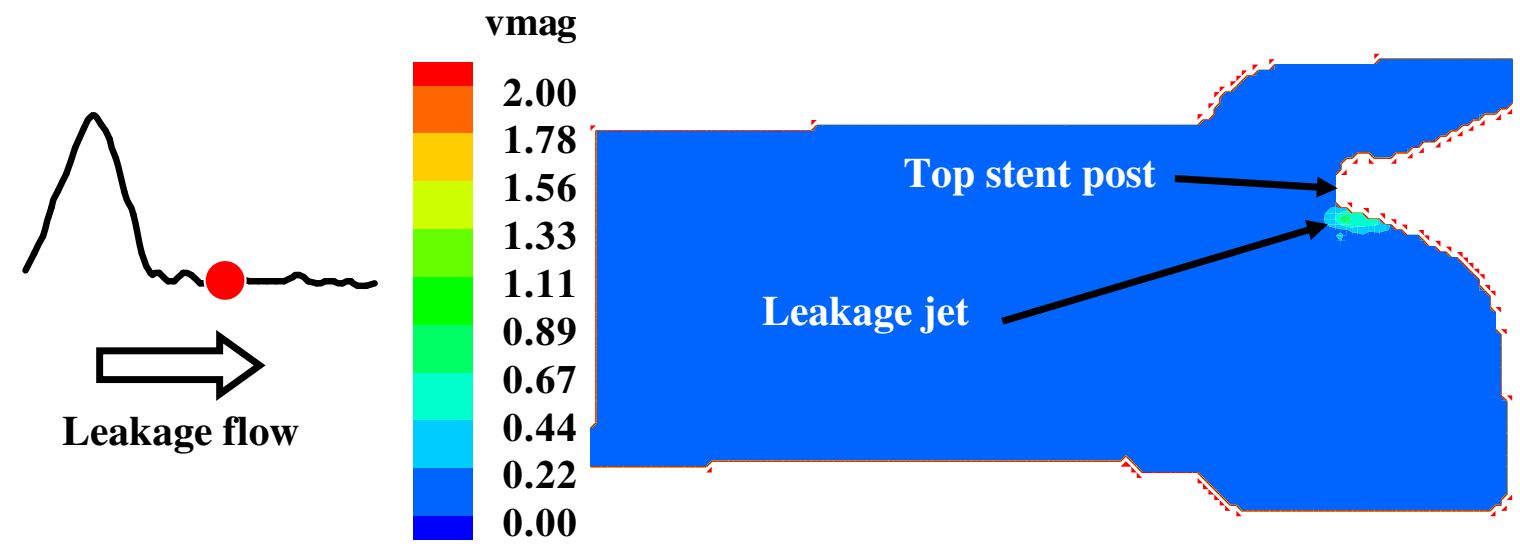

Figure 7-174 Velocity fields at the $+8 \mathrm{~mm}$ offset plane of prototype B during mid diastole, time bin 21; a small leakage jet of $0.6 \mathrm{~m} / \mathrm{s}$ was observed near the top stent post of the valve and persisted throughout the diastole (avi, 614k, B_+8mm_plane_vmag.avi)

\subsubsection{4 \pm 12 mm Plane}

Animations $\quad$ B_+12mm_plane_vmag.avi (B_-12mm_plane_vmag.avi) and B_+12mm_plane_rss.avi (B_-12mm_plane_rss.avi) show the velocity magnitude $(\mathrm{m} / \mathrm{s})$ and Reynolds shear stress (dynes/ $\mathrm{cm}^{2}$ ) contour plots for the $12 \mathrm{~mm}$ offset measurement 
planes of prototype B throughout the cardiac cycle, respectively. Refer to Appendix $\mathrm{H}$ for the list of animations. The flow patterns in the two offset measurement planes were similar, hence only flow fields of one offset measurement plane are shown.

\section{Systole}

Figure 7-175 shows the flow field for the $+12 \mathrm{~mm}$ offset plane of prototype B during the acceleration phase (time bin 5). The flow field inside the valve was not captured by the $+12 \mathrm{~mm}$ offset plane. A forward flow velocity of $0.46 \mathrm{~m} / \mathrm{s}$ was measured $35 \mathrm{~mm}$ downstream from the valve sewing ring. The velocity immediately downstream of the valve was less than $0.05 \mathrm{~m} / \mathrm{s}$, while that inside the sinus region was less than $0.02 \mathrm{~m} / \mathrm{s}$. The flow field downstream from the leaflet trailing edge at peak systole was dominated by a central jet of $0.95 \mathrm{~m} / \mathrm{s}$ occupying the upper part of the flow chamber, and a reverse flow of $0.90 \mathrm{~m} / \mathrm{s}$ in the lower part of the flow chamber (time bin 9, Figure 7-176). A vortex was created between the two flow regions, with a velocity $0.29 \mathrm{~m} / \mathrm{s}$ that persisted to the end of the systole. During this time phase (time bin 14), the forward flow decelerated to a velocity of $0.37 \mathrm{~m} / \mathrm{s}$, and the reverse flow to a velocity of $0.43 \mathrm{~m} / \mathrm{s}$.

Figure 7-177 shows the Reynolds shear stress distribution for the $+12 \mathrm{~mm}$ offset plane of prototype B at peak systole (time bin 9). Reynolds shear stress values of approximately 2,000 dynes $/ \mathrm{cm}^{2}$ and 500 dynes $/ \mathrm{cm}^{2}$ were observed inside the forward flow jet and the reverse flow, respectively, at peak systole. Reynolds shear stress values of 900 dynes $/ \mathrm{cm}^{2}$ were recorded in the region between the forward and the reverse flows at peak systole. The Reynolds shear stress inside the sinus region at peak systole was close to zero. 


\section{$\underline{\text { Diastole }}$}

Figure 7-178 shows the flow field for the $+12 \mathrm{~mm}$ offset plane of prototype B during mid diastole (time bin 21). The velocity downstream from the leaflet trailing edge was typically less than $0.09 \mathrm{~m} / \mathrm{s}$, while that inside the sinus region was less than $0.03 \mathrm{~m} / \mathrm{s}$. A vortex was observed occasionally at the edge of the sinus region during diastole. The Reynolds shear stress throughout the measurement plane during diastole was less than 25 dynes $/ \mathrm{cm}^{2}$.

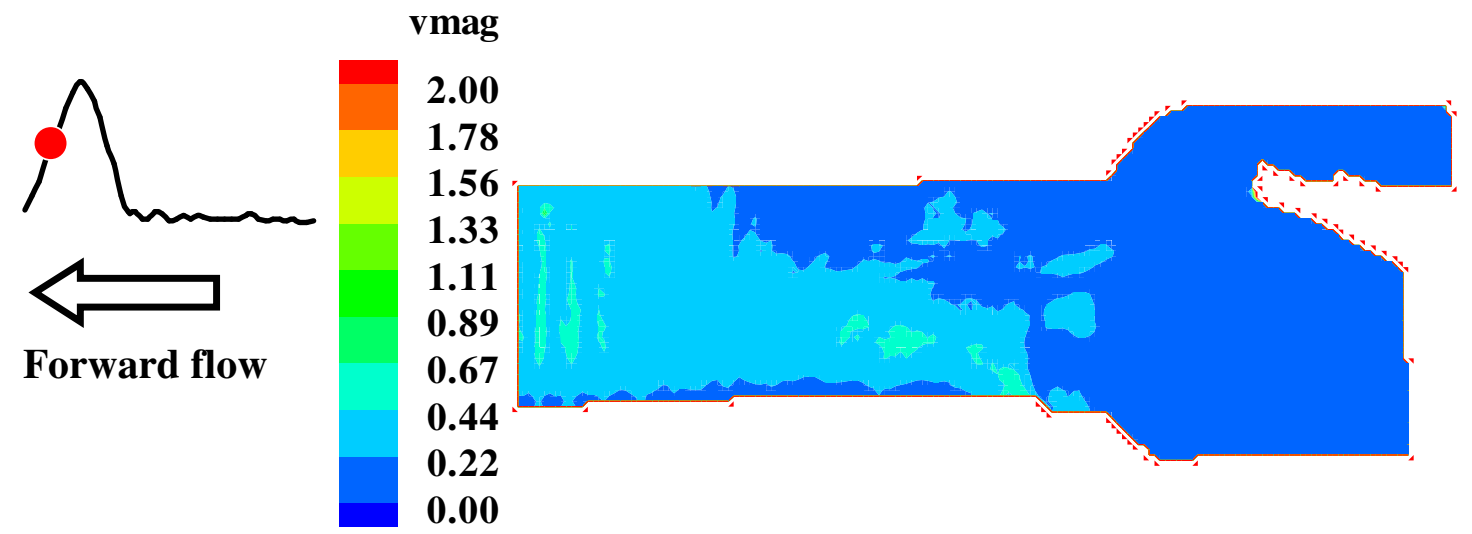

Figure 7-175 Velocity fields at the $\pm 12 \mathrm{~mm}$ offset plane of prototype B during the acceleration phase, time bin 5; forward flow velocity of $0.46 \mathrm{~m} / \mathrm{s}$ was observed $35 \mathrm{~mm}$ downstream from the valve sewing ring (avi, 559k, B_+12mm_plane_vmag.avi) 


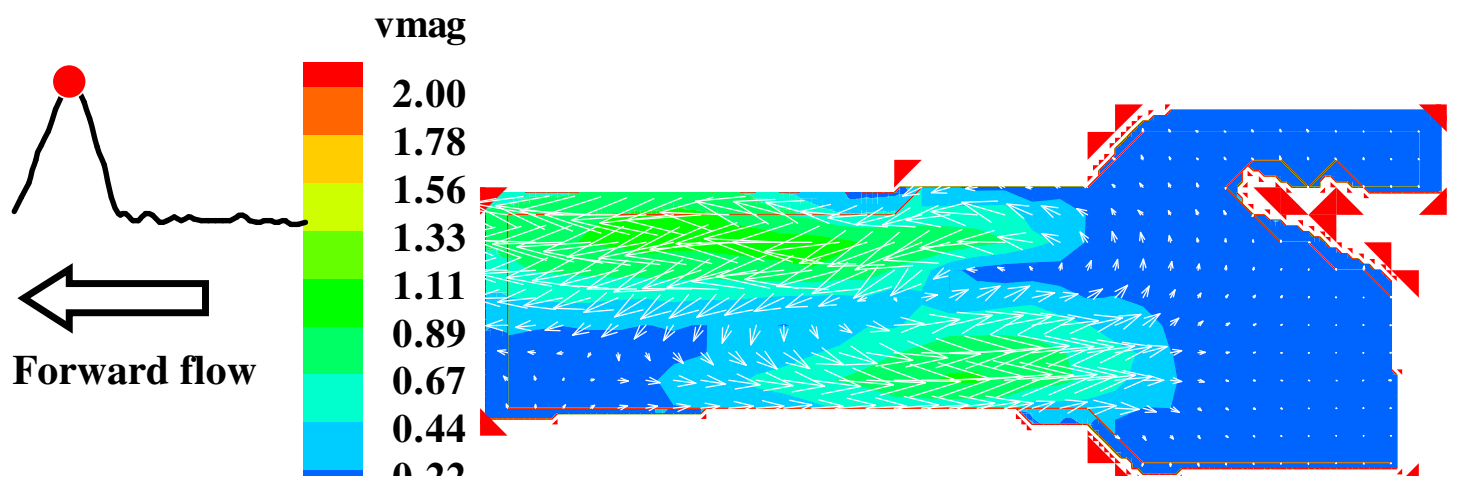

Figure 7-176 Velocity fields at the $+12 \mathrm{~mm}$ offset plane of prototype B during peak systole, time bin 9; opposing flows of approximately $0.90 \mathrm{~m} / \mathrm{s}$ evident at the downstream region of the valve (avi, 559k, B_+12mm_plane_vmag.avi)
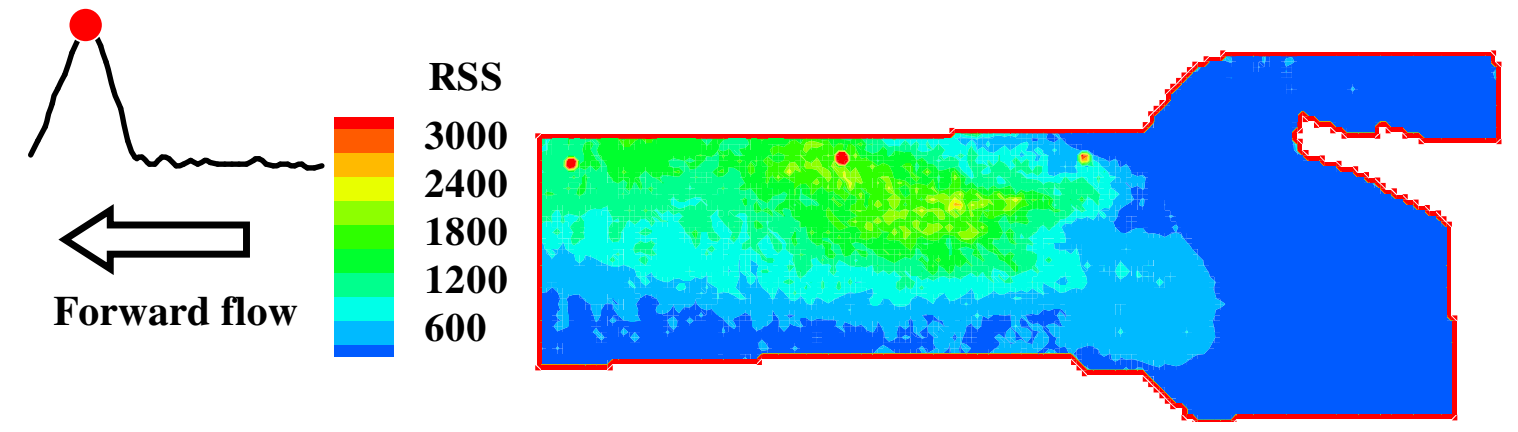

Figure 7-177 Reynolds shear stress contour plot of the $+12 \mathrm{~mm}$ offset plane of prototype B peak systole, time bin 9; elevated Reynolds shear stress was observed $45 \mathrm{~mm}$ downstream of the valve sewing ring at the location where the forward flow mix with the surrounding flow (avi, 610k, B_+12mm_plane_rss.avi) 


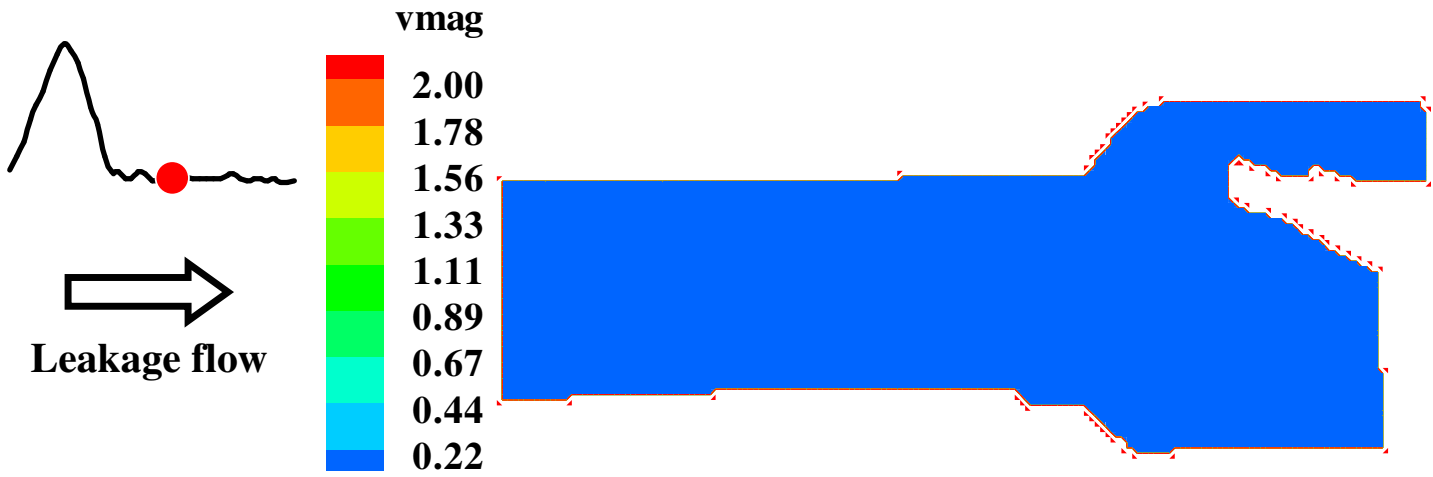

Figure 7-178 Velocity vectors at the $+12 \mathrm{~mm}$ offset plane of prototype B during diastole, time bin 21; flow was typically lower than $0.09 \mathrm{~m} / \mathrm{s}$ downstream from the leaflet trailing edge and $0.03 \mathrm{~m} / \mathrm{s}$ inside the sinus region (avi, 559k, B_+12mm_plane_vmag.avi)

\subsubsection{Semi-Open Commissure Valve With 120 um Leaflet Thickness (Prototype C)}

\subsection{Center Plane}

Animations C_center_plane_vmag.avi and C_center_plane_rss.avi show the velocity magnitude $(\mathrm{m} / \mathrm{s})$ and Reynolds shear stress $\left(\right.$ dynes $\left./ \mathrm{cm}^{2}\right)$ contour plots for the center plane measurement of prototype $\mathrm{C}$ throughout the cardiac cycle, respectively. Animation C_isosurface_vmag.avi shows the 3D reconstructed iso-surface of the velocity magnitude of prototype $\mathrm{C}$ throughout the cardiac cycle. Refer to Appendix $\mathrm{H}$ for the list of animations. Refer to Figure 7-138 for the terminology pertinent to the following description of the prototype $\mathrm{C}$ results.

\section{Systole}

Figure 7-179 shows the center plane flow field downstream of prototype $\mathrm{C}$ during the acceleration phase (time bin 5). The flow characteristics at this time phase were similar to those seen in prototype B. A velocity of $1.35 \mathrm{~m} / \mathrm{s}$ was observed inside the central orifice jet, $3 \mathrm{~mm}$ downstream from the leaflet trailing edge. A vortex ring was evident along the 
edge of the central jet approximately $30 \mathrm{~mm}$ from the valve sewing ring. At peak systole (time bin 9, Figure 7-180) the central orifice jet reached a maximum velocity of $2.1 \mathrm{~m} / \mathrm{s}$ and a diameter of approximately $16 \mathrm{~mm}$. The central orifice jet had a flat profile when exiting from the valve orifice, and became parabolic as the central jet traveled approximately $50 \mathrm{~mm}$ downstream from the valve sewing ring. As shown in Figure 7-181 (time bin 9), the velocity iso-surface of the central orifice jet of prototype $\mathrm{C}$ did not display the distinct three lobe feature seen in prototypes A and B. From Figure 7-181, the flow profile became circular in shape as it approached the distal part of the flow chamber (65 $\mathrm{mm}$ downstream of the valve sewing ring). Flow separation was observed at the trailing edge of the leaflet. The re-attachment point in the upper part of the flow chamber appeared to have occurred beyond the measurement plane, while re-acttachment point in the lower part of the flow chamber occurred at $45 \mathrm{~mm}$ downstream from the valve sewing ring at peak systole. A velocity of $0.5 \mathrm{~m} / \mathrm{s}$ was seen inside the recirculation zone. Vortex structures were not observed in the sinus region, which had a velocity of generally less than $0.04 \mathrm{~m} / \mathrm{s}$ during systole. During the deceleration phase (time bin 14), the velocity in the central orifice jet decelerated from $2.1 \mathrm{~m} / \mathrm{s}$ to $1.2 \mathrm{~m} / \mathrm{s}$. However, the velocity of the recirculation zone in the upper part of flow chamber maintained at a velocity of $0.5 \mathrm{~m} / \mathrm{s}$. The flow inside prototype $\mathrm{C}$ was similar to that observed in prototypes $\mathrm{A}$ and $\mathrm{B}$ at peak systole. A maximum flow velocity of $1.8 \mathrm{~m} / \mathrm{s}$ was reached inside the valve along the trailing edge of the leaflet. A region of low flow with a velocity of $0.78 \mathrm{~m} / \mathrm{s}$ was observed along the stent inflow region inside the valve. The flow velocity at the rear part of the valve was approximately $1.4 \mathrm{~m} / \mathrm{s}$. 
Figure 7-182 shows the Reynolds shear stress distribution for the center plane measurement of prototype $C$ at peak systole (time bin 9). Reynolds shear stress values of more than 2,000 dynes $/ \mathrm{cm}^{2} \mathrm{~m} / \mathrm{s}$ were observed along the edge of the central orifice and at the commissural region of the valve. In contrast, the Reynolds shear stress inside the central orifice jet was typically less than 45 dynes $/ \mathrm{cm}^{2}$, while that at the sinus region was close to zero.

\section{Diastole}

Figure 7-183 shows the center plane flow field downstream of prototype $\mathrm{C}$ during mid diastole (time bin 21). The velocity downstream from the leaflet trailing edge was between 0.08 and $0.12 \mathrm{~m} / \mathrm{s}$. A vortex was occasionally seen inside the sinus region during diastole, and the velocity inside the sinus region was typically less than $0.02 \mathrm{~m} / \mathrm{s}$. Flow patterns inside the valve during diastole were similar to those seen in prototype B. Two leakage jets were observed inside the valve during diastole: one in the coaptation region and one in the commissural region of the valve. The leakage jet at the coaptation point had a velocity of $1.39 \mathrm{~m} / \mathrm{s}$, while the jet in the commissural region had a velocity of 0.7 $\mathrm{m} / \mathrm{s}$. In addition to these leakage jets, retrograde flow of $0.57 \mathrm{~m} / \mathrm{s}$ was seen occasionally along the trailing edge of the leaflet. Similar to prototype A, prototype C displayed split flow during diastole, in which the flow was directed toward both the stent inflow and high central regions. The Reynolds shear stress downstream from the leaflet trailing edge during diastole was less than 50 dynes $/ \mathrm{cm}^{2}$. Conversely, a Reynolds shear stress of 4,000 dynes $/ \mathrm{cm}^{2}$ was measured in the leakage jets inside the valve during diastole Figure 7-184). 


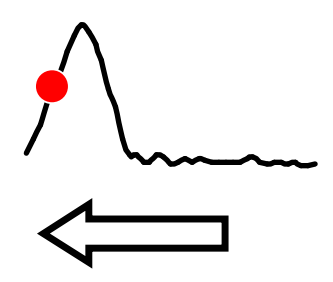

Forward flow

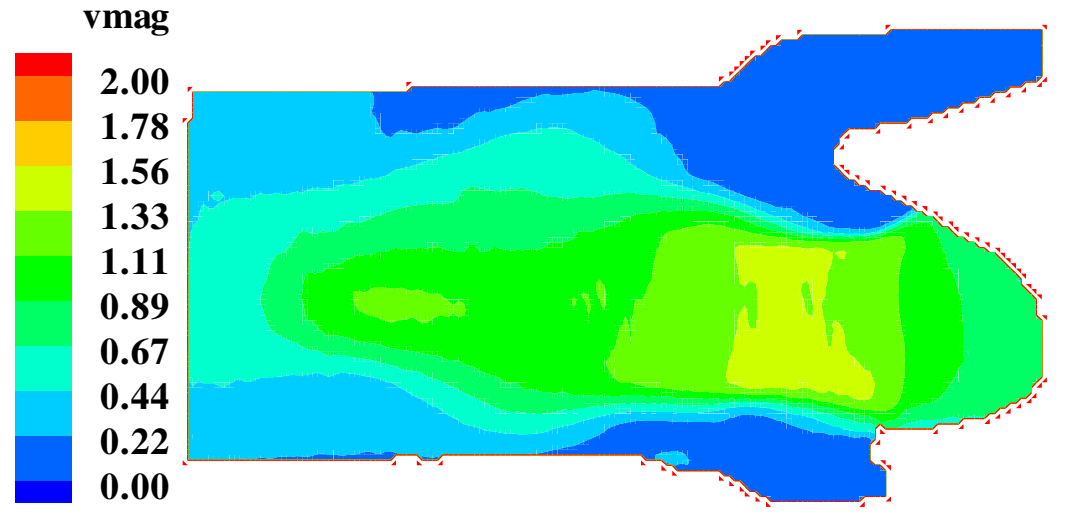

Figure 7-179 Velocity fields at the center plane of prototype $\mathrm{C}$ during acceleration phase, time bin 5 ; central orifice jet of $1.35 \mathrm{~m} / \mathrm{s}$ was observed emanating from the valve orifice with the formation of vortex ring was seen along the edge of the central jet (avi, 599k, C_center_plane_vmag.avi)

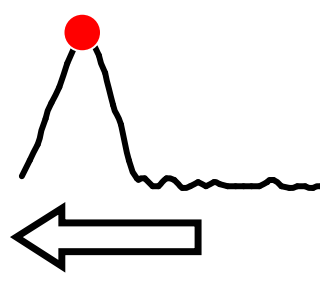

Forward flow

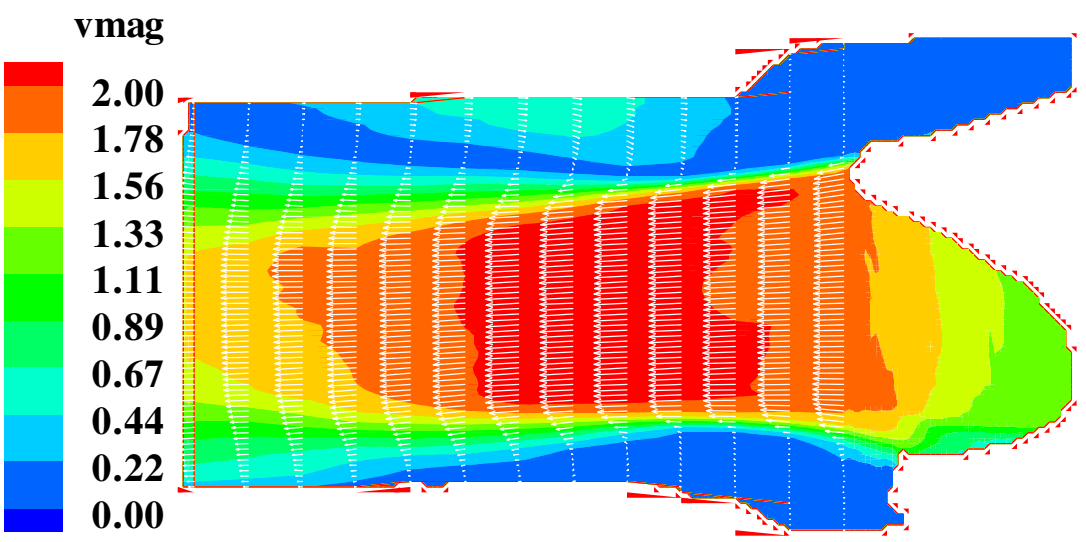

Figure 7-180 Velocity fields at the center plane of prototype $\mathrm{C}$ during peak systole, time bin 9 ; a maximum velocity of $2.1 \mathrm{~m} / \mathrm{s}$ was recorded inside the central orifice jet during peak systole (avi, 599k, C_center_plane_vmag.avi) 

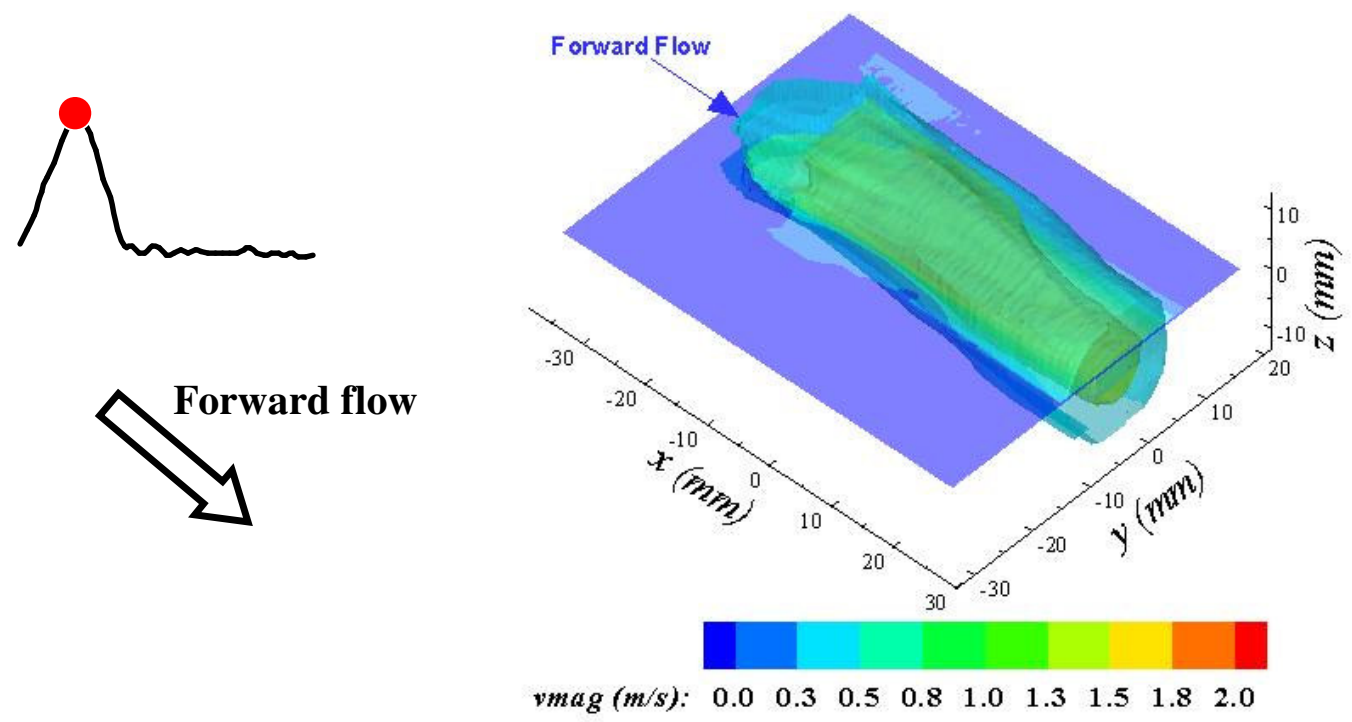

Figure 7-181 Iso-surface of velocity magnitude of prototype $\mathrm{C}$ at peak systole, time bin 9; the velocity iso-surface of prototype $\mathrm{C}$ did not have the distinct three-lobe feature as seen in prototype $\mathrm{A}$ and $\mathrm{B}$. The flow profile was much more circular as the central jet approached the distal end of the flow chamber (avi, 929k, C_isosurface_vmag.avi)

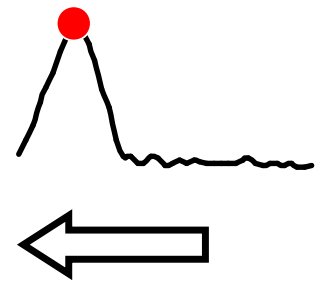

Forward flow
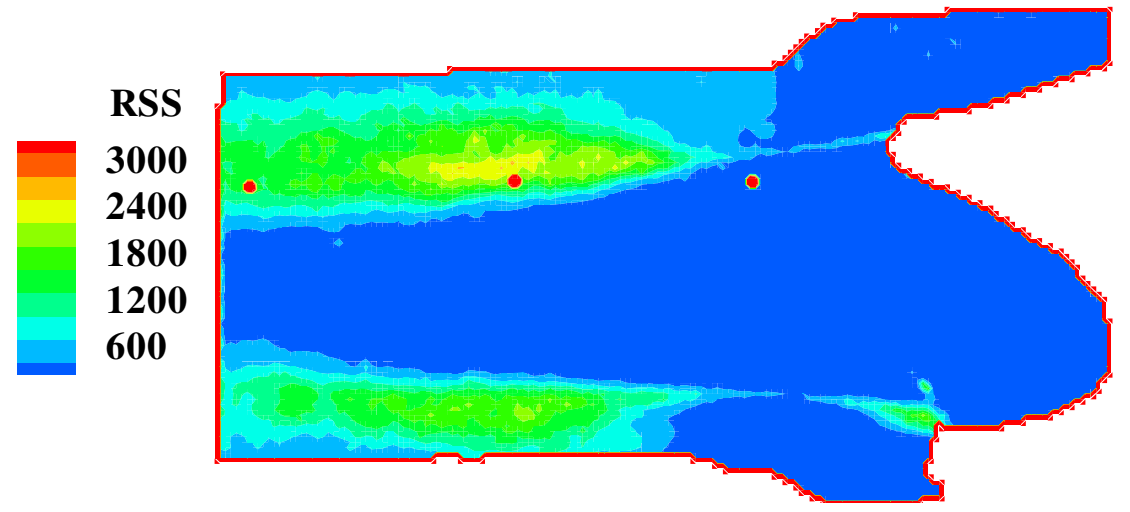

Figure 7-182 Reynolds shear stress contour plot of the center plane of prototype $\mathrm{C}$ during peak systole, time bin 9; high Reynolds shear stress values observed along the edge of the central orifice jet and at the commissural region inside the valve (avi, 635k, C_center_plane_rss.avi) 


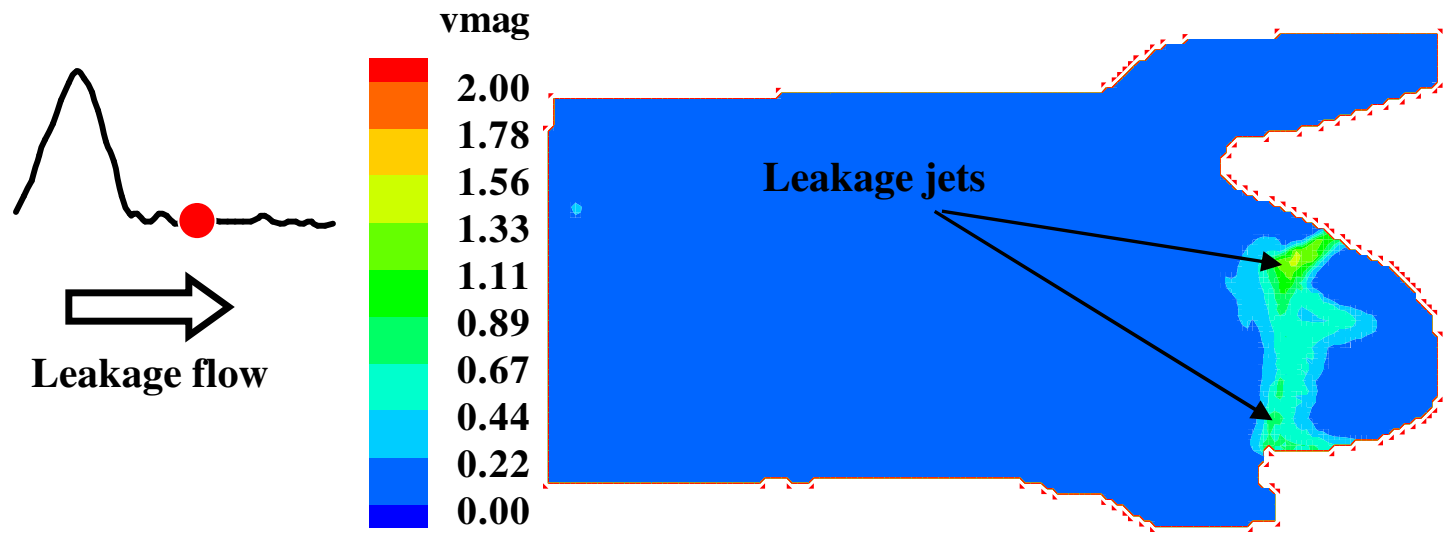

Figure 7-183 Velocity fields at the center plane of prototype $\mathrm{C}$ during diastole, time bin 21; leakage jets of more than $0.7 \mathrm{~m} / \mathrm{s}$ were seen at both the coaptation and the commissural region in the valve (avi, 599k, C_center_plane_vmag.avi)

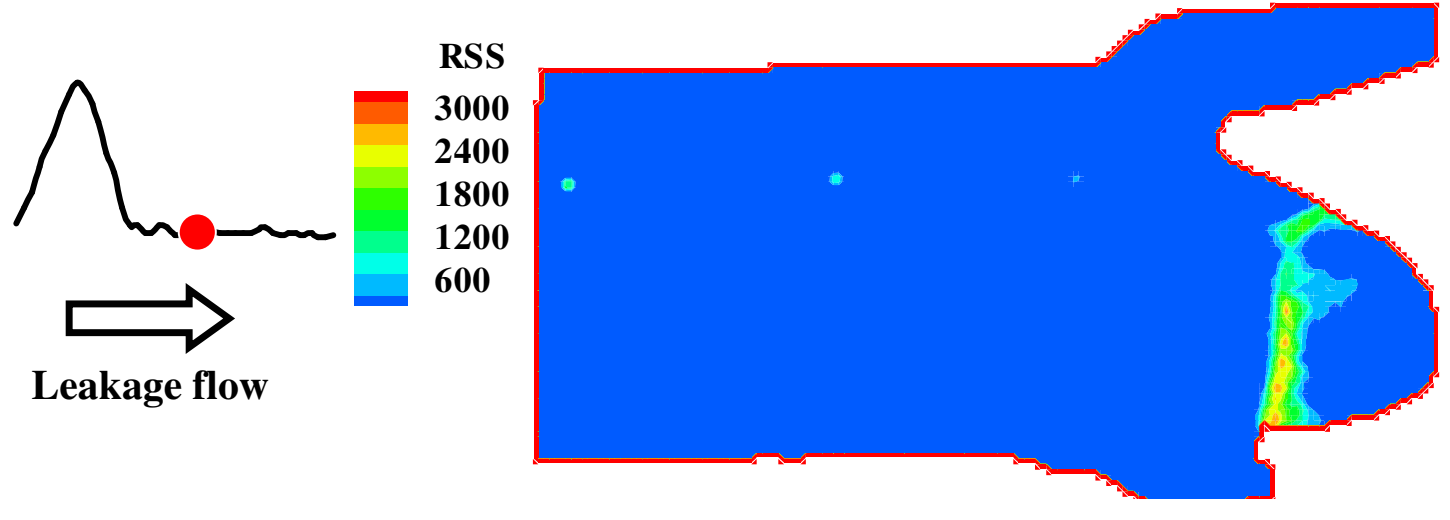

Figure 7-184 Reynolds shear stress contour plot of the center plane of prototype C during diastole, time bin 21; elevated Reynolds shear stress levels of more than 4,000 dynes $/ \mathrm{cm}^{2}$ observed along the trailing edge of the valve (avi, 635k, C_center_plane_rss.avi)

\subsubsection{2 $\pm 4 \mathrm{~mm}$ Planes}

Animations $\quad$ C_+4mm_plane_vmag.avi (C_-4mm_plane_vmag.avi) and C_+4mm_plane_rss.avi (C_-4mm_plane_rss.avi) show the velocity magnitude $(\mathrm{m} / \mathrm{s})$ and Reynolds shear stress (dynes $/ \mathrm{cm}^{2}$ ) contour plots for the $4 \mathrm{~mm}$ offset measurement planes 
of prototype $\mathrm{C}$ throughout the cardiac cycle, respectively. Refer to Appendix $\mathrm{H}$ for the list of animations. The flow patterns in the two offset measurement planes were similar, hence only flow fields of one offset measurement plane are shown.

\section{Systole}

Figure 7-185 shows the $+4 \mathrm{~mm}$ offset plane downstream from the leaflet trailing edge of prototype $\mathrm{C}$ during the acceleration phase (time bin 5). The flow field for prototype $\mathrm{C}$ was similar to that for prototype B during the acceleration phase. A velocity of $1.5 \mathrm{~m} / \mathrm{s}$ was observed inside the central orifice jet during the acceleration phase. A vortex ring was created along the edge of the central jet $20 \mathrm{~mm}$ downstream from the valve sewing ring. At peak systole (time bin 9) the velocity of the central orifice jet increased to 2.1 $\mathrm{m} / \mathrm{s}$ (Figure 7-186). The central jet had a diameter of about $13 \mathrm{~mm}$. Flow separation was observed at the trailing edge of the leaflet, while the re-attachment points occurred 60 $\mathrm{mm}$ and $40 \mathrm{~mm}$ downstream from the valve sewing ring in the upper and lower parts of the flow chamber, respectively. The velocity inside the recirculation zone ranged between 0.1 and $0.15 \mathrm{~m} / \mathrm{s}$, while flow inside the sinus region was typically less than $0.03 \mathrm{~m} / \mathrm{s}$. No vortices were observed in the sinus region. The velocity of the central orifice jet decelerated to approximately $1.2 \mathrm{~m} / \mathrm{s}$ during the deceleration phase (time bin 14 ), while the recirculation zone in the upper part of the flow chamber accelerated to a velocity of $0.3 \mathrm{~m} / \mathrm{s}$. The flow field inside the valve was similar to those seen in both prototypes A and B during systole. A maximum flow velocity of $1.8 \mathrm{~m} / \mathrm{s}$ was recorded along the trailing edge of the leaflet at peak systole, and the flow along the stent inflow region reached a velocity of $0.5 \mathrm{~m} / \mathrm{s}$. Figure 7-187 shows the Reynolds shear stress distribution 
for the $4 \mathrm{~mm}$ offset plane of prototype $\mathrm{C}$ at peak systole (time bin 9). Reynolds shear stress values of more than 3,000 dynes $/ \mathrm{cm}^{2}$ were observed along the edge of the central orifice jet and also near the top stent post at peak systole. The Reynolds shear stress inside the central jet was typically less than $50 \mathrm{dynes} / \mathrm{cm}^{2}$, while that inside the sinus region was close to zero.

\section{Diastole}

Figure 7-188 shows the flow field for the $+4 \mathrm{~mm}$ offset plane of prototype $\mathrm{C}$ during mid diastole (time bin 21). The velocity downstream from the leaflet trailing edge was between 0.05 and $0.1 \mathrm{~m} / \mathrm{s}$, while the flow inside the sinus region during diastole was typically less than $0.03 \mathrm{~m} / \mathrm{s}$. A vortex with a velocity of less than $0.01 \mathrm{~m} / \mathrm{s}$ was observed at the top right corner of Figure 7-188 and persisted throughout the diastole. Leakage jets of approximately 0.4 and $0.3 \mathrm{~m} / \mathrm{s}$ were observed, respectively, at the coaptation region and near the rear the valve during diastole. Two vortex structures were also seen inside prototype $\mathrm{C}$, similar to those observed with prototype B during diastole (see time bin 21 , Figure 7-169). One vortex was located next to the leakage jet at the coaptation region $(0.37 \mathrm{~m} / \mathrm{s})$, and the other was at the rear of the valve $(0.15 \mathrm{~m} / \mathrm{s})($ lower right corner of Figure 7-188). The two leakage jets and vortex structures persisted throughout diastole. The Reynolds shear stress values downstream of the leaflet trailing edge and inside the sinus region were less than 50 dyesn $/ \mathrm{cm}^{2}$ during diastole. The Reynolds shear stress levels inside the valve during diastole were generally less than $20 \mathrm{dynes} / \mathrm{cm}^{2}$, except for those recorded in the leakage jet inside the valve, which had a maximum Reynolds shear stress of 329 dynes $/ \mathrm{cm}^{2}$. 


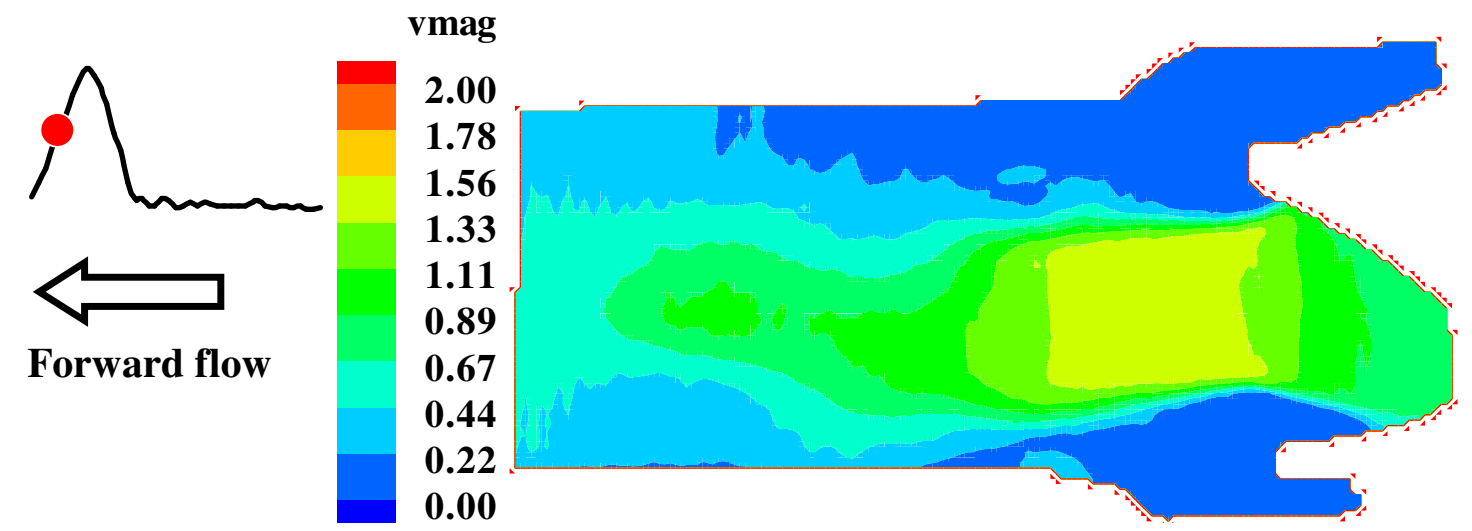

Figure 7-185 Velocity fields at the $+4 \mathrm{~mm}$ offset plane of prototype $\mathrm{C}$ acceleration phase, time bin 5; velocity of $1.5 \mathrm{~m} / \mathrm{s}$ was measured inside the central orifice jet (avi, 600k, C_+4mm_plane_vmag.avi)

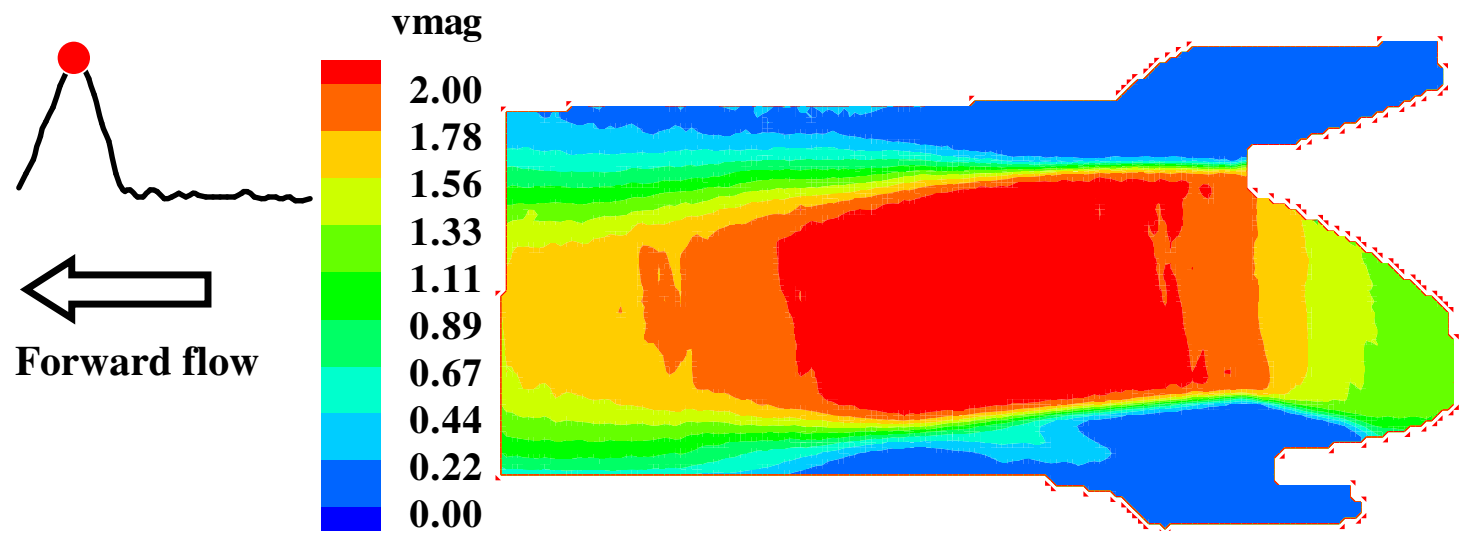

Figure 7-186 Velocity fields at the $+4 \mathrm{~mm}$ offset plane of prototype $\mathrm{C}$ during peak systole, time bin 9; maximum flow velocity of $2.1 \mathrm{~m} / \mathrm{s}$ was reached inside the central orifice jet at peak systole (avi, 600k, C_+4mm_plane_vmag.avi) 


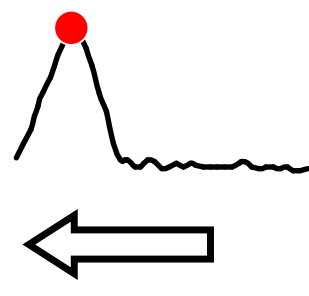

Forward flow

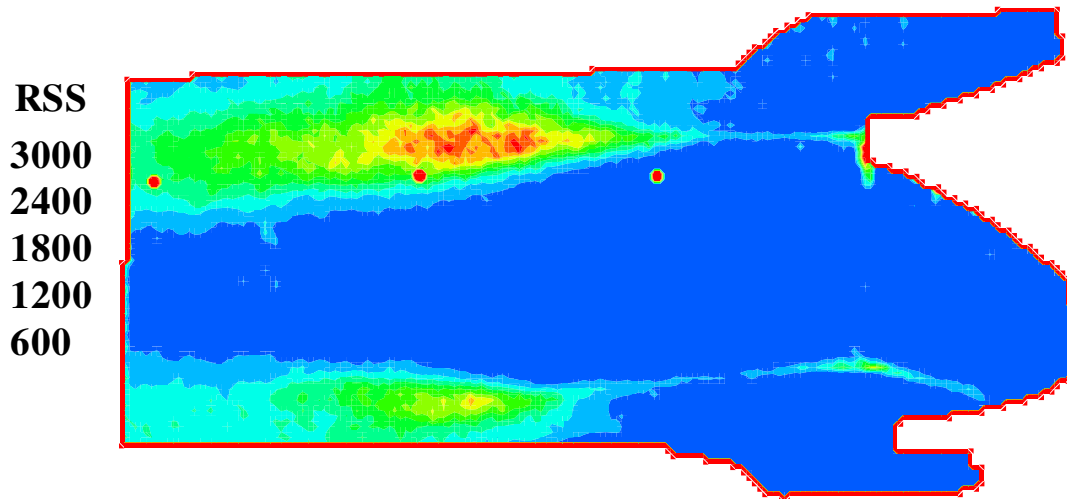

Figure 7-187 Reynolds shear stress contour plot of the $+4 \mathrm{~mm}$ offset plane of prototype C during peak systole, time bin 9; high Reynolds shear stress values observed along the edge of the central orifice jet and at the top stent post of the valve (avi, 767k, C_+4mm_plane_rss.avi)
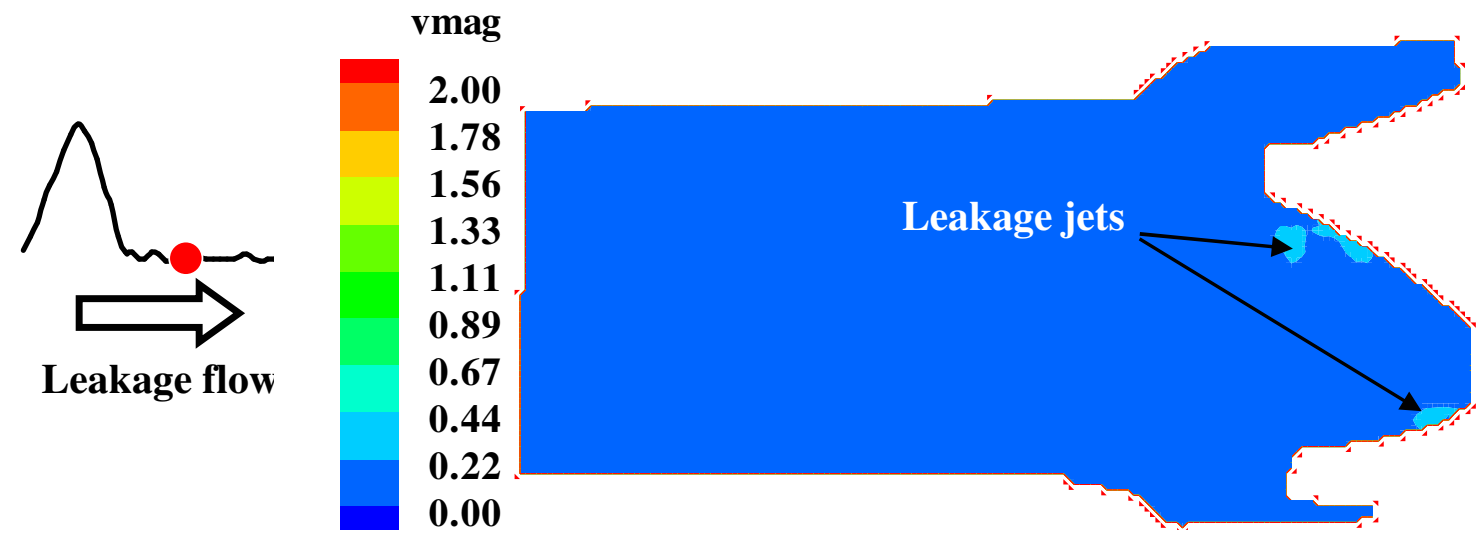

Figure 7-188 Velocity fields at the $+4 \mathrm{~mm}$ offset plane of prototype $\mathrm{C}$ during mid diastole, time bin 21 ; leakage jets of approximately $0.3 \mathrm{~m} / \mathrm{s}$ were seen at both the coaptation region and near the rear part of the valve during diastole (avi, 600k, C_+4mm_plane_vmag.avi)

\subsubsection{3 $\pm 8 \mathrm{~mm}$ Planes}

Animations $\quad$ C_+8mm_plane_vmag.avi $\quad$ (C_-8mm_plane_vmag.avi) and C_+8mm_plane_rss.avi (C_-8mm_plane_rss.avi) show the velocity magnitude $(\mathrm{m} / \mathrm{s})$ and Reynolds shear stress (dynes $/ \mathrm{cm}^{2}$ ) contour plots for the $8 \mathrm{~mm}$ offset measurement planes 
of prototype $\mathrm{C}$ throughout the cardiac cycle, respectively. Refer to Appendix $\mathrm{H}$ for the list of animations. The flow patterns in the two offset measurement planes were similar, hence only flow fields of one offset measurement plane are shown.

\section{Systole}

Figure 7-189 shows the flow field for the $+8 \mathrm{~mm}$ offset plane of prototype $\mathrm{C}$ during the acceleration phase (time bin 5). A central orifice jet of approximately $1.2 \mathrm{~m} / \mathrm{s}$ was observed emanating from the valve orifice. A vortex ring was observed forming along the edge of the central jet approximately $25 \mathrm{~mm}$ downstream from the valve sewing ring. At peak systole (time bin 9), the velocity inside the central jet accelerated to $2.0 \mathrm{~m} / \mathrm{s}$ (Figure 7-190). Unlike the flow pattern seen in the $+8 \mathrm{~mm}$ offset plane in prototype $\mathrm{B}$, the flow downstream of the leaflet trailing edge in prototype $\mathrm{C}$ was predominantly in the forward direction. The velocity of the central jet decreased to $1.42 \mathrm{~m} / \mathrm{s} 55 \mathrm{~mm}$ from the valve sewing ring. The flow velocity in the sinus region was typically less than $0.04 \mathrm{~m} / \mathrm{s}$. Flow separation was observed at the trailing edge of the leaflet, while the re-attachment points were observed $50 \mathrm{~mm}$ and $40 \mathrm{~mm}$ downstream of the valve sewing ring in the upper and lower parts of the flow chambers, respectively. The flow pattern inside prototype $\mathrm{C}$ was similar to that observed in prototypes A and B. A maximum flow velocity of approximately $1.7 \mathrm{~m} / \mathrm{s}$ was recorded along the leaflet trailing edge. The flow patterns during the deceleration phase (time bin 14) were similar to those observed at peak systole, however, both the central orifice jet and the flow along the leaflet trailing edge inside the valve had decelerated to a velocity of $0.7 \mathrm{~m} / \mathrm{s}$. Figure $7-191$ shows the Reynolds shear stress distribution for the $8 \mathrm{~mm}$ offset plane of prototype $\mathrm{C}$ at peak systole (time bin 9 ). 
Reynolds shear stress levels of more than 3,000 dynes $/ \mathrm{cm}^{2}$ were recorded in the middle of the flow chamber where mixing between the high velocity central jet and low velocity surrounding fluid occurred.

\section{$\underline{\text { Diastole }}$}

Figure 7-192 shows the flow field of the $+8 \mathrm{~mm}$ offset plane for prototype $\mathrm{C}$ during mid diastole (time bin 21). The flow velocity throughout the entire investigated region was less than $0.1 \mathrm{~m} / \mathrm{s}$ during diastole, except for a leakage jet of $0.26 \mathrm{~m} / \mathrm{s}$ observed near the top stent post. The leakage jet persisted throughout diastole. A maximum Reynolds shear stress of 2,250 dynes $/ \mathrm{cm}^{2}$ was recorded at the top stent post during diastole, coinciding with the location of this leakage jet (Figure 7-193).

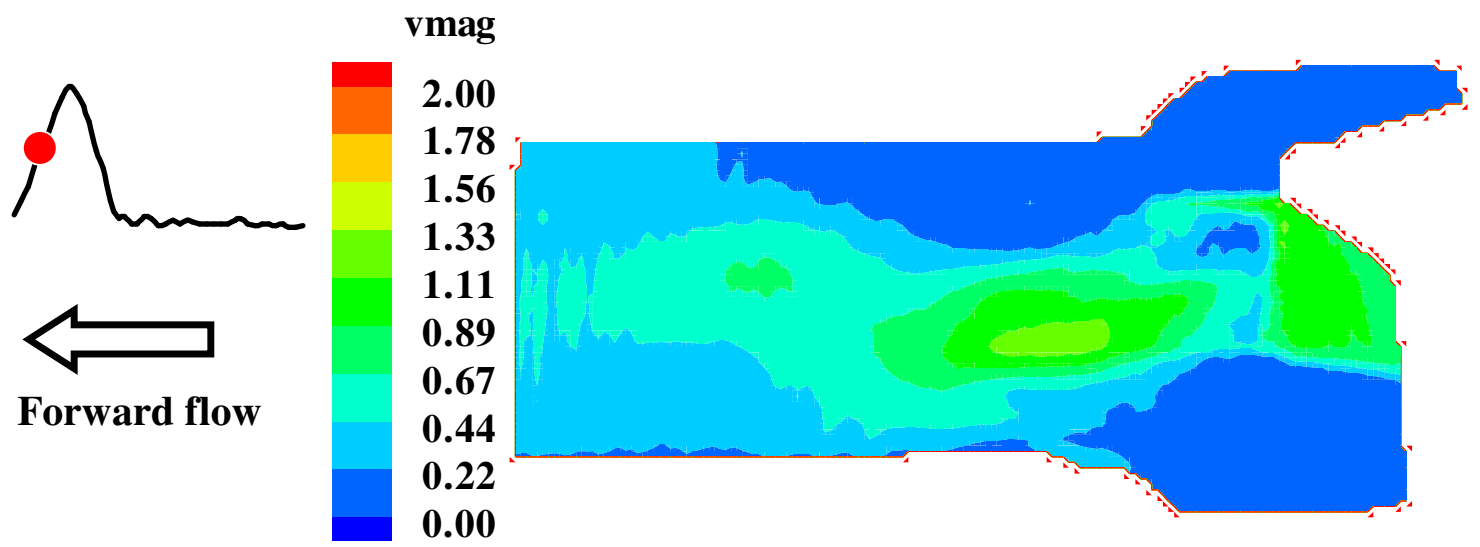

Figure 7-189 Velocity fields at the $+8 \mathrm{~mm}$ offset plane of prototype $\mathrm{C}$ during the acceleration phase, time bin 5; a velocity of $1.2 \mathrm{~m} / \mathrm{s}$ was observed inside the central orifice jet with vortex ring formation at the edge of the jet (avi, 576k, C_+8mm_plane_vmag.avi) 


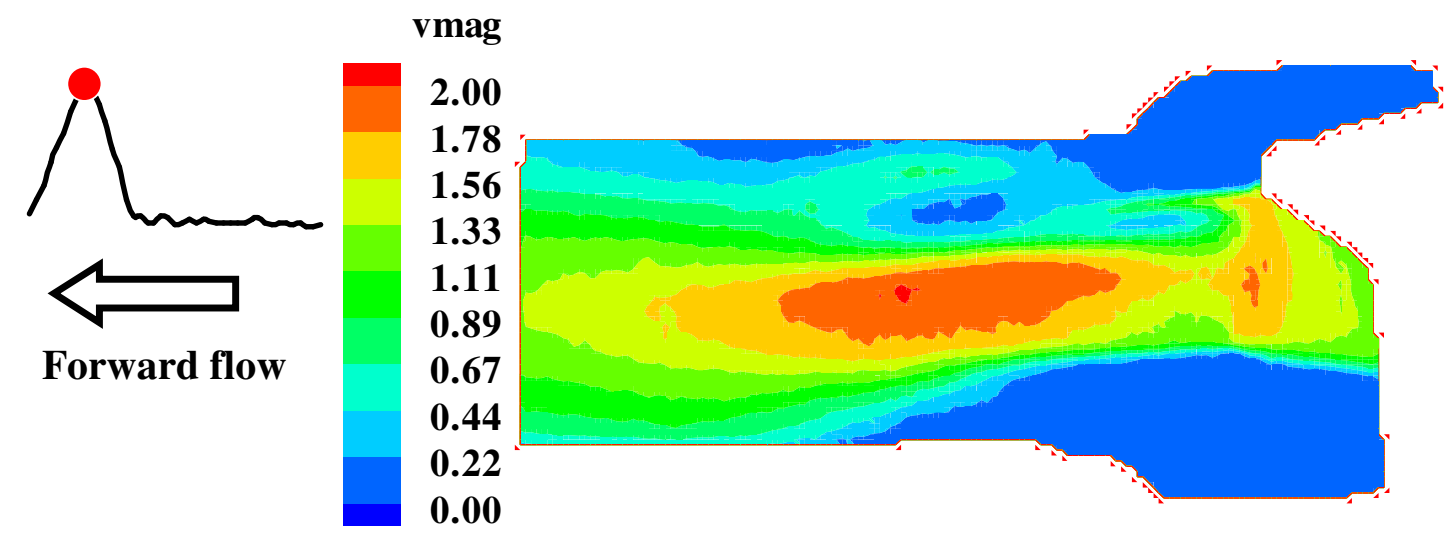

Figure 7-190 Velocity fields at the $+8 \mathrm{~mm}$ offset plane of prototype $\mathrm{C}$ during peak systole, time bin 9; a maximum velocity of $2.0 \mathrm{~m} / \mathrm{s}$ was recorded inside the central orifice jet at peak systole (avi, 576k, C_+8mm_plane_vmag.avi)

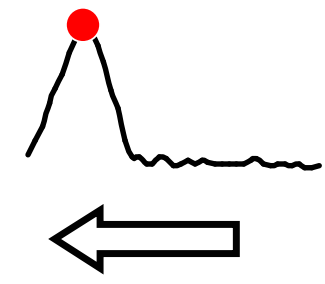

Forward flow

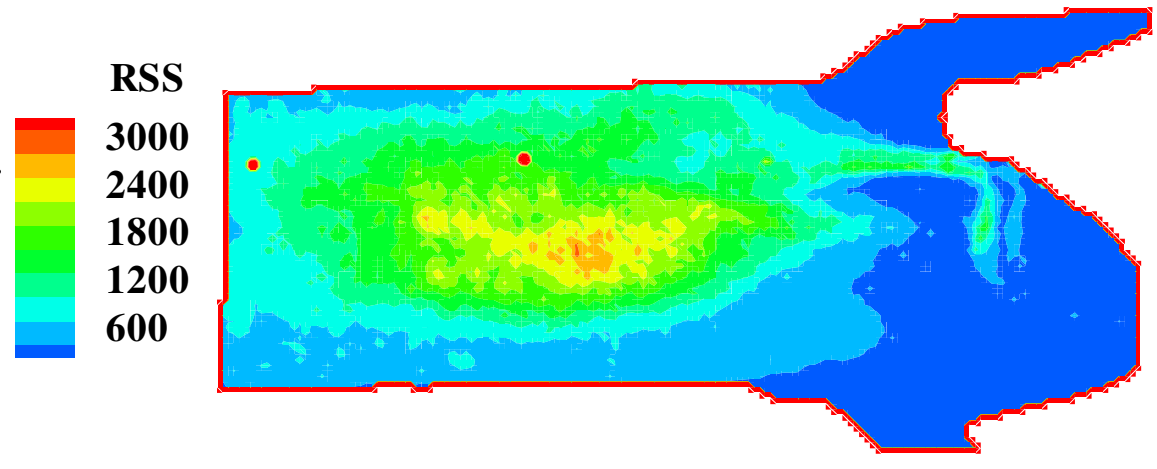

Figure 7-191 Reynolds shear stress contour plot of the $+8 \mathrm{~mm}$ offset plane of prototype C during peak systole, time bin 9; high Reynolds shear stress values coincided with regions mixing between the central orifice jet and the surrounding fluid (avi, 667k, C_+8mm_plane_rss.avi) 


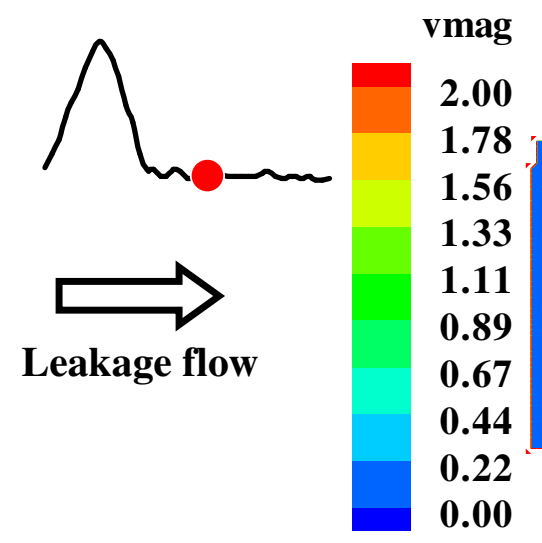

Figure 7-192 Velocity fields at the $+8 \mathrm{~mm}$ offset plane of prototype $\mathrm{C}$ during mid diastole, time bin 21; a small leakage jet of $0.26 \mathrm{~m} / \mathrm{s}$ was observed near the top stent post of the valve and persisted throughout the diastole (avi, 576k, C_+8mm_plane_vmag.avi)
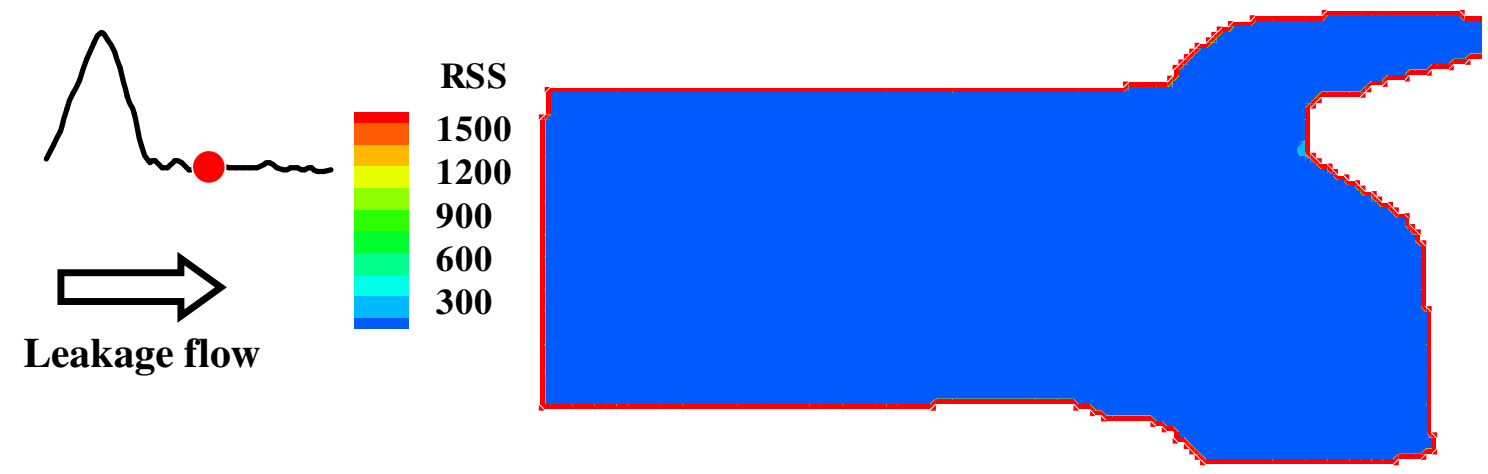

Figure 7-193 Reynolds shear stress contour plot of the $+8 \mathrm{~mm}$ offset plane of prototype C during mid diastole, time bin 21; elevated Reynolds shear stress values were observed at the top stent post coinciding with the location of the leakage jet (avi, 667k, C_+8mm_plane_rss.avi)

\subsubsection{4 $\pm 12 \mathrm{~mm}$ Planes}

Animations $\quad$ C_+12mm_plane_vmag.avi (C_-12mm_plane_vmag.avi) and C_+12mm_plane_rss.avi (C_-12mm_plane_rss.avi) show the velocity magnitude (m/s) and Reynolds shear stress (dynes $/ \mathrm{cm}^{2}$ ) contour plots for the $12 \mathrm{~mm}$ offset measurement 
planes of prototype $\mathrm{C}$ throughout the cardiac cycle, respectively. Refer to Appendix $\mathrm{H}$ for the list of animations. The flow patterns in the two offset measurement planes were similar, hence only flow fields of one offset measurement plane are shown.

\section{Systole}

Figure 7-194 shows the flow field for the $+12 \mathrm{~mm}$ offset plane of prototype $\mathrm{C}$ during the acceleration phase (time bin 5). A velocity of $0.5 \mathrm{~m} / \mathrm{s}$ was observed in the central orifice jet $35 \mathrm{~mm}$ downstream from the valve sewing ring. Vortices were observed at the edge of the sinus region approximately $10 \mathrm{~mm}$ from the leaflet edge. The velocity inside the sinus region was typically less than $0.01 \mathrm{~m} / \mathrm{s}$. At peak systole (time bin 9) the central jet accelerated to $0.65 \mathrm{~m} / \mathrm{s} 40 \mathrm{~mm}$ downstream from the leaflet edge (Figure 7-195). The vortex at the edge of the sinus region during the acceleration phase traveled $20 \mathrm{~mm}$ downstream from the leaflet edge and moved to the middle part of the flow chamber at peak systole. A weaker vortex of $0.03 \mathrm{~m} / \mathrm{s}$ was observed inside the sinus region and persisted throughout the systole. The velocity throughout the measurement plane during the deceleration phase (time bin 14) was less than $0.1 \mathrm{~m} / \mathrm{s}$, except in the central orifice jet, which had a velocity of $0.3 \mathrm{~m} / \mathrm{s}$. Figure 7-196 shows the Reynolds shear stress distribution for the $12 \mathrm{~mm}$ offset plane of prototype $\mathrm{C}$ at peak systole (time bin 9). A maximum Reynolds shear stress of 1,900 dynes $/ \mathrm{cm}^{2}$ was recorded in the central orifice jet at peak systole. The Reynolds shear stress in the sinus region was typically less than 50 dynes $/ \mathrm{cm}^{2}$. 


\section{$\underline{\text { Diastole }}$}

Figure 7-197 (time bin 21) shows the flow field for the $+12 \mathrm{~mm}$ offset plane of prototype

C during mid diastole. The flow velocity throughout the measurement plane was less than $0.1 \mathrm{~m} / \mathrm{s}$. A vortex was occasionally observed at the sinus region. The Reynolds shear stress levels were typically less than 50 dynes $/ \mathrm{cm}^{2}$, throughout the investigated region.

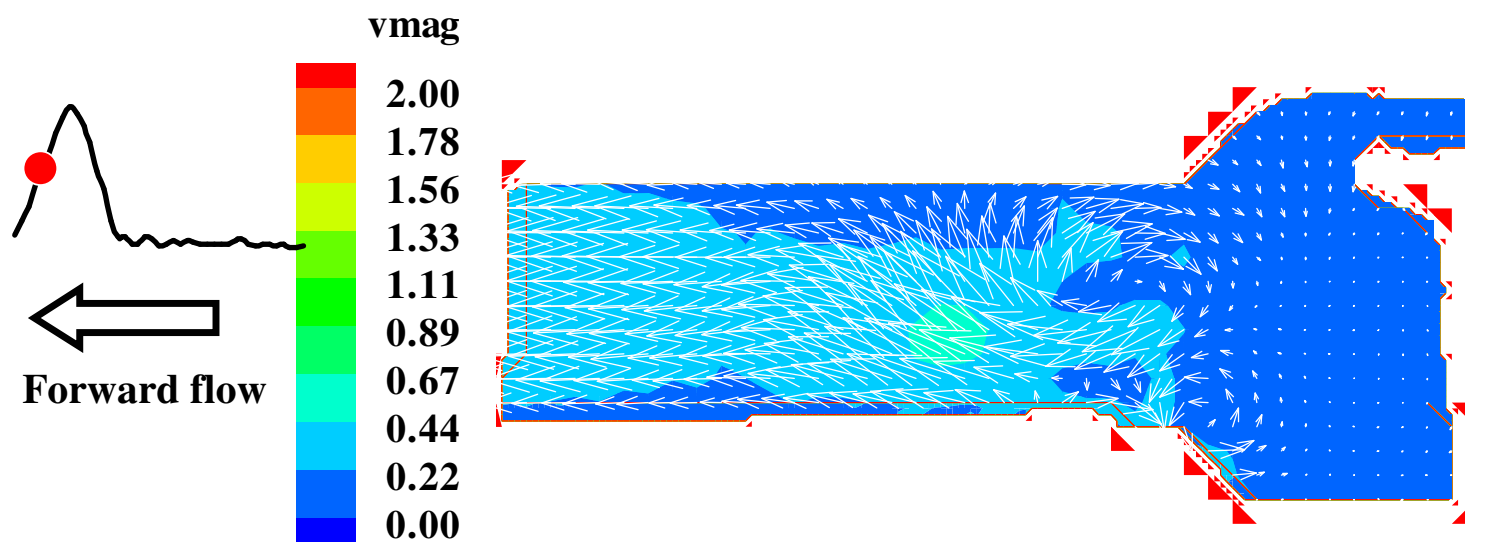

Figure 7-194 Velocity fields at the $+12 \mathrm{~mm}$ offset plane of prototype $\mathrm{C}$ during the acceleration phase, time bin 5; vortex was observed at the edge of the sinus region during the acceleration phase (avi, 550k, C_+12mm_plane_vmag.avi) 


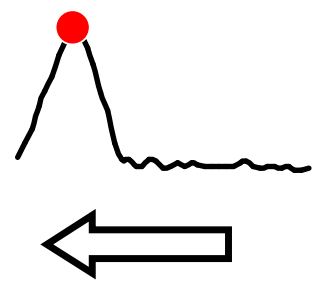

Forward flow

vmag

2.00

1.78

1.56

1.33

1.11

0.89

0.67

0.44

0.22

0.00

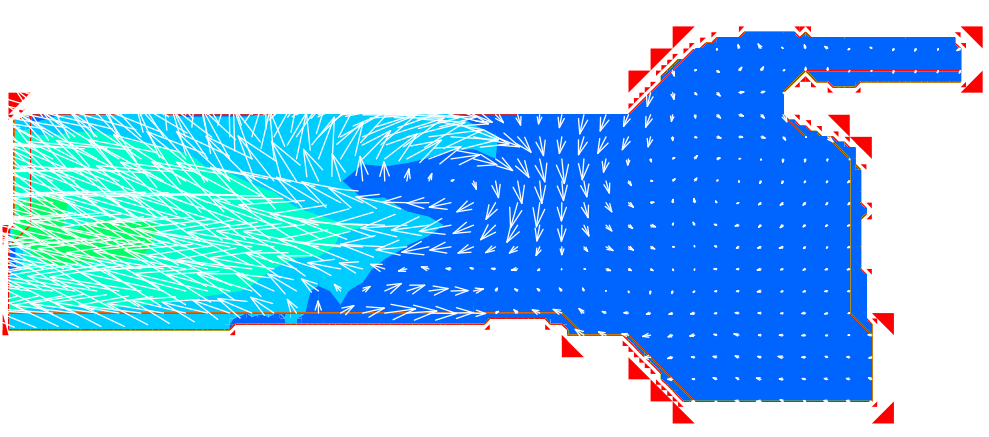

Figure 7-195 Velocity fields at the $+12 \mathrm{~mm}$ offset plane of prototype $\mathrm{C}$ during peak systole, time bin 9 ; maximum flow velocity of $0.65 \mathrm{~m} / \mathrm{s}$ was recorded at location $55 \mathrm{~mm}$ from the valve sewing ring (avi, 550k, C_+12mm_plane_vmag.avi)

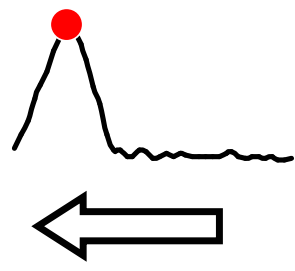

Forward flow

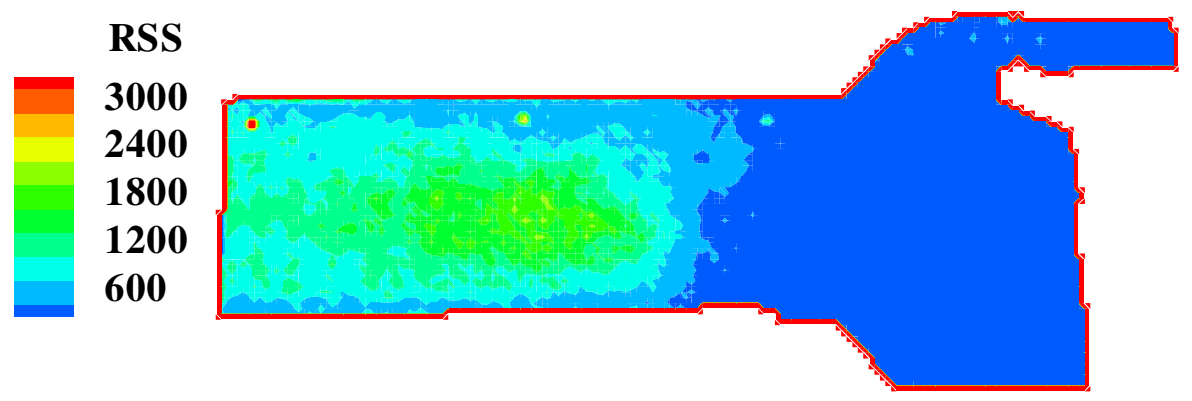

Figure 7-196 Reynolds shear stress contour plot of the $+12 \mathrm{~mm}$ offset plane of prototype C peak systole, time bin 9; elevated Reynolds shear stress was observed $45 \mathrm{~mm}$ downstream of the valve sewing ring at the location where the forward flow mix with the surrounding flow (avi, 602k, C_+12mm_plane_rss.avi) 


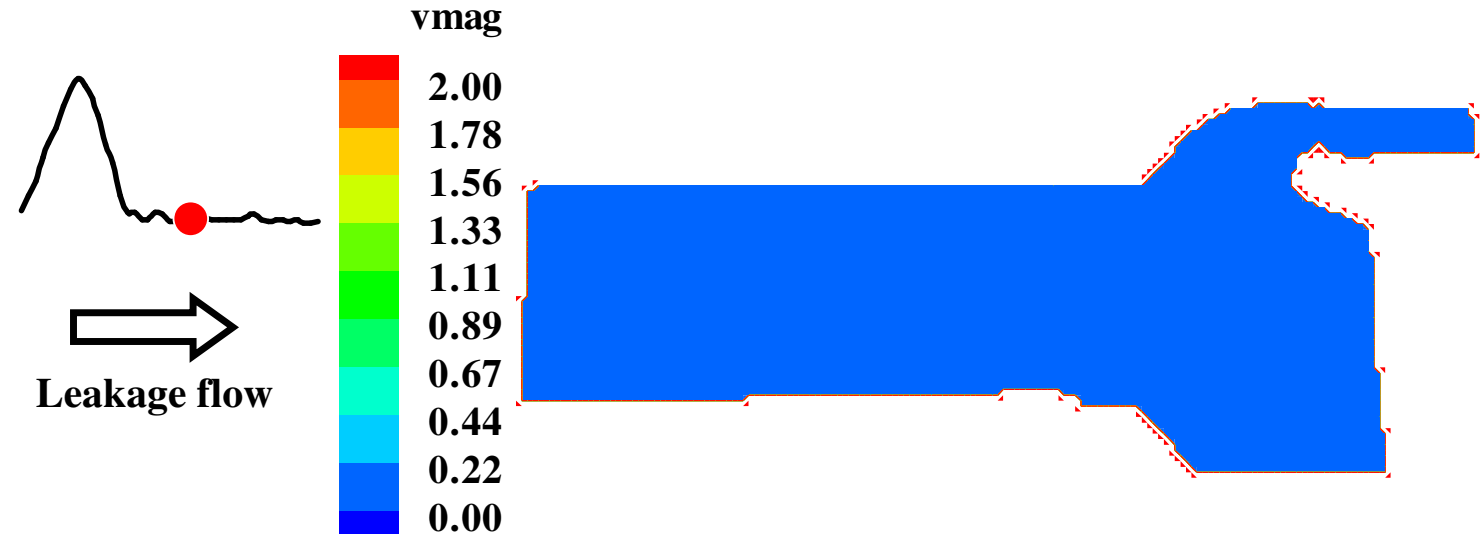

Figure 7-197 Velocity vectors at the $+12 \mathrm{~mm}$ offset plane of prototype C during diastole, time bin 21; flow velocity was typically lower than $0.1 \mathrm{~m} / \mathrm{s}$ throughout the entire measurement plane during diastole (avi, 550k, C_+12mm_plane_vmag.avi)

\subsubsection{Leaflets Surface Topography Study}

Figure 7-198 compares the 3D surface topography of the leaflets of both the bileaflet mechanical and trileaflet polymeric (prototype B) heart valves. The figure shows that the 3D surface topography of the bileaflet mechanical heart valve is smoother than the leaflet surfaces of the prototype $B$. Table 7-2 summarized the $R_{a}$ and $R_{q}$ values of the bileaflet mechanical heart valve and the polymeric heart valve (refer to section 5.6 for the definition of $\mathrm{R}_{\mathrm{a}}$ and $\mathrm{R}_{\mathrm{q}}$ ). The $\mathrm{R}_{\mathrm{a}}$ and $\mathrm{R}_{\mathrm{q}}$ values of the leaflet outer surface of prototype $\mathrm{B}$ are much smaller compare to those of its leaflet inner surface. On the other hand, the surface of the bileaflet mechanical heart valve leaflet was smoother than both the inner and outer surfaces of prototype B. 


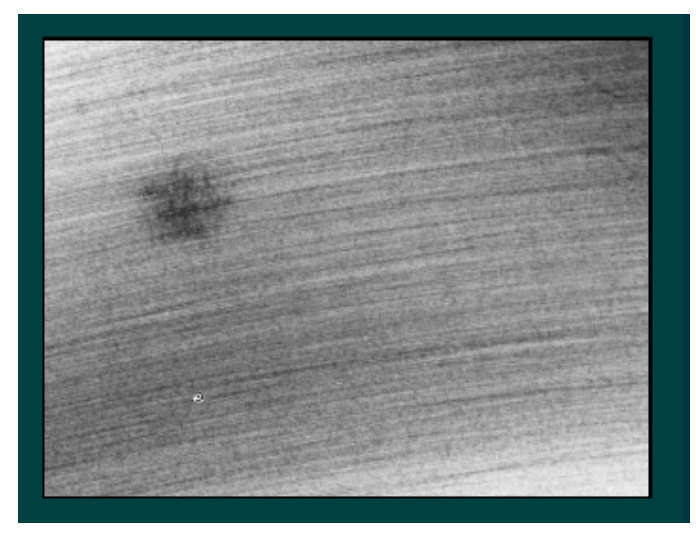

(a)

Figure 7-198 3D surface topography of the leaflet of heart valve prostheses; (a) the leaflet surface of the pyrolytic carbon in the bileaflet mechanical valve, (b) the leaflet surface of the polymeric heart valve (prototype B)

Table 7-2 The $R_{a}$ and $R_{q}$ values of the leaflet surfaces of the two heart valve prostheses

\begin{tabular}{c|cc}
\hline & $R_{a}(\mathrm{~nm})$ & $R_{q}(\mathrm{~nm})$ \\
\hline Bileaflet inner/outer surface & $5-7$ & $7-9$ \\
Tri-leaflet outer surface & $14-16$ & $17-19$ \\
Tri-leaflet inner surface & $900-1500$ & $1100-1700$ \\
\hline
\end{tabular}

Figure 7-199 show the surface roughness of the inner side of the leaflet for prototype B: the surface topogragphy was characterized by a series of 'bumps'. It can be seen that the peak of each crest was spaced approximately $0.1 \mathrm{~mm}$ apart. 


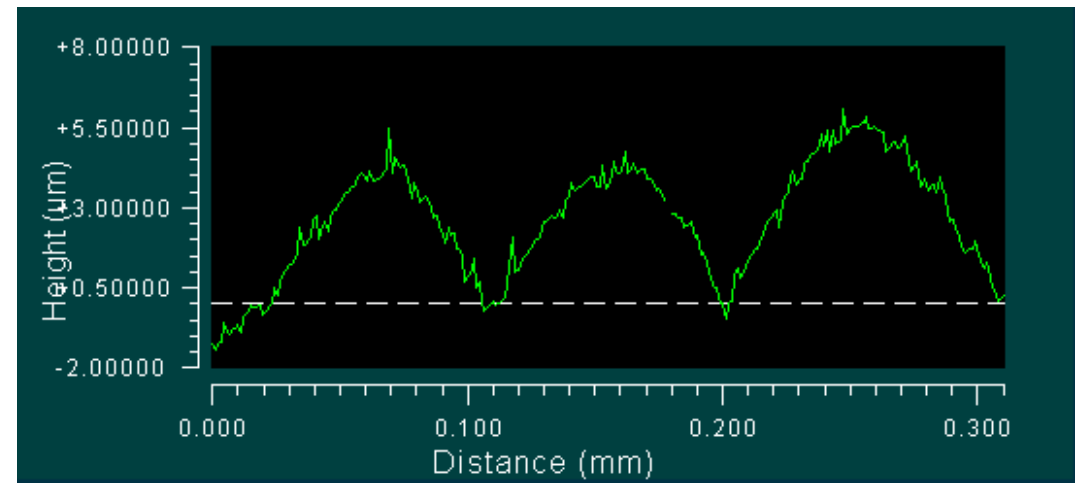

Figure 7-199 The inner surface roughness of the polymeric heart valve 


\section{CHAPTER 8: DISCUSSION}

As seen in the previous Chapter, detailed flow fields measurements in the hinge region of the four prosthetic bileaflet valves were obtained in this study. Measurements in the hinge region of these valves revealed complex and unsteady flow structures under physiological conditions. The calculated peak Reynolds shear stresses (RSS) in the hinge were well above the critical threshold required to damage blood elements. These studies proved to be a significant improvement over previous studies by Ellis et al [Ellis, 1996a; Ellis, 2000a; Ellis, 2000b] that were limited due to low spatial resolution. The improved spatial resolution of the current study permitted precise identification of intricate flow structures at different locations inside the hinge over different phases of the cardiac cycle. This allowed for a more accurate assessment of the flow fields in the various hinge geometry designs. In addition, the study also provided sufficient data to correlate the observed flow structures in the three $27 \mathrm{~mm}$ bileaflet valves to platelet activation levels previously measured.

The detailed assessment of the hemodynamic performance of the three prototype polymeric valves demonstrated the importance of the valve design parameters on the potential for thrombus formation on the valve superstructure. The dynamics of the valve leaflet motion is shown to significantly contribute to the complex flow fields inside the valves. The results of these studies showed that flow fields created in the vicinity of the polymeric valves should determine their success. In addition, the elevated Reynolds shear 
stress calculated in these valves were above the levels for platelet activation and hemolysis.

The objective of this Chapter is to discuss the results in the context of the three specific aims outlined in Chapter 3. The discussion is presented in the following order: 1) the effect of gap width tolerances on hinge flow fields in bileaflet MHVs; 2) the effect of design characteristics on hinge flow fields in bileaflet MHVs; and 3) the effect of leaflet thickness and commissural designs on the flow through prototype PHVs. Finally a review of the limitations of the study is provided.

\section{$\underline{8.1 \text { Effect of Hinge Gap Width Tolerance on Hinge Flow }}$}

This section addresses the specific aim: To study the flow fields in the hinge region of a bileaflet mechanical heart valve design with varying hinge gap widths and relate these flow fields to their respective thrombogenic potentials. The objective of this specific aim is to demonstrate the influence of hinge gap width on the spatial and temporal characteristics of the hinge micro flow fields.

\subsubsection{Effect On Static Leakage Flow}

The static leakage performance of the three $27 \mathrm{~mm}$ bileaflet valves, corresponding to a pressure drop of $120 \mathrm{mmHg}$ across the valve, with respect to hinge gap width tolerance is shown in Figure 7-1. It is clear that leakage flow rate increases with hinge gap width. Closer comparison between the leakage flow rates (Figure 7-1) show that both the SJM $27 \mathrm{~mm}$ Standard and $27 \mathrm{~mm}$ prototype high leaker valves had leakage rates that were one 
order of magnitude higher than that of the $27 \mathrm{~mm}$ prototype low leaker. Similar experiments on static leakage performance with respect to hinge gap width were conducted by Travis [Travis, 2001c] for three $27 \mathrm{~mm}$ carbon MHVs. Results by Travis [Travis, 2001c] showed the leakage rate of the $27 \mathrm{~mm}$ prototype carbon high and low leaker carbon valves were $0.84 \pm 0.08$ and $0.147 \pm 0.011$ liter/min, respectively [Travis, 2001c]; the leakage rate of the former almost double that of the current prototype high leaker clear valve model. This difference is attributed to differences in the hinge tolerance between the clear and carbon prototype high leaker valves. The leakage results for the SJM $27 \mathrm{~mm}$ Standard carbon valve obtained by Travis was $0.423 \pm 0.034$ liter/min [Travis, 2001c]. In assessing these results, the current $27 \mathrm{~mm}$ prototype low leaker valve presents an extreme case for very low hinge gap width, while the $27 \mathrm{~mm}$ prototype high leaker valve represents a moderately higher gap width. In contrast, CM carbon valves have higher leakage rates compared to other current bileaflet mechanical heart prostheses [Akins, 1995]. Leakage rate from the CM $23 \mathrm{~mm}$ clear valve model was observed to be equivalent to that of the $27 \mathrm{~mm}$ prototype high leaker valve model (see Figure 7-1).

\subsubsection{Effect On Flow Structures In Hinge}

This section demonstrates that the hinge gap width influences the washout and Reynolds shear stress levels inside the hinge region. The hinge gap width of the SJM $27 \mathrm{~mm}$ Standard valve provided optimum hemodynamic performance in comparison to the prototype low and high leaker valves. In contrast, the two valves with large and small gap widths have higher thromboembolic potential. This section first discusses the common 
flow features observed in the three $27 \mathrm{~mm}$ bileaflet valves followed by a detailed comparison between the valves that allow us to draw the above conclusions.

\subsubsection{Common Flow Features}

The dimension of the hinge geometry at the primary flat level (Figure 5.11) is identical for the three $27 \mathrm{~mm}$ clear valves (approximately $3.5 \times 3.5 \mathrm{~mm}^{2}$ ). The only variable in this study is the hinge gap width, i.e. the distance between the tip of the leaflet ear and the top of the hinge recess inside the housing (Figure 4-4). The gap-widths estimated using micro-computer tomography were approximately $200 \mu \mathrm{m}, 100 \mu \mathrm{m}$, and $50 \mu \mathrm{m}$ for the 27 mm prototype high leaker, SJM $27 \mathrm{~mm}$ Standard, and the $27 \mathrm{~mm}$ prototype low leaker, respectively (See Section 4.1.1 for details).

As seen in section 7.1.2, velocities between 0.70 and $2.0 \mathrm{~m} / \mathrm{s}$ were typically observed in the leakage jets at the atrial and adjacent corners at the flat level of the hinge geometry, during early systole. Even though the size of the jet at the adjacent corner is smaller than the jet at the atrial corner, its recorded Reynolds shear stress levels were one order of magnitude higher (Table 8-1, Table 8-2). An additional leakage jet was also observed at the lateral corner of the SJM $27 \mathrm{~mm}$ Standard and the $27 \mathrm{~mm}$ prototype high leaker hinges. Even though the studies were conducted with 2D LDV, it became apparent that the flow structures seen inside the hinge mechanism had a dominant out-of-plane velocity component. The common flow features seen in section 7.1.2 are sketched in Figure 8-1. The flow features observed during systole (leakage phase) are shown in Figure 8-1a, 
while the features observed during diastole (forward phase) are summarized in Figure $8-1 b$.

During systole, the leakage jet at the atrial corner consisted of flow coming from the upper region inside the hinge pocket, while the leakage jet at the lateral corner was mainly from the flow below the flat level outside the hinge pocket. The leakage jet at the adjacent corner comprised of flow along the lower edge of the hinge wall from the ventricular side. Flow diversion, seen on the ventricular side of the hinge geometry is attributed to the obstruction caused by the leaflet ear, thus directing the flow towards both the adjacent and lateral corners during the leakage phase resulting in the formation of the leakage jets as shown in Figure 8-1a.

Table 8-1 Peak phase averaged velocities $(\mathrm{m} / \mathrm{s})$ measured within the hinge region of the three $27 \mathrm{~mm}$ bileaflet valves. $\mathrm{NA}=$ not available

\begin{tabular}{c|cccc|cccc}
\hline & \multicolumn{5}{|c|}{ Systole } & \multicolumn{5}{c}{ Diastole } \\
\hline & Flat & $\begin{array}{c}190 \mu \mathrm{m} \\
\text { above }\end{array}$ & $\begin{array}{c}390 \mu \mathrm{m} \\
\text { above }\end{array}$ & $\begin{array}{c}585 \mu \mathrm{m} \\
\text { above }\end{array}$ & $\begin{array}{c}500 \mu \mathrm{m} \\
\text { below }\end{array}$ & $\begin{array}{c}1 \mathrm{~mm} \\
\text { below }\end{array}$ & $\begin{array}{c}3 \mathrm{~mm} \\
\text { below }\end{array}$ & $\begin{array}{c}\text { Thumb } \\
\text {-nail }\end{array}$ \\
\hline $\begin{array}{c}\text { SJM 27 } \\
\text { mm }\end{array}$ & 1.57 & 1.34 & 0.72 & 0.04 & 2.1 & 1.39 & 1.44 & 0.46 \\
$\begin{array}{c}\text { Standard } \\
27 \mathrm{~mm} \\
\text { prototype } \\
\text { low leaker } \\
27 \text { mm } \\
\text { prototype } \\
\text { high leaker }\end{array}$ & 1.85 & 1.94 & 0.84 & 0.06 & 1.31 & 1.42 & 1.41 & 0.41 \\
\hline
\end{tabular}


Table 8-2 Maximum phased averaged Reynolds shear stresses (dynes $/ \mathrm{cm}^{2}$ ) measured within the hinge region of the three $27 \mathrm{~mm}$ valves. $\mathrm{NA}=$ not available

\begin{tabular}{c|ccccccccc}
\hline & Flat & $\begin{array}{c}\text { Systole } \\
\text { above }\end{array}$ & $\begin{array}{c}390 \mu \mathrm{m} \\
\text { above }\end{array}$ & $\begin{array}{c}585 \mu \mathrm{m} \\
\text { above }\end{array}$ & $\begin{array}{c}500 \mu \mathrm{m} \\
\text { below }\end{array}$ & $\begin{array}{c}1 \mathrm{~mm} \\
\text { below }\end{array}$ & $\begin{array}{c}3 \mathrm{~mm} \\
\text { below }\end{array}$ & $\begin{array}{c}\text { Thumb- } \\
\text { nail }\end{array}$ \\
\hline $\begin{array}{c}\text { SJM 27 } \\
\text { mm }\end{array}$ & 7006 & 3808 & 1261 & 8.3 & 2592 & 2073 & 443 & 533 \\
$\begin{array}{c}\text { Standard } \\
27 \text { mm } \\
\text { prototype } \\
\text { low leaker } \\
27 \text { mm } \\
\text { prototype } \\
\text { high leaker }\end{array}$ & 13315 & 7625 & 1147 & 115 & 1320 & 800 & 446 & 247 \\
\hline
\end{tabular}

Low flow velocity of magnitude less than $0.05 \mathrm{~m} / \mathrm{s}$ were observed at both the extreme edges of the atrial and ventricular corners and they may be the result of the flow separation occurring along the edge of the hinge geometry. Fluid in these corners experienced low velocity flow for an extended periods of time and may assist platelet aggregation. The Reynolds shear stress values at the extreme edges of the atrial and ventricular corners were less than $100 \mathrm{dynes} / \mathrm{cm}^{2}$. Low flow was also seen at the adjacent corner next to the hinge wall during systole (Figure 8-1a); however, this region experienced good washout during diastole, which would prevent the buildup of clot elements.

The leakage flow patterns at $190 \mu \mathrm{m}$ above the flat level were similar to those seen at the primary flat level. Leakage jets observed at the atrial and adjacent corners had velocities of $0.54-1.95 \mathrm{~m} / \mathrm{s}$ (Table $8-1$ ). Flow diversion as a result of the obstruction due to the 
leaflet ear was still apparent at the ventricular side of the hinge together with a region of low flow $(<0.05 \mathrm{~m} / \mathrm{s})$ along the edge of the ventricular inflow wall.

During diastole, flow recirculation was observed near the adjacent corner of the hinge at both the primary flat and the $190 \mu \mathrm{m}$ above the flat levels in all the three $27 \mathrm{~mm}$ bileaflet valves (Figure 8-1b). Flows from the atrial and adjacent corners were directed into this recirculation region as a result of the obstruction of the leaflet ear in the open position. Closer inspection of the forward flow patterns at the flat level revealed a second smaller region of flow recirculation at the lateral corner of the hinge that persisted throughout diastole. Low forward flow velocity (less than $0.05 \mathrm{~m} / \mathrm{s}$ ) was observed at the higher measurement levels. However, just like the flat and $190 \mu \mathrm{m}$ above flat level, the pulsatility in the velocity was still evident at the $390 \mu \mathrm{m}$ level, but was absent at the 585 $\mu \mathrm{m}$ above flat level.

The flow patterns below the primary flat level in all three $27 \mathrm{~mm}$ bileaflet valves showed that the fluid behavior was strongly influenced by the fluid structure interaction from the leaflet. Disturbed flow was observed between the two hinge pivots at the $500 \mu \mathrm{m}$ and $1 \mathrm{~mm}$ below flat levels at the start of systole when the leaflets closed. During mid systole, high velocity leakage jets of approximately $1.0 \mathrm{~m} / \mathrm{s}$ were seen emanating from the hinge pockets and from between the closed leaflets. No discernable leakage jets were evident at the $3 \mathrm{~mm}$ below flat level. The reduced number of measurement locations at the $3 \mathrm{~mm}$ level was caused by the obstruction of the laser beams by the leaflets. 


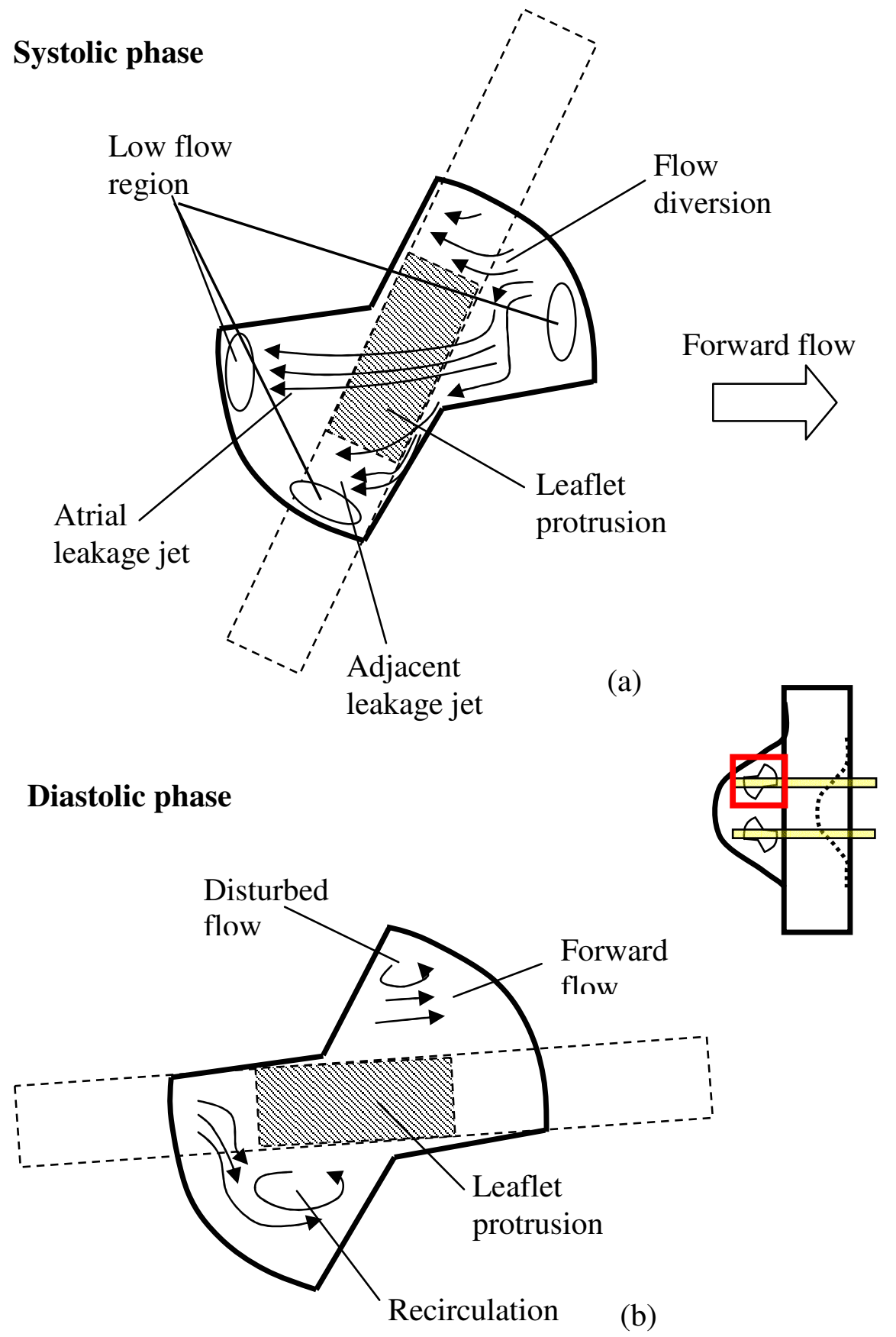

Figure 8-1 Flow features inside the hinge pocket of the three $27 \mathrm{~mm}$ bileaflet clear housing valve models; (a) leakage jets at the atrial side of the hinge socket during systole; (b) recirculation near the adjacent corner during diastole 
The forward flow patterns in the measurement planes below the primary flat during diastole were characterized by three forward flow jets; one central orifice jet bounded by two lateral jets. This flow pattern is typical of the mechanical bileaflet heart valve and was a result of the open leaflet configuration during diastole. A peak forward velocity of approximately $1.0 \mathrm{~m} / \mathrm{s}$ was recorded in these forward flow jets (Table 8-1).

The three $27 \mathrm{~mm}$ bileaflet valves demonstrated complex flow patterns downstream of the thumbnail region. Velocity measurement inside the thumbnail was not possible due to optical inaccessibility (Figure 4-12). The measurement plane at the downstream region of the thumbnail, as shown in Figure 5-14, spanned the entire central orifice region and extended slightly into the lateral flow regions. The measurement plane was taken on the same plane as the primary flat level and was not obstructed by the movement of the leaflets. Flow fields during the acceleration phase of diastole were characterized by strong lateral flows attributed to the lateral orifice jets issuing from the two side orifices when the leaflets were in the open position (see Figure 7-47). These jets imparted on the lateral flows a momentum that led to the subsequent inversion of the lateral jet velocity vectors during the deceleration phase. A retrograde flow of approximately $0.3 \mathrm{~m} / \mathrm{s}$ was seen at the onset of systole when the leaflet closed. This retrograde flow then decreased to a velocity of less than $0.05 \mathrm{~m} / \mathrm{s}$ when the valve fully shut, leaving the gap between the leaflets and the hinge pockets as the only flow pathway for the retrograde jets.

The Reynolds shear stress levels calculated in the current hinge studies during mid systole were above the critical threshold required for the damage of red blood cells and 
platelet activation (Table 8-2). It is generally accepted that the local activation of blood elements is related to both the magnitude of the shear stress and exposure time, and these properties in turn depend on the local fluid dynamics and geometry. [Turitto, 1998] Regions of high shear stress can lead to platelet activation and the destruction of red blood cells [Nemerson, 1991; Slack, 1993; Slack, 1994a; Slack, 1994b; Turitto, 1998]. Damaged and activated blood elements may also accumulate in low flow regions, thus enhancing the formation and deposition of thrombi. Research in recent years involving various mechanical and tissue heart valves has shown the importance of Reynolds shear stress calculations as a means of quantifying the damage to blood elements [Ellis, 1996a; Ellis, 2000a; Hung, 1976; Lu, 2001; Ramstack, 1979; Sallam, 1984]. These studies have reported that hemolysis can occur after exposure to Reynolds shear stress ranging from as low as 400 dyne $/ \mathrm{cm}^{2}$ to 5,600 dyne $/ \mathrm{cm}^{2}$ with exposure times ranging from $10^{2}$ to $10^{-4} \mathrm{~s}$. For platelet activation, the reported Reynolds shear stress threshold is between 100 dyne $/ \mathrm{cm}^{2}$ and 1,000 dyne $/ \mathrm{cm}^{2}$ with exposure times varying from $10^{2}$ to $10^{-2} \mathrm{~s}$. Given that in the current study, the velocity was phase-averaged over a time bin of $20 \mathrm{~ms}\left(10^{-2} \mathrm{~s}\right)$, it is likely that coupled with the elevated Reynolds shear stress observed in the three $27 \mathrm{~mm}$ valves, the flow characteristics in the valve were detrimental to the blood elements.

Results from the these hinge studies identified two regions of high Reynolds shear stress, i.e. the leakage jets at the atrial and adjacent corners. The former experienced Reynolds shear stress in the range between 500 and 1500 dynes $/ \mathrm{cm}^{2}$, while the latter encountered Reynolds shear stress exceeding 5,000 dynes $/ \mathrm{cm}^{2}$. Table 8-3 and Table 8-4 summarize the velocity and Reynolds shear stress levels of the leakage jets at both the atrial and 
adjacent corners of the hinge in the three $27 \mathrm{~mm}$ valves during mid systole. These leakage jets are likely to cause the activation of platelets and hemolysis. Regions conducive to the buildup of blood clots include the flow areas that encounter persistent low flow throughout the entire cardiac cycle, i.e. the regions adjacent to the hinge wall at both the atrial and ventricular sides of hinge, and at the region higher up inside the hinge recess.

The flow velocities and Reynolds shear stress measured below the flat levels were typically lower than those within the hinge pocket. Regions that were most likely to cause platelet activation and hemolysis include the leakage jets observed during systole. The Reynolds shear stress measured downstream of the thumbnail region were typically less than 200 dynes $/ \mathrm{cm}^{2}$, which is unlikely to cause hemolysis. Previous studies by Ellis et al showed that recirculation flows with Reynolds shear stress less than 500 dynes $/ \mathrm{cm}^{2}$ were created in the thumbnail region during mid-diastole [Ellis, 2000a], and it was believed that this may lead to the buildup of micro thombi. It was suggested that the thumbnail region acts as a means of increasing the orifice area for the valve [Ellis, 2000a], but it may simply be a manufacturing artifact (personal communication, Dr Yi-Ren Woo). On hindsight, the thumbnail may act to prevent lockup of the leaflet in SJM bileaflet valves. Lockup of leaflets, i.e. the immobilization of the occluder as a result of the wedging of chordae, suture material, a thrombus, or catheters between the leaflets and the valve housing, tends to occur in bileaflet mechanical heart valves. Indeed, immobilization of the occluders in mechanical prostheses, which can happen either in the open or close position, have been reported in several studies [Hylen, 1972; Starek, 1981]. For bileaflet 
valves, lockup usually occurs between the hinge mechanism and the thumbnail region (Figure 8-2) [Starek, 1981].

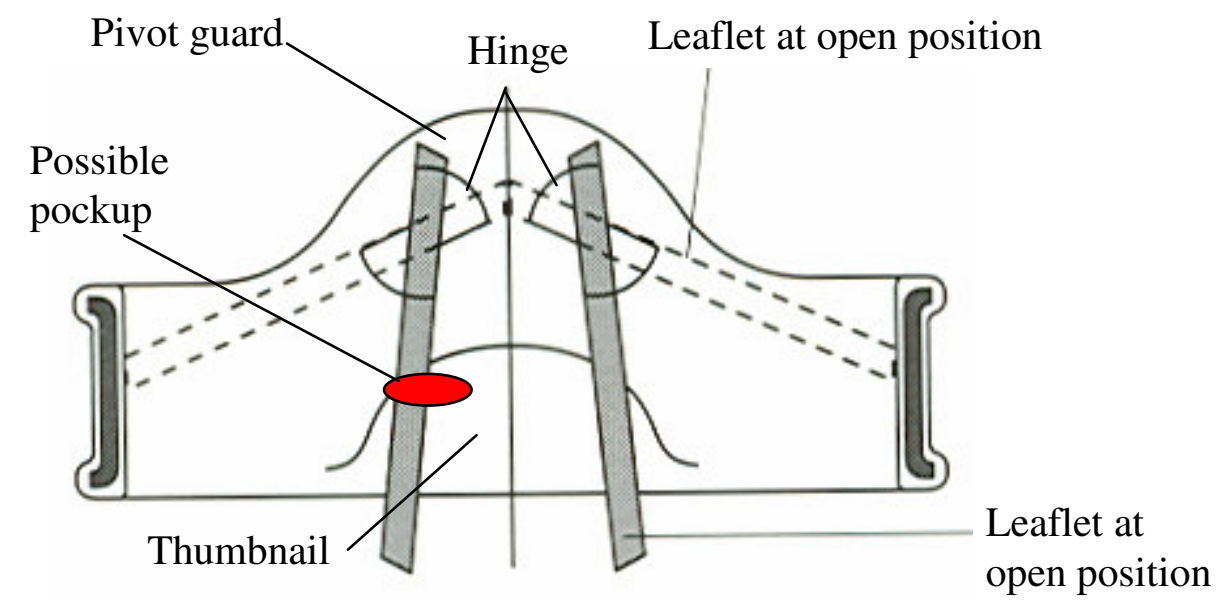

Figure 8-2 Location of the lockup in the SJM bileaflet mechanical heart valve

\subsubsection{Differences In Flow Features And Reynolds Shear Stresses}

The flow characteristics below the primary flat level in the three $27 \mathrm{~mm}$ bileaflet valves were similar, and most of the observed differences in the flow patterns occurred inside the hinge pocket. For that reason the following discussion is based mainly on the measurements in the flat and $190 \mu \mathrm{m}$ above the flat levels. The size and velocity magnitude of the leakage jet during mid systole at the flat level in the $27 \mathrm{~mm}$ prototype high leaker valve were larger and higher than those observed in the SJM $27 \mathrm{~mm}$ Standard and $27 \mathrm{~mm}$ prototype low leaker valves (Table 8-3). The results at $190 \mu \mathrm{m}$ above the flat level showed that the size and velocity magnitude of the leakage jets in the Standard valve were between those of the prototype high and low leaker valves (Table 8-4). The size and velocity magnitude of the atrial leakage jet in the SJM $27 \mathrm{~mm}$ Standard valve 
were comparable to those observed for the prototype high leaker valve, while its adjacent jet had characteristics was similar to those observed in the prototype low leaker valve.

Both the SJM $27 \mathrm{~mm}$ Standard and prototype high leaker valves experienced Reynolds shear stresses that were higher than that observed for the prototype low leaker valve. This observation may be attributed to the larger hinge gap width of the prototype high leaker. Measurement levels in both the SJM $27 \mathrm{~mm}$ Standard and prototype high leaker valves were able to reach up to the $585 \mu \mathrm{m}$ level inside the hinge pocket, while for the prototype low leaker the highest measurement plane inside the hinge region was $390 \mu \mathrm{m}$ above the flat.

The larger gap width in both the SJM $27 \mathrm{~mm}$ Standard and prototype high leaker valves permitted a larger flow volume through the hinge, giving rise to slightly higher flow velocities. Occasionally, air bubbles were observed to be trapped inside the prototype high leaker valve during the LDV experiments (LDV experiments would have to be stopped to purge the air bubbles). This observation demonstrated that the larger gap width in the prototype high leaker may affect the efficient washout of blood elements that may accumulate inside the hinge region. Efficient washout in the hinge region was usually associated with good leakage flow during the systolic phase [Ellis, 2000a]. 
Table 8-3 Comparison of the number of measurement locations in the atrial and adjacent leakage jets at the flat level with their corresponding velocity and RSS range

\begin{tabular}{c|cccc}
\hline Valve Type & Leakage Jets & $\begin{array}{c}\text { \# of } \\
\text { Measurement } \\
\text { Locations }\end{array}$ & $\begin{array}{c}\text { Velocity } \\
\text { range }(\mathrm{m} / \mathrm{s})\end{array}$ & $\begin{array}{c}\text { RSS range } \\
\left.\text { (dynes/cm }^{2}\right)\end{array}$ \\
\hline $\begin{array}{c}\text { SJM } 27 \mathrm{~mm} \\
\text { Standard }\end{array}$ & Atrial Leakage Jet & 34 & $0.35-1.57$ & $160-2359$ \\
$27 \mathrm{~mm}$ & Adjacent Leakage Jet & 10 & $0.27-1.1$ & $130-3475$ \\
$\begin{array}{c}\text { prototype low } \\
\text { leaker }\end{array}$ & Atrial Leakage Jet & 32 & $0.26-0.71$ & $110-1000$ \\
$\begin{array}{c}\text { Adjacent Leakage Jet } \\
\text { prototype high } \\
\text { leaker }\end{array}$ & Atrial Leakage Jet & 8 & $0.28-2.08$ & $200-3051$ \\
& Adjacent Leakage Jet & 19 & $0.35-1.85$ & $400-2825$ \\
& & & $0.34-1.57$ & $1017-4829$ \\
\hline
\end{tabular}

Table 8-4 Comparison of the number of measurement locations in the atrial and adjacent leakage jets at the $190 \mu \mathrm{m}$ above flat level with their corresponding velocity and RSS range

\begin{tabular}{|c|c|c|c|c|}
\hline Valve Type & Leakage Jets & $\begin{array}{c}\text { \# of } \\
\text { Measurement } \\
\text { Locations }\end{array}$ & $\begin{array}{c}\text { Velocity } \\
\text { range }(\mathrm{m} / \mathrm{s})\end{array}$ & $\begin{array}{l}\text { RSS range } \\
\left(\text { dynes } / \mathrm{cm}^{2}\right)\end{array}$ \\
\hline \multirow{2}{*}{$\begin{array}{l}\text { SJM } 27 \mathrm{~mm} \\
\text { Standard }\end{array}$} & Atrial Leakage Jet & 24 & $0.35-1.29$ & $100-1373$ \\
\hline & Adjacent leakage Jet & 7 & $0.33-0.9$ & $100-3072$ \\
\hline \multirow{2}{*}{$\begin{array}{l}27 \text { mm prototype } \\
\text { low leaker }\end{array}$} & Atrial Leakage Jet & 19 & $0.31-0.55$ & $100-1496$ \\
\hline & Adjacent Leakage Jet & 7 & $0.3-0.91$ & $190-3827$ \\
\hline \multirow{2}{*}{$\begin{array}{l}27 \text { mm prototype } \\
\text { high leaker }\end{array}$} & Atrial Leakage Jet & 26 & $0.36-1.24$ & $640-4259$ \\
\hline & Adjacent Leakage Jet & 12 & $0.56-0.84$ & $500-3523$ \\
\hline
\end{tabular}

A high forward flow velocity of approximately $0.4 \mathrm{~m} / \mathrm{s}$ was observed at the atrial side of the hinge geometry in both SJM $27 \mathrm{~mm}$ Standard and prototype low leaker valves. The latter valve also reported similar forward flow velocity on the ventricular side. On the 
other hand, the forward flow velocity in the prototype high leaker valve was typically less than $0.25 \mathrm{~m} / \mathrm{s}$. The strong flow observed in both the SJM $27 \mathrm{~mm}$ Standard and prototype low leaker valves may provide a means of washing out thrombotic materials inside the hinge region and prevent the subsequent accumulation of thrombus. This strong forward flow in the SJM $27 \mathrm{~mm}$ Standard valve may explain why no air bubbles were seen trapped inside the hinge region even though both the SJM $27 \mathrm{~mm}$ Standard and prototype high leaker valves had equivalent leakage jet size and velocity magnitudes during systole.

Additional problem could arise in valves, especially those with larger gap widths, implanted in the mitral position; the washout of the hinge region in the mitral valve was typically less efficient than that in the aortic position [Simon, 2004]. In an adult the mitral flow rate is approximately 12 liter/min, whereas in the aortic position the maximum flow volume attained can be as high as $25 \mathrm{liter} / \mathrm{min}$. The valve in the mitral position experiences a lower forward flow velocity than in the aortic position due to the fact that the mitral valve diameter is usually larger than that of the aortic valve and that diastole is twice as long as systole at a normal heart rate. The lower forward flow rate in the mitral valve makes it vulnerable to the thrombus buildup in the hinge region. This may explain the observation that mitral valves have higher incidence rate of thromboembolic and bleeding complications [Cannegieter, 1994; Steegers, 1999]. In a review of the clinical data on the thromboemobolic and bleeding complications in patients with mechanical heart valves, Cannegieter et al observed that the incidence rates for valve thrombosis and embolism of the mitral valve compared with that of the aortic valve were five times and about 1.5 times as high, respectively [Cannegieter, 1994; Steegers, 1999]. Steeger et al, 
reviewed data from clinical and animal reports and observed that more significant thrombus formation occurred in valves implanted in the mitral than in the aortic position [Steegers, 1999]. Interestingly, it has been reported that the rate of thrombotic occlusion is the highest in the tricuspid mechanical prostheses at 20 percent compared to those in the left side of the heart at 0.5 percent and 0.8 percent [Craver, 1999]. The case in point is that both the tricuspid and mitral valves encounter flow conditions that are conducive to the buildup of thrombi materials. Therefore, valve with a larger hinge gap width implanted in the mitral position may have an increased risk of thrombus buildup in the hinge region.

Besides having a lower forward flow rate, the mitral valve also has the harshest leakage conditions among the four valves. The closing pressure across the aortic valve is about 80 $\mathrm{mmHg}$, while that of mitral valve is $120 \mathrm{mmHg}$. The loading rate for a resting mitral valve is between 750 and 2,000 $\mathrm{mmHg} / \mathrm{s}$, which translates to a higher leaflet closing speed that may further initiate the generation of microbubbles (cavitations) [Chandran, 1998; Guyton, 1991]. Akin reported that valves in mitral position have high a transient signal (HITS) that is twice as high as for those in the aortic position [Akins, 1995]. It is believed that this could be due to the higher pressure drop during the closed phase in the mitral position, which may lead to an increased rate of cavitations, and/or increased blood damage in the hinge region. The prototype low leaker valve had the smallest leakage jet among the three valves due to a more constricted flow area (gap width of approximately $50 \mu \mathrm{m})$ in the hinge pocket. However, a velocity of more than $2.0 \mathrm{~m} / \mathrm{s}$ observed at the adjacent corner (Table 8-3) may constitute an increased risk of cavitations. A low 
pressure point could be created within the high velocity jet. As a result of which, the boiling temperature of the working medium reduces, leading to the local formation of vapor bubbles. The subsequent implosion of these vapor bubbles could lead to destruction of the blood elements as well as pitting of the valve surface.

Most of the cavitations research with heart valve prostheses are dedicated to studying squeeze flow through the interstitial gap between the closing occluder and the valve housing. This squeeze flow has been linked to cavitations seen in current mechanical heart valves [Bluestein, 1994; Makhijani, 1996]. Numerical and in vitro experiments have shown that the pattern of squeeze flow is related to the moving boundaries, i.e. the approaching leaflets toward the valve housing, and the velocity of the closing leaflets [Bluestein, 1994; Graf, 1991; Makhijani, 1996]. Typical closing velocities at the occluder tip range from 1 to $3 \mathrm{~m} / \mathrm{s}$. With a gap width of approximately $50 \mu \mathrm{m}$, the peak squeezeflow velocity has been estimated to be as high as $30-40 \mathrm{~m} / \mathrm{s}$ with corresponding negative pressures of approximately $15 \mathrm{MPa}$ [Bluestein, 1994; Graf, 1991; Makhijani, 1996]. With a gap width of $48 \mu \mathrm{m}$ and an occluder tip velocity of $4 \mathrm{~m} / \mathrm{s}$, Bluestein et al obtained a peak squeeze-flow velocity of approximately $30 \mathrm{~m} / \mathrm{s}$. Makhijani demonstrated a peak squeeze-flow velocity as high as $150 \mathrm{~m} / \mathrm{s}$ with gap width of only $0.3 \mu \mathrm{m}$. On the other hand, He showed a peak squeeze-flow velocity of less than $10 \mathrm{~m} / \mathrm{s}$ through a gap width of approximately $100 \mu \mathrm{m}[\mathrm{He}, 2001]$. At present, no experiments have been conducted to study cavitations in the hinge region of the bileaflet mechanical heart valves. 


\subsubsection{Comparison Of Current $27 \mathrm{~mm}$ Prototype Valves Studies With Previous Hydrogen Bubble Experiments}

In a recent study, Shu et al made use of hydrogen bubbles to visualize the hinge flow structures of the Medtronic Advantage and SJM valves [Shu, 2003]. The experiment provided an excellent corroboration to the observed flow structures in the current $27 \mathrm{~mm}$ SJM prototype hinge studies. The hydrogen bubble experiments showed slow moving fluid at the thumbnail region was drawn toward the ventricular side of the hinge geometry during systole, and then once inside the hinge pocket, the flow was accelerated toward the atrial corner of the hinge geometry giving rise to the leakage jet at the atrial corner (Figure 8-3). The leakage jet at the adjacent corner was not visible in these experiments because of the presence of the leaflet ear. However, a leakage jet was evident issuing from the gap between the closed leaflets. During diastole, a strong recirculation structure was created at the adjacent corner of the hinge geometry, with low retrograde flow seen along the leaflet wall flowing toward the atrial side of the hinge. A forward jet was also seen issuing from the recirculation zone into the central orifice flow mixing with the retrograde flow inside the thumbnail region. The flow reversal observed downstream of the thumbnail region was also evident in the hydrogen bubble experiments and was attributed to both the opening motion of the leaflets as well as the high velocity jets from the lateral orifices of the valve. 

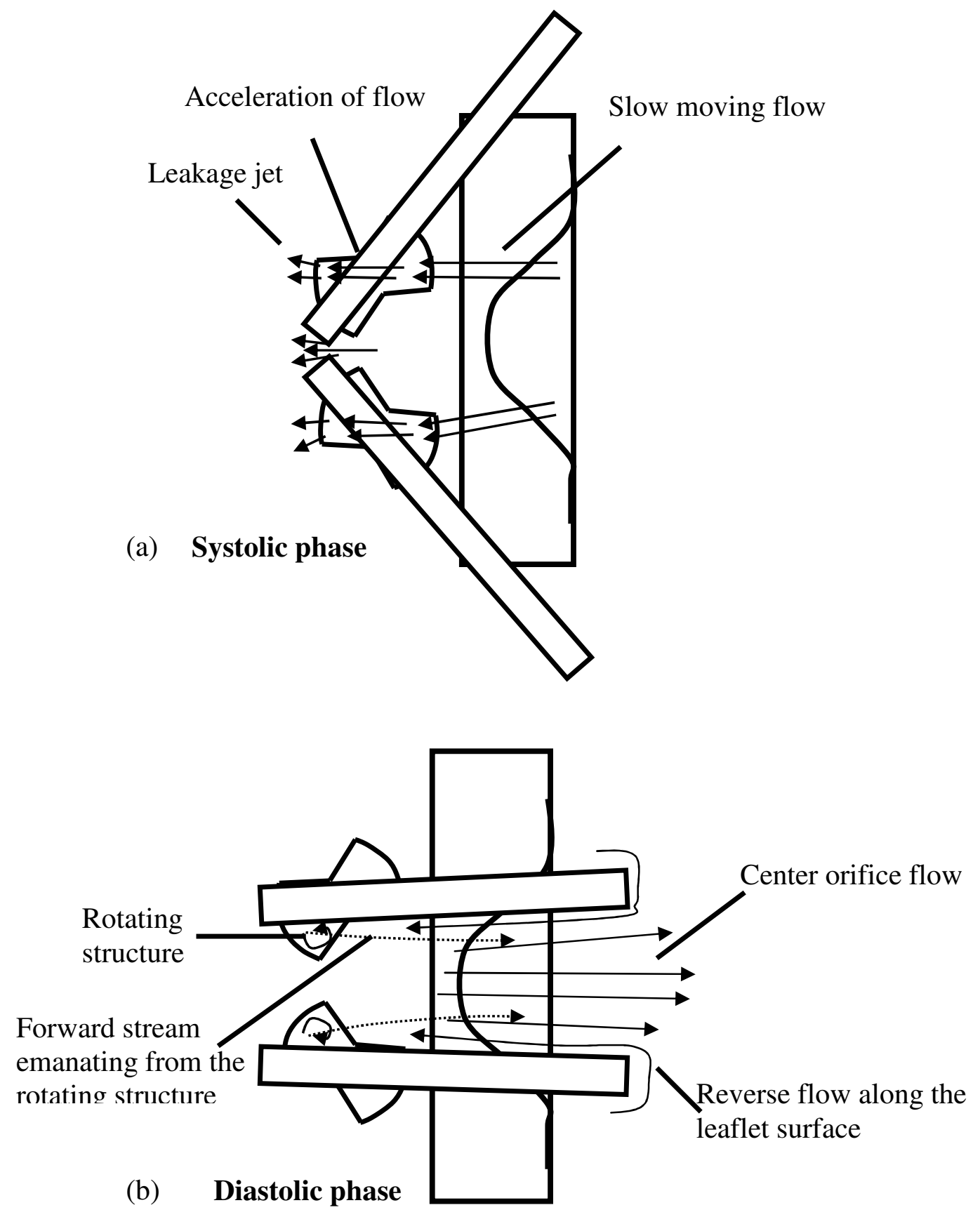

Figure 8-3 Sketch of the flow characteristics of the SJM bileaflet mechanical heart valve as observed in the hydrogen bubble experiments [Shu, 2003] 


\subsubsection{Integration Of Previous Blood Experiments With Observed Flow Structures}

Travis et al performed blood studies comparing the effect of the hinge gap width of three $27 \mathrm{~mm}$ bileaflet carbon valves during steady leakage flow (the static leakage rate from these three valves was discussed in Section 8.1) [Travis, 2001a]. The authors demonstrated that the leakage gap width had a significant effect on both platelet activation and anionic phospholipid exposure initiated by leakage flow through a prosthetic heart valve, i.e. both $27 \mathrm{~mm}$ prototype high and low leaker valves had higher activated platelet counts compared with the SJM $27 \mathrm{~mm}$ Standard valve. Even though the activated platelet count difference between the SJM $27 \mathrm{~mm}$ Standard and prototype low leaker valves was not significant, the author did show that the prototype high leaker valve produced significantly more platelet activation than the SJM $27 \mathrm{~mm}$ Standard valve. These findings are consistent with the findings of the current research, which showed that the prototype high leaker valve had comparatively larger leakage regions with higher Reynolds shear stress than both the SJM $27 \mathrm{~mm}$ Standard and prototype low leaker valves. Travis et al postulated that different mechanisms were responsible for the observed elevated platelet activation seen in both the $27 \mathrm{~mm}$ prototype high and low leaker valves [Travis, 2001b]. In the prototype high leaker, the increase in platelet activation may be due to increased turbulence inside the hinge pocket as a result of increased hinge gap width, while in the prototype low leaker, the dominant force behind the observed platelet activation may be the viscous shear stresses. The leakage jet turbulence is governed by the pivot geometry and the dimensionless Reynolds number:

$$
R_{e}=\frac{\rho U l}{\mu}
$$


where $l(\mathrm{~m})$ is the governing length scale and is equivalent to the hinge gap width, $\rho\left(\mathrm{kg} / \mathrm{m}^{3}\right)$ is the density of the working medium, $\mu\left(\mathrm{m}^{2} / \mathrm{s}\right)$ is the kinematic viscosity of the working medium and $U(\mathrm{~m} / \mathrm{s})$ is the measured mean velocity. Using the maximum velocity obtained in the current LDV hinge studies, the peak Reynolds numbers for the $27 \mathrm{~mm}$ prototype high leaker, SJM $27 \mathrm{~mm}$ Standard and $27 \mathrm{~mm}$ prototype low leaker valves are 105, 44 and 29, respectively. Even though these Reynolds numbers are well below the customary threshold for turbulent flow, the irregular geometry of the hinge pivot may induce turbulence at very low Reynolds numbers. Reynolds shear stresses encountered in both the hinge gap as well as in the emitted leakage jets may disrupt the blood cells leading to an increased risk of thrombosis. Assuming the CM valve has the same gap width as the $27 \mathrm{~mm}$ prototype high leaker valve, its calculated Reynolds number is approximately 180.

In addition to its influence on leakage flow rate and turbulence, the leakage gap width also influences the viscous stresses that blood cells may experience when flowing through the hinge pocket. It has been estimated that blood flow through the hinge gap has a shear rate of over $100 \mathrm{~s}^{-1}$ and can be modeled as Newtonian fluid [Schneck, 1995]. Assuming that flow within the leakage gap can be modeled as Newtonian flow through a long, narrow slit, where $l$ is the pivot gap width:

$\tau_{\max }=\frac{4 \mu U_{\max }}{l}$

where $U_{\max }$ is the maximum leakage velocity measured inside the hinge region. Table 8-5 compares the maximum viscous shear calculated inside the hinge region of the three 27 
$\mathrm{mm}$ bileaflet heart valves. The largest viscous shear stresses in the $27 \mathrm{~mm}$ prototype high, SJM $27 \mathrm{~mm}$ Standard and $27 \mathrm{~mm}$ prototype low leaker valves are, respectively, 1,350, 2,300 and 5,880 dynes/ $\mathrm{cm}^{2}$. The prototype low leaker valve had a significantly higher viscous shear stress level, which may be responsible for the observed increase in platelet activation. The calculated viscous shear stress level for the CM valve, assuming the same gap width as the prototype high leaker valve, was approximately $2,330 \mathrm{dynes} / \mathrm{cm}^{2}$, which is equivalent to that of the SJM $27 \mathrm{~mm}$ Standard valve. It should be noted that equation 81 assumed only laminar and steady conditions, while the flow conditions inside the hinge recess were unsteady. Therefore, equation 8-1 may have underestimated the viscous shear stress values inside these hinge recess.

Table 8-5 Comparison of the calculated maximum viscous shear stress inside the hinge recess for the three $27 \mathrm{~mm}$ bileaflet mechanical heart valves

\begin{tabular}{|c|c|c|c|}
\hline Valve Type & $\begin{array}{c}\text { Hinge gap width } \\
(\mu \mathrm{m})\end{array}$ & $\begin{array}{l}U_{\max } \\
(\mathrm{m} / \mathrm{s})\end{array}$ & $\begin{array}{c}\tau_{\max } \\
\left(\text { dynes } / \mathrm{cm}^{2}\right)\end{array}$ \\
\hline $\begin{array}{l}\text { SJM } 27 \mathrm{~mm} \\
\text { Standard }\end{array}$ & 100 & 24 & 2,300 \\
\hline $\begin{array}{l}27 \text { mm prototype } \\
\text { low leaker }\end{array}$ & 50 & 19 & 5,880 \\
\hline $\begin{array}{l}27 \text { mm prototype } \\
\text { high leaker }\end{array}$ & 200 & 26 & 1,350 \\
\hline $\mathrm{CM} 23 \mathrm{~mm}$ & 200 & 3.2 & 2,330 \\
\hline
\end{tabular}

\section{$\underline{8.2 \text { Effect of Valve Designs on Hinge Flows }}$}

This section addresses the specific aim: To study the flow fields in the hinge region of different bileaflet mechanical heart valve designs and to evaluate their potential to 
cause thrombus formation. The objective of this specific aim is to identify the hemodynamic significance of the micro flow structures within the hinge region of the CarboMedics bileaflet MHV. Cross valve assessment was performed by comparing the micro flow fields between the current CM, with the SJM Regent, Medtronic Advantage (MA), and Medtronic Parallel (MP) bileaflet valve studies conducted in our laboratory.

\subsubsection{Hinge Flow Structures in CM 23 mm}

This study shows that the different hinge profile could significantly influence the fluid dynamics inside the hinge geometry, and that these complex hinge flow fields could determine the success of the bileaflet valve. The order of this discussion section begins with the general flow features of the CM $23 \mathrm{~mm}$ hinge flow, followed by a detailed comparison of the flow structures in various hinge designs, including the current three 27 mm bileaflet clear housing valves, and how these geometries influence the valve's potential for thrombus formation.

\subsubsection{General Flow Features}

For purpose of comparison, the flow field measurements in the CM hinge study were made in selected regions at similar locations to those in previous SJM valve hinge studies [Ellis, 1999; Ellis, 2000a; Ellis, 2000b]. The leakage flow field within the hinge region of the $\mathrm{CM}$ valve was characterized by a dynamic washout flow pattern, which effectively minimized the formation of vortex structures and complex flows. During systole, the highest leakage velocity and its corresponding Reynolds shear stresses tended to occur in the regions near the atrial and lateral corners of the hinge recess. These peak values 
usually occurred the moment after the impact of the valve leaflets against the valve housing during closure. It is postulated that this sudden closure of the valve leaflets may contribute to the effective washout of any deposited blood elements or pooled coagulative factors in vivo.

During the forward flow phase, the peak velocities through the hinge were in the range of 0.3 to $0.77 \mathrm{~m} / \mathrm{s}$ with the highest forward flow velocity observed at the $390 \mu \mathrm{m}$ level (Table 8-6). Forward flow velocities were typically low, mostly on the order of $0.3 \mathrm{~m} / \mathrm{s}$ in areas close to the boundary of the hinge. This was because most of the forward flow was through the open leaflets with less volume being directed into the hinge. At the $190 \mu \mathrm{m}$ level, three distinct regions of flow were observed during mid diastole even though the average velocity throughout the hinge was low. The forward flow at the adjacent corner of the hinge was observed only at the $190 \mu \mathrm{m}$ level within the hinge. This may be because the flow of the fluid particles at this level, unlike those at $390 \mu \mathrm{m}$ and primary flat, were being blocked and directed over the side of the leaflet pivot. This 'channeled' flow may have caused the elevated velocity magnitudes observed at this phase of the cycle.

The leakage jets observed at the atrial, adjacent and lateral corners of the CM hinge geometry at the flat level recorded Reynolds shear stress levels (approximately 5,000 dynes $/ \mathrm{cm}^{2}$ ) that exceeded the thresholds required for the destruction of red blood cell and the activation of platelets. Similar elevated Reynolds shear stress levels were also observed at the atrial and lateral corners at $190 \mu \mathrm{m}$ above the flat level. However, the 
Reynolds shear stress obtained during the diastolic phase were much lower at approximately 200 dynes $/ \mathrm{cm}^{2}$.

Table 8-6 Peak phase averaged velocities and RSS measured within the hinge region of the CM $23 \mathrm{~mm}$ clear housing valve

\begin{tabular}{c|ccc}
\hline \multirow{2}{*}{ Elevation } & $\begin{array}{c}\text { Peak phase } \\
\text { averaged forward } \\
\text { flow velocity }(\mathrm{m} / \mathrm{s})\end{array}$ & $\begin{array}{c}\text { Peak phase averaged } \\
\text { leakage velocity } \\
(\mathrm{m} / \mathrm{s})\end{array}$ & $\begin{array}{c}\text { Peak phase averaged } \\
\text { leakage } R S S \\
\left(\text { dynes } / \mathrm{cm}^{2}\right)\end{array}$ \\
\hline Flat & 0.54 & 3.17 & 5,510 \\
$190 \mu \mathrm{m}$ above & 0.77 & 2.91 & 5,640 \\
$390 \mu \mathrm{m}$ above & 0.3 & 2.52 & 4,380 \\
$1 \mathrm{~mm}$ below & 1.0 & 0.5 & 543 \\
\hline
\end{tabular}

Since the CM valve lacks the thumbnail region, the measurements taken $1 \mathrm{~mm}$ below the flat level showed only the hinge mechanism. The flow at $1 \mathrm{~mm}$ below the flat level is outside the hinge. The flow velocity at this level was relatively low in both the forward and backward directions with the highest velocity magnitude observed close to the central gap (b-datum line) between the two leaflets. The flow arrows in Figure 7-83, Figure 7-84, Figure 7-85 showed that the position of the leaflets had a more dominant effect on the flow at this level than it did in the levels within the hinge recess. The energy of the leakage jet within the hinge may have already dissipated before it could have any visible effect on the flow $1 \mathrm{~mm}$ below the flat. It is speculated that a measurement level closer to the primary flat may show the influence of the hinge flow. Two regions of high shear stress were observed at this level; one at the gap between the two leaflets and another at the right lateral corner. The former may be due to the decreasing gap area between the closing leaflets, while the latter may be a result of the constricting flow area between the 
housing wall and the moving leaflet. However, this phenomenon was not observed at the left lateral side of the flow, which probably meant that it may be unique to the particular valve studied. This slight flow asymmetry observed at $1 \mathrm{~mm}$ below the flat could be due to asynchronous leaflet motion. The Reynolds shear stress levels at $1 \mathrm{~mm}$ below the flat level were less than those observed in the hinge region, ranging between $50-600$ dynes $/ \mathrm{cm}^{2}$.

\subsubsection{Comparison of The CM $23 \mathrm{~mm}$ With The Three $27 \mathrm{~mm}$ Bileaflet Heart Valves}

Direct comparison of the hinge flow structures observed in the current SJM $27 \mathrm{~mm}$ Standard and CM $23 \mathrm{~mm}$ clear valve models was possible due to the similar incremental resolution of the measurement locations. Both valve designs displayed a strong atrial jet during systole, but the SJM $27 \mathrm{~mm}$ valve had much larger leakage jets at both the adjacent and lateral corners (see sections 7.1.2 and 7.2.1). On the other hand, the CM valve recorded higher peak leakage velocities in the hinge region than the SJM valve (see Table 8-1 and Table 8-6). During diastole, the CM valve demonstrated a higher forward velocity at the lateral corner but a weaker recirculation flow at the adjacent corner than the SJM valve, and this may be due to the different hinge geometry of the CM valve. The hinge profile of the $\mathrm{CM}$ can be likened to that of a parallel plate channel; hence the measurement level at $390 \mathrm{~mm}$ above flat had more measurement locations (110 locations) than in the SJM valve (approximately 20 locations). Consequently, the number of measurement locations above the flat level inside the $\mathrm{CM}$ hinge pocket ended more abruptly than in the SJM valve, which had a more gradual reduction in the number of measurement locations above the flat (See Table 5-1, Table 5-2 for comparison). Flow 
measurements below the flat level demonstrated that both the $\mathrm{CM}$ and three $27 \mathrm{~mm}$ valves had similar flow profiles during diastole and systole.

The major design differences between the CM and the SJM (Regent and Standard) valves include: (i) differences in the valve hinge geometry; (ii) incorporation of the pivot guards in the SJM design; (iii) enlargement of the internal orifice diameter in the Regent design; (iv) variations in gap width tolerances between the different manufacturers; and (v) probably to a lesser extent, the incorporation of the thumbnail region in the SJM valve design. The SJM hinge mechanism has a streamlined and curved profile that allows the fluid traveling through it to follow the contour of the inside surface, thereby effectively reducing the creation of a vortex within the hinge. The $\mathrm{CM}$ valve design has a hinge geometry that is similar to that of the SJM valve, except that it has sharper corners and less streamlined edges. It also does not have a true thumbnail region.

A closer inspection of the hinge geometry of the two valves revealed more than a subtle difference in the hinge designs (Figure 4-7). A cross-sectional view of the hinge showed that instead of a smooth, curved recess machined into the valve housing as seen in the SJM valve designs, the CM hinge geometry has an angulated recess. Additionally, in the $\mathrm{CM}$ design the leaflet projections within the recess were observed not to sweep all the way to the edge of the hinge geometry. Therefore visible regions of 'unmated' spaces were left at each corner of the hinge geometry during both the fully closed and fully open positions of the leaflets. Thus angulated recess may offer more gap space in between the leaflet projection and the valve housing, thereby allowing more fluid to flow through the 
hinge during the systolic phase giving rise to higher leakage jet velocities and corresponding Reynolds shear stresses. The presence of these 'unmated' spaces at the hinge corners may further aggravate the leakage problem leading to elevated leakage jet velocities.

The basic valve construction and hinge design of the prototype $27 \mathrm{~mm}$ high and low leaker valves were similar to those of the SJM $27 \mathrm{~mm}$ Standard, except for the varying hinge gap width. Like the CM valve, both the prototype leaker valves also demonstrated a strong leakage jet at the atrial corner; however, as in the SJM $27 \mathrm{~mm}$ Standard valve, the two prototype valves had larger leakage jets at the adjacent and lateral corners. The leakage velocities recorded in the two prototype valves were lower than those measured in the CM valve, but the Reynolds shear stress values recorded in the leakage jets of the $\mathrm{CM}$ valve were comparable to those measured in the $27 \mathrm{~mm}$ prototype high leaker valve. This may be explained by the fact that the two valves had similar static leakage rates (Figure 7-1). These findings were consistent with those reported by Akin et al, who showed that the CM carbon valves typically have higher static leak rates than other valves such as the Starr-Edwards, Omniscience and Medtronic-Hall [Akins, 1995]. However, an air bubble was observed trapped inside the prototype high leaker but not inside the CM valve. The flat and angulated profile of the CM hinge socket may enable better washout characteristics during systole, while the concave hinge profile of the prototype high leaker valve could increase the potential for thrombi buildup at the top region of the hinge socket (Figure 8-4). During diastole, the CM displayed a stronger forward flow in the lateral corner but a weaker recirculation flow in the adjacent corner 
than the two leaker valves (see Table 8-1 and Table 8-6). Such observations can be attributed to the different hinge geometry for the two valves. Flow measurements below the flat level demonstrated that both the $\mathrm{CM}$ and the two $27 \mathrm{~mm}$ leaker valves had similar flow profiles during diastole and systole.

(a)

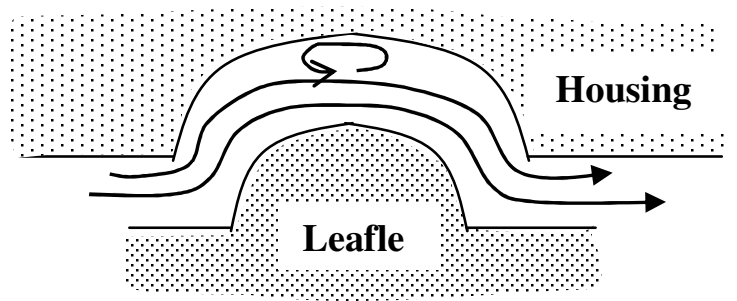

(b)

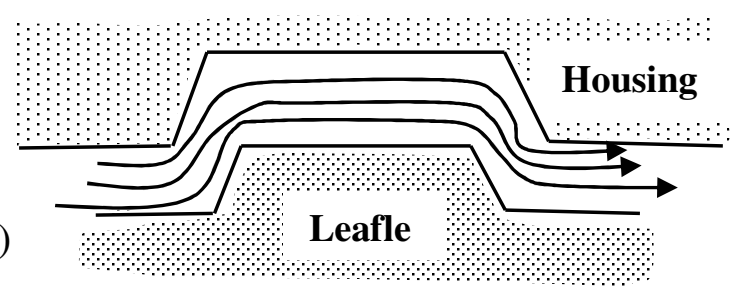

Figure 8-4 The comparison of the washout flow characteristics during $\mathrm{s}$ in the mitral hinge studies; (a) The $27 \mathrm{~mm}$ prototype high leaker valve had a less efficient forward washout and a higher propensity for air bubbles to recirculate inside the pivot region, (b) The CM $23 \mathrm{~mm}$ valve had better washout due to a flatter hinge profile

An interesting aspect of the LDV hinge studies was the observed leaflet motion during the cardiac cycle. These observation applied to both the CM $23 \mathrm{~mm}$ and the three $27 \mathrm{~mm}$ bileaflet clear housing valves. Besides the sweeping motion, the leaflets were also seen to translate in motion during both the opening and closing phases of the cycle. At valve closure the leaflets bounced against the valve housing before coming to rest. This slamming motion, as discussed earlier may initiate cavitations. In addition to the sweeping and translating motion, the leaflets also moved vertically up and down inside the hinge pocket. This was evident during the closing and opening phases of the cardiac 
cycle. (see Figure 7-7, time bin 1). The transition period, which corresponded to the accelerating phase of the cardiac cycle, was characterized by low flow and lasted for about 40 - $60 \mathrm{~ms}$. During this time, the leaflets appeared to be 'floating' in the valve housing. The influence of these leaflet motions in combination with the complex hinge geometry contributed to the observed complex hinge flow fields. In addition, it was apparent from the hydrogen bubble experiments that the closing speed of the leaflets was much higher than the opening speed.

\subsubsection{Comparison Of Current Hinge Studies With Previous Experiments}

The importance of the influence of hinge geometry has been shown by several studies. A recent LDV study by Steeger et al compared the leakage fluid dynamic characteristics of a SJM Standard to those of a CM bileaflet valve of similar tissue annulus size in the mitral position [Steegers, 1999]. They reported a higher leakage jet velocity of $1.7 \mathrm{~m} / \mathrm{s}$ and a maximum Reynolds shear stress of 800 dyne $/ \mathrm{cm}^{2}$ for the $\mathrm{CM}$ design and corresponding values of $1.0 \mathrm{~m} / \mathrm{s}$ and $450 \mathrm{dyne} / \mathrm{cm}^{2}$ for the SJM Standard design. This is consistent with the measurements obtained in the present CM valve study. Higher leakage velocities and Reynolds shear stress values were obtained in the hinge region of a $23 \mathrm{~mm} \mathrm{CM}$ valve $\left(3.17 \mathrm{~m} / \mathrm{s}\right.$ and 5,640 dyne $\left./ \mathrm{cm}^{2}\right)$ compared to results obtained in a previous study in our laboratory with a $23 \mathrm{~mm} \mathrm{SJM} \mathrm{Regent} \mathrm{valve}(1.5 \mathrm{~m} / \mathrm{s}$ and 2, 600 dyne/ $\mathrm{cm}^{2}$ [Ellis, 1999] see Table 8-7). Current SJM $27 \mathrm{~mm}$ Standard valve had a velocity magnitude of $1.57 \mathrm{~m} / \mathrm{s}$ and corresponding Reynolds shear stress of 7,006 dyne/ $\mathrm{cm}^{2}$. A similar study by Meyer et al reported equivalent order of magnitude velocities and stresses in both valve designs [Meyer, 2001]. They observed a maximum leakage jet 
velocity of $2.2 \mathrm{~m} / \mathrm{s}$ and a maximum Reynolds shear stress of 3,600 dyne $/ \mathrm{cm}^{2}$ for the CM valve, with corresponding values of $0.7 \mathrm{~m} / \mathrm{s}$ and 1,800 dyne $/ \mathrm{cm}^{2}$ for the SJM Standard valve. Studies with the MP valve reported high leakage velocity and Reynolds shear stress up to $4 \mathrm{~m} / \mathrm{s}$ and 8,000 dynes $/ \mathrm{cm}^{2}$, respectively. These studies [Ellis, 1996a; Gross, 1996; Healy, 1998] suggested that the abrupt changes in the MP hinge geometry lead to the formation of stagnation sites and regions of elevated levels of turbulence. Hinge study with the Medtronic Advantage (MA) $29 \mathrm{~mm}$ valve by Saxena and coworkers recorded maximum leakage jet velocity of $0.9 \mathrm{~m} / \mathrm{s}, 1.6 \mathrm{~m} / \mathrm{s}$ and $1.8 \mathrm{~m} / \mathrm{s}$, respectively, in the hinge at zero depth, one-third depth, and two-thirds depth levels into the hinge recess [Saxena, 2003]. The locations of the measured velocities are in contrast with those observed in all current hinge studies, where the maximum velocity is typically recorded at the flat levels. This observation may be explained by the geometry of the MA hinge mechanism; the MA bileaflet valve incorporates a butterfly hinge design similar to the SJM valve with a unique bi-level flat design feature. The new feature incorporates a secondary flat at the inflow (ventricular side for valve at mitral position) region, which helps to promote flow washing characteristics through the hinge during the forward and leakage flow phases. Elevated leakage velocities were typically observed at the atrial and lateral corners of the MA hinge geometry. This flow trend is similar to that observed in the current CM valve study, but in contrast to SJM $27 \mathrm{~mm}$ studies (see Figure 8-1). The forward flow velocities observed in the CM $23 \mathrm{~mm}$ and SJM $27 \mathrm{~mm}$ Standard valves $(0.5 \mathrm{~m} / \mathrm{s})$ were comparable to those observed in the SJM $23 \mathrm{~mm}$ Regent and the MA $29 \mathrm{~mm}$ valves $(0.5 \mathrm{~m} / \mathrm{s})$. 
Table 8-7 Comparison of the velocity magnitudes and Reynolds shear stresses from various valve designs

\begin{tabular}{c|cc}
\hline & $\begin{array}{c}\text { Peak phase averaged } \\
\text { leakage velocity }(\mathrm{m} / \mathrm{s})\end{array}$ & $\begin{array}{c}\text { Peak phase averaged leakage } \\
\text { TSS }\left(\text { dynes } / \mathrm{cm}^{2}\right)\end{array}$ \\
\hline MP 27 mm & 4.0 & 8,000 [Ellis, 1996a] \\
SJM 23 mm SHP & 1.5 & 2,600 [Ellis, 2000b] \\
(Regent) \\
MA 29 mm
\end{tabular}

Given the lower spatial resolution in previous hinge studies, it was sometimes difficult to make a direct comparison of the flow structures that were observed inside the various hinge designs. However, general flow field patterns could still be derived from these studies to provide some insight into the dynamics of the hinge flows. The results from present mitral hinge studies were consistent with those from previous experiments [Ellis, 1999; Ellis, 1996a; Ellis, 1996b]. High leakage jets were typically observed at the atrial side of the hinge geometry. A hinge study with the SJM $17 \mathrm{~mm}$ HP showed the leakage jet at the adjacent corner [Ellis, 2000a], while study with the SJM $23 \mathrm{~mm}$ Regent valve recorded a leakage jet at the atrial corner during mid systole [Ellis, 2000b]. Noticeable recirculation structures could also be seen at the adjacent corner in both of these hinge studies during mid-diastole.

The Reynolds shear stress levels measured in the hinge region of the MA $29 \mathrm{~mm}$ were comparable to those obtained for present SJM and CM hinge studies, typically in the range 3,000 to 6,900 dynes $/ \mathrm{cm}^{2}$. The leakage jet velocities for the three $27 \mathrm{~mm}$ bileaflet 
heart valves were comparable to those obtained in previous SJM hinge studies [Ellis, 1996a; Ellis, 2000a; Ellis, 2000b], but the Reynolds shear stress values were not; they were twice as high as those reported in previous studies. On the other hand, the peak Reynolds shear stress $\left(8,000\right.$ dynes $\left./ \mathrm{cm}^{2}\right)$ measured in the MP $27 \mathrm{~mm}$ hinge study was comparable to that found in the current $27 \mathrm{~mm}$ bileaflet valve studies. However, the velocity magnitude $(4 \mathrm{~m} / \mathrm{s}$ ) obtained in the MP hinge was almost double that for all the present SJM and CM hinge studies. One main difference between the current study compared with previous hinge studies was that the spatial resolution used in this research was much higher than that in previous hinge experiments. As many as 250 measurement locations were acquired at the primary flat level for the current SJM $27 \mathrm{~mm}$ hinge studies (168 measurement locations were taken at the flat level in the CM $23 \mathrm{~mm}$ clear valve). This constitutes approximately 10 times more measurement locations than in previous studies (approximately 20 measurement locations at the flat level) [Ellis, 1999; Ellis, 1996a; Ellis, 1996b]. The larger number of measurement locations provide better spatial resolution and are able to resolve smaller flow structures. Therefore it is more likely that higher Reynolds shear stress values would be obtained. Indeed, hinge studies performed by Simon have shown that higher Reynolds shear stress levels were typically observed in hinge studies with more measurement locations, even though the velocity magnitudes were was equivalent [Simon, 2004]. It appears that increased spatial resolution leads to increased Reynolds shear stress values, but not necessarily higher velocity magnitudes. It therefore possible that the previous hinge studies underestimated the Reynolds shear stress levels. 


\subsection{Flow Quantification in Aortic Polymeric Valves}

This section addresses the specific aim: To quantify and analyze the flow fields in the immediate vicinity of a polymeric heart valve with different leaflet thicknesses and commissural designs. The flow fields are then related to their potential to cause blood damage and thromboembolic complications, including thrombus deposition on the valve super structure. The objective of this specific aim is to assess the significance of the polymeric valve design on the valve's potential for thrombus formation.

\subsubsection{Static Leakage}

The static leakage rates for the three Aortech prototype polymeric heart valves with a pressure drop of $120 \mathrm{mmHg}$ across the valve, with respect to the leaflet thickness and commissural designs are shown in Figure 7-87. These leakage rates were measured at both room $\left(22^{\circ} \mathrm{C}\right)$ and physiological body $\left(37^{\circ} \mathrm{C}\right)$ temperatures. An additional testing condition was the inclusion of valve oscillations during the experiments to account for the oscillations observed with these polymeric valves during valve closure in the pulsatile flow loop. The static leakage study demonstrated that the temperature has a smaller effect on the leakage volume than the valve oscillations.

Three parameters are investigated in the static leakage experiments, namely: (1) valve types; (2) fluid temperature; and (3) valve oscillation. Figure 7-87 demonstrated that both valve types and valve oscillation influence the leakage volume, and that the fluid temperature has a lesser effect than the valve oscillation on the leakage volume. The 
leakage volumes of the three prototype valves tested without oscillation were significantly different at the two temperatures $(\mathrm{p}<0.05)$ (Figure 7-87). At the higher temperature the leaflets became more compliant consequently forming a better seal to reduce the leakage flow through the valve. However, at room temperature the less compliant leaflets could not close sufficiently to reduce leakage flow. The effect of temperature was decreased when the valves were tested with oscillations resulting in more comparable leakage volumes at the two temperatures $(\mathrm{p}<0.05)$. In addition, the leakage volume obtained with oscillations was significantly higher than that obtained without oscillations $(\mathrm{p}<0.05)$.

\subsubsection{Effect Of Valve Design on Flow Fields Inside and in the Vicinity of the Polymeric Valves}

The polymeric valve studies, to our knowledge, present for the first time a detailed characterization of the flow fields both inside and outside a polymeric heart valve. Design parameters such as the leaflet thickness and commissural design influenced the flow structures inside and downstream of the valve. It was found that the valve with thicker leaflets tended to have greater leakage flow during the diastolic phase, and that neither an open nor a closed commissural design was able to ensure efficient washout during systole (to prevent thrombus formation) near the stent inside the valve. Thus, the flow patterns inside the valve may provide an explanation for the observed blood clots found along the inflow stent region of the valve in the animal experiments [Daebritz, 2003; Daebritz, 2004]. This study made use of 2D LDV and 3D PIV techniques to investigate the flow 
field patterns inside three polymeric heart valves. A detailed comparison of the two techniques can be found in section 8.4 of the Discussion chapter.

The polymeric valve studies are discussed in the following sequence; 1) the common flow structures of the three polymeric valves; 2) the differences in the flow fields between the three valves; 3) the comparison of the current polymeric valve studies with previous experiments; and lastly 4) a discussion on the influence of valve design on potential thrombus formation.

\subsubsection{Common Flow Structures}

For ease of discussion, the flow patterns outside the valve and downstream of the leaflet trailing edge and inside the sinus are discussed first, followed by those inside the valve, and lastly, the regions with elevated Reynolds shear stresses.

\subsection{Common Flow Features Outside and Downstream of Aortic Polymeric Valves}

The velocity profile downstream of the leaflet edge during systole was characterized by an asymmetrical central orifice jet, shown schematically in Figure 7-95. The central jet has a diameter of approximately $15 \mathrm{~mm}$. Peak forward velocity of higher than $2.0 \mathrm{~m} / \mathrm{s}$ was recorded inside the central orifice jet of the three polymeric valves during peak systole (Table 8-8, Table 8-10, Table 8-11, Table 8-12). Prototype A displayed a slightly higher velocity downstream of the valve due to the smaller effective flow orifice resulting from its closed commissural design (Table 8-8, Table 8-10, Table 8-11, Table 8-12). The central orifice jet in the three polymeric valves displayed a flat velocity profile 
immediately downstream of the leaflet trailing edge and became more parabolic in shape $45 \mathrm{~mm}$ downstream from the sewing ring of the valve.

Table 8-8 Maximum phased averaged velocity magnitudes $(\mathrm{m} / \mathrm{s})$ inside and in the immediate vicinity of the three polymeric heart valves during systole and diastole (2D

LDV). Refer to section 5.3.2 for the locations of the 2D LDV measurement planes (parenthisis refer to the distance from the edge of the valve sewing ring marked as $\mathrm{x}=0$, see Figure 7-93)

\begin{tabular}{c|ccc|ccc}
\hline & \multicolumn{3}{|c|}{$\begin{array}{c}\text { Peak phase averaged systolic } \\
\text { velocities }(\mathrm{m} / \mathrm{s}) \text { at various } \\
\text { measurement plane }\end{array}$} & \multicolumn{2}{c}{$\begin{array}{c}\text { Peak phase averaged diastolic } \\
\text { velocities }(\mathrm{m} / \mathrm{s}) \text { at various } \\
\text { measurement planes }\end{array}$} \\
\hline & $\begin{array}{c}\text { Down- } \\
\text { stream flow }\end{array}$ & $\begin{array}{c}\text { Stent } \\
\text { downstream }\end{array}$ & $\begin{array}{c}\text { Inside } \\
\text { valve }\end{array}$ & $\begin{array}{c}\text { Down- } \\
\text { stream } \\
\text { flow }\end{array}$ & $\begin{array}{c}\text { Stent } \\
\text { downstream }\end{array}$ & $\begin{array}{c}\text { Inside } \\
\text { valve }\end{array}$ \\
\hline prototype A & 2.84 & 0.99 & 2.63 & 0.51 & 0.67 & 0.53 \\
& $(25 \mathrm{~mm})$ & $(15 \mathrm{~mm})$ & $(15 \mathrm{~mm})$ & $(60 \mathrm{~mm})$ & $(15 \mathrm{~mm})$ & $(11 \mathrm{~mm})$ \\
prototype B & 2.47 & 2.35 & 2.00 & 0.48 & 0.47 & 3.62 \\
& $(25 \mathrm{~mm})$ & $(15 \mathrm{~mm})$ & $(15 \mathrm{~mm})$ & $(55 \mathrm{~mm})$ & $(15 \mathrm{~mm})$ & $(10 \mathrm{~mm})$ \\
prototype C & 2.30 & 2.07 & 1.91 & 0.41 & 0.53 & 2.45 \\
& $(21 \mathrm{~mm})$ & $(15 \mathrm{~mm})$ & $(15 \mathrm{~mm})$ & $(54 \mathrm{~mm})$ & $(15 \mathrm{~mm})$ & $(12 \mathrm{~mm})$ \\
\hline
\end{tabular}

Table 8-9 Maximum Reynolds shear stresses (dynes $\left./ \mathrm{cm}^{2}\right)$ inside and in the immediate vicinity of the three polymeric heart valves (2D LDV). Refer to section 5.3.2 for the locations of the 2D LDV measurement planes (parenthisis refer to the distance from the edge of the valve sewing ring marked as $\mathrm{x}=0$, see Figure 7-93)

\begin{tabular}{c|ccc}
\hline & \multicolumn{3}{|c}{ Maximum Reynolds shear stresses $\left(\right.$ dynes $\left./ \mathrm{cm}^{2}\right)$} \\
& measurement planes & atious \\
& Downstream flow & Stent downstream & Inside valve \\
\hline prototype A & 9,650 & 1,425 & 9,200 \\
& $(25 \mathrm{~mm})$ & $(15 \mathrm{~mm})$ & $(11 \mathrm{~mm})$ \\
prototype B & 1,725 & 5,956 & 13,968 \\
& $(40 \mathrm{~mm})$ & $(15 \mathrm{~mm})$ & $(10 \mathrm{~mm})$ \\
prototype C & 1,327 & 1,399 & 4,902 \\
& $(45 \mathrm{~mm})$ & $(15 \mathrm{~mm})$ & $(12 \mathrm{~mm})$ \\
\hline
\end{tabular}


Table 8-10 Maximum phased averaged velocity magnitudes $(\mathrm{m} / \mathrm{s})$ and corresponding RSS values (dynes/cm2) inside and downstream of prototype A during peak systole and diastole (3D PIV). Refer to section 5.4.1 for the locations of the 3D PIV measurement planes (parenthisis refer to the distance from the edge of the valve sewing ring marked as $\mathrm{x}=0$, see Figure 7-93)

\begin{tabular}{c|cc|cc}
\hline $\begin{array}{c}\text { Measurement } \\
\text { planes }\end{array}$ & \multicolumn{2}{|c|}{$\begin{array}{c}\text { Peak phased averaged } \\
\text { velocities }(\mathrm{m} / \mathrm{s})\end{array}$} & $\begin{array}{c}\text { Peak phased averaged RSS } \\
\left(\text { dynes } / \mathrm{cm}^{2}\right)\end{array}$ \\
\hline \multirow{3}{*}{ Center } & systole & diastole & systole & diastole \\
\cline { 2 - 5 } & 2.4 & 1.6 & 3,372 & 9,000 \\
$\pm 4 \mathrm{~mm}$ & $(20 \mathrm{~mm})$ & $(7 \mathrm{~mm})$ & $(40 \mathrm{~mm})$ & $(7 \mathrm{~mm})$ \\
& 2.4 & 0.3 & 4,194 & 641 \\
$\pm 8 \mathrm{~mm}$ & $(22 \mathrm{~mm})$ & $(15 \mathrm{~mm})$ & $(45 \mathrm{~mm})$ & $(15 \mathrm{~mm})$ \\
& 2.1 & 0.3 & 3,108 & 200 \\
$\pm 12 \mathrm{~mm}$ & $(25 \mathrm{~mm})$ & $(15 \mathrm{~mm})$ & $(45 \mathrm{~mm})$ & $(15 \mathrm{~mm})$ \\
& 1.0 & $<0.1$ & 2,804 & 45 \\
\hline
\end{tabular}

Table 8-11 Maximum velocity magnitudes (m/s) and corresponding RSS values $\left(\right.$ dynes $\left./ \mathrm{cm}^{2}\right)$ inside and downstream of prototype B during peak systole and diastole (3D PIV). Refer to section 5.4.1 for the locations of the 3D PIV measurement planes (parenthisis refer to the distance from the edge of the valve sewing ring marked as $\mathrm{x}=0$, see Figure 7-93)

\begin{tabular}{c|cc|cc}
\hline $\begin{array}{c}\text { Measurement } \\
\text { planes }\end{array}$ & \multicolumn{2}{|c|}{$\begin{array}{c}\text { Peak phased averaged } \\
\text { velocities }(\mathrm{m} / \mathrm{s})\end{array}$} & $\begin{array}{r}\text { Peak phased averaged RSS } \\
\left(\text { dynes } / \mathrm{cm}^{2}\right)\end{array}$ \\
\hline \multirow{3}{*}{ Center } & systole & diastole & systole & diastole \\
\cline { 2 - 5 } & 2.1 & 2.0 & 5,184 & 12,650 \\
& $(25 \mathrm{~mm})$ & $(6 \mathrm{~mm})$ & $(45 \mathrm{~mm})$ & $(6 \mathrm{~mm})$ \\
$\pm 4 \mathrm{~mm}$ & 2.1 & 0.5 & 3,154 & 2,297 \\
& $(21 \mathrm{~mm})$ & $(15 \mathrm{~mm})$ & $(40 \mathrm{~mm})$ & $(15 \mathrm{~mm})$ \\
$\pm 8 \mathrm{~mm}$ & 2.0 & 0.3 & 3,614 & 1,592 \\
& $(25 \mathrm{~mm})$ & $(15 \mathrm{~mm})$ & $(40 \mathrm{~mm})$ & $(15 \mathrm{~mm})$ \\
$\pm 12 \mathrm{~mm}$ & 1.0 & $<0.1$ & 2,182 & 34 \\
& $(45 \mathrm{~mm})$ & & $(45 \mathrm{~mm})$ & \\
\hline
\end{tabular}


Table 8-12 Maximum velocity magnitudes (m/s) and corresponding RSS values $\left(\right.$ dynes $\left./ \mathrm{cm}^{2}\right)$ inside and downstream of prototype C during peak systole and diastole (3D

PIV). Refer to section 5.4.1 for the locations of the 3D PIV measurement planes (parenthisis refer to the distance from the edge of the valve sewing ring marked as $\mathrm{x}=0$, see Figure 7-93)

\begin{tabular}{c|cc|cc}
\hline \multirow{2}{*}{$\begin{array}{c}\text { Measurement } \\
\text { planes }\end{array}$} & \multicolumn{2}{|c|}{$\begin{array}{c}\text { Peak phased averaged } \\
\text { velocities }(\mathrm{m} / \mathrm{s})\end{array}$} & \multicolumn{2}{c}{$\begin{array}{c}\text { Peak phased averaged } \\
\text { RSS }\left(\text { dynes } / \mathrm{cm}^{2}\right)\end{array}$} \\
\hline \multirow{3}{*}{ Center } & systole & diastole & systole & diastole \\
\cline { 2 - 5 } & 2.1 & 0.73 & 2,500 & 4,500 \\
& $(25 \mathrm{~mm})$ & $(15 \mathrm{~mm})$ & $(40 \mathrm{~mm})$ & $(15 \mathrm{~mm})$ \\
$\pm 4 \mathrm{~mm}$ & 2.1 & 0.8 & 3,275 & 2,079 \\
& $(26 \mathrm{~mm})$ & $(15 \mathrm{~mm})$ & $(42 \mathrm{~mm})$ & $(15 \mathrm{~mm})$ \\
$\pm 8 \mathrm{~mm}$ & 2.6 & 0.3 & 3,775 & 150 \\
& $(23 \mathrm{~mm})$ & $(15 \mathrm{~mm})$ & $(40 \mathrm{~mm})$ & $(15 \mathrm{~mm})$ \\
$\pm 12 \mathrm{~mm}$ & 0.8 & $<0.4$ & 1,871 & 55 \\
& $(45 \mathrm{~mm})$ & & $(45 \mathrm{~mm})$ & \\
\hline
\end{tabular}

Vortex rings was observed along the edge and trailing behind the front of the central orifice jet during the acceleration phase at a velocity of approximately $0.6 \mathrm{~m} / \mathrm{s}$. This vortex was created when the boundary layer at the edge of the valve orifice separated and rolled up into a vortex ring. The vortex formation was exacerbated by the opening sequence of the leaflets as illustrated in Figure 8-5. A crest was formed on the leaflet when the valve opened fully during the acceleration phase. This produced a sudden increase in the flow area inside the valve and a concomitant contraction of the flow area at the edge of the leaflets. The change in the shape of the leaflet caused a redirection of the flow inside the valve, thus giving rise to the vortex ring at systole.

Flow separation occurred at the edge of the leaflets when the central orifice jet emerged from the valve orifice into the sinus region. The central jet reattached approximately 50 
mm downstream from the valve sewing ring in the lower part of the flow chamber. However, it appeared that the location of flow reattachment in the upper part of the chamber occurred beyond the measurement plane. The difference in reattachment points between the upper and lower parts of the flow chamber is attributed to the asymmetrical nature of the central jet. LDV measurements taken immediately downstream of the stent at peak systole showed a forward jet with a velocity of more than $1.5 \mathrm{~m} / \mathrm{s}$ emanating from the commissural region. The flow inside the sinus region was between $0.02-0.07 \mathrm{~m} / \mathrm{s}$ throughout systole. A recirculation region of approximately $0.5 \mathrm{~m} / \mathrm{s}$ was observed in the upper part of the flow chamber during peak systole. The flow in the recirculation zone decelerated to approximately $0.4 \mathrm{~m} / \mathrm{s}$ in late systole.

The velocity downstream of the leaflet trailing edge was typically less than $0.1 \mathrm{~m} / \mathrm{s}$ during diastole and was predominantly in the reverse direction flowing upstream toward the valve. A vortex was observed inside the sinus region and persisted throughout diastole. Retrograde flow with a velocity between $0.2-0.4 \mathrm{~m} / \mathrm{s}$ was observed along the trailing edge of the leaflet throughout diastole. The location of this retrograde flow coincided with that of the coaptation region of the valve during diastole. LDV measurements downstream of the stent showed leakage flow between $0.3-0.5 \mathrm{~m} / \mathrm{s}$ occurring at the commissural region throughout diastole.

\subsection{Common Flow Features Inside the Aortic Polymeric Valves}

The flow fields inside the three polymeric valves were uniform throughout systole with a peak velocity greater than $2.0 \mathrm{~m} / \mathrm{s}$ typically observed along the trailing edge of the leaflet 
at peak systole. A velocity of $0.5 \mathrm{~m} / \mathrm{s}$ was observed along the stent inflow region during the first half of systole decelerating to less than $0.1 \mathrm{~m} / \mathrm{s}$ in late systole. Complex flow structures were observed inside the valves during diastole. Leakage jets ranging between $0.5-2.0 \mathrm{~m} / \mathrm{s}$ were measured at the coaptation region throughout diastole. Retrograde flows were also observed intermittently at the commissural region as well as along the trailing edge of the leaflet. The flow patterns inside the three valves were directed toward the stent inflow region and the high central region of the valve throughout diastole. This flow typically had a velocity of approximately $0.5 \mathrm{~m} / \mathrm{s}$.

\subsection{Reynolds Shear Stress Distribution}

The results from the polymeric valve experiments enable identification of four regions of high shear stress where the potential for platelet activation and hemolysis and subsequent thrombus formation is high: 1) the leakage jet inside the valve during diastole; 2) the trailing edge of the leaflet during systole; 3) along the edge of the central orifice jet during systole, which extended from the inside of the valve and into the distal part of the flow chamber; and 4) in the distal region of the flow chamber where turbulence mixing occurred.

During peak systole, elevated Reynolds shear stress values of more than 3,000 dynes $/ \mathrm{cm}^{2}$ were observed along the edge of the central orifice jet, along the trailing edge of the leaflet, and at the distal region of the flow chamber where the central orifice jet mixed with the surrounding fluid. During diastole, high Reynolds shear stresses exceeding 4,000 dynes $/ \mathrm{cm}^{2}$ were observed inside the valve in the leakage jets at the high central region. In 
contrast, Reynolds shear stress values of less than 100 dynes $/ \mathrm{cm}^{2}$ were measured outside the valve during diastole. In the regions of elevated shear stress, the exposure time of the fluid to the high Reynolds shear stress was between 120 and 300 ms. The magnitude and duration of these shear stresses, ranging from 2,000 dyne $/ \mathrm{cm}^{2}$ to more than 13,000 dyne $/ \mathrm{cm}^{2}$ are above the threshold values reported in the literature for the destruction of red blood cells and platelets and platelet activation ([Ellis, 1996a; Ellis, 2000a; Hung, 1976; Lu, 2001; Ramstack, 1979; Sallam, 1984]

\subsubsection{Leaflet Dynamics}

The temperature dependence study of polymeric prototype valve A in the pulsatile loop showed no significant difference in leakage volume at room $\left(20.5^{\circ} \mathrm{C}\right)$ and physiological body $\left(37^{\circ} \mathrm{C}\right)$ temperatures. The oscillation of the polymeric valves during diastole appeared to play a more significant role than the temperature in influencing leakage flow through the polymeric valves.

The temperature dependence study also showed no difference in the leaflet dynamics at the two temperatures $\left(20.5^{\circ} \mathrm{C}\right.$ and $\left.37^{\circ} \mathrm{C}\right)$. The highest velocity tended to occur at the edge of the leaflet and at the high central region of the leaflet during the opening and closing phases, respectively. This observation is consistent with the opening pattern shown in Figure 7-91 and Figure 7-92. Figure 8-5 illustrates the opening sequence of the valve leaflets during the acceleration phase. The leaflets first opened gradually during early systole. Then at mid acceleration, the edge of the leaflet suddenly lifted up and opened faster than the middle portion of the leaflet. This phenomenon is caused by the 
characteristics of the jet issuing from the valve; at this time phase, the emerging central jet encountered an increased flow area in the sinus region leading to a diverging central jet that 'pushed' the edge of the leaflet to the fully open position. Subsequently, there was an elevated velocity at the edge of the leaflet during the opening phase. Towards peak systole, the middle portion of the leaflets lifted up to form a crest, which interrupted the development of the forward flow jet creating vortex rings around the central orifice jet. The opening dynamics of the leaflets during systole, as shown in Figure 7-92, suggested the presence of extreme bending stresses at the trailing edge of the leaflets. This observation may explain the reported leaflet tear typically seen in the middle of the leaflets [Butterfield, 2000]. The high_speed_camera_upstream.avi also showed that during valve opening, the top leaflet opened $26 \mathrm{~ms}$ earlier than the remaining two leaflets (Figure 7-92b). This could be due to the non-symmetrical inlet flow profile caused by the uneven deformation of the pumping bulb farther upstream of the polymeric valve.

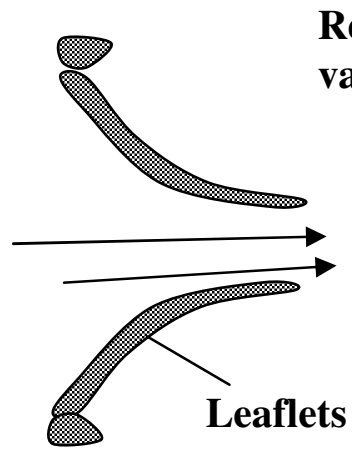

(a)

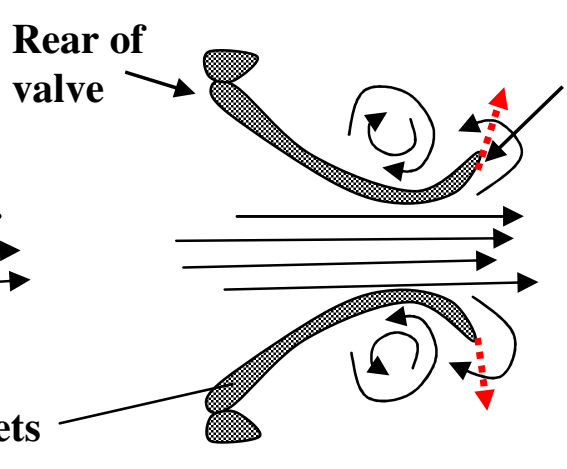

(b)

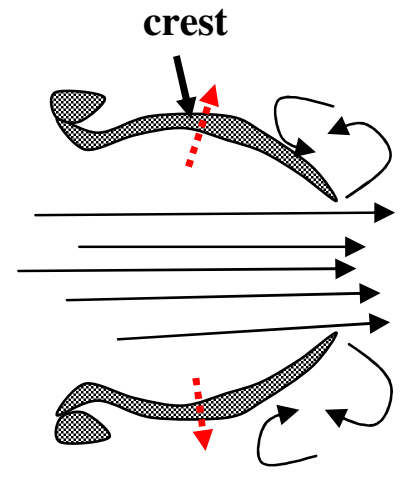

(c)

Figure 8-5 The opening configuration of the polymeric valve's leaflets during the acceleration phase; (a) gradual opening of the leaflets, (b) the edge of the leaflets opens faster than the middle portion, and (c) the formation of a crest at peak systole. Dotted arrows show the motion of the leaflets and bold arrows the fluid motion/behavior 
In Figure 7-91d, the leaflet was fully open and was beginning to close. Since the leaflet is curved, the highest point in the leaflet occurs at the crest of its curvature in the center of the leaflet (marker \# 13). Therefore, marker \#13 in the high central region of the leaflet must travel a greater distance to close. Consequently, during the closing phase the peak leaflet velocity is in the high central region of the leaflet (marker \#13). The valve leaflets were observed to 'bounce' for a period of 100 to $120 \mathrm{~ms}$ after valve closure. This oscillation can be attributed to the viscoelastic properties of the polymeric material, which allows the leaflets and stents to flex under flow-induced loading. The fluid is affected by the motion of the leaflets and stents, and both the initial unsteadiness of the flow inside the valve and the oscillation of the leakage jet can be attributed to the bouncing of the valve.

\subsubsection{Differences in Flow Features and Reynolds Shear Stresses}

For ease of discussion, the flow patterns downstream of the leaflet trailing edge are discussed first, followed by those inside the polymeric valves.

\subsection{Flow Features Downstream of the Aortic Polymeric Valves}

The 3D PIV reconstructed iso-surface of the velocity magnitudes at peak systole show that the central orifice jet in the three polymeric heart valves has a three-lobe flow configuration (Figure 8-6), in which each lobe represents a high velocity flow discharging from the commissural region. The three-lobe flow feature was most evident $50 \mathrm{~mm}$ downstream from the valve sewing ring, and was much more distinct in prototype A than in prototypes $\mathrm{B}$ and $\mathrm{C}$. This is due to the closed commissural design of prototype 
A, which resulted in a smaller effective flow area compared to prototypes B and C. This observation also explains the fact that a higher central orifice jet velocity was recorded in prototype A than in prototypes B and C. Accordingly, prototype A produced a smaller central orifice jet with a diameter of $13 \mathrm{~mm}$ compared with the $16 \mathrm{~mm}$ diameter central orifice jet seen in both prototypes B and C.

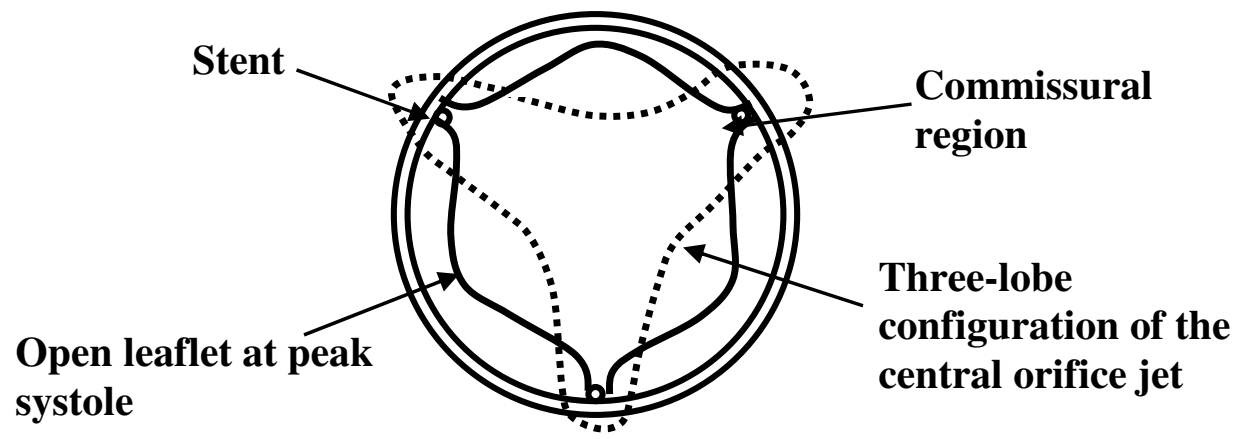

Figure 8-6 Three-lobe flow features common in the three prototype polymeric heart valves. This flow feature was more distinct in the prototype $\mathrm{A}$ than prototypes $\mathrm{B}$ and $\mathrm{C}$ as a result of the closed commissural design in prototype $\mathrm{A}$

In all three prototype valves, the central orifice jet reattached to the lower part of the flow chamber approximately $50 \mathrm{~mm}$ downstream from the valve sewing ring at peak systole. However, for prototype B the jet appeared to reattach to the upper part of the flow chamber closer to the valve $50 \mathrm{~mm}$ downstream from the valve sewing ring. Conversely, in prototypes $\mathrm{A}$ and $\mathrm{C}$ the jet reattached to the upper part of the flow chamber at a location beyond the measurement plane. It is possible that the opened commissural design of prototype B caused the central orifice jet to be directed more toward the upper part of the flow chamber at peak systole so that the central jet reattached at a location closer to the valve. 
LDV measurements made downstream of the commissural region in the three prototype polymeric valves showed that the various commissural designs markedly influence the flow patterns immediately downstream of the stent during both systole and diastole. Figure 8-7, Figure 8-8, Figure 8-9 show schematics of the flow through the commissural region for the three prototype valves during systole and diastole. During systole, a jet with a maximum velocity of approximately $1.0 \mathrm{~m} / \mathrm{s}$ and a corresponding Reynolds shear stress level of $1,260 \mathrm{dynes} / \mathrm{cm}^{2}$ was seen in the gap channel of prototype A, giving rise to vortex structures downstream of the stent. Unlike prototype A (closed commissural design), prototype B had an open commissure design, which produced a relatively unobstructed forward flow through the commissural region. As a result, the maximum forward flow velocity at the stent tip of prototype B $(2.3 \mathrm{~m} / \mathrm{s}$ and a corresponding Reynolds shear stress of 6,000 dynes $/ \mathrm{cm}^{2}$ ) was more than double that of prototype A. In prototype C (semi-open commissural design), two forward flow jets were observed emanating from the commissural region (see Figure 7-124): the upper jet had a velocity of $1.4 \mathrm{~m} / \mathrm{s}$ and a corresponding Reynolds shear stress of 130 dynes $/ \mathrm{cm}^{2}$, while the lower jet a velocity of $0.78 \mathrm{~m} / \mathrm{s}$ and a corresponding Reynolds shear stress of 1,050 dynes $/ \mathrm{cm}^{2}$. The upper jet corresponded to part of the central orifice jet, and the lower jet corresponded to the flow issuing from the gap channel at the commissural region.

During diastole a leakage jet was observed at the commissural region in both prototypes A and B. The leakage jet in prototype A had a velocity of $0.7 \mathrm{~m} / \mathrm{s}$, while the leakage jet in prototype B a velocity of $0.4 \mathrm{~m} / \mathrm{s}$. In contrast, two leakage jets were observed in prototype C: the smaller leakage jet had a velocity of $0.4 \mathrm{~m} / \mathrm{s}$, while the upper leakage jet 
had a velocity of $0.6 \mathrm{~m} / \mathrm{s}$. The location of the smaller leakage jet corresponded to the retrograde flow at the gap channel, and the larger leakage jet corresponded to the flow slightly above the commissural region.

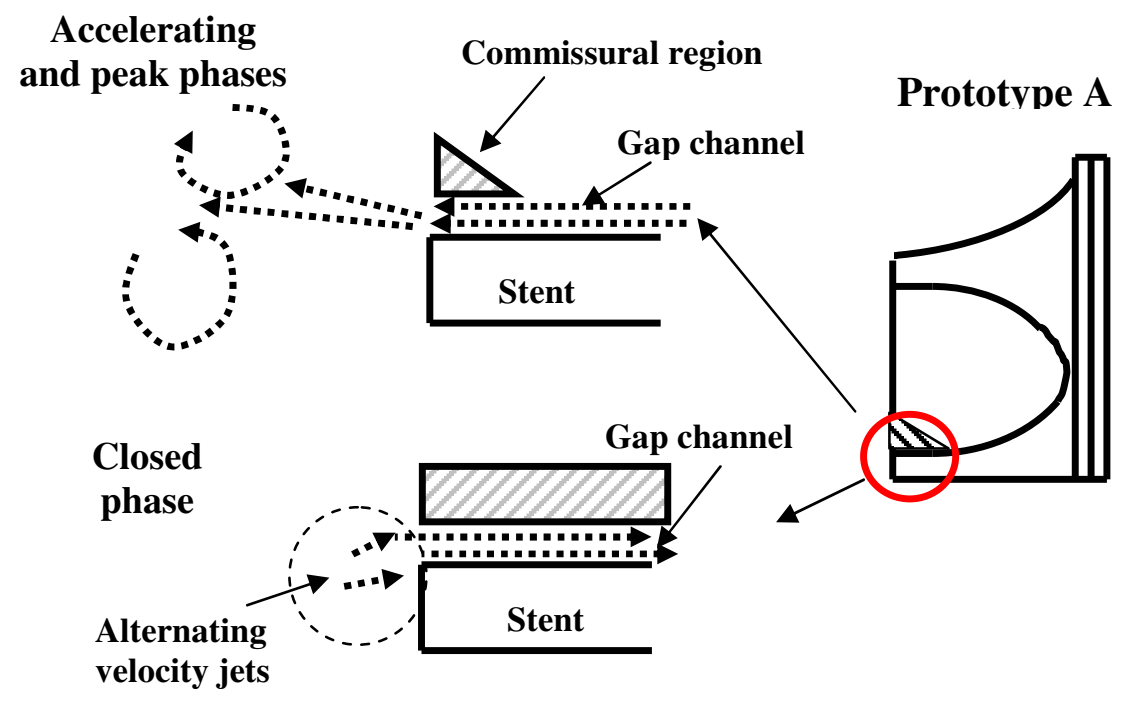

Figure 8-7 Schematic of the flow field at the stent during the acceleration and early diastolic phases for prototypes A. The closed commissural design of prototype A produced a forward and leakage jets at the gap channel during systole and diastole, respectively. The cross-hatched areas depict the regions where the adjacent leaflets at the commissural region come together 


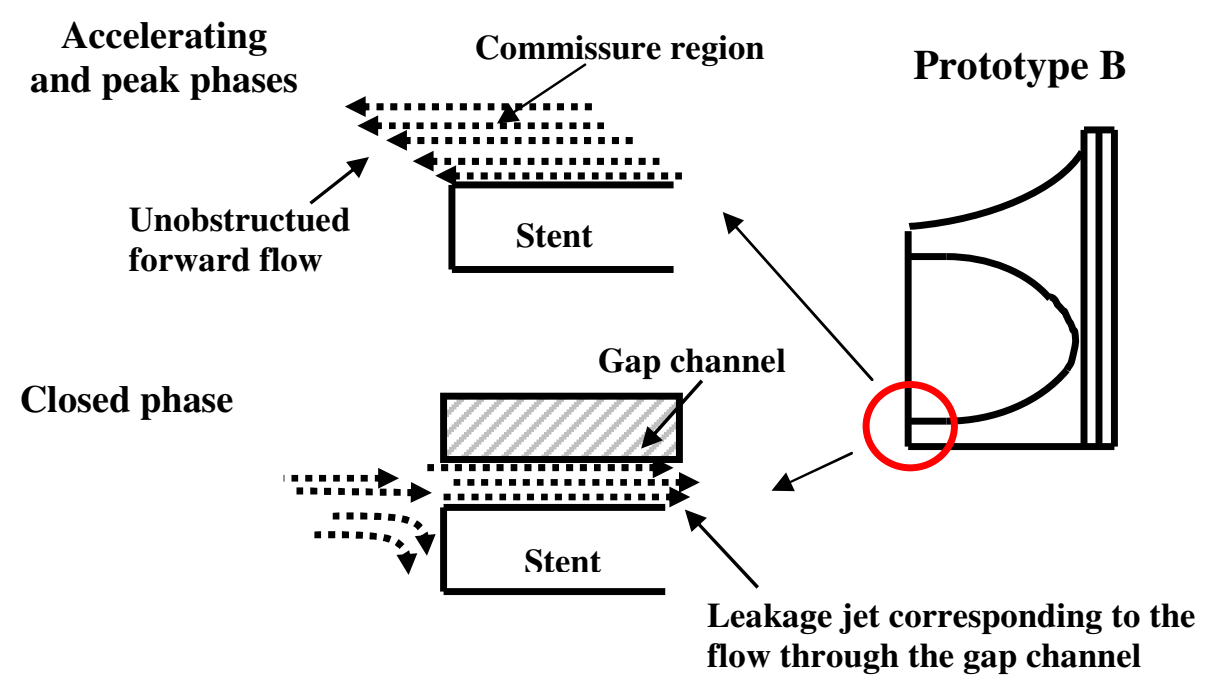

Figure 8-8 Schematic of the flow field at the stent during the acceleration and early diastolic phases for prototypes B. An unobstructed forward flow was observed at the commissural region in prototype B during systole, while a leakage jet was observed next to the gap channel during diastole. The cross-hatched areas depict the regions where the adjacent leaflets at the commissural region come together

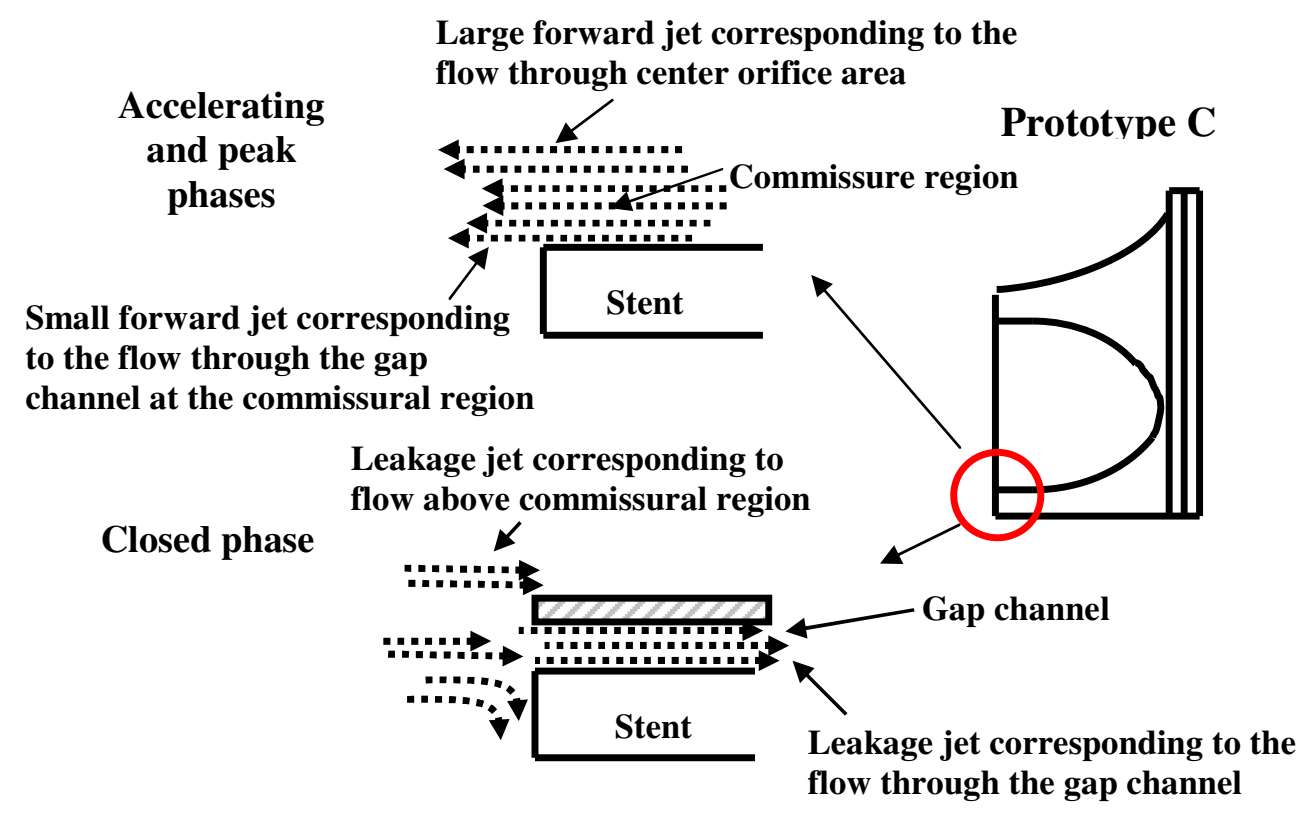

Figure 8-9 Schematic of the flow field at the stent during the acceleration and early diastolic phases for prototypes C. A small forward and leakage jet observed during systole and diastole, respectively, corresponded to the flow at the gap channel. The crosshatched areas depict the regions where the adjacent leaflets at the commissural region come together 


\subsection{Flow Features Inside the Aortic Polymeric Valves}

The closed commissural design in prototype A gave rise to an area of stagnation (shown in Figure 7-102) at the valve commissural region throughout systole. This stagnation region led to an area of low flow $(<0.5 \mathrm{~m} / \mathrm{s})$ along the stent inflow region during the acceleration and deceleration phases. In contrast, the open and semi-open commissural designs of prototypes $\mathrm{B}$ and $\mathrm{C}$ produced relatively unobstructed flow along the stent inflow region throughout systole. During the deceleration phase the velocity at the stent inflow region of prototype B $(1.4 \mathrm{~m} / \mathrm{s})$ was double that of prototype A $(0.8 \mathrm{~m} / \mathrm{s})$, while the stent flow in prototype $\mathrm{C}$ had a velocity of approximately $1.1 \mathrm{~m} / \mathrm{s}$.

A leakage jet was observed in the high central region for all three prototype valves. The velocity and size of the leakage jet for prototype B $\left(2.0 \mathrm{~m} / \mathrm{s}, 13,968\right.$ dynes $\left./ \mathrm{cm}^{2}\right)$ were higher and larger than in prototype A $\left(1.0 \mathrm{~m} / \mathrm{s}, 9,200\right.$ dynes $\left./ \mathrm{cm}^{2}\right)$. This may be due to the thicker, and therefore less compliant leaflets of prototype B, which may inhibit complete valve closure during diastole. Furthermore, the leakage jet in prototype B continued to increase in both magnitude and size $220 \mathrm{~ms}$ after valve closure, while the leakage jet in prototype A became stable at early diastole. The leakage jets observed in prototypes A and $\mathrm{B}$ were comparatively more well-defined than that observed in prototype $\mathrm{C}$. This is mainly because the leakage jet $\left(1.4 \mathrm{~m} / \mathrm{s}, 1,000\right.$ dynes $\left./ \mathrm{cm}^{2}\right)$ at the high central region of prototype $\mathrm{C}$ appeared only intermittently throughout diastole, and tended to mix with the retrograde flow along the trailing edge of the valve. An additional leakage jet $(0.8 \mathrm{~m} / \mathrm{s}$,

1,000 dynes $/ \mathrm{cm}^{2}$ ) was observed at the commissural region of prototypes B and C. The observed leakage jet is attributed to the open and semi-open commissural designs of 
prototypes B and C. It is possible that the leaflets at the commissural region of prototypes $\mathrm{B}$ and $\mathrm{C}$ formed a large gap channel at the stent during diastole, thereby permitting leakage flow to occur along the stent inflow region.

\subsubsection{Comparison Of Current Aortic Polymeric Valves Studies With Previous Experiments}

Important studies investigating the in vitro hemodynamic characteristics of the tissue and polymeric heart valves performed in the past two decades have revealed complex flow structures in the vicinity of the trileaflet valve prostheses, corroborating the findings of the current experiments with the three polymeric heart valves [Woo, 1983a; Woo, 1983b; Yoganathan, 1983; Yoganathan, 1986a; Yoganathan, 1986b]. Using flow visualization and LDV, the authors demonstrated that these trileaflet bioprostheses and polymer prostheses produced jet-type flow fields like those observed in the current three polymeric heart valves [Woo, 1983a; Woo, 1983b; Yoganathan, 1983; Yoganathan, 1986a; Yoganathan, 1986b]. In general, the pericardial and Aortech polymeric heart valve designs created flatter velocity profiles than the porcine and Abiomed valve designs. All three Aortech polymeric heart valves produced jets with flat velocity profiles at peak systole, identical to those observed in the Ionescus-Shirley pericardial, CarpentierEdwards 2650 porcine, Hancock pericardial, and Carpentier-Edwards pericardial valves. In contrast, the velocity profiles of the central jets in the Carpentier-Edwards 2625 porcine, Hancock modified-orifice porcine, Hancock II porcine, and the Abiomed valves were more parabolic in shape. 
The diameters of the central jets in the tissue bioprostheses at peak systole is typically larger than that in the polymeric heart valves. The bioprosthetic valves studied have larger tissue annular diameters compared with the polymeric prostheses (see Table 8-13). For a given cardiac output, valves with larger tissue annular diameters typically have larger jet diameter compared to those with smaller tissue annular diameters. Central orifice jet diameters between $15-25 \mathrm{~mm}$ were observed in the bioprostheses at peak systole with the smallest occurring in Carpentier-Edwards 2625 and Hancock standardorifice porcine valves, and the largest in the Hancock pericardial and Carpentier-Edwards pericardial valves [Yoganathan, 1986a]. The jet diameters for three Aortech polymeric heart valves were comparable to that of the Abiomed trileaflet polymeric valve prosthesis, which had a jet diameter of $14 \mathrm{~mm}$ [Woo, 1983a; Woo, 1983b]. The Aortech prototype A had a jet diameter of $13 \mathrm{~mm}$, while both Aortech prototypes $\mathrm{B}$ and $\mathrm{C}$ a jet diameter of 16 mm.

Flow separation was observed at the edges of the valve leaflets and around the jet. Flow reattachment occurred when the central orifice jet diverged and impinged on the chamber wall downstream of the valve. The location of reattachment points varies from valve to valve. Under the same cardiac output, valves with larger orifice sizes tend to have reattachment points closer to the trailing edge of the valve leaflet. For instance, the central orifice jets of the Abiomed $25 \mathrm{~mm}$ and $21 \mathrm{~mm}$ valves were observed to reattach $40 \mathrm{~mm}$ and $60 \mathrm{~mm}$ downstream from the valves, respectively [Woo, 1983b]. A common observation pertaining to the reattachment points of the central orifice downstream of the valve was made in the flow visualization studies as well as in current studies. 
Reattachment of the orifice jet occurred at different downstream locations in the upper and lower parts of the flow chamber. This observation is attributed to the asymmetric nature of the central orifice jet. In the Abiomed $25 \mathrm{~mm}$ and $21 \mathrm{~mm}$ valves, the central orifice jet reattached approximately $75 \mathrm{~mm}$ and $40 \mathrm{~mm}$ downstream of the sewing ring in the upper and lower parts of the flow chamber, respectively [Woo, 1983b]. For the tissue bioprostheses, the jet typically reattached in the upper part of the flow chamber at a location between 55 - $90 \mathrm{~mm}$ downstream from the valve sewing ring, and at a location between $35-50 \mathrm{~mm}$ in the lower part of the flow chamber. These findings are consistent with those made for the three current Aortech polymeric heart valves; the central jet in the three prototypes valves reattached at a location between 45 - $55 \mathrm{~mm}$ downstream from the valve sewing ring in the lower part of the flow chamber. For both prototypes A and $\mathrm{C}$, the jet reattachment point in the upper part of the flow chamber seem to occur beyond the measurement plane, which only extended $77.5 \mathrm{~mm}$ downstream from the valve sewing ring. The exception to the jet reattachment location in the upper part of the flow chamber was observed in both the Hancock pericardial and the Aortech prototype B valves. The central orifice jets in both valves were observed to reattach closer to the valve at approximately $55 \mathrm{~mm}$ downstream from the valve sewing ring in the upper part of the flow chamber.

The leaflet profile and flow patterns of the three Aortech polymeric heart valves at peak systole was similar to that seen in the pericardial heart valve where a small recirculation flow was observed at the edge of the leaflet during systole (Figure 8-10) [Black, 1991]. The shape of the open leaflet may explain the formation of a vena contracta in the central 
orifice jet. This narrowing of the central orifice jet immediately downstream of the valve was due to the opening configuration of the valve during systole where the shape of the leaflet was such that the flow area at the leaflet's trailing edge was more constricted than that inside the valve (Figure 8-10).

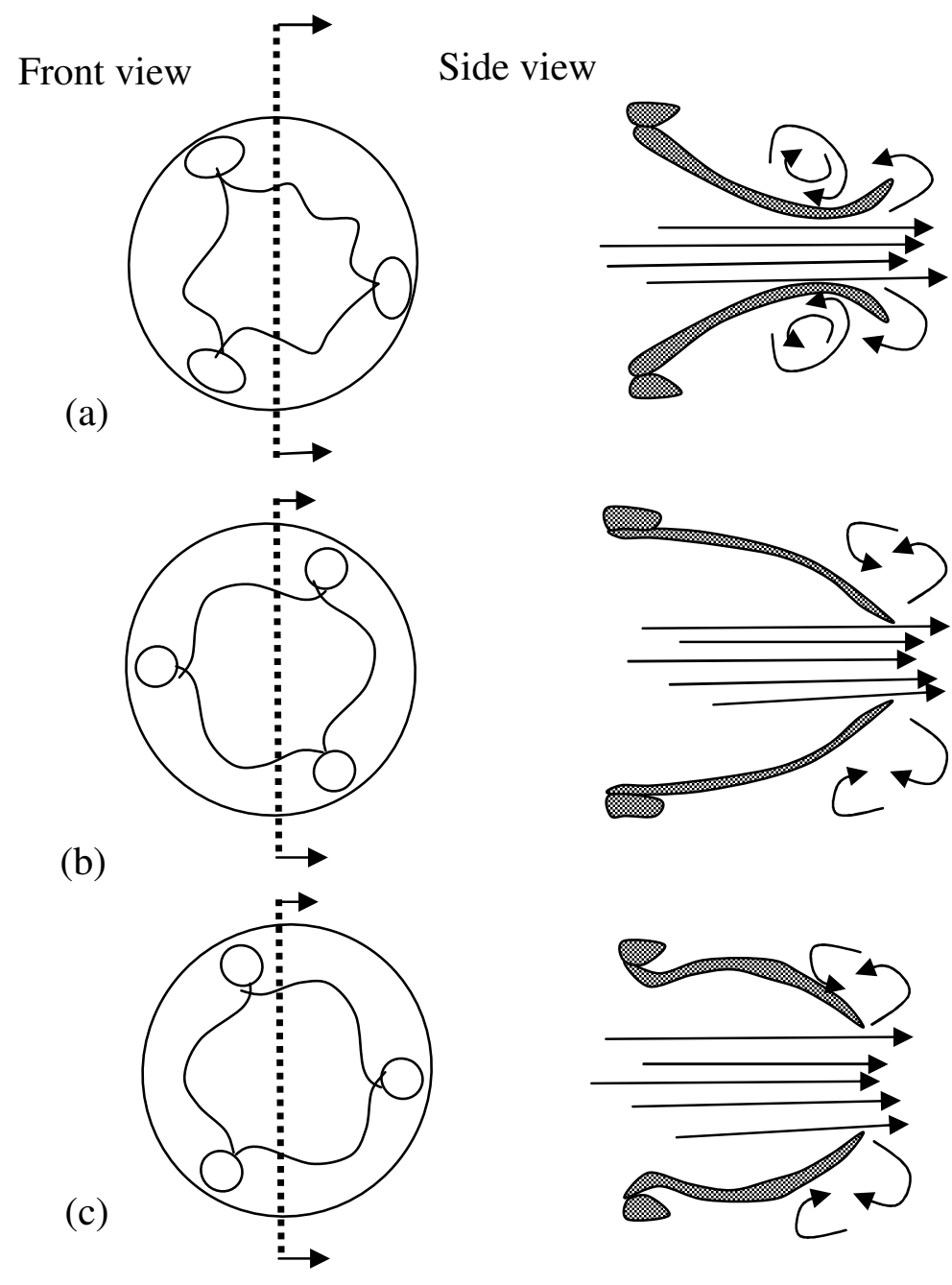

Figure 8-10 Comparison of flow patterns and leaflet configuration at peak systole among the three heart valve prostheses: (a) porcine bioprosthesis, (b) pericardial bioprosthesis, and (c) Aortech polymeric heart valve [Black, 1991; Woo, 1983a]

Table 8-13 compares the peak velocities and Reynolds shear stress values of previous valve studies with those obtained in the current polymeric valve experiments. It can be 
seen that the peak forward velocities obtained from previous studies were comparable to those recorded in the current study. The peak Reynolds shear stress levels calculated downstream of all valves during systole was typically between $1,000-2,900$ dynes $/ \mathrm{cm}^{2}$. The highest Reynolds shear stress of 8,347 dynes $/ \mathrm{cm}^{2}$ was recorded in Aortech prototype A, while both the Abiomed $21 \mathrm{~mm}$ and the Carpentier-Edwards $27 \mathrm{~mm} 2625$ porcine valves recorded Reynolds shear stresses of 4,500 dynes $/ \mathrm{cm}^{2}$. Reynolds shear stress studies performed with the trileaflet bioprostheses revealed a Reynolds shear stress distribution identical to those from the current polymeric valves. Elevated Reynolds shear stress levels were typically observed along the edge of the central orifice jet and were spread out over a wider area farther downstream from the valve as the energy of the central jet become dissipated. All of the aortic valves studied produced Reynolds shear stress in excess of 200 dynes $/ \mathrm{cm}^{2}$ during the majority portion of systole. It is therefore clear that the elevated Reynolds shear stress levels could lead to sub-lethal and/or lethal damage to the blood elements. 
Table 8-13 Comparison of the velocity magnitudes and Reynolds shear stresses from various trileaflet valve designs

\begin{tabular}{|c|c|c|c|}
\hline & $\begin{array}{c}\text { Effective annular } \\
\text { orifice diameter } \\
(\mathrm{mm})\end{array}$ & $\begin{array}{c}\text { Maximum phase } \\
\text { averaged velocity } \\
\text { during systole }(\mathrm{m} / \mathrm{s})\end{array}$ & $\begin{array}{c}\text { Maximum phase } \\
\text { averaged TSS during } \\
\text { systole }\left(\text { dynes } / \mathrm{cm}^{2}\right)\end{array}$ \\
\hline $\begin{array}{l}\text { Carpentier-Edwards } \\
2625 \text { porcine valve } \\
(27 \mathrm{~mm})\end{array}$ & 23.0 & 3.3 & 4,500 \\
\hline $\begin{array}{l}\text { Hancock modified } \\
\text { orifice porcine valve } \\
\text { (25 mm) }\end{array}$ & 21.8 & 3.0 & 2,900 \\
\hline $\begin{array}{l}\text { Ionesuc-Shiley } \\
\text { pericardial valve ( } 27 \\
\mathrm{~mm})\end{array}$ & 23.4 & 2.3 & 2,500 \\
\hline $\begin{array}{l}\text { Carpentier-Edwards } \\
2650 \text { porcine valve } \\
(27 \mathrm{~mm})\end{array}$ & 25.0 & 2.0 & 2,000 \\
\hline $\begin{array}{l}\text { Hancock II porcine } \\
\text { valve } \\
(27 \mathrm{~mm})\end{array}$ & 24.0 & 2.6 & 2,500 \\
\hline $\begin{array}{l}\text { Hancock pericardial } \\
\text { valve } \\
(27 \mathrm{~mm})\end{array}$ & 23.3 & 1.8 & 2,100 \\
\hline $\begin{array}{l}\text { Ionescuc-Shiley low } \\
\text { profile pericardial } \\
\text { valve }(27 \mathrm{~mm})\end{array}$ & 23.0 & 2.2 & 2,400 \\
\hline $\begin{array}{l}\text { Carpentier-Edwards } \\
\text { pericardial valve }(27 \\
\mathrm{mm})\end{array}$ & 25.7 & 1.8 & 1,000 \\
\hline Abiomed $(21 \mathrm{~mm})$ & 18.6 & 3.7 & 4,500 \\
\hline Abiomed $(25 \mathrm{~mm})$ & 22.8 & 2.2 & 2,200 \\
\hline $\begin{array}{l}\text { Aortech prototype A } \\
\qquad(23 \mathrm{~mm})\end{array}$ & 23.0 & 2.8 & 8,347 \\
\hline $\begin{array}{l}\text { Aortech prototype B } \\
\qquad(23 \mathrm{~mm})\end{array}$ & 23.0 & 2.5 & 1,725 \\
\hline $\begin{array}{c}\text { Aortech prototype C } \\
(23 \mathrm{~mm})\end{array}$ & 23.0 & 2.3 & 1,327 \\
\hline
\end{tabular}

\subsubsection{Integration of Previous In Vivo Studies with Observed Flow Structures}

Animal studies using sheep have shown that polymeric valves are prone to material degradation as a result of extrinsic calcification of the attached host biological material on the leaflet surface [Daebritz, 2003; Daebritz, 2004; Wheatley, 2001]. These studies 
found local fibrin deposits in the commissural regions, which was attributed to inefficient washout (Figure 8-11). Preliminary in vivo experiments involving prototypes A and B also showed thrombus formation along the commissures in the stent inflow region of both designs and in the high central region of prototype B. Thrombus deposition along the stent inflow region of prototype A was initially attributed to inefficient washout of the closed commissural region. However, subsequent animal experiments with prototype B showed similar thrombus formation along the stent region. Hence, the low flow region may not be the sole contributor to the observed thrombus. It is likely that (1) the flow structures observed inside the valve during diastole, and (2) the location of elevated Reynolds shear stress levels in the vicinity of the prototype valves during systole both contribute to the observed clots in prototypes A and B. Currently, there are no animal results available for prototype $\mathrm{C}$.

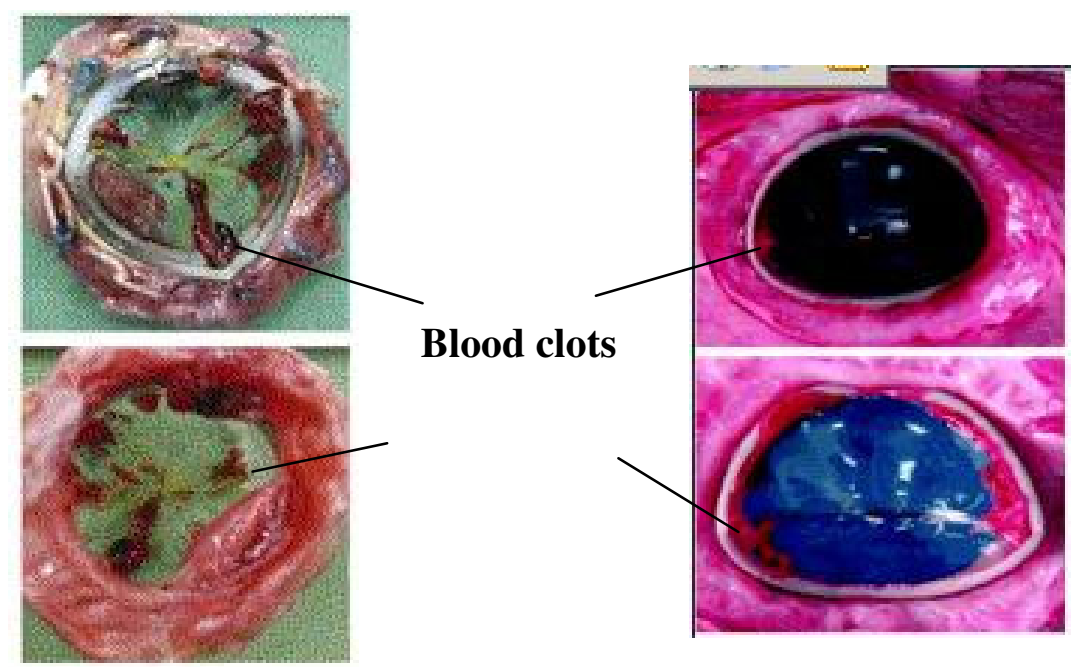

Figure 8-11 Thrombi observed at the commissural region and along the stent inflow region of implanted polymeric heart valves; (a) aortic position [Daebritz, 2004], (b) mitral position [Daebritz, 2003] 
Figure 8-12 shows the stream traces of the flow inside prototype A during diastole. The general flow feature inside the three prototype valves was that of flow 'splitting' with a portion of the flow directed towards the center of the valve, i.e. towards the leakage jet at the high central region, and the remainder of the flow moving towards the stent inflow region. This flow pattern was unsteady during early diastole, but stabilized $100 \mathrm{~ms}$ after valve closure and persisted throughout diastole. In both prototypes $\mathrm{B}$ and $\mathrm{C}$, the strength of the split flow increased intermittently when leakage jets were observed at the high central and the commissural regions. Therefore, it is likely that these leakage jets may be partially responsible for the formation of split flow.

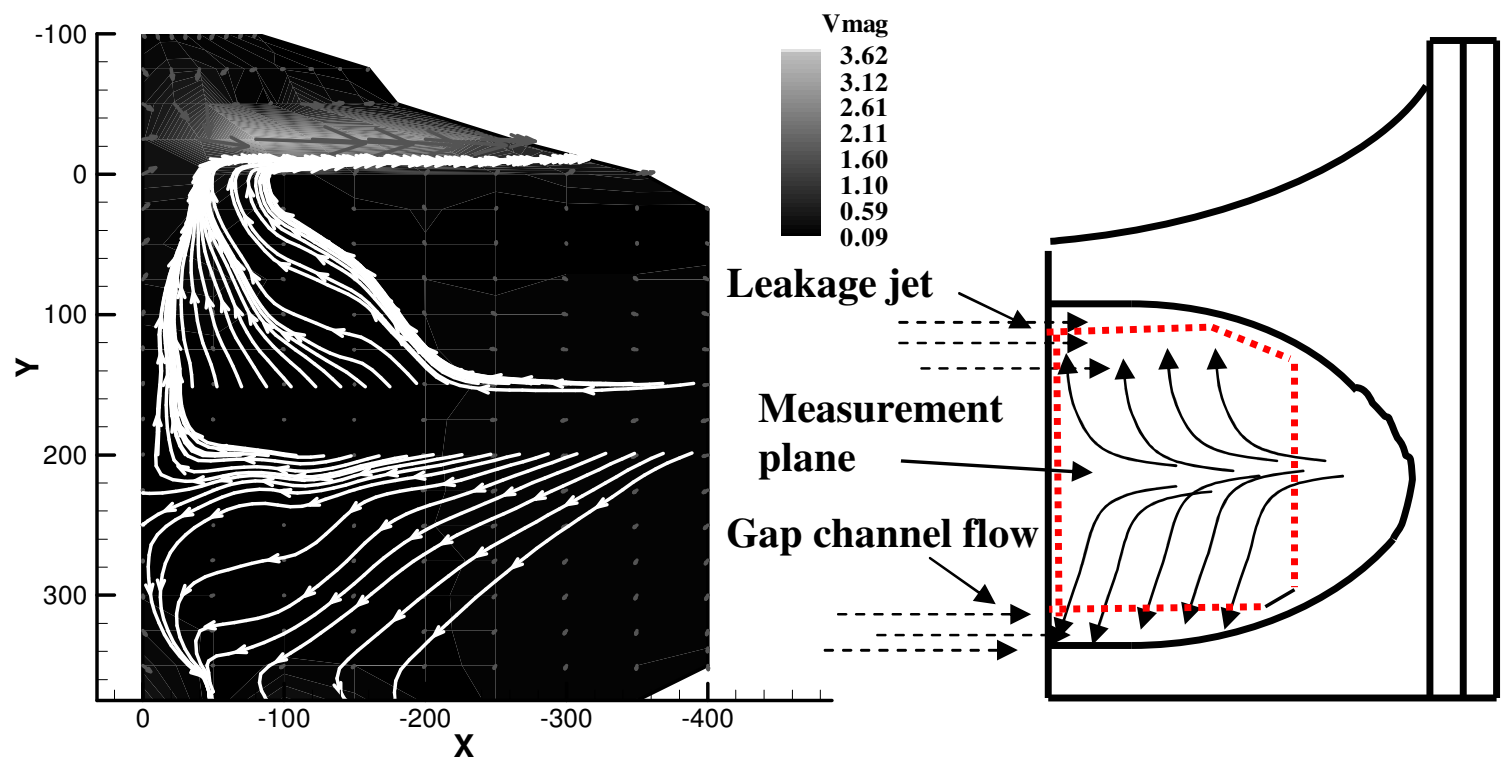

Figure 8-12 Stream traces of the flow inside prototype A during diastole

Another factor that may contribute to the formation of flow 'splitting' phenomena is the dynamics of the valve leaflet during diastole. The closing motion of prototype A, depicted in Figure 8-13, indicates that the valve closed first at the lateral side of the 
leaflets (dotted arrows) forcing fluid towards the coaptation and stent inflow regions. In addition, the tracing of the marker array in Figure 7-91 and the upstream high speed video of the valve in Figure 7-92 show that small oscillations actually persist into late diastole. The closing dynamics of the valve and the persistence of the oscillations may give rise to the flow 'splitting' phenomenon that may enhance the transportation of activated blood elements towards the stent inflow and high central regions of the valve during diastole.

(a)

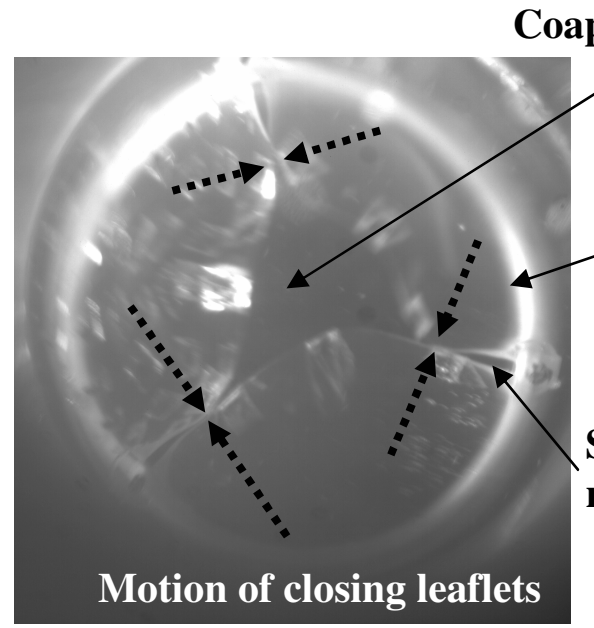

Coaptation region

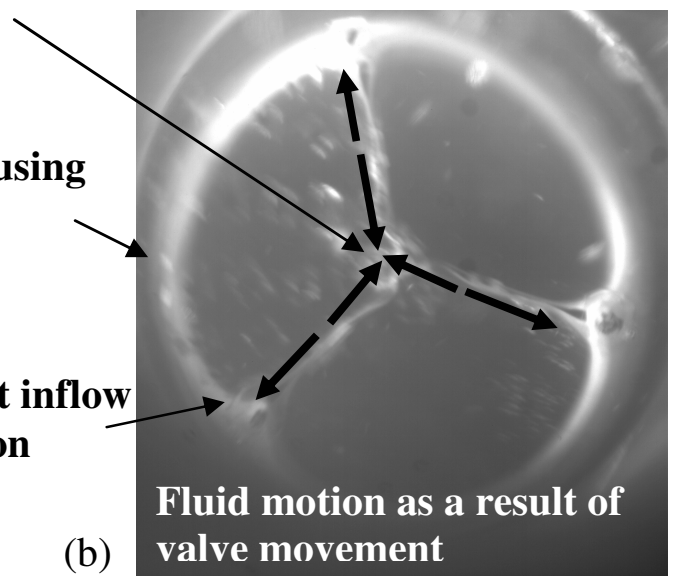

Figure 8-13 High speed images of prototype A illustrating the closing motion of the valve. (a) The valve closes first at the lateral side of the leaflets (dotted arrows) (b) forcing fluid

A factor that may exacerbate the deposition of activated blood elements is the high shear stress experienced by the blood during systole. 2D, 3D LDV and 3D PIV contour plot of the Reynolds shear stress distribution of the three prototype valves show that high shear rates tended to occur at the following locations: 1) edge of the central orifice jet;2) at the commissural region; and 3) the distal region of the flow chamber. The elevated Reynolds 
shear stress levels observed in locations (1) and (2) may explain the observed thrombi at the commissural and stent inflow regions of prototypes A and B in animal studies.

In addition to the clots formation at the stent inflow and commissural regions in prototypes A and B, thrombi were also observed in the high central region of prototype B. It is likely that the higher shear stresses encountered in the leakage jet of prototype B may be partially responsible for these clot buildups. The thinner leaflets of prototype A may render the valve more flexible hence enabling better valve closure during diastole, which would result in lower leakage jet velocities. Conversely, the stiffer and thicker leaflets of prototypes B may prevent complete valve closure resulting in higher leakage jet velocities.

Additionally, the thrombus depositions appear to correlate with the machining lines on prototype B. These machining lines, not evident on prototype A, were created during the fabrication process in which the polyurethane frames of the leaflets are mounted onto steel formers leaving indentations on the inflow side of the leaflets [Mackay, 1996]. Apparently, the steel former used for the fabrication of leaflets in prototype B is not identical to that used for prototype A. The surface topography at the high central region of prototype B in Figure 7-199 clearly shows ridge patterns on the inner surface of prototype $\mathrm{B}$. The peak ridge separation was approximately $100 \mu \mathrm{m}$ and thrombus deposition was believed to have occurred at the troughs of the ridge pattern. Figure 8-14 shows the orientation of the indentation on the inner surface of the polymeric valve. 
Coupled with a rougher inner leaflet surface, the thicker leaflets in prototype B likely led to the observed thrombus deposition in the high central region.
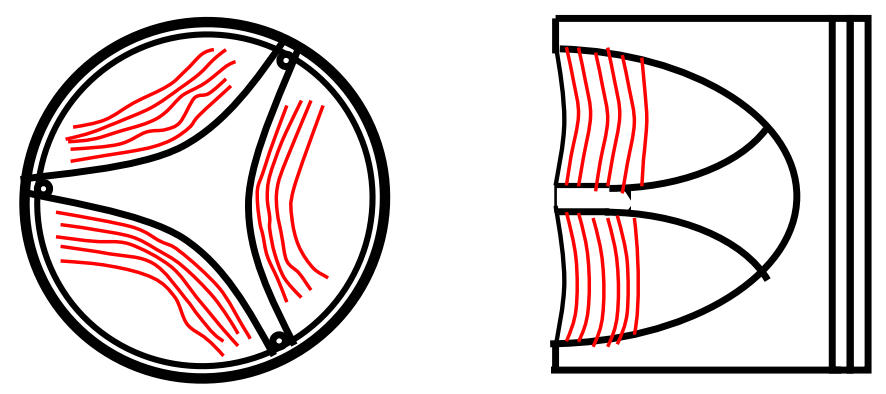

Figure 8-14 Crest and trough pattern in the inner surface of polymeric heart valve

Current studies implicate hemodynamics as one of the major contributors to the observed thrombus formation in the Aortech prototype valves. This observation may explain the blood clots found in current polymeric and bioprothetic heart valves. At present hemodynamics is not highlighted as one of the reasons for the observed calcific deposits in bioprosthetic heart valves. Indeed, failure of current heart valve bioprostheses is usually attributed to material deterioration and associated calcium phosphate deposits at the commissural and stent inflow regions [Butterfield, 1991; Goldsmith, 1998; Thubrikar, 1983]. Some authors argue that cyclical bending stresses encountered at these regions could damage the collagen framework and predispose the leaflets to premature commissural tears and calcification resulting in valve failure [Goldsmith, 1998; Thubrikar, 1983; Vyavahare, 1999]. Leat et al demonstrated that maximal stresses located at the commissural attachment of the polymeric valves are consistent with the findings from bioprosthetic valves, suggesting that the failure mechanism of polymeric heart valves is similar to that of tissue heart valves [Leat, 1995]. However, in vivo 
experiments with the polymeric valves showed that the thrombi and pannus growth observed at the commissural regions are extrinsic in nature, and explanted valves show no degradation of the polyurethane material [Daebritz, 2003; Daebritz, 2004]. Studies with bioprosthetic valves have also revealed that calcium deposits at the commissural regions of explanted tissue valves were not intrinsic in nature with no destruction of the biological structures. Hence, it is likely that hemodynamics may be one of the contributors to the observed calcium and thrombi deposits at the commissural and stent inflow regions of the current bioprosthetic and polymeric valves. The elevated fluid shear along the edge of the central orifice jet during systole may lead to hemolysis and activation of platelets. Subsequent thrombus buildup along the commissural and stent inflow regions may be further exacerbated by flow structures such as the split flow observed in the polymeric valves during diastole, thereby increasing the potential for subsequent valve failure.

Beyond the scope of the hemodynamic investigation of the three prototype valves is the discussion of the material properties and the manufacturing process of the polymeric heart valves. These factors have equal weight in the success of a polymer valve. This is exemplified by the fact that clots found at the high central region of prototype B took on the pattern of the steel former used for the leaflets during the fabrication process. In addition, by controlling the elastic modulus properties of the leaflets and the valve stent, it may be possible to minimize the oscillations of the valve leaflets during diastole and thereby reduce the size and magnitude leakage jets and the subsequent formation of clots along the stent. 


\section{$\underline{\text { 8.4 Comparison of the LDV and PIV Techniques }}$}

The purpose of this section is to determine the extent of agreement between the mean velocities and maximum Reynolds principle shear stresses measured using LDV and PIV. Both laser techniques non-invasively measure velocity flow fields. However, LDV is a point-wise method that provides excellent temporal resolution. In contrast, PIV is a field measurement technique that permits instantaneous measurement of the velocity fields at a much lower temporal resolution. Data acquisition time and data storage are the limiting factors for LDV and PIV, respectively. While it is extremely time-consuming to acquire enough data points using LDV to yield a flow field with good spatial and temporal resolution, PIV requires a huge amount of data storage for its global velocity information.

In this section, comparisons are made between the results obtained by 2D LDV, 3D LDV and 3D PIV for flow through the three polymeric heart valves. Results from the three methods good agreement with respect to flow features and Reynolds shear stress distributions for the three polymeric vheart valves. Comparison of the Reynolds shear stress $(-\overline{\rho u v})$ and the root-mean-square of the $U$ velocity component $\left(U_{r m s}\right)$ values between the three valves both inside and downstream of the valve showed excellent agreement between the three laser techniques. This section first compare the flow profile and Reynolds shear stress profile of the central orifice jet at location immediately downstream of the leaflet of prototype A during peak systole, followed by comparison of the flow profile, Reynolds shear stress $(-\overline{\rho u v})$ and $U_{r m s}$ values both along the leaflet trailing edge inside the valve and $40 \mathrm{~mm}$ downstream from the valve sewing ring. 


\subsubsection{Comparison of Velocity Magnitude and Reynolds Shear Stress Profiles at Immediate Downstream of the Valve}

Figure 8-15 illustrates a point to point comparison between the 2D LDV, 3D LDV and 3D PIV velocity magnitudes at the location immediately downstream of the leaflet during peak systole. All three techniques show a flat profile in the center with a velocity of approximately $2 \mathrm{~m} / \mathrm{s}$.
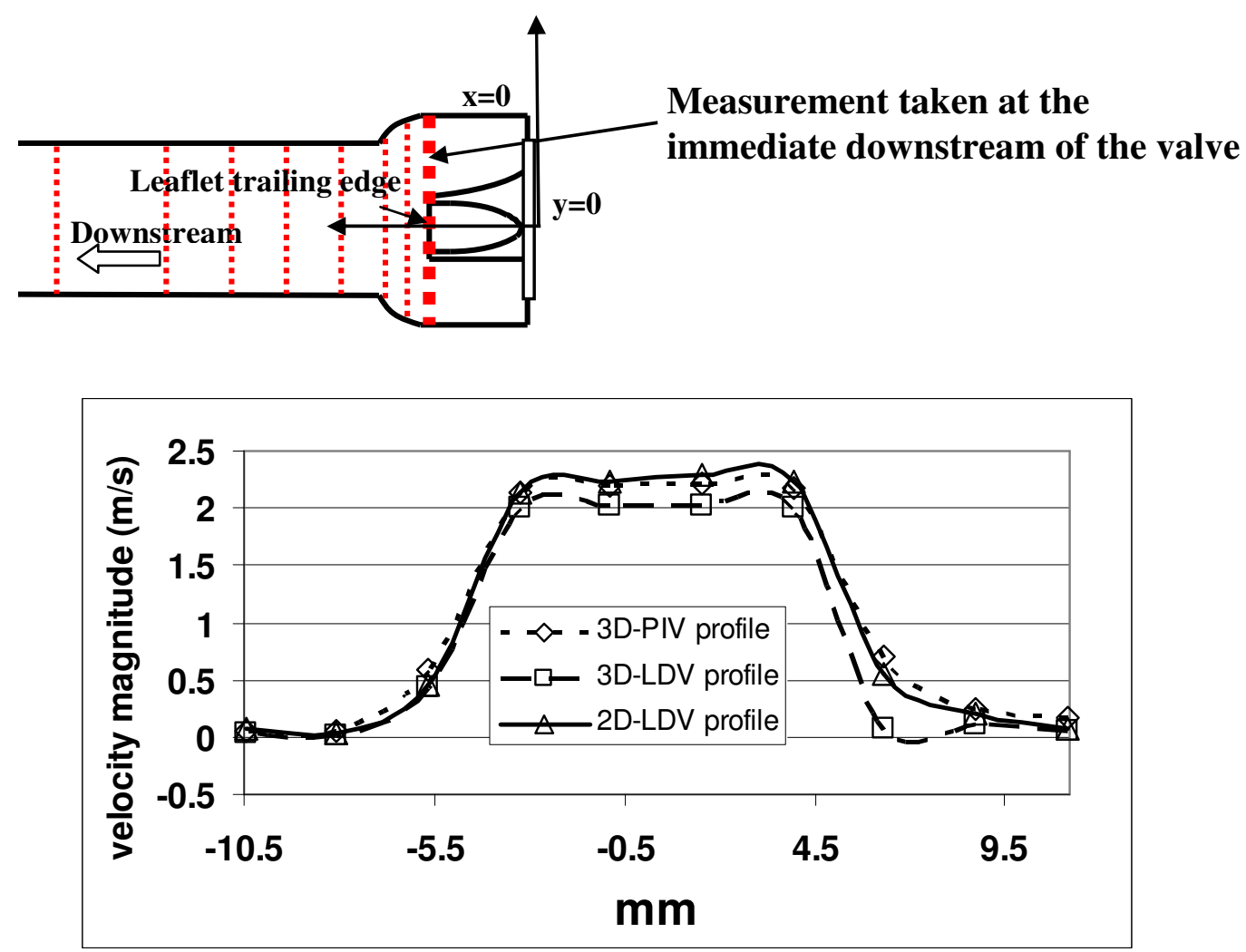

Figure 8-15 Comparison of velocity magnitude profile obtained by the 2D LDV, 3D LDV and the 3D PIV immediately downstream from the leaflet trailing edge of prototype A at peak systole, time bin 9

Figure 8-16 illustrates a point to point comparison between the 2D LDV, 3D LDV and 3D PIV Reynolds shear stress values at the location immediately downstream of the leaflet during peak systole. All three techniques yielded similar profiles with elevated 
shear stress observed along the edge of the central orifice jet. The three laser techniques yielded maximum Reynolds shear stress of approximately 900 dynes $/ \mathrm{cm}^{2}$.

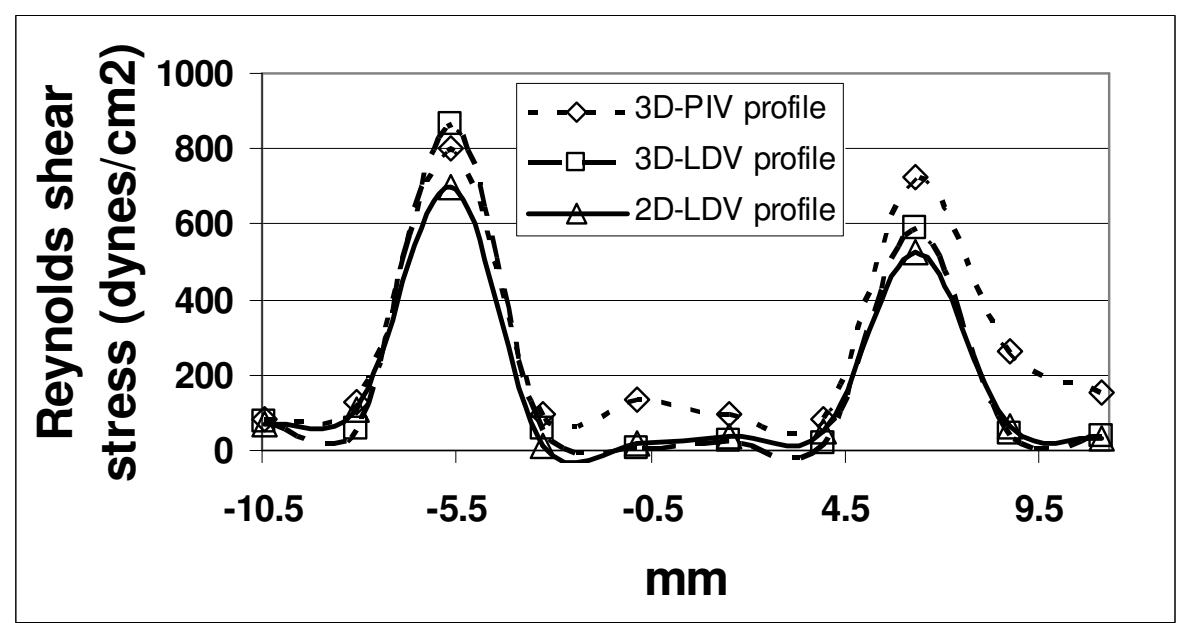

Figure 8-16 Comparison of Reynolds shear stress profile obtained by the 2D LDV, 3D LDV and the 3D PIV immediately downstream of the leaflet trailing edge of prototype A at peak systole, time bin 9

\subsubsection{Comparison of Velocity Magnitude, Reynolds Shear Stress $(-\overline{\rho u v})$ and $\boldsymbol{U}_{r m s}$ Profiles $40 \mathrm{~mm}$ Downstream of the Three Polymeric Heart Valves}

Comparison of the velocity magnitude, Reynolds shear stress (- $\overline{\rho u v})$ and root-meansquare of the $U$ component $\left(U_{r m s}\right)$ were made between the three valves at location $40 \mathrm{~mm}$ downstream from valve sewing ring during peak systole (time bin 9). Figure 8-17, Figure 8-18 and Figure 8-19, respectively, show the velocity magnitude, Reynolds shear stress and $U_{r m s}$ profiles of the central orifice jet $40 \mathrm{~mm}$ downstream from sewing ring of prototype A during peak systole. The three laser techniques yielded maximum velocity magnitude, Reynolds shear stress (- $\overline{\rho u v})$ and $U_{r m s}$ values of approximately $2.4 \mathrm{~m} / \mathrm{s}, 900$ dynes $/ \mathrm{cm}^{2}$ and $0.3 \mathrm{~m} / \mathrm{s}$, respectively. 

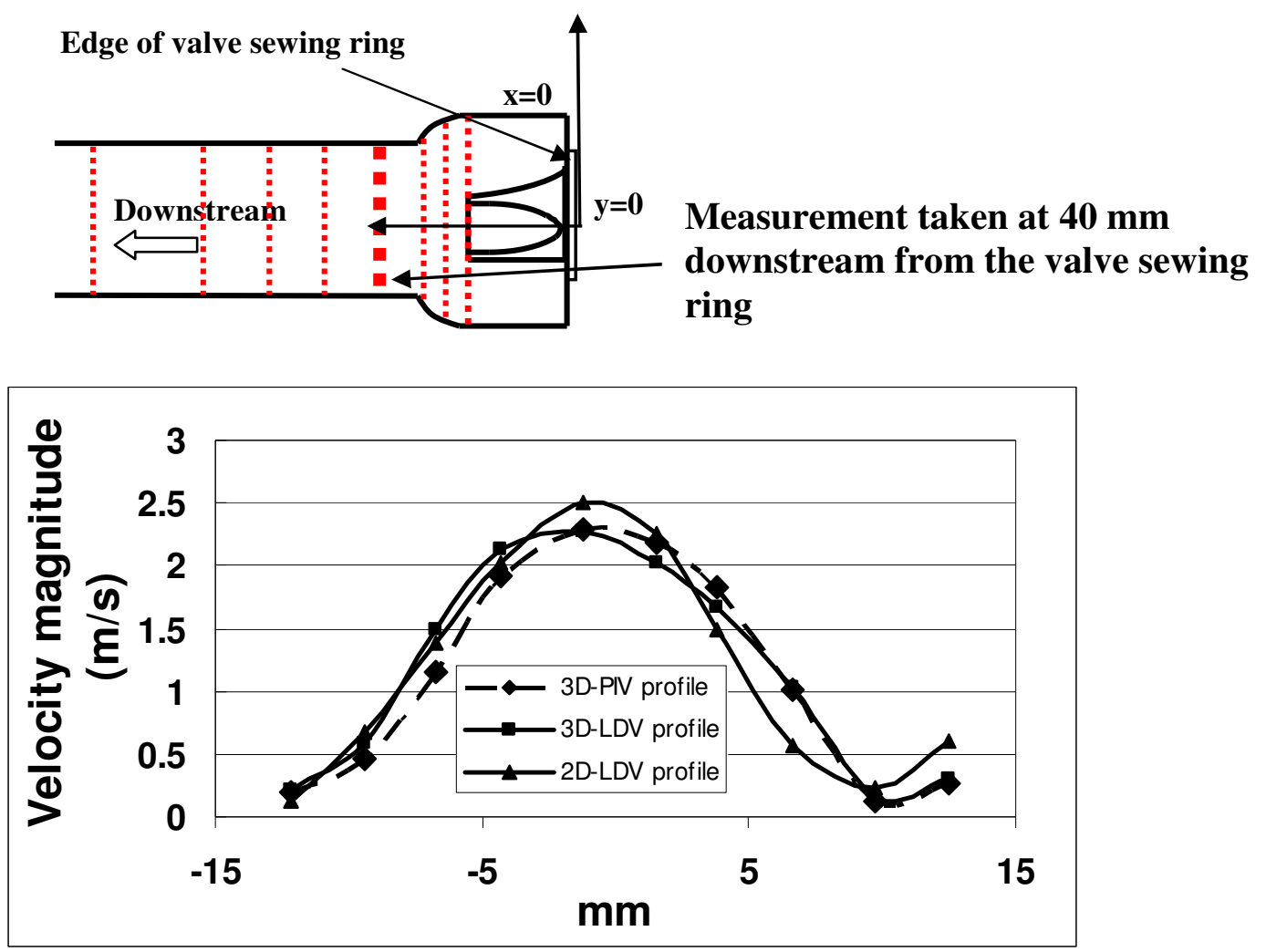

Figure 8-17 Comparison of velocity magnitude profile obtained by the 2D LDV, 3D LDV and the 3D PIV $40 \mathrm{~mm}$ downstream from the sewing ring of prototype A at peak systole, time bin 9

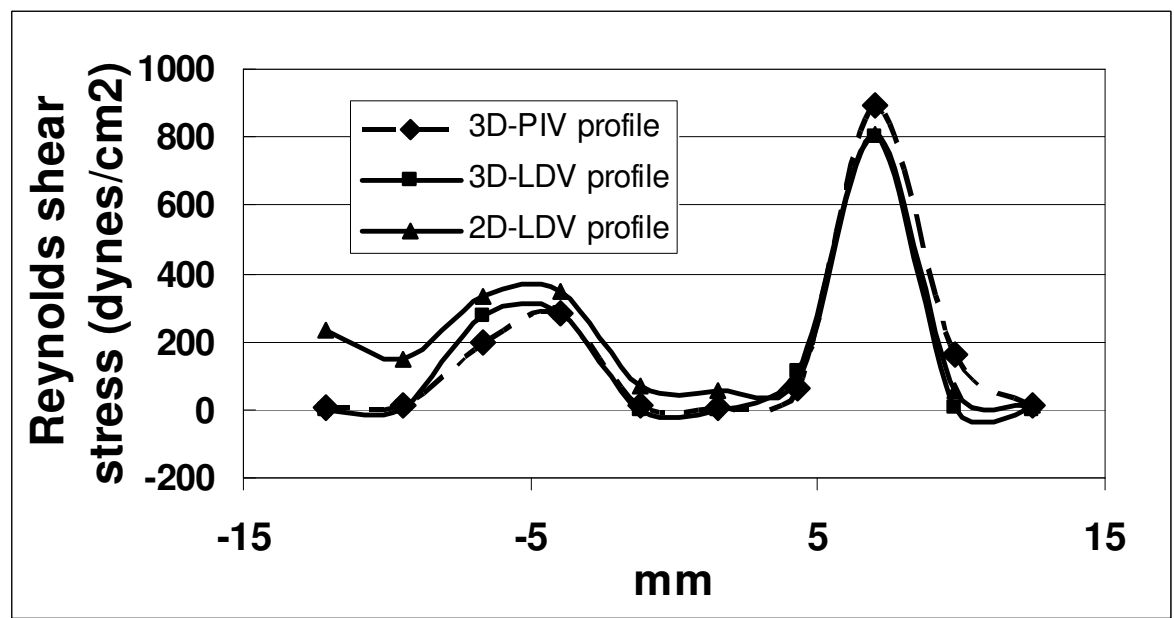

Figure 8-18 Comparison of Reynolds shear stress profile obtained by the 2D LDV, 3D LDV and the 3D PIV $40 \mathrm{~mm}$ downstream from the sewing ring of prototype A at peak systole, time bin 9 


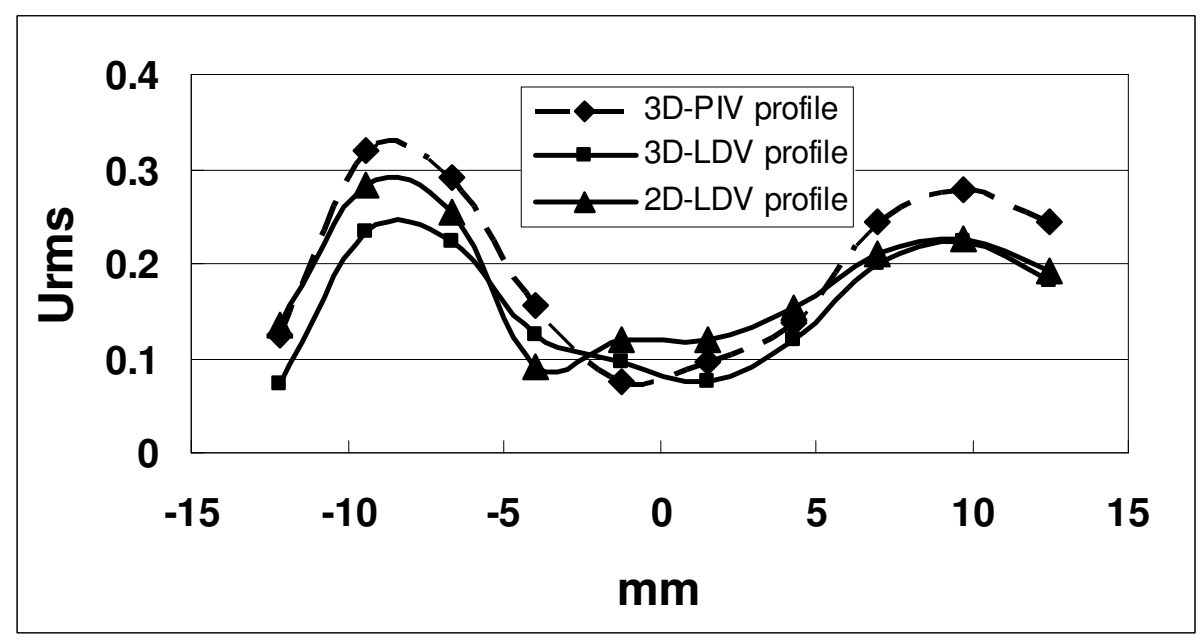

Figure 8-19 Comparison of $U_{r m s}$ profile obtained by the 2D LDV, 3D LDV and the 3D PIV $40 \mathrm{~mm}$ downstream from the sewing ring of prototype A at peak systole, time bin 9

Figure 8-20, Figure 8-21 and Figure 8-22, respectively, show the velocity magnitude, Reynolds shear stress and $U_{r m s}$ profiles of the central orifice jet $40 \mathrm{~mm}$ downstream from sewing ring of prototype $\mathrm{B}$ during peak systole. The two laser techniques yielded maximum velocity magnitude, Reynolds shear stress $(-\overline{\rho u v})$ and $U_{r m s}$ values of approximately $2.0 \mathrm{~m} / \mathrm{s}, 600 \mathrm{dynes} / \mathrm{cm}^{2}$ and $0.45 \mathrm{~m} / \mathrm{s}$, respectively. 


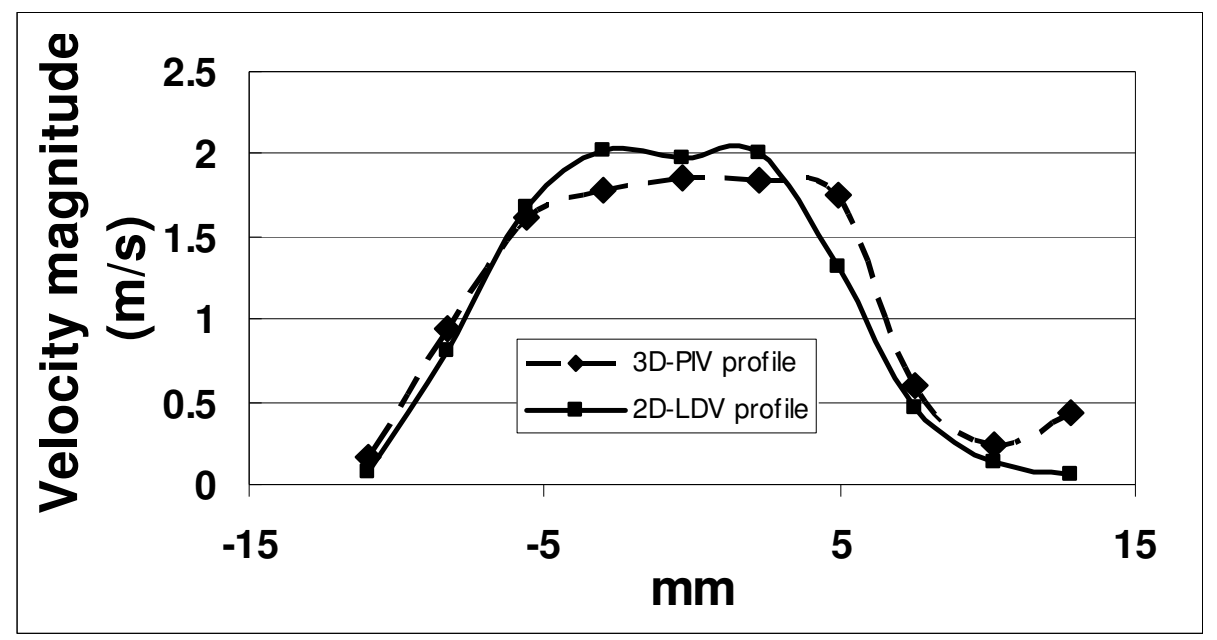

Figure 8-20 Comparison of velocity magnitude profile obtained by the 2D LDV and the 3D PIV $40 \mathrm{~mm}$ downstream from the sewing ring of prototype $\mathrm{B}$ at peak systole, time bin 9

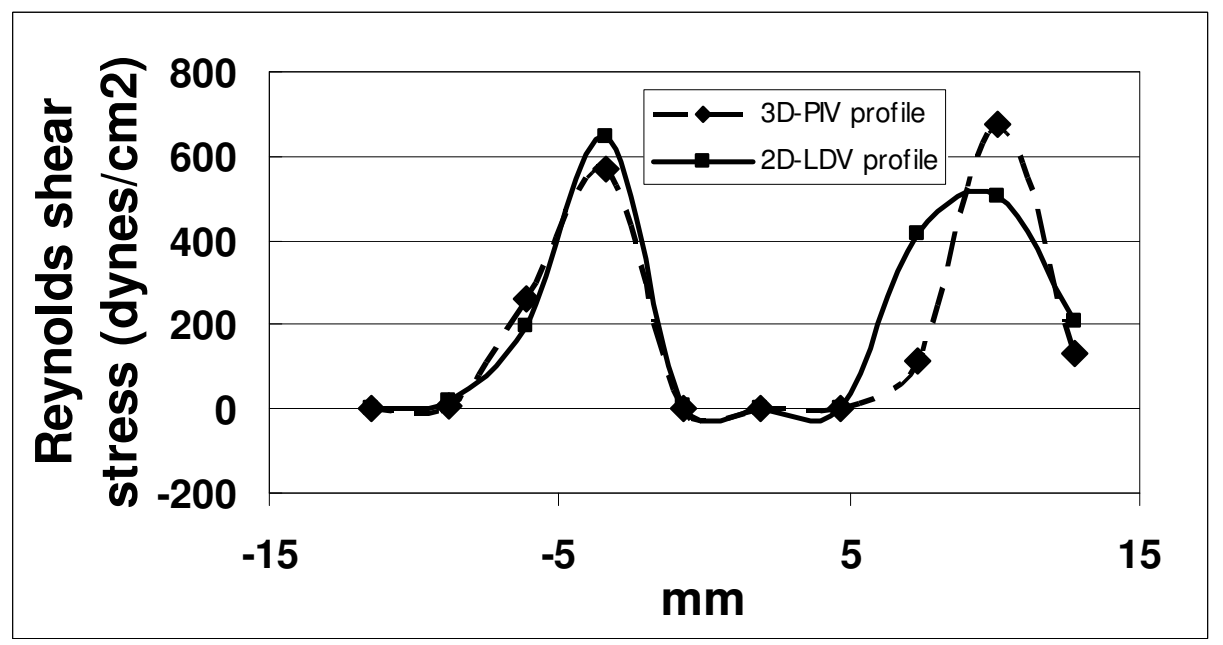

Figure 8-21 Comparison of Reynolds shear stress profile obtained by the 2D LDV and the 3D PIV $40 \mathrm{~mm}$ downstream from the sewing ring of prototype B at peak systole, time bin 9 


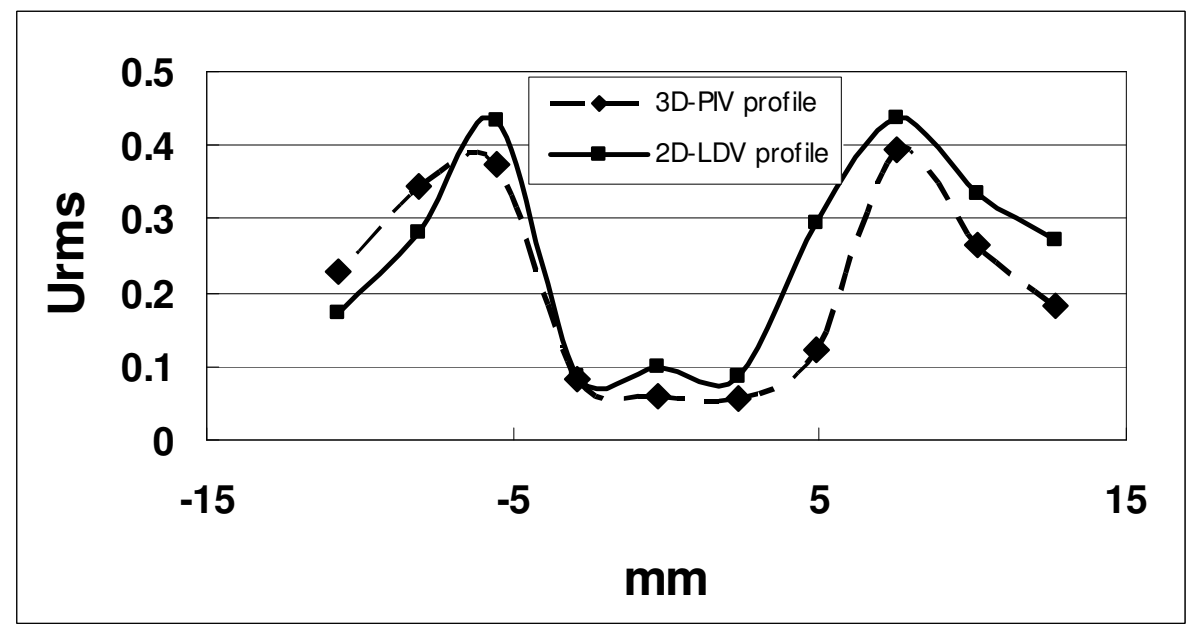

Figure 8-22 Comparison of $U_{r m s}$ profile obtained by the 2D LDV and the 3D PIV $40 \mathrm{~mm}$ downstream from the sewing ring of prototype $\mathrm{B}$ at peak systole, time bin 9

Figure 8-23, Figure 8-24 and Figure 8-25, respectively, show the velocity magnitude, Reynolds shear stress and $U_{r m s}$ profiles of the central orifice jet $40 \mathrm{~mm}$ downstream from sewing ring of prototype $\mathrm{C}$ during peak systole. The two laser techniques yielded maximum velocity magnitude, Reynolds shear stress $(-\overline{\rho u v})$ and $U_{r m s}$ values of approximately $2.0 \mathrm{~m} / \mathrm{s}, 900$ dynes $/ \mathrm{cm}^{2}$ and $0.5 \mathrm{~m} / \mathrm{s}$, respectively. 


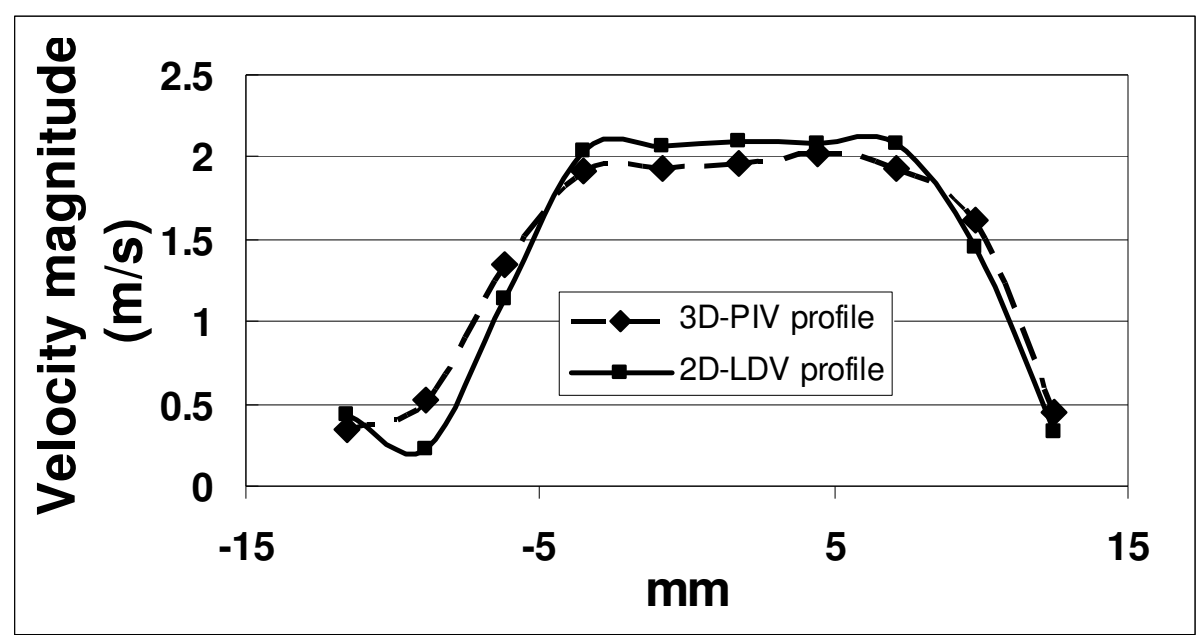

Figure 8-23 Comparison of velocity magnitude profile obtained by the 2D LDV and the 3D PIV $40 \mathrm{~mm}$ downstream from the sewing ring of prototype $\mathrm{C}$ at peak systole, time bin 9

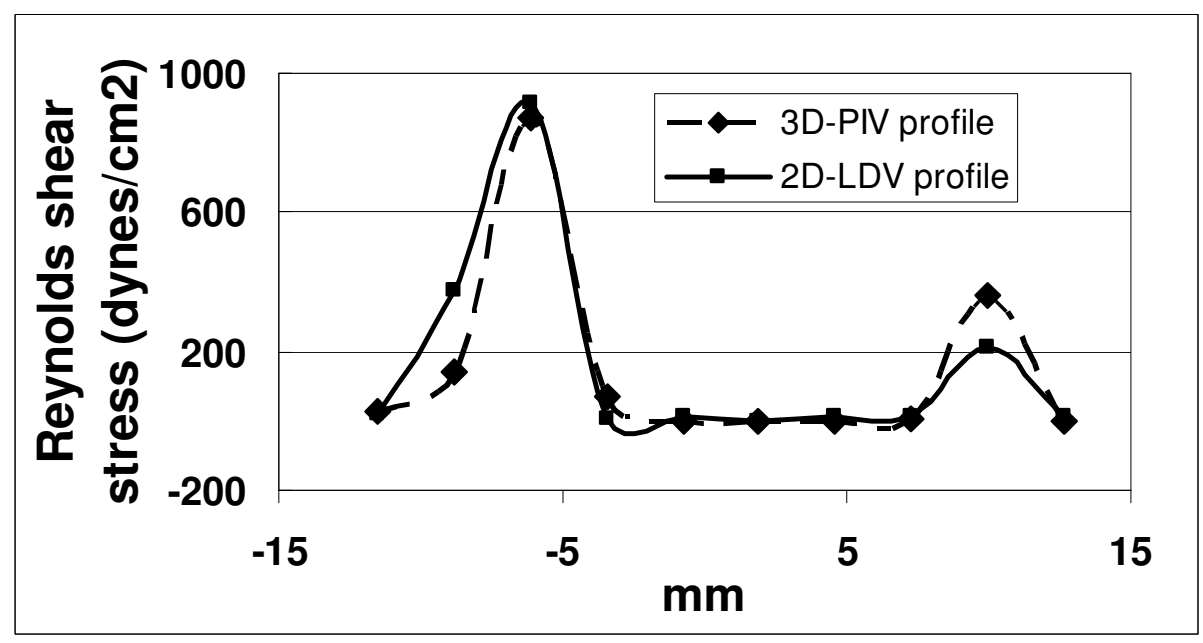

Figure 8-24 Comparison of Reynolds shear stress profile obtained by the 2D LDV and the 3D PIV $40 \mathrm{~mm}$ downstream from the sewing ring of prototype $\mathrm{C}$ at peak systole, time bin 9 


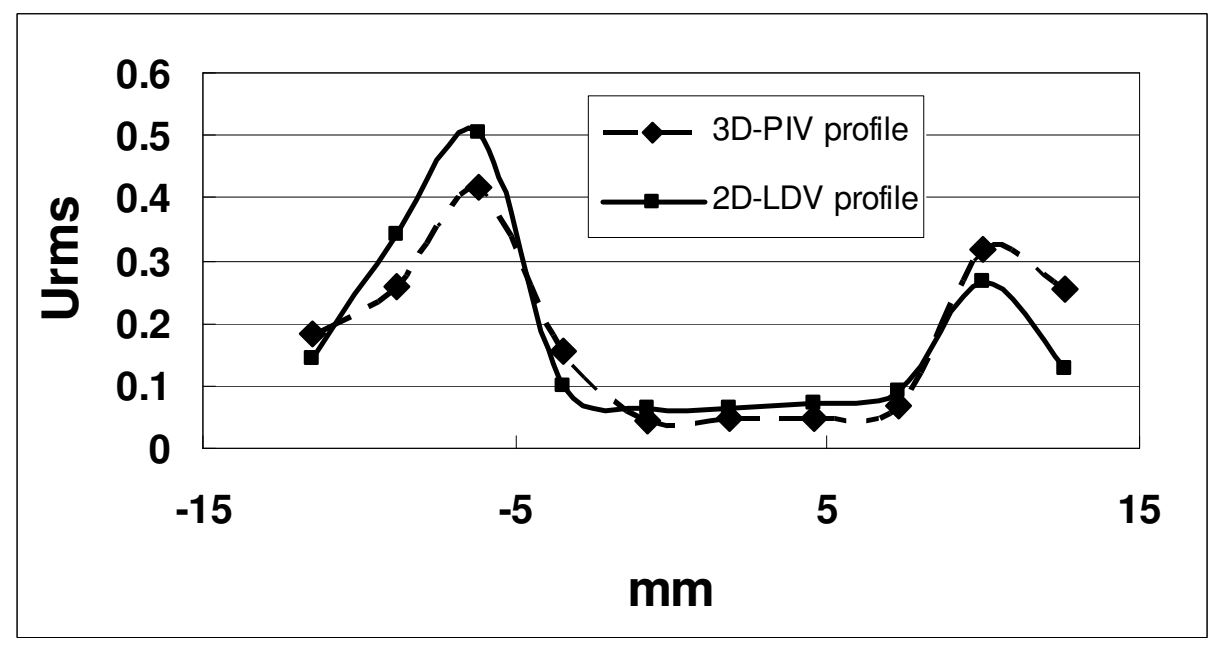

Figure 8-25 Comparison of $U_{r m s}$ profile obtained by the 2D LDV and the 3D PIV $40 \mathrm{~mm}$ downstream from the sewing ring of prototype $\mathrm{C}$ at peak systole, time bin 9

\subsubsection{Comparison of Velocity Magnitude, Reynolds Shear Stress $(-\overline{\rho u v})$ and $\boldsymbol{U}_{r m s}$ Inside the Three Polymeric Heart Valves}

Comparison of the velocity magnitude, Reynolds shear stress $(-\overline{\rho u v})$ and root-meansquare of the $U$ component $\left(U_{r m s}\right)$ were made between the three valves along the leaflet trailing edge inside the valve during peak systole (time bin 9). Figure 8-26, Figure 8-27 and Figure 8-28, respectively, show the velocity magnitude, Reynolds shear stress and $U_{r m s}$ profiles along the leaflet trailing edge inside prototype A during peak systole. The two laser techniques yielded maximum velocity magnitude, Reynolds shear stress $(-\overline{\rho u v})$ and $U_{r m s}$ values of approximately $2.2 \mathrm{~m} / \mathrm{s}, 120$ dynes $/ \mathrm{cm}^{2}$ and $0.22 \mathrm{~m} / \mathrm{s}$, respectively. 

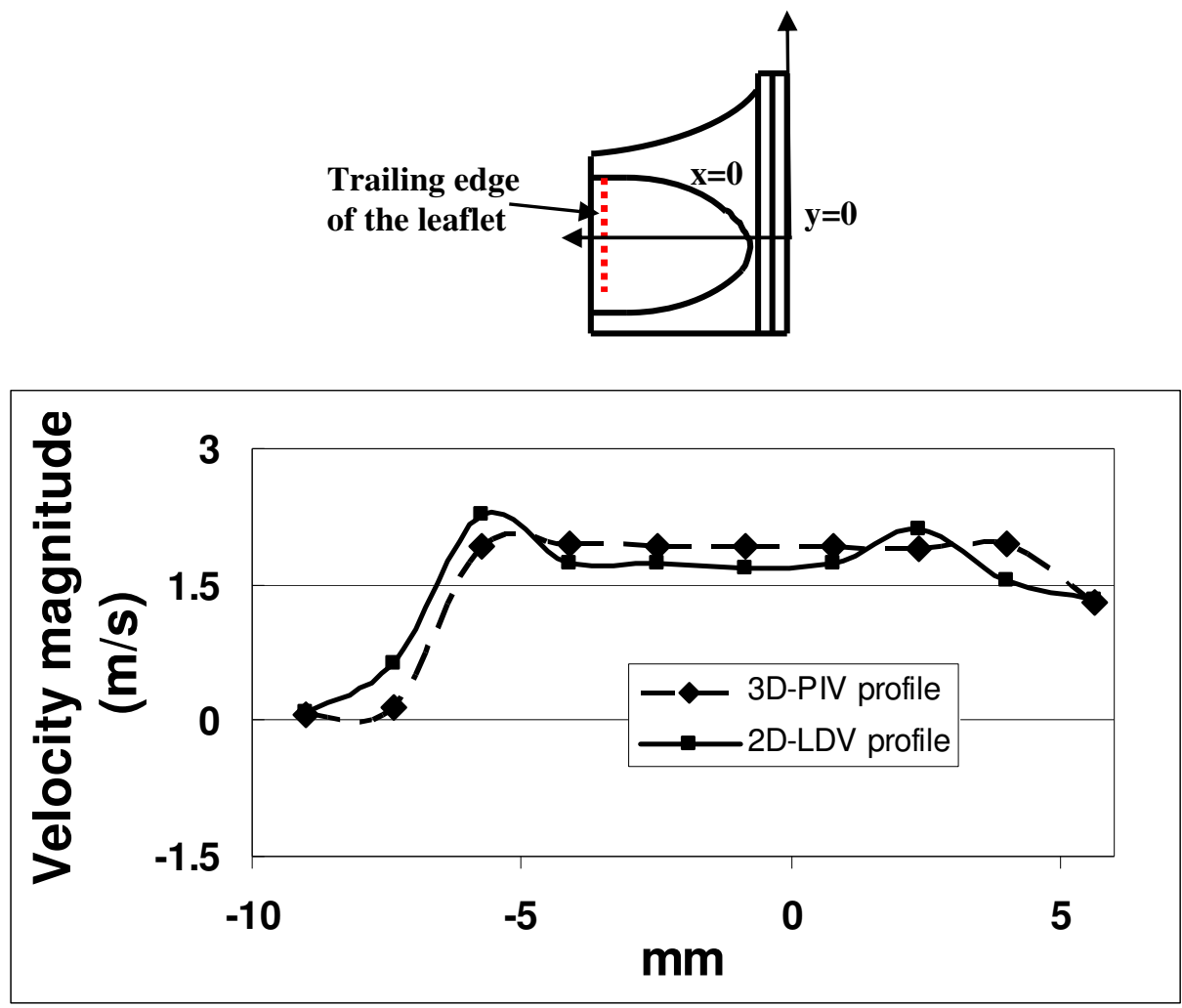

Figure 8-26 Comparison of velocity magnitude profile obtained by the 2D LDV and the 3D PIV along the trailing edge of prototype A at peak systole, time bin 9

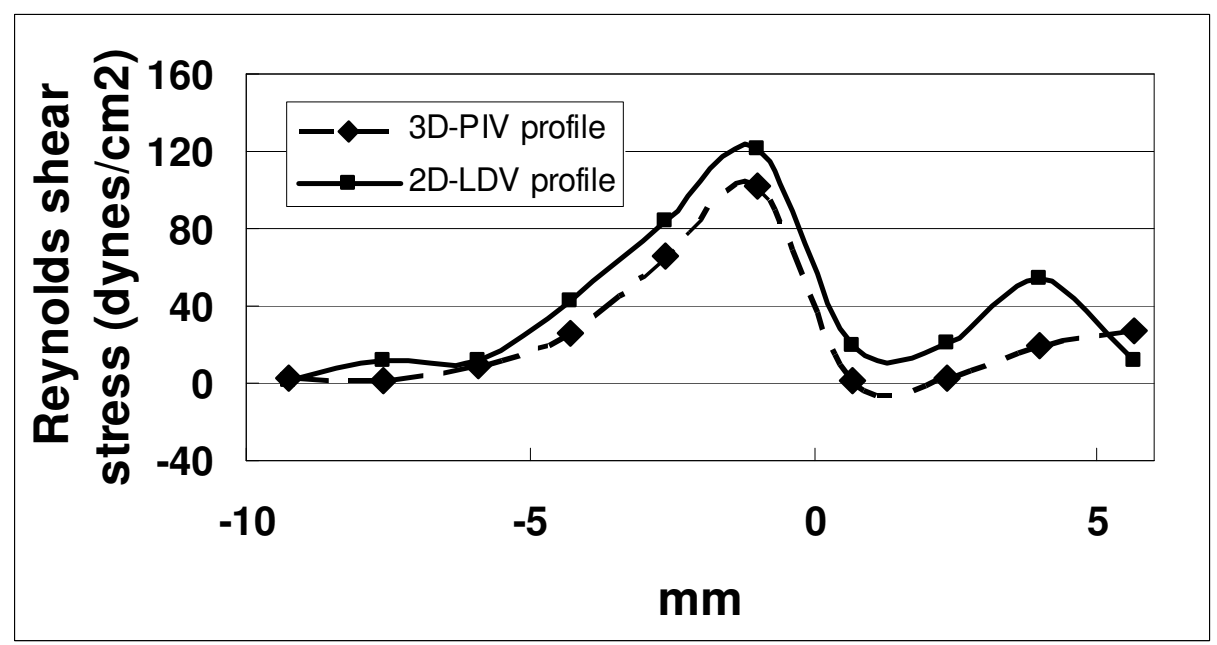

Figure 8-27 Comparison of Reynolds shear stress profile obtained by the 2D LDV and the 3D PIV along the leaflet trailing edge of prototype $A$ at peak systole, time bin 9 


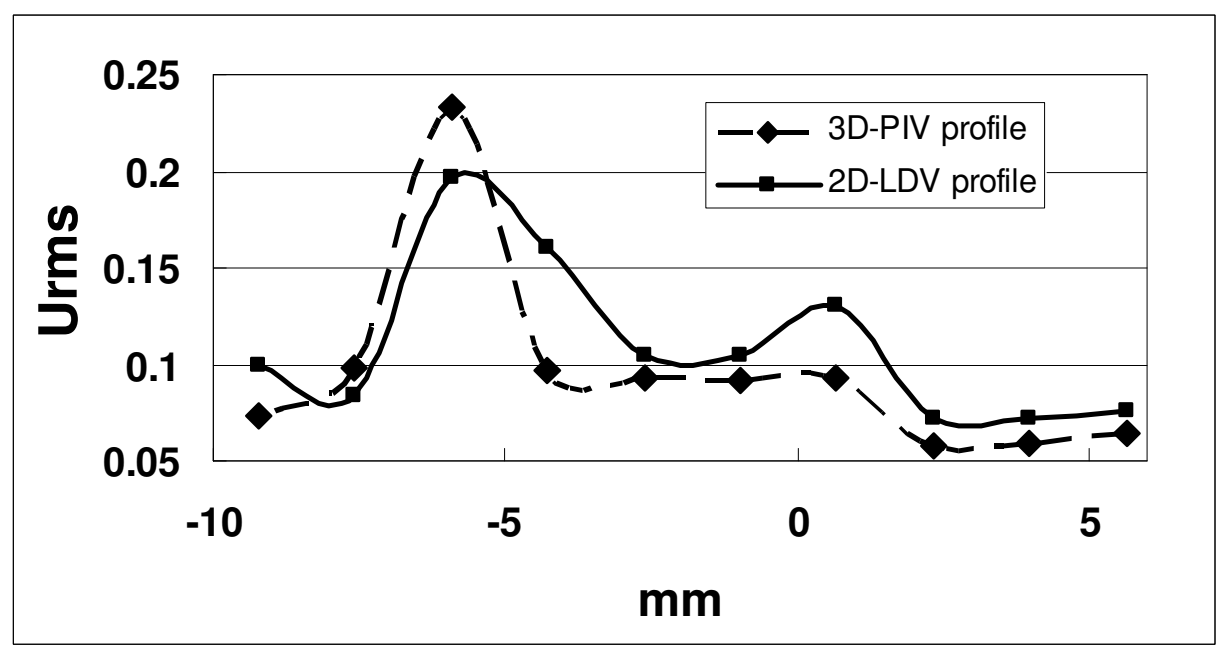

Figure 8-28 Comparison of $U_{r m s}$ profile obtained by the 2D LDV and the 3D PIV along the leaflet trailing edge of prototype $\mathrm{A}$ at peak systole, time bin 9

Figure 8-29, Figure 8-30 and Figure 8-31, respectively, show the velocity magnitude, Reynolds shear stress $(-\overline{\rho u v})$ and $U_{r m s}$ profiles along the leaflet trailing edge inside prototype B during peak systole. The two laser techniques yielded maximum velocity magnitude, Reynolds shear stress $(-\overline{\rho u v})$ and $U_{r m s}$ values of approximately $1.8 \mathrm{~m} / \mathrm{s}, 12$ dynes $/ \mathrm{cm}^{2}$ and $0.06 \mathrm{~m} / \mathrm{s}$, respectively. 


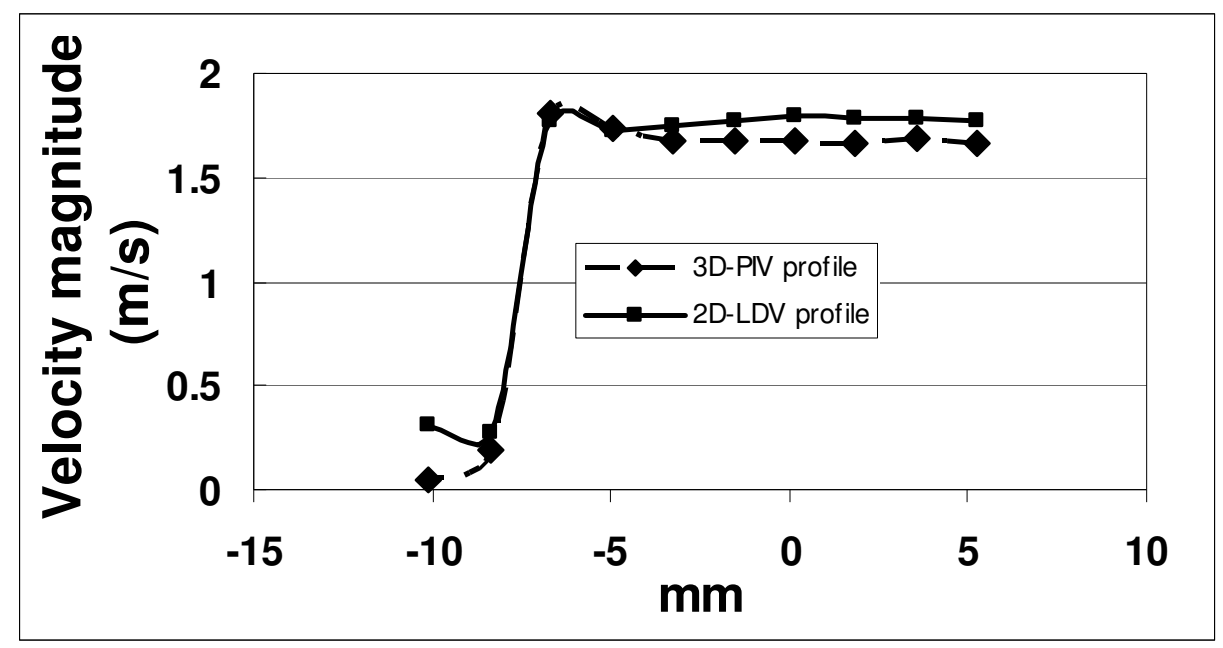

Figure 8-29 Comparison of velocity magnitude profile obtained by the 2D LDV and the 3D PIV along the trailing edge of prototype B at peak systole, time bin 9

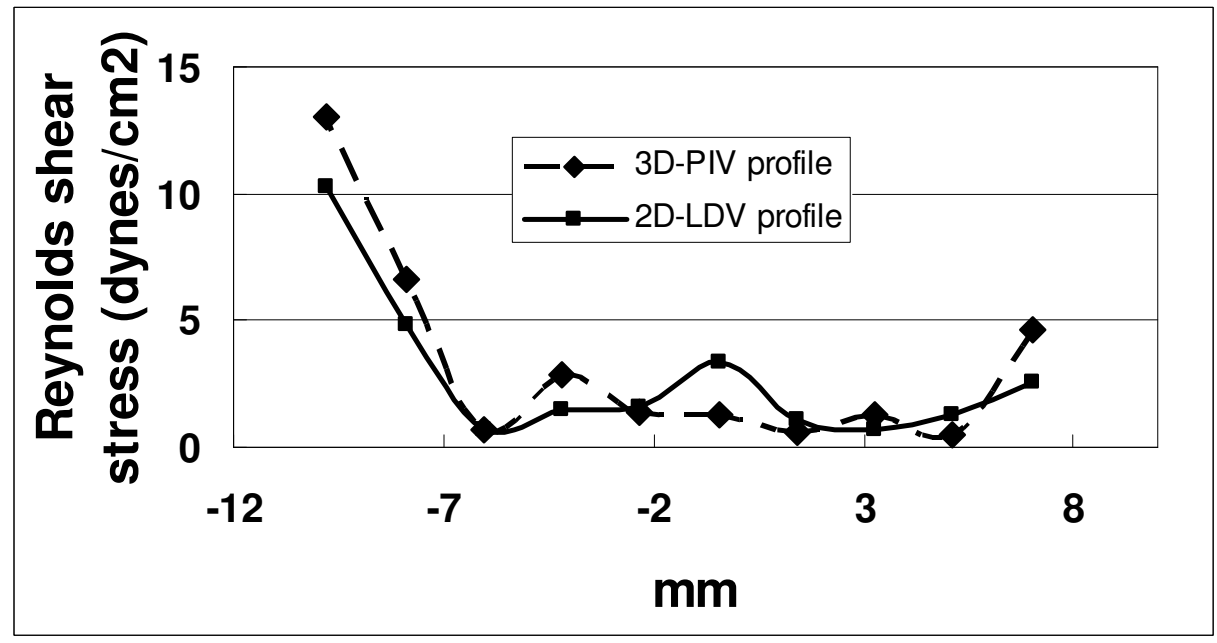

Figure 8-30 Comparison of Reynolds shear stress profile obtained by the 2D LDV and the 3D PIV along the leaflet trailing edge of prototype B at peak systole, time bin 9 


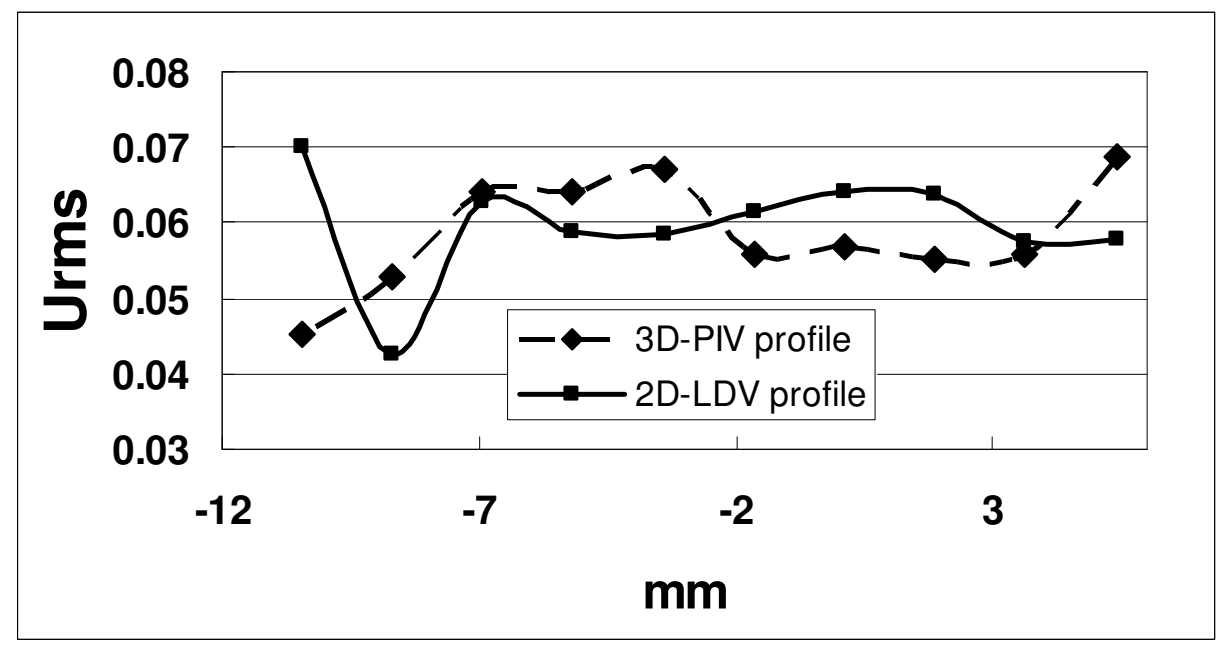

Figure 8-31 Comparison of $U_{r m s}$ profile obtained by the 2D LDV and the 3D PIV along the leaflet trailing edge of prototype B at peak systole, time bin 9

Figure 8-32, Figure 8-33 and Figure 8-34, respectively, show the velocity magnitude, Reynolds shear stress $(-\overline{\rho u v})$ and $U_{r m s}$ profiles along the leaflet trailing edge inside prototype $\mathrm{C}$ during peak systole. The two laser techniques yielded maximum velocity magnitude, Reynolds shear stress $(-\overline{\rho u v})$ and $U_{r m s}$ values of approximately $2.0 \mathrm{~m} / \mathrm{s}, 30$ $\mathrm{m}^{2} / \mathrm{s}^{2}$ and $0.06 \mathrm{~m} / \mathrm{s}$, respectively. 


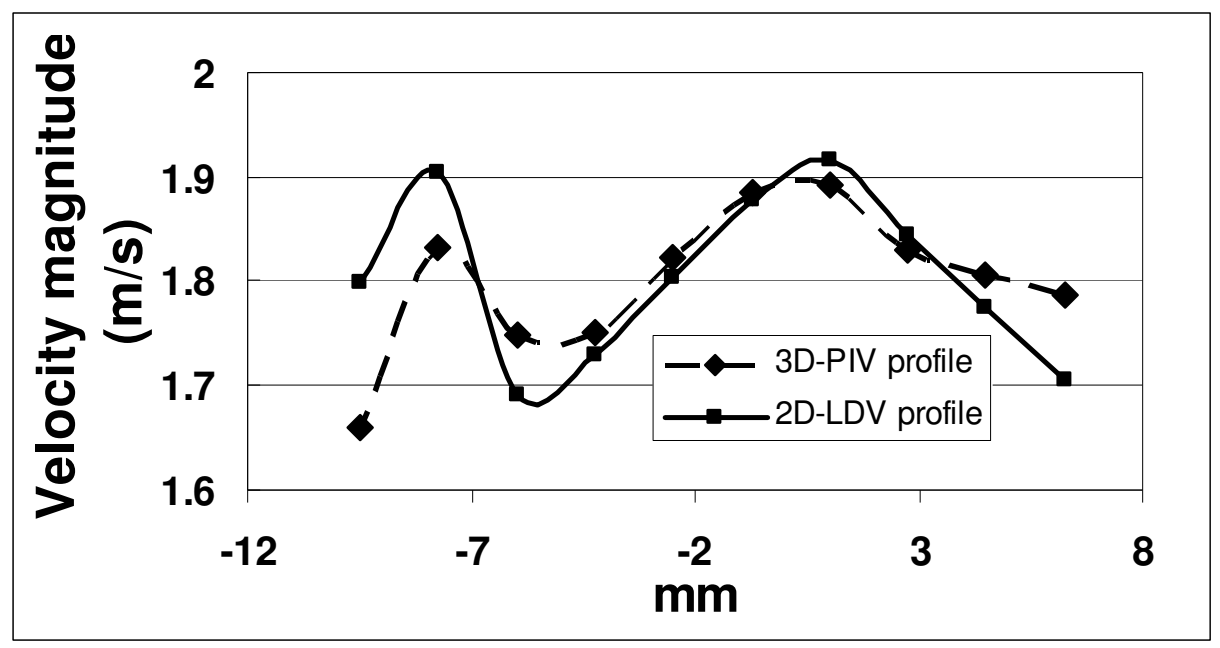

Figure 8-32 Comparison of velocity magnitude profile obtained by the 2D LDV and the 3D PIV along the trailing edge of prototype $\mathrm{C}$ at peak systole, time bin 9

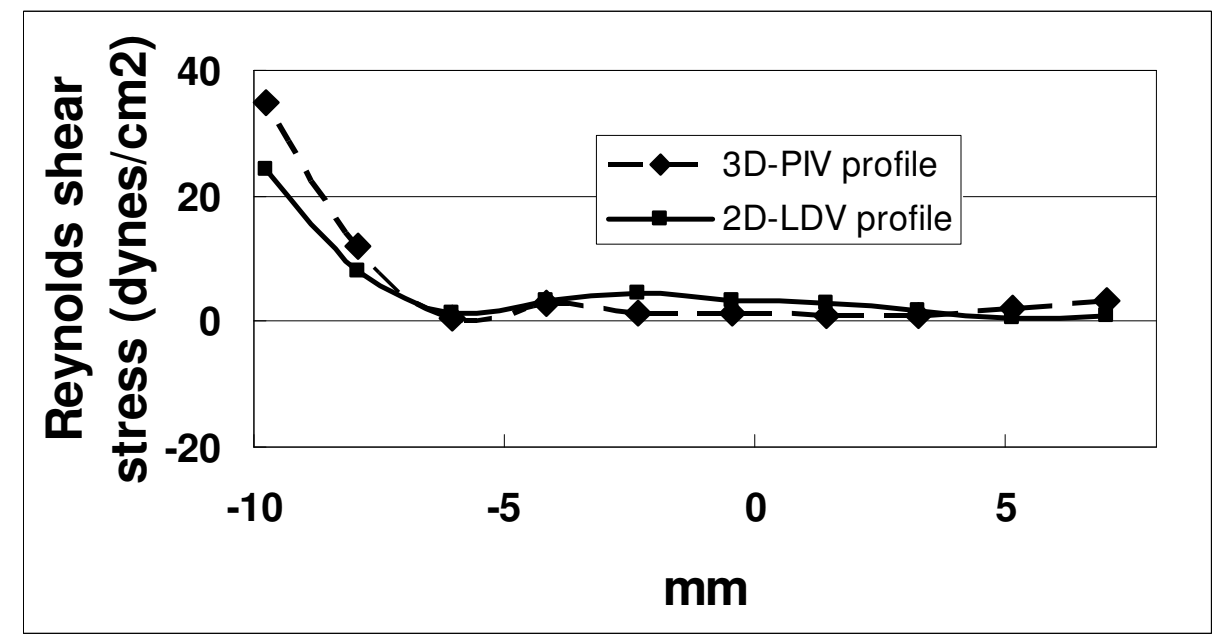

Figure 8-33 Comparison of Reynolds shear stress profile obtained by the 2D LDV and the $3 \mathrm{D}$ PIV along the leaflet trailing edge of prototype $\mathrm{C}$ at peak systole, time bin 9 


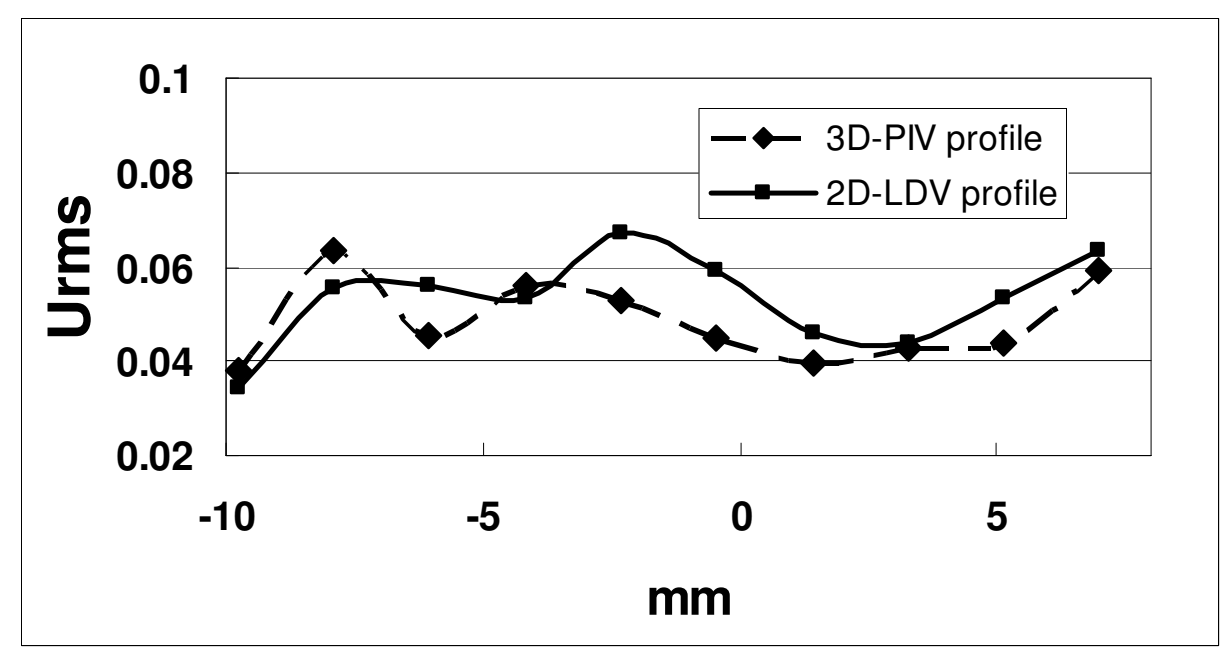

Figure 8-34 Comparison of $U_{r m s}$ profile obtained by the 2D LDV and the 3D PIV along the leaflet trailing edge of prototype $\mathrm{C}$ at peak systole, time bin 9

\subsubsection{Review of the LDV and PIV Techniques}

Comparison of the velocity magnitude, Reynolds shear stress $(-\overline{\rho u v})$ and $U_{r m s}$ profiles in sections 8.4.1, 8.42 and 8.4.3 show good agreement between the LDV and PIV results. The main reason for the observed difference between the LDV and PIV results could be due to the different in data reduction between the two laser techniques. All experiments discussed in this section were performed under pulsatile conditions. In LDV, a total of 21,500 data points were acquired continuously over approximately 150 cardiac cycles at each measurement location. The entire cardiac cycle was then divided into 43 time bins, and the velocity data were phase averaged over a period of $20 \mathrm{~ms}$, which corresponds to approximately 500 data point. In contrast, no binning was performed for the PIV results. Instead, 250 image pairs were sampled at the same instant in the cardiac cycle and then used for subsequent data reduction. LDV is capable of measuring a higher density of points than PIV, but becomes impractical for studies that involved details spatial flow field interrogations. The main limitation of the PIV is that only 250 image pairs were 
obtained at each location as compared to the 500 samples attained by the LDV at each location in each time bin. The number of image pairs in PIV is constraint by the storage capacity of the PIV system. Based on the error calculation using equation 4-3 in section 4.8.3.4, approximately 10,000 image pairs are needed to achieve an error that is lower than 3 percent for the out-of-plane measurements $(z)$. This amount of data would require an inordinate amount of storage space, which is deemed infeasible for current studies.

Another possible source of error that could cause the observed differences between the LDV and PIV results is the difficulty associated with the acquisition of an accurate outof-plane velocity component in the current PIV system. The accuracy of the out-of-plane velocity measurement in 3D PIV, besides CCD camera resolution and particle density, is largely influenced by the angle between the two CCD cameras $(\alpha)$. Lawson et al has shown that significant errors in the $z$ component (out-of-plane) begin to occur when the camera angle is decreased from $45^{\circ}$ to $10^{\circ}$ [Lawson, 1997]. For instance, the study revealed that the rms error for the out-of-plane measurement at $45^{\circ}$ is 4 percent with an increase to a maximum of 13 percent at $10^{\circ}$. Conversely, the $x$ component (stream-wise) error increases gradually with camera angle from around 1 percent at $10^{\circ}$ to $3-4$ percent at $45^{\circ}$. It is believed that these counteracting effects are caused by the geometric and imaging properties of the system. The error in the out-of-plane measurements is mainly determined by the camera geometry, since an increase in camera angle will result in greater imaging sensitivity to a $z$ displacement and hence a lower measurement error. In contrast, for the stream-wise component, $x$, greater camera angles will invariably lead to an increase in imaging problems, such as aberrations, with a resultant increase in the $x$ 
error. Hence, a compromise must be sought between acceptable imaging quality and the required $z$ accuracy. Based on the initial results, Lawson et al suggested an optimum camera of between $20^{\circ}$ and $30^{\circ}$. For the studies presented in this thesis, the camera angle was set at approximately $30^{\circ}$.

The obvious differences between the LDV and PIV results are presented in sections 7.3.3, 7.3.4 and 7.3.5 is the different field of view for the two laser techniques. In LDV, the field of view is constrained by the approaching angle of the laser beam, hence velocity measurements that are close to chamber wall or the stent of the polymeric valve were not obtained. In contrast, the flow structures in the PIV was captured by a CCD camera placed perpendicular to the laser sheet. This configuration allows velocity measurements to be obtained very close to the wall of the flow chamber as well as to the stationary stent of the polymeric heart valve. Therefore, 3D PIV was able to capture leakage jets along the stent inflow region that could not be captured by 2D LDV or 3D LDV due to interference by the stent oscillation during diastole. This interference significantly reduced the data rate near the leaflet and valve stent surface. Figure 8-35 shows the overlapping of the measurement planes for both the LDV and PIV methods. The 2D LDV and 3D LDV measurement planes downstream of the valve had a length of about 62.2 $\mathrm{mm}$ measured from the trailing edge of the valve leaflet, while the 3D PIV measurement plane had a length of about $56 \mathrm{~mm}$ measured from the rear edge of the valve. Therefore, the 3D PIV measurement plane could effectively acquire only flow fields $44 \mathrm{~mm}$ downstream from the trailing edge of the valve leaflet. 
Another obvious difference can be seen in the vector density plots in sections 7.3.3, 7.3.4 and 7.3.5. The results from 3D PIV were able to provide more detailed global flow field information than those obtained by 2D LDV and 3D LDV. The PIV results have a spatial resolution of $0.4 \mathrm{~mm} \times 0.4 \mathrm{~mm}$ in the $\mathrm{x}$ and y directions, respectively. Approximately 7,500 velocity vectors were obtained in a single 3D PIV measurement plane, while only 80-180 measurement locations were obtained in a single 2D LDV or 3D LDV measurement plane. This explains why a vortex ring was observed only in the 3D PIV results and not in the $2 \mathrm{D}$ LDV or 3D LDV results. On the other hand, the differences in flow characteristics observed in the 3D PIV results were not as distinct as those observed in the 2D LDV and 3D LDV studies. Indeed, comparison of the 3D PIV results revealed no significant difference in the flow field distribution inside and downstream of the polymeric heart valves. This is due to the fact that the size of the LDV probe volume (20 $\mathrm{x} 140 \mu \mathrm{m})$ is much smaller than the laser sheet thickness $(1.5 \mathrm{~mm})$ of the PIV measurement. Consequently, the 2D LDV and 3D LDV measurements can better detect the micro flow structures in the vicinity of the polymeric heart valves. For instance, the 2D LDV flow field measurements downstream of the stent revealed forward jets and leakage jets through the commissural region of the three polymeric valves during peak systole and diastole, respectively. These jets were not observed in the 3D PIV results. 


\section{Field of view of 3D LDV}

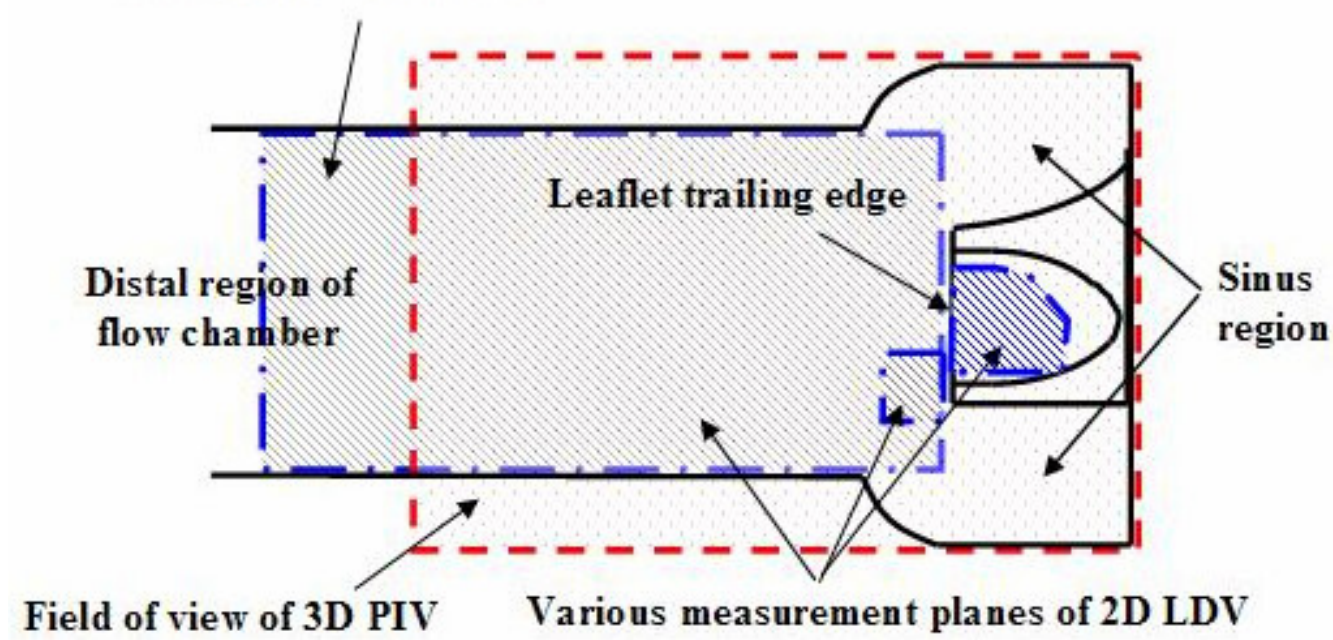

Figure 8-35 Shows the overlapping of field of view for 2D, 3D LDV and 3D PIV. The different field of view constitute one of the main reasons for the observed different in the different flow features inside and in the vicinity of the polymeric heart valves

In conclusion, it is important to note that given the time consuming nature of taking LDV measurements, it is impractical to use it to capture large-scale coherent structures. For this purpose PIV is superior. Previous comparison of the LDV and PIV techniques under steady flow conditions concluded that the PIV method may not be able to accurately resolve the turbulent fluctuations in fluid flow [Browne, 2000]; however, comparison of the velocity magnitude, shear stress $(-\overline{\rho u v})$ and $U_{r m s}$ profiles in sections 8.4.1, 8.4.2 and 8.4.3 show that the results from PIV are compatible to those obtained by the LDV technique. Current studies demonstrated that with sufficient PIV image pairs, it is possble to achieve accurate PIV measurements comparable to those of the LDV. 


\section{$\underline{\text { 8.5 Experimental Limitations }}$}

2D LDV mitral hinge studies - The LDV measurements were conducted only at selected locations and thus do not completely define the flow fields within the hinge regions of the valve. Also, the measurements were acquired in a 2D plane, and any out of plane velocities (i.e. change in the orientation of the jet) are not represented. It is apparent that the confined hinge flow fields may indeed be 3D because of the complex geometries formed by the mating of the leaflet and hinge. Other experimental techniques [Gao, 1999; Healy, 1998], such as high-speed flow visualization or the use of scaled-up models, may be useful in assessing the flow fields more completely. The primary experimental limitations that prevented more detailed interrogations were the optical access of the models and the width of the gap between leaflet and housing. The low data rate sometimes encountered during data acquisition could contribute to the cyclic variation error for the Reynolds shear stress. Furthermore, the measurement sites in the hinge pocket are very close to where the leaflets mate against the hinge stop during opening or closing. In such a small, confined area it is possible that vibrations or pressure wave reflections caused by the impact of the leaflets against the hinge stop caused the probe volume to shift slightly into an adjacent region. Although no study was conducted to investigate the effect of vibration on the velocity measurements, preliminary tests were performed at two levels, namely the $390 \mu \mathrm{m}$ and $190 \mu \mathrm{m}$ levels within the hinge socket in the CM $23 \mathrm{~mm}$ valve, and the velocity data were shown to be similar to those obtained during the actual experiment. An important consideration is the accuracy of probe volume translation through the very small hinge. The traversing mechanisms have an accuracy of 0.001 inch $(0.003 \mathrm{~mm})$ and it is possible that some spatial errors in locating 
the measurement sites could have been resulted. However, the use of the oscilloscope to identify the appearance of the Doppler bursts was helpful as a means of locating the datum surfaces for each study.

Another limitation of the mitral hinge studies is that of the use of rigid mounted models because it does not take into account the deformation of the valve wall during each cardiac cycle. However, the present simplified setup helped isolate various factors that might contribute to the influence of the hinge flow dynamics. Further by ensuring that the waveforms obtained were compatible with that of the physiological conditions, the author is confident that the in vitro set up could, to a very good approximation, mimic the flow dynamics of the in vivo conditions. Although only one set of test valves was used, the static flow leakage data were representative of the particular type of valve designs and it should provide reliable flow information in regards to the fluid dynamic performance. In addition, although the clear housing valves used in the thesis were a reproduction of a clinical quality carbon bileaflet valves, the dimensional accuracy of the reproduction should be considered in evaluating the limitations of this study. As stated earlier the static leakage flow rate through the reproduced clear housing valves were similar to that through the clinical quality carbon valves, thereby providing confidence in the reproduction technique [Ellis, 1996a; Ellis, 2000a; Ellis, 2000b]. Furthermore, based on prior experience with LDV experimental technique, the transparent valve models do accurately reproduce the critical dimensions of the hinge regions of the clinical quality valves. 
2D, 3D-LDV and 3D PIV polymeric valve studies - A major constraint to the current aortic valve studies was that only three different valve designs were investigated. Further work involving more valve designs would provide a much broader understanding of the effect of design parameters on the flow fields of polymeric valves. However, the author believes that the current studies set the ground work for further in vitro studies that focus on the hemodynamic aspects of polymeric heart valve design.

One of the major challenges in the LDV technique is the optimization of the data rate. Unlike other engineering research where the data rate for LDV could easily go beyond 1,000 Hz [Gizzi, 1999; Glas, 2000; Szabo, 2001], the typical data rate for a 2D LDV fluid flow interrogation at the downstream regions of valve prostheses was $400 \mathrm{~Hz}$. With the use of 3D LDV, the data rate could go down to $20 \mathrm{~Hz}$; the inclusion of an additional velocity component invariably reduced the temporal resolution of the data, with a concomitant increase in the time needed for the data acquisition. Therefore, prior to conducting the LDV experiments, it is necessary to consider if the advantage for having more velocity information outweigh the need for better data accuracy and the time needed to acquire the velocity data. Another consideration, which is related to all the experiments but most importantly to the LDV studies, was the matching of the refractive indices between the clear housing models and the blood analog solution. Care was taken to match the indices of refraction, so as to minimize the error that could arise due to the difference in the model and fluid indices. 
As discussed in section 8.4.4, the accuracy of the 3D PIV velocity measurement can be effected by the number of image pairs taken. For current thesis works, the PIV velocity information was phased averaged from 250 image pairs, which was able to resolve stream-wise velocity to an error of less than 3 percent. Comparison of the shear stress $(u v)$ and $U_{r m s}$ profiles in section 8.4.1, 8.4.2 and 8.4.3 show that PIV stream-wise turbulence measurements were comparable to those obstained by the LDV method. However, to obtain an equivalent accuracy for the out-of-plane velocity component would require more than 10,000 image pairs, which would incur an inordinate amount of data storage space. Therefore, balance need to be strike between the acceptable accuracy for each velocity component and the investment on the amount of disk space for storing these information. 


\section{CHAPTER 9: CONCLUSION}

\subsection{Mitral Hinge Studies}

Hemolysis and platelet activation in artificial heart valves are primarily associated with regions of high shear stress in leakage jets and along the edge of high velocity forward flow. Damaged or activated blood elements initiate a biochemical cascade resulting in thrombus formation, which may be further exacerbated by regions of low and recirculating flow. Therefore, it is imperative to highlight flow regions that may affect the thrombogenic potential of prosthetic heart valves.

The 2D LDV mitral hinge studies in this thesis identified several areas in the valve as potential locations for platelet activation and/or thrombus formation. The regions where high Reynolds shear stresses occurred were: 1) the leakage jets at the atrial, adjacent and lateral hinge corners during systole; 2) the gap between the closed leaflets during systole; and 3) flow next to the inner surface of the open leaflets during diastole. These high Reynolds shear stress levels may damage red blood cells and/or activate platelets. Regions of low flow were: 1) the low flow regions next to the atrial and ventricular wall of the pivot hinge during the entire cardiac cycle; and 2) the vortex in the adjacent corner of the hinge geometry during diastole. These low flow regions may enhance the interaction between the activated/lysed blood elements thereby increasing the potential for thrombus formation inside the hinge region. 
With improved spatial resolution the current mitral hinge study of the CM $23 \mathrm{~mm}$ clear valve was able to provide a detailed assessment of the micro flow hinge profile. Based on the peak leakage velocities and the maximum Reynolds shear stresses, the CM mitral hinge study demonstrated that the hinge flow dynamics of the CM valve lie somewhere in between those of the SJM Regent and the MP bileaflet valve designs. The leakage velocities in the CM valve were higher than those in the MA valve; however, the Reynolds shear stress levels in both valves were comparable. The fluid dynamics of the $\mathrm{CM}$ valve were found to be similar to those of the SJM Regent valves, although with higher leakage velocities and Reynolds shear stresses. These differences are mainly attributed to the differences in the hinge geometries of the three valve designs as well as to the larger hinge gap width of the CM valve. The hinge design of the CM valve is characterized by a sharper profile, which creates visible regions of 'unmated' spaces at each corner of the hinge geometry, during both the fully closed and fully open positions of the leaflets. The angulated recess may result in more gap space between the leaflet projection and the valve housing thereby allowing more fluid to flow through the hinge during the systolic phase giving rise to higher leakage jet velocities and corresponding Reynolds shear stresses. Furthermore, it is possible that these 'unmated' spaces at the hinge corners may aggravate the leakage problem by disrupting the hinge flow, which may lead to increased levels of turbulent shear stresses.

The comparison of the hinge flow fields between the SJM $27 \mathrm{~mm}$ Standard and the two $27 \mathrm{~mm}$ prototype high and low leaker valves demonstrated the existence of an optimum leakage gap. The performance of the SJM $27 \mathrm{~mm}$ Standard valves was found to lie 
between that of the high and low leaker valves. The SJM $27 \mathrm{~mm}$ Standard valve had regions of elevated Reynolds shear stress and high velocity similar to those observed in the prototype high leaker but of smaller magnitudes. Air bubbles were observed inside the prototype high leaker valve suggesting a potential for inefficient washout of the blood elements in valves with a large hinge gap width. In addition, the prototype high leaker valve was also plagued by elevated velocity magnitude and Reynolds shear stress levels in the hinge region during the leakage phase. The prototype low leaker valve recorded smaller regions of high Reynolds shear stress and elevated velocities compared to the SJM $27 \mathrm{~mm}$ Standard and prototype high leaker. However, its smaller gap width may make it more prone than other two valves to cavitation that is also detrimental to the blood elements. The decreased hinge gap width in the low leaker valve may predispose it to cause high viscous shear stresses. These hinge studies highlighted the importance of the pivot mechanism in determining the success of a bileaflet heart valve. A proper hinge design as well as an optimal hinge gap width with precise manufacturing tolerances can significantly reduce the potential for thrombus formation.

\subsection{Aortic Polymeric Valve Studies}

The results of the present study indicated that commisural design and leaflet thickness can influence the thrombogenic potential of tri-leaflet polymeric valves. The studies identified the following regions of high shear stress and high velocity flow in the three polymeric heart valves: 1) the leakage jet inside the valve during diastole; 2) the trailing edge of the valve; 3) the vortex ring surrounding the forward jet during the acceleration phase; 4) the edge of the central orifice jet, which extended from the inside of the valve 
into the distal part of the flow chamber; and 5) the distal region of the flow chamber where turbulence mixing occurred during systole. Low flow velocities were observed in the following regions of the three polymeric heart valves: 1) the split flow inside the valve during diastole; 2) a vortex inside the sinus region; and 3) flow inside the recirculation zone along the chamber wall during diastole. Additional low flow regions were observed in prototype A: 1) the inflow stent region during late systole; and 2) the closed commissural region during systole.

Reconstruction of the the 3D PIV results yielded a three-lobe iso-surface of the flow profile downstream of the valve. This observed profile can be attributed to the open configuration of the leaflets at systole. The forward flow in the polymeric heart valve was characterized by a central orifice jet that has a flat profile very similar to that observed in bioprosthesic valves. A split flow phenomenon was observed inside the three polymeric valves and was believed to be caused by the leakage jets and the oscillation of the valve leaflets during early diastole. During diastole, this split flow may enhance the transportation of activated/lysed blood elements towards the stent regions. The findings of the 2D LDV and 3D PIV studies were qualitatively corroborated in preliminary animal studies performed by Dr D. J. Wheatley at the University of Glasgow. Figure 9-1 shows an explanted polymeric valve similar in design to prototype B. Clots were found along the stent inflow region as well as at the high central region of the leaflets. In animal trials with prototype $\mathrm{A}$, besides the stent inflow region, clots were formed at the closed commissural regions. LDV results with prototype A showed a stagnation zone at the 
commissural region as well as a region of low flow that extended along the stent inflow region inside the valve.

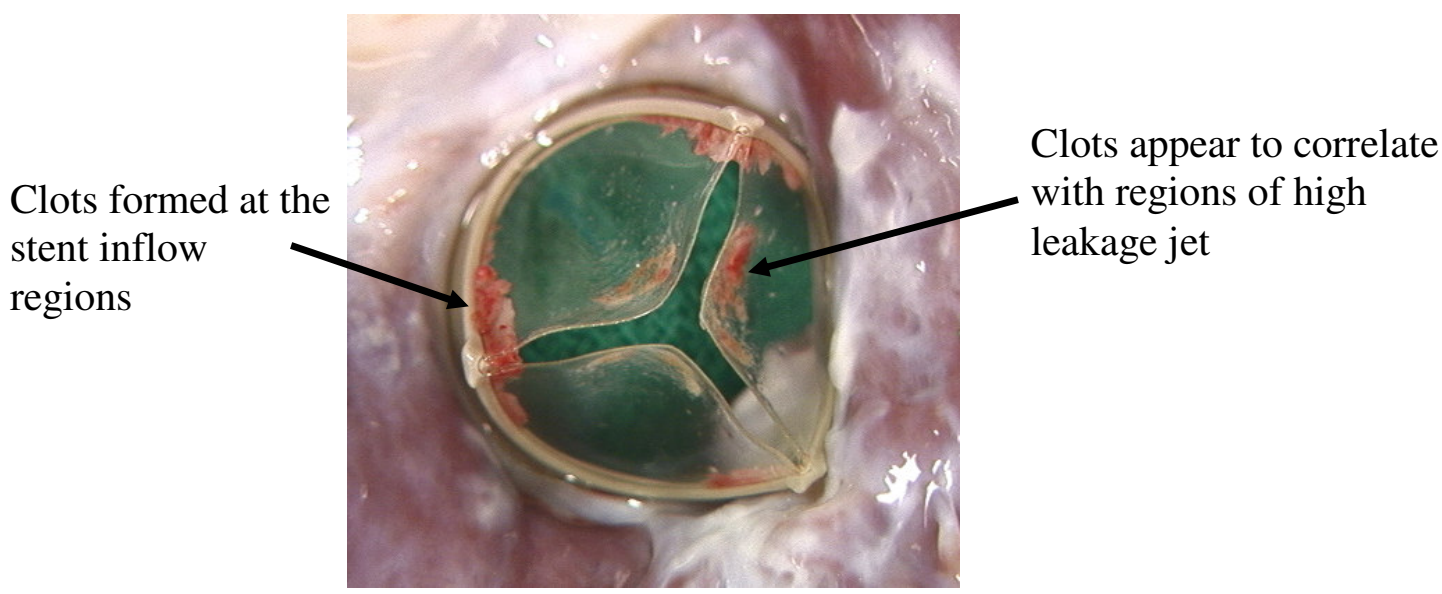

Figure 9-1 Clot locations inside an explanted polymeric valve (prototype B). Figure was obtained from preliminary animal trials performed with sheep by Dr Wheatley DJ of the University of Glasgow. Courtesy of Aortech, Inc.

Prototype A, which had thinner leaflets $(80 \mu \mathrm{m})$ than prototypes B and C $(120 \mu \mathrm{m})$, was able to form a better seal against retrograde flow. In contrast, the thicker leaflets of prototypes $\mathrm{B}$ and $\mathrm{C}$ prevented complete valve closure producing higher volumes of retrograde flow through the valves. This was further aggravated by the oscillations observed in the three the valves, which intermittently increased the flow area between the leaflets, and thus allowing more leakage flows.

In summary, the leaflet thickness was found to influence the closing dynamics of the valve leaflets during diastole. While the commissural design may aggravate thrombus formation at the commissure region, it does not necessarily affect clot formation along the stent inflow region. Instead, the high shear stress along the edge of the central orifice 
jet at systole and the split flow observed inside all of the valves during diastole were identified as possible factors for the observed blood clots along the stent.

In conclusion, current valve studies with LDV and PIV illuminated regions of stagnation and high shear stresses in both the bileaflet mechanical and tri-leaflet polymeric heart valves. Since these in vitro results are supported by clinical evidence they have the capability to predict potential complication or success rates before in vivo animal or clinical trials are initiated. Thus, hemodynamic studies conducted for this thesis should be implemented as part of routine in vitro testing and analysis of valve prototypes that are either already in the clinical use or still in the design stage. 


\section{CHAPTER 10: RECOMMENDATIONS}

\section{$\underline{\text { 10.1 Mitral Hinge Studies }}$}

Hinge gap width - In the mitral hinge studies with the SJM $27 \mathrm{~mm}$ Standard and the two $27 \mathrm{~mm}$ prototype leaker valves, the range of the available hinge gap width was not well optimize to provide a more detailed comparison of the hinge flow fields between various hinge tolerance. The only controlled variable for differentiating the three $27 \mathrm{~mm}$ valves was through their static leakage volume performed at St Jude Medical and later at Georgia Tech. The exact hinge tolerances were never made known. It was only through micro-Computed Tomography that the hinge tolerance could be elucidated; the measured hinge gap width for the SJM $27 \mathrm{~mm}$ Standard, and the $27 \mathrm{~mm}$ prototype high and low leakers valves were approximately $100 \mu \mathrm{m}, 200 \mu \mathrm{m}$ and $50 \mu \mathrm{m}$, respectively. Better control of the hinge gap width with known gradations, ranging from the extreme low to extreme high leakers, would provide the investigator with a better comparison tool in terms of the relationship between hinge flow fields and hinge gap width. Besides testing the valves in the mitral position, an additional set of data should also be collected at the aortic position. This would provide an opportunity to directly observe how various flow conditions could influence the hinge flow fields and to determine the susceptibility of each hinge tolerance to the thrombus formation. In addition, pulsatile blood studies could also be performed to provide information regarding the clotting potential in relation to the hinge gap width. Clotting locations could then be accurately correlated with the flow profiles. 
PIV hinge study - Viscous shear stress is an important parameter not thoroughly investigated in the current hinge studies due to the limitation of the LDV technique. Besides the effect of the alleged turbulent flow in the hinge region, viscous shear rate may also play an important role in influencing the potential for thrombus formation. To account for this, a PIV system could be employed since this measurement technique is based on the correlation between an image pair, which produces an instantaneous global velocity map that enables the calculation of viscous shear stresses across the hinge geometry. In addition, the PIV technique could be used to map the spatial and temporal shear history by tracking particles or following specific blood cells through the hinge region. Preliminary hinge studies with PIV was performed and was deemed feasible with the current setup. The experiments were conducted under steady flow conditions, with the followings 2D PIV settings;

1) A $5 \mathrm{X}$ objective lens was attached to a CCD camera to magnify the hinge region. The objective lens had a depth of field of approximately $14 \mu \mathrm{m}$. Algorithm was used to filter out any out-of-focus particle, thereby ensuring accurate velocity measurement. The CCD camera and its objective lens assembly was placed on a traversing mechanism that has a traversing resolution of $10 \mu \mathrm{m}$.

2) $2 \mu \mathrm{m}$ particles were used to seed the flow; conventional particles (larger than $20 \mu \mathrm{m}$ ) were deemed too big for the micro-PIV setup.

3) The flow loop was reduced to accommodate approximately 1.5 liters of blood analog (aqueous solution of $\mathrm{NaI}$ ) solution for the purpose of increasing the particle density

4) The entire hinge region was volume illuminated by the laser sheet. 
5) The valve was placed in he closed position and the flow rate adjusted to approximately 5 litre/min

Figure 10-1 shows the raw image acquired in the hinge region of a SJM $23 \mathrm{~mm}$ Regent clear valve model. The field of view was $5 \times 5 \mathrm{~mm}^{2}$, and the pulse separation was set at $1.5 \mu$ s to ensure that velocity magnitude of more than $2 \mathrm{~m} / \mathrm{s}$ could be resolved. Figure 10-2 shows the corresponding velocity fields inside the hinge region.

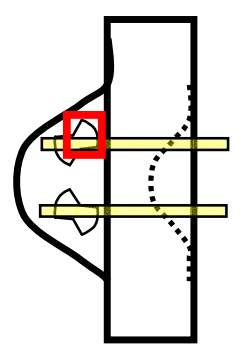

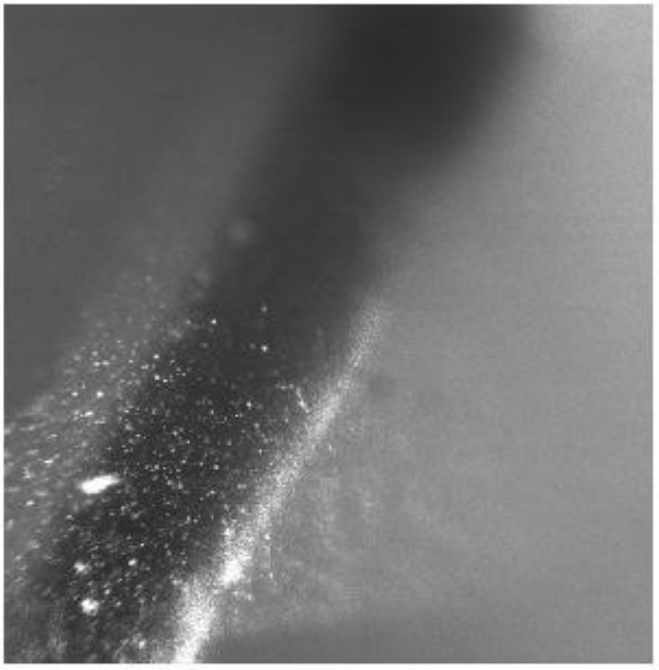

(a)

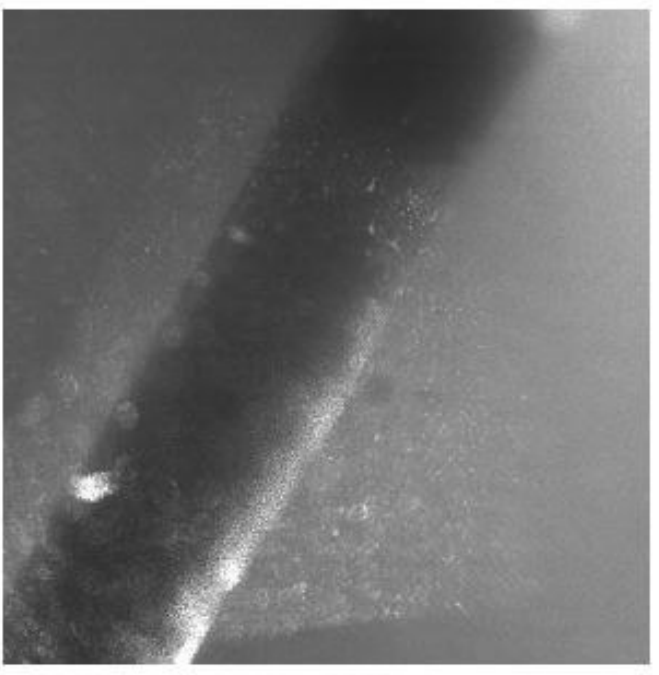

(b)

Figure 10-1 Raw 2D PIV image inside a SJM $23 \mathrm{~mm}$ Regent clear valve housing. About half of the hinge region was captured at: a) $200 \mu \mathrm{m}$ above the flat level and b) at the primary flat level 

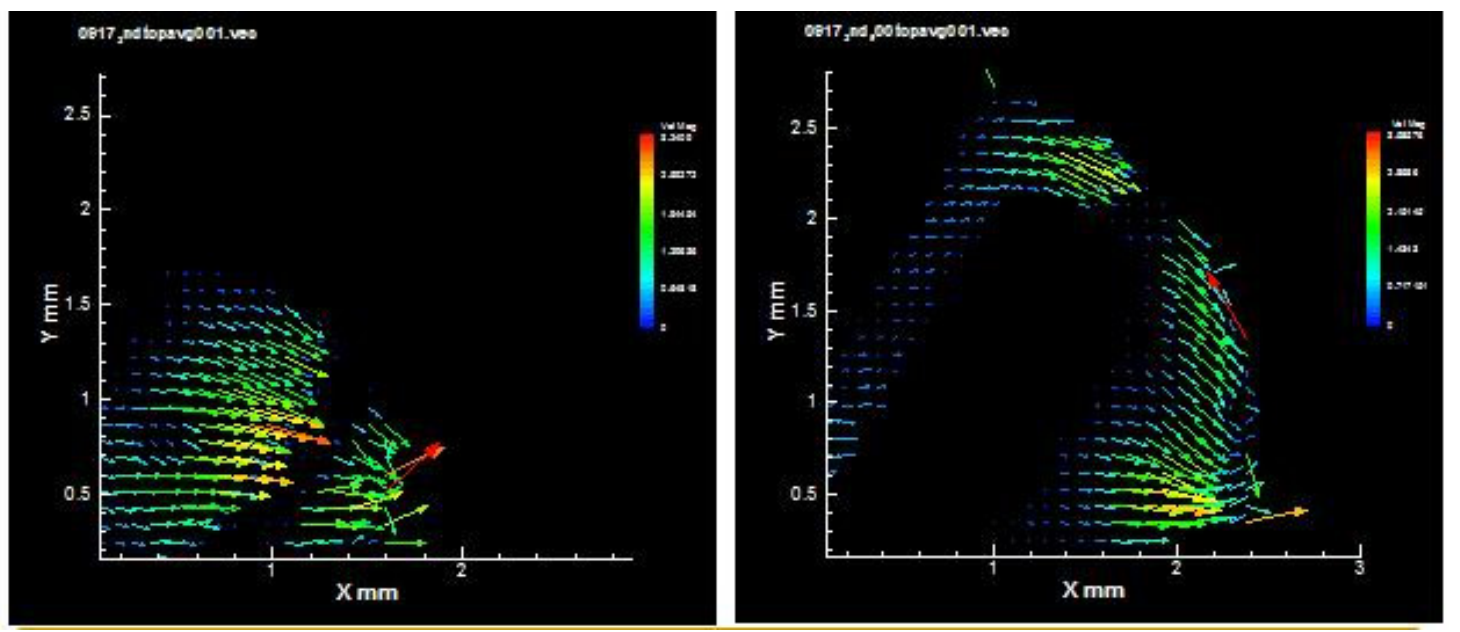

Figure 10-2 Velocity map inside the hinge region of a SJM $23 \mathrm{~mm}$ Regent clear valve housing; a) at $200 \mu \mathrm{m}$ above the flat level and b) at the primary flat level

Computational Fluid Dynamics (CFD) hinge study - Another promising thrust in the hinge studies is the numerical investigation of the flow inside the hinge mechanism. Recent work by Zhi et al compared the flow inside a $5 \mathrm{X}$ enlarged hinge pocket with that from a numerical simulation. They were able to demonstrate the existence of complex flow structures inside the hinge pocket. However, the numerical simulations of the hinge micro flow fields could not be validated with the experimental results due to poor resolution; the PIV vectors were averaged over only 5 image pairs. Currently, the Georgia Tech Fluid Mechanics lab with Dr Sotirlopoulos' lab are pursuing a similar direction with numerical studies of the hinge flow fields. Hinge geometry could be modeled accurately using the micro CT tool to ensure that the CFD mesh matches exactly the geometry of the hinge used in the experimental studies. Figure 10-3 depicts the hinge profile obtained from the micro-CT scan. 


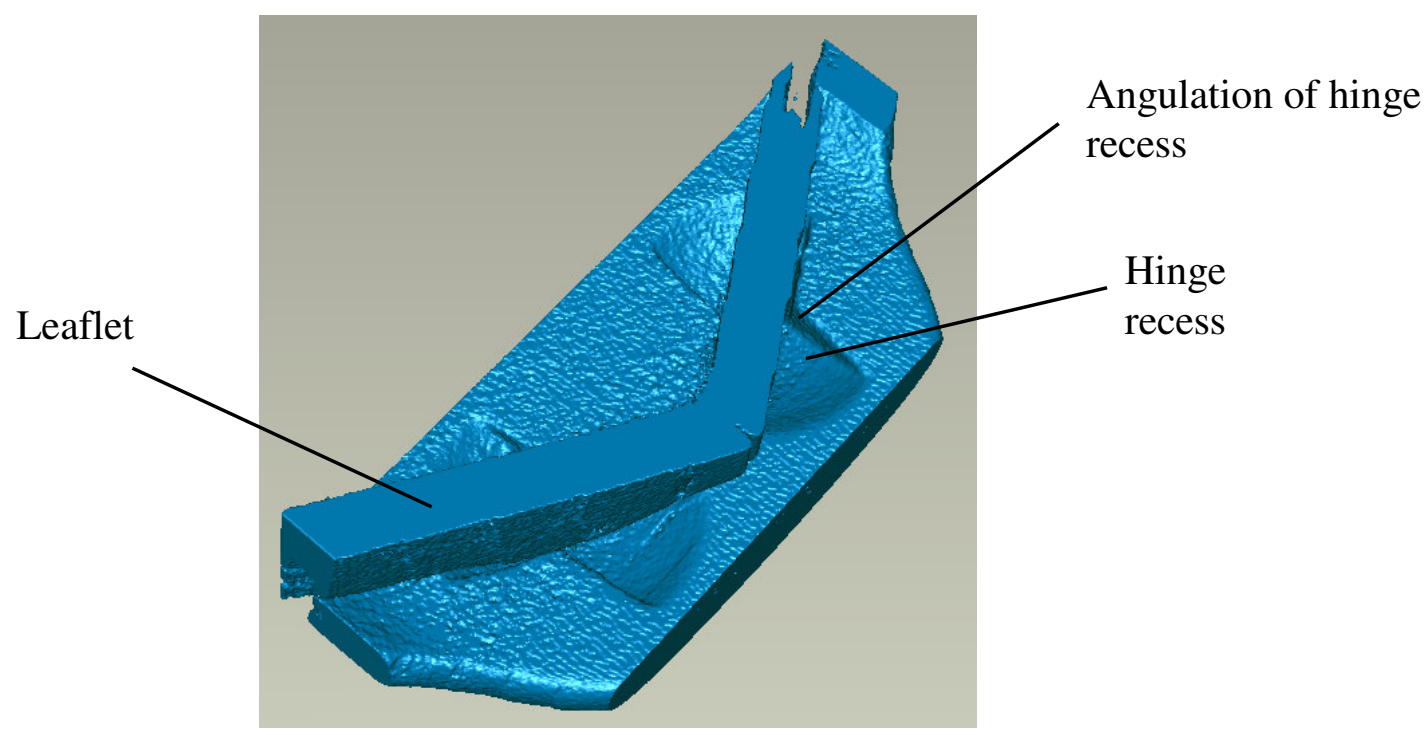

Figure 10-3 Micro CT scan image of the hinge region of a SJM $23 \mathrm{~mm}$ Regent valve

\section{$\underline{10.2 \text { Polymeric Valves Studies }}$}

Blood Studies - Detailed mapping of the flow fields in the vicinity of the tri-leaflet polymeric valve was accomplished in the current thesis. Another useful future study would involve blood experiments similar to those conducted by Travis [Travis, 2001c]. Pulsatile blood experiments could help isolate and determine the extent of the effect of the valve oscillation on its potential for thrombus formation. For instance, using a piston pump, it would be possible to control the magnitude and frequency of the valve oscillation by importing custom flow curves with varying degrees of pulsatility.

CFD studies of the polymeric valves - Another promising extension of the current thesis works is the use of Computational Fluid Dynamics to study the flow through polymeric heart valves. This study would make use of high speed camera to record the 
complex motion of the polymeric leaflets so that these experimental data could be implemented into the numerical simulation to ensure an accurate representation of the valve motion. In addition, the complex geometry of the polymeric valve could be accurately modeled using the micro-CT tools (Figure 10-4).

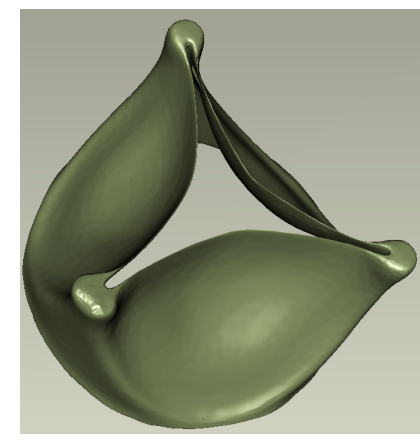

Figure 10-4 CT-scan image of polymeric valve A

Particle tracking - Particle tracking technique could be used on the 3D PIV measurements to estimate the amount of hemolysis and platelet activation when the blood goes through the valve. This is useful since particle tracking provide a Lagragian perspective of the possible pathway taken by a single blood element as it flow through the heart valve in a single cardiac cycle. This technique provide a more precise index to assess the damage of blood elements through valve prostheses.

1D LDV measurements - An important aspect of the LDV measurement technique is its ability to yield excellent temporal information. Power spectral analysis of all current 2D LDV data acquired from the pulsatile loop experiments were plagued by noise, and it is suggested that all future works include also the 1D LDV measurements. 1D LDV tends to 
have a higher data rate compared with the higher dimension measurement and hence may provide the frequency information with less noise contamination. 


\section{Appendix A: Program Code for Autotracking.m}

Figure A-1 shows the flow chart for tracking the marker array on the leaflet surface in the high speed camera experiments.

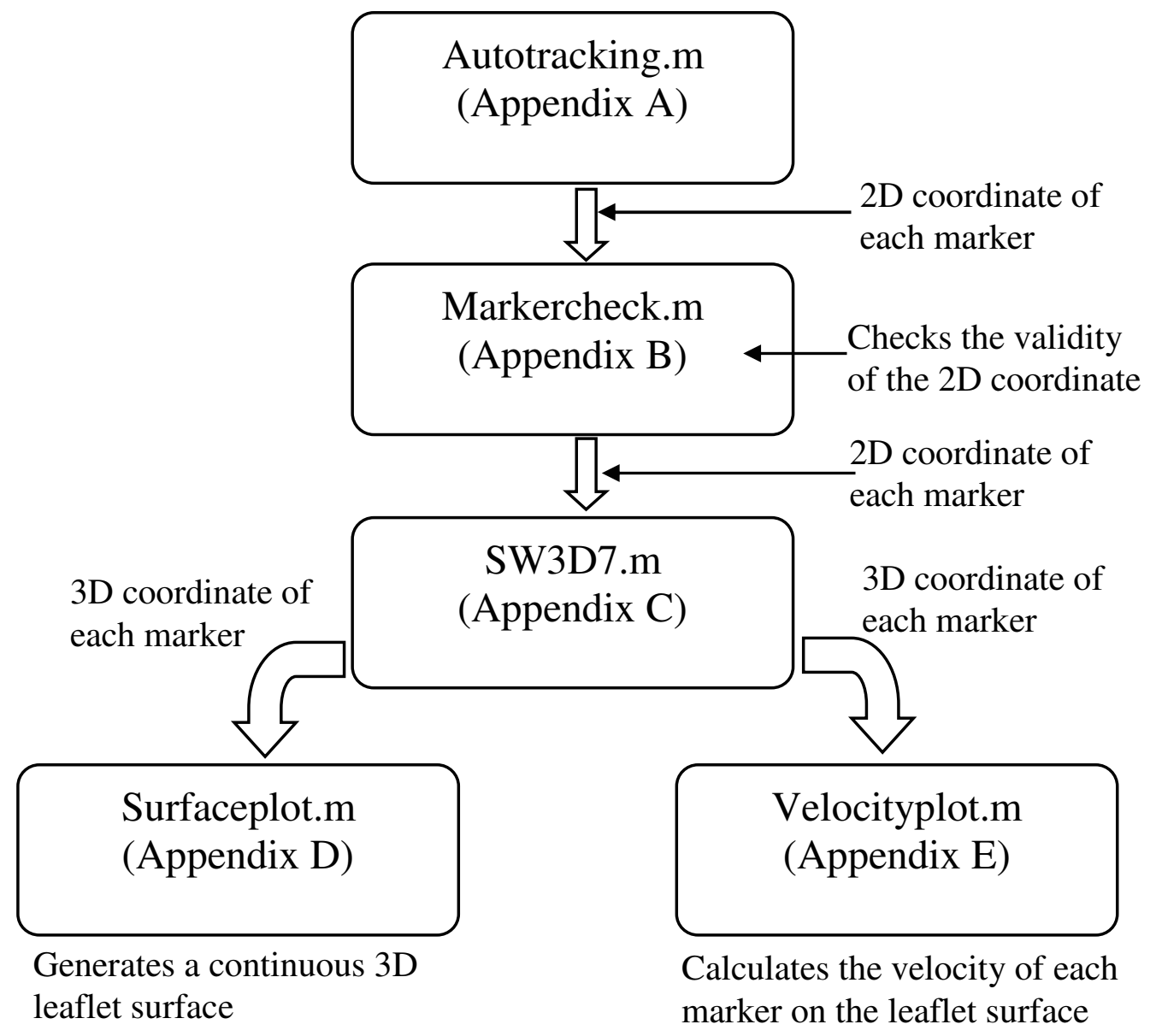

Figure A-1 shows the flow chart for the tracking and processing of the leaflet surface marker array. The 2D position of the markers are first tracked and checked by Autotracking.m and Markercheck.m, respectively. The 3D coordinate of the markers are obtained using the SW3D7.m code. The 3D coordinate information is then used by Surfaceplot.m to generate a continuous leaflet surface. The velocity of each individual marker is calculated using Velocityplot.m 
Autotracking.m. is a Matlab program developed 'in-house' by Dennis Soerensen. This Matlab code tracks the motion of individual markers on the leaflet surface. In this code, each marker is identified in the paired image set by the marker placement within the matrix. At a mean marker size of 40 pixels the autotracking was able to determine the 2D positions of the center of the markers with a mean error in distance of 0.5 pixels. Shown here is an incomplete sample of Autotracking.m. The complete code is at the Cardiovascular Fluid Mechanics Laboratory at Georgia Institute of Technology.

function marker_check(string_part, num_first, num_last )

if nargin $\sim=3$

error('Wrong number of input arguments. Use the following format: autotracking( string part of image.tif, number of first image to check, number of last image to check )')

end

load markerarray

$\%$ The following is done to make it possible for the user to choose certain frames to check so the user wont have to go through the entire marker_array

for $\mathrm{n}=$ num_first:1:num_last

if $\mathrm{n}<10$

image $=$ imread $(\operatorname{sprintf}(' \%$ s00\%d.tif', string_part , n ) );

elseif $n>=100$

image $=$ imread $(\operatorname{sprintf}(' \%$ s\%d.tif', string_part, $\mathrm{n}))$;

else

image $=$ imread $(\operatorname{sprintf}(' \%$ s0\%d.tif', string_part, $\mathrm{n}))$;

end

X_pos = center_pixel_coordinates_in_array $(1,1,:$, n-first_frame +1$)$;

$\mathrm{Y} \_$pos = center_pixel_coordinates_in_array $\left(1,2,:, \mathrm{n}-\mathrm{first} \_\right.$frame +1$)$; 


\section{Appendix B: Program Code for Markercheck.m}

Markercheck.m is a Matlab program developed 'in-house' by Dennis Soerensen. It enables the visualization of the position of the markers tracked by Autotracking.m. This program double checks to ensure that each individual marker is tracked correctly and not confused with air bubbles that may also be present. Markercheck.m lets the user check the marker locations found in Autotracking.m, and if the locations are satisfactory the Markercheck.m file converts the marker 2D coordinates into the appropriate output format for further calculations. Shown here is an incomplete sample of Markercheck.m. The complete code is at the Cardiovascular Fluid Mechanics Laboratory at Georgia Institute of Technology.

function autotracking(tif_image,num_of_first, num_of_last, num_of_markers)

if nargin $\sim=4$

error('Wrong number of input arguments. Use the following format: autotracking( first part of image.tif name, number of first image, number of last image, number of markers)')

end

first_frame = num_of_first;

num_of_frames = num_of_last - num_of_first + 1;

ThreeByThree $=$ ones(3)/9;

$\mathrm{j}=1$;

if num_of_first $<10$

image $=$ imread $(\operatorname{sprintf}(' \%$ s00\%d.tif', tif_image , num_of_first $))$;

elseif num_of_first $<100$

image $=$ imread $(\operatorname{sprintf}(' \%$ s0\%d.tif', tif_image , num_of_first $))$;

elseif num_of_first $>=100$

image $=$ imread $(\operatorname{sprintf}(' \% s \%$ d.tif', tif_image, num_of_first $))$; 


\section{Appendix C: Program Code for SW3D7.m}

SW3D7.m is a Matlab program developed by Dr. Michael Sacks at the University of Pittsburgh. It converts the 2D coordinates obtained by Autotracking.m of an individual marker from images taken by cameras A and B and converts them into a single set of 3D coordinates. Shown here is an incomplete sample of SW3D7.m. The complete code is at the Cardiovascular Fluid Mechanics Laboratory at Georgia Institute of Technology.

$\%$ PROGRAM

SW3D7

\% This program computes 3D coordinates $(\mathrm{X}, \mathrm{Y}, \mathrm{Z})$ from $2 \mathrm{D}$ coordinates $(\mathrm{U}, \mathrm{V})$ by Direct $\%$ Linear Transform (DLT). The program utilizes the functions dltfu.m and reconfu.m \%originally written by Christoph Reinschmidt from the University of Calgary (). $\%$

$\%$ There are 4 phases: Loading the 2D data sets, Calibrating camera coordinates, $\%$ Computing 3D coordinates, and Writing output file.

$\%$

$\%$ (1)Loading 2D data sets- The program will ask for 2 filenames. These are where $\%$ the 2D coordinates are stored for each camera. Press ENTER for defaults (acoord $\% \&$ bcoord).

$\%$

$\%$ (2)Calibrating camera coordinates- The program will ask for 3 filenames. These

$\%$ are where the 2D coordinates of cube corners (acal \& bcal) and known 3D

$\%$ coordinates of cube (reference) are stored.

$\%$

$\%(3)$ Computing 3D coordinates- X,Y,Z coordinates will be calculated using the

$\%$ least squares method.

$\%$

$\%(4)$ Writing output file- The program will ask for a filename for the output $\%$ (markers3d). The program will also ask for 2 pressure files (ventricular and

$\%$ transmitral). The output will be in the following format:

$\% \quad$ Row 1: \#frames, \#markers

$\% \quad$ Row n: frame\#, time, LVpressure, TMpressure

$\% \quad$ Row $\mathrm{n}+1: \mathrm{X}, \mathrm{Y}, \mathrm{Z}$

$\%$ END HELP

TEXT 


\section{Appendix D: Program Code for Surfaceplot.m}

Surfaceplot.m is a Matlab code developed 'in-house' by Steffan Finnegan. It constructs a continuous leaflet surface from the 3D spatial coordinates generated by SW3D7.m. The discrete data points of the dynamic surface were interpolated into a smooth, contiguous surface using a Delaunay triangulation algorithm to yield a smooth 3D surface. Shown here is an incomplete sample of Surfaceplot.m. The complete code is at the Cardiovascular Fluid Mechanics Laboratory at Georgia Institute of Technology.

\section{PROGRAM}

\section{SURFACEPLOT}

This program was written to be used with the output from sw3d7.m. The program reads in the 3D coordinates and plots the markers and generates a surface in space frameby-frame.

The program calculates marker velocity, marker displacement and marker acceleration for every frame and plots the top $20 \%$ of the fastest moving markers for a desired frame.

$\mathrm{H} \quad$ = matrix name for the loaded data, user writes filename to be loaded

$\mathrm{fpm} \quad=$ Frames per minute $=$ user input

frmlim = frame limits $=$ number of first and last frame $=$ values from $\mathrm{H}$

mkrnum = number of markers $=$ values from $\mathrm{H}$

frmnum $\quad=$ size of image sequence $=$ calculated from frmlim

f1 $\quad=$ renaming marker number one in every frame

flast $\quad=$ renaming last marker in every frame

$\mathrm{x}, \mathrm{y}, \mathrm{z} \quad=$ reading $\mathrm{x}, \mathrm{y}, \mathrm{z}$ coordinates from $\mathrm{H}$

$\mathrm{xpos}, \mathrm{ypos}, \mathrm{zpos} \quad=$ saving $\mathrm{x}, \mathrm{y}, \mathrm{z}$ coordinates into separate matrices

$\mathrm{Fx}, \mathrm{Fy}, \mathrm{Fz}=$ MATLAB calculated gradient factors

xlin, ylin = line spacing in the evenly spaced grid

positionvector $=$ rearranging $\mathrm{xpos}$, ypos, zpos into one matrix

velocity = matrix holding all the calculated velocity data for every

marker in every frame 


\section{Appendix E: Program Code for Velocityplot.m}

Velocityplot.m is a Matlab code developed 'in-house' by Steffan Finnegan. It computes the speed of individual markers on the surface of the leaflet. This program is an extension of Surfaceplot.m. The input for this program is the output from SW3D7.m. The program plots the top 'user defined' percent of the fastest or slowest moving markers for a desired frame. Shown here is an incomplete sample of Velocityplot.m. The complete code is at the Cardiovascular Fluid Mechanics Laboratory at Georgia Institute of Technology.

\section{PROGRAM}

\section{SURFACEPLOT}

This program was written to be used as an extension of surfaceplot.m. The input for this program is the output from sw3d7.m. The program plots the top 'user defined' - percent of the fastest or slowest moving markers for a desired frame.

Nomenclature:

$\mathrm{H} \quad$ = matrix name for the loaded data, user writes filename to be loaded

fpm $\quad=$ Frames per minute $=$ user input

frmlim = frame limits $=$ number of first and last frame $=$ values from $\mathrm{H}$

mkrnum = number of markers $=$ values from $\mathrm{H}$

frmnum $\quad=$ size of image sequence $=$ calculated from frmlim

f1 $\quad=$ renaming marker number one in every frame

flast $\quad=$ renaming last marker in every frame

$\mathrm{x}, \mathrm{y}, \mathrm{z} \quad=$ reading $\mathrm{x}, \mathrm{y}, \mathrm{z}$ coordinates from $\mathrm{H}$

$\mathrm{xpos}, \mathrm{ypos}, \mathrm{zpos} \quad=$ saving $\mathrm{x}, \mathrm{y}, \mathrm{z}$ coordinates into separate matrices

$\mathrm{Fx}, \mathrm{Fy}, \mathrm{Fz}=$ MATLAB calculated gradient factors

xlin, ylin $\quad=$ line spacing in the evenly spaced grid

positionvector $=$ rearranging $\mathrm{xpos}$, ypos, zpos into one matrix

velocity = matrix holding all the calculated velocity data for every marker in every frame

meanvelocity = matrix with values for the calculated mean surface velocity

Mvel = rearranging and renaming meanvelocity matrix

acceleration $=$ matrix holding all the calculated acceleration data for every marker in every frame 


\section{Appendix F: Comparison of Phase-averaging and Cyclic Removal techniques}

Appendix F compares and discusses two different analysis methods for the removal of cyclic variation from the 2D LDV results. The 2D LDV results include the mitral hinge and polymeric heart valve studies. The hinge results obtained in this study are comparable to those obtained earlier by Brandon Travis [Travis, 2001c]. The present study demonstrated that the Reynolds shear stress magnitudes calculated by cyclic averaging were a mean of 15.0 percent lower than Reynolds shear stress calculated by phase window averaging. Appendix F will first compare the results obtained by the two analysis techniques followed by a discussion of the observations. The comparison of the RSS levels in this section pertain to the spatial averaged values calculated by the phase averaging and cyclic removal methods.

\section{F.1 RESULTS FOR REMOVAL OF CYCLIC VARIATION}

\section{F.1.1 CM Hinge Study}

In general, the velocity magnitude and Reynolds shear stress values obtained using the cycle-resolved technique were lower than those calculated by the traditional phase averaging method. As many as 20 leakage jet measurement locations were used for the comparison. The Reynolds shear stress magnitudes calculated by cyclic averaging were a mean of 17.0 percent and 13.0 percent lower than Reynolds shear stress calculated by phase window averaging at the flat and $1 \mathrm{~mm}$ below the flat levels, respectively. 
Figure F-1 shows the scatter plot for the Reynolds shear stress difference at the primary flat level at early systole (time bin 2). Large Reynolds shear stress differences were typically observed along the edge of the hinge geometry and occasionally in the leakage jets at the atrial side of the hinge during systole. Figure F-2 shows the data rate scatter plot at the flat level. A data rate of $150 \mathrm{~Hz}$ or higher was seen at the atrial, adjacent, and lateral corners of the hinge geometry. The first two corners coincided with the locations where leakage jets were seen during systole, while the third was associated with the region of the forward jet during diastole.

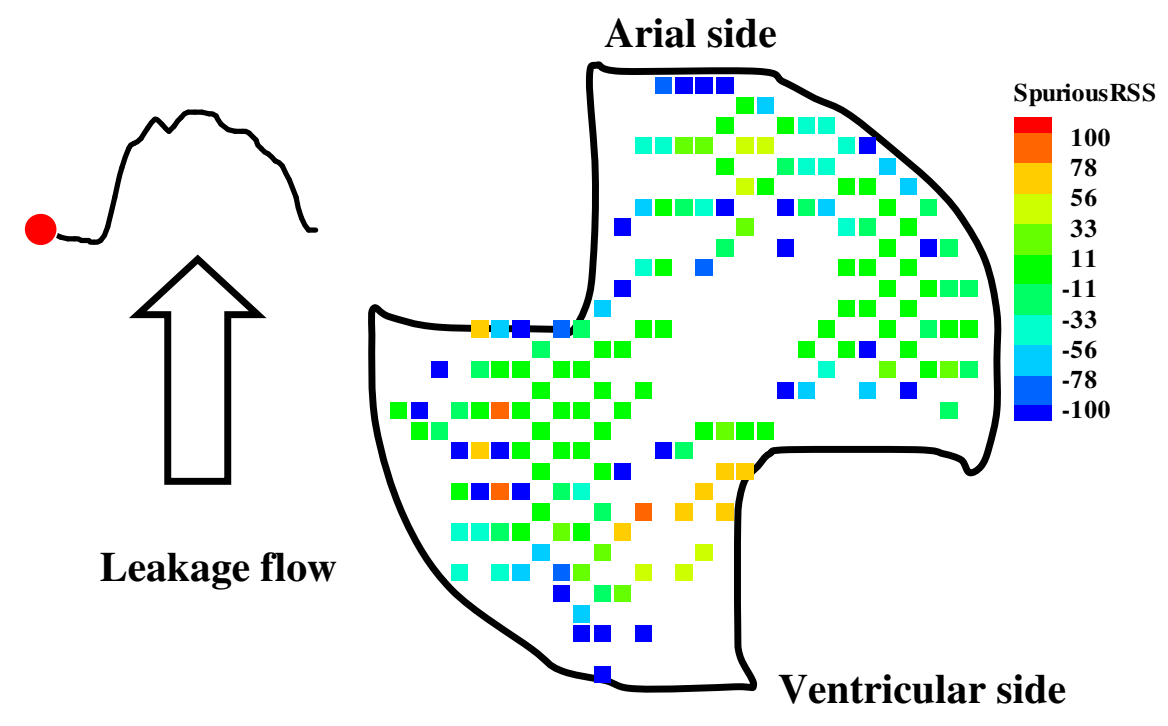

Figure F-1 Scatter plot of the percentage Reynolds shear stress difference between the phase averaging and cyclic removal methods at the flat level of CM $23 \mathrm{~mm}$ during early systole, time bin 2; large Reynolds shear stress difference were typically observed along the edge of the hinge geometry 


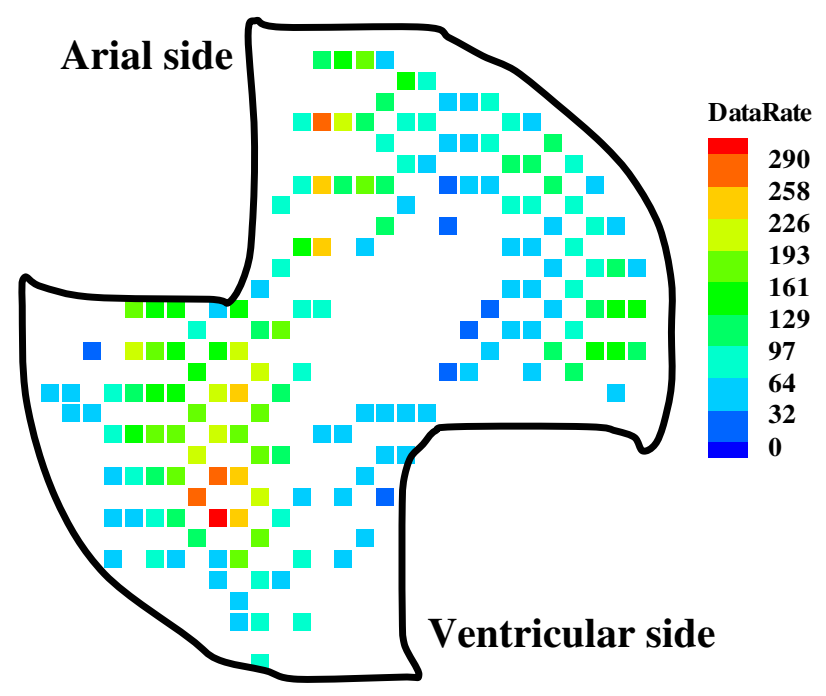

Figure F-2 Scatter plot of the data rate at the flat level of the CM $23 \mathrm{~mm}$; regions of high data rate coincided with regions of leakage and forward flows

Figure F-3 and Figure F-4 show the scatter plot of the Reynolds shear stress differences at the $1 \mathrm{~mm}$ below the flat level at peak diastole (time bin 23) and early systole (time bin 1), respectively. The Reynolds shear stress levels calculated by the phase averaging method were approximately 15.0 percent higher than those calculated by the cyclic removal method. Figure F-5 shows the scatter plot for the $1 \mathrm{~mm}$ below flat level. A data rate of $500 \mathrm{~Hz}$ or higher typically occurred in area where the high velocity forward jet was seen. The data rate at the leakage jet locations was less than $100 \mathrm{~Hz}$. 

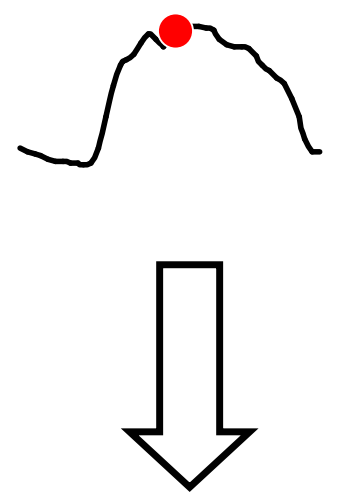

Forward flow

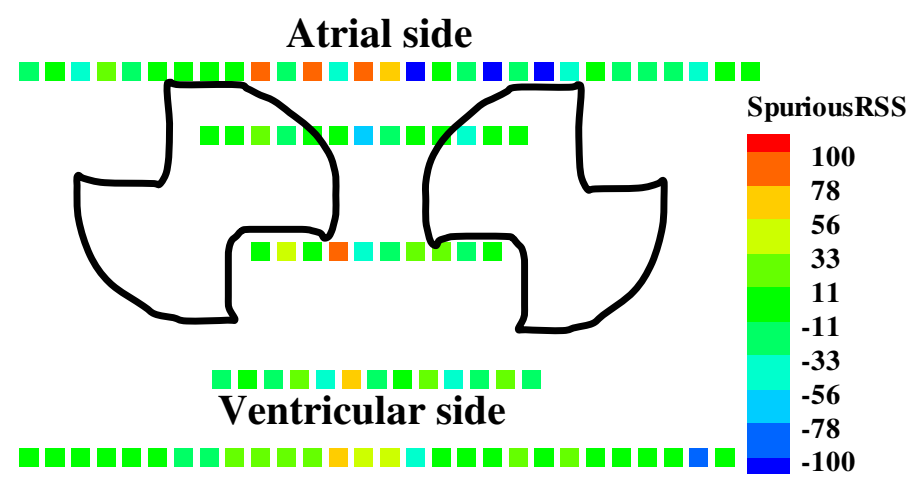

Figure F-3 Scatter plot of the percentage Reynolds shear stress difference between the phase averaging and cyclic removal methods at the $1 \mathrm{~mm}$ below the flat level of CM 23 $\mathrm{mm}$ valve during peak diastole, time bin 23; elevated Reynolds shear stress was observed at the atrial side of the hinge mechanism

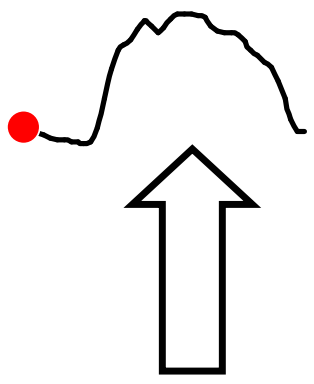

Leakage flow

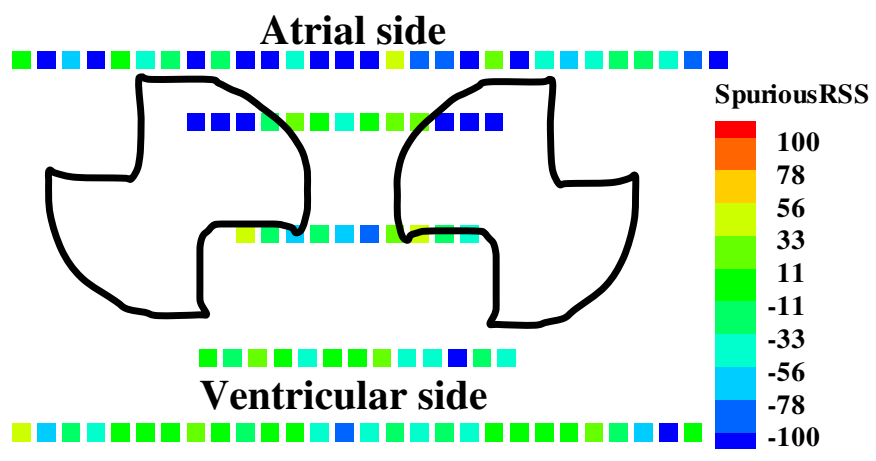

Figure F-4 Scatter plot of the percentage Reynolds shear stress difference between the phase averaging and cyclic removal methods at the $1 \mathrm{~mm}$ below the flat level of CM 23 $\mathrm{mm}$ valve during early systole, time bin 1; elevated Reynolds shear stress difference was observed in the leakage jet between the closing leaflets, i.e. B-datum line 


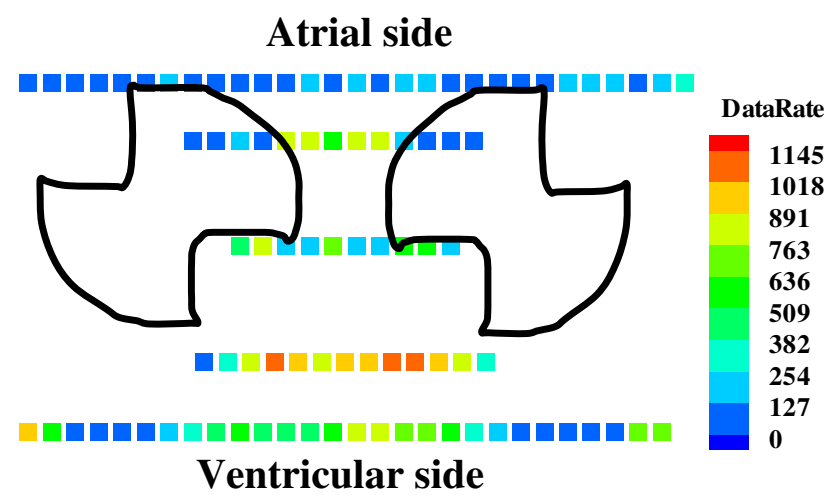

Figure F-5 Scatter plot of the data rate at the $1 \mathrm{~mm}$ below the flat level of the CM $23 \mathrm{~mm}$ valve; regions of high data rate coincided with regions of forward flow as well as the shear region

\section{F.1.2 SJM Hinge Study}

Similar observations can be made from the SJM $27 \mathrm{~mm}$ Standard and the two $27 \mathrm{~mm}$ prototype bileaflet valves; the regions of higher Reynolds shear stress difference typically occurred at the leakage jets measurement locations. For ease of presentation, only the plots from the Standard valve are displayed. The Reynolds shear stress values calculated by cyclic averaging were a mean of 15.0 percent and 16.0 percent lower than Reynolds shear stress values calculated by phase window averaging at the flat and $500 \mu \mathrm{m}$ below flat levels, respectively.

Figure F-6 shows the scatter plot for the Reynolds shear stress difference at the flat level approaching mid systole (time bin 4). Elevated Reynolds shear stress differences usually occurred along the edge of the hinge geometry and also in the leakage jets at the atrial side of the hinge region during systole. Figure F-7 shows the data rate at various 
measurement locations for the primary flat level of the SJM $27 \mathrm{~mm}$ Standard valve. A data rate of more than $450 \mathrm{~Hz}$ was seen at the adjacent and ventricular corners coinciding with the location of the leakage jet during systole and with the forward flow during diastole, respectively.
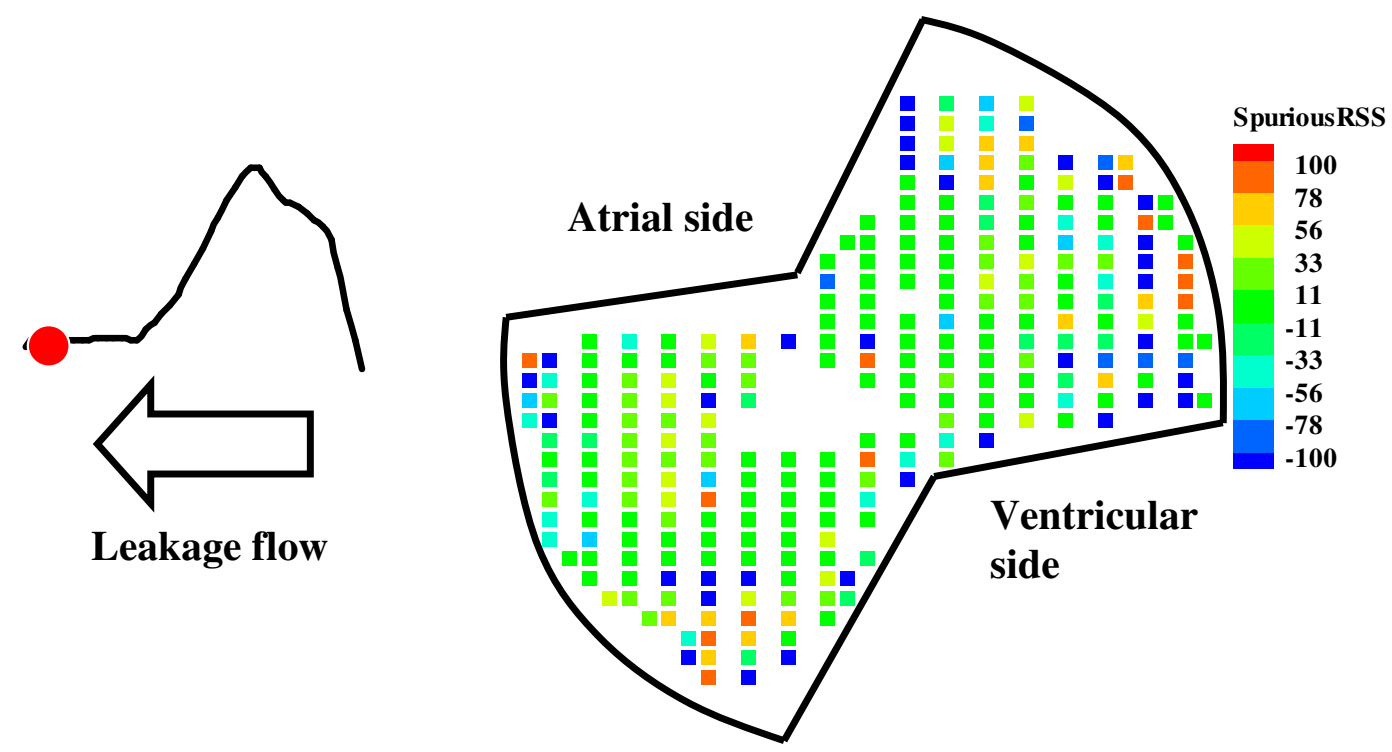

Figure F-6 Scatter plot of the percentage Reynolds shear stress difference between the phase averaging and cyclic removal methods at the flat level of SJM $27 \mathrm{~mm}$ Standard valve approaching mid systole, time bin 4; elevated Reynolds shear stress difference was observed along the edge of the measurement plane 


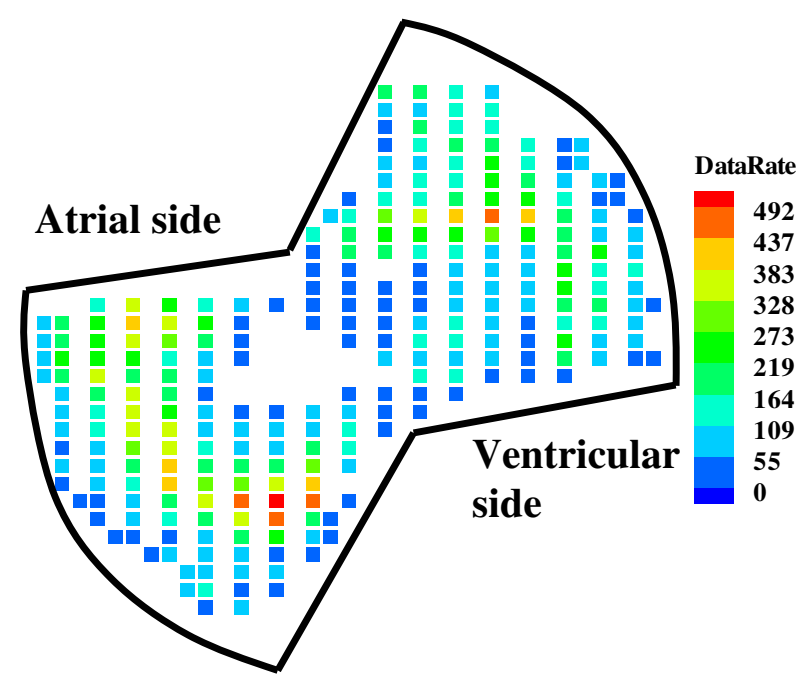

Figure F-7 Scatter plot of the data rate at the flat measurement level of the SJM $27 \mathrm{~mm}$ Standard valve; regions of high data rate coincided with regions of leakage and forward flows

Figure F-8 shows the scatter plot for the measurement levels below the primary flat level approaching mid systole (time bin 4). Phase averaging method produced spurious Reynolds shear stress values that was apprximately 10.0 percent higher than those obtained using the cyclic removal technique. The data rate for the measurement locations below the flat level was typically higher than that inside the hinge pocket (Figure F-9). Data rates between 300 to $1000 \mathrm{~Hz}$ were observed at the ventricular side of the hinge mechanism and coincided with the strong forward jet observed during diastole. A region of high data rate $(>900 \mathrm{~Hz})$ was also observed at the atrial side, where the three leakage jets were observed during systole. 


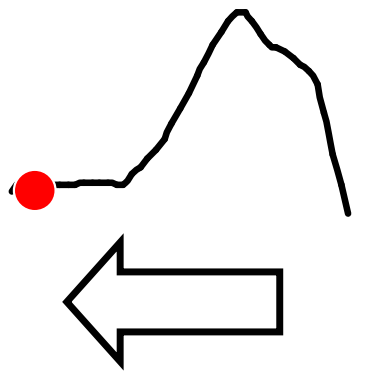

Leakage flow

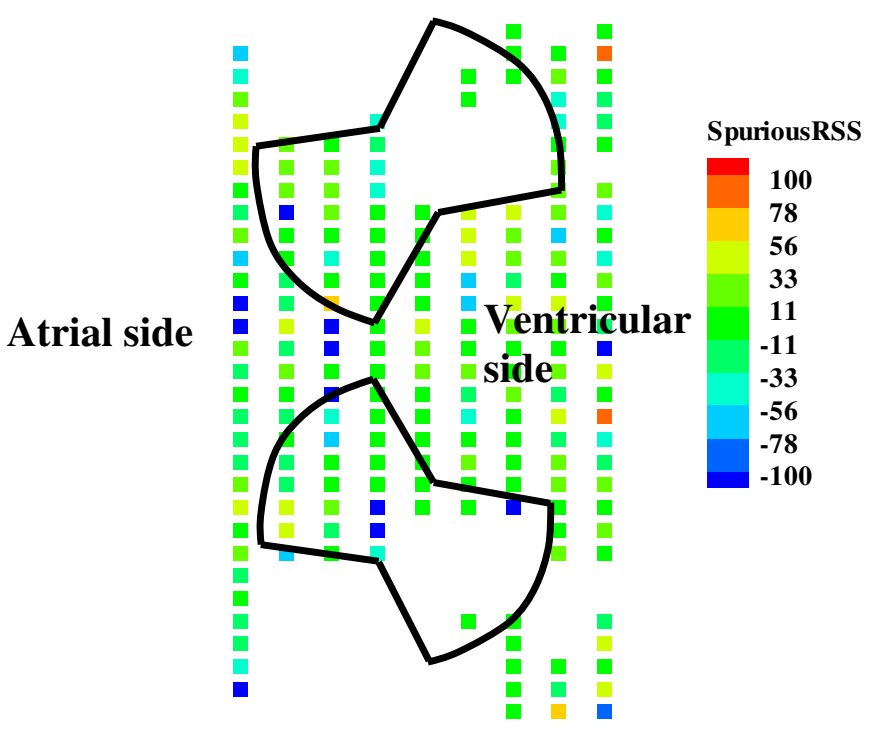

Figure F-8 Scatter plot of the percentage Reynolds shear stress difference between the phase averaging and cyclic removal methods at the $500 \mu \mathrm{m}$ below the flat level of SJM $27 \mathrm{~mm}$ Standard valve approaching mid systole, time bin 4; the average Reynolds shear stress difference throughout the measurement plane is less than 10.0 percent

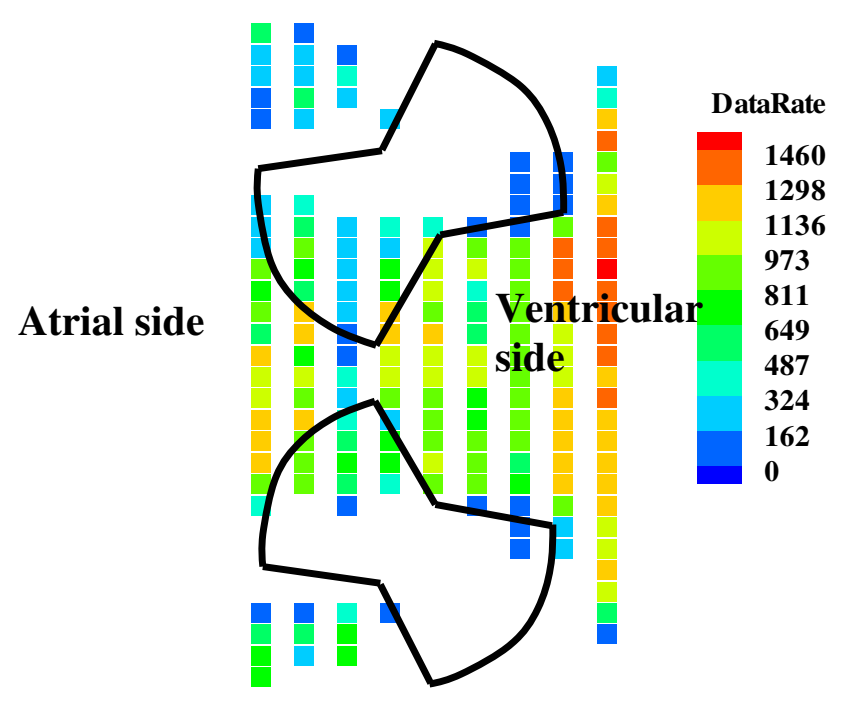

Figure F-9 Scatter plot of the data rate at the $500 \mu \mathrm{m}$ below the flat level of the SJM 27 $\mathrm{mm}$ Standard valve; regions of high data rate coincided with regions of forward and leakage flows 


\section{F.1.3 Polymeric Heart Valves Studies}

The three polymeric valves demonstrated similar trends in the distribution of high Reynolds shear stress difference. Figure F-10 shows the scatter plot for the Reynolds shear stress percentage difference downstream of valve A at peak systole (time bin 9). Comparison of 20 measurement locations showed that the phase averaging method produced spurious Reynolds shear stress values with a mean of 31.0 percent higher than those calculated by the cyclic removal method. The measurement data rate downstream of the valve was more than $850 \mathrm{~Hz}$ at the distal region of the flow chamber where the turbulence mixing of the center orifice jet and the surrounding fluid occurred (Figure F11). A data rate of more than $600 \mathrm{~Hz}$ was also observed at the region inside the central orifice jet as well as along the trailing edge of the valve, while values of less than $200 \mathrm{~Hz}$ was seen near the top sinus region of the flow chamber.

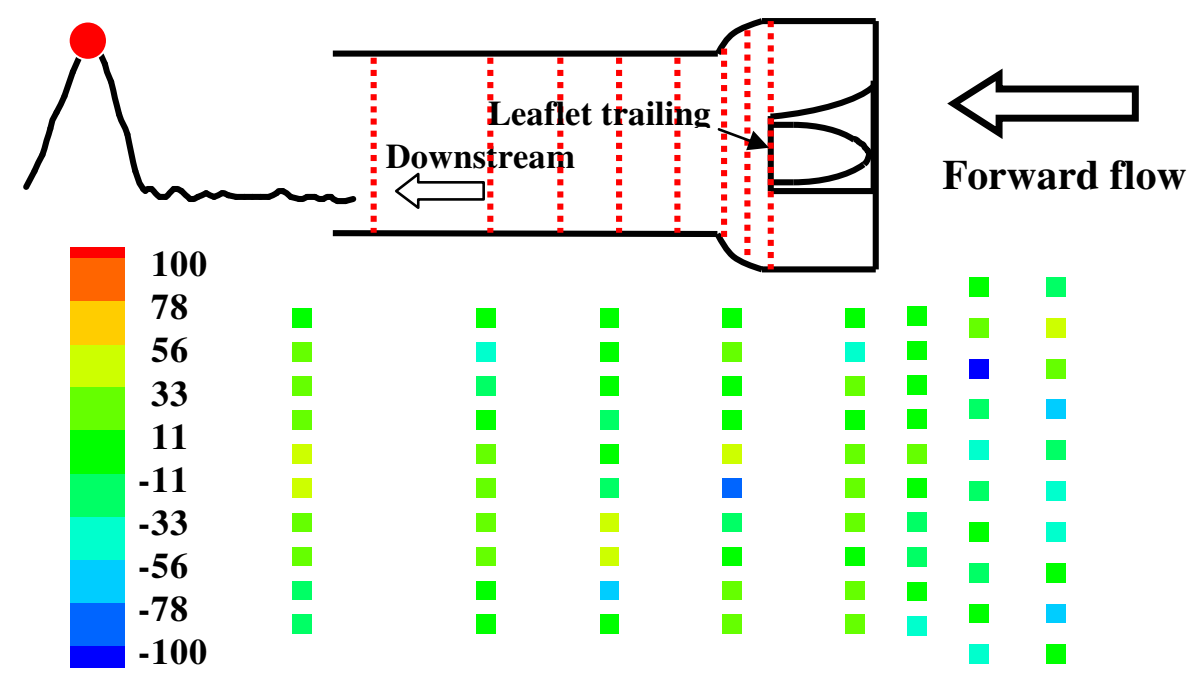

Figure F-10 Scatter plot of the percentage Reynolds shear stress difference between the phase averaging and cyclic removal methods at downstream of prototype A at peak systole, time bin 9; the observed Reynolds shear stress percentage difference throughout the measurement plane was approximately 10.0 percent 


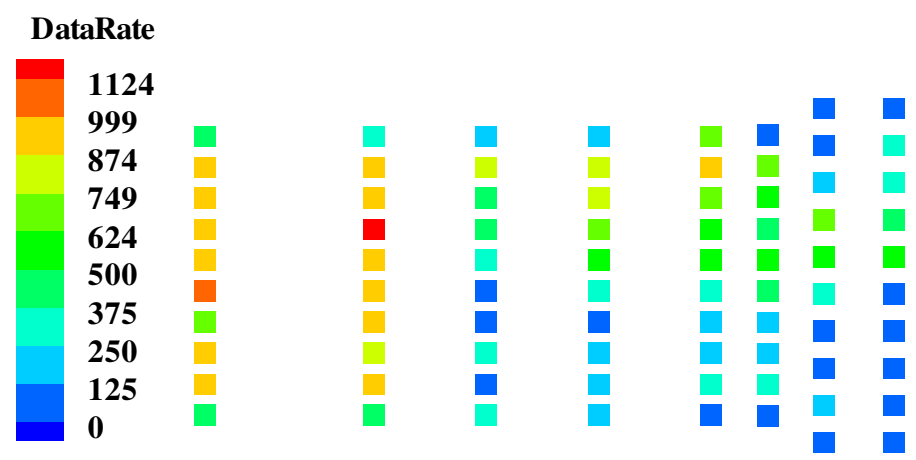

Figure F-11 Scatter plot of the data rate at the downstream measurement of prototype A; regions of high data rate coincided with regions of turbulence mixing

Figure F-12 shows the scatter plot of the percentage Reynolds shear stress difference between the phase averaging and cyclic removal methods inside prototype $\mathrm{A}$ at mid diasotle (time bin 21). Comparison of 20 measurement locations showed that the phase averaging method produced spurious Reynolds shear stress values with a mean of 88.0 percent higher than those calculated by the cyclic removal method. However, comparison of flow inside the valve at locations other than the leakage jet at the high central region showed that phase averaging method produced spurious Reynolds shear stress values that were 18.0 percent higher than those obtained by the cyclic removal method. Figure F-13 shows the data rate plot at the measurement plane inside the valve. A data rate of more than $250 \mathrm{~Hz}$ was observed throughout the measurement plane except at the region close to the top stent post, which recorded values of typically less than $100 \mathrm{~Hz}$. 

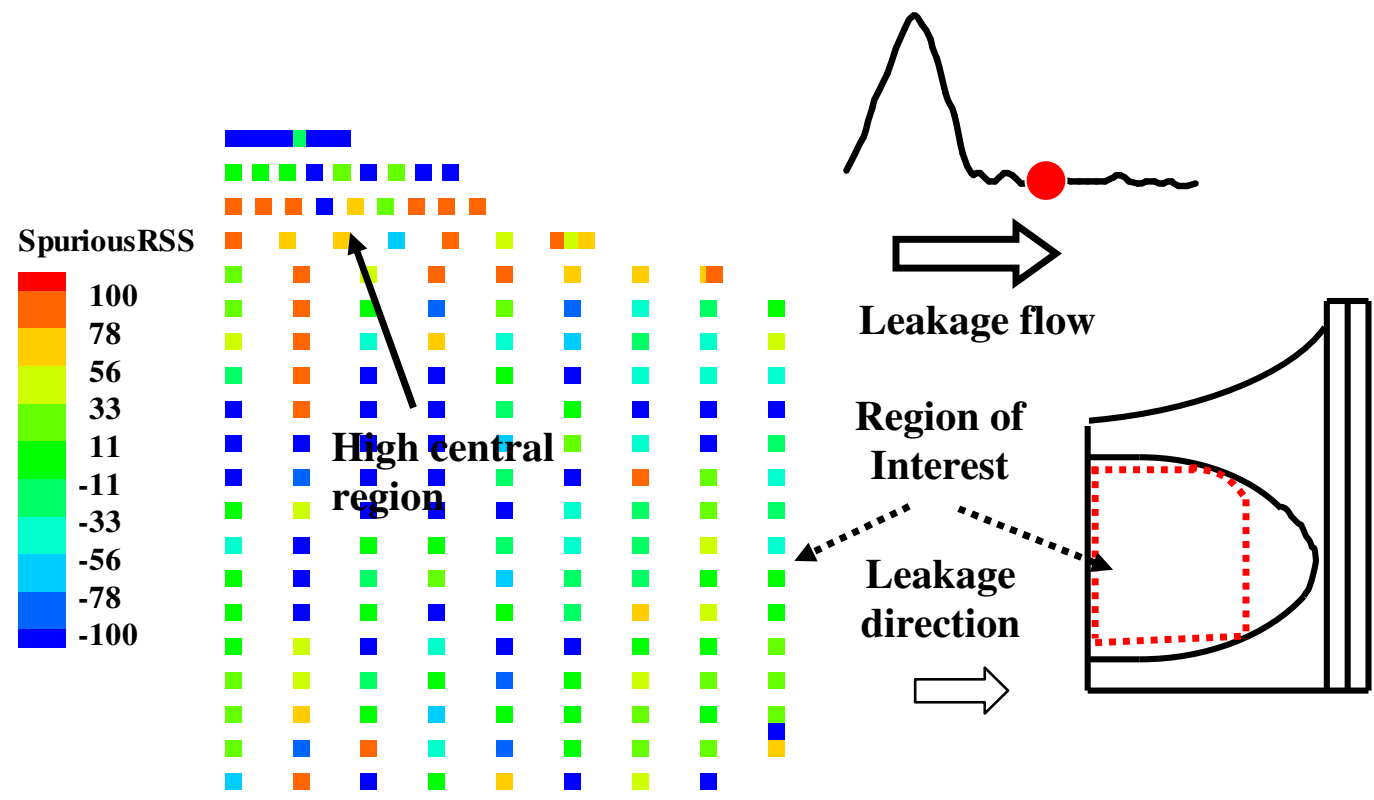

Figure F-11 Scatter plot of the percentage Reynolds shear stress difference between the phase averaging and cyclic removal methods inside prototype A at mid diasotle, time bin 21; highest Reynolds shear stress difference was observed in the leakage jet at the high central region inside the valve during diastole

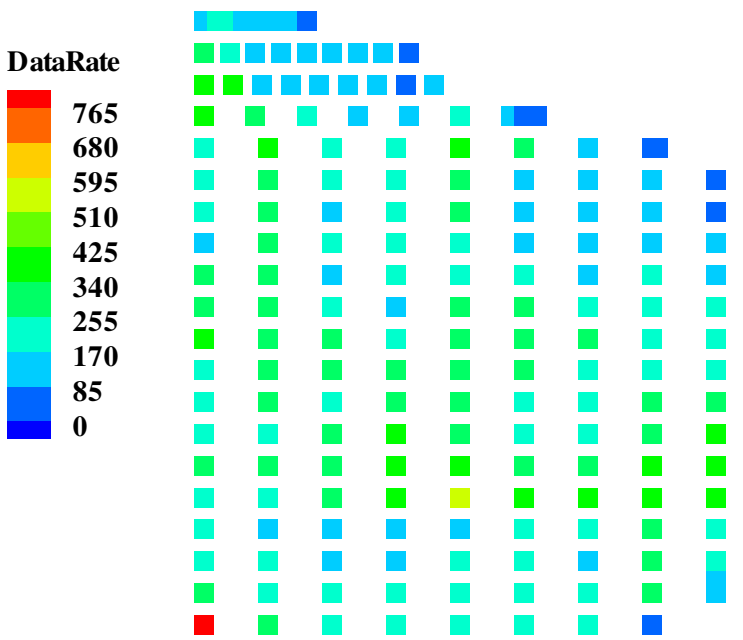

Figure F-12 Scatter plot of the data rate inside prototype A; the data rate was evenly distributed throughout the measurement plane 


\section{F.2 DISCUSSION OF THE EFFECTS OF CYCLIC VARIATION REMOVAL IN}

\section{D LDV DATA}

According to recent studies, cyclic variations exist in the pulsatile flow [Liu, 2000;

Tiederman, 1988]. The accuracy of the phased averaged velocity information can be easily influenced by these variations and, hence, the calculation of the Reynolds shear stress may be overestimated. On the other hand, cycle-resolved analysis is not affected by the cyclic variation and thereby provides more accurate results (see section 6.1). For the mitral hinge studies, the Reynolds shear stress levels were obtained from both the atrial leakage jets and the adjacent leakage jets inside the hinge pocket and at the gap between the closed leaflets. For the polymeric valves, the Reynolds shear stress levels was obtained at the high central region inside the valve and at the shear region downtream of the valve.

Typically, the Reynolds shear stress magnitudes calculated by cyclic averaging were lower than Reynolds shear stress calculated by phase window averaging. The average percent difference between the Reynolds shear stresses calculated by phase window averaging and cyclic averaging at the flat and $1 \mathrm{~mm}$ below flat levels for the CM $23 \mathrm{~mm}$ clear valve were 17.0 and 13.0 percent, respectively. The percent difference at the flat and $500 \mu \mathrm{m}$ below flat levels for the SJM $27 \mathrm{~mm}$ Standard clear valve were 15.0 and 16.0 percent, respectively. The Reynolds shear stress percent difference calculated inside the valve at the high central region and downstream of the polymeric heart valve were 88.0 and 31.0 percent, respectively. However, inside the valve at locations other than the leakage jet at the high central region during systole showed a spurious Reynolds shear 
stress difference of 18.0 percent. This discrepancy could be due to the dynamics of the valve during diastole where the cyclic oscillation of the leaflets may have had more influence on the flow at the high central region in comparison to flow at other locations during diastole.

The results obtained in this research are comparable with those previously obtained by Travis for his leakage jet experiments with the SJM $27 \mathrm{~mm}$ Standard (14.0 percent) and SJM 17 mm Regent (16.5 percent) carbon valves [Travis, 2001c]. Current results showed that regions of high RSS percent difference typically coincided with regions of low data rate such as those along the edge of the hinge geometry and occasionally in regions of high data rate such as the hinge atrial leakage jets during systole. This research also demonstrated that, in some measurement locations, the cyclic averaged Reynolds shear stresses were larger than the phase averaged Reynolds shear stresses.

The RSS percent difference obtained in the present research was consistent with findings by Tiederman et al and Liu et al who investigated the effects of cyclic variation on Reynolds shear stress during the forward flow phase of prosthetic heart valves using LDV [Liu, 2000; Tiederman, 1988]. Both groups recorded spurious Reynolds shear stress percent differences between 10.0 and 90.0 percent when cyclic variation was removed from the Reynolds shear stress calculations. Removal of the cycle-to-cycle effects in the current studies resulted in estimates of Reynolds shear stress that were slightly lower than those obtained using simple phase averaging estimates. However, cycle-to-cycle variation 
can significantly affect turbulent statistics in pulsatile flows, and should be removed or shown to be small when reporting such statistics. 


\section{Appendix G: Tabulated Data for the Static Leakage Experiments}

Appendix $\mathrm{G}$ records the tabulated static leakage rate data for all valve prostheses studied in this research.

Table G-1 tabulated static leakage data for the SJM $27 \mathrm{~mm}$ Standard valve

\begin{tabular}{|c|c|c|}
\hline \multicolumn{2}{|c|}{ Regular Leakage } & \\
\hline liters & sec & $\begin{array}{c}\text { Leakage flowrate } \\
\text { (liter/min) }\end{array}$ \\
\hline 0.425 & 60 & 0.425 \\
\hline 0.35 & 60 & 0.350 \\
\hline 0.29 & 60 & 0.290 \\
\hline 0.33 & 60 & 0.330 \\
\hline 0.36 & 60 & 0.360 \\
\hline avg & & $\mathbf{0 . 3 5 1}$ \\
\hline stdev & & 0.049 \\
\hline
\end{tabular}

Table G-2 tabulated static leakage data for transparent SJM $27 \mathrm{~mm}$ prototype low leaker

\begin{tabular}{|c|c|c|}
\hline \multicolumn{2}{|c|}{ Low Leaker } & \\
\hline liters & sec & $\begin{array}{c}\text { Leakage flowrate } \\
\text { (liter/min) }\end{array}$ \\
\hline 0.065 & 90 & 0.043 \\
\hline 0.075 & 90 & 0.050 \\
\hline 0.077 & 90 & 0.051 \\
\hline 0.077 & 90 & 0.051 \\
\hline 0.075 & 90 & 0.050 \\
\hline avg & & $\mathbf{0 . 0 4 9}$ \\
\hline stdev & & 0.003 \\
\hline
\end{tabular}


Table G-3 tabulated static leakage data for transparent SJM 27 mm prototype high leaker

\begin{tabular}{|c|c|c|}
\hline \multicolumn{2}{|c|}{ High Leaker } & \\
\hline liters & sec & $\begin{array}{c}\text { Leakage flowrate } \\
\text { (liter/min) }\end{array}$ \\
\hline 0.45 & 60 & 0.450 \\
\hline 0.44 & 60 & 0.440 \\
\hline 0.56 & 60 & 0.560 \\
\hline 0.5 & 60 & 0.500 \\
\hline 0.5 & 60 & 0.500 \\
\hline avg & & $\mathbf{0 . 4 9 0}$ \\
\hline stdev & & 0.048 \\
\hline
\end{tabular}

Table G-4 tabulated static leakage data for transparent CM 23 mm valve

\begin{tabular}{|c|c|c|}
\hline \multicolumn{2}{|c|}{ CM 23mm } & \\
\hline liters & sec & $\begin{array}{c}\text { Leakage } \\
\text { flowrate } \\
\text { (liter/min) }\end{array}$ \\
\hline 0.55 & 60 & 0.5 \\
\hline 0.55 & 60 & 0.5 \\
\hline 0.55 & 60 & 0.5 \\
\hline 0.44 & 60 & 0.44 \\
\hline 0.49 & 60 & 0.49 \\
\hline avg & & $\mathbf{0 . 4 8 6}$ \\
\hline stdev & & 0.026 \\
\hline
\end{tabular}


Table G-5 tabulated static leakage data for Aortech $23 \mathrm{~mm}$ prototype A at $20.5^{\circ} \mathrm{C}$ and $37^{\circ} \mathrm{C}$ with no oscillations

\begin{tabular}{|c|c|c|c|c|c|}
\hline \multicolumn{2}{|c|}{ Prototype A (no oscillation) } & & & \\
\hline $\begin{array}{c}20.5 \\
\mathrm{C}\end{array}$ & & $37 \mathrm{C}$ & & \\
\hline liters & $\mathrm{sec}$ & $\begin{array}{c}\text { leakage flowrate } \\
\text { (liter/min) }\end{array}$ & liters & sec & $\begin{array}{c}\text { leakage flowrate } \\
\text { (liter/min) }\end{array}$ \\
\hline 0.476 & 30 & 0.952 & 0.3 & 30 & 0.6 \\
\hline 0.505 & 30 & 1.01 & 0.288 & 30 & 0.575 \\
\hline 0.495 & 30 & 0.99 & 0.29 & 30 & 0.58 \\
\hline 0.505 & 30 & 1.01 & 0.275 & 30 & 0.55 \\
\hline 0.515 & 30 & 1.03 & 0.275 & 30 & 0.55 \\
\hline avg & & $\mathbf{0 . 9 9 8 4}$ & & & $\mathbf{0 . 5 7 1}$ \\
\hline stdev & & 0.03 & & & 0.021 \\
\hline
\end{tabular}

Table G-6 tabulated static leakage data for Aortech $23 \mathrm{~mm}$ prototype A at $20.5^{\circ} \mathrm{C}$ and $37^{\circ} \mathrm{C}$ with oscillations

\begin{tabular}{|c|c|c|c|c|c|}
\hline \multicolumn{3}{|c|}{ Prototype A (oscillation) } & & & \\
\hline $\begin{array}{c}20.5 \\
\mathrm{C}\end{array}$ & & $37 \mathrm{C}$ & & \\
\hline liters & $\mathrm{sec}$ & $\begin{array}{c}\text { leakage flowrate } \\
\text { (liter/min) }\end{array}$ & liters & sec & $\begin{array}{c}\text { leakage flowrate } \\
\text { (liter/min) }\end{array}$ \\
\hline 0.525 & 30 & 1.05 & 0.688 & 30 & 1.375 \\
\hline 0.509 & 30 & 1.017 & 0.688 & 30 & 1.371 \\
\hline 0.553 & 30 & 1.105 & 0.664 & 30 & 1.327 \\
\hline 0.529 & 30 & 1.057 & 0.679 & 30 & 1.357 \\
\hline 0.529 & 30 & 1.058 & 0.688 & 30 & 1.375 \\
\hline avg & & $\mathbf{1 . 0 5 7}$ & & & $\mathbf{1 . 3 6 1}$ \\
\hline stdev & & 0.031 & & & 0.0203 \\
\hline
\end{tabular}


Table G-7 tabulated static leakage data for Aortech $23 \mathrm{~mm}$ prototype $\mathrm{B}$ at $20.5^{\circ} \mathrm{C}$ and $37^{\circ} \mathrm{C}$ with no oscillations

\begin{tabular}{|c|c|c|c|c|c|}
\hline \multicolumn{2}{|c|}{ Prototype B (no oscillation) } & & & \\
\hline $\begin{array}{c}20.5 \\
\mathrm{C}\end{array}$ & & $37 \mathrm{C}$ & & \\
\hline liters & sec & $\begin{array}{c}\text { leakage flowrate } \\
\text { (liter/min) }\end{array}$ & liters & sec & $\begin{array}{c}\text { leakage flowrate } \\
\text { (liter/min) }\end{array}$ \\
\hline 0.495 & 30 & 0.989 & 0.35 & 30 & 0.7 \\
\hline 0.505 & 30 & 1.01 & 0.346 & 30 & 0.692 \\
\hline 0.434 & 30 & 0.868 & 0.332 & 30 & 0.663 \\
\hline 0.473 & 30 & 0.946 & 0.335 & 30 & 0.669 \\
\hline 0.468 & 30 & 0.935 & 0.33 & 30 & 0.66 \\
\hline avg & & $\mathbf{0 . 9 5}$ & & & $\mathbf{0 . 6 7 7}$ \\
\hline stdev & & 0.055 & & & 0.018 \\
\hline
\end{tabular}

Table G-8 tabulated static leakage data for Aortech $23 \mathrm{~mm}$ prototype B at $20.5^{\circ} \mathrm{C}$ and $37^{\circ} \mathrm{C}$ with oscillations

\begin{tabular}{|c|c|c|c|c|c|}
\hline \multicolumn{2}{|c|}{ Prototype B (oscillation) } & & & \\
\hline $20.5 \mathrm{C}$ & & $\begin{array}{c}\text { leakage flowrate } \\
\text { (liter/min) }\end{array}$ & liters & sec & $\begin{array}{c}\text { leakage flowrate } \\
\text { (liter/min) }\end{array}$ \\
\hline liters & sec & 1.311 & 0.71 & 30 & 1.42 \\
\hline 0.656 & 30 & 1.318 & 0.63 & 30 & 1.26 \\
\hline 0.659 & 30 & 1.347 & 0.585 & 30 & 1.17 \\
\hline 0.674 & 30 & 1.325 & 0.61 & 30 & 1.22 \\
\hline 0.663 & 30 & 1.311 & 0.615 & 30 & 1.23 \\
\hline 0.656 & 30 & $\mathbf{1 . 3 2 2}$ & & & $\mathbf{1 . 2 6}$ \\
\hline avg & & 0.015 & & & 0.095 \\
\hline stdev & & & & & \\
\hline
\end{tabular}


Table G-9 tabulated static leakage data for Aortech $23 \mathrm{~mm}$ prototype $\mathrm{C}$ at $20.5^{\circ} \mathrm{C}$ and $37^{\circ} \mathrm{C}$ with no oscillations

\begin{tabular}{|c|c|c|c|c|c|}
\hline \multicolumn{2}{|c|}{ Prototype C (no oscillation) } & & & \\
\hline $\begin{array}{c}20.5 \\
\mathrm{C}\end{array}$ & & $37 \mathrm{C}$ & & \\
\hline liters & $\mathrm{sec}$ & $\begin{array}{c}\text { leakage flowrate } \\
\text { (liter/min) }\end{array}$ & liters & sec & $\begin{array}{c}\text { leakage flowrate } \\
\text { (liter/min) }\end{array}$ \\
\hline 2 & 30 & 4 & 1.79 & 30 & 3.58 \\
\hline 1.9 & 30 & 3.8 & 1.8 & 30 & 3.6 \\
\hline 1.9 & 30 & 3.8 & 1.85 & 30 & 3.7 \\
\hline 1.95 & 30 & 3.9 & 1.824 & 30 & 3.6 \\
\hline 1.9 & 30 & 3.8 & 1.85 & 30 & 3.7 \\
\hline avg & & $\mathbf{3 . 8 6}$ & & & $\mathbf{3 . 6 4 6}$ \\
\hline stdev & & 0.089 & & & 0.055 \\
\hline
\end{tabular}

Table G-10 tabulated static leakage data for Aortech $23 \mathrm{~mm}$ prototype $\mathrm{C}$ at $20.5^{\circ} \mathrm{C}$ and $37^{\circ} \mathrm{C}$ with oscillations

\begin{tabular}{|c|c|c|c|c|c|}
\hline \multicolumn{3}{|c|}{ Prototype C (oscillation) } & & & \\
\hline $\begin{array}{c}20.5 \\
\mathrm{C}\end{array}$ & & $37 \mathrm{C}$ & & \\
\hline liters & sec & $\begin{array}{c}\text { leakage flowrate } \\
\text { (liter/min) }\end{array}$ & liters & sec & $\begin{array}{c}\text { leakage flowrate } \\
\text { (liter/min) }\end{array}$ \\
\hline 1.874 & 30 & 3.747 & 1.75 & 30 & 3.5 \\
\hline 1.808 & 30 & 3.616 & 1.735 & 30 & 3.47 \\
\hline 1.748 & 30 & 3.495 & 1.665 & 30 & 3.33 \\
\hline 1.748 & 30 & 3.495 & 1.653 & 30 & 3.305 \\
\hline 1.645 & 30 & 3.29 & 1.706 & 30 & 3.401 \\
\hline avg & & $\mathbf{3 . 5 2 9}$ & & & $\mathbf{3 . 4 0 1}$ \\
\hline stdev & & 0.169 & & & 0.085 \\
\hline
\end{tabular}




\section{Appendix H: Catalog of Animations}

Appendix $\mathrm{H}$ is a list of the velocity magnitude $(\mathrm{m} / \mathrm{s})$ and the Reynolds shear stress (dynes $/ \mathrm{cm}^{2}$ ) animations for all valve prostheses studied in this thesis.

\section{LIST OF ANIMATIONS}

\section{H-1 Temperature-dependent xperiments}

1) 3D_reconstructed_leaflet_motion_room_temp.avi

2) 3D_reconstructed_leaflet_motion_physiological_temp.avi

3) high_speed_camera_upstream_room_temp.avi

4) high_speed_camera_upstream_physiological_temp.avi

\section{H-2 Mitral hinge studies}

\section{H-2-1 Clear valve model of CM $23 \mathrm{~mm}$ valve}

1) CM_flat_vmag.avi

2) CM_flat_rss.avi

3) CM_190um_above_flat_vmag.avi

4) CM_190um_above_flat_rss.avi

5) CM_390um_above_flat_vmag.avi

6) CM_390um_above_flat_rss.avi

7) CM_1mm_below_flat_vmag.avi

8) CM_1mm_below_flat_rss.avi

\section{H-2-2 Three $27 \mathrm{~mm}$ clear models}

H-2-2-1 SJM 27 mm Standard valve

1) reg_flat_vmag.avi

2) reg_flat_rss.avi

3) reg_190um_above_flat_vmag.avi

4) reg_190um_above_flat_rss.avi

5) reg_390um_above_flat_vmag.avi

6) reg_390um_above_flat_rss.avi

7) reg_585um_above_flat_vmag.avi

8) reg_585um_above_flat_rss.avi

9) reg_500um_below_flat_vmag.avi

10) reg_500um_below_flat_rss.avi

11) reg_1mm_below_flat_vmag.avi

12) reg_1mm_below_flat_rss.avi

13) reg_3mm_below_flat_vmag.avi 
14) reg_3mm_below_flat_rss.avi

15) reg_thumbnail_vmag.avi

16)reg_thumbnail_rss.avi

\section{H-2-2-2 27 $\mathrm{mm}$ prototype low leaker valve}

1) low_flat_vmag.avi

2) low_flat_rss.avi

3) low_190um_above_flat_vmag.avi

4) low_190um_above_flat_rss.avi

5) low_390um_above_flat_vmag.avi

6) low_390um_above_flat_rss.avi

7) low_500um_below_flat_vmag.avi

8) low_500um_below_flat_rss.avi

9) low_1mm_below_flat_vmag.avi

10) low_1mm_below_flat_rss.avi

11)low_3mm_below_flat_vmag.avi

12) low_3mm_below_flat_rss.avi

13) low_thumbnail_vmag.avi

14) low_thumbnail_rss.avi

\section{H-2-2-3 $27 \mathrm{~mm}$ prototype high leaker valve}

1) high_flat_vmag.avi

2) high_flat_rss.avi

3) high_190um_above_flat_vmag.avi

4) high_190um_above_flat_rss.avi

5) high_390um_above_flat_vmag.avi

6) high_390um_above_flat_rss.avi

7) high_585um_above_flat_vmag.avi

8) high_585um_above_flat_rss.avi

9) high_500um_below_flat_vmag.avi

10)high_500um_below_flat_rss.avi

11)high_1mm_below_flat_vmag.avi

12)high_1mm_below_flat_rss.avi

13)high_3mm_below_flat_vmag.avi

14)high_3mm_below_flat_rss.avi

15)high_thumbnail_vmag.avi

16)high_thumbnail_rss.avi

\section{H-3 2D LDV Aortic polymeric valve studies}

\section{H-3-1 Aortech $23 \mathrm{~mm}$ prototype $A$}

1) A_parallel_downstream_vmag.avi

2) A_parallel_downstream_rss.avi

3) A_perpend_downstream_vmag.avi

4) A_perpend_downstream_rss.avi

5) A_stent_downstream_vmag.avi 
6) A_stent_downstream_rss.avi

7) A_inside_valve_vmag.avi

8) A_inside_valve_rss.avi

9) A_parallel_downstream_3DLDV_vmag.avi

10) A_parallel_downstream_3DLDV_rss.avi

\section{H-3-2 Aortech $23 \mathrm{~mm}$ prototype B}

1) B_parallel_downstream_vmag.avi

2) B_parallel_downstream_rss.avi

3) B_perpend_downstream_vmag.avi

4) B_perpend_downstream_rss.avi

5) B_stent_downstream_vmag.avi

6) B_stent_downstream_rss.avi

7) B_inside_valve_vmag.avi

8) B_inside_valve_rss.avi

\section{H-3-3 Aortech $23 \mathrm{~mm}$ prototype $\mathrm{C}$}

1) C_parallel_downstream_vmag.avi

2) C_parallel_downstream_rss.avi

3) C_perpend_downstream_vmag.avi

4) C_perpend_downstream_rss.avi

5) C_stent_downstream_vmag.avi

6) C_stent_downstream_rss.avi

7) C_inside_valve_vmag.avi

8) C_inside_valve_rss.avi

\section{H-4 3D PIV Aortic polymeric valve studies}

\section{H-4-1 Aortech $23 \mathrm{~mm}$ prototype A}

1) A_center_plane_vmag.avi

2) A_center_plane_rss.avi

3) A_+4mm_plane_vmag.avi

4) A_-4mm_plane_vmag.avi

5) A_+4mm_plane_rss.avi

6) A_-4mm_plane_rss.avi

7) A_+8mm_plane_vmag.avi

8) A_-8mm_plane_vmag.avi

9) A_+8mm_plane_rss.avi

10) A_-8mm_plane_rss.avi

11) A_+12mm_plane_vmag.avi

12) A_-12mm_plane_vmag.avi

13) A_+12mm_plane_rss.avi

14) A_-12mm_plane_rss.avi

\section{H-4-2 Aortech $23 \mathrm{~mm}$ prototype B}

1) B_center_plane_vmag.avi

2) B_center_plane_rss.avi 
3) B_+4mm_plane_vmag.avi

4) B_-4mm_plane_vmag.avi

5) B_+4mm_plane_rss.avi

6) B_-4mm_plane_rss.avi

7) B_+8mm_plane_vmag.avi

8) B_-8mm_plane_vmag.avi

9) B_+8mm_plane_rss.avi

10) B_-8mm_plane_rss.avi

11) B_+12mm_plane_vmag.avi

12) B_-12mm_plane_vmag.avi

13) B_+12mm_plane_rss.avi

14) B_-12mm_plane_rss.avi

\section{H-4-3 Aortech $23 \mathrm{~mm}$ prototype C}

1) C_center_plane_vmag.avi

2) C_center_plane_rss.avi

3) C_+4mm_plane_vmag_1.avi

4) C_-4mm_plane_vmag_2.avi

5) C_+4mm_plane_rss_1.avi

6) C_-4mm_plane_rss_2.avi

7) C_+8mm_plane_vmag_1.avi

8) C_-8mm_plane_vmag_2.avi

9) C_+8mm_plane_rss_1.avi

10) C_-8mm_plane_rss_2.avi

11) C_+12mm_plane_vmag_1.avi

12) C_-12mm_plane_vmag_2.avi

13) C_+12mm_plane_rss_1.avi

14) C_-12mm_plane_rss_2.avi 


\section{Appendix I: Leaflet Motion Results at Physiological Temperature}
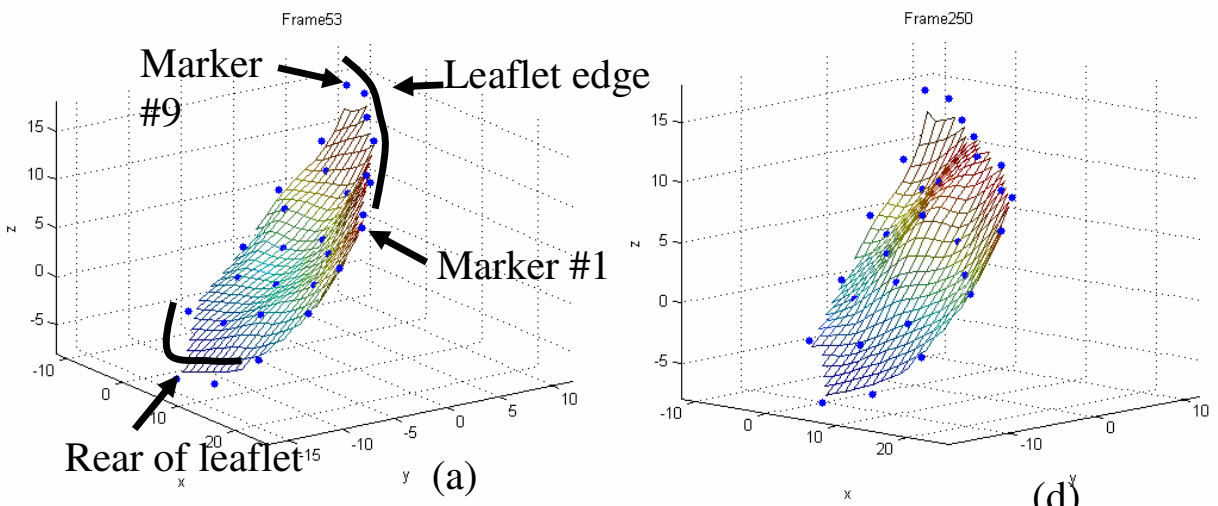

Frame115

(d)

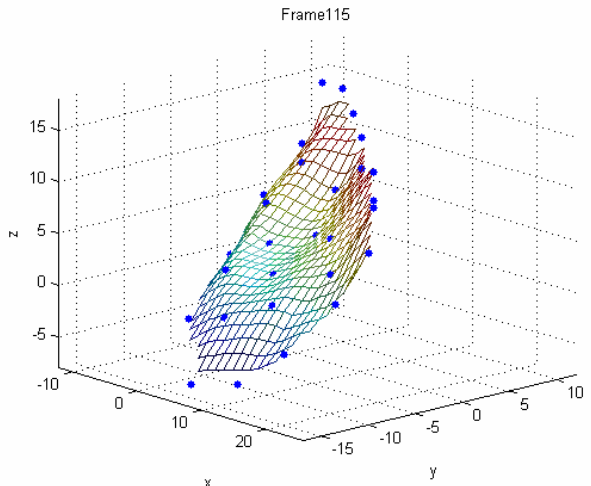

(b)
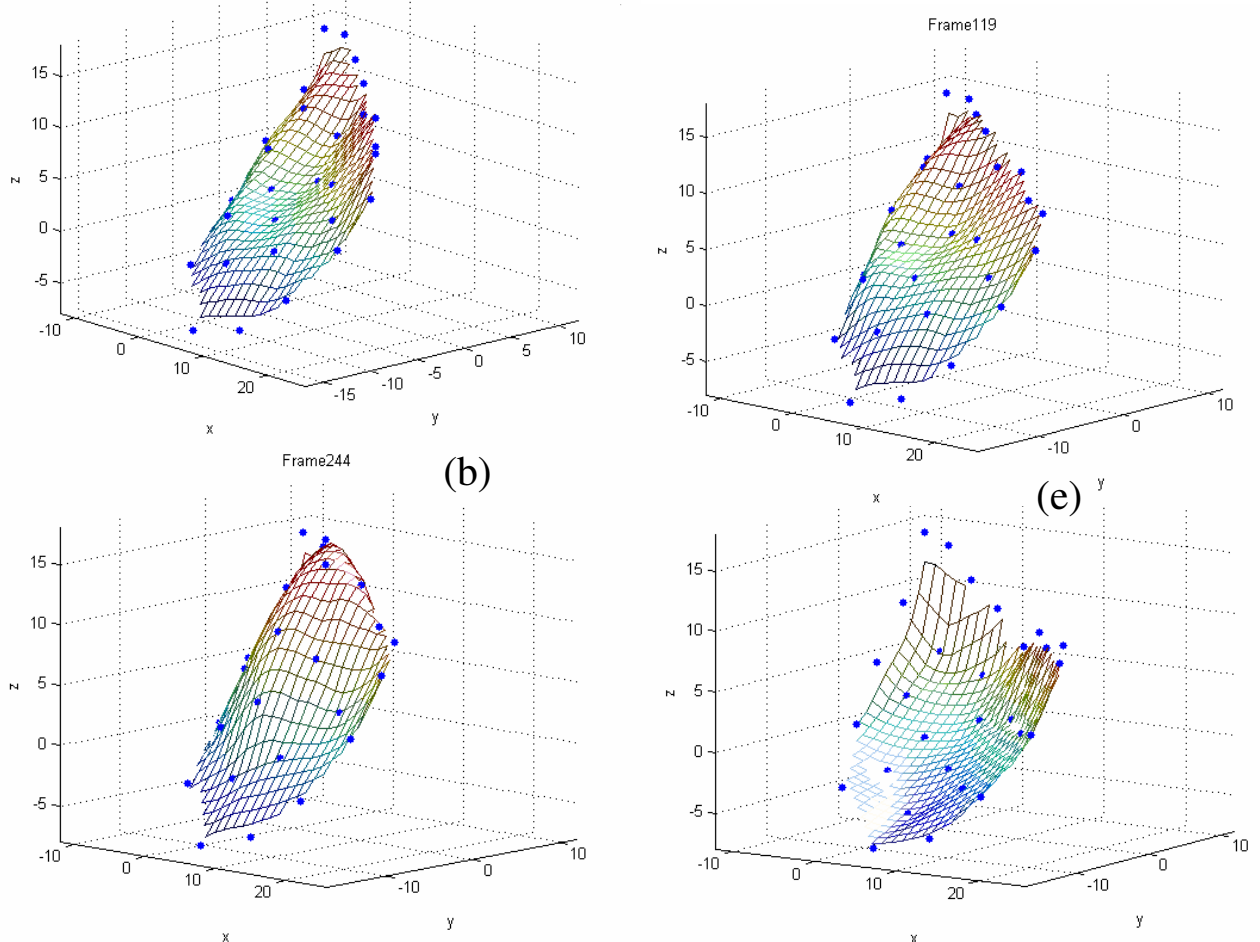

(c)

(f)

Figure J-1 The motion of the leaflet through a single cardiac cycle (temperature $-37^{\circ} \mathrm{C}$ ). (a) The beginning of the opening phase; (b) Leaflet at the verge of opening; (c) Leaflet at fully opened position; (d) The beginning of the closing phase; (e) The closing phase; and (f) Leaflet at fully closed position (wmv, 6,202k, 3D_reconstructed_leaflet_motion_physiological_temp.wmv) 


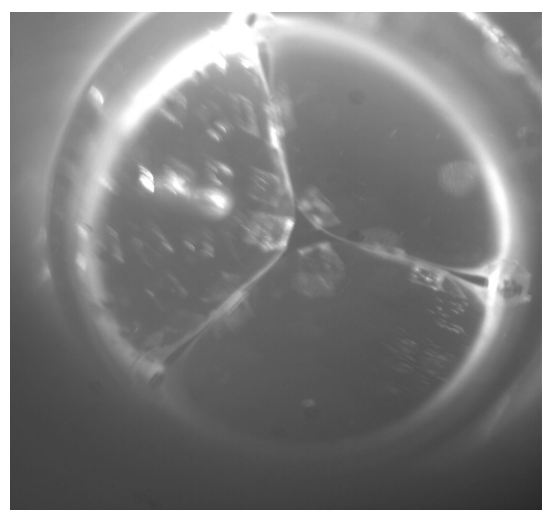

(a)

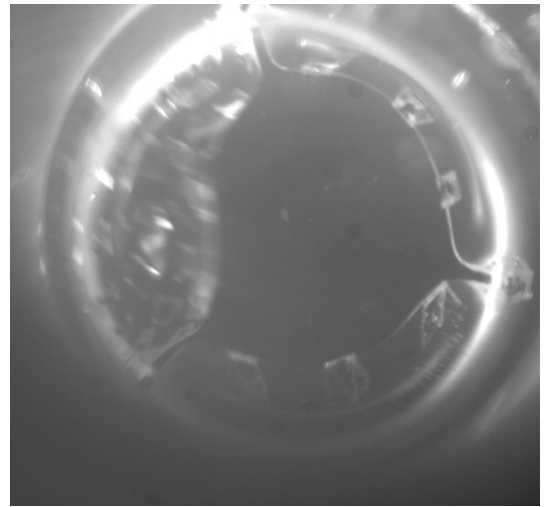

(b)

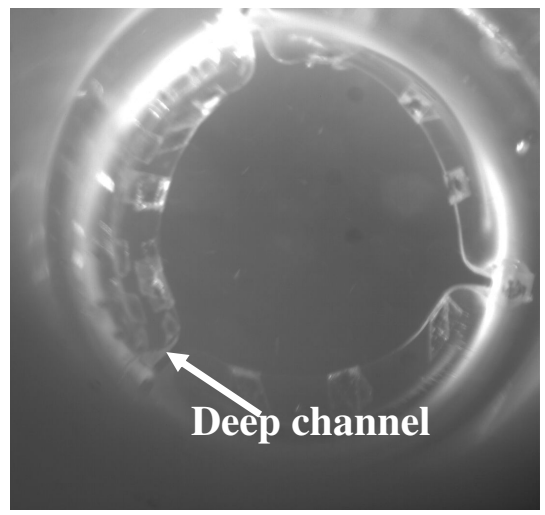

(c)

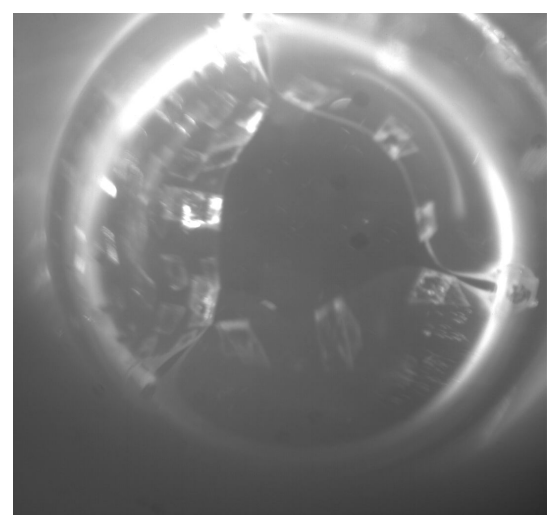

(d)

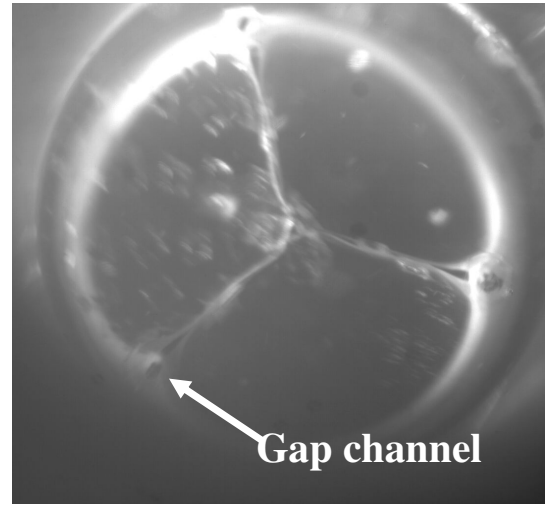

(e)

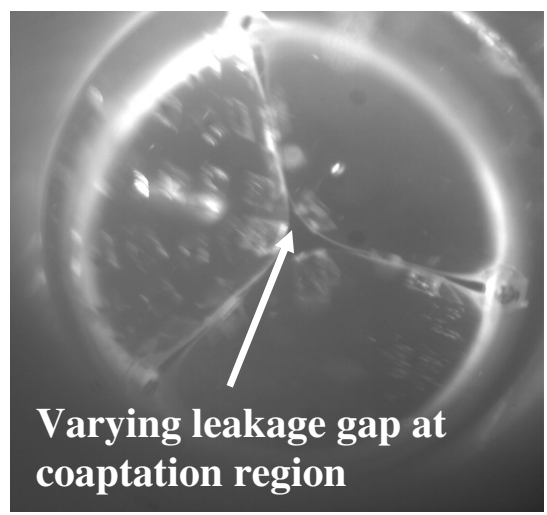

(f)

Figure J-2 Upstream high speed camera image of the valve through the dogleg (temperature $-37^{\circ} \mathrm{C}$ ). The opening sequence of the valve leaflet at $37^{\circ} \mathrm{C}$ was similar to that at $20.5^{\circ} \mathrm{C}$. (a) The beginning of opening phase; (b) valve opening; (c) valve when full open; (d) valve closing; (e) valve when closed; and e) oscillation of valve leaflet during diastolic phase (wmv, 1,858k, high_speed_camera_upstream_physiological_temp.wmv) 


\section{REFERENCES}

1. Aagaard, J., Tingleff, J., Hansen, C.N., Noergaard, M.A., and Rasmussen, C.E., Twelve years' clinical experience with the CarboMedics prosthetic heart valve. $J$ Heart Valve Dis. 10(2): p. 177-84, 2001.

2. Akins, C.W., Results with mechanical cardiac valvular prostheses. Ann Thorac Surg. 60(6): p. 1836-44, 1995.

3. Akutsu, T., Dreyer, B., and Kolff, W.J., Polyurethane artificial heart valves in animals. J Appl Physiol. 14: p. 1045-8, 1959.

4. Alferiev, I., Vyavahare, N., Song, C., Connolly, J., Hinson, J.T., Lu, Z., Tallapragada, S., Bianco, R., and Levy, R., Bisphosphonate derivatized polyurethanes resist calcification. Biomaterials. 22(19): p. 2683-93, 2001.

5. Baudet, E.M., Puel, V., McBride, J.T., Grimaud, J.P., Roques, F., Clerc, F., Roques, X., and Laborde, N., Long-term results of valve replacement with the St. Jude Medical prosthesis. J Thorac Cardiovasc Surg. 109(5): p. 858-70, 1995.

6. Bernacca, G.M., Mackay, T.G., Wilkinson, R., and Wheatley, D.J., Calcification and fatigue failure in a polyurethane heart value. Biomaterials. 16(4): p. 279-85, 1995.

7. Bernacca, G.M., Mackay, T.G., and Wheatley, D.J., In vitro function and durability of a polyurethane heart valve: material considerations. J Heart Valve Dis. 5(5): p. 538-42, 1996.

8. Bernacca, G.M., Mackay, T.G., Gulbransen, M.J., Donn, A.W., and Wheatley, D.J., Polyurethane heart valve durability: effects of leaflet thickness and material. Int J Artif Organs. 20(6): p. 327-31, 1997a.

9. Bernacca, G.M., Mackay, T.G., Wilkinson, R., and Wheatley, D.J., Polyurethane heart valves: fatigue failure, calcification, and polyurethane structure. J Biomed Mater Res. 34(3): p. 371-9, 1997b.

10. Bernacca, G.M., Gulbransen, M.J., Wilkinson, R., and Wheatley, D.J., In vitro blood compatibility of surface-modified polyurethanes. Biomaterials. 19(13): p. 1151-65, 1998. 
11. Bernacca, G.M., O'Connor, B., Williams, D.F., and Wheatley, D.J., Hydrodynamic function of polyurethane prosthetic heart valves: influences of Young's modulus and leaflet thickness. Biomaterials. 23(1): p. 45-50, 2002a.

12. Bernacca, G.M., Straub, I., and Wheatley, D.J., Mechanical and morphological study of biostable polyurethane heart valve leaflets explanted from sheep. $J$ Biomed Mater Res. 61(1): p. 138-45, 2002b.

13. Bhushan, B., Wyant, J.C., and Koliopoulos, C., Measurement of surface topography of magnetic tapes by Mirau Interferometry. Applied Optics. 24(10): p. 1489-1497, 1985.

14. Black, M.M., Cochrane, T., Lawford, P.V., Reul, H., and Yoganathan, A.P., Design and flow characteristics, in Replacement cardiac valves. 1991, Pergamon press: New York. p. 482.

15. Black, M.M. and Drury, P.J., Mechanical and other problems of artificial valves. Curr Top Pathol. 86: p. 127-59, 1994.

16. Blackman, B.R., Barbee, K.A., and Thibault, L.E., In vitro cell shearing device to investigate the dynamic response of cells in a controlled hydrodynamic environment. Ann Biomed Eng. 28(4): p. 363-72, 2000.

17. Bluestein, D., Einav, S., and Hwang, N.H., A squeeze flow phenomenon at the closing of a bileaflet mechanical heart valve prosthesis. J Biomech. 27(11): p. 1369-78, 1994.

18. Bodnar, E. and Frater, R., eds. Replacement cardiac valves. 1991, Pergamon press: New York. 482.

19. Browne, P., Ramuzat, A., Saxena, R., and Yoganathan, A.P., Experimental investigation of the steady flow downstream of the St. Jude bileaflet heart valve: a comparison between laser Doppler velocimetry and particle image velocimetry techniques. Ann Biomed Eng. 28(1): p. 39-47, 2000.

20. Butterfield, M., Fisher, J., Davies, G.A., and Kearney, J.M., Leaflet geometry and function in porcine bioprostheses. Eur J Cardiothorac Surg. 5(1): p. 27-32; discussion 33, 1991.

21. Butterfield, M. and Fisher, J., Fatigue analysis of clinical bioprosthetic heart valves manufactured using photooxidized bovine pericardium. J Heart Valve Dis. 9(1): p. 161-6; discussion 167, 2000.

22. Cannegieter, S.C., Rosendaal, F.R., and Briet, E., Thromboembolic and bleeding complications in patients with mechanical heart valve prostheses. Circulation. 89(2): p. 635-41, 1994. 
23. Cebi, N. and Bozkurt, E., Mid-term results of mitral valve repair for complicated active bacterial endocarditis in high-risk patients. Heart Vessels. 19(4): p. 179-82, 2004.

24. Chandran, K.B., Khalighi, B., and Chen, C.J., Experimental study of physiological pulsatile flow past valve prostheses in a model of human aorta--I. Caged ball valves. J Biomech. 18(10): p. 763-72, 1985a.

25. Chandran, K.B., Khalighi, B., and Chen, C.J., Experimental study of physiological pulsatile flow past valve prosthesis in a model of human aorta--II. Tilting disc valves and the effect of orientation. J Biomech. 18(10): p. 773-80, $1985 b$.

26. Chandran, K.B., Fatemi, R., Schoephoerster, R., Wurzel, D., Hansen, G., Pantalos, G., Yu, L.S., and Kolff, W.J., In vitro comparison of velocity profiles and turbulent shear distal to polyurethane trileaflet and pericardial prosthetic valves. Artif Organs. 13(2): p. 148-54, 1989.

27. Chandran, K.B., Dexter, E.U., Aluri, S., and Richenbacher, W.E., Negative pressure transients with mechanical heart-valve closure: correlation between in vitro and in vivo results. Ann Biomed Eng. 26(4): p. 546-56, 1998.

28. Chetta, G.E. and Lloyd, J.R., The design, fabrication and evaluation of a trileaflet prosthetic heart valve. J Biomech Eng. 102(1): p. 34-41, 1980.

29. Christy, J.R. and Macleod, N., The role of stasis in the clotting of blood and milk flows around solid objects. Cardiovasc Res. 23(11): p. 949-59, 1989.

30. Christy, J.R. and Marosek, K.W., Ultrasonic determination of clot deposition rates in a milk-based, in-vitro procedure for thrombogenicity assessment. J Heart Valve Dis. 9(3): p. 379-88, 2000.

31. Cooley, D.A., The quest for the perfect prosthetic heart valve. Med Instrum. 11(2): p. 82-4, 1977.

32. Corden, J., David, T., and Fisher, J., Determination of the curvatures and bending strains in open trileaflet heart valves. Proc Inst Mech Eng [H]. 209(2): p. 121-8, 1995a.

33. Corden, J., David, T., and Fisher, J., In vitro determination of the curvatures and bending strains acting on the leaflets of polyurethane trileaflet heart valves during leaflet motion. Proc Inst Mech Eng [H]. 209(4): p. 243-53, 1995b. 
34. Cosgrove, D.M., Lytle, B.W., and Williams, G.W., Hemodynamic performance of the Carpentier-Edwards pericardial valve in the aortic position in vivo.

Circulation. 72(3 Pt 2): p. II146-52, 1985.

35. Craver, J., CarboMedics Prosthetic Heart Valve. Eur J Cardiothorac Surg. 15 Suppl 1: p. S3-11; discussion S39-43, 1999.

36. Daebritz, S.H., Sachweh, J.S., Hermanns, B., Fausten, B., Franke, A., Groetzner, J., Klosterhalfen, B., and Messmer, B.J., Introduction of a flexible polymeric heart valve prosthesis with special design for mitral position. Circulation. 108 Suppl 1: p. II134-9, 2003.

37. Daebritz, S.H., Fausten, B., Hermanns, B., Schroeder, J., Groetzner, J., Autschbach, R., Messmer, B.J., and Sachweh, J.S., Introduction of a flexible polymeric heart valve prosthesis with special design for aortic position. Eur J Cardiothorac Surg. 25(6): p. 946-52, 2004.

38. Daffertshofer, M., Ries, S., Schminke, U., and Hennerici, M., High-intensity transient signals in patients with cerebral ischemia. Stroke. 27(10): p. 1844-9, 1996.

39. Dalrymple-Hay, M.J., Pearce, R., Dawkins, S., Haw, M.P., Lamb, R.K., Livesey, S.A., and Monro, J.L., A single-center experience with 1,378 CarboMedics mechanical valve implants. Ann Thorac Surg. 69(2): p. 457-63, 2000.

40. Demtröder, W., Laser spectroscopy: basic concepts and instrumentation. 3rd ed Berlin ed. 2003, New York: Springer.

41. DeWall, R.A., Qasim, N., and Carr, L., Evolution of mechanical heart valves. Ann Thorac Surg. 69(5): p. 1612-21, 2000.

42. Diamond, S.L., Eskin, S.G., and McIntire, L.V., Fluid flow stimulates tissue plasminogen activator secretion by cultured human endothelial cells. Science. 243(4897): p. 1483-5, 1989.

43. D'Souza, S.S., Butterfield, M., and Fisher, J., Kinematics of synthetic flexible leaflet heart valves during accelerated testing. J Heart Valve Dis. 12(1): p. 110-9; discussion 119-20, 2003.

44. Eckstein, E.C. and Belgacem, F., Model of platelet transport in flowing blood with drift and diffusion terms. Biophys J. 60(1): p. 53-69, 1991.

45. Ellis, J., An in vitro investigation of the leakage and hinge flow fields through bileafet mechanical heart valves, in Chemical Engineering Department. 1999, Georgia Institute of Technology: Atlanta, GA. p. 367. 
46. Ellis, J.T., Healy, T.M., Fontaine, A.A., Saxena, R., and Yoganathan, A.P., Velocity measurements and flow patterns within the hinge region of a Medtronic Parallel bileaflet mechanical valve with clear housing. J Heart Valve Dis. 5(6): p. 591-9, 1996a.

47. Ellis, J.T., Healy, T.M., Fontaine, A.A., Weston, M.W., Jarret, C.A., Saxena, R., and Yoganathan, A.P., An in vitro investigation of the retrograde flow fields of two bileaflet mechanical heart valves. J Heart Valve Dis. 5(6): p. 600-6, 1996 b.

48. Ellis, J.T. and Yoganathan, A.P., A comparison of the hinge and near-hinge flow fields of the St Jude medical hemodynamic plus and regent bileaflet mechanical heart valves. J Thorac Cardiovasc Surg. 119(1): p. 83-93, 2000a.

49. Ellis, J.T., Travis, B.R., and Yoganathan, A.P., An in vitro study of the hinge and near-field forward flow dynamics of the St. Jude Medical Regent bileaflet mechanical heart valve. Ann Biomed Eng. 28(5): p. 524-32, 2000 b.

50. Figliola, R.S. and Mueller, T.J., On the hemolytic and thrombogenic potential of occluder prosthetic heart valves from in-vitro measurements. J Biomech Eng. 103(2): p. 83-90, 1981.

51. Fisher, J., Reece, I.J., and Wheatley, D.J., In vitro evaluation of six mechanical and six bioprosthetic valves. Thorac Cardiovasc Surg. 34(3): p. 157-62, 1986.

52. Fung, Y.C., Biodynamics : circulation. 1984, New York: Springer-Verlag. xi, 404.

53. Gao, Z.B., Hosein, N., Dai, F.F., and Hwang, N.H., Pressure and flow fields in the hinge region of bileaflet mechanical heart valves. J Heart Valve Dis. 8(2): p. 197205, 1999.

54. Gencbay, M., Turan, F., Yaymaci, B., Degertekin, M., Basaran, Y., Dindar, I., and Izgi, A., Prevalence of microbubbles associated with mechanical prosthetic cardiac valves: a prospective transesophageal echocardiography study. J Heart Valve Dis. 7(3): p. 340-4, 1998.

55. George, W. Limitations to measuring acurracy inherent in the laser dopple signal. in Proceedings of the 1975 LDV Symposium. 1975. Copenhagen, Denmark.

56. Ghista, D.N. and Reul, H., Optimal prosthetic aortic leaflet valve: design parametric and longevity analyses: development of the Avcothane-51 leaflet valve based on the optimum design analysis. J Biomech. 10(5-6): p. 313-24, 1977.

57. Gizzi, W.P., Roduner, C., Stahlecker, D., Koppel, P., and Gyarmathy, G., Timeresolved measurements with fast-response probes and laser Doppler velocimetry at the impeller exit of a centrifugal compressor: a comparison of two 
measurement techniques. Proceedings of the Institution of Mechanical Engineers Part a-Journal of Power and Energy. 213(A4): p. 291-308, 1999.

58. Glas, W., Forstner, M., Kuhn, K., and Jaberg, H., Smoothing and statistical evaluation of laser Doppler velocimetry data of turbulent flows in rotating and reciprocating machinery. Experiments in Fluids. 29(5): p. 411-417, 2000.

59. Goldsmith, H.L. and Turitto, V.T., Rheological aspects of thrombosis and haemostasis: basic principles and applications. ICTH-Report--Subcommittee on Rheology of the International Committee on Thrombosis and Haemostasis. Thromb Haemost. 55(3): p. 415-35, 1986.

60. Goldsmith, I., Mukundan, S., Nugent, A., and Rosin, M.D., Early clinical experience with the Tissuemed porcine bioprosthesis. Ann Thorac Surg. 66(6 Suppl): p. S259-63, 1998.

61. Goldsmith, I., Turpie, A.G., and Lip, G.Y., Valvar heart disease and prosthetic heart valves. Bmj. 325(7374): p. 1228-31, 2002.

62. Graf, T., Fischer, H., Reul, H., and Rau, G., Cavitation potential of mechanical heart valve prostheses. Int J Artif Organs. 14(3): p. 169-74, 1991.

63. Gregoric, I.D., Frazier, O.H., Tamez, D., Tuzun, E., Shah, N.L., Clubb, F.J., Chee, H.K., Eya, K., Byler, D., Conger, J., Cervera, R., and Kadipasaoglu, K.A., Thrombogenicity of mechanical aortic valves in an animal model: site specific testing is crucial. Asaio J. 50(4): p. 376-80, 2004.

64. Gross, J.M., Shu, M.C., Dai, F.F., Ellis, J., and Yoganathan, A.P., A microstructural flow analysis within a bileaflet mechanical heart valve hinge. $J$ Heart Valve Dis. 5(6): p. 581-90, 1996.

65. Guezennec, Y.G., Brodkey, R.S., Trigui, N., and Kent, J.C., Algorithms for fully automated three-dimensional particle tracking velocimetry. Experimental Fluids. 17: p. 209-19, 1994.

66. Guyton, A., Textbook of Medical Physiology. 1991, Philadelphia, PA: WB Sanders Company.

67. Hashimoto, K., Arai, T., Takeuchi, N., Sasaki, T., Higashi, S., Onoguchi, K., Tanaka, K., and Shibata, T., Valvular (external hinge) leakage of the CarboMedics mitral valve. J Thorac Cardiovasc Surg. 111(1): p. 277-8, 1996.

68. He, Z., Xi, B., Zhu, K., and Hwang, N.H., Mechanisms of mechanical heart valve cavitation: investigation using a tilting disk valve model. J Heart Valve Dis. 10(5): p. 666-74, 2001. 
69. Healy, T.M., Ellis, J.T., Fontaine, A.A., Jarrett, C.A., and Yoganathan, A.P., An automated method for analysis and visualization of laser Doppler velocimetry data. Ann Biomed Eng. 25(2): p. 335-43, 1997.

70. Healy, T.M., Fontaine, A.A., Ellis, J.T., Walton, S., and Yoganathan, A.P., Visualization of the hinge flow in a 5:1 scaled model of the Medtronic ParallelTM bileaflet heart valve prosthesis. Exp. Fluids. (25): p. 512-518, 1998.

71. Herold, M., Lo, H.B., Reul, H., Muckter, H., Taguchi, K., Giesiepen, M., Birkle, G., Hollweg, G., Rau, G., and Messmer, B.J., The Helmoltz-institute-tri-leafletpolyurethane-heart valve prosthesis: design, manufacturing and first in-vitro and in vivo results, in Polyurethanes in Biomedical Engineering II, Planck, H.e.a., Editor. 1987, Elsevier Science Publishers. p. 231-268.

72. Hessel, E.A., 2nd, Anderson, H.N., and Merendino, K.A., Evaluation of materials for use as prosthetic heart valve leaflets in a simple biologic model. Ann Surg. 164(6): p. 1013-20, 1966.

73. Hidgon, A., Ohlsen, E., Stiles, W., Weese, J., and Riley, W., Mechanics of materials, fourth edition. 1985, New York: John Wiley and Sons.

74. Hufnagel, C.A., Gillespie, J.F., Conrad, P.W., Mercier, C., and Evangelist, F.A., Cardiac valve replacement-its current status. Med Ann Dist Columbia. 35(9): p. 457-62, 1966.

75. Hung, T.C., Hochmuth, R.M., Joist, J.H., and Sutera, S.P., Shear-induced aggregation and lysis of platelets. Trans Am Soc Artif Intern Organs. 22: p. 28591, 1976.

76. Hyde, J.A., Chinn, J.A., and Phillips, R.E., Jr., Polymer heart valves. J Heart Valve Dis. 8(3): p. 331-9, 1999.

77. Hylen, J.C., Mechanical malfunction and thrombosis of prosthetic heart valves. Am J Cardiol. 30(4): p. 396-404, 1972.

78. Jamieson, W.R., Burr, L.H., Anderson, W.N., Jr., Chambers, J.B., Gams, J.P., and Dowd, C.M., Prosthesis-related complications: first-year annual rates. J Heart Valve Dis. 11(6): p. 758-63, 2002.

79. Jansen, J., Willeke, S., Reiners, B., Harbott, P., Reul, H., Lo, H.B., Dabritz, S., Rosenbaum, C., Bitter, A., and Ziehe, K., Advances in design principle and fluid dynamics of a flexible polymeric heart valve. ASAIO Trans. 37(3): p. M451-3, 1991. 
80. Joist, J.H., Bauman, J.E., and Sutera, S.P., Platelet adhesion and aggregation in pulsatile shear flow: effects of red blood cells. Thromb Res. 92(6 Suppl 2): p. S47-52, 1998.

81. Keane, R.D. and Adrian, R.J., Optimisation of particle image velocimetry. Measurement Science Technology. 2: p. 1202-1215, 1990.

82. Keggen, L.A., Black, M.M., Lawford, P.V., Hose, D.R., and Strachan, J.R., The use of enzyme activated milk for in vitro simulation of prosthetic valve thrombosis. J Heart Valve Dis. 5(1): p. 74-83, 1996.

83. Kleine, P., Perthel, M., Hasenkam, J.M., Nygaard, H., Hansen, S.B., and Laas, J., Downstream turbulence and high intensity transient signals (HITS) following aortic valve replacement with Medtronic Hall or St. Jude Medical valve substitutes. Eur J Cardiothorac Surg. 17(1): p. 20-4, 2000.

84. Korossis, S.A., Fisher, J., and Ingham, E., Cardiac valve replacement: a bioengineering approach. Biomed Mater Eng. 10(2): p. 83-124, 2000.

85. Laas, J., Kseibi, S., Perthel, M., Klingbeil, A., El-Ayoubi, L., and Alken, A., Impact of high intensity transient signals on the choice of mechanical aortic valve substitutes. Eur J Cardiothorac Surg. 23(1): p. 93-6, 2003.

86. Lawson, N.J. and Wu, J., Three-dimensional particle image velocimetry: experimental error analysis of a digital angular stereoscopic system. Measurement Science Technology. 8: p. 1455-1464, 1997.

87. Leat, M.E. and Fisher, J., The influence of manufacturing methods on the function and performance of a synthetic leaflet heart valve. Proc Inst Mech Eng [H]. 209(1): p. 65-9, 1995.

88. Leo, H.L., He, Z., Ellis, J.T., and Yoganathan, A.P., Microflow fields in the hinge region of the CarboMedics bileaflet mechanical heart valve design. $J$ Thorac Cardiovasc Surg. 124(3): p. 561-74, 2002.

89. Lievense, A.M., Bakker, S.L., Dippel, D.W., Taams, M.A., Koudstaal, P.J., and Bogers, A.J., Intracranial high-intensity transient signals after homograft or mechanical aortic valve replacement. J Cardiovasc Surg (Torino). 39(5): p. 613-7, 1998.

90. Liu, J.S., Lu, P.C., and Chu, S.H., Turbulence characteristics downstream of bileaflet aortic valve prostheses. J Biomech Eng. 122(2): p. 118-24, 2000.

91. Lu, P.C., Lai, H.C., and Liu, J.S., A reevaluation and discussion on the threshold limit for hemolysis in a turbulent shear flow. J Biomech. 34(10): p. 1361-4, 2001. 
92. Mackay, T.G., Wheatley, D.J., Bernacca, G.M., Fisher, A.C., and Hindle, C.S., New polyurethane heart valve prosthesis: design, manufacture and evaluation. Biomaterials. 17(19): p. 1857-63, 1996.

93. Makhijani, V.B., Siegel, J.M., Jr., and Hwang, N.H., Numerical study of squeezeflow in tilting disc mechanical heart valves. J Heart Valve Dis. 5(1): p. 97-103, 1996.

94. Meyer, R.S., Deutsch, S., Bachmann, C.B., and Tarbell, J.M., Laser Doppler velocimetry and flow visualization studies in the regurgitant leakage flow region of three mechanical mitral valves. Artif Organs. 25(4): p. 292-9, 2001.

95. Mori, H., Hessel, E.A., 2nd, Nelson, R.J., Anderson, H.N., Dillard, D.H., and Merendino, K.A., Design and durability test of Silastic trileaflet aortic valve prostheses. J Thorac Cardiovasc Surg. 65(4): p. 576-82, 1973.

96. Nemerson, Y. and Turitto, V.T., The effect of flow on hemostasis and thrombosis. Thromb Haemost. 66(3): p. 272-6, 1991.

97. Okazaki, Y., Furukawa, K., Higuchi, S., and Itoh, T., Thrombus at the pivot of a mechanical heart valve. Ann Thorac Surg. 75(6): p. 1978, 2003.

98. Raco, L., Belcher, P.R., Sim, I., McGarrity, A., Bernacca, G.M., and Wheatley, D.J., Platelet aggregation and high-intensity transient signals (HITS) in a sheep model of mitral valve replacement. J Heart Valve Dis. 8(5): p. 476-80; discussion 481, 1999.

99. Rambod, E., Beizaie, M., Shusser, M., Milo, S., and Gharib, M., A physical model describing the mechanism for formation of gas microbubbles in patients with mitral mechanical heart valves. Ann Biomed Eng. 27(6): p. 774-92, 1999.

100. Ramstack, J.M., Zuckerman, L., and Mockros, L.F., Shear-induced activation of platelets. J Biomech. 12(2): p. 113-25, 1979.

101. Rashtian, M.Y., Stevenson, D.M., Allen, D.T., Yoganathan, A.P., Harrison, E.C., Edmiston, W.A., Faughan, P., and Rahimtoola, S.H., Flow characteristics of four commonly used mechanical heart valves. Am J Cardiol. 58(9): p. 743-52, 1986.

102. Roe, B.B., Owsley, J.W., and Boudoures, P.C., Experimental results with a prosthetic aortic valve. J Thorac Surg. 36(4): p. 563-70, 1958.

103. Roe, B.B., Kelly, P.B., Jr., Myers, J.L., and Moore, D.W., Tricuspid leaflet aortic valve prosthesis. Circulation. 33(4 Suppl): p. I124-30, 1966. 
104. Roudaut, R., Roques, X., Lafitte, S., Choukroun, E., Laborde, N., Madona, F., Deville, C., and Baudet, E., Surgery for prosthetic valve obstruction. A single center study of 136 patients. Eur J Cardiothorac Surg. 24(6): p. 868-72, 2003.

105. Sallam, A.M. and Hwang, N.H., Human red blood cell hemolysis in a turbulent shear flow: contribution of Reynolds shear stresses. Biorheology. 21(6): p. 783-97, 1984.

106. Saxena, R., Lemmon, J., Ellis, J., and Yoganathan, A., An in vitro assessment by means of laser Doppler velocimetry of the medtronic advantage bileaflet mechanical heart valve hinge flow. J Thorac Cardiovasc Surg. 126(1): p. 90-8, 2003.

107. Schneck, D., An Outline of Cardiovascular Structure and Function. The Biomedical Engineering Handbook., Bronzino, J., Editor. 1995, CRC Press: Boca Raton, FL. p. 3-14.

108. Schoen, F.J., Role of device retrieval and analysis in the evaluation of substitute heart valves, in Clinical Evaluation of Medical Devices, Witken, K.B., Editor. 1988, Humana Press Inc: New Jersey. p. 23.

109. Schoephoerster, R.T. and Chandran, K.B., Velocity and turbulence measurements past mitral valve prostheses in a model left ventricle. J Biomech. 24(7): p. 549-62, 1991.

110. Seifalian, A.M., Salacinski, H.J., Tiwari, A., Edwards, A., Bowald, S., and Hamilton, G., In vivo biostability of a poly(carbonate-urea)urethane graft. Biomaterials. 24(14): p. 2549-57, 2003.

111. Shipkowitz, T., Rodgers, V.G., Frazin, L.J., and Chandran, K.B., Numerical study on the effect of secondary flow in the human aorta on local shear stresses in abdominal aortic branches. J Biomech. 33(6): p. 717-28, 2000.

112. Shu, M.C., Gross, J.M., O'Rourke, K.K., and Yoganathan, A.P., An integrated macro/micro approach to evaluating pivot flow within the Medtronic ADVANTAGE bileaflet mechanical heart valve. J Heart Valve Dis. 12(4): p. 503$12,2003$.

113. Shu, M.C., O'Rourke, K.K., Coppin, C.M., and Lemmon, J.D., Flow characterization of the ADVANTAGE and St. Jude Medical bileaflet mechanical heart valves. J Heart Valve Dis. 13(5): p. 814-22, 2004.

114. Simon, H., Influence of the implant location on the hinge and leakage flow fields through bileaflet mechanical heart valves, in Chemical Engineering Department. 2004, Georgia Institute of Technology: Atlanta, GA. p. 243. 
115. Slack, S.M., Cui, Y., and Turitto, V.T., The effects of flow on blood coagulation and thrombosis. Thromb Haemost. 70(1): p. 129-34, 1993.

116. Slack, S.M., Jennings, L.K., and Turitto, V.T., Platelet size distribution measurements as indicators of shear stress-induced platelet aggregation. Ann Biomed Eng. 22(6): p. 653-9, 1994a.

117. Slack, S.M. and Turitto, V.T., Flow chambers and their standardization for use in studies of thrombosis. On behalf of the Subcommittee on Rheology of the Scientific and Standardization Committee of the ISTH. Thromb Haemost. 72(5): p. 777-81, 1994b.

118. Smith, R.L., Blick, E.F., Coalson, J., and Stein, P.D., Thrombus production by turbulence. J Appl Physiol. 32(2): p. 261-4, 1972.

119. Starek, P.J., Immobilization of disc heart valves by unraveled sutures. Ann Thorac Surg. 31(1): p. 66-9, 1981.

120. Starr, A., Fessler, C.L., Grunkemeier, G., and He, G.W., Heart valve replacement surgery: past, present and future. Clin Exp Pharmacol Physiol. 29(8): p. 735-8, 2002.

121. Steegers, A., Paul, R., Reul, H., and Rau, G., Leakage flow at mechanical heart valve prostheses: improved washout or increased blood damage? J Heart Valve Dis. 8(3): p. 312-23, 1999.

122. Sutera, S.P., Nowak, M.D., Joist, J.H., Zeffren, D.J., and Bauman, J.E., A programmable, computer-controlled cone-plate viscometer for the application of pulsatile shear stress to platelet suspensions. Biorheology. 25(3): p. 449-59, 1988.

123. Szabo, S. and Kecke, H.J., Experimental investigation of the velocity field in a turbomachine passage by using Laser Doppler Velocimetry (LDV). Technisches Messen. 68(3): p. 131-139, 2001.

124. Thubrikar, M.J., Deck, J.D., Aouad, J., and Nolan, S.P., Role of mechanical stress in calcification of aortic bioprosthetic valves. J Thorac Cardiovasc Surg. 86(1): p. 115-25, 1983.

125. Tiederman, W.G., Privette, R.M., and Phillips, W.M., Cycle-to-Cycle variation effects on turbulent shear stress measurment in pulsatile flows. Experiments in Fluids. 6: p. 265-272, 1988.

126. Travis, B.R., Marzec, U.M., Leo, H.L., Momin, T., Sanders, C., Hanson, S.R., and Yoganathan, A.P., Bileaflet aortic valve prosthesis pivot geometry influences platelet secretion and anionic phospholipid exposure. Ann Biomed Eng. 29(8): p. 657-64, 2001a. 
127. Travis, B.R., Marzec, U.M., Ellis, J.T., Davoodi, P., Momin, T., Hanson, S.R., Harker, L.A., and Yoganathan, A.P., The sensitivity of indicators of thrombosis initiation to a bileaflet prosthesis leakage stimulus. J Heart Valve Dis. 10(2): p. 228-38, 2001b.

128. Travis, B.R., The effects of bileaflet prosthesis pivot geometry on turbulence and blood damage potential, in Chemcial Engineering Department. 2001c, Georgia Institute of Technology: Atlanta, GA. p. 352.

129. Turitto, V.T. and Hall, C.L., Mechanical factors affecting hemostasis and thrombosis. Thromb Res. 92(6 Suppl 2): p. S25-31, 1998.

130. Vongpatanasin, W., Hillis, L.D., and Lange, R.A., Prosthetic heart valves. $N$ Engl J Med. 335(6): p. 407-16, 1996.

131. Vyavahare, N., Ogle, M., Schoen, F.J., Zand, R., Gloeckner, D.C., Sacks, M., and Levy, R.J., Mechanisms of bioprosthetic heart valve failure: fatigue causes collagen denaturation and glycosaminoglycan loss. J Biomed Mater Res. 46(1): p. 44-50, 1999.

132. Walker, P.G. and Yoganathan, A.P., In vitro pulsatile flow hemodynamics of five mechanical aortic heart valve prostheses. Eur J Cardiothorac Surg. 6 Suppl 1: p. S113-23, 1992.

133. Wheatley, D.J., Raco, L., Bernacca, G.M., Sim, I., Belcher, P.R., and Boyd, J.S., Polyurethane: material for the next generation of heart valve prostheses? Eur J Cardiothorac Surg. 17(4): p. 440-8, 2000.

134. Wheatley, D.J., Bernacca, G.M., Tolland, M.M., O'Connor, B., Fisher, J., and Williams, D.F., Hydrodynamic function of a biostable polyurethane flexible heart valve after six months in sheep. Int J Artif Organs. 24(2): p. 95-101, 2001.

135. Wieneke, B. Stereo-PIV using self-calibration on particle images. in 5th International symposium on PIV. 2003. Busan, Korea.

136. Williams, A.R., Shear-induced fragmentation of human erythrocytes. Biorheology. 10(3): p. 303-11, 1973.

137. Wisman, C.B., Pierce, W.S., Donachy, J.H., Pae, W.E., Myers, J.L., and Prophet, G.A., A polyurethane trileaflet cardiac valve prosthesis: in vitro and in vivo studies. Trans Am Soc Artif Intern Organs. 28: p. 164-8, 1982.

138. Woo, Y.R., Williams, F.P., and Yoganathan, A.P., In-vitro fluid dynamic characteristics of the abiomed trileaflet heart valve prosthesis. $J$ Biomech Eng. 105(4): p. 338-45, 1983a. 
139. Woo, Y.R., Williams, F.P., and Yoganathan, A.P., Steady and pulsatile flow studies on a trileaflet heart valve prosthesis. Scand J Thorac Cardiovasc Surg. 17(3): p. 227-36, $1983 b$.

140. Woo, Y.R. and Yoganathan, A.P., Two-component laser Doppler anemometer for measurement of velocity and turbulent shear stress near prosthetic heart valves. Med Instrum. 19(5): p. 224-31, 1985.

141. Yoganathan, A.P., Woo, Y.R., Williams, F.P., Stevenson, D.M., Franch, R.H., and Harrison, E.C., In vitro fluid dynamic characteristics of Ionescu-Shiley and Carpentier-Edwards tissue bioprostheses. Artif Organs. 7(4): p. 459-69, 1983.

142. Yoganathan, A.P., Chaux, A., Gray, R.J., Woo, Y.R., DeRobertis, M., Williams, F.P., and Matloff, J.M., Bileaflet, tilting disc and porcine aortic valve substitutes: in vitro hydrodynamic characteristics. J Am Coll Cardiol. 3(2 Pt 1): p. 313-20, 1984.

143. Yoganathan, A.P., Woo, Y.R., Sung, H.W., Williams, F.P., Franch, R.H., and Jones, M., In vitro hemodynamic characteristics of tissue bioprostheses in the aortic position. J Thorac Cardiovasc Surg. 92(2): p. 198-209, 1986 .

144. Yoganathan, A.P., Woo, Y.R., and Sung, H.W., Turbulent shear stress measurements in the vicinity of aortic heart valve prostheses. J Biomech. 19(6): p. 433-42, $1986 b$.

145. Yoganathan, A.P., Cardiac Valve Prostheses, in The Biomedical Engineering Handbook. 2000, CRC Press LLC. p. 127-1 - 127-23.

146. Zdrahala, R.J. and Zdrahala, I.J., Biomedical applications of polyurethanes: a review of past promises, present realities, and a vibrant future. J Biomater Appl. 14(1): p. 67-90, 1999.

147. Zhao, J.B., Shi, Y.B., Yeo, T.J., and Hwang, N.H., Digital particle image velocimetry investigation of the pulsating flow around a simplified 2-D model of a bileaflet heart valve. J Heart Valve Dis. 10(2): p. 239-53, 2001. 